Supporting Information

\title{
Enantioselective Rh-catalyzed hydroboration of Silyl Enol Ethers
}

\author{
Wenke Dong, ${ }_{+, *}^{+}$Xin Xu, ${ }^{+, \#}$ Honghui Ma, ${ }^{+}$Yaqin Lei ${ }^{+}$Zhenyang Lin, ${ }^{+}$and Wanxiang Zhao ${ }^{+, *}$ \\ + State Key Laboratory of Chemo/Biosensing and Chemometrics, College of Chemistry and Chemical \\ Engineering, Hunan University, Changsha, Hunan 410082, China. \\ + Department of Chemistry, The Hong Kong University of Science and Technology, Clear Water Bay, \\ Kowloon, Hong Kong, P. R. China. \\ E-mail : zhaowanxiang@hnu.edu.cn.
}

\section{Table of Contents}

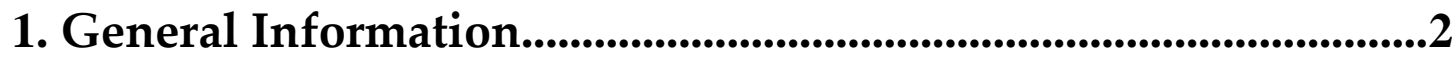

2. Ligand Screening ..........................................................................................3

3. Ligand Synthesis.......................................................................................................4

4. Synthesis of Starting Materials.....................................................6

5. Asymmetric Hydroboration of Silyl Enol Ethers .........................34

6. Control Experiments .........................................................................................101

7. Gram-scale Reaction and Applications .............................................103

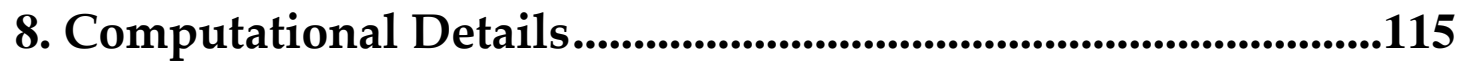

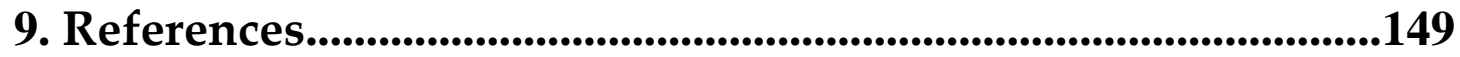

10. NMR Spectra......................................................................................152 


\section{General Information}

Unless otherwise noted, all reactions were conducted in oven-dried vials with a magnetic stir bar under nitrogen atmosphere. Solvents were purified under nitrogen using a solvent purification system. Analytical thin layer chromatography (TLC) was performed using silica gel plates. Visualisation was by ultraviolet fluorescence, and/or phosphomolybdic acid, and/or $\mathrm{KMnO}_{4}$. Flash column chromatography was performed using EM Science (200-300 mesh) silica gel.

${ }^{1} \mathrm{H}-$ Nuclear Magnetic Resonance $\left({ }^{1} \mathrm{H} \mathrm{NMR}\right)$ and ${ }^{13} \mathrm{C}$ Nuclear Magnetic Resonance $\left({ }^{13} \mathrm{C}\right.$ NMR) spectra were recorded on Bruker $400 \mathrm{MHz}$ at $20{ }^{\circ} \mathrm{C}$ with $\mathrm{CDCl}_{3}$ as solvent, and were reported in ppm referenced to tetramethylsilane $(\delta=$ $0 \mathrm{ppm}$ for $\left.{ }^{1} \mathrm{H}-\mathrm{NMR}\right)$ and deuteriochloroform $\left(\delta=77.16 \mathrm{ppm}\right.$ for $\left.{ }^{13} \mathrm{C}-\mathrm{NMR}\right)$. The data are reported as follows: chemical shift (ppm), multiplicity ( $\mathrm{s}=$ singlet, $\mathrm{d}=$ doublet, $\mathrm{dd}=$ doublet of doublet, $\mathrm{t}=$ triplet, $\mathrm{m}=$ multiplet, $\mathrm{m}_{\mathrm{c}}=$ centered multiplet, br $=$ broad), coupling constant $J(\mathrm{~Hz})$, and integration. High resolution mass spectra were recorded on a Bruker Maxis System using Electron Spray Ionization (ESI). IR spectra were collected on a Spectrum QATR-S from SHIMADZU and reported in unit of $\mathrm{cm}^{-1}$.

The optical rotation of chiral compounds was determined on a Jasco P-1010 apparatus and transformed to the specific optical rotation for a given temperature according to the following formula:

$$
[\alpha]_{D}{ }^{T}=\frac{\alpha * 100}{c * d}
$$

$$
\begin{array}{ll}
\mathrm{T}=\text { temperature }\left[{ }^{\circ} \mathrm{C}\right] & d=\text { length of cuvette }[\mathrm{dm}] \\
a=\text { measured value for optical rotation }\left[{ }^{\circ}\right] & c=\text { concentration }[\mathrm{g} / 100 \mathrm{~mL}]
\end{array}
$$

Chiral HPLC (High Pressure Liquid Chromatography) was performed on an Agilent HPLC apparatus with columns (Chiralpak AD-H, IC, IA, AS-H; Chiralcel OD-H, $250 \mathrm{~mm} \times 4.6 \mathrm{~mm}$ ).

Ligands, bases, pinacolborane (HBpin), and aryl acetic acids were purchased from Energy Chemicals Inc. or Macklin Inc. and used as received. $[\mathrm{RhCl}(\mathrm{cod})]_{2}$ was prepared according to literature procedure and all characterization data are in accordance with the literature ${ }^{1}$. 


\section{Ligand Screening}

Table S1. Ligand Screening for Aryl Silyl Enol Ethera.

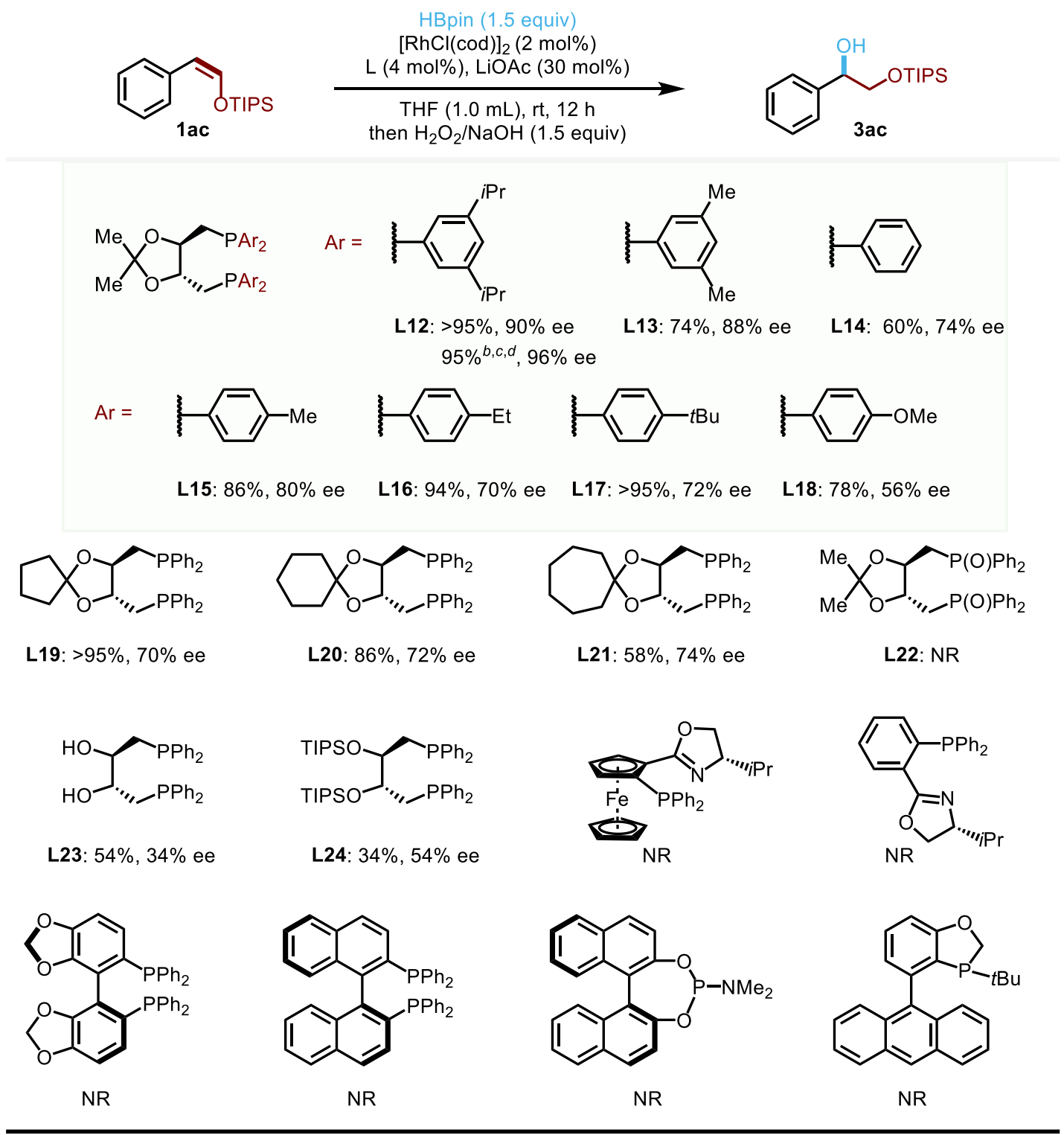

aReactions conditions: 1ac (0.1 mmol), HBpin (1.5 equiv), [ $\mathrm{RhCl}(\mathrm{cod})]_{2}(2 \mathrm{~mol} \%)$, L (4 mol\%), LiOAc (30 mol\%), THF (0.5 mL), rt, 12 h, ${ }^{1} \mathrm{H}$ NMR yield. Enantiomeric ratios were determined by chiral HPLC. ${ }^{b} \mathrm{~T}=-20{ }^{\circ} \mathrm{C}$. ${ }^{c}$ Toluene as solvent. ${ }^{d}$ Isolated yield for 2 steps. 


\section{Ligand Synthesis}
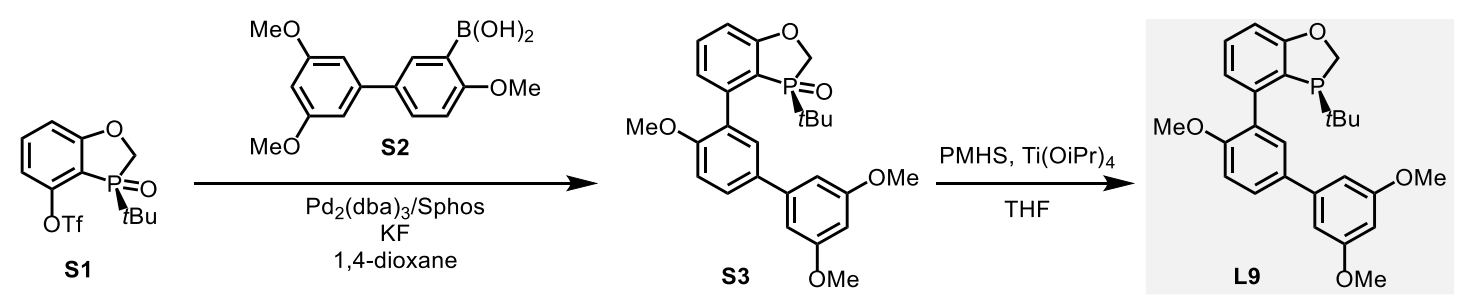

Following the literature procedure ${ }^{2}$, to a sealed tube were added S1 (0.68 g, $2.00 \mathrm{mmol}), \mathrm{S2}^{3}$ (1.16 g, $\left.4.00 \mathrm{mmol}\right), \mathrm{Pd}_{2}(\mathrm{dba})_{3}(55.0 \mathrm{mg}, 0.06 \mathrm{mmol}$ ), S-Phos $(164.0 \mathrm{mg}, 0.40 \mathrm{mmol})$ and KF $(0.46 \mathrm{~g}, 8.00 \mathrm{mmol})$, followed by the addition of 1,4-dioxane. The resulting mixture was then heated to $100{ }^{\circ} \mathrm{C}$ and stirred for 24 $h$, then cooled to room temperature, extracted with EtOAc, dried with $\mathrm{Na}_{2} \mathrm{SO}_{4}$, filtered and concentrated. The residue was purified by silica gel flash chromatography to afford compound S3 as a white solid in 75\% yield $(0.68 \mathrm{~g}$, eluent: EtOAc).

To a solution of S3 $(0.68 \mathrm{~g}, 1.50 \mathrm{mmol})$ in THF was added PMHS (3.5 mL) and $\mathrm{Ti}(\mathrm{OiPr})_{4}(1.99 \mathrm{~g}, 7.00 \mathrm{mmol})$ at room temperature. The resulting mixture was then heated to $70{ }^{\circ} \mathrm{C}$ and stirred for $24 \mathrm{~h}$, then cooled to room temperature and quenched with degassed $30 \% \mathrm{NaOH}$. The resulting mixture was further stirred at $60{ }^{\circ} \mathrm{C}$ for $0.5 \mathrm{~h}$. The aqueous layer was removed and subsequently extracted with $\mathrm{Et}_{2} \mathrm{O}$ under nitrogen atmosphere. The combined organicphase were dried with $\mathrm{Na}_{2} \mathrm{SO}_{4}$ and filtered through nitrogen-purged neutral alumina with $\mathrm{Et}_{2} \mathrm{O}$. The solvents were removed under reduced pressure to afford ligand L9 as a white solid in 62\% yield (0.42 g).

$\mathbf{R}_{f}=0.43$ (petroleum ether/EtOAc $=10 / 1$ )

${ }^{1} \mathrm{H}$ NMR (400 MHz, C6 $\left.\mathrm{D}_{6}\right) \delta 8.15(\mathrm{~s}, 1 \mathrm{H}), 7.61(\mathrm{~d}, J=8.5 \mathrm{~Hz}, 1 \mathrm{H}), 7.28-7.19(\mathrm{~m}$, 2H), $7.10(\mathrm{~s}, 2 \mathrm{H}), 7.06-7.00(\mathrm{~m}, 1 \mathrm{H}), 6.77(\mathrm{~d}, J=8.5 \mathrm{~Hz}, 1 \mathrm{H}), 6.64(\mathrm{~s}, 1 \mathrm{H}), 4.72$ $4.61(\mathrm{~m}, 1 \mathrm{H}), 4.50-4.33(\mathrm{~m}, 1 \mathrm{H}), 3.55-3.36(\mathrm{~m}, 9 \mathrm{H}), 0.77(\mathrm{~d}, J=11.9 \mathrm{~Hz}, 9 \mathrm{H})$.

${ }^{13} \mathrm{C}$ NMR (100 MHz, C6 $\left.\mathrm{D}_{6}\right) \delta 164.6,161.9,156.7,143.5,142.9$ (d, J = 15.4 Hz), 134.0, 132.0, $131.8(\mathrm{~d}, J=5.1 \mathrm{~Hz}), 130.9,127.7,124.3(\mathrm{~d}, J=15.7 \mathrm{~Hz}), 123.8(\mathrm{~d}, J=3.4 \mathrm{~Hz})$, 111.7, 110.3, 105.7, 99.4, $70.1(\mathrm{~d}, J=27.5 \mathrm{~Hz}), 55.2,55.0,31.5(\mathrm{~d}, J=20.5 \mathrm{~Hz}), 26.8$ $(\mathrm{d}, J=14.3 \mathrm{~Hz})$.

${ }^{31 P}$ NMR (162 MHz, $\left.\mathrm{C}_{6} \mathrm{D}_{6}\right) \delta-10.2$.

HRMS (ESI ${ }^{+}$) calcd for $\mathrm{C}_{26} \mathrm{H}_{29} \mathrm{NaO}_{4} \mathrm{P}^{+}[\mathrm{M}+\mathrm{Na}]^{+}: 459.1696$, found: 459.1693 . IR (neat, $\mathrm{cm}^{-1}$ ): 2926, 2931, 1515, 1592, 1418, 1235, 1067, 1030, 872, 808. 


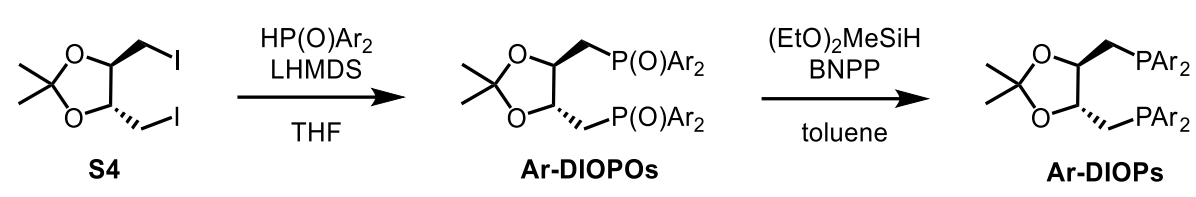

Following a modified procedure ${ }^{4}$, to a solution of diarylphosphine oxide (2.2 equiv) and $(R, R)$-4,5-bis(iodomethyl)-2,2-dimethyl-1,3-dioxolane ( $\mathrm{S} 4)^{4}(1.0$ equiv) in THF was added LHMDS dropwise (1.0 M in THF, 2.0 equiv) at $0{ }^{\circ} \mathrm{C}$. The resulting mixture was then warmed up to room temperature and stirred for $15 \mathrm{~h}$, then quenched with saturated $\mathrm{NH}_{4} \mathrm{Cl}$, extracted with EtOAc, dried with $\mathrm{Na}_{2} \mathrm{SO}_{4}$, filtered and concentrated. The residue was purified by silica gel flash chromatography to afford the corresponding Ar-DIOPOs.

Following the literature procedure ${ }^{5}$, to a solution of bis(4nitrophenyl)hydrogen phosphate (BNPP, 0.3 equiv) and Ar-DIOPOs (1.0 equiv) in toluene was added (EtO) ${ }_{2} \mathrm{MeSiH}$ (8.0 equiv) at room temperature. The resulting mixture was then heated to $110^{\circ} \mathrm{C}$ and stirred for $12 \mathrm{~h}$. After cooled to room temperature, the reaction mixture was quenched with $\mathrm{H}_{2} \mathrm{O}$, extracted with EtOAc, dried with $\mathrm{Na}_{2} \mathrm{SO}_{4}$, filtered and concentrated. The residue was purified by silica gel flash chromatography to afford the corresponding ArDIOPs.

The data of $(R, R)-4-\mathrm{Me}-\mathrm{DIOP}^{6},(R, R)-4-\mathrm{OMe}^{\mathrm{DIOP}}{ }^{7},(R, R)-4-\mathrm{tBu}-\mathrm{DIOP}{ }^{6}$, $(R, R)-3,5-\mathrm{Me}_{2}-\mathrm{DIOP}^{7}, \quad(R, R)-\mathrm{Cp}-\mathrm{DIOP}{ }^{7}, \quad(R, R)-\mathrm{Cy}^{-\mathrm{DIOP}}{ }^{7}, \quad(R, R)$-Chept-DIOP${ }^{7}$, $(R, R)-4$-Et-DIOP ${ }^{6}$ are consistent with the literatures.

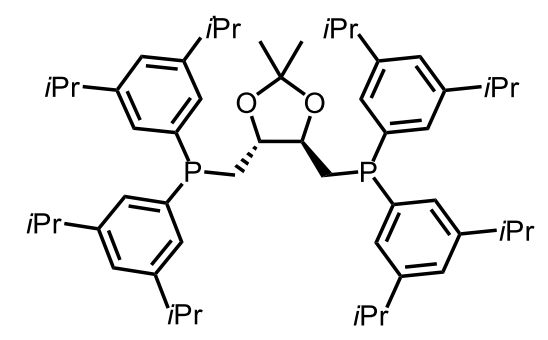

Ligand L12 was prepared as a colorless oil in 25\% yield (2 steps) following the above procedure.

$\mathbf{R}_{f}=0.65$ (petroleum ether $/$ EtOAc $=10 / 1$ )

${ }^{1} \mathrm{H}$ NMR (400 MHz, $\left.\mathrm{CDCl}_{3}\right) \delta$ 8.20-7.15 (m, 4H), 7.14-7.08 (m, 4H), 7.04-6.96 (m, $4 \mathrm{H}), 3.91-3.80(\mathrm{~m}, 2 \mathrm{H}), 2.93-2.74(\mathrm{~m}, 8 \mathrm{H}), 2.50-2.38(\mathrm{~m}, 2 \mathrm{H}), 2.34-2.24(\mathrm{~m}, 2 \mathrm{H})$, $1.36(\mathrm{~s}, 6 \mathrm{H}), 1.23-1.15(\mathrm{~m}, 48 \mathrm{H})$.

${ }^{13} \mathrm{C}$ NMR (100 MHz, $\mathrm{CDCl}_{3}$ ) $\delta 148.8$ (dd, $\left.J=13.6,6.9 \mathrm{~Hz}\right), 138.0(\mathrm{dd}, J=85.4,11.7$ 
$\mathrm{Hz}), 128.6(\mathrm{dd}, J=47.9,19.4 \mathrm{~Hz}), 125.3(\mathrm{~m}), 108.7,80.1(\mathrm{~m}), 34.4(\mathrm{~m}), 32.8(\mathrm{~m})$, 27.5, $24.1(\mathrm{~m})$.

${ }^{31} \mathrm{P}$ NMR (162 MHz, $\left.\mathrm{CDCl}_{3}\right) \delta$-21.9.

HRMS (ESI ${ }^{+}$) calcd for $\mathrm{C}_{55} \mathrm{H}_{81} \mathrm{O}_{2} \mathrm{P}_{2}{ }^{+}[\mathrm{M}+\mathrm{H}]^{+}:$835.5706, found: 835.5705.

IR (neat, $\mathrm{cm}^{-1}$ ): 2959, 2931, 2870, 1592, 1464, 1102, 1067, 1040, 872, 710.

\section{Synthesis of Starting Materials}

\section{General Procedure A}

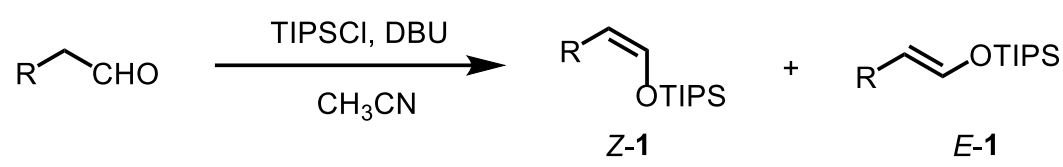

Following the literature procedure ${ }^{8}$, to a solution of the aldehyde (1.0 equiv) and TIPSCl (1.2 equiv) in $\mathrm{CH}_{3} \mathrm{CN}$ was added $\mathrm{DBU}$ (1.2 equiv) at $0{ }^{\circ} \mathrm{C}$. The resulting mixture was then heated to $65^{\circ} \mathrm{C}$ and stirred for $12 \mathrm{~h}$. After cooled to room temperature, the reaction mixture was quenched with $\mathrm{H}_{2} \mathrm{O}$ at room temperature, extracted with EtOAc, dried with $\mathrm{Na}_{2} \mathrm{SO}_{4}$, filtered and concentrated. The residue was purified by silica gel flash chromatography to afford pure $(Z)$ - and $(E)$-silyl enol ethers.

\section{General Procedure B}

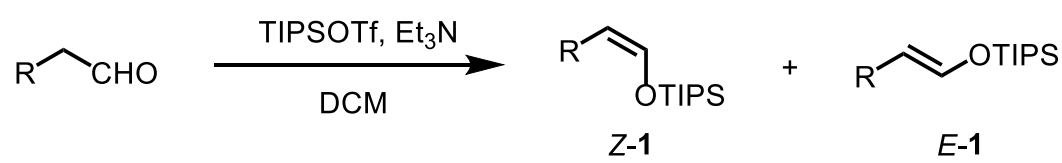

Following the literature procedure ${ }^{8}$, to a solution of the corresponding aldehyde (1.0 equiv), Et $3 \mathrm{~N}$ (2.0 equiv) in DCM was added TIPSOTf (1.2 equiv) at $0{ }^{\circ} \mathrm{C}$. The resulting mixture was then stirred for $5 \mathrm{~h}$, then quenched with $\mathrm{H}_{2} \mathrm{O}$ at room temperature, extracted with EtOAc, dried with $\mathrm{Na}_{2} \mathrm{SO}_{4}$, filtered and concentrated. The residue was purified by silica gel flash chromatography to afford pure $(Z)$ - and (E)-silyl enol ethers.

\section{General Procedure C}




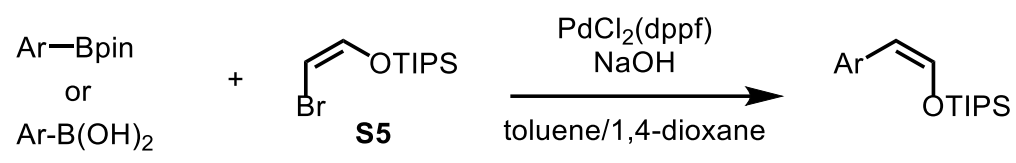

To a solution of aryl boronic acid (or ester, 2.0 equiv), (Z)-((2bromovinyl)oxy)triisopropylsilane (S5) ${ }^{9}$ (1.0 equiv), and $\mathrm{PdCl}_{2}$ (dppf) $(1.5 \mathrm{~mol} \%)$ in toluene/1,4-dioxane (1:1) was added 3.0 $\mathrm{M} \mathrm{NaOH}$ solution (3.0 equiv) at room temperature. The resulting mixture was then heated to $70{ }^{\circ} \mathrm{C}$ and stirred for $12 \mathrm{~h}$. After cooled to room temperature, the reaction mixture was quenched with $\mathrm{H}_{2} \mathrm{O}$ at room temperature, extracted with EtOAc, dried with $\mathrm{Na}_{2} \mathrm{SO}_{4}$, filtered and concentrated. The residue was purified by silica gel flash chromatography to afford pure (Z)-silyl enol ethers.

\section{General Procedure D:}

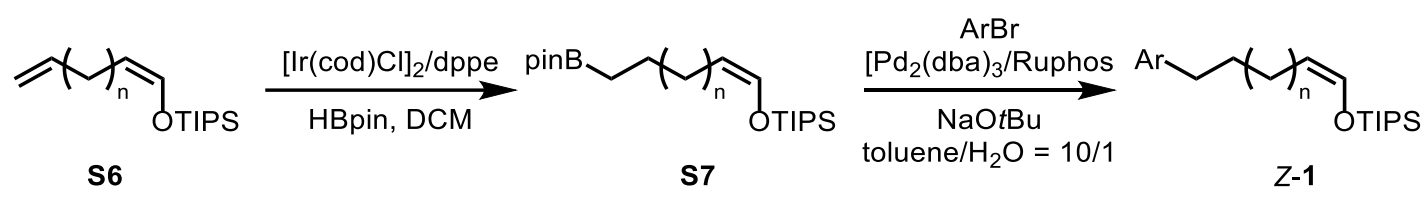

Following the literature procedure ${ }^{10}$, to a solution of $[\operatorname{Ir}(\mathrm{cod}) \mathrm{Cl}]_{2}(1.0 \mathrm{~mol} \%)$ and dppe $(2.0 \mathrm{~mol} \%)$ in DCM was added pinacolborane (1.2 equiv) and $\mathbf{S 6}$ (synthesized via General procedure B, 1.0 equiv) at room temperature. The resulting mixture was then stirred for $24 \mathrm{~h}$ at room temperature, then quenched with $\mathrm{H}_{2} \mathrm{O}$ at room temperature, extracted with EtOAc, dried with $\mathrm{Na}_{2} \mathrm{SO}_{4}$, filtered and concentrated. The residue was purified by silica gel flash chromatography to afford the compound S7.

Following the literature procedure ${ }^{11}$, to a solution of $\mathrm{Pd}_{2}(\mathrm{dba})_{3}(2 \mathrm{~mol} \%)$, Ruphos (4 mol\%), $\mathrm{NaO}$ tBu (3.0 equiv) and $\mathrm{ArX}$ (1.0 equiv) in toluene/ $\mathrm{H}_{2} \mathrm{O}$ (10:1) was added $\mathbf{S 7}$ (1.2 equiv) at room temperature. The resulting mixture was then heated to $80^{\circ} \mathrm{C}$ and stirred for $24 \mathrm{~h}$. After cooled to room temperature, the reation mixture was filtered and concentrated. The residue was purified by silica gel flash chromatography to afford ( $Z$ )-silyl enol ethers.

\section{(Z)-Triisopropyl((4-phenylbut-1-en-1-yl)oxy)silane (1a)}

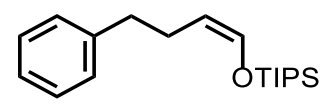

Compound 1a was prepared as a colorless oil in $42 \%$ yield $(0.64 \mathrm{~g}$, eluent: petroleum ether) from 4-phenylbutanal $(0.74 \mathrm{~g}, 5.00 \mathrm{mmol})$, TIPSCl (1.16 g, 6.00 $\mathrm{mmol})$, DBU $(0.91 \mathrm{~g}, 6.00 \mathrm{mmol})$ following the above general procedure $\mathrm{A}$, 
which was previously reported ${ }^{12}$

$\mathbf{R}_{f}=0.80(Z), 0.68(E)$ (petroleum ether)

${ }^{1} \mathrm{H}$ NMR (400 MHz, $\left.\mathrm{CDCl}_{3}\right)$ \& 7.30-7.10 (m, 5H), 6.36-6.20 (m, 1H), 4.51-4.36 (m, $1 \mathrm{H}), 2.71-2.61(\mathrm{~m}, 2 \mathrm{H}), 2.48-2.38(\mathrm{~m}, 2 \mathrm{H}), 1.19-0.97(\mathrm{~m}, 21 \mathrm{H})$.

${ }^{13} \mathrm{C}$ NMR (100 MHz, $\left.\mathrm{CDCl}_{3}\right) \delta$ 142.7, 139.6, 128.6, 128.3, 125.7, 109.0, 36.1, 25.7, $17.9,12.1$.

(Z)-Triisopropyl((5-(4-(trifluoromethyl)phenyl)pent-1-en-1-yl)oxy)silane (1b)<smiles>FC(F)(F)CCCCO/C=C\CCCc1ccc(C(F)(F)F)cc1</smiles>

Compound $1 \mathbf{b}$ was prepared as a colorless oil in $35 \%$ yield $(0.21 \mathrm{~g}$, eluent: petroleum ether) from 5-(4-(trifluoromethyl)phenyl)pentanal (0.36 g, 1.54 $\mathrm{mmol}), \operatorname{TIPSCl}(0.36 \mathrm{~g}, 1.85 \mathrm{mmol}), \mathrm{DBU}(0.13 \mathrm{~g}, 1.85 \mathrm{mmol})$ following the above general procedure A, which was previously reported ${ }^{12}$.

$\mathbf{R}_{f}=0.80(Z), 0.68(E)$ (petroleum ether)

${ }^{1} \mathrm{H}$ NMR (400 MHz, $\left.\mathrm{CDCl}_{3}\right) \delta 7.51(\mathrm{~d}, J=7.8 \mathrm{~Hz}, 2 \mathrm{H}), 7.28(\mathrm{~d}, J=7.8 \mathrm{~Hz}, 2 \mathrm{H})$, $6.31(\mathrm{~d}, J=5.6 \mathrm{~Hz}, 1 \mathrm{H}), 4.48-4.36(\mathrm{~m}, 1 \mathrm{H}), 2.76-2.62(\mathrm{~m}, 2 \mathrm{H}), 2.22-2.09(\mathrm{~m}, 2 \mathrm{H})$, $1.75-1.62(\mathrm{~m}, 2 \mathrm{H}), 1.18-0.99(\mathrm{~m}, 21 \mathrm{H})$.

${ }^{13} \mathrm{C}$ NMR (100 MHz, $\left.\mathrm{CDCl}_{3}\right) \delta$ 147.1, 139.8, 128.9, 128.1 (q, J = 31.9 Hz), 125.3 (q, $J=3.9 \mathrm{~Hz}), 124.6(\mathrm{q}, J=271 \mathrm{~Hz}), 109.0,35.6,31.3,23.3,17.9,12.1$.

${ }^{19} \mathrm{~F}$ NMR $\left(377 \mathrm{MHz}, \mathrm{CDCl}_{3}\right) \delta-62.3$.

(Z)-((5-(4-Fluorophenyl)pent-1-en-1-yl)oxy)triisopropylsilane (1c)

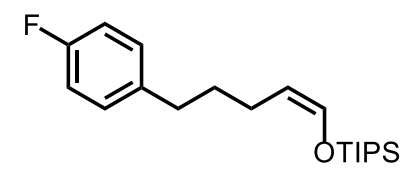

Compound 1c was prepared as a colorless oil in $52 \%$ yield $(0.35 \mathrm{~g}$, eluent: petroleum ether) from 5-(4-fluorophenyl)pentanal $(0.36 \mathrm{~g}, 2.00 \mathrm{mmol}), \mathrm{TIPSCl}$ $(0.46 \mathrm{~g}, 2.40 \mathrm{mmol})$, DBU $(0.36 \mathrm{~g}, 2.40 \mathrm{mmol})$ following the above general procedure $\mathrm{A}$, which was previously reported ${ }^{12}$.

$\mathbf{R}_{f}=0.79(Z), 0.66(E)$ (petroleum ether)

${ }^{1} \mathrm{H}$ NMR (400 MHz, $\left.\mathrm{CDCl}_{3}\right)$ \& 7.16-7.09 (m, 2H), 6.98-6.89 (m, 2H), 6.33-6.25 (m, $1 \mathrm{H}), 4.47-4.36(\mathrm{~m}, 1 \mathrm{H}), 2.63-2.54(\mathrm{~m}, 2 \mathrm{H}), 2.18-2.10(\mathrm{~m}, 2 \mathrm{H}), 1.71-1.59(\mathrm{~m}, 2 \mathrm{H})$, $1.19-0.99(\mathrm{~m}, 21 \mathrm{H})$. 
${ }^{13} \mathrm{C}$ NMR (100 MHz, $\left.\mathrm{CDCl}_{3}\right) \delta 161.25$ (d, $\left.J=242.6 \mathrm{~Hz}\right), 139.59,138.57$ (d, $J=3.2$ $\mathrm{Hz}), 129.86(\mathrm{~d}, J=7.7 \mathrm{~Hz}), 114.99(\mathrm{~d}, J=21.0 \mathrm{~Hz}), 109.33,34.92,31.77,23.29,17.88$, 12.08 .

${ }^{19}$ F NMR (377 MHz, $\left.\mathrm{CDCl}_{3}\right) \delta-118.4$.

(Z)-Triisopropyl((5-(4-methoxyphenyl)pent-1-en-1-yl)oxy)silane (1d)

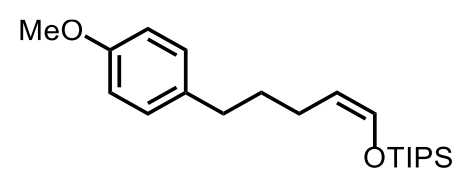

Compound $\mathbf{1 b}$ was prepared as a colorless oil in $72 \%$ yield $(0.25 \mathrm{~g}$, eluent: petroleum ether) from 1-bromo-4-methoxybenzene $(0.19 \mathrm{~g}, 1.00 \mathrm{mmol}),(Z)$ triisopropyl((5-(4,4,5,5-tetramethyl-1,3,2-dioxaborolan-2-yl)pent-1-en-1-

yl)oxy)silane (0.44 g, $1.20 \mathrm{mmol}), \mathrm{Pd}_{2}(\mathrm{dba})_{3}(18.3 \mathrm{mg}, 0.02 \mathrm{mmol})$, Ruphos (18.7 $\mathrm{mg}, 0.04 \mathrm{mmol})$ and $\mathrm{NaOtBu}(0.29 \mathrm{~g}, 3.00 \mathrm{mmol})$ following the above general procedure $\mathrm{D}$, which was previously reported ${ }^{12}$

$\mathbf{R}_{f}=0.45$ (petroleum ether)

${ }^{1} \mathrm{H}$ NMR (400 MHz, $\left.\mathrm{CDCl}_{3}\right) \delta 7.09(\mathrm{~d}, J=7.9 \mathrm{~Hz}, 2 \mathrm{H}), 6.81(\mathrm{~d}, J=7.9 \mathrm{~Hz}, 2 \mathrm{H})$, $6.29(\mathrm{~d}, J=5.8 \mathrm{~Hz}, 1 \mathrm{H}), 4.47-4.36(\mathrm{~m}, 1 \mathrm{H}), 3.78(\mathrm{~s}, 3 \mathrm{H}), 2.56(\mathrm{t}, J=7.8 \mathrm{~Hz}, 2 \mathrm{H})$, 2.19-2.08 (m, 2H), 1.68-1.57 (m, 2H), 1.18-0.99 (m, 21H).

${ }^{13} \mathrm{C}$ NMR (100 MHz, $\left.\mathrm{CDCl}_{3}\right) \delta$ 157.7, 139.4, 135.2, 129.4, 113.8, 109.6, 55.4, 34.9, $31.9,23.4,17.9,12.1$.

\section{(Z)-Triisopropyl((4-(thiophen-2-yl)but-1-en-1-yl)oxy)silane (1g)}

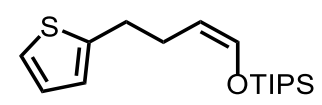

Compound $1 \mathrm{~g}$ was prepared as a colorless oil in 38\% yield (0.64 g, eluent: petroleum ether) from 4-(thiophen-2-yl)butanal (0.84 g, $5.40 \mathrm{mmol})$, TIPSOTf $(1.99 \mathrm{~g}, 6.48 \mathrm{mmol}), \mathrm{Et}_{3} \mathrm{~N}(1.10 \mathrm{~g}, 10.80 \mathrm{mmol})$ following the above general procedure B, which was previously reported ${ }^{12}$.

$\mathbf{R}_{f}=0.85(Z), 0.72(E)$ (petroleum ether)

${ }^{1} \mathrm{H}$ NMR (400 MHz, $\left.\mathrm{CDCl}_{3}\right) \delta 7.09$ (d, $\left.J=5.1 \mathrm{~Hz}, 1 \mathrm{H}\right), 6.95-6.86(\mathrm{~m}, 1 \mathrm{H}), 6.84-$ $6.77(\mathrm{~m}, 1 \mathrm{H}), 6.30(\mathrm{~d}, J=5.8 \mathrm{~Hz}, 1 \mathrm{H}), 4.52-4.41(\mathrm{~m}, 1 \mathrm{H}), 2.88(\mathrm{t}, J=7.8 \mathrm{~Hz}, 2 \mathrm{H})$, 2.57-2.43 (m, 2H), 1.31-0.97 (m, 21H).

${ }^{13} \mathrm{C}$ NMR (100 MHz, $\left.\mathrm{CDCl}_{3}\right) \delta$ 145.6, 140.1, 126.7, 124.1, 122.9, 108.4, 30.1, 26.1, $17.9,12.1$. 


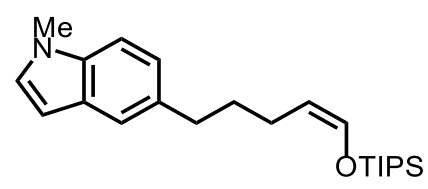

Compound 1h was prepared as a colorless oil in $46 \%$ yield $(0.22 \mathrm{~g}$, eluent: petroleum ether/Et $2 \mathrm{O}=100 / 3)$ from 5-bromo-1-methyl-1H-indole $(0.27 \mathrm{~g}, 1.28$ mmol), (Z)-triisopropyl((5-(4,4,5,5-tetramethyl-1,3,2-dioxaborolan-2-yl)pent-1en-1-yl)oxy)silane (0.57 g, $1.54 \mathrm{mmol}), \mathrm{Pd}_{2}(\mathrm{dba})_{3}(23.4 \mathrm{mg}, 0.026 \mathrm{mmol})$, Ruphos (23.9 $\mathrm{mg}, 0.051 \mathrm{mmol})$ and $\mathrm{NaOtBu}(0.37 \mathrm{~g}, 3.84 \mathrm{mmol})$ following the above general procedure $\mathrm{D}$.

$\mathbf{R}_{f}=0.23$ (petroleum ether)

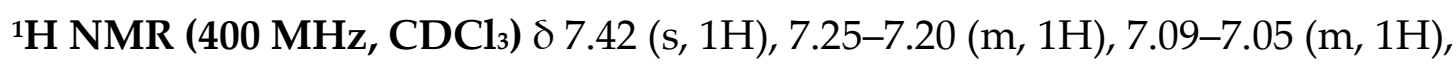
$7.00(\mathrm{~d}, J=3.1 \mathrm{~Hz}, 1 \mathrm{H}), 6.42-6.38(\mathrm{~m}, 1 \mathrm{H}), 6.32-6.28(\mathrm{~m}, 1 \mathrm{H}), 4.50-4.40(\mathrm{~m}, 1 \mathrm{H})$, $3.76(\mathrm{~s}, 3 \mathrm{H}), 2.76-2.67(\mathrm{~m}, 2 \mathrm{H}), 2.23-2.13(\mathrm{~m}, 2 \mathrm{H}), 1.77-1.66(\mathrm{~m}, 2 \mathrm{H}), 1.19-1.01$ $(\mathrm{m}, 21 \mathrm{H})$.

${ }^{13} \mathrm{C}$ NMR (101 MHz, $\left.\mathrm{CDCl}_{3}\right) \delta$ 139.3, 136.0, 135.4, 133.9, 128.9, 122.8, 120.1, 109.9, $108.9,100.5,35.9,33.0,32.6,23.6,17.9,12.1$.

HRMS (ESI ${ }^{+}$) calcd for $\mathrm{C}_{23} \mathrm{H}_{37} \mathrm{NNaOSi}^{+}[\mathrm{M}+\mathrm{Na}]^{+}:$394.2537, found: 394.2535.

IR (neat, cm$^{-1}$ ): 2939, 2865, 1147, 1106, 881, 716, 683.

(Z)-Triisopropyl((5-(naphthalen-2-yl)pent-1-en-1-yl)oxy)silane (1j)

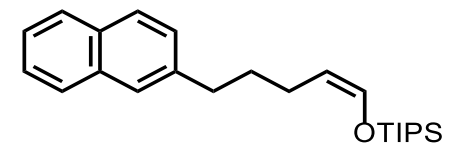

Compound $\mathbf{1} \mathbf{j}$ was prepared as a colorless oil in $39 \%$ yield $(0.29 \mathrm{~g}$, eluent: petroleum ether) from 2-bromonaphthalene $(0.41 \mathrm{~g}, 2.00 \mathrm{mmol}),(Z)-$ triisopropyl((5-(4,4,5,5-tetramethyl-1,3,2-dioxaborolan-2-yl)pent-1-en-1yl)oxy)silane (0.88 g, $2.40 \mathrm{mmol}), \mathrm{Pd}_{2}(\mathrm{dba})_{3}(36.6 \mathrm{mg}, 0.04 \mathrm{mmol})$, Ruphos (37.4 $\mathrm{mg}, 0.08 \mathrm{mmol})$ and $\mathrm{NaOtBu}(0.58 \mathrm{~g}, 6.00 \mathrm{mmol})$ following the above general procedure $\mathrm{D}$, which was previously reported ${ }^{12}$.

$\mathbf{R}_{f}=0.45$ (petroleum ether)

${ }^{1} \mathrm{H}$ NMR (400 MHz, $\left.\mathrm{CDCl}_{3}\right) \delta$ 7.82-7.71 (m, 3H), 7.61 (s, 1H), 7.46-7.36 (m, 2H), $7.33(\mathrm{~d}, J=8.4 \mathrm{~Hz}, 1 \mathrm{H}), 6.34-6.28(\mathrm{~m}, 1 \mathrm{H}), 4.51-4.40(\mathrm{~m}, 1 \mathrm{H}), 2.78(\mathrm{t}, J=7.8 \mathrm{~Hz}$, 
2H), 2.25-2.15 (m, 2H), 1.81-1.70 (m, 2H), 1.21-0.93 (m, 21H).

${ }^{13} \mathrm{C}$ NMR (100 MHz, $\left.\mathrm{CDCl}_{3}\right) \delta$ 140.6, 139.6, 133.8, 132.0, 127.8, 127.69, 127.67, $127.5,126.4,125.9,125.1,109.5,36.0,31.6,23.6,17.9,12.1$.

(Z)-Triisopropyl((3-phenylprop-1-en-1-yl)oxy)silane (1k)<smiles>CC=CO[Hg]</smiles>

Compound $1 \mathbf{k}$ was prepared as a colorless oil in $40 \%$ yield $(0.58 \mathrm{~g}$, eluent: petroleum ether) from 3-phenylpropanal $(0.67 \mathrm{~g}, 5.00 \mathrm{mmol})$, TIPSCl (1.16 g, $6.00 \mathrm{mmol})$, DBU $(0.91 \mathrm{~g}, 6.00 \mathrm{mmol})$ following the above general procedure A, which was previously reported ${ }^{12}$

$\mathbf{R}_{f}=0.80(Z), 0.69(E)$ (petroleum ether)

${ }^{1} \mathrm{H}$ NMR (400 MHz, CDCl 3 ) \& 7.30-7.20 (m, 4H), 7.19-7.13 (m, 1H), 6.40 (d, J = $5.7 \mathrm{~Hz}, 1 \mathrm{H}), 4.67-4.57(\mathrm{~m}, 1 \mathrm{H}), 3.47(\mathrm{~d}, J=7.3 \mathrm{~Hz}, 2 \mathrm{H}), 1.28-1.04(\mathrm{~m}, 21 \mathrm{H})$.

${ }^{13} \mathrm{C}$ NMR (100 MHz, $\mathrm{CDCl}_{3}$ ) ठ 142.3, 139.7, 128.5, 128.4, 125.7, 108.6, 30.1, 17.9, 12.1.

(Z)-Triisopropyl(prop-1-en-1-yloxy)silane (11)<smiles>[13CH2]O[13CH2]O</smiles>

Compound 11 was prepared as a colorless oil in $22 \%$ yield $(0.47 \mathrm{~g}$, eluent: petroleum ether) from propionaldehyde $(0.58 \mathrm{~g}, 10.00 \mathrm{mmol})$, TIPSCl (2.30 g, $12.00 \mathrm{mmol})$, DBU $(1.83 \mathrm{~g}, 12.00 \mathrm{mmol})$ following the above general procedure A, which was previously reported ${ }^{12}$.

$\mathbf{R}_{f}=0.91(Z), 0.82(E)$ (petroleum ether)

${ }^{1} \mathrm{H}$ NMR (400 MHz, $\left.\mathrm{CDCl}_{3}\right) \delta 6.28(\mathrm{~d}, J=5.0 \mathrm{~Hz}, 1 \mathrm{H}), 4.50-4.39(\mathrm{~m}, 1 \mathrm{H}), 1.59$ (d, $J=6.6 \mathrm{~Hz}, 3 \mathrm{H}), 1.21-0.98(\mathrm{~m}, 21 \mathrm{H})$.

${ }^{13} \mathrm{C}$ NMR (100 MHz, $\left.\mathrm{CDCl}_{3}\right)$ \& 139.9, 104.2, 17.9, 12.2, 9.0.

(Z)-(Hex-1-en-1-yloxy)triisopropylsilane (1m)

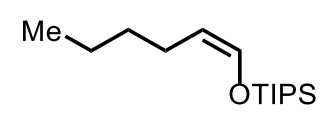

Compound $1 \mathrm{~m}$ was prepared as a colorless oil in $45 \%$ yield $(2.31 \mathrm{~g}$, eluent: petroleum ether) from hexanal ( $2.00 \mathrm{~g}, 20.00 \mathrm{mmol})$, TIPSCl $(4.60 \mathrm{~g}, 24.00 \mathrm{mmol})$, 
DBU (3.60 g, $24.00 \mathrm{mmol})$ following the above general procedure A, which was previously reported ${ }^{12}$.

$\mathbf{R}_{f}=0.91(Z), 0.82(E)$ (petroleum ether)

${ }^{1} \mathrm{H}$ NMR (400 MHz, $\left.\mathrm{CDCl}_{3}\right) \delta 6.26(\mathrm{~d}, J=5.6 \mathrm{~Hz}, 1 \mathrm{H}), 4.45-4.34(\mathrm{~m}, 1 \mathrm{H}), 2.16-$ $2.05(\mathrm{~m}, 2 \mathrm{H}), 1.38-1.27(\mathrm{~m}, 4 \mathrm{H}), 1.19-1.01(\mathrm{~m}, 21 \mathrm{H}), 0.89(\mathrm{t}, J=5.8 \mathrm{~Hz}, 3 \mathrm{H})$.

${ }^{13} \mathrm{C}$ NMR (100 MHz, $\left.\mathrm{CDCl}_{3}\right) \delta$ 139.0, 110.2, 32.1, 23.4, 22.5, 17.9, 14.1, 12.2.

\section{(Z)-(Hexadec-1-en-1-yloxy)triisopropylsilane (1n)}

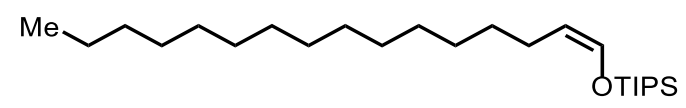

Compound 1n was prepared as a colorless oil in 62\% yield (1.23 g, eluent: petroleum ether) from palmitaldehyde $(1.07 \mathrm{~g}, 4.43 \mathrm{mmol})$, TIPSOTf $(1.63 \mathrm{~g}$, $5.32 \mathrm{mmol}), \mathrm{Et}_{3} \mathrm{~N}(0.89 \mathrm{~g}, 8.86 \mathrm{mmol})$ following the above general procedure $\mathrm{B}$, which was previously reported ${ }^{13}$.

$\mathbf{R}_{f}=0.88(Z), 0.75(E)$ (petroleum ether)

${ }^{1} \mathrm{H}$ NMR (400 MHz, $\left.\mathrm{CDCl}_{3}\right) \delta 6.26(\mathrm{~d}, J=5.8 \mathrm{~Hz}, 1 \mathrm{H}), 4.44-4.33(\mathrm{~m}, 1 \mathrm{H}), 2.16-$ $2.05(\mathrm{~m}, 2 \mathrm{H}), 1.36-1.22(\mathrm{~m}, 24 \mathrm{H}), 1.18-1.04(\mathrm{~m}, 21 \mathrm{H}), 0.88(\mathrm{t}, J=6.8 \mathrm{~Hz}, 3 \mathrm{H})$.

${ }^{13} \mathrm{C}$ NMR (100 MHz, $\left.\mathrm{CDCl}_{3}\right) \delta$ 139.0, 110.2, 32.1, 29.95-29.75 (m, 8C), 29.7, 29.5, $23.7,22.9,17.9,14.3,12.1$.

(Z)-((4-Cyclohexylbut-1-en-1-yl)oxy)triisopropylsilane (1o)<smiles>[AlH2]OC/C=C\CC[Hg]</smiles>

Compound 1o was prepared as a colorless oil in 47\% yield (1.46 g, eluent: petroleum ether) from 4-cyclohexylbutanal (1.54 g, $10.00 \mathrm{mmol})$, TIPSCl (2.32g $\mathrm{g}, 12.00 \mathrm{mmol}), \operatorname{DBU}(1.83 \mathrm{~g}, 12.00 \mathrm{mmol})$ following the above general procedure A.

$\mathbf{R}_{f}=0.91(Z), 0.82(E)$ (petroleum ether)

${ }^{1} \mathbf{H}$ NMR (400 MHz, $\left.\mathrm{CDCl}_{3}\right) \delta 6.25(\mathrm{~d}, J=5.6 \mathrm{~Hz}, 1 \mathrm{H}), 4.42-4.32$ (m, 1H), 2.16$2.06(\mathrm{~m}, 2 \mathrm{H}), 1.78-1.57(\mathrm{~m}, 5 \mathrm{H}), 1.31-0.98(\mathrm{~m}, 27 \mathrm{H}), 0.94-0.79(\mathrm{~m}, 2 \mathrm{H})$.

${ }^{13} \mathrm{C}$ NMR (100 MHz, $\left.\mathrm{CDCl}_{3}\right) \delta$ 138.9, 110.4, 37.6, 37.4, 33.5, 27.0, 26.6, 21.1, 17.9, 12.2 .

HRMS (ESI ${ }^{+}$) calcd for $\mathrm{C}_{19} \mathrm{H}_{38} \mathrm{NaOSi}^{+}[\mathrm{M}+\mathrm{Na}]^{+}: 333.2584$, found: 333.2583 .

IR (neat, cm-1): 2922, 2856, 1655, 1107, 883, 684 . 
(Z)-((5,5-Dimethylhex-1-en-1-yl)oxy)triisopropylsilane (1p)<smiles></smiles>

Compound $1 \mathrm{p}$ was prepared as a colorless oil in $33 \%$ yield $(0.21 \mathrm{~g}$, eluent: petroleum ether) from 5,5-dimethylhexanal $(0.28 \mathrm{~g}, 2.16 \mathrm{mmol}), \operatorname{TIPSCl}(0.50 \mathrm{~g}$, $2.59 \mathrm{mmol}), \mathrm{DBU}(0.39 \mathrm{~g}, 2.59 \mathrm{mmol})$ following the above general procedure A. $\mathbf{R}_{f}=0.91(Z), 0.82(E)$ (petroleum ether)

${ }^{1} \mathrm{H}$ NMR (400 MHz, $\left.\mathrm{CDCl}_{3}\right) \delta 6.25(\mathrm{~d}, J=5.7 \mathrm{~Hz}, 1 \mathrm{H}), 4.46-4.34(\mathrm{~m}, 1 \mathrm{H}), 2.12-$ $2.03(\mathrm{~m}, 2 \mathrm{H}), 1.28-1.03(\mathrm{~m}, 23 \mathrm{H}), 0.89(\mathrm{~s}, 9 \mathrm{H})$.

${ }^{13} \mathrm{C}$ NMR (100 MHz, $\mathrm{CDCl}_{3}$ ) $\delta$ 138.8, 110.9, 44.2, 30.6, 29.5, 19.1, 17.9, 12.1.

HRMS (ESI') calcd for $\mathrm{C}_{17} \mathrm{H}_{36} \mathrm{NaOSi}^{+}[\mathrm{M}+\mathrm{Na}]^{+}: 307.2428$, found: 307.2427 .

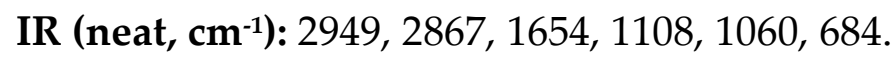

tert-Butyl (Z)-(6-((triisopropylsilyl)oxy)hex-5-en-1-yl)carbamate (1w)<smiles>CC(C)(C)OC(=O)NCCCC/C=C\O[Na]</smiles>

Compound 1w was prepared as a colorless oil in $34 \%$ yield $(0.44 \mathrm{~g}$, eluent: petroleum ether/EtOAc $=20 / 1)$ from tert-butyl (6-oxohexyl)carbamate $(0.66 \mathrm{~g}$, $3.50 \mathrm{mmol})$, TIPSOTf $(1.20 \mathrm{~g}, 4.20 \mathrm{mmol}), \mathrm{Et}_{3} \mathrm{~N}(0.70 \mathrm{~g}, 7.00 \mathrm{mmol})$ following the above general procedure $\mathrm{B}$.

$\mathbf{R}_{f}=0.35$ (petroleum ether $/$ EtOAc $=20 / 1$ )

${ }^{1} \mathrm{H}$ NMR (400 MHz, CDCl $)$ d $6.27(\mathrm{~d}, J=5.8 \mathrm{~Hz}, 1 \mathrm{H}), 4.53(\mathrm{~s}, 1 \mathrm{H}), 4.42-4.33(\mathrm{~m}$, $1 \mathrm{H}), 3.21-2.98(\mathrm{~m}, 2 \mathrm{H}), 2.18-2.04(\mathrm{~m}, 2 \mathrm{H}), 1.52-1.34(\mathrm{~m}, 11 \mathrm{H}), 1.23-0.94(\mathrm{~m}$, $23 \mathrm{H})$.

${ }^{13} \mathrm{C}$ NMR (100 MHz, $\mathrm{CDCl}_{3}$ ) ठ 156.1, 139.4, 109.4, 79.0, 40.7, 29.8, 28.5, 27.0, 23.3, 17.9, 12.1 .

HRMS (ESI+) calcd for $\mathrm{C}_{20} \mathrm{H}_{41} \mathrm{O}_{3} \mathrm{NNaSi}^{+}[\mathrm{M}+\mathrm{Na}]^{+}: 394.2748$, found: 394.2742 .

IR (neat, cm ${ }^{-1}$ ): 2943, 2867, 1694, 1249, 1173, 1089, 1068, 882, 684, 666.

(Z)-Triisopropyl((5-(4,4,5,5-tetramethyl-1,3,2-dioxaborolan-2-yl)pent-1-en-1yl)oxy)silane (1x)

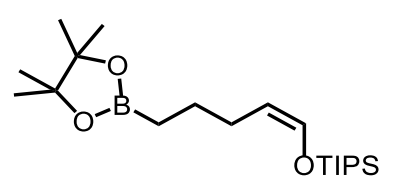


Compound 1x was prepared as a colorless oil in $68 \%$ yield ( $2.38 \mathrm{~g}$, eluent: petroleum ether) from (Z)-triisopropyl(penta-1,4-dien-1-yloxy)silane (2.28 $\mathrm{g}$, $9.50 \mathrm{mmol})$, HBpin (1.46 g, $11.40 \mathrm{mmol})$, [ $\mathrm{IrCl}(\mathrm{cod})]_{2}(63.7 \mathrm{mg}, 0.10 \mathrm{mmol})$ and dppe $(75.5 \mathrm{mg}, 0.19 \mathrm{mmol})$ following the above general procedure $\mathrm{D}$.

$\mathbf{R}_{f}=0.55$ (petroleum ether $/$ EtOAc $=20 / 1$ )

${ }^{1} \mathrm{H}$ NMR (400 MHz, $\left.\mathrm{CDCl}_{3}\right)$ ठ 6.31-6.20 (m, 1H), 4.43-4.34 (m, 1H), 2.17-2.05 (m, 2H), 1.53-1.38 (m, 2H), $1.24(\mathrm{~s}, 12 \mathrm{H}), 1.18-1.01(\mathrm{~m}, 21 \mathrm{H}), 0.80(\mathrm{t}, J=7.9 \mathrm{~Hz}, 2 \mathrm{H})$. ${ }^{13} \mathrm{C}$ NMR (100 MHz, $\left.\mathrm{CDCl}_{3}\right) \delta$ 139.1, 110.0, 82.9, 26.4, 24.9, 24.3, 17.9, 12.1.

The carbon signal attached to $\mathrm{B}$ atom was not observed due to low intensity; HRMS (ESI ${ }^{+}$) calcd for $\mathrm{C}_{20} \mathrm{H}_{41} \mathrm{BNaO}_{3} \mathrm{Si}^{+}[\mathrm{M}+\mathrm{Na}]^{+}: 391.2810$, found: 391.2808. IR (neat, cm-1): 2939, 2867, 1372, 1316, 1146, 1100, 882, 684.

\section{(Z)-((6-Chlorohex-1-en-1-yl)oxy)triisopropylsilane (1y)}<smiles>COC=CCCCCCl</smiles>

Compound 1y was prepared as a colorless oil in $72 \%$ yield $(2.09 \mathrm{~g}$, eluent: petroleum ether) from 6-chlorohexanal $(1.35 \mathrm{~g}, 10.00 \mathrm{mmol})$, TIPSOTf (3.67 $\mathrm{g}$, $12.00 \mathrm{mmol}), \mathrm{Et}_{3} \mathrm{~N}(0.20 \mathrm{~g}, 20.00 \mathrm{mmol})$ following the above general procedure B.

$\mathbf{R}_{f}=0.72(Z), 0.48(E)$ (petroleum ether)

${ }^{1} \mathrm{H}$ NMR $\left(400 \mathrm{MHz}, \mathrm{CDCl}_{3}\right) \delta 6.29(\mathrm{~d}, J=5.8 \mathrm{~Hz}, 1 \mathrm{H}), 4.43-4.33(\mathrm{~m}, 1 \mathrm{H}), 3.55(\mathrm{t}$, $J=6.8 \mathrm{~Hz}, 2 \mathrm{H}), 2.19-2.09(\mathrm{~m}, 2 \mathrm{H}), 1.86-1.73(\mathrm{~m}, 2 \mathrm{H}), 1.54-1.45(\mathrm{~m}, 2 \mathrm{H}), 1.20-$ $1.02(\mathrm{~m}, 21 \mathrm{H})$.

${ }^{13} \mathrm{C}$ NMR (100 MHz, $\mathrm{CDCl}_{3}$ ) $\delta$ 139.7, 109.0, 45.2, 32.3, 26.9, 22.8, 17.9, 12.1.

HRMS (ESI+) calcd for $\mathrm{C}_{15} \mathrm{H}_{31} \mathrm{NaClOSi}^{+}[\mathrm{M}+\mathrm{Na}]^{+}: 313.1725$, found: 313.1724 .

IR (neat, cm ${ }^{-1}$ ): 2943, 2867, 1111, 1064, 883, 685, 661.

\section{(Z)-Triisopropyl((12,12,12-trifluorododec-1-en-1-yl)oxy)silane (1z)}

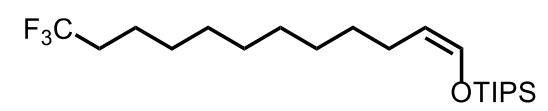

Compound $1 \mathrm{z}$ was prepared as a colorless oil in $65 \%$ yield $(0.56 \mathrm{~g}$, eluent: petroleum ether) from 12,12,12-trifluorododecanal (0.52 g, $2.20 \mathrm{mmol})$, TIPSOTf $(0.81 \mathrm{~g}, 2.64 \mathrm{mmol}), E t_{3} \mathrm{~N}(0.45 \mathrm{~g}, 4.40 \mathrm{mmol})$ following the above general procedure $\mathrm{B}$.

$\mathbf{R}_{f}=0.72(Z), 0.48(E)$ (petroleum ether) 
${ }^{1} \mathbf{H}$ NMR (400 MHz, $\left.\mathrm{CDCl}_{3}\right) \delta 6.27$ (d, $\left.J=5.8 \mathrm{~Hz}, 1 \mathrm{H}\right), 4.43-4.35$ (m, 1H), 2.16$1.97(\mathrm{~m}, 4 \mathrm{H}), 1.59-1.49(\mathrm{~m}, 2 \mathrm{H}), 1.40-1.24(\mathrm{~m}, 12 \mathrm{H}), 1.20-0.98(\mathrm{~m}, 21 \mathrm{H})$.

${ }^{13} \mathrm{C}$ NMR (100 MHz, $\left.\mathrm{CDCl}_{3}\right) \delta 139.1,127.5(\mathrm{q}, J=276.3 \mathrm{~Hz}), 110.1,33.9(\mathrm{q}, J=$ $28.2 \mathrm{~Hz}), 29.8,29.6,29.5,29.4,29.3,28.9,23.7,22.0(\mathrm{q}, J=2.9 \mathrm{~Hz}), 17.9,12.1$.

${ }^{19}$ F NMR (377 MHz, $\left.\mathrm{CDCl}_{3}\right) \delta-66.4(\mathrm{t}, J=11.1 \mathrm{~Hz})$.

HRMS (ESI ${ }^{+}$) calcd for $\mathrm{C}_{21} \mathrm{H}_{41} \mathrm{~F}_{3} \mathrm{NaOSi}^{+}[\mathrm{M}+\mathrm{Na}]^{+}:$417.2771, found: 417.2769.

IR (neat, cm-1): 2927, 2863, 1252, 1136, 1093, 1067, 882, 684, 660.

(Z)-((11-Fluoroundec-1-en-1-yl)oxy)triisopropylsilane (1aa)

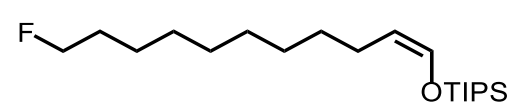

Compound 1aa was prepared as a colorless oil in $58 \%$ yield $(0.74 \mathrm{~g}$, eluent: petroleum ether) from 11-fluoroundecanal ( $0.70 \mathrm{~g}, 3.70 \mathrm{mmol})$, TIPSOTf (1.36 g, $4.44 \mathrm{mmol}), \mathrm{Et}_{3} \mathrm{~N}(0.75 \mathrm{~g}, 7.40 \mathrm{mmol})$ following the above general procedure $\mathrm{B}$. $\mathbf{R}_{f}=0.52(Z), 0.35(E)$ (petroleum ether)

${ }^{1}$ H NMR (400 MHz, $\left.\mathrm{CDCl}_{3}\right) \delta$ 6.30-6.22 (m, 1H), $4.49(\mathrm{t}, J=6.2 \mathrm{~Hz}, 1 \mathrm{H}), 4.42-$ $4.34(\mathrm{~m}, 2 \mathrm{H}), 2.13-2.05(\mathrm{~m}, 2 \mathrm{H}), 1.76-1.60$ (m, 2H), 1.43-1.25 (m, 12H), 1.21-1.01 $(\mathrm{m}, 21 \mathrm{H})$.

${ }^{13} \mathrm{C}$ NMR (100 MHz, $\left.\mathrm{CDCl}_{3}\right) \delta$ 139.1, 110.1, 84.4 (d, $\left.J=163.9 \mathrm{~Hz}\right), 30.6(\mathrm{~d}, J=19.3$ Hz), 29.8, 29.7, 29.6, 29.5, 29.4, 25.3 (d, J = 5.7 Hz), 23.7, 17.9, 12.1.

${ }^{19}$ F NMR (377 MHz, $\mathrm{CDCl}_{3}$ ) $\delta-218.0(\mathrm{~m})$.

HRMS (ESI ${ }^{+}$) calcd for $\mathrm{C}_{20} \mathrm{H}_{41} \mathrm{FNaOSi}^{+}[\mathrm{M}+\mathrm{Na}]^{+}:$367.2803, found: 367.2801 .

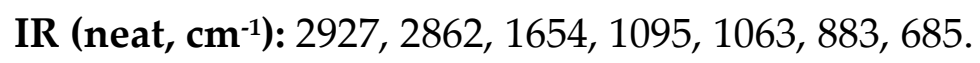

\section{Compound $1 \mathrm{ab}$}

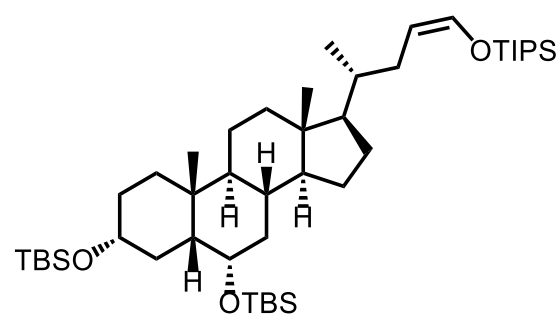

Compound 1ab was prepared as a colorless oil in 76\% yield (1.03 g, eluent: petroleum ether/EtOAc $=50 / 1)$ from $(R)-4-((3 R, 5 R, 6 S, 8 S, 9 S, 10 R, 13 R, 14 S, 17 R)-$ 3,6-bis((tert-butyldimethylsilyl)oxy)-10,13-dimethylhexadecahydro- $1 \mathrm{H}$ cyclopenta[a]phenanthren-17-yl)pentanal ${ }^{14}(1.06 \mathrm{~g}, 1.74 \mathrm{mmol})$, TIPSOTf $(0.64$ 
g, $2.09 \mathrm{mmol}), \mathrm{Et}_{3} \mathrm{~N}(0.35 \mathrm{~g}, 3.48 \mathrm{mmol})$ following the above general procedure B.

$\mathbf{R}_{f}=0.50(Z), 0.30(E)$ (petroleum ether)

${ }^{1} \mathrm{H}$ NMR (400 MHz, $\left.\mathrm{CDCl}_{3}\right) \delta 6.31(\mathrm{~d}, J=5.9 \mathrm{~Hz}, 1 \mathrm{H}), 4.44-4.32(\mathrm{~m}, 1 \mathrm{H}), 4.02-$ $3.93(\mathrm{~m}, 1 \mathrm{H}), 3.59-3.45(\mathrm{~m}, 1 \mathrm{H}), 2.20-2.09(\mathrm{~m}, 1 \mathrm{H}), 1.99-1.83(\mathrm{~m}, 4 \mathrm{H}), 1.79-1.68$ (m, 1H), 1.62-1.51 (m, 3H), 1.50-1.25 (m, 9H), 1.21-1.01 (m, 26H), 0.99-0.77 (m, $25 \mathrm{H}), 0.62(\mathrm{~s}, 3 \mathrm{H}), 0.08--0.01(\mathrm{~m}, 12 \mathrm{H})$.

${ }^{13} \mathrm{C}$ NMR (100 MHz, $\left.\mathrm{CDCl}_{3}\right) \delta$ 139.7, 107.8, 73.1, 68.9, 56.3, 56.2, 49.7, 42.9, 40.0, 39.7, 36.5, 36.1, 36.1, 35.6, 35.1, 31.2, 30.0, 29.9, 28.4, 26.2, 26.0, 24.4, 23.7, 20.9, $19.0,18.6,18.3,17.9,12.2,12.1,-4.3,-4.5,-4.6,-4.6$.

HRMS (ESI') calcd for $\mathrm{C}_{45} \mathrm{H}_{88} \mathrm{O}_{3} \mathrm{NaSi}_{3}{ }^{+}[\mathrm{M}+\mathrm{Na}]^{+}:$783.5933, found: 783.5928 .

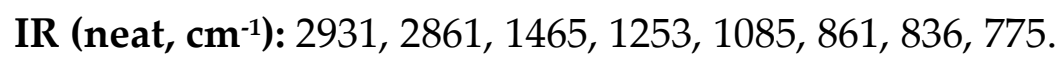

\section{Synthesis of 1e}
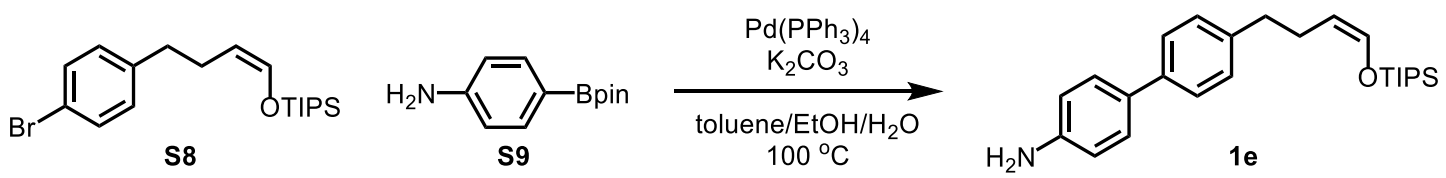

To a sealed tube were added $\mathrm{Pd}\left(\mathrm{PPh}_{3}\right)_{4}(115.5 \mathrm{mg}, 0.10 \mathrm{mmol}), \mathrm{K}_{2} \mathrm{CO}_{3}(0.97$ g, $7.00 \mathrm{mmol})$, S8 (0.92 g, $2.40 \mathrm{mmol})$ and S9 (0.44 g, $2.00 \mathrm{mmol})$. Toluene (12.5 $\mathrm{mL}), \mathrm{EtOH}(5.0 \mathrm{~mL})$ and $\mathrm{H}_{2} \mathrm{O}(2.5 \mathrm{~mL})$ were then added. The resulting mixture was heated to $100{ }^{\circ} \mathrm{C}$ and stirred for $15 \mathrm{~h}$. After cooled to room temperature, the reaction mixture was extracted with EtOAc, dried with $\mathrm{Na}_{2} \mathrm{SO}_{4}$, filtered, concentrated. The residue was purified by silica gel flash chromatography to afford compound $1 \mathrm{e}$ as a red oil in $79 \%$ yield $(0.63 \mathrm{~g}$, eluent: petroleum ether $/ \mathrm{EtOAc}=5 / 1)$.

$\mathbf{R}_{f}=0.60$ (petroleum ether $/$ EtOAc $=3 / 1$ )

${ }^{1} \mathrm{H}$ NMR (400 MHz, $\left.\mathrm{CDCl}_{3}\right) \delta 7.44(\mathrm{~d}, J=8.1 \mathrm{~Hz}, 2 \mathrm{H}), 7.39(\mathrm{~d}, J=8.4 \mathrm{~Hz}, 2 \mathrm{H})$, $7.27-7.19(\mathrm{~m}, 2 \mathrm{H}), 6.73(\mathrm{~d}, J=8.5 \mathrm{~Hz}, 2 \mathrm{H}), 6.29(\mathrm{~d}, J=5.8 \mathrm{~Hz}, 1 \mathrm{H}), 4.51-4.42$ $(\mathrm{m}, 1 \mathrm{H}), 3.67(\mathrm{~s}, 2 \mathrm{H}), 2.75-2.64(\mathrm{~m}, 2 \mathrm{H}), 2.52-2.39(\mathrm{~m}, 2 \mathrm{H}), 1.20-0.93(\mathrm{~m}, 21 \mathrm{H})$. ${ }^{13} \mathrm{C}$ NMR (100 MHz, $\left.\mathrm{CDCl}_{3}\right) \delta 145.6,140.7,139.6,138.7,131.8,128.9,128.0,126.3$, $115.5,109.0,35.6,25.7,17.9,12.1$.

HRMS (ESI') calcd for $\mathrm{C}_{25} \mathrm{H}_{38} \mathrm{ONSi}^{+}[\mathrm{M}+\mathrm{H}]^{+}:$396.2717, found: 396.2710 .

IR (neat, cm-1): 2943, 2865, 1621, 1500, 1116, 1103, 882, 815, 685, 666 


\section{Synthesis of $1 \mathrm{f}$}
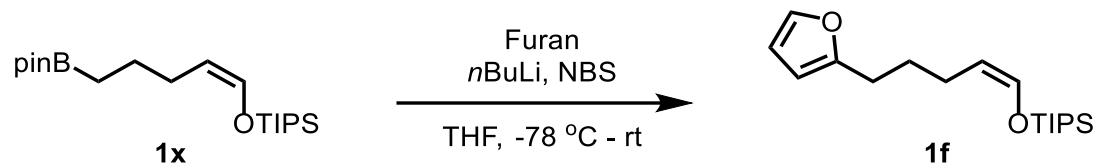

Following the literature procedure ${ }^{15}$, to the solution of furan $(0.27 \mathrm{~g}, 4.00$ $\mathrm{mmol})$ in THF was added $n \mathrm{BuLi}\left(2.5 \mathrm{M}\right.$ in hexane, 2.0 equiv) at $-78^{\circ} \mathrm{C}$. After stirred for $1 \mathrm{~h}$ at room temperature, $1 \times 1 \times(0.74 \mathrm{~g}, 2.00 \mathrm{mmol})$ was added. The reaction was vigorously stirred at $-78{ }^{\circ} \mathrm{C}$ for $5 \mathrm{~h}$ and a solution of NBS $(0.71 \mathrm{~g}$, $4.00 \mathrm{mmol}$ ) in THF was added. The resulting mixture was then warmed up to room temperature and stirred for $5 \mathrm{~h}$, then quenched with saturated $\mathrm{NH}_{4} \mathrm{Cl}$, extracted with EtOAc, dried with $\mathrm{Na}_{2} \mathrm{SO}_{4}$, filtered and concentrated. The residue was purified by silica gel flash chromatography to afford compound $\mathbf{1 f}$ as a colorless oil in $67 \%$ yield $(0.21 \mathrm{~g}$, eluent: petroleum ether).

$\mathbf{R}_{f}=0.80$ (petroleum ether)

${ }^{1} \mathrm{H}$ NMR (400 MHz, $\left.\mathrm{CDCl}_{3}\right) \delta$ 7.33-7.21 (m, 1H), 6.37-6.23 (m, 2H), 6.01-5.93 (m, $1 \mathrm{H}), 4.46-4.33(\mathrm{~m}, 1 \mathrm{H}), 2.63(\mathrm{t}, J=7.8 \mathrm{~Hz}, 2 \mathrm{H}), 2.23-2.06(\mathrm{~m}, 2 \mathrm{H}), 1.78-1.62(\mathrm{~m}$, 2H), $1.21-0.96(\mathrm{~m}, 21 \mathrm{H})$.

${ }^{13} \mathrm{C}$ NMR (100 MHz, $\left.\mathbf{C D C l}_{3}\right) \delta$ 156.8, 140.7, 139.7, 110.1, 109.1, 104.7, 28.2, 27.8, $23.4,17.9,12.1$.

HRMS (ESI ${ }^{+}$) calcd for $\mathrm{C}_{18} \mathrm{H}_{32} \mathrm{O}_{2} \mathrm{NaSi}^{+}[\mathrm{M}+\mathrm{Na}]^{+}:$331.2064, found: 331.2062.

IR (neat, cm-1): 2942, 2866, 1111, 1059, 882, 726, 684 .

\section{Synthesis of $1 \mathrm{i}$}
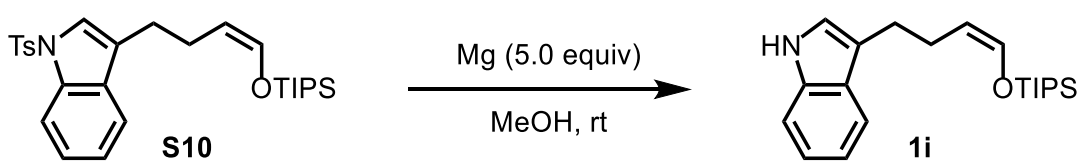

Following a modified procedure ${ }^{16}$, to a solution of $\mathbf{S 1 0}(1.00 \mathrm{~g}, 2.00 \mathrm{mmol})$ in $\mathrm{MeOH}$ was added $\mathrm{Mg}$ powder $(0.24 \mathrm{~g}$, $10.00 \mathrm{mmol})$. The resulting suspension was sonicated for $0.5 \mathrm{~h}$. The reaction was diluted with DCM, filtered and concentrated. The residue was purified by silica gel flash chromatography to afford compound $\mathbf{1 i}$ as a colorless oil in $22 \%$ yield $(0.15 \mathrm{~g}$, eluent: petroleum ether $\left./ \mathrm{Et}_{2} \mathrm{O}=40 / 1\right)$.

$\mathbf{R}_{f}=0.48$ (petroleum ether $/$ EtOAc $=10 / 1$ )

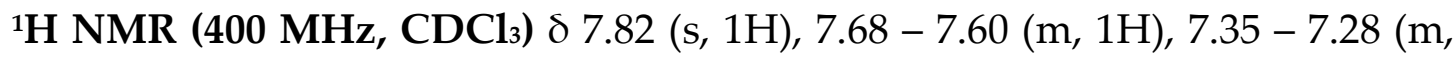
1H), $7.20-7.13(\mathrm{~m}, 1 \mathrm{H}), 7.13-7.05(\mathrm{~m}, 1 \mathrm{H}), 7.02-6.95(\mathrm{~m}, 1 \mathrm{H}), 6.30(\mathrm{~d}, J=5.8$ 
$\mathrm{Hz}, 1 \mathrm{H}), 4.58-4.47(\mathrm{~m}, 1 \mathrm{H}), 2.85-2.77(\mathrm{~m}, 2 \mathrm{H}), 2.59-2.49(\mathrm{~m}, 2 \mathrm{H}), 1.18-1.12$ $(\mathrm{m}, 3 \mathrm{H}), 1.11-1.03(\mathrm{~m}, 18 \mathrm{H})$.

${ }^{13} \mathrm{C}$ NMR (100 MHz, $\left.\mathrm{CDCl}_{3}\right) \delta$ 139.4, 136.4, 127.8, 121.9, 121.2, 119.2, 119.1, 117.0, $111.1,109.8,25.4,24.4,17.9,12.1$.

HRMS (ESI') calcd for $\mathrm{C}_{21} \mathrm{H}_{34} \mathrm{ONSi}^{+}[\mathrm{M}+\mathrm{H}]^{+}: 344.2404$, found: 344.2399 .

IR (neat, cm-1): 3420, 2943, 2866, 1655, 1457, 1110, 1068, 1012, 883, 740, 685, 665

\section{Synthesis of $1 \mathrm{q}$}

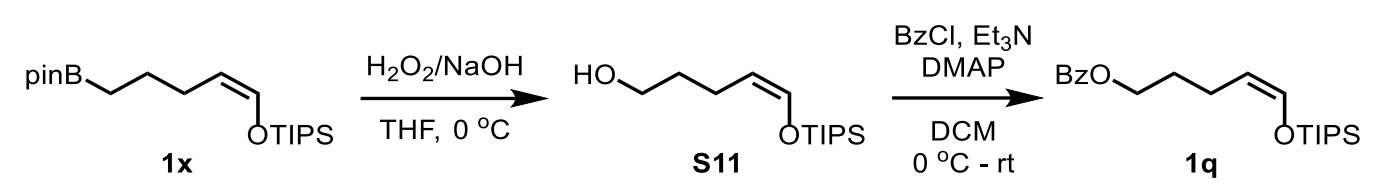

To the solution of $\mathbf{1 x}(3.68 \mathrm{~g}, 10.00 \mathrm{mmol})$ in THF was added $\mathrm{NaOH}(3.0 \mathrm{M}$, 1.5 equiv), $\mathrm{H}_{2} \mathrm{O}_{2}\left(30 \%, 1.5\right.$ equiv) at $0{ }^{\circ} \mathrm{C}$. The resulting mixture was stirred for $0.5 \mathrm{~h}$, then extracted with EtOAc, dried with $\mathrm{Na}_{2} \mathrm{SO}_{4}$, filtered and concentrated. The residue was purified by silica gel chromatography to afford compound $\mathbf{S 1 1}$ as a colorless oil in 95\% yield (2.46 g, eluent: petroleum ether/EtOAc $=20 / 1$ ).

To the solution of $\mathbf{S 1 1}(0.51 \mathrm{~g}, 2.00 \mathrm{mmol}), \mathrm{Et}_{3} \mathrm{~N}(0.61 \mathrm{~g}, 6.00 \mathrm{mmol})$ and DMAP (48.8 mg, $0.40 \mathrm{mmol})$ in DCM was added $\mathrm{BzCl}(0.42 \mathrm{~g}, 3.00 \mathrm{mmol})$ at 0 ${ }^{\circ} \mathrm{C}$. The resulting mixture was then warmed up to room temperature and stirred for $5 \mathrm{~h}$, then quenched with saturated $\mathrm{NH}_{4} \mathrm{Cl}$, extracted with $\mathrm{EtOAc}$, dried with $\mathrm{Na}_{2} \mathrm{SO}_{4}$, filtered and concentrated. The residue was purified by silica gel flash chromatography to afford compound $1 \mathbf{q}$ as a colorless oil in $72 \%$ yield $(0.52 \mathrm{~g}$, eluent: petroleum ether/EtOAc $=30 / 1)$.

$\mathbf{R}_{f}=0.65$ (petroleum ether $/ \mathrm{EtOAc}=30 / 1$ )

${ }^{1} \mathbf{H}$ NMR (400 MHz, $\left.\mathrm{CDCl}_{3}\right) \delta 8.05(\mathrm{~d}, J=7.8 \mathrm{~Hz}, 2 \mathrm{H}), 7.58-7.51(\mathrm{~m}, 1 \mathrm{H}), 7.43(\mathrm{t}$, $J=7.7 \mathrm{~Hz}, 2 \mathrm{H}), 6.32(\mathrm{~d}, J=5.8 \mathrm{~Hz}, 1 \mathrm{H}), 4.49-4.41(\mathrm{~m}, 1 \mathrm{H}), 4.33(\mathrm{t}, J=6.5 \mathrm{~Hz}, 2 \mathrm{H})$, 2.34-2.24 (m, 2H), 1.89-1.77 (m, 2H), 1.18-1.10 (m, 3H), 1.08-1.04 (m, 18H).

${ }^{13} \mathrm{C}$ NMR (100 MHz, $\left.\mathrm{CDCl}_{3}\right) \delta$ 166.8, 140.0, 132.9, 130.7, 129.7, 128.4, 108.3, 65.0, $28.9,20.4,17.9,12.1$.

HRMS (ESI') calcd for $\mathrm{C}_{21} \mathrm{H}_{34} \mathrm{O}_{3} \mathrm{NaSi}^{+}[\mathrm{M}+\mathrm{Na}]^{+}$: 385.2169, found: 385.2167.

IR (neat, cm-1): 2945, 2867, 1721, 1272, 1116, 1069, 711, 686.

\section{Synthesis of $1 \mathbf{r}$}



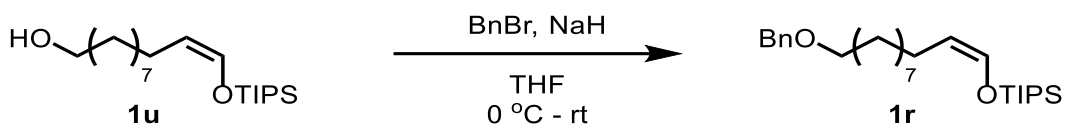

To the solution of $1 \mathbf{u}(0.34 \mathrm{~g}, 1.00 \mathrm{mmol})$ in THF was added $\mathrm{NaH}(60 \%, 60$ $\mathrm{mg}, 1.50 \mathrm{mmol})$ at $0{ }^{\circ} \mathrm{C}$. After stirred for $1 \mathrm{~h}$ at room temperature, $\mathrm{BnBr}(0.34 \mathrm{~g}$, $2.00 \mathrm{mmol}$ ) was added. The resulting mixture was then stirred for $12 \mathrm{~h}$, then quenched with $\mathrm{H}_{2} \mathrm{O}$, extracted with EtOAc, dried with $\mathrm{Na}_{2} \mathrm{SO}_{4}$, filtered and concentrated. The residue was purified by silica gel flash chromatography to afford compound $1 \mathrm{r}$ as a colorless oil in $51 \%$ yield $(0.19 \mathrm{~g}$, eluent: petroleum ether $/ \mathrm{EtOAc}=20 / 1)$.

$\mathbf{R}_{f}=0.80$ (petroleum ether/EtOAc $=10 / 1$ )

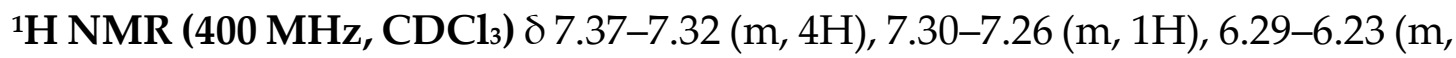
$1 \mathrm{H}), 4.50(\mathrm{~s}, 2 \mathrm{H}), 4.42-4.34(\mathrm{~m}, 1 \mathrm{H}), 3.46(\mathrm{t}, J=6.7 \mathrm{~Hz}, 2 \mathrm{H}), 2.14-2.04(\mathrm{~m}, 2 \mathrm{H})$, $1.64-1.59(\mathrm{~m}, 2 \mathrm{H}), 1.36-1.25(\mathrm{~m}, 12 \mathrm{H}), 1.19-1.02(\mathrm{~m}, 21 \mathrm{H})$.

${ }^{13} \mathrm{C}$ NMR (100 MHz, $\left.\mathrm{CDCl}_{3}\right) \delta$ 139.0, 138.8, 128.5, 127.8, 127.6, 110.2, 73.0, 70.7, 29.9, 29.8, 29.7, 29.64, 29.63, 29.5, 26.4, 23.7, 17.9, 12.1 .

HRMS (ESI') calcd for $\mathrm{C}_{27} \mathrm{H}_{48} \mathrm{O}_{2} \mathrm{NaSi}^{+}[\mathrm{M}+\mathrm{Na}]^{+}$: 455.3316, found: 455.3313 .

IR (neat, cm-1): 2926, 2857, 1461, 1096, 883, 736, 690.

\section{Synthesis of $1 \mathrm{~s}$}
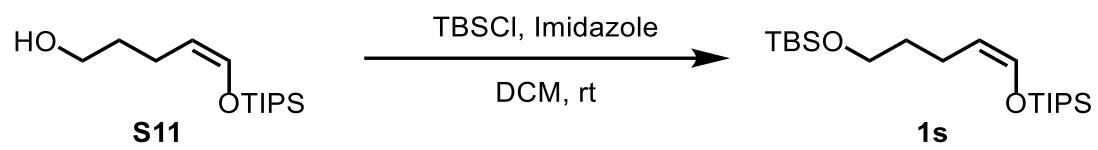

To the solution of $\mathbf{S 1 1}(0.31 \mathrm{~g}, 1.22 \mathrm{mmol})$, imidazole $(0.10 \mathrm{~g}, 1.46 \mathrm{mmol})$ in DCM was added TBSCl $(0.22 \mathrm{~g}, 1.46 \mathrm{mmol})$ at room temperature. The resulting mixture was then stirred for $0.5 \mathrm{~h}$, then quenched with saturated $\mathrm{NH}_{4} \mathrm{Cl}$, extracted with EtOAc, dried with $\mathrm{Na}_{2} \mathrm{SO}_{4}$, filtered and concentrated. The residue was purified by silica gel flash chromatography to afford compound $1 \mathrm{~s}$ as a colorless oil in $92 \%$ yield (0.42 g, eluent: petroleum ether/EtOAc $=50 / 1)$. $\mathbf{R}_{f}=0.80$ (petroleum ether $/$ EtOAc $=50 / 1$ )

${ }^{1} \mathrm{H}$ NMR (400 MHz, $\left.\mathrm{CDCl}_{3}\right) \delta 6.27(\mathrm{~d}, J=5.8 \mathrm{~Hz}, 1 \mathrm{H}), 4.45-4.35$ (m, 1H), 3.67$3.57(\mathrm{~m}, 2 \mathrm{H}), 2.20-2.07(\mathrm{~m}, 2 \mathrm{H}), 1.66-1.54(\mathrm{~m}, 2 \mathrm{H}), 1.20-1.01(\mathrm{~m}, 21 \mathrm{H}), 0.89$ (s, $9 \mathrm{H}), 0.05(\mathrm{~s}, 6 \mathrm{H})$.

${ }^{13} \mathrm{C}$ NMR (100 MHz, $\left.\mathrm{CDCl}_{3}\right) \delta$ 139.3, 109.4, 63.4, 33.1, 26.1, 20.2, 18.5, 17.9, 12.1, -5.1 . 
HRMS (ESI ${ }^{+}$) calcd for $\mathrm{C}_{20} \mathrm{H}_{44} \mathrm{O}_{2} \mathrm{NaSi}_{2}{ }^{+}[\mathrm{M}+\mathrm{Na}]^{+}$: 395.2772, found: 395.2770.

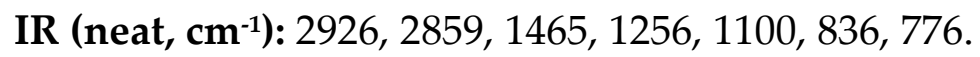

\section{Synthesis of $\mathbf{1 t}$}
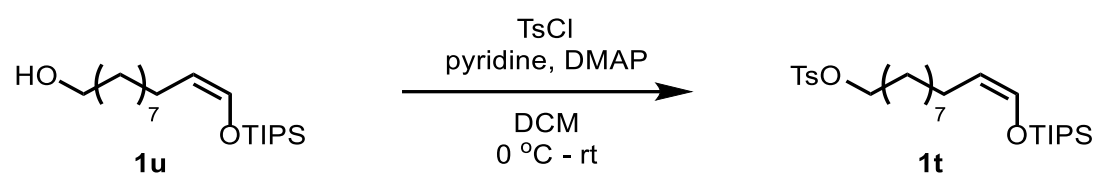

To the solution of $1 \mathbf{u}(0.40 \mathrm{~g}, 1.17 \mathrm{mmol})$, pyridine $(0.19 \mathrm{~g}, 1.75 \mathrm{mmol})$ and DMAP (14.7 mg, $0.12 \mathrm{mmol})$ in DCM was added TsCl $(0.33 \mathrm{~g}, 1.75 \mathrm{mmol})$ at room temperature. The resulting mixture was then stirred for $12 \mathrm{~h}$, then quenched with $\mathrm{H}_{2} \mathrm{O}$, extracted with EtOAc, dried with $\mathrm{Na}_{2} \mathrm{SO}_{4}$, filtered and concentrated. The residue was purified by silica gel flash chromatography to afford compound $\mathbf{1 t}$ as a colorless oil in $46 \%$ yield $(0.27 \mathrm{~g}$, eluent: petroleum ether $/ \mathrm{EtOAc}=30 / 1)$.

$\mathbf{R}_{f}=0.73$ (petroleum ether/EtOAc $=30 / 1$ )

${ }^{1} \mathrm{H}$ NMR (400 MHz, $\left.\mathrm{CDCl}_{3}\right) \delta 7.79(\mathrm{~d}, J=7.8 \mathrm{~Hz}, 2 \mathrm{H}), 7.34(\mathrm{~d}, J=8.0 \mathrm{~Hz}, 2 \mathrm{H})$, $6.26(\mathrm{~d}, J=5.8 \mathrm{~Hz}, 1 \mathrm{H}), 4.43-4.33(\mathrm{~m}, 1 \mathrm{H}), 4.07-3.96(\mathrm{~m}, 2 \mathrm{H}), 2.45(\mathrm{~s}, 3 \mathrm{H}), 2.15-$ $2.03(\mathrm{~m}, 2 \mathrm{H}), 1.68-1.56(\mathrm{~m}, 2 \mathrm{H}), 1.37-1.01(\mathrm{~m}, 33 \mathrm{H})$.

${ }^{13} \mathrm{C}$ NMR (100 MHz, $\left.\mathrm{CDCl}_{3}\right) \delta$ 144.7, 139.1, 133.5, 129.9, 128.0, 110.1, 70.8, 29.8, 29.5, 29.4, 29.1, 29.0, 25.5, 23.7, 21.8, 17.9, 12.1 .

HRMS (ESI ${ }^{+}$) calcd for $\mathrm{C}_{27} \mathrm{H}_{48} \mathrm{O}_{4} \mathrm{NaSSi}^{+}[\mathrm{M}+\mathrm{Na}]^{+}$: 519.2935, found: 519.2931.

IR (neat, cm$\left.^{-1}\right)$ : 2927, 2862, 1364, 1178, 1096, 663.

\section{Synthesis of $1 \mathrm{u}$}
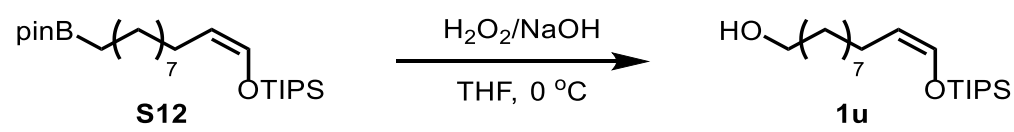

To the solution of $\mathbf{S 1 2}(2.26 \mathrm{~g}, 5.00 \mathrm{mmol})$ in THF was added $\mathrm{NaOH}$ (3.0 M, 1.5 equiv), $\mathrm{H}_{2} \mathrm{O}_{2}\left(30 \%, 1.5\right.$ equiv) at $0{ }^{\circ} \mathrm{C}$. The resulting mixture was stirred for $0.5 \mathrm{~h}$, then extracted with EtOAc, dried with $\mathrm{Na}_{2} \mathrm{SO}_{4}$, filtered and concentrated. The residue was purified by silica gel chromatography to afford compound $\mathbf{1} \mathbf{u}$ as a colorless oil in 95\% yield (1.63 g, eluent: petroleum ether/EtOAc $=20 / 1)$. $\mathbf{R}_{f}=0.35$ (petroleum ether/EtOAc $=10 / 1$ )

${ }^{1} \mathrm{H}$ NMR (400 MHz, $\left.\mathrm{CDCl}_{3}\right) \delta 6.26(\mathrm{~d}, J=5.8 \mathrm{~Hz}, 1 \mathrm{H}), 4.44-4.34(\mathrm{~m}, 1 \mathrm{H}), 3.63$ $(\mathrm{t}, J=6.7 \mathrm{~Hz}, 2 \mathrm{H}), 2.14-2.04(\mathrm{~m}, 2 \mathrm{H}), 1.62-1.52(\mathrm{~m}, 2 \mathrm{H}), 1.43-1.23(\mathrm{~m}, 13 \mathrm{H})$, 
$1.18-0.99(\mathrm{~m}, 21 \mathrm{H})$.

${ }^{13}$ C NMR (100 MHz, $\left.\mathrm{CDCl}_{3}\right) \delta$ 139.0, 110.1, 63.2, 33.0, 29.8, 29.7, 29.60, 29.58, 29.5, 25.9, 23.7, 17.9, 12.1 .

HRMS (ESI+) calcd for $\mathrm{C}_{20} \mathrm{H}_{43} \mathrm{O}_{2} \mathrm{Si}^{+}[\mathrm{M}+\mathrm{H}]^{+}: 343.3027$, found: 343.3018 .

IR (neat, $\mathrm{cm}^{-1}$ ): 2924, 2866, 2855, 1092, 882, 684, 666

\section{Synthesis of $1 \mathbf{v}^{17}$}
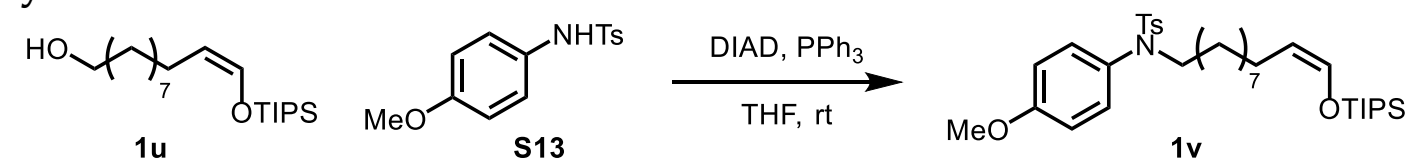

To the solution of $1 \mathbf{u}(0.51 \mathrm{~g}, 1.50 \mathrm{mmol}), \mathrm{S}^{13}{ }^{18}(0.83 \mathrm{~g}, 3.00 \mathrm{mmol})$, and $\mathrm{PPh}_{3}(1.14 \mathrm{~g}$, $4.35 \mathrm{mmol})$ in THF was added DIAD $(0.89 \mathrm{~g}, 4.50 \mathrm{mmol})$ at $0{ }^{\circ} \mathrm{C}$. The resulting mixture was then warmed up to room temperature and stirred for $15 \mathrm{~h}$, then quenched with saturated $\mathrm{NH}_{4} \mathrm{Cl}$, extracted with EtOAc, dried with $\mathrm{Na}_{2} \mathrm{SO}_{4}$, filtered and concentrated. The residue was purified by silica gel flash chromatography to afford compound $1 \mathbf{v}$ as a colorless oil in $81 \%$ yield ( 0.73 g, eluent: petroleum ether/EtOAc $=10 / 1)$.

$\mathbf{R}_{f}=0.50$ (petroleum ether/EtOAc $=10 / 1$ )

${ }^{1} \mathrm{H}$ NMR (400 MHz, $\left.\mathrm{CDCl}_{3}\right)$ \& 7.50-7.44 (m, 2H), $7.24(\mathrm{~d}, J=8.0 \mathrm{~Hz}, 2 \mathrm{H}), 6.96-$ $6.90(\mathrm{~m}, 2 \mathrm{H}), 6.84-6.78(\mathrm{~m}, 2 \mathrm{H}), 6.29-6.23(\mathrm{~m}, 1 \mathrm{H}), 4.43-4.33(\mathrm{~m}, 1 \mathrm{H}), 3.80(\mathrm{~s}, 3 \mathrm{H})$, 3.50-3.41 (m, 2H), $2.42(\mathrm{~s}, 3 \mathrm{H}), 2.12-2.02(\mathrm{~m}, 2 \mathrm{H}), 1.39-1.18(\mathrm{~m}, 14 \mathrm{H}), 1.18-1.01$ $(\mathrm{m}, 21 \mathrm{H})$.

${ }^{13} \mathrm{C}$ NMR (100 MHz, CDCl $)$ ) $\delta$ 159.0, 143.2, 139.0, 135.5, 131.8, 130.1, 129.4, 127.9, 114.2, 110.1, 55.5, 50.8, 29.8, 29.63, 29.56, 29.5, 29.3, 28.3, 26.6, 23.7, 21.7, 17.9, 12.1 . HRMS (ESI+) calcd for $\mathrm{C}_{34} \mathrm{H}_{55} \mathrm{NO}_{4} \mathrm{NaSSi}^{+}[\mathrm{M}+\mathrm{Na}]^{+}: 624.3513$, found: 624.3509 .

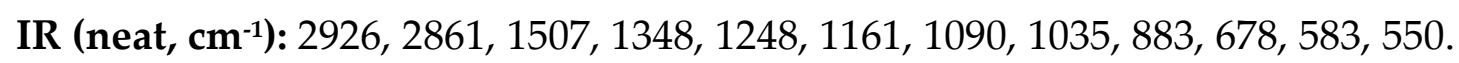

\section{(Z)-Triisopropyl(styryloxy)silane (1ac)}

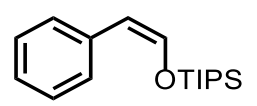

Compound 1ac was prepared as a colorless oil in 35\% yield (0.48 g, eluent: petroleum ether) from 2-phenylacetaldehyde ( $0.60 \mathrm{~g}, 5.00 \mathrm{mmol}), \mathrm{TIPSCl}$ (1.16 $\mathrm{g}, 6.00 \mathrm{mmol}), \mathrm{DBU}(0.91 \mathrm{~g}, 6.00 \mathrm{mmol})$ following the above general procedure A, which was previously reported ${ }^{19}$. $\mathbf{R}_{f}=0.85(Z), 0.68(E)$ (petroleum ether) 
${ }^{1} \mathrm{H}$ NMR (400 MHz, $\left.\mathrm{CDCl}_{3}\right) \delta 7.64(\mathrm{~d}, J=7.6 \mathrm{~Hz}, 2 \mathrm{H}), 7.35-7.25(\mathrm{~m}, 2 \mathrm{H}), 7.23$ $7.03(\mathrm{~m}, 1 \mathrm{H}), 6.50(\mathrm{~d}, J=6.4 \mathrm{~Hz}, 1 \mathrm{H}), 5.27(\mathrm{~d}, J=6.4 \mathrm{~Hz}, 1 \mathrm{H}), 1.32-1.18(\mathrm{~m}, 3 \mathrm{H})$, $1.16-1.05(\mathrm{~m}, 18 \mathrm{H})$.

${ }^{13} \mathrm{C}$ NMR (100 MHz, $\left.\mathrm{CDCl}_{3}\right) \delta$ 141.1, 136.3, 128.2, 128.0, 125.5, 108.5, 17.7, 11.8.

(Z)-Triisopropyl((4-methylstyryl)oxy)silane (1ad)<smiles></smiles>

Compound 1ad was prepared as a colorless oil in 53\% yield $(0.23 \mathrm{~g}$, eluent: petroleum ether) from $(Z)-((2$-bromovinyl)oxy)triisopropylsilane $(0.42 \mathrm{~g}, 1.50$ mmol), p-tolylboronic acid (0.41 g, $3.00 \mathrm{mmol}), \mathrm{PdCl}_{2}(\mathrm{dppf})(16.4 \mathrm{mg}, 0.023$ $\mathrm{mmol}$ ) following the above general procedure $\mathrm{C}$.

$\mathbf{R}_{f}=0.85(Z), 0.68(E)$ (petroleum ether)

${ }^{1} \mathbf{H}$ NMR (400 MHz, $\left.\mathrm{CDCl}_{3}\right) \delta 7.55(\mathrm{~d}, J=7.8 \mathrm{~Hz}, 2 \mathrm{H}), 7.10(\mathrm{~d}, J=7.8 \mathrm{~Hz}, 2 \mathrm{H})$, $6.46(\mathrm{~d}, J=5.4 \mathrm{~Hz}, 1 \mathrm{H}), 5.24(\mathrm{~d}, J=6.5 \mathrm{~Hz}, 1 \mathrm{H}), 2.32(\mathrm{~s}, 3 \mathrm{H}), 1.30-1.21(\mathrm{~m}, 3 \mathrm{H})$, $1.17-1.07(\mathrm{~m}, 18 \mathrm{H})$.

${ }^{13} \mathrm{C}$ NMR (100 MHz, $\left.\mathrm{CDCl}_{3}\right) \delta 140.5,135.2,133.6,128.9,128.3,108.5,21.3,17.9$, 12.0 .

HRMS (ESI ${ }^{+}$) calcd for $\mathrm{C}_{18} \mathrm{H}_{31} \mathrm{OSi}^{+}[\mathrm{M}+\mathrm{H}]^{+}:$291.2139, found: 291.2138.

IR (neat, cm-1): 2944, 2867, 1643, 1464, 1422, 1259, 1085, 1069, 995, 885, 823, 675.

(Z)-Triisopropyl((2-methylstyryl)oxy)silane (1ae)<smiles>Cc1ccccc1/C=C\O[SnH3]</smiles>

Compound 1ae was prepared as a colorless oil in $42 \%$ yield $(0.33 \mathrm{~g}$, eluent: petroleum ether) from 2-(o-tolyl)acetaldehyde ${ }^{20}(0.36 \mathrm{~g}, 2.70 \mathrm{mmol})$, TIPSCl $(0.62 \mathrm{~g}, 3.24 \mathrm{mmol}), \mathrm{DBU}(0.49 \mathrm{~g}, 3.24 \mathrm{mmol})$ following the above general procedure A.

$\mathbf{R}_{f}=0.85(Z), 0.68(E)$ (petroleum ether)

${ }^{1} \mathbf{H}$ NMR (400 MHz, $\left.\mathrm{CDCl}_{3}\right) \delta 8.07$ (d, $\left.J=7.9 \mathrm{~Hz}, 1 \mathrm{H}\right), 7.17-7.08$ (m, 2H), 7.07$6.99(\mathrm{~m}, 1 \mathrm{H}), 6.56(\mathrm{~d}, J=6.7 \mathrm{~Hz}, 1 \mathrm{H}), 5.37(\mathrm{~d}, J=6.8 \mathrm{~Hz}, 1 \mathrm{H}), 2.30(\mathrm{~s}, 3 \mathrm{H}), 1.28-$ $1.17(\mathrm{~m}, 3 \mathrm{H}), 1.16-1.05(\mathrm{~m}, 18 \mathrm{H})$.

${ }^{13} \mathrm{C}$ NMR (100 MHz, $\left.\mathrm{CDCl}_{3}\right) \delta$ 140.9, 134.8, 134.6, 129.9, 129.3, 125.7, 125.6, 105.5, $20.3,17.9,12.0$. 
HRMS (ESI ${ }^{+}$) calcd for $\mathrm{C}_{18} \mathrm{H}_{30} \mathrm{NaOSi}^{+}[\mathrm{M}+\mathrm{Na}]^{+}:$313.1958, found: 313.1957.

IR (neat, cm-1): 2944, 2867, 1636, 1462, 1264, 1220, 1108, 1084, 1069, 994, 883, 775, $729,673,489$.

\section{(Z)-Triisopropyl((4-methoxy-3,5-dimethylstyryl)oxy)silane (1af)}<smiles>COc1c(C)cc(/C=C\OC(=O)OCc2ccccc2)cc1C</smiles>

Compound 1af was prepared as a colorless oil in $40 \%$ yield $(0.40 \mathrm{~g}$, eluent: petroleum ether/Et $\left.t_{2} \mathrm{O}=100 / 3\right)$ from $(Z)-((2$-bromovinyl)oxy)triisopropylsilane (0.83 g, $3.00 \mathrm{mmol})$, 2-(4-methoxy-3,5-dimethylphenyl)-4,4,5,5-tetramethyl1,3,2-dioxaborolane ${ }^{21}$ (1.57 g, $\left.6.00 \mathrm{mmol}\right), \mathrm{PdCl}_{2}(\mathrm{dppf})(32.9 \mathrm{mg}, 0.045 \mathrm{mmol})$ following the above general procedure $C$.

$\mathbf{R}_{f}=0.65$ (petroleum ether $/ \mathrm{Et}_{2} \mathrm{O}=20 / 1$ )

${ }^{1} \mathrm{H}$ NMR (400 MHz, $\left.\mathrm{CDCl}_{3}\right) \delta 7.35$ (s, 2H), $6.43(\mathrm{~d}, J=6.5 \mathrm{~Hz}, 1 \mathrm{H}), 5.16(\mathrm{~d}, J=6.5$ $\mathrm{Hz}, 1 \mathrm{H}), 3.70$ (s, 3H), 2.25 (s, 6H), 1.30-1.20 (m, 3H), 1.19-1.09 (m, 18H).

${ }^{13} \mathrm{C}$ NMR (100MHz, $\left.\mathrm{CDCl}_{3}\right) \delta$ 155.0, 140.2, 132.1, 130.3, 128.9, 108.2, 59.8, 17.9, $16.2,12.0$.

HRMS (ESI+) calcd for $\mathrm{C}_{20} \mathrm{H}_{34} \mathrm{NaO}_{2} \mathrm{Si}^{+}[\mathrm{M}+\mathrm{Na}]^{+}$: 357.2220, found: 357.2219.

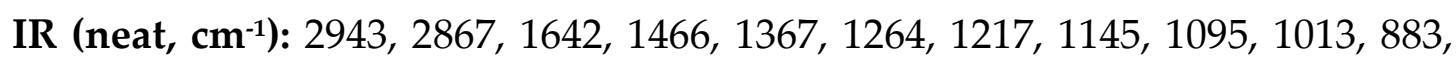
$858,687$.

(Z)-N,N-Dimethyl-4-(2-((triisopropylsilyl)oxy)vinyl)aniline (1ag)<smiles>CN(C)c1ccc(/C=C\[OH+][PH3])cc1</smiles>

Compound 1ag was prepared as a colorless oil in $26 \%$ yield $(0.17 \mathrm{~g}$, eluent: petroleum ether/EtOAc = 100/3) from (Z)-((2bromovinyl)oxy)triisopropylsilane (0.56 g, $2.0 \mathrm{mmol}), N, N$-dimethyl-4-(4,4,5,5tetramethyl-1,3,2-dioxaborolan-2-yl)aniline ${ }^{22}$ (0.99 g, $\left.4.00 \mathrm{mmol}\right), \mathrm{PdCl}_{2}(\mathrm{dppf})$ (21.9 $\mathrm{mg}, 0.030 \mathrm{mmol}$ ) following the above general procedure $\mathrm{C}$.

$\mathbf{R}_{f}=0.62$ (petroleum ether $/ \mathrm{Et}_{2} \mathrm{O}=20 / 1$ )

${ }^{1} \mathrm{H}$ NMR (400 MHz, $\left.\mathrm{CDCl}_{3}\right) \delta 7.56(\mathrm{~d}, J=8.8 \mathrm{~Hz}, 2 \mathrm{H}), 6.69(\mathrm{~d}, J=8.9 \mathrm{~Hz}, 2 \mathrm{H})$, $6.37(\mathrm{~d}, J=6.5 \mathrm{~Hz}, 1 \mathrm{H}), 5.18(\mathrm{~d}, J=6.5 \mathrm{~Hz}, 1 \mathrm{H}), 2.93(\mathrm{~s}, 6 \mathrm{H}), 1.31-1.19(\mathrm{~m}, 3 \mathrm{H})$, $1.18-1.05(\mathrm{~m}, 18 \mathrm{H})$. 
${ }^{13} \mathrm{C}$ NMR (100 MHz, $\left.\mathrm{CDCl}_{3}\right) \delta$ 148.7, 138.4, 129.3, 125.6, 112.7, 108.5, 40.9, 17.9, 12.1 .

HRMS (ESI ${ }^{+}$) calcd for $\mathrm{C}_{19} \mathrm{H}_{33} \mathrm{NNaOSi}^{+}[\mathrm{M}+\mathrm{Na}]^{+}: 342.2224$, found: 342.2223 .

IR (neat, cm ${ }^{-1}$ ): 2943, 2866, 1643, 1610, 1519, 1348, 1260, 1163, 1083, 1065, 884, $819,690,657,628$.

\section{(Z)-Triisopropyl((4-methoxystyryl)oxy)silane (1ah)}<smiles></smiles>

Compound 1ah was prepared as a colorless oil in 56\% yield ( $0.86 \mathrm{~g}$, eluent: petroleum ether/ $\mathrm{Et}_{2} \mathrm{O}=100 / 3$ to 20/1) from (Z)-((2bromovinyl)oxy)triisopropylsilane (1.39 g, $5.00 \mathrm{mmol})$, 2-(4-methoxyphenyl)4,4,5,5-tetramethyl-1,3,2-dioxaborolane ${ }^{23}$ (2.34 g, $\left.10.00 \mathrm{mmol}\right), \mathrm{PdCl}_{2}$ (dppf) (54.8 $\mathrm{mg}, 0.075 \mathrm{mmol})$ following the above general procedure C.

$\mathbf{R}_{f}=0.65$ (petroleum ether $/ \mathrm{Et}_{2} \mathrm{O}=20 / 1$ )

${ }^{1} \mathrm{H}$ NMR (400 MHz, $\left.\mathrm{CDCl}_{3}\right) \delta 7.59(\mathrm{~d}, J=8.8 \mathrm{~Hz}, 2 \mathrm{H}), 6.83(\mathrm{~d}, J=8.8 \mathrm{~Hz}, 2 \mathrm{H})$, $6.42(\mathrm{~d}, J=6.6 \mathrm{~Hz}, 1 \mathrm{H}), 5.22(\mathrm{~d}, J=6.5 \mathrm{~Hz}, 1 \mathrm{H}), 3.79(\mathrm{~s}, 3 \mathrm{H}), 1.32-1.18(\mathrm{~m}, 3 \mathrm{H})$, $1.18-1.05(\mathrm{~m}, 18 \mathrm{H})$.

${ }^{13} \mathrm{C}$ NMR (100 MHz, $\left.\mathrm{CDCl}_{3}\right) \delta$ 157.6, 139.5, 129.5, 129.4, 113.6, 108.1, 55.3, 17.9, 12.0 .

HRMS (ESI') calcd for $\mathrm{C}_{18} \mathrm{H}_{30} \mathrm{NaO}_{2} \mathrm{Si}^{+}[\mathrm{M}+\mathrm{Na}]^{+}$: 329.1907, found: 329.1906.

IR (neat, cm-1): 2942, 2866, 1644, 1607, 1510, 1462, 1241, 1176, 1083, 1068, 1036, $883,832,807,708,671$.

(Z)-((4-(Benzyloxy)styryl)oxy)triisopropylsilane (1ai)

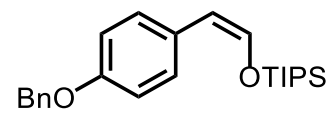

Compound 1ai was prepared as a colorless oil in 73\% yield ( $0.70 \mathrm{~g}$, eluent: petroleum ether/ $\mathrm{Et}_{2} \mathrm{O}=100 / 3$ to 20/1) from (Z)-((2bromovinyl)oxy)triisopropylsilane (0.70 g, $2.5 \mathrm{mmol})$, 2-(4-(benzyloxy)phenyl)4,4,5,5-tetramethyl-1,3,2-dioxaborolane ${ }^{24}$ (1.55 g, $\left.5.00 \mathrm{mmol}\right), \mathrm{PdCl}_{2}(\mathrm{dppf})$ (27.4 $\mathrm{mg}, 0.038 \mathrm{mmol}$ ) following the above general procedure C.

$\mathbf{R}_{f}=0.75$ (petroleum ether/ $\mathrm{Et}_{2} \mathrm{O}=20 / 1$ ) 
${ }^{1} \mathbf{H}$ NMR (400 MHz, $\left.\mathrm{CDCl}_{3}\right) \delta 7.59$ (d, $\left.J=8.8 \mathrm{~Hz}, 2 \mathrm{H}\right), 7.46-7.41$ (m, 2H), 7.40$7.34(\mathrm{~m}, 2 \mathrm{H}), 7.34-7.28(\mathrm{~m}, 1 \mathrm{H}), 6.97-6.86(\mathrm{~m}, 2 \mathrm{H}), 6.42(\mathrm{~d}, J=6.6 \mathrm{~Hz}, 1 \mathrm{H}), 5.22$ $(\mathrm{d}, J=6.6 \mathrm{~Hz}, 1 \mathrm{H}), 5.06(\mathrm{~s}, 2 \mathrm{H}), 1.31-1.19(\mathrm{~m}, 3 \mathrm{H}), 1.18-1.07(\mathrm{~m}, 18 \mathrm{H})$.

${ }^{13} \mathrm{C}$ NMR (100 MHz, $\left.\mathrm{CDCl}_{3}\right) \delta$ 156.9, 139.6, 137.4, 129.64, 129.56, 128.7, 128.0, 127.6, 114.6, 108.1, 70.1, 17.9, 12.0 .

HRMS (ESI') calcd for $\mathrm{C}_{24} \mathrm{H}_{34} \mathrm{NaO}_{2} \mathrm{Si}^{+}[\mathrm{M}+\mathrm{Na}]^{+}:$405.2220, found: 405.2219 .

IR (neat, cm ${ }^{-1}$ ): 2943, 2866, 1643, 1508, 1461, 1234, 1174, 1084, 1017, 884, 832, 727, 677,630 .

(Z)-((3-(1,3-Dioxolan-2-yl)styryl)oxy)triisopropylsilane (1aj)

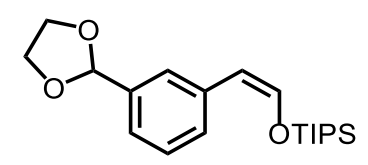

Compound 1aj was prepared as a colorless oil in 34\% yield ( $0.19 \mathrm{~g}$, eluent: petroleum ether/EtOAc = 100/3) from (Z)-((2bromovinyl)oxy)triisopropylsilane $(0.44 \mathrm{~g}, 1.60 \mathrm{mmol}), 2-(3-(1,3-$ dioxolan-2yl)phenyl)- 4,4,5,5-tetramethyl-1,3,2-dioxaborolane $(0.88 \mathrm{~g}$, $3.20 \mathrm{mmol})$, $\mathrm{PdCl}_{2}$ (dppf) (17.5 mg, $0.024 \mathrm{mmol}$ ) following the above general procedure $\mathrm{C}$.

$\mathbf{R}_{f}=0.65$ (petroleum ether $/ \mathrm{EtOAc}=20 / 1$ )

${ }^{1} \mathbf{H}$ NMR (400 MHz, C6 $\left.\mathrm{D}_{6}\right) \delta 8.19(\mathrm{~s}, 1 \mathrm{H}), 7.71(\mathrm{~d}, J=7.7 \mathrm{~Hz}, 1 \mathrm{H}), 7.42(\mathrm{~d}, J=7.7$ $\mathrm{Hz}, 1 \mathrm{H}), 7.30-7.21(\mathrm{~m}, 1 \mathrm{H}), 6.39(\mathrm{~d}, J=6.5 \mathrm{~Hz}, 1 \mathrm{H}), 5.88(\mathrm{~s}, 1 \mathrm{H}), 5.34(\mathrm{~d}, J=6.6$ $\mathrm{Hz}, 1 \mathrm{H}), 3.70-3.58(\mathrm{~m}, 2 \mathrm{H}), 3.57-3.43(\mathrm{~m}, 2 \mathrm{H}), 1.11-1.02(\mathrm{~m}, 21 \mathrm{H})$.

${ }^{13} \mathrm{C}$ NMR (100 MHz, C6 $\left.\mathrm{D}_{6}\right) \delta$ 141.3, 139.1, 136.8, 129.3, 128.3, 127.2, 124.5, 109.4, 104.3, 65.1, 17.9, 12.1 .

HRMS (ESI+) calcd for $\mathrm{C}_{20} \mathrm{H}_{33} \mathrm{O}_{3} \mathrm{Si}^{+}[\mathrm{M}+\mathrm{H}]^{+}: 349.2193$, found: 349.2193 .

IR (neat, cm ${ }^{-1}$ ): 2945, 2867, 1640, 1464, 1262, 1167, 1074, 993, 882, 801, 680.

(Z)-((4-Fluorostyryl)oxy)triisopropylsilane (1al)<smiles>Fc1ccc(/C=C\[OH+][SnH3])cc1</smiles>

Compound 1al was prepared as a colorless oil in $49 \%$ yield ( $0.55 \mathrm{~g}$, eluent: petroleum ether) from 2-(4-fluorophenyl)acetaldehyde ${ }^{25}(0.53 \mathrm{~g}, 3.76 \mathrm{mmol})$, TIPSCl (0.87 g, $4.50 \mathrm{mmol})$, DBU (0.69 $\mathrm{g}, 4.50 \mathrm{mmol})$ following the above general procedure A. 
$\mathbf{R}_{f}=0.85(Z), 0.68(E)$ (petroleum ether)

${ }^{1} \mathrm{H}$ NMR (400 MHz, $\left.\mathrm{CDCl}_{3}\right) \delta$ 7.67-7.53 (m, 2H), 7.04-6.88 (m, 2H), $6.46(\mathrm{~d}, J=$ $6.5 \mathrm{~Hz}, 1 \mathrm{H}), 5.23(\mathrm{~d}, J=6.5 \mathrm{~Hz}, 1 \mathrm{H}), 1.31-1.18(\mathrm{~m}, 3 \mathrm{H}), 1.17-1.01(\mathrm{~m}, 18 \mathrm{H})$.

${ }^{13} \mathrm{C}$ NMR (100 MHz, $\left.\mathrm{CDCl}_{3}\right) \delta 160.9$ (d, $\left.J=244.7 \mathrm{~Hz}\right), 140.7$ (d, J=2.3 Hz), 132.6 $(\mathrm{d}, J=3.3 \mathrm{~Hz}), 129.8(\mathrm{~d}, J=7.5 \mathrm{~Hz}), 114.9(\mathrm{~d}, J=21.2 \mathrm{~Hz}), 107.6,17.8,12.0$.

${ }^{19}$ F NMR (377 MHz, $\mathrm{CDCl}_{3}$ ) $\delta-116.7(\mathrm{~m})$.

HRMS (ESI') calcd for $\mathrm{C}_{17} \mathrm{H}_{28} \mathrm{FOSi}^{+}[\mathrm{M}+\mathrm{H}]^{+}:$295.1888, found: 295.1887.

IR (neat, cm-1): 2945, 2868, 1645, 1507, 1260, 1224, 1085, 1068, 884, 836, 714, 670, 625.

(Z)-((4-Chlorostyryl)oxy)triisopropylsilane (1am)<smiles></smiles>

Compound 1am was prepared as a colorless oil in $42 \%$ yield ( $0.18 \mathrm{~g}$, eluent: petroleum ether) from 2-(4-chlorophenyl)acetaldehyde ${ }^{26}(0.22 \mathrm{~g}, 1.40 \mathrm{mmol})$, TIPSCl (0.32 g, $1.68 \mathrm{mmol}), \mathrm{DBU}(0.26 \mathrm{~g}, 1.68 \mathrm{mmol})$ following the above general procedure A.

$\mathbf{R}_{f}=0.85(Z), 0.68(E)$ (petroleum ether)

${ }^{1} \mathrm{H}$ NMR (400 MHz, $\left.\mathrm{CDCl}_{3}\right) \delta 7.57(\mathrm{~d}, J=8.6 \mathrm{~Hz}, 2 \mathrm{H}), 7.23(\mathrm{~d}, J=8.5 \mathrm{~Hz}, 2 \mathrm{H})$, $6.50(\mathrm{~d}, J=6.5 \mathrm{~Hz}, 1 \mathrm{H}), 5.23(\mathrm{~d}, J=6.5 \mathrm{~Hz}, 1 \mathrm{H}), 1.31-1.18(\mathrm{~m}, 3 \mathrm{H}), 1.16-1.04(\mathrm{~m}$, $18 \mathrm{H})$.

${ }^{13} \mathrm{C}$ NMR (100 MHz, $\left.\mathrm{CDCl}_{3}\right) \delta$ 141.7, 134.9, 131.0, 129.6, 128.3, 107.6, 17.8, 12.0. HRMS (ESI') calcd for $\mathrm{C}_{17} \mathrm{H}_{28} \mathrm{ClOSi}^{+}[\mathrm{M}+\mathrm{H}]^{+}$: 311.1592, found: 311.1592 .

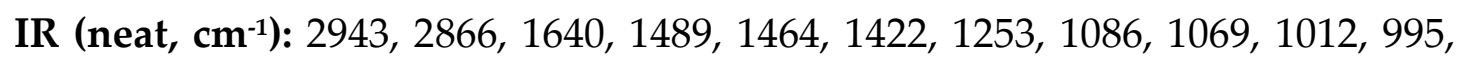
$883,831,776,682,600$.

(Z)-((4-Bromostyryl)oxy)triisopropylsilane (1an)<smiles></smiles>

Compound 1an was prepared as a colorless oil in $44 \%$ yield (1.17 g, eluent: petroleum ether) from 2-(4-bromophenyl)acetaldehyde (1.48 g, $7.50 \mathrm{mmol})$, TIPSCl (1.74 g, $9.00 \mathrm{mmol})$, DBU (1.37 g, $9.00 \mathrm{mmol})$ following the above general procedure $\mathrm{A}$. 
$\mathbf{R}_{f}=0.85(Z), 0.68(E)$ (petroleum ether)

${ }^{1} \mathrm{H}$ NMR (400 MHz, $\left.\mathrm{CDCl}_{3}\right) \delta 7.51(\mathrm{~d}, J=8.5 \mathrm{~Hz}, 2 \mathrm{H}), 7.39(\mathrm{~d}, J=8.7 \mathrm{~Hz}, 2 \mathrm{H})$, $6.52(\mathrm{~d}, J=6.5 \mathrm{~Hz}, 1 \mathrm{H}), 5.22(\mathrm{~d}, J=6.5 \mathrm{~Hz}, 1 \mathrm{H}), 1.31-1.19(\mathrm{~m}, 3 \mathrm{H}), 1.19-0.88(\mathrm{~m}$, $18 \mathrm{H})$.

${ }^{13} \mathrm{C}$ NMR (100 MHz, $\left.\mathrm{CDCl}_{3}\right) \delta$ 141.9, 135.3, 131.2, 129.9, 119.0, 107.6, 17.8, 11.9.

HRMS (ESI ${ }^{+}$) calcd for $\mathrm{C}_{17} \mathrm{H}_{28} \mathrm{BrOSi}^{+}[\mathrm{M}+\mathrm{H}]^{+}$: 355.1087, found: 355.1087 .

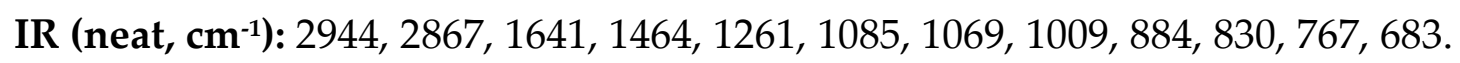

(Z)-Triisopropyl((4-(trifluoromethyl)styryl)oxy)silane (1ap)<smiles>FC(F)(F)c1ccc(/C=C\CO[SnH3])cc1</smiles>

Compound 1ap was prepared as a colorless oil in $28 \%$ yield $(0.17 \mathrm{~g}$, eluent: petroleum ether) from 2-(4-(trifluoromethyl)phenyl)acetaldehyde ${ }^{27}(0.34 \mathrm{~g}, 1.80$ $\mathrm{mmol}), \operatorname{TIPSCl}(0.42 \mathrm{~g}, 2.16 \mathrm{mmol}), \mathrm{DBU}(0.33 \mathrm{~g}, 2.16 \mathrm{mmol})$ following the above general procedure $\mathrm{A}$.

$\mathbf{R}_{f}=0.85(Z), 0.68(E)$ (petroleum ether)

${ }^{1} \mathrm{H}$ NMR (400 MHz, $\left.\mathrm{CDCl}_{3}\right) \delta 7.73(\mathrm{~d}, J=7.9 \mathrm{~Hz}, 2 \mathrm{H}), 7.52(\mathrm{~d}, J=8.2 \mathrm{~Hz}, 2 \mathrm{H})$, $6.59(\mathrm{~d}, J=6.5 \mathrm{~Hz}, 1 \mathrm{H}), 5.31(\mathrm{~d}, J=6.5 \mathrm{~Hz}, 1 \mathrm{H}), 1.32-1.21(\mathrm{~m}, 3 \mathrm{H}), 1.19-1.04(\mathrm{~m}$, $18 \mathrm{H})$.

${ }^{13} \mathrm{C}$ NMR (100 MHz, CDCl $)$ \& 143.4, $140.0(\mathrm{~d}, J=1.5 \mathrm{~Hz}), 128.3,127.3(\mathrm{q}, J=32.1$

$\mathrm{Hz}), 125.1(\mathrm{q}, J=3.9 \mathrm{~Hz}), 124.6(\mathrm{q}, J=269 \mathrm{~Hz}) 107.5,17.8,12.0$.

${ }^{19} \mathrm{~F}$ NMR (377 MHz, $\left.\mathrm{CDCl}_{3}\right) \delta$ - 62.3 .

HRMS (ESI') calcd for $\mathrm{C}_{18} \mathrm{H}_{28} \mathrm{~F}_{3} \mathrm{OSi}^{+}[\mathrm{M}+\mathrm{H}]^{+}:$345.1856, found: 345.1856 .

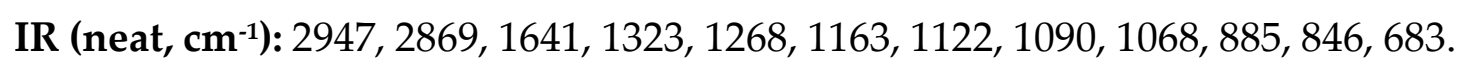

\section{(Z)-((5-Fluoro-2-methoxystyryl)oxy)triisopropylsilane (1ar)}<smiles>COc1ccc(OC)c(F)c1</smiles>

Compound 1ar was prepared as a colorless solid in $24 \%$ yield $(0.15 \mathrm{~g}$, eluent: petroleum ether/EtOAc $=20 / 1)$ from $(Z)-((2$-bromovinyl $)$ oxy $)$ triisopropylsilane $(0.53 \mathrm{~g}, 1.90 \mathrm{mmol})$, (5-fluoro-2-methoxyphenyl)boronic acid $(0.65 \mathrm{~g}, 3.80$ $\mathrm{mmol}), \mathrm{PdCl}_{2}(\mathrm{dppf})(20.8 \mathrm{mg}, 0.029 \mathrm{mmol})$ following the above general 
procedure C.

$\mathbf{R}_{f}=0.75$ (petroleum ether $/$ EtOAc $=20 / 1$ )

${ }^{1} \mathrm{H}$ NMR (400 MHz, CDCl$)$ ) 8 8.00-7.90 (m, 1H), 6.87-6.68 (m, 2H), 6.58 (d, J = $6.7 \mathrm{~Hz}, 1 \mathrm{H}), 5.68(\mathrm{~d}, J=6.7 \mathrm{~Hz}, 1 \mathrm{H}), 3.79(\mathrm{~s}, 3 \mathrm{H}), 1.32-1.20(\mathrm{~m}, 3 \mathrm{H}), 1.17-1.04(\mathrm{~m}$, $18 \mathrm{H})$.

${ }^{13} \mathrm{C}$ NMR (100 MHz, $\left.\mathrm{CDCl}_{3}\right) \delta 157.1(\mathrm{~d}, J=235.8 \mathrm{~Hz}), 151.9$ (d, $\left.J=1.9 \mathrm{~Hz}\right), 142.2$, $126.6(\mathrm{~d}, J=8.9 \mathrm{~Hz}), 116.3(\mathrm{~d}, J=25.0 \mathrm{~Hz}), 112.3(\mathrm{~d}, J=23.4 \mathrm{~Hz}), 111.1(\mathrm{~d}, J=8.7$ $\mathrm{Hz}), 100.9(\mathrm{~d}, J=2.2 \mathrm{~Hz}), 56.3,17.8,12.0$.

${ }^{19}$ F NMR (377 MHz, $\mathrm{CDCl}_{3}$ ) $\delta$-124.2.

HRMS (ESI ${ }^{+}$) calcd for $\mathrm{C}_{18} \mathrm{H}_{30} \mathrm{FO}_{2} \mathrm{Si}^{+}[\mathrm{M}+\mathrm{H}]^{+}:$325.1994, found: 325.1993 .

IR (neat, cm cm: $^{-1}$ 2945, 2868, 1637, 1485, 1465, 1266, 1250, 1201, 1180, 1154, 1067, 966, 882, 749, 706, 677.

(Z)-1-Methyl-5-(2-((triisopropylsilyl)oxy)vinyl)-1H-indole (1as)<smiles></smiles>

Compound 1as was prepared as a colorless oil in 32\% yield (0.53 g, eluent: petroleum ether) from $(Z)-((2-$ bromovinyl)oxy)triisopropylsilane (1.39 $\mathrm{g}, 5.00$ mmol), 1-methyl-5-(4,4,5,5-tetramethyl-1,3,2-dioxaborolan-2-yl)-1H-indole ${ }^{28}$ (2.57 g, $10.00 \mathrm{mmol}), \mathrm{PdCl}_{2}$ (dppf) (54.8 mg, $0.075 \mathrm{mmol}$ ) following the above general procedure $C$.

$\mathbf{R}_{f}=0.82$ (petroleum ether)

${ }^{1} \mathrm{H}$ NMR (400 MHz, CDCl $)$ d $7.94(\mathrm{~s}, 1 \mathrm{H}), 7.59(\mathrm{~d}, J=8.6 \mathrm{~Hz}, 1 \mathrm{H}), 7.28-7.16(\mathrm{~m}$, $1 \mathrm{H}), 6.97(\mathrm{~d}, J=3.0 \mathrm{~Hz}, 1 \mathrm{H}), 6.45(\mathrm{~d}, J=6.6 \mathrm{~Hz}, 1 \mathrm{H}), 6.41(\mathrm{~d}, J=3.0 \mathrm{~Hz}, 1 \mathrm{H}), 5.38$ $(\mathrm{d}, J=6.5 \mathrm{~Hz}, 1 \mathrm{H}), 3.74(\mathrm{~s}, 3 \mathrm{H}), 1.33-1.21(\mathrm{~m}, 3 \mathrm{H}), 1.19-1.09(\mathrm{~m}, 18 \mathrm{H})$.

${ }^{13} \mathrm{C}$ NMR (100 MHz, $\mathrm{CDCl}_{3}$ ) $\delta$ 138.8, 135.4, 128.9, 128.6, 128.0, 123.1, 120.7, 109.7, 108.7, 101.1, 32.9, 18.0, 12.1 .

HRMS (ESI ${ }^{+}$) calcd for $\mathrm{C}_{20} \mathrm{H}_{32} \mathrm{NOSi}^{+}[\mathrm{M}+\mathrm{H}]^{+}: 330.2248$, found: 330.2247 .

IR (neat, cm$^{-1}$ ) 2942, 2866, 1642, 1464, 1413, 1296, 1261, 1145, 1103, 1064, 880, 804, 684 .

(Z)-((2-(Furan-3-yl)vinyl)oxy)triisopropylsilane (1at)<smiles>C[AsH2]OCc1ccoc1</smiles> 
Compound 1at was prepared as a colorless oil in 31\% yield $(0.33 \mathrm{~g}$, eluent: petroleum ether) from $(Z)-((2$-bromovinyl)oxy)triisopropylsilane $(1.11 \mathrm{~g}, 4.00$ mmol), furan-3-ylboronic acid ( $0.90 \mathrm{~g}, 8.00 \mathrm{mmol}), \mathrm{PdCl}_{2}(\mathrm{dppf})(43.9 \mathrm{mg}, 0.060$ $\mathrm{mmol}$ ) following the above general procedure $\mathrm{C}$.

$\mathbf{R}_{f}=0.85$ (petroleum ether)

${ }^{1} \mathrm{H}$ NMR (400 MHz, $\left.\mathrm{CDCl}_{3}\right)$ $\delta$ 7.44-7.39 (m, 1H), 7.33-7.30 (m, 1H), 7.23-7.19 (m, $1 \mathrm{H}), 6.44(\mathrm{~d}, J=6.2 \mathrm{~Hz}, 1 \mathrm{H}), 5.41(\mathrm{~d}, J=6.3 \mathrm{~Hz}, 1 \mathrm{H}), 1.33-1.19(\mathrm{~m}, 3 \mathrm{H}), 1.17-1.06$ (m, 18H).

${ }^{13} \mathrm{C}$ NMR (100 MHz, $\mathrm{CDCl}_{3}$ ) $\delta$ 140.0, 136.9, 128.6, 124.3, 121.2, 103.6, 17.9, 12.0.

HRMS (ESI+) calcd for $\mathrm{C}_{15} \mathrm{H}_{27} \mathrm{O}_{2} \mathrm{Si}^{+}[\mathrm{M}+\mathrm{H}]^{+}:$267.1775, found: 267.1774.

IR (neat, cm-1): 2945, 2867, 1650, 1464, 1262, 1096, 1059, 1019, 881, 787, 689, 648.

(Z)-Triisopropyl((2-(6-methoxynaphthalen-2-yl)vinyl)oxy)silane (1av)

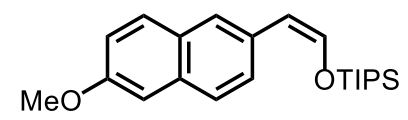

Compound 1av was prepared as a colorless oil in $43 \%$ yield $(0.31 \mathrm{~g}$, eluent: petroleum ether/EtOAc $=20 / 1)$ from $(Z)-((2$-bromovinyl $)$ oxy $)$ triisopropylsilane (0.56 g, $2.00 \mathrm{mmol}), 2-(6-m e t h o x y n a p h t h a l e n-2-y l)-4,4,5,5$-tetramethyl-1,3,2dioxaborolane $^{29}(1.14 \mathrm{~g}, 4.00 \mathrm{mmol}), \mathrm{PdCl}_{2}(\mathrm{dppf})(21.9 \mathrm{mg}, 0.030 \mathrm{mmol})$ following the above general procedure $\mathrm{C}$.

$\mathbf{R}_{f}=0.55$ (petroleum ether $/ \mathrm{EtOAc}=20 / 1$ )

${ }^{1} \mathrm{H}$ NMR (400 MHz, CDCl $)$ d $8.11(\mathrm{~s}, 1 \mathrm{H}), 7.77-7.69(\mathrm{~m}, 1 \mathrm{H}), 7.67-7.60(\mathrm{~m}, 2 \mathrm{H})$, 7.14-7.05 (m, 2H), $6.55(\mathrm{~d}, J=6.5 \mathrm{~Hz}, 1 \mathrm{H}), 5.39(\mathrm{~d}, J=6.5 \mathrm{~Hz}, 1 \mathrm{H}), 3.90(\mathrm{~s}, 3 \mathrm{H})$, $1.32-1.24(\mathrm{~m}, 3 \mathrm{H}), 1.20-1.10(\mathrm{~m}, 18 \mathrm{H})$.

${ }^{13} \mathrm{C}$ NMR (100 MHz, CDCl $) \delta$ d 157.4, 141.0, 133.0, 131.9, 129.5, 129.3, 127.9, 126.6, 126.3, 118.6, 108.8, 105.8, 55.4, 17.9, 12.0 .

HRMS (ESI') calcd for $\mathrm{C}_{22} \mathrm{H}_{33} \mathrm{O}_{2} \mathrm{Si}^{+}[\mathrm{M}+\mathrm{H}]^{+}:$357.2244, found: 357.2243 .

IR (neat, cm ${ }^{-1}$ ): 2943, 2866, 1640, 1603, 1463, 1267, 1217, 1170, 1084, 1067, 1034, $883,853,685$.

(E/Z)-2-(7-((Triisopropylsilyl)oxy)hept-6-en-1-yl)pyridine (1aw)<smiles>COC=CCCCCCCc1ccccn1</smiles> 
Compound 1aw was prepared as a colorless oil in $82 \%$ yield $(0.57 \mathrm{~g}, E / Z=1 / 1.1$; eluent: petroleum ether/EtOAc $=20 / 1)$ from 7-(pyridin-2-yl)heptanal $(0.54 \mathrm{~g}$, $2.00 \mathrm{mmol})$, TIPSCl $(0.46 \mathrm{~g}, 2.40 \mathrm{mmol})$, DBU $(0.46 \mathrm{~g}, 3.00 \mathrm{mmol})$ following the above general procedure $\mathrm{A}$.

$\mathbf{R}_{f}=0.72$ (petroleum ether $/$ EtOAc $=5 / 1$ )

${ }^{1} \mathrm{H}$ NMR (400 MHz, $\left.\mathrm{CDCl}_{3}\right) \delta 8.51(\mathrm{~d}, J=4.6 \mathrm{~Hz}, 1 \mathrm{H}), 7.57(\mathrm{t}, J=7.6 \mathrm{~Hz}, 1 \mathrm{H})$, $7.17-7.03(\mathrm{~m}, 2 \mathrm{H}), 6.35-6.21(\mathrm{~m}, 1 \mathrm{H}), 5.03-4.93(\mathrm{~m}, 0.46 \mathrm{H}), 4.38(\mathrm{q}, J=7.1 \mathrm{~Hz}$, $0.51 \mathrm{H}), 2.87-2.72(\mathrm{~m}, 2 \mathrm{H}), 2.16-2.05(\mathrm{~m}, 1 \mathrm{H}), 1.97-1.80(\mathrm{~m}, 1 \mathrm{H}), 1.79-1.65$ (m, 2H), $1.46-1.30(\mathrm{~m}, 4 \mathrm{H}), 1.21-0.94(\mathrm{~m}, 21 \mathrm{H})$.

${ }^{13} \mathrm{C}$ NMR (100 MHz, $\left.\mathrm{CDCl}_{3}\right) \delta$ 162.7, 162.6, 149.30, 149.28, 140.7, 139.1, 136.34, 136.31, 122.80, 122.78, 121.0, 120.9, 111.2, 109.9, 38.57, 38.56, 30.5, 30.0, 29.9, 29.7, $29.3,29.0,27.3,23.6,17.90,17.88,12.2,12.1$.

HRMS (ESI') calcd for $\mathrm{C}_{21} \mathrm{H}_{38} \mathrm{NOSi}^{+}[\mathrm{M}+\mathrm{H}]^{+}:$348.2717, found: 348.2710.

IR (neat, cm$^{-1}$ ): 2956, 2925, 2857, 1454, 1061, 759, 700

\section{Synthesis of 1ak}
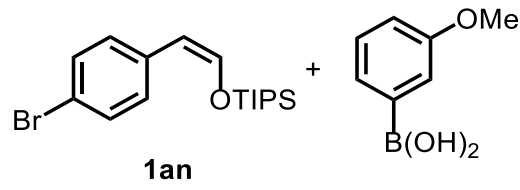

To a sealed tube were added $\mathrm{Pd}\left(\mathrm{PPh}_{3}\right)_{4}(35.0 \mathrm{mg}, 0.03 \mathrm{mmol}), \mathrm{K}_{2} \mathrm{CO}_{3}(0.41$ g, $3.00 \mathrm{mmol})$, 1an (0.36 g, $1.00 \mathrm{mmol})$ and (3-methoxyphenyl)boronic acid (0.3 $\mathrm{g}$, $2.00 \mathrm{mmol})$. Toluene and $\mathrm{H}_{2} \mathrm{O}(10: 1,1.0 \mathrm{~mL})$ were then added. The resulting mixture was heated to $90{ }^{\circ} \mathrm{C}$ and stirred for $12 \mathrm{~h}$. After cooled to room temperature, the reaction mixture was extracted with EtOAc, dried with $\mathrm{Na}_{2} \mathrm{SO}_{4}$, filtered, concentrated. The residue was purified by silica gel flash chromatography to afford compound 1ak as a colorless oil in $72 \%$ yield ( $0.28 \mathrm{~g}$, petroleum ether $/ \mathrm{EtOAc}=30 / 1)$.

$\mathbf{R}_{f}=0.55$ (petroleum ether $/$ EtOAc $=20 / 1$ )

${ }^{1} \mathrm{H}$ NMR (400 MHz, $\left.\mathrm{CDCl}_{3}\right) \delta 7.72(\mathrm{~d}, J=8.4 \mathrm{~Hz}, 2 \mathrm{H}), 7.53(\mathrm{~d}, J=8.4 \mathrm{~Hz}, 2 \mathrm{H})$, 7.37-7.28 (m, 1H), 7.24-7.17 (m, 1H), 7.17-7.11 (m, 1H), 6.88-6.82 (m, 1H), 6.52 $(\mathrm{d}, J=6.5 \mathrm{~Hz}, 1 \mathrm{H}), 5.31(\mathrm{~d}, J=6.5 \mathrm{~Hz}, 1 \mathrm{H}), 3.83(\mathrm{~s}, 3 \mathrm{H}), 1.32-1.22(\mathrm{~m}, 3 \mathrm{H}), 1.17-$ $1.10(\mathrm{~m}, 18 \mathrm{H})$.

${ }^{13} \mathrm{C}$ NMR (100 MHz, $\left.\mathrm{CDCl}_{3}\right) \delta$ 160.1, 142.8, 141.5, 138.1, 135.8, 129.8, 128.7, 126.9, $119.5,112.6,112.5,108.2,55.4,17.9,12.0$. 
HRMS (ESI') calcd for $\mathrm{C}_{24} \mathrm{H}_{35} \mathrm{O}_{2} \mathrm{Si}^{+}$[M+H] $]^{+}: 383.2401$, found: 383.2400 .

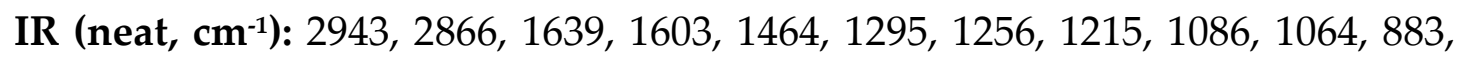
$837,777,690$.

\section{Synthesis of 1ao}
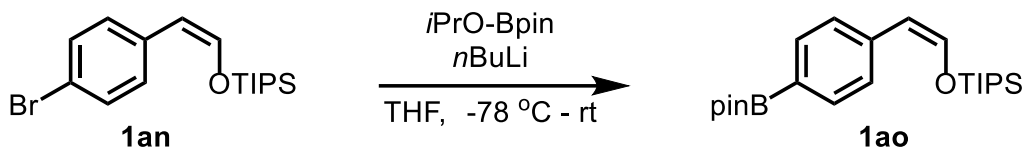

To the solution of $1 \mathrm{an}(0.36 \mathrm{~g}, 1.00 \mathrm{mmol})$ in THF was added $n \operatorname{BuLi}(2.5 \mathrm{M}$ in hexane, $0.44 \mathrm{~mL}, 1.1$ equiv) at $-78{ }^{\circ} \mathrm{C}$. After stirred for $1 \mathrm{~h}$ at $-78{ }^{\circ} \mathrm{C}$, 2isopropoxy-4,4,5,5-tetramethyl-1,3,2-dioxaborolane $(0.22 \mathrm{~g}$, $1.20 \mathrm{mmol}, 1.2$ equiv) was added. The resulting mixture was then warmed up to room temperature and stirred for $12 \mathrm{~h}$, then quenched with saturated $\mathrm{NH}_{4} \mathrm{Cl}$, extracted with EtOAc, dried with $\mathrm{Na}_{2} \mathrm{SO}_{4}$, filtered and concentrated. The residue was purified by silica gel flash chromatography to afford compound 1 ao as a colorless oil in $74 \%$ yield $(0.45$ g, eluent: petroleum ether $/ E t O A c=100 / 3)$ $\mathbf{R}_{f}=0.45$ (petroleum ether $/$ EtOAc $=20 / 1$ )

${ }^{1} \mathrm{H}$ NMR $\left(400 \mathrm{MHz}, \mathrm{CDCl}_{3}\right) \delta 7.74(\mathrm{~d}, J=8.1 \mathrm{~Hz}, 2 \mathrm{H}), 7.66(\mathrm{~d}, J=8.1 \mathrm{~Hz}, 2 \mathrm{H})$, $6.53(\mathrm{~d}, J=6.4 \mathrm{~Hz}, 1 \mathrm{H}), 5.29(\mathrm{~d}, J=6.6 \mathrm{~Hz}, 1 \mathrm{H}), 1.33(\mathrm{~s}, 12 \mathrm{H}), 1.30-1.18(\mathrm{~m}, 3 \mathrm{H})$, $1.18-1.03(\mathrm{~m}, 18 \mathrm{H})$.

${ }^{13} \mathrm{C}$ NMR (100 MHz, $\left.\mathrm{CDCl}_{3}\right) \delta$ 142.2, 139.3, 134.7, 127.6, 108.8, 83.7, 25.0, 17.8, 11.9.

The carbon signal attached to $\mathrm{B}$ atom was not observed due to low intensity;

HRMS (ESI') calcd for $\mathrm{C}_{23} \mathrm{H}_{40} \mathrm{BO}_{3} \mathrm{Si}^{+}$[M+Na] $]^{+}$: 403.2834, found: 403.2833 .

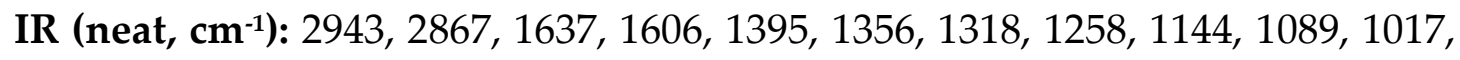
996, 962, 884, 856, 754, 677, 656.

\section{Synthesis of 1aq}
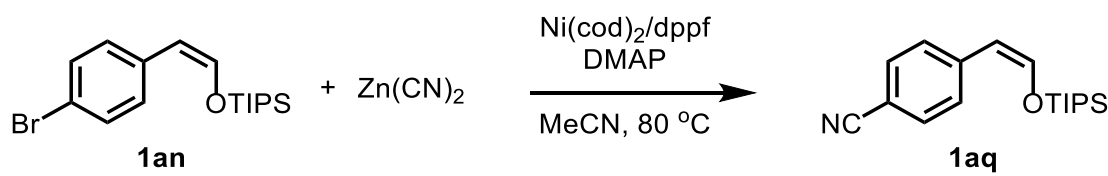

Following the literature procedure ${ }^{30}$, to a sealed tube were added $\mathrm{Ni}(\mathrm{cod})_{2}$ (13.8 mg, $0.05 \mathrm{mmol})$, dppf ( $33.3 \mathrm{mg}, 0.06 \mathrm{mmol})$ and $\mathrm{MeCN}(2.0 \mathrm{~mL})$. The reaction mixture was stirred for $20 \mathrm{~min}$ at room temperature. Then DMAP (122.2 mg, $1.00 \mathrm{mmol}), \mathrm{Zn}(\mathrm{CN})_{2}(93.9 \mathrm{mg}, 0.80 \mathrm{mmol})$, 1 an $(355.6 \mathrm{mg}, 1.00 \mathrm{mmol})$ 
and $\mathrm{MeCN}(2.0 \mathrm{~mL})$ were added. The resulting mixture was then heated to 80 ${ }^{\circ} \mathrm{C}$ and stirred for $20 \mathrm{~h}$. After cooled to room temperature, the reaction mixture was filtered through a short pad of silica gel and washed with EtOAc. The solvent was evaporated under the reduced pressure and the residue was purified by column chromatography on silica gel to afford compound 1aq as a colorless oil in $36 \%$ yield ( $0.11 \mathrm{~g}$, eluent: petroleum ether/DCM $=20 / 1)$.

$\mathbf{R}_{f}=0.45$ (petroleum ether/DCM $=5 / 1$ )

${ }^{1} \mathrm{H}$ NMR (400 MHz, $\left.\mathrm{CDCl}_{3}\right) \delta 7.70(\mathrm{~d}, J=8.5 \mathrm{~Hz}, 2 \mathrm{H}), 7.54(\mathrm{~d}, J=8.5 \mathrm{~Hz}, 2 \mathrm{H})$, $6.63(\mathrm{~d}, J=6.5 \mathrm{~Hz}, 1 \mathrm{H}), 5.30(\mathrm{~d}, J=6.5 \mathrm{~Hz}, 1 \mathrm{H}), 1.33-1.21(\mathrm{~m}, 3 \mathrm{H}), 1.17-1.05(\mathrm{~m}$, $18 \mathrm{H})$.

${ }^{13} \mathrm{C}$ NMR (100 MHz, $\left.\mathrm{CDCl}_{3}\right) \delta$ 144.6, 141.1, 132.0, 128.5, 119.6, 108.4, 107.4, 17.8, 11.9 .

HRMS (ESI') calcd for $\mathrm{C}_{18} \mathrm{H}_{28} \mathrm{NOSi}^{+}[\mathrm{M}+\mathrm{H}]^{+}:$302.1935, found: 302.1934 .

IR (neat, cm-1): 2945, 2867, 1636, 1603, 1265, 1088, 1069, 995, 883, 843, 685, 629, 566.

\section{Synthesis of 1au}

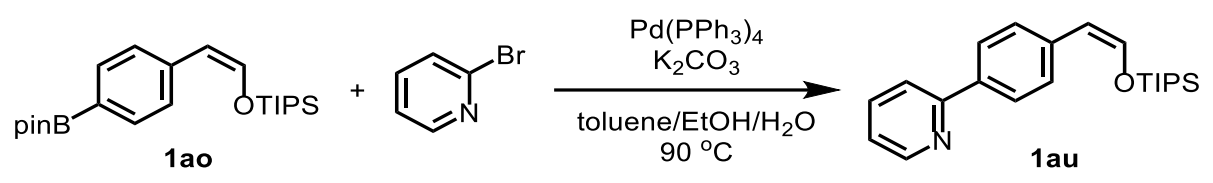

To a sealed tube were added $\mathrm{Pd}\left(\mathrm{PPh}_{3}\right)_{4}(21.0 \mathrm{mg}, 0.02 \mathrm{mmol}), \mathrm{K}_{2} \mathrm{CO}_{3}(0.15$ $\mathrm{g}, 1.10 \mathrm{mmol})$, 1 ao $(0.15 \mathrm{~g}, 0.37 \mathrm{mmol})$ and 2-bromopyridine (58.5 mg, 0.37 $\mathrm{mmol})$. Toluene $(1.9 \mathrm{~mL}), \mathrm{EtOH}(0.75 \mathrm{~mL})$ and $\mathrm{H}_{2} \mathrm{O}(0.40 \mathrm{~mL})$ were then added. The resulting mixture was heated to $90{ }^{\circ} \mathrm{C}$ and stirred for $15 \mathrm{~h}$. After cooled to room temperature, the reaction mixture was extracted with EtOAc, dried with $\mathrm{Na}_{2} \mathrm{SO}_{4}$, filtered, concentrated. The residue was purified by silica gel flash chromatography to afford compound 1au as a colorless oil in $90 \%$ yield $(0.12 \mathrm{~g}$, eluent: petroleum ether/EtOAc $=30 / 1)$.

$\mathbf{R}_{f}=0.74$ (petroleum ether $/$ EtOAc $=5 / 1$ )

${ }^{1} \mathrm{H}$ NMR (400 MHz, $\left.\mathrm{CDCl}_{3}\right) \delta 8.74-8.59(\mathrm{~m}, 1 \mathrm{H}), 7.94(\mathrm{~d}, J=8.1 \mathrm{~Hz}, 2 \mathrm{H}), 7.80-$ $7.58(\mathrm{~m}, 4 \mathrm{H}), 7.21-7.12(\mathrm{~m}, 1 \mathrm{H}), 6.56(\mathrm{~d}, J=6.4 \mathrm{~Hz}, 1 \mathrm{H}), 5.34(\mathrm{~d}, J=6.5 \mathrm{~Hz}, 1 \mathrm{H})$, $1.33-1.22(\mathrm{~m}, 3 \mathrm{H}), 1.20-1.08(\mathrm{~m}, 18 \mathrm{H})$.

${ }^{13} \mathrm{C}$ NMR (100 MHz, $\left.\mathrm{CDCl}_{3}\right) \delta$ 157.5, 149.7, 142.0, 137.3, 136.7, 136.3, 128.7, 126.6, $121.8,120.3,108.3,17.9,11.9$. 
HRMS (ESI') calcd for $\mathrm{C}_{22} \mathrm{H}_{32} \mathrm{NOSi}^{+}[\mathrm{M}+\mathrm{H}]^{+}:$354.2248, found: 354.2251 .

IR (neat, cm-1): 2943, 2867, 1637, 1465, 1276, 1086, 883, 787, 681.

\section{Synthesis of 1aw}

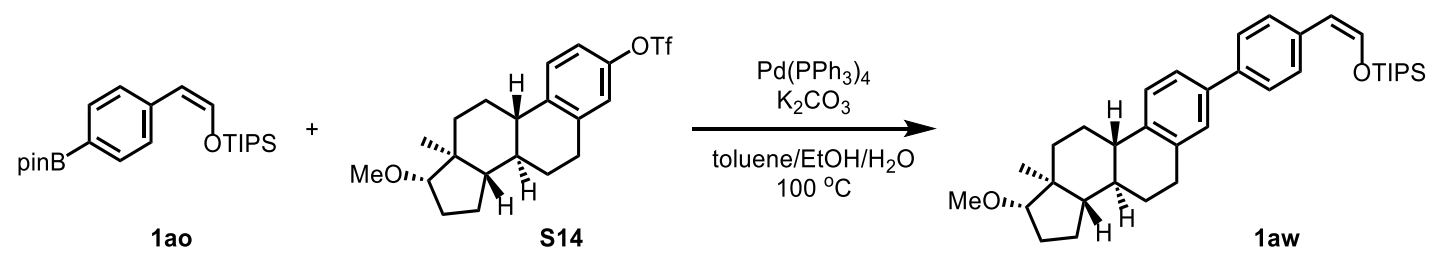

To a sealed tube were added $\mathrm{Pd}\left(\mathrm{PPh}_{3}\right)_{4}(35.0 \mathrm{mg}, 0.03 \mathrm{mmol}), \mathrm{K}_{2} \mathrm{CO}_{3}(0.41$ g, $3.00 \mathrm{mmol})$, 1 ao $(0.42 \mathrm{~g}, 1.20 \mathrm{mmol})$ and $\mathbf{S 1 4}{ }^{31}(0.42 \mathrm{~g}, 1.00 \mathrm{mmol})$. Toluene (1.3 mL), EtOH $(0.5 \mathrm{~mL})$ and $\mathrm{H}_{2} \mathrm{O}(0.25 \mathrm{~mL})$ were then added. The resulting mixture was heated to $100{ }^{\circ} \mathrm{C}$ and stirred for $15 \mathrm{~h}$. After cooled to room temperature, the reaction mixture was extracted with EtOAc, dried with $\mathrm{Na}_{2} \mathrm{SO}_{4}$, filtered, concentrated. The residue was purified by silica gel flash chromatography to afford compound 1aw as a colorless mucus in $82 \%$ yield $(0.45$ g, eluent: petroleum ether/EtOAc $=30 / 1)$.

$\mathbf{R}_{f}=0.55$ (petroleum ether/EtOAc $=20 / 1$ )

${ }^{1} \mathrm{H}$ NMR (400 MHz, $\left.\mathrm{CDCl}_{3}\right) \delta 7.70(\mathrm{~d}, J=9.8 \mathrm{~Hz}, 2 \mathrm{H}), 7.51(\mathrm{~d}, J=8.4 \mathrm{~Hz}, 2 \mathrm{H})$, 7.43-7.37 (m, 1H), 7.37-7.31 (m, 2H), $6.52(\mathrm{~d}, J=6.4 \mathrm{~Hz}, 1 \mathrm{H}), 5.30(\mathrm{~d}, J=6.5 \mathrm{~Hz}$, $1 \mathrm{H}), 3.38(\mathrm{~s}, 3 \mathrm{H}), 3.35-3.28(\mathrm{~m}, 1 \mathrm{H}), 2.96-2.88(\mathrm{~m}, 2 \mathrm{H}), 2.39-2.31(\mathrm{~m}, 1 \mathrm{H}), 2.30$ $2.21(\mathrm{~m}, 1 \mathrm{H}), 2.13-2.01(\mathrm{~m}, 2 \mathrm{H}), 1.95-1.86(\mathrm{~m}, 1 \mathrm{H}), 1.76-1.65(\mathrm{~m}, 1 \mathrm{H}), 1.64-1.32$ $(\mathrm{m}, 6 \mathrm{H}), 1.32-1.20(\mathrm{~m}, 4 \mathrm{H}), 1.19-1.07(\mathrm{~m}, 18 \mathrm{H}), 0.80(\mathrm{~s}, 3 \mathrm{H})$.

${ }^{13} \mathrm{C}$ NMR (100 MHz, $\left.\mathrm{CDCl}_{3}\right) \delta$ 141.3, 139.4, 138.5, 138.2, 137.1, 135.3, 128.7, 127.5, $126.7,125.9,124.3,108.3,90.9,58.0,50.6,44.5,43.4,38.6,38.2,29.8,27.9,27.4,26.4$, 23.2, 17.9, 12.0, 11.7 .

HRMS (ESI+) calcd for $\mathrm{C}_{36} \mathrm{H}_{53} \mathrm{O}_{2} \mathrm{Si}^{+}[\mathrm{M}+\mathrm{H}]^{+}: 545.3809$, found: 545.3808 .

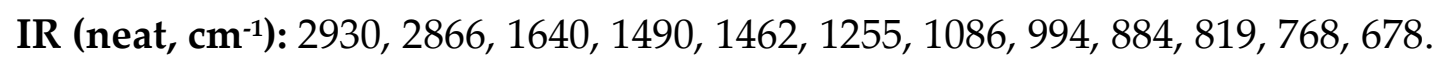




\section{Asymmetric Hydroboration of Silyl Enol Ethers}
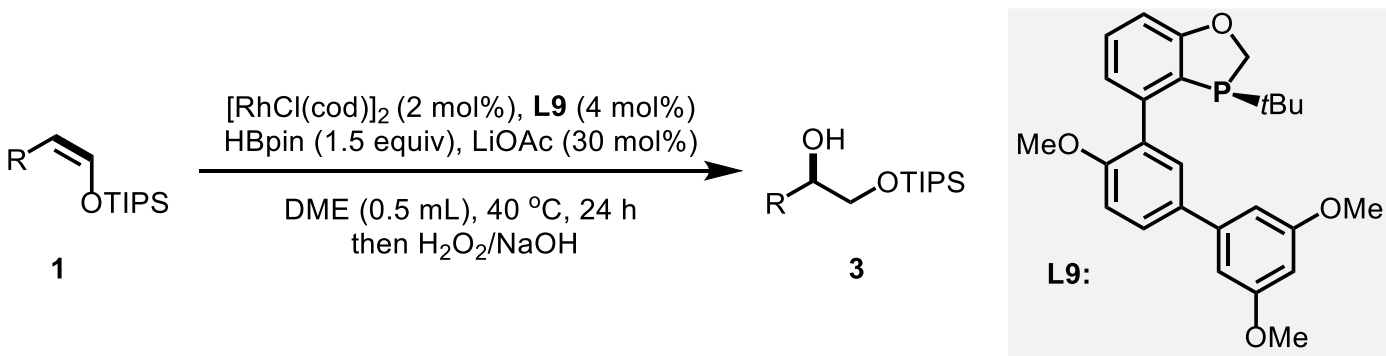

\section{General Procedure E:}

In a glove box, to an oven-dried $10-\mathrm{mL}$ vial were added $[\mathrm{RhCl}(\mathrm{cod})]_{2}(2.0$ $\mathrm{mg}, 0.004 \mathrm{mmol}), \mathbf{L 9}(3.5 \mathrm{mg}, 0.008 \mathrm{mmol})$ and anhydrous DME (0.5 mL). The resulting solution was stirred for $10 \mathrm{~min}$ at room temperature, at which time HBpin (38.4 mg, $0.30 \mathrm{mmol}$ ) was added. After the resulting mixture was stirred for an additional $10 \mathrm{~min}, \mathrm{LiOAc}(4.0 \mathrm{mg}, 0.06 \mathrm{mmol})$ was added and stirred for 30 min. (Z)-Silyl enol ether was then added at room temperature and stirred for $24 \mathrm{~h}$ at $40{ }^{\circ} \mathrm{C}$. The resulting suspension was quenched with $\mathrm{H}_{2} \mathrm{O}$ and cooled to room temperature, filtered through a silica gel pad and washed with EtOAc, concentrated. The residue was dissolved in THF $(1.0 \mathrm{~mL})$ and cooled to $0{ }^{\circ} \mathrm{C}$, then $\mathrm{NaOH}$ (1.5 equiv, $3.0 \mathrm{M}$ ), $\mathrm{H}_{2} \mathrm{O}_{2}$ (1.5 equiv, 30\%) was added. The resulting mixture was stirred for $0.5 \mathrm{~h}$, then extracted with EtOAc, dried with $\mathrm{Na}_{2} \mathrm{SO}_{4}$, filtered and concentrated. The residue was purified by silica gel chromatography to afford the desired products.
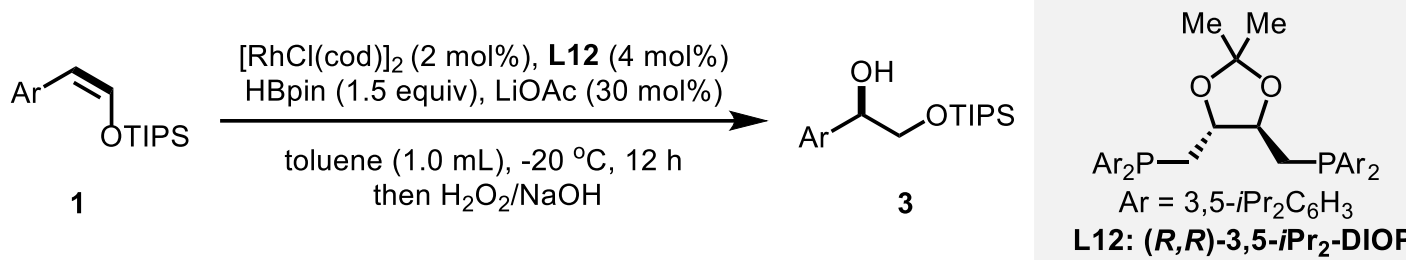

\section{General Procedure F (LiOAc as base):}

In a glove box, to an oven-dried $10-\mathrm{mL}$ vial were added $[\mathrm{RhCl}(\operatorname{cod})]_{2}(3.0$ $\mathrm{mg}, 0.006 \mathrm{mmol}),(R, R)-3,5-i \operatorname{Pr}_{2}$-DIOP $(10.0 \mathrm{mg}, 0.012 \mathrm{mmol})$ and anhydrous toluene $(1.0 \mathrm{~mL})$. The resulting solution was stirred for $10 \mathrm{~min}$ at room temperature, at which time HBpin $(57.6 \mathrm{mg}, 0.45 \mathrm{mmol})$ was added. After stirred for an additional $10 \mathrm{~min}$, LiOAc $(6.0 \mathrm{mg}, 0.09 \mathrm{mmol})$ was added and stirred for $30 \mathrm{~min}$. (Z)-Silyl enol ether was then added at $-20{ }^{\circ} \mathrm{C}$ and stirred for 
$12 \mathrm{~h}$. The resulting suspension was quenched with $\mathrm{H}_{2} \mathrm{O}$ and warmed up to room temperature, filtered through a silica gel pad and washed with EtOAc, concentrated. The residue was dissolved in THF $(1.0 \mathrm{~mL})$ and cooled to $0{ }^{\circ} \mathrm{C}$, then $\mathrm{NaOH}$ (1.5 equiv, 3.0 M), $\mathrm{H}_{2} \mathrm{O}_{2}$ (1.5 equiv, 30\%) was added. The resulting mixture was stirred for $0.5 \mathrm{~h}$, then extracted with EtOAc, dried with $\mathrm{Na}_{2} \mathrm{SO}_{4}$, filtered and concentrated. The residue was purified by silica gel chromatography to afford the desired products.

\section{General Procedure G (AgOAc as base):}

In a glove box, to an oven-dried $10-\mathrm{mL}$ vial were added $[\mathrm{RhCl}(\mathrm{cod})]_{2}(3.0$ $\mathrm{mg}, 0.006 \mathrm{mmol}),(R, R)-3,5-i \mathrm{Pr}_{2}$-DIOP $(10.0 \mathrm{mg}, 0.012 \mathrm{mmol})$ and anhydrous toluene $(1.0 \mathrm{~mL})$. The resulting solution was stirred for $10 \mathrm{~min}$ at room temperature, AgOAc (15.0 mg, $0.09 \mathrm{mmol})$ was added and stirred for $30 \mathrm{~min}$. After removed the precipitate by filtration, HBpin $(57.6 \mathrm{mg}, 0.45 \mathrm{mmol})$ was added and stirred for $10 \mathrm{~min}$. (Z)-Silyl enol ether was then added at $-20{ }^{\circ} \mathrm{C}$ and stirred for $12 \mathrm{~h}$. The resulting suspension was quenched with $\mathrm{H}_{2} \mathrm{O}$ and warmed up to room temperature, filtered through a silica gel pad and washed with EtOAc, concentrated. The crude residue was dissolved in THF (1.0 mL) and cooled to $0{ }^{\circ} \mathrm{C}$, then $\mathrm{NaOH}(1.5$ equiv, $3.0 \mathrm{M}), \mathrm{H}_{2} \mathrm{O}_{2}(1.5$ equiv, 30\%) was added. The resulting mixture was stirred for $0.5 \mathrm{~h}$, then extracted with EtOAc, dried with $\mathrm{Na}_{2} \mathrm{SO}_{4}$, filtered and concentrated. The residue was purified by silica gel chromatography to afford the desired products.
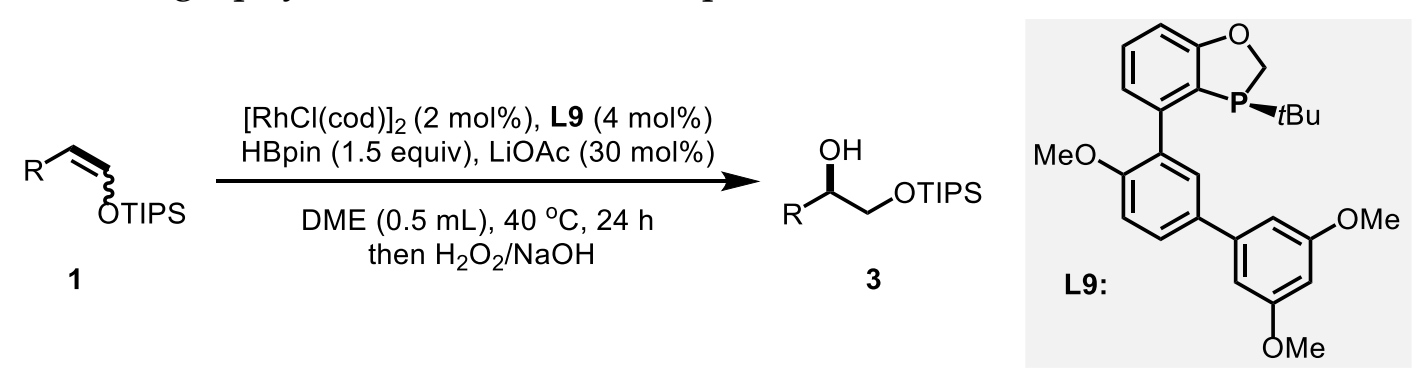

\section{General Procedure H:}

In a glove box, to an oven-dried $10-\mathrm{mL}$ vial were added $[\mathrm{RhCl}(\mathrm{cod})]_{2}(2.0$ mg, $0.004 \mathrm{mmol}), \mathbf{L 9}(3.5 \mathrm{mg}, 0.008 \mathrm{mmol})$ and anhydrous DME (0.5 mL). The resulting solution was stirred for $10 \mathrm{~min}$ at room temperature, at which time HBpin (38.4 mg, $0.30 \mathrm{mmol}$ ) was added. After the resulting mixture was stirred for an additional $10 \mathrm{~min}, \mathrm{LiOAc}(4.0 \mathrm{mg}, 0.06 \mathrm{mmol})$ was added and stirred for $30 \mathrm{~min}$. (E/Z)-Silyl enol ether was then added at room temperature and stirred 
for $24 \mathrm{~h}$ at $40{ }^{\circ} \mathrm{C}$. The resulting suspension was quenched with $\mathrm{H}_{2} \mathrm{O}$ and cooled to room temperature, filtered through a silica gel pad and washed with EtOAc, concentrated. The residue was dissolved in THF $(1.0 \mathrm{~mL})$ and cooled to $0{ }^{\circ} \mathrm{C}$, then $\mathrm{NaOH}\left(1.5\right.$ equiv, $3.0 \mathrm{M}$ ), $\mathrm{H}_{2} \mathrm{O}_{2}$ (1.5 equiv, 30\%) was added. The resulting mixture was stirred for $0.5 \mathrm{~h}$, then extracted with EtOAc, dried with $\mathrm{Na}_{2} \mathrm{SO}_{4}$, filtered and concentrated. The residue was purified by silica gel chromatography to afford the desired products.

\section{General procedure I:}

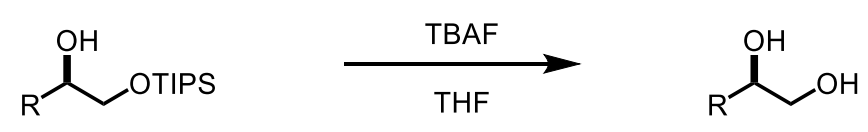

To the solution of silyl ether (1.0 equiv) in THF was added TBAF (1.0 M in $\mathrm{THF}, 1.5$ equiv) at $0{ }^{\circ} \mathrm{C}$. The resulting mixture was stirred for $0.5 \mathrm{~h}$, then extracted with EtOAc, dried with $\mathrm{Na}_{2} \mathrm{SO}_{4}$, filtered and concentrated. The residue was purified by silica gel flash chromatography to afford the pure alcohol.

\section{General procedure J:}<smiles>[R]C(O)C[OH+][SnH3]</smiles>

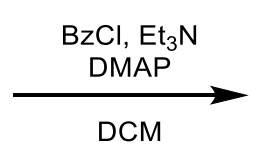<smiles>[R]C([2H])C[OH+][SnH3]</smiles>

To the solution of alcohol (1.0 equiv), $\mathrm{Et}_{3} \mathrm{~N}$ (2.0 equiv) and DMAP (0.1 equiv) in $\mathrm{DCM}$ was added $\mathrm{BzCl}$ (1.5 equiv) at $0{ }^{\circ} \mathrm{C}$. The resulting mixture was then warmed up to room temperature and stirred for $5 \mathrm{~h}$, then quenched with saturated $\mathrm{NH}_{4} \mathrm{Cl}$, extracted with EtOAc, dried with $\mathrm{Na}_{2} \mathrm{SO}_{4}$, filtered and concentrated. The residue was purified by silica gel flash chromatography to afford the benzoyl ester.

\section{Sythesis of $(R)-3 a:$}

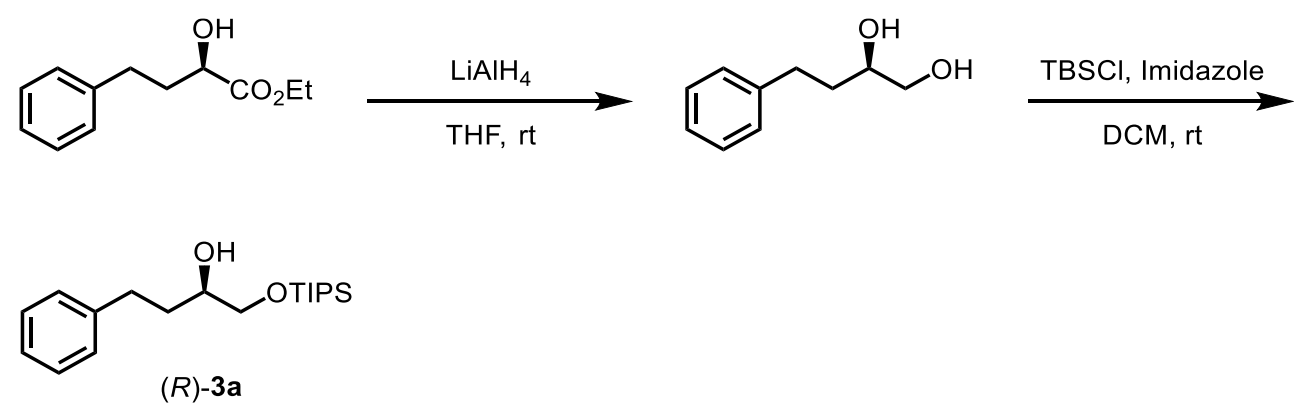


To a solution of $\mathrm{LiAlH}_{4}(0.19 \mathrm{~g}, 5.00 \mathrm{mmol})$ in THF was added ethyl $(R)-2-$ hydroxy-4-phenylbutanoate $(0.42 \mathrm{~g}, 2.00 \mathrm{mmol})$ at $0{ }^{\circ} \mathrm{C}$. The resulting mixture was then warmed up to room temperature and stirred for $12 \mathrm{~h}$, then quenched with $\mathrm{H}_{2} \mathrm{O}$ at $0{ }^{\circ} \mathrm{C}$, extracted with EtOAc, dried with $\mathrm{Na}_{2} \mathrm{SO}_{4}$, filtered and concentrated. The residue was purified by silica gel flash chromatography to afford ( $R$ )-4-phenylbutane-1,2-diol as a colorless oil in 66\% yield ( $0.26 \mathrm{~g}$, eluent: petroleum ether $/$ EtOAc $=1 / 1$ ).

To a solution of (R)-4-phenylbutane-1,2-diol $(0.26 \mathrm{~g}$, $1.54 \mathrm{mmol})$ and imidazole $(0.11 \mathrm{~g}, 1.54 \mathrm{mmol})$ in DCM was added TIPSCl $(0.30 \mathrm{~g}, 1.54 \mathrm{mmol})$ at room temperature. The resulting mixture was then stirred for $0.5 \mathrm{~h}$, then quenched with saturated $\mathrm{NH}_{4} \mathrm{Cl}$, extracted with EtOAc, dried with $\mathrm{Na}_{2} \mathrm{SO}_{4}$, filtered and concentrated. The residue was purified by silica gel flash chromatography to afford compound (R)-3a as a colorless oil in 73\% yield ( 0.36 g, eluent: petroleum ether $/ \mathrm{EtOAc}=20 / 1)$.

Sythesis of $(R)-3 a c:$<smiles>OCC(O)c1ccccc1</smiles>

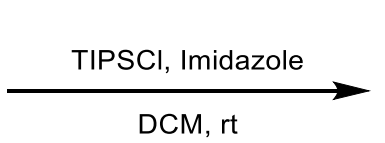<smiles>OCC(O)c1ccccc1</smiles>

(R)-3ac

To a solution of $(R)-1$-phenylethane-1,2-diol $(0.13 \mathrm{~g}, 1.00 \mathrm{mmol})$, imidazole $(81.6 \mathrm{mg}, 1.20 \mathrm{mmol})$ in DCM was added TIPSCl $(0.19 \mathrm{~g}, 1.00 \mathrm{mmol})$ at room temperature. The resulting mixture was then stirred for $0.5 \mathrm{~h}$, then quenched with saturated $\mathrm{NH}_{4} \mathrm{Cl}$, extracted with EtOAc, dried with $\mathrm{Na}_{2} \mathrm{SO}_{4}$, filtered and concentrated. The residue was purified by silica gel flash chromatography to afford compound $(R)$-3ac as a colorless oil in $68 \%$ yield $(0.22 \mathrm{~g}$, eluent: petroleum ether $/ \mathrm{EtOAc}=20 / 1$ ).

\section{(S)-4-Phenyl-1-((triisopropylsilyl)oxy)butan-2-ol (3a)}<smiles>OC(CCc1ccccc1)CO[Na]</smiles>

Compound 3a was prepared as a colorless oil in $72 \%$ yield $(46.4 \mathrm{mg}$, eluent: petroleum ether/EtOAc $=100 / 3$ to 20/1), from $(Z)$-triisopropyl((4-phenylbut-1en-1-yl)oxy)silane 1a (60.8 mg, $0.20 \mathrm{mmol})$, [ $\mathrm{RhCl}(\mathrm{cod})]_{2}(2.0 \mathrm{mg}, 0.004 \mathrm{mmol})$, (S)-L9 (3.5 mg, $0.008 \mathrm{mmol})$, HBpin ( $38.4 \mathrm{mg}, 0.30 \mathrm{mmol})$ and LiOAc $(4.0 \mathrm{mg}$, $0.06 \mathrm{mmol}$ ) following the above general procedure $\mathrm{E}$. 
$\mathbf{R}_{f}=0.62$ (petroleum ether/EtOAc $\left.=10 / 1\right)$

${ }^{1} \mathrm{H}$ NMR (400 MHz, $\left.\mathrm{CDCl}_{3}\right)$ $\delta$ 7.34-7.14 (m, 5H), 3.77-3.62 (m, 2H), 3.57-3.46 (m, 1H), 2.90-2.76 (m, 1H), 2.76-2.63 (m, 1H), 2.58 (s, 1H), 1.87-1.66 (m, 2H), 1.19$0.97(\mathrm{~m}, 21 \mathrm{H})$.

${ }^{13} \mathrm{C}$ NMR (100 MHz, $\left.\mathrm{CDCl}_{3}\right) \delta$ 142.2, 128.6, 128.5, 125.9, 71.4, 67.6, 34.7, 32.0, 18.1, 12.0 .

HRMS (ESI ${ }^{+}$) calcd for $\mathrm{C}_{19} \mathrm{H}_{34} \mathrm{O}_{2} \mathrm{NaSi}^{+}[\mathrm{M}+\mathrm{Na}]^{+}$: 345.2220, found: 345.2219.

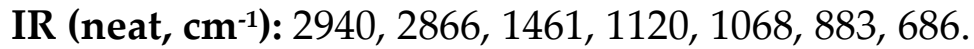

$[\alpha]_{\mathrm{D}^{26.2}}=-4.81\left(\mathrm{c}=0.92, \mathrm{CHCl}_{3}, 95 \%\right.$ ee $)$; Enantiomeric excess was determined by chiral HPLC (Daicel Chiralcel OD-H, hexane/isopropyl alcohol =95/5, flow rate $=1.0 \mathrm{~mL} / \mathrm{min}, \lambda=220 \mathrm{~nm}$ ),$t^{1}=4.1 \mathrm{~min}$ (major),$t^{2}=5.7 \mathrm{~min}$ (minor).

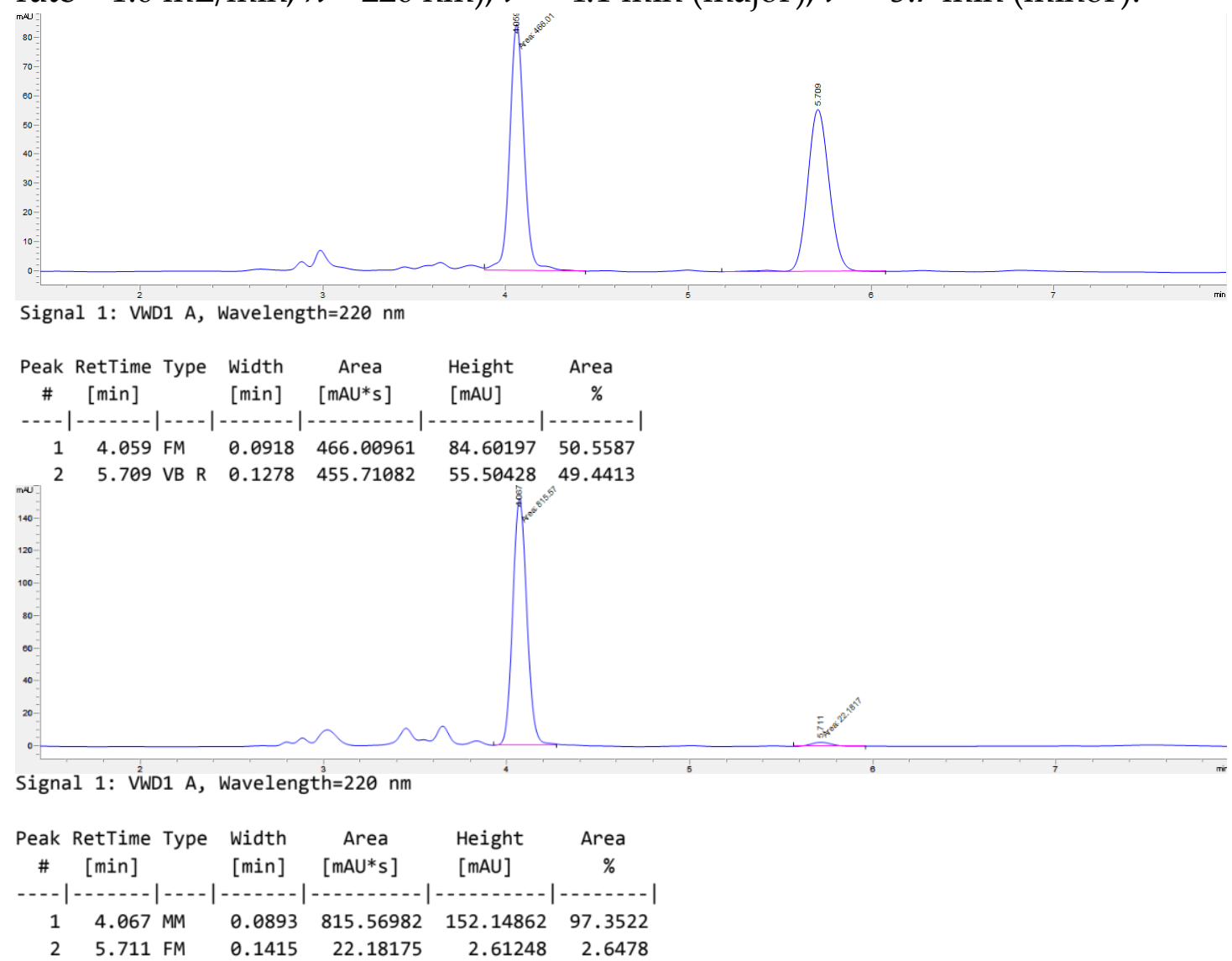

Compound 3a was prepared in 70\% yield $(45.1 \mathrm{mg})$ and $85 \%$ ee from $(E)-\mathbf{1 a}$ (60.8 mg, $0.20 \mathrm{mmol})$, [RhCl(cod) $]_{2}(2.0 \mathrm{mg}, 0.004 \mathrm{mmol}),(S)-\mathrm{L} 9$ (3.5 mg, 0.008 $\mathrm{mmol})$, HBpin (38.4 mg, $0.30 \mathrm{mmol})$ and LiOAc $(4.0 \mathrm{mg}, 0.06 \mathrm{mmol})$ following the above general procedure $\mathrm{E}$. 


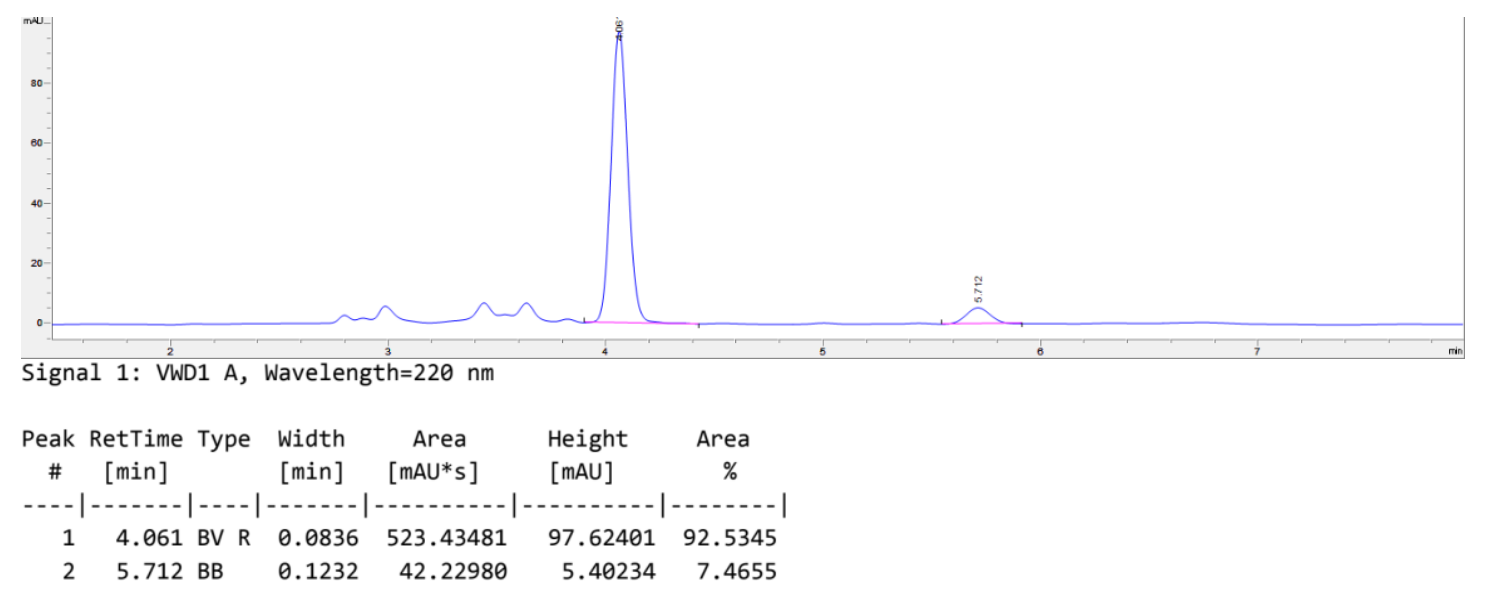

Compound 3a was prepared in $68 \%$ yield $(43.8 \mathrm{mg})$ and $90 \%$ ee from $1 \mathbf{a}(E / Z=$ 1/1) $(60.8 \mathrm{mg}, 0.20 \mathrm{mmol})$, [RhCl(cod) $]_{2}(2.0 \mathrm{mg}, 0.004 \mathrm{mmol}),(S)-\mathrm{L} 9$ (3.5 mg, $0.008 \mathrm{mmol}), \mathrm{HBpin}(38.4 \mathrm{mg}, 0.30 \mathrm{mmol})$ and LiOAc $(4.0 \mathrm{mg}, 0.06 \mathrm{mmol})$ following the above general procedure $\mathrm{E}$.

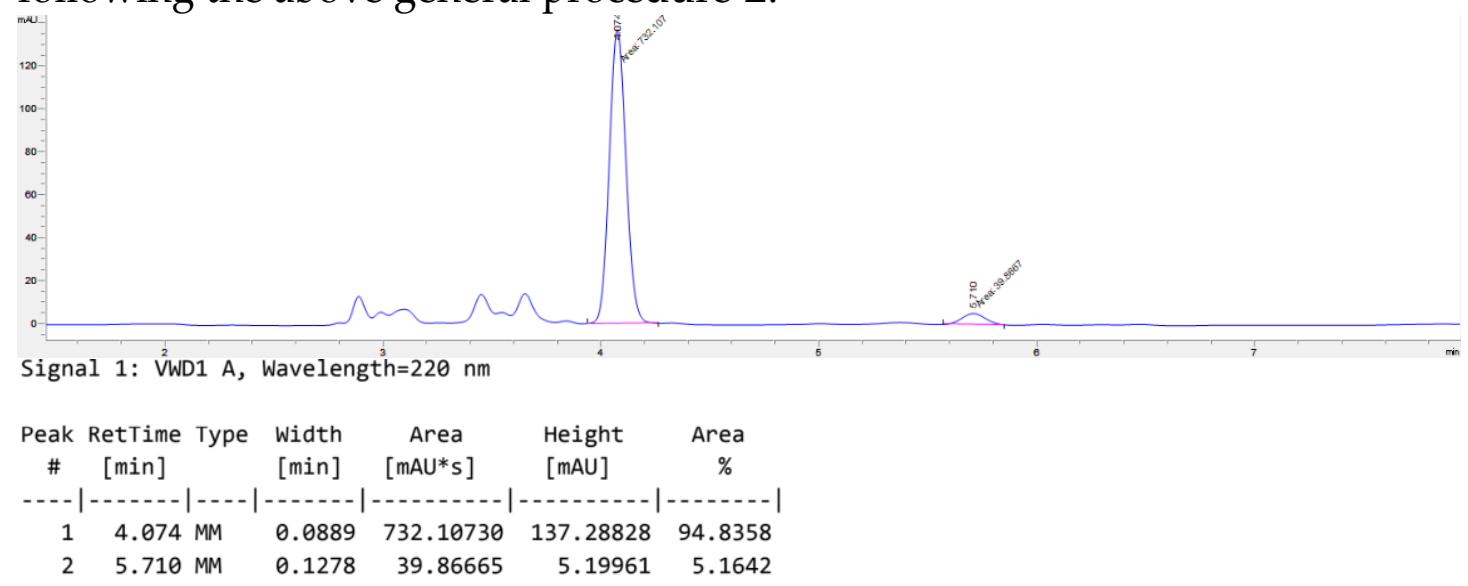

Compound 3a was prepared in $68 \%$ yield $(43.8 \mathrm{mg})$ and $92 \%$ ee from $1 \mathrm{a}(E / \mathrm{Z}=$ $1 / 9)(60.8 \mathrm{mg}, 0.20 \mathrm{mmol}),[\mathrm{RhCl}(\mathrm{cod})]_{2}(2.0 \mathrm{mg}, 0.004 \mathrm{mmol}),(S)-\mathrm{L} 9$ (3.5 mg, $0.008 \mathrm{mmol})$, HBpin $(38.4 \mathrm{mg}, 0.30 \mathrm{mmol})$ and LiOAc $(4.0 \mathrm{mg}, 0.06 \mathrm{mmol})$ following the above general procedure $\mathrm{E}$.

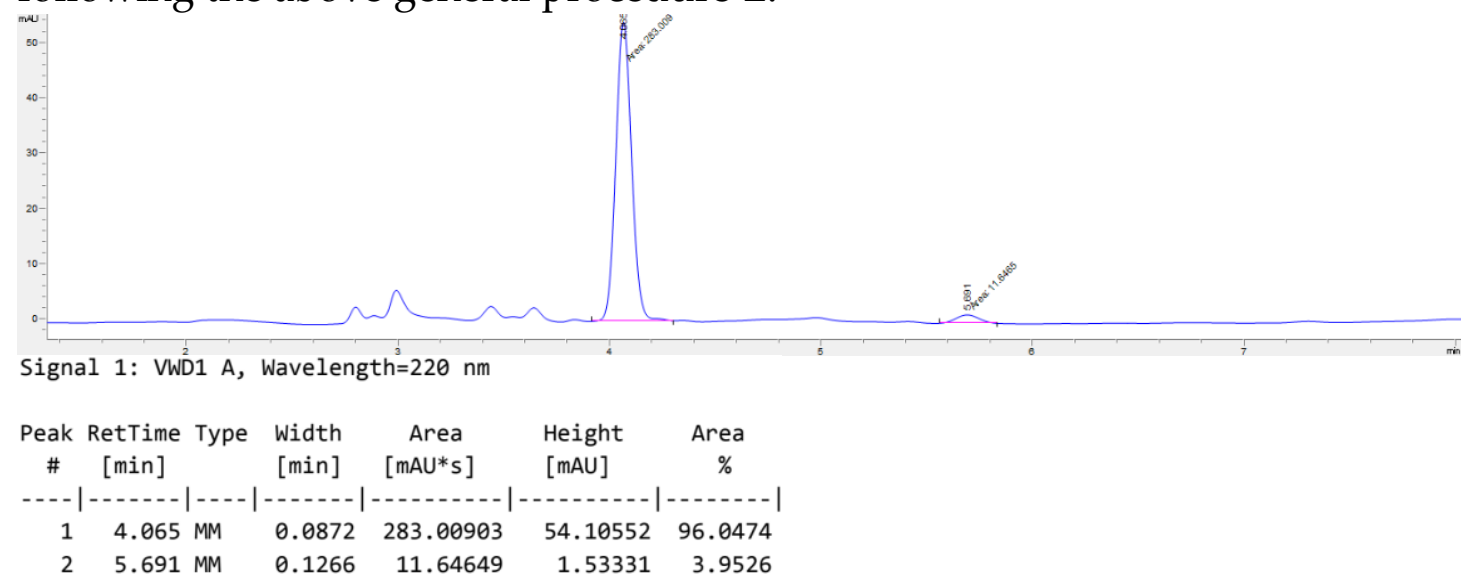


(S)-5-(4-(Trifluoromethyl)phenyl)-1-((triisopropylsilyl)oxy)pentan-2-ol (3b)

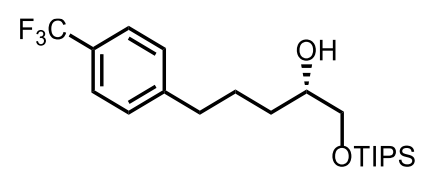

Compound $3 \mathbf{b}$ was prepared as a colorless oil in $83 \%$ yield $(65.0 \mathrm{mg}$, eluent: petroleum ether/EtOAc $=100 / 3$ to 20/1), from (Z)-triisopropyl((5-(4(trifluoromethyl)phenyl)pent-1-en-1-yl)oxy)silane $\mathbf{1 b}(74.8 \mathrm{mg}, 0.20 \mathrm{mmol})$, $[\mathrm{RhCl}(\mathrm{cod})]_{2}(2.0 \mathrm{mg}, 0.004 \mathrm{mmol}),(S)-\mathrm{L} 9$ (3.5 mg, $\left.0.008 \mathrm{mmol}\right), \mathrm{HBpin}$ (38.4 mg, $0.30 \mathrm{mmol})$ and LiOAc $(4.0 \mathrm{mg}, 0.06 \mathrm{mmol})$ following the above general procedure E.

$\mathbf{R}_{f}=0.65$ (petroleum ether/EtOAc $=10 / 1$ )

${ }^{1} \mathrm{H}$ NMR (400 MHz, $\left.\mathrm{CDCl}_{3}\right) \delta 7.52(\mathrm{~d}, J=7.8 \mathrm{~Hz}, 2 \mathrm{H}), 7.28(\mathrm{~d}, J=7.9 \mathrm{~Hz}, 2 \mathrm{H})$, 3.82-3.63 (m, 2H), 3.53-3.37 (m, 1H), 2.79-2.65 (m, 2H), $2.55(\mathrm{~s}, 1 \mathrm{H}), 1.92-1.77$ (m, 1H), 1.77-1.59 (m, 1H), 1.56-1.36 (m, 2H), 1.17-0.93 (m, 21H).

${ }^{13} \mathrm{C}$ NMR (100 MHz, $\left.\mathrm{CDCl}_{3}\right) \delta$ 146.6, 128.9, 128.3 (q, J = 32.1 Hz), 125.4 (q, J = 4.0 $\mathrm{Hz}), 124.5(\mathrm{q}, J=270 \mathrm{~Hz}), 71.9,67.6,35.9,32.4,27.3,18.1,12.1$.

${ }^{19}$ F NMR (377 MHz, $\left.\mathrm{CDCl}_{3}\right) \delta-62.3$.

HRMS (ESI') calcd for $\mathrm{C}_{21} \mathrm{H}_{35} \mathrm{~F}_{3} \mathrm{NaO}_{2} \mathrm{Si}^{+}[\mathrm{M}+\mathrm{Na}]^{+}: 427.2251$, found: 427.2248 .

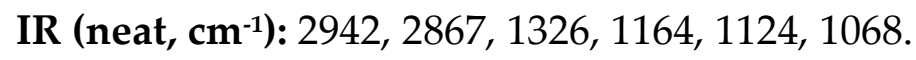

$[\alpha]_{\mathrm{D}^{26.2}}=3.80\left(\mathrm{c}=0.60, \mathrm{CHCl}_{3}, 93 \%\right.$ ee $)$.

Compound $\mathbf{3 b}$ was subjected to general procedure I and the resulting alcohol was analyzed by chiral HPLC. Enantiomeric excess was determined by chiral HPLC (Daicel Chiralcel OD-H, hexane/isopropyl alcohol $=95 / 5$, flow rate $=1.0$ $\mathrm{mL} / \mathrm{min}, \lambda=220 \mathrm{~nm}$ ) $t^{1}=14.9 \mathrm{~min}$ (minor),$t^{2}=15.6 \mathrm{~min}$ (major).

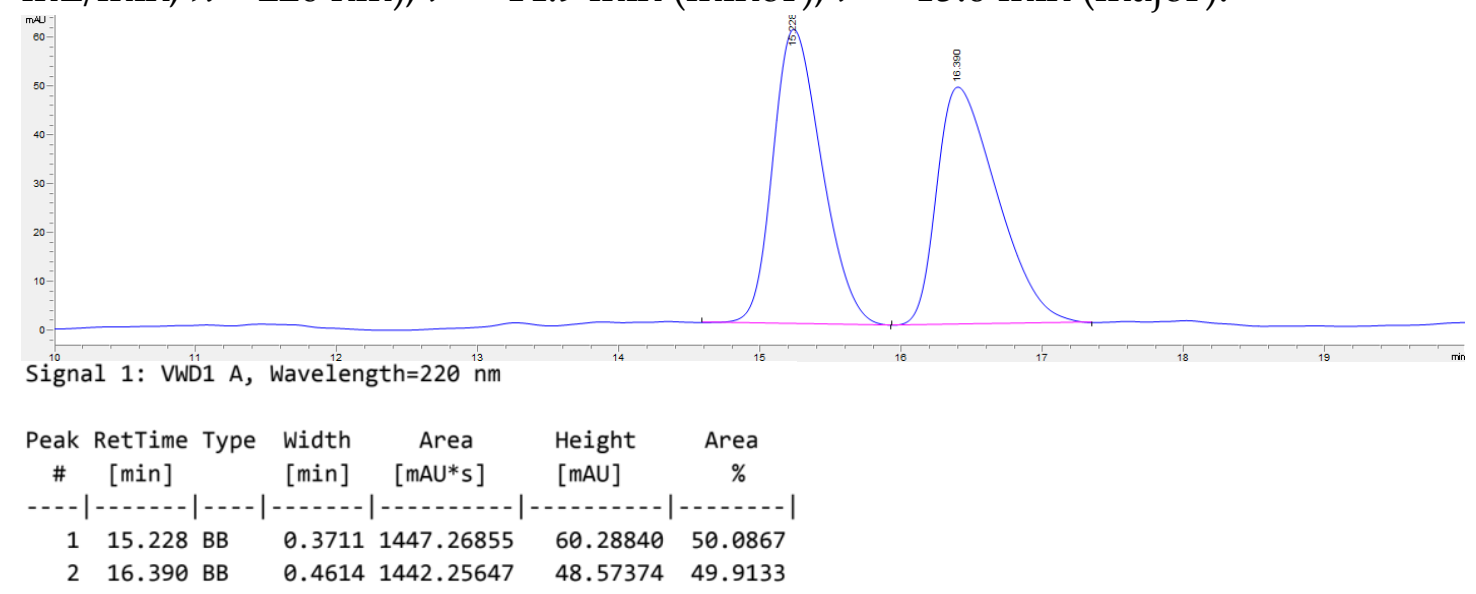




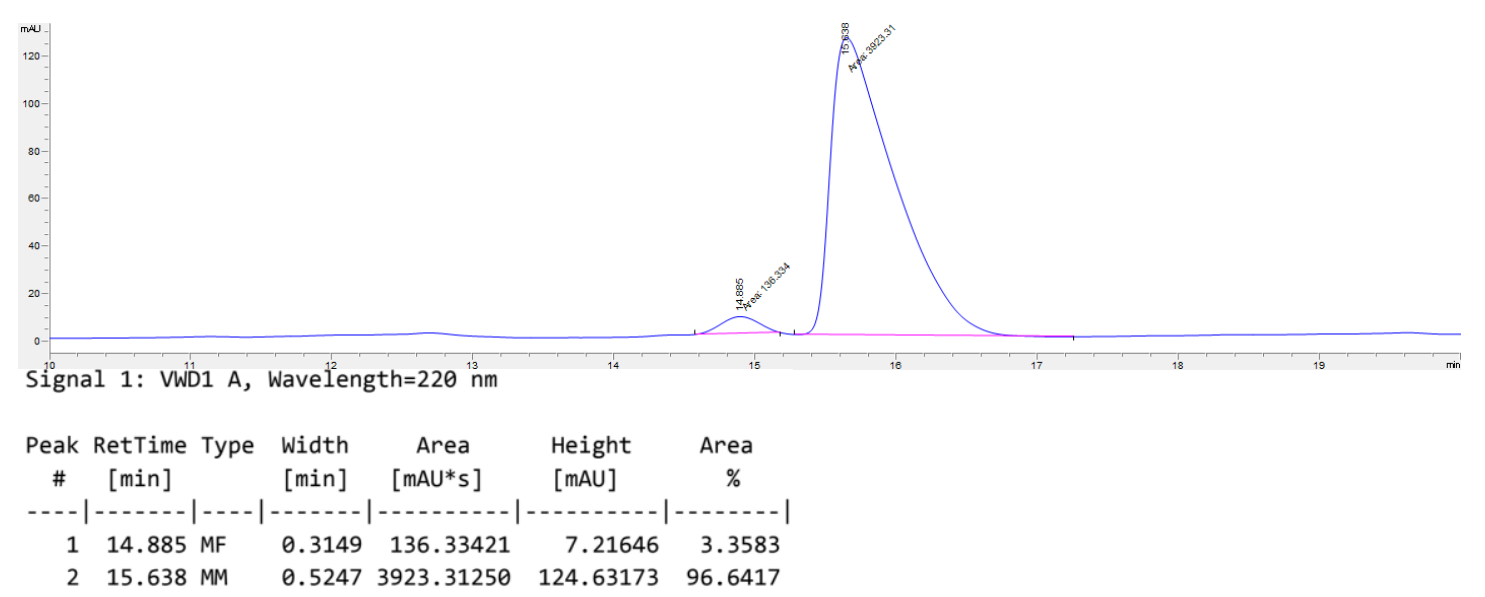

(S)-5-(4-Fluorophenyl)-1-((triisopropylsilyl)oxy)pentan-2-ol (3c)

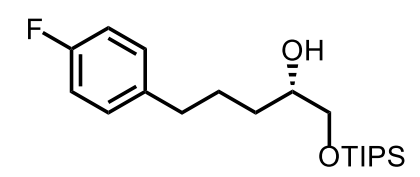

Compound 3c was prepared as a colorless oil in $89 \%$ yield $(63.3 \mathrm{mg}$, eluent: petroleum ether/EtOAc $=100 / 3$ to 20/1), from (Z)-((5-(4-fluorophenyl)pent-1en-1-yl)oxy)triisopropylsilane $1 \mathrm{c}(67.2 \mathrm{mg}, 0.20 \mathrm{mmol}),[\mathrm{RhCl}(\mathrm{cod})]_{2}(2.0 \mathrm{mg}$, $0.004 \mathrm{mmol}),(S)-\mathbf{L} 9(3.5 \mathrm{mg}, 0.008 \mathrm{mmol}), \mathrm{HBpin}(38.4 \mathrm{mg}, 0.30 \mathrm{mmol})$ and $\mathrm{LiOAc}(4.0 \mathrm{mg}, 0.06 \mathrm{mmol})$ following the above general procedure $\mathrm{E}$.

$\mathbf{R}_{f}=0.65$ (petroleum ether $/$ EtOAc $=10 / 1$ )

${ }^{1} \mathrm{H}$ NMR (400 MHz, $\left.\mathrm{CDCl}_{3}\right)$ \& 7.18-7.06 (m, 2H), 7.01-6.87 (m, 2H), 3.77-3.60 (m, $2 \mathrm{H}), 3.56-3.37(\mathrm{~m}, 1 \mathrm{H}), 2.71-2.45(\mathrm{~m}, 3 \mathrm{H}), 1.88-1.72(\mathrm{~m}, 1 \mathrm{H}), 1.72-1.55(\mathrm{~m}, 1 \mathrm{H})$, $1.54-1.35(\mathrm{~m}, 2 \mathrm{H}), 1.17-0.94(\mathrm{~m}, 21 \mathrm{H})$.

${ }^{13} \mathrm{C}$ NMR (100 MHz, $\left.\mathrm{CDCl}_{3}\right)$ o $161.4(\mathrm{~d}, J=243.2 \mathrm{~Hz}), 138.0(\mathrm{~d}, J=3.1 \mathrm{~Hz}), 129.8$ $(\mathrm{d}, J=7.7 \mathrm{~Hz}), 115.1(\mathrm{~d}, J=21.1 \mathrm{~Hz}), 71.9,67.7,35.2,32.4,27.7,18.1,12.0$.

${ }^{19}$ F NMR (377 MHz, $\left.\mathrm{CDCl}_{3}\right) \delta$-118.0.

HRMS (ESI ${ }^{+}$) calcd for $\mathrm{C}_{20} \mathrm{H}_{35} \mathrm{FO}_{2} \mathrm{NaSi}^{+}[\mathrm{M}+\mathrm{Na}]^{+}: 377.2283$, found: 377.2281 .

IR (neat, cm$^{-1}$ ): 2939, 2866, 1510, 1223, 1119, 1068, 883, 684.

$[\alpha]_{\mathrm{D}^{26.2}}=2.30\left(\mathrm{c}=1.26, \mathrm{CHCl}_{3}, 91 \%\right.$ ee); Enantiomeric excess was determined by chiral HPLC (Daicel Chiralpak IA, hexane/isopropyl alcohol $=98.5 / 1.5$, flow rate $=0.5 \mathrm{~mL} / \mathrm{min}, \lambda=220 \mathrm{~nm}$ ),$t^{1}=10.5 \mathrm{~min}$ (minor),$t^{2}=10.9 \min$ (major).

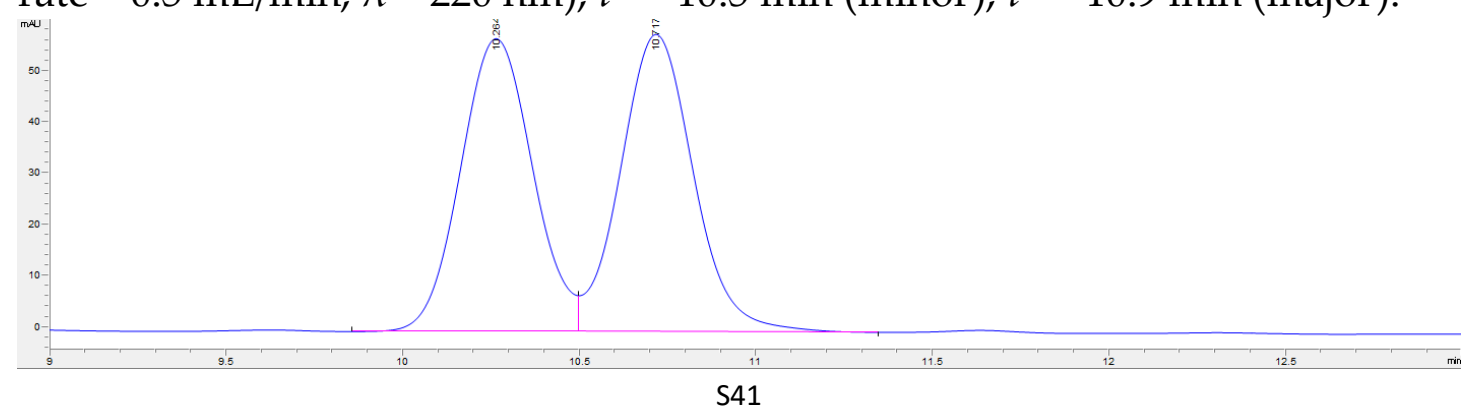




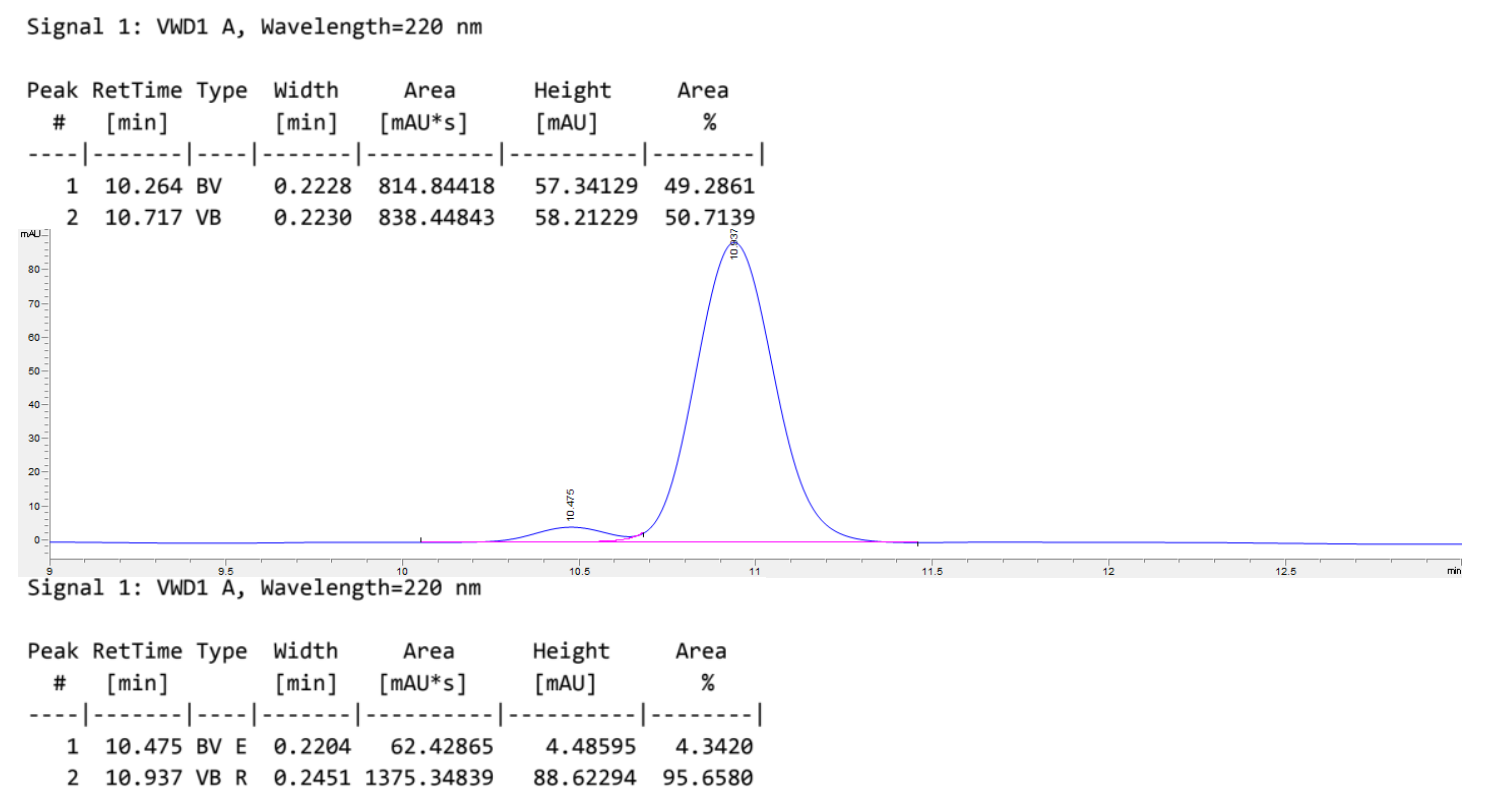

(S)-5-(4-Methoxyphenyl)-1-((triisopropylsilyl)oxy)pentan-2-ol (3d)

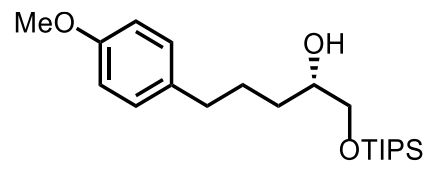

Compound 3d was prepared as a colorless oil in $74 \%$ yield $(54.4 \mathrm{mg}$, eluent: petroleum ether/EtOAc $=100 / 3$ to 20/1), from (Z)-triisopropyl((5-(4methoxyphenyl)pent-1-en-1-yl)oxy)silane $\quad \mathbf{1 d} \quad(69.6 \quad \mathrm{mg}, \quad 0.20 \quad \mathrm{mmol})$, [RhCl(cod)]2 (2.0 mg, $0.004 \mathrm{mmol})$, (S)-L9 (3.5 mg, $0.008 \mathrm{mmol})$, HBpin (38.4 mg, $0.30 \mathrm{mmol})$ and LiOAc $(4.0 \mathrm{mg}, 0.06 \mathrm{mmol})$ following the above general procedure $\mathrm{E}$.

$\mathbf{R}_{f}=0.32$ (petroleum ether $/$ EtOAc $=10 / 1$ )

${ }^{1} \mathrm{H}$ NMR $\left(400 \mathrm{MHz}, \mathrm{CDCl}_{3}\right) \delta 7.09(\mathrm{~d}, J=7.9 \mathrm{~Hz}, 2 \mathrm{H}), 6.82(\mathrm{~d}, J=7.9 \mathrm{~Hz}, 2 \mathrm{H})$, $3.78(\mathrm{~s}, 3 \mathrm{H}), 3.73-3.63(\mathrm{~m}, 2 \mathrm{H}), 3.52-3.42(\mathrm{~m}, 1 \mathrm{H}), 2.68-2.41(\mathrm{~m}, 3 \mathrm{H}), 1.86-1.72$ (m, 1H), 1.72-1.56 (m, 1H), 1.54-1.36 (m, 2H), 1.18-0.95 (m, 21H).

${ }^{13} \mathrm{C}$ NMR (100 MHz, CDCl $)$ ) $157.8,134.6,129.4,113.8,72.0,67.7,55.4,35.2,32.4$, $27.8,18.1,12.0$.

HRMS (ESI') calcd for $\mathrm{C}_{21} \mathrm{H}_{38} \mathrm{O}_{3} \mathrm{NaSi}^{+}$[M+Na] $]^{+}: 389.2482$, found: 389.2481 .

IR (neat, $\left.\mathbf{c m}^{-1}\right)$ : 2939, 2865, 1513, 1245, 1117, 883, 683.

$[\alpha]_{\mathrm{D}^{26.2}}=3.27$ ( $\mathrm{c}=0.61, \mathrm{CHCl}_{3}, 92 \%$ ee); Enantiomeric excess was determined by chiral HPLC (Daicel Chiralpak IA, hexane/isopropyl alcohol $=97 / 3$, flow rate $=$ $1.0 \mathrm{~mL} / \mathrm{min}, \lambda=220 \mathrm{~nm}$ ), $t^{1}=5.3 \mathrm{~min}$ (minor),$t^{2}=5.6 \mathrm{~min}$ (major). 


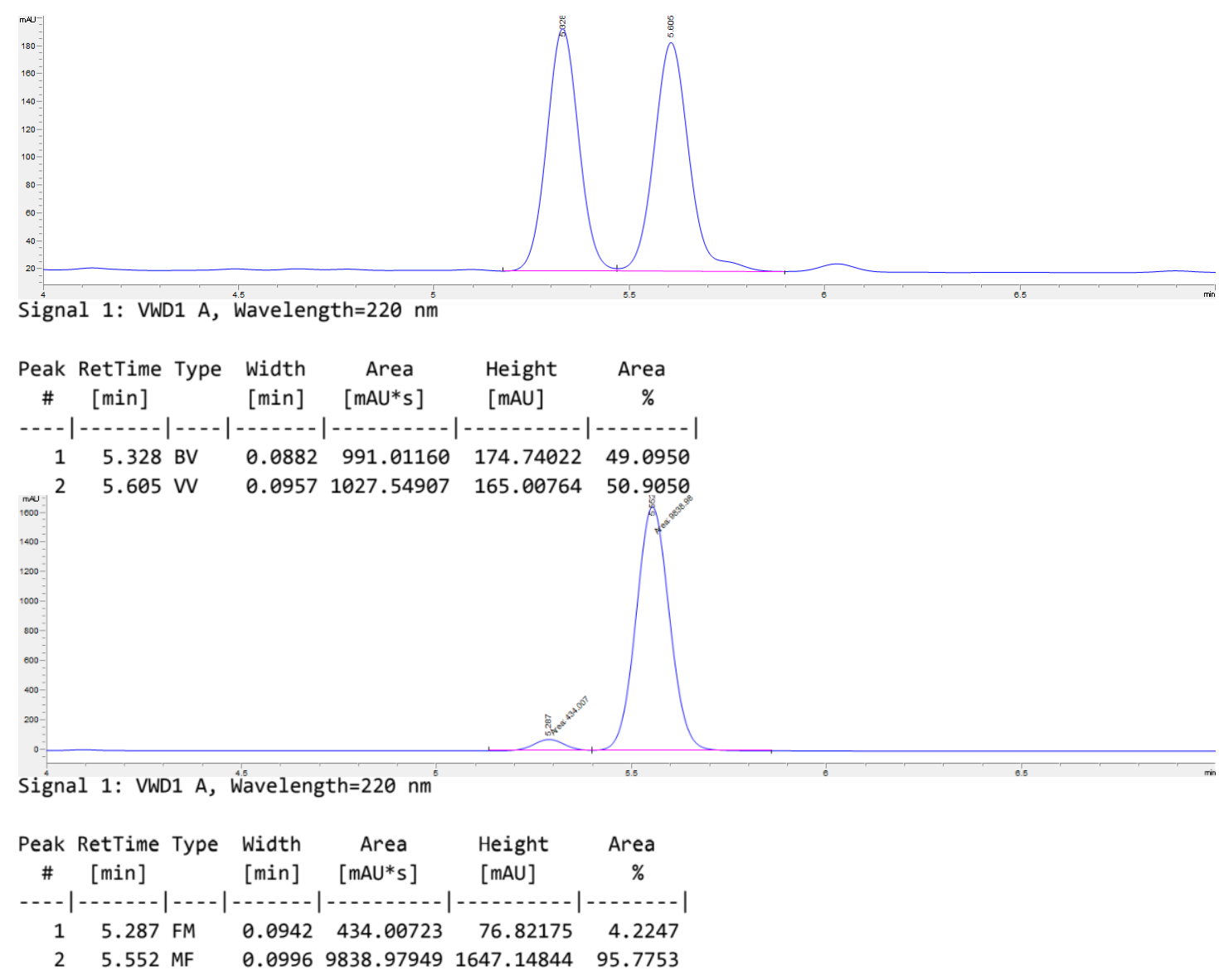

Compound $3 \mathbf{d}$ was prepared in $80 \%$ yield $(58.6 \mathrm{mg})$ and $88 \%$ ee from $1 \mathbf{d}(E / Z=$ 1/1.2) (69.6 mg, $0.20 \mathrm{mmol})$, [ RhCl(cod)]2 (2.0 mg, $0.004 \mathrm{mmol}),(S)-\mathrm{L} 9$ (3.5 mg, $0.008 \mathrm{mmol}), \mathrm{HBpin}(38.4 \mathrm{mg}, 0.30 \mathrm{mmol})$ and LiOAc $(4.0 \mathrm{mg}, 0.06 \mathrm{mmol})$ following the above general procedure $\mathrm{E}$.

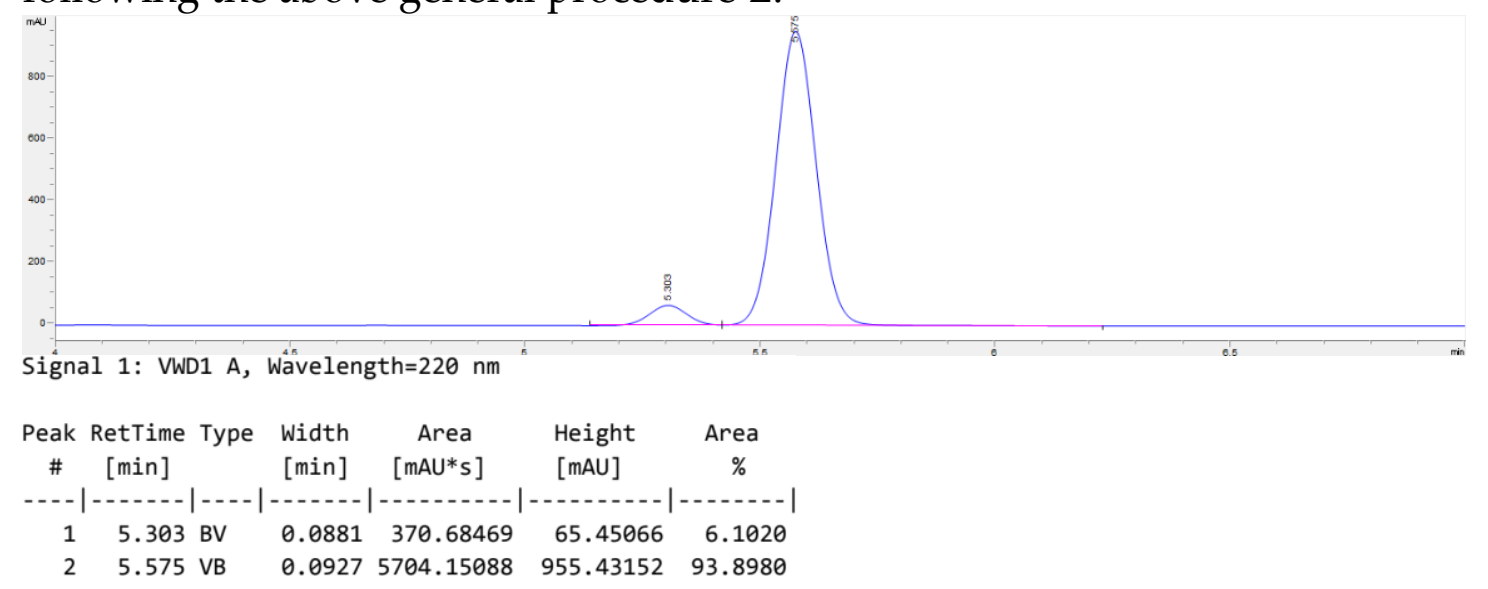

(S)-4-(4'-Amino-[1,1'-biphenyl]-4-yl)-1-((triisopropylsilyl)oxy)butan-2-ol (3e) 


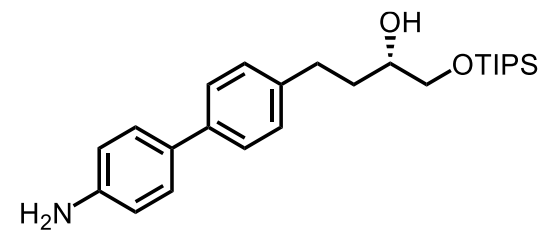

Compound 3e was prepared as a yellow oil in $66 \%$ yield $(55.1 \mathrm{mg}$, eluent: petroleum ether/EtOAc = 5/1 to 3/1), from (Z)-4'-(4-((triisopropylsilyl)oxy)but3-en-1-yl)-[1,1'-biphenyl]-4-amine 1 e (79.8 mg, $0.20 \mathrm{mmol})$, [RhCl(cod)]2 $(4.0 \mathrm{mg}$, $0.008 \mathrm{mmol})$, (S)-L9 (7.0 mg, $0.016 \mathrm{mmol})$, HBpin ( $89.6 \mathrm{mg}, 0.70 \mathrm{mmol})$ and $\mathrm{LiOAc}(4.0 \mathrm{mg}, 0.06 \mathrm{mmol})$ following the above general procedure $\mathrm{E}\left(30^{\circ} \mathrm{C}, 48\right.$ h).

$\mathbf{R}_{f}=0.40$ (petroleum ether $/$ EtOAc $=3 / 1$ )

${ }^{1} \mathrm{H}$ NMR (400 MHz, $\left.\mathrm{CDCl}_{3}\right) \delta 7.45(\mathrm{~d}, J=8.2 \mathrm{~Hz}, 2 \mathrm{H}), 7.39(\mathrm{~d}, J=8.5 \mathrm{~Hz}, 2 \mathrm{H})$, $7.23(\mathrm{~d}, J=9.1 \mathrm{~Hz}, 2 \mathrm{H}), 6.73(\mathrm{~d}, J=8.5 \mathrm{~Hz}, 2 \mathrm{H}), 3.85-3.61(\mathrm{~m}, 4 \mathrm{H}), 3.58-3.46$ $(\mathrm{m}, 1 \mathrm{H}), 2.94-2.79(\mathrm{~m}, 1 \mathrm{H}), 2.76-2.65(\mathrm{~m}, 1 \mathrm{H}), 2.60(\mathrm{~s}, 1 \mathrm{H}), 1.86-1.66(\mathrm{~m}, 2 \mathrm{H})$, $1.20-0.82(\mathrm{~m}, 21 \mathrm{H})$.

${ }^{13} \mathrm{C}$ NMR (100 MHz, $\left.\mathrm{CDCl}_{3}\right) \delta$ 145.7, 140.2, 138.9, 131.6, 128.9, 127.9, 126.5, 115.5, 71.4, 67.6, 34.6, 31.6, 18.1, 12.0.

HRMS (ESI') calcd for $\mathrm{C}_{25} \mathrm{H}_{3} \mathrm{O}_{2} \mathrm{NNaSi}^{+}[\mathrm{M}+\mathrm{Na}]^{+}: 436.2642$, found: 436.2633 .

IR (neat, cm cm: $^{-1}$ 2942, 2865, 1500, 1122, 883, 808, 683

$[\alpha]_{\mathrm{D}^{23.2}}=-2.80\left(\mathrm{c}=1.01, \mathrm{CHCl}_{3}, 85 \%\right.$ ee); Enantiomeric excess was determined by chiral HPLC (Daicel Chiralpak IA, hexane/isopropyl alcohol =95/5, flow rate $=0.5 \mathrm{~mL} / \mathrm{min}, \lambda=220 \mathrm{~nm}$ ), $t^{1}=44.8 \mathrm{~min}$ (minor),$t^{2}=45.6 \mathrm{~min}$ (major).

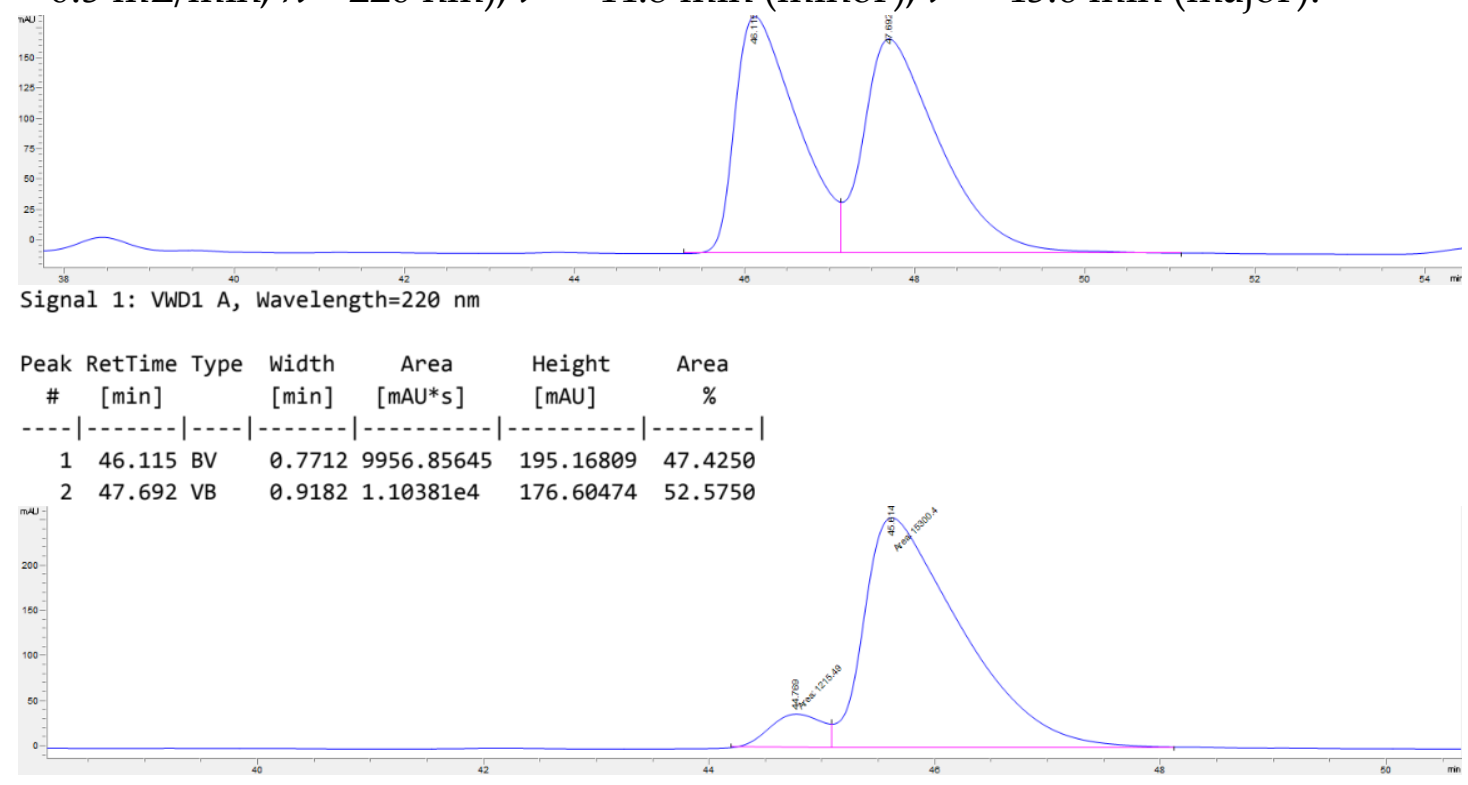




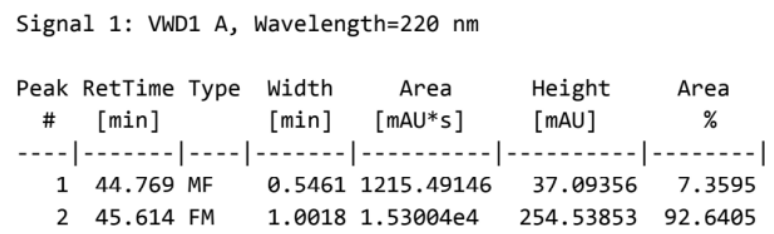

(S)-5-(Furan-2-yl)-1-((triisopropylsilyl)oxy)pentan-2-ol (3f)

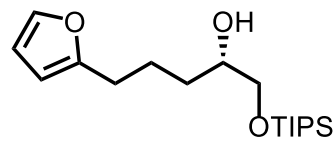

Compound $3 \mathrm{f}$ was prepared as a colorless oil in $68 \%$ yield $(44.5 \mathrm{mg}$, eluent: petroleum ether/EtOAc $=100 / 3$ to $20 / 1)$, from $(Z)-((5-($ furan-2-yl)pent-1-en-1yl)oxy)triisopropylsilane $1 f(61.6 \mathrm{mg}, 0.20 \mathrm{mmol})$, [ $\mathrm{RhCl}(\mathrm{cod})]_{2}(2.0 \mathrm{mg}, 0.004$ mmol), (S)-L9 (3.5 mg, $0.008 \mathrm{mmol})$, HBpin (38.4 mg, $0.30 \mathrm{mmol}$ ) and LiOAc $(4.0 \mathrm{mg}, 0.06 \mathrm{mmol})$ following the above general procedure $\mathrm{E}$.

$\mathbf{R}_{f}=0.70$ (petroleum ether/EtOAc $=10 / 1$ )

${ }^{1}$ H NMR (400 MHz, $\mathrm{CDCl}_{3}$ ) $\delta$ 7.32-7.27 (m, 1H), 6.31-6.22 (m, 1H), 6.04-5.94 (m, $1 \mathrm{H}), 3.74-3.64(\mathrm{~m}, 2 \mathrm{H}), 3.52-3.43(\mathrm{~m}, 1 \mathrm{H}), 2.71-2.63(\mathrm{~m}, 2 \mathrm{H}), 2.57$ (s, 1H), 1.91$1.78(\mathrm{~m}, 1 \mathrm{H}), 1.78-1.59(\mathrm{~m}, 1 \mathrm{H}), 1.56-1.39(\mathrm{~m}, 2 \mathrm{H}), 1.18-0.94(\mathrm{~m}, 21 \mathrm{H})$.

${ }^{13} \mathrm{C}$ NMR (100 MHz, $\left.\mathrm{CDCl}_{3}\right) \delta$ 156.1, 140.9, 110.2, 105.0, 71.8, 67.6, 32.3, 28.1, 24.3, 18.1, 12.0.

HRMS (ESI') calcd for $\mathrm{C}_{18} \mathrm{H}_{34} \mathrm{O}_{3} \mathrm{NaSi}^{+}[\mathrm{M}+\mathrm{Na}]^{+}$: 349.2169, found: 349.2168.

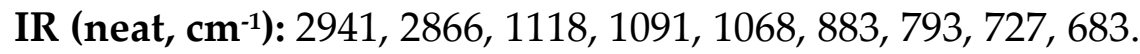

$[\alpha]_{\mathrm{D}^{26.2}}=1.09\left(\mathrm{c}=0.78, \mathrm{CHCl}_{3}, 91 \%\right.$ ee $)$.

Compound $3 \mathbf{f}$ was subjected to general procedure $\mathrm{H}$ and the resulting alcohol was analyzed by chiral HPLC. Enantiomeric excess was determined by chiral HPLC (Daicel Chiralcel OD-H, hexane/isopropyl alcohol $=95 / 5$, flow rate $=0.5$ $\mathrm{mL} / \mathrm{min}, \lambda=220 \mathrm{~nm}$ ),$t^{1}=32.2 \mathrm{~min}$ (minor),$t^{2}=33.5 \mathrm{~min}$ (major).

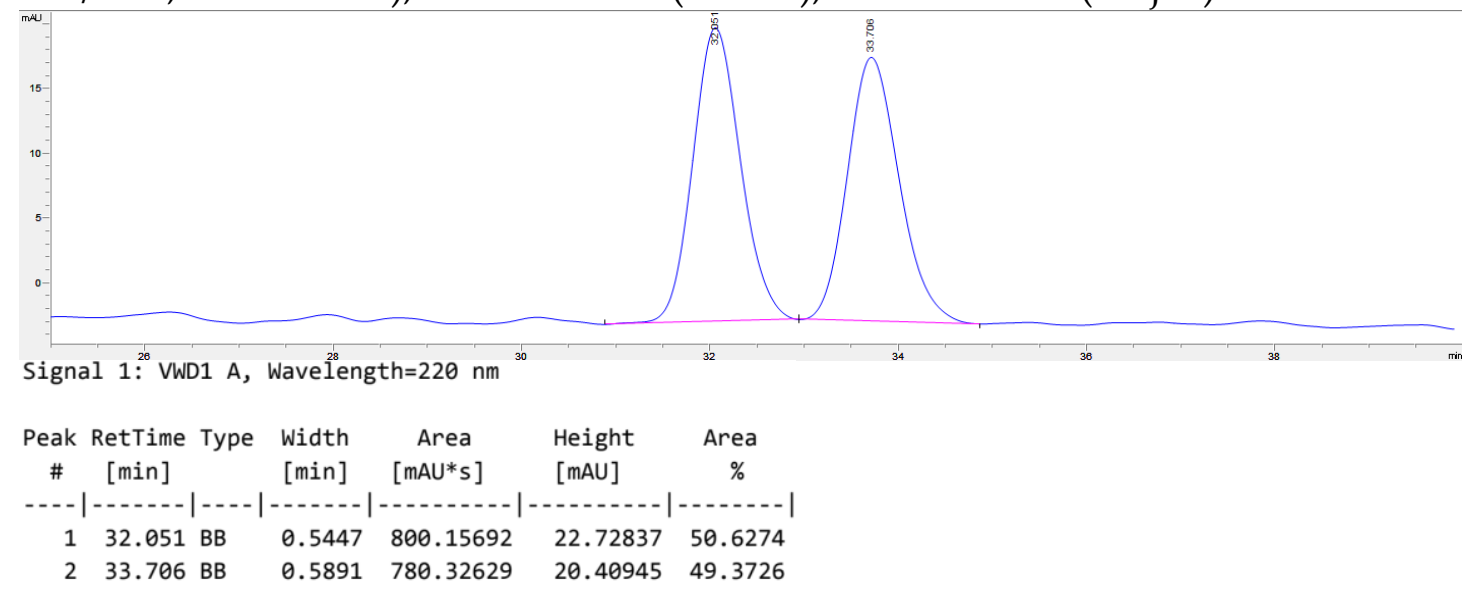




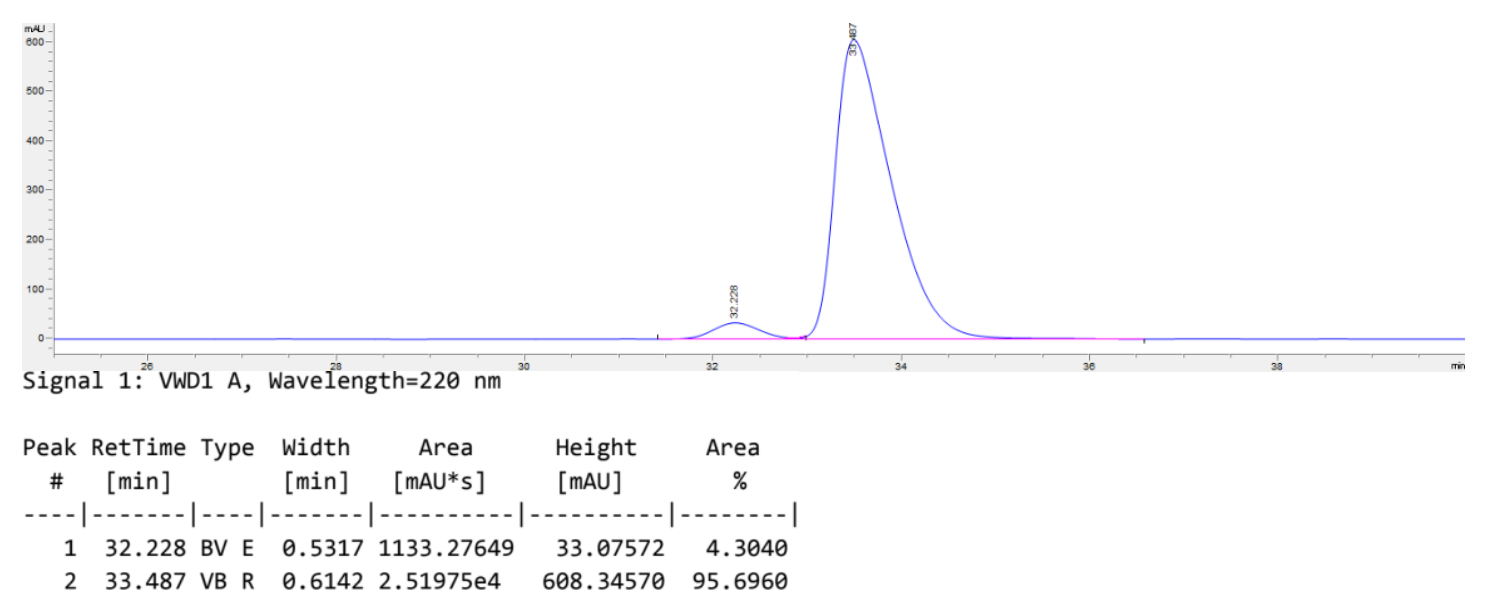

(S)-4-(Thiophen-2-yl)-1-((triisopropylsilyl)oxy)butan-2-ol (3g)<smiles>C[Hg]OC(O)CCCc1cccs1</smiles>

Compound 3g was prepared as a colorless oil in $74 \%$ yield (48.4 $\mathrm{mg}$, eluent: petroleum ether/EtOAc $=100 / 3$ to 20/1), from $(Z)$-triisopropyl((4-(thiophen-2yl)but-1-en-1-yl)oxy)silane $1 \mathrm{~g}$ (62.0 mg, $0.20 \mathrm{mmol})$, [RhCl(cod)]2 (2.0 mg, 0.004 mmol), (S)-L9 (3.5 mg, $0.008 \mathrm{mmol})$, HBpin $(38.4 \mathrm{mg}, 0.30 \mathrm{mmol})$ and LiOAc $(4.0 \mathrm{mg}, 0.06 \mathrm{mmol})$ following the above general procedure $\mathrm{E}$.

$\mathbf{R}_{f}=0.70$ (petroleum ether $/$ EtOAc $=10 / 1$ )

${ }^{1} \mathrm{H}$ NMR (400 MHz, $\left.\mathrm{CDCl}_{3}\right) \delta$ 7.16-7.08 (m, 1H), 6.95-6.88 (m, 1H), 6.85-6.78 (m, $1 \mathrm{H}), 3.79-3.66(\mathrm{~m}, 2 \mathrm{H}), 3.58-3.45(\mathrm{~m}, 1 \mathrm{H}), 3.11-3.00(\mathrm{~m}, 1 \mathrm{H}), 2.99-2.87(\mathrm{~m}, 1 \mathrm{H})$, $2.57(\mathrm{~s}, 1 \mathrm{H}), 1.90-1.72(\mathrm{~m}, 2 \mathrm{H}), 1.20-0.95(\mathrm{~m}, 21 \mathrm{H})$.

${ }^{13} \mathrm{C}$ NMR (100 MHz, CDCl $)$ ) $145.1,126.9,124.4,123.1,71.1,67.6,35.0,26.2,18.1$, 12.0.

HRMS (ESI ${ }^{+}$) calcd for $\mathrm{C}_{17} \mathrm{H}_{32} \mathrm{O}_{2} \mathrm{NaSSi}^{+}[\mathrm{M}+\mathrm{Na}]^{+}:$351.1784, found: 351.1783 .

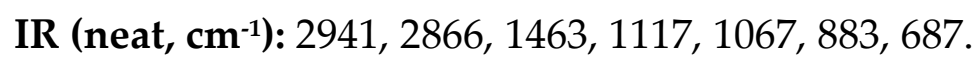

$[\alpha]_{D^{26.2}}=-5.29\left(\mathrm{c}=0.67, \mathrm{CHCl}_{3}, 91 \%\right.$ ee); Enantiomeric excess was determined by chiral HPLC (Daicel Chiralcel OD-H, hexane/isopropyl alcohol $=95 / 5$, flow rate $=1.0 \mathrm{~mL} / \mathrm{min}, \lambda=220 \mathrm{~nm}$ ) $t^{1}=4.2 \mathrm{~min}$ (major),$t^{2}=4.6 \mathrm{~min}$ (minor).

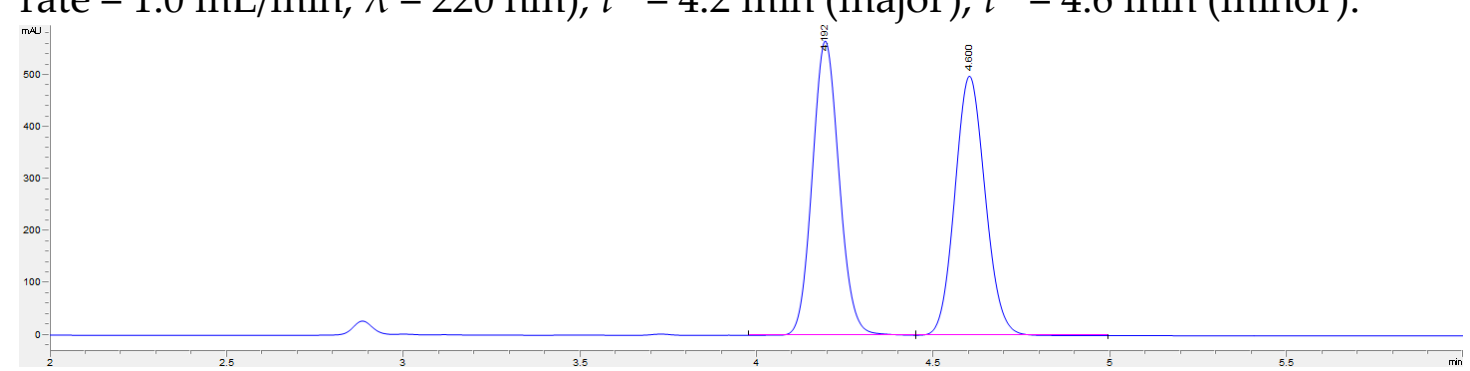


Signal 1: VWD1 A, Wavelength $=220 \mathrm{~nm}$

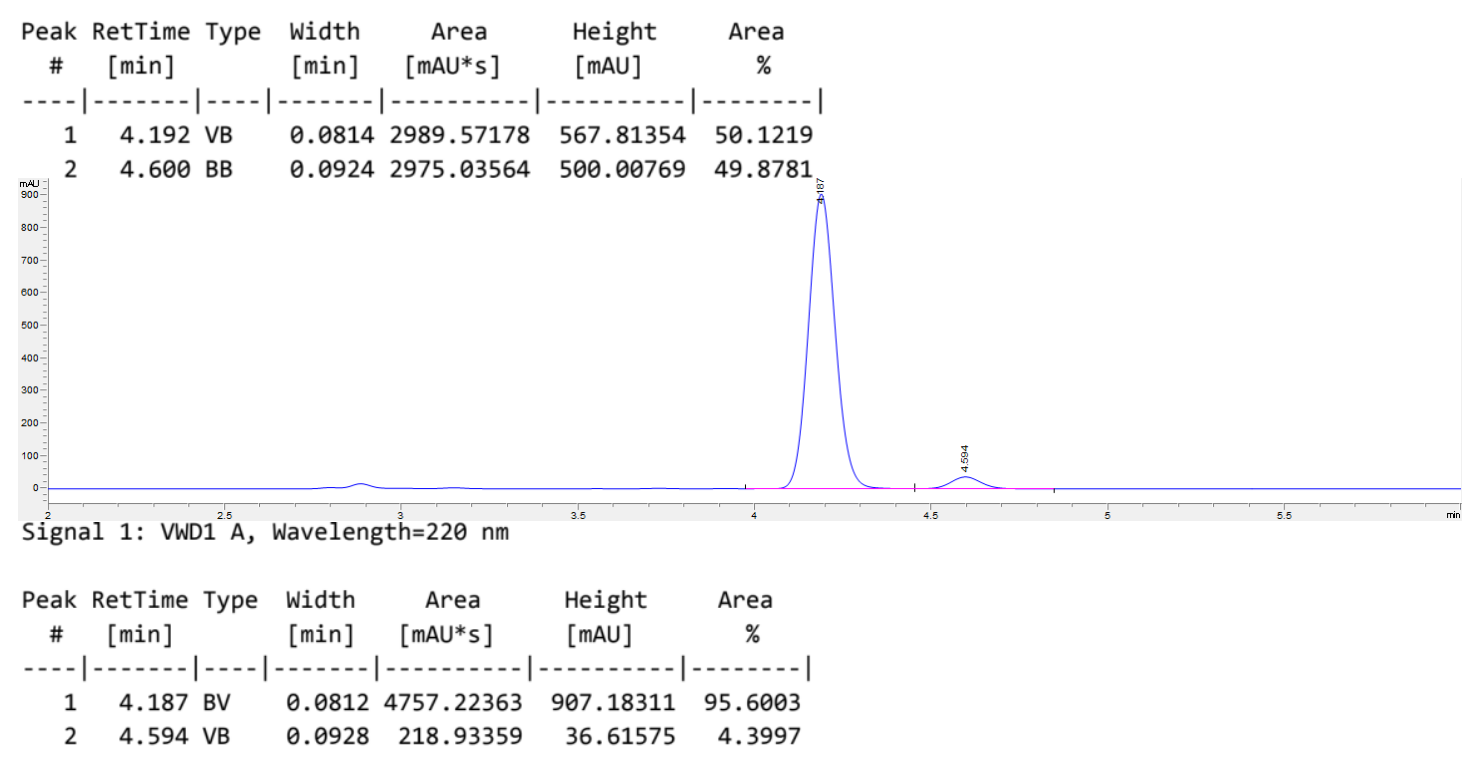

Compound 3g was prepared in $68 \%$ yield $(44.6 \mathrm{mg})$ and $88 \%$ ee from $1 \mathrm{~g}(E / \mathrm{Z}=$ 1/1.9) (62.0 mg, $0.20 \mathrm{mmol})$, [RhCl(cod) $]_{2}(2.0 \mathrm{mg}, 0.004 \mathrm{mmol}),(S)-\mathrm{L} 9$ (3.5 mg, $0.008 \mathrm{mmol})$, HBpin $(38.4 \mathrm{mg}, 0.30 \mathrm{mmol})$ and LiOAc $(4.0 \mathrm{mg}, 0.06 \mathrm{mmol})$ following the above general procedure $\mathrm{E}$.

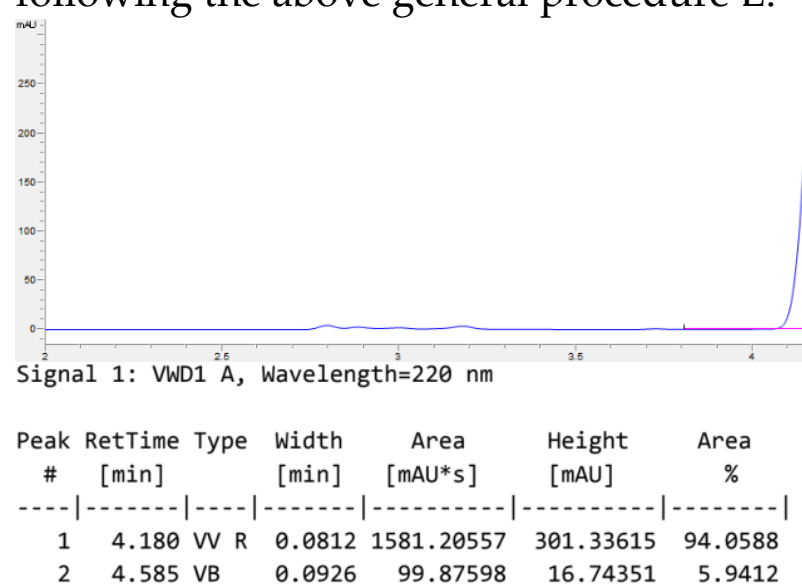

(S)-5-(1-Methyl-1H-indol-5-yl)-1-((triisopropylsilyl)oxy)pentan-2-ol (3h)<smiles>OC(O)CCCc1ccc2cc[nH]cc1-2</smiles>

Compound $3 \mathrm{~h}$ was prepared as a colorless oil in $81 \%$ yield $(62.9 \mathrm{mg}$, eluent: petroleum ether/EtOAc $=50 / 1$ to 10/1), from (Z)-1-methyl-5-(5((triisopropylsilyl)oxy)pent-4-en-1-yl)-1H-indole $1 \mathrm{~h}$ (74.4 mg, $0.20 \mathrm{mmol})$, $[\mathrm{RhCl}(\mathrm{cod})]_{2}(2.0 \mathrm{mg}, 0.004 \mathrm{mmol}),(S)-\mathrm{L} 9(3.5 \mathrm{mg}, 0.008 \mathrm{mmol}), \mathrm{HBpin}(38.4 \mathrm{mg}$, 
$0.30 \mathrm{mmol})$ and LiOAc $(4.0 \mathrm{mg}, 0.06 \mathrm{mmol})$ following the above general procedure $\mathrm{E}$.

$\mathbf{R}_{f}=0.50$ (petroleum ether $/ \mathrm{EtOAc}=5 / 1$ )

${ }^{1} \mathrm{H}$ NMR (400 MHz, $\mathrm{CDCl}_{3}$ ) \& 7.44-7.39 (m, 1H), 7.26-7.21 (m, 1H), 7.09-7.04 (m, 1H), 7.03-6.97 (m, 1H), 6.44-6.36 (m, 1H), $3.76(\mathrm{~s}, 3 \mathrm{H}), 3.73-3.64(\mathrm{~m}, 2 \mathrm{H}), 3.49-$ $3.40(\mathrm{~m}, 1 \mathrm{H}), 2.81-2.68(\mathrm{~m}, 2 \mathrm{H}), 2.58(\mathrm{~s}, 1 \mathrm{H}), 1.93-1.78(\mathrm{~m}, 1 \mathrm{H}), 1.77-1.62(\mathrm{~m}, 1 \mathrm{H})$, $1.56-1.39$ (m, 2H), 1.17-0.92 (m, 21H).

${ }^{13} \mathrm{C}$ NMR (100 MHz, CDCl $)$ ) $135.4,133.3,129.0,128.7,122.7,120.2,109.1,100.5$, 72.1, 67.7, 36.2, 33.0, 32.5, 28.3, 18.1, 12.0 .

HRMS (ESI') calcd for $\mathrm{C}_{23} \mathrm{H}_{39} \mathrm{NO}_{2} \mathrm{NaSi}^{+}[\mathrm{M}+\mathrm{Na}]^{+}: 412.2642$, found: 412.2640 .

IR (neat, cm ${ }^{-1}$ ): 2939, 2865, 1462, 1117, 882, 790, 717, 683.

$[\alpha]_{\mathrm{D}^{24.3}}=3.24\left(\mathrm{c}=0.93, \mathrm{CHCl}_{3}, 92 \%\right.$ ee); Enantiomeric excess was determined by chiral HPLC (Daicel Chiralpak IA, hexane/isopropyl alcohol $=98 / 2$, flow rate $=$ $1.0 \mathrm{~mL} / \mathrm{min}, \lambda=220 \mathrm{~nm}$ ), $t^{1}=9.6 \mathrm{~min}$ (minor),$t^{2}=11.8 \mathrm{~min}$ (major).

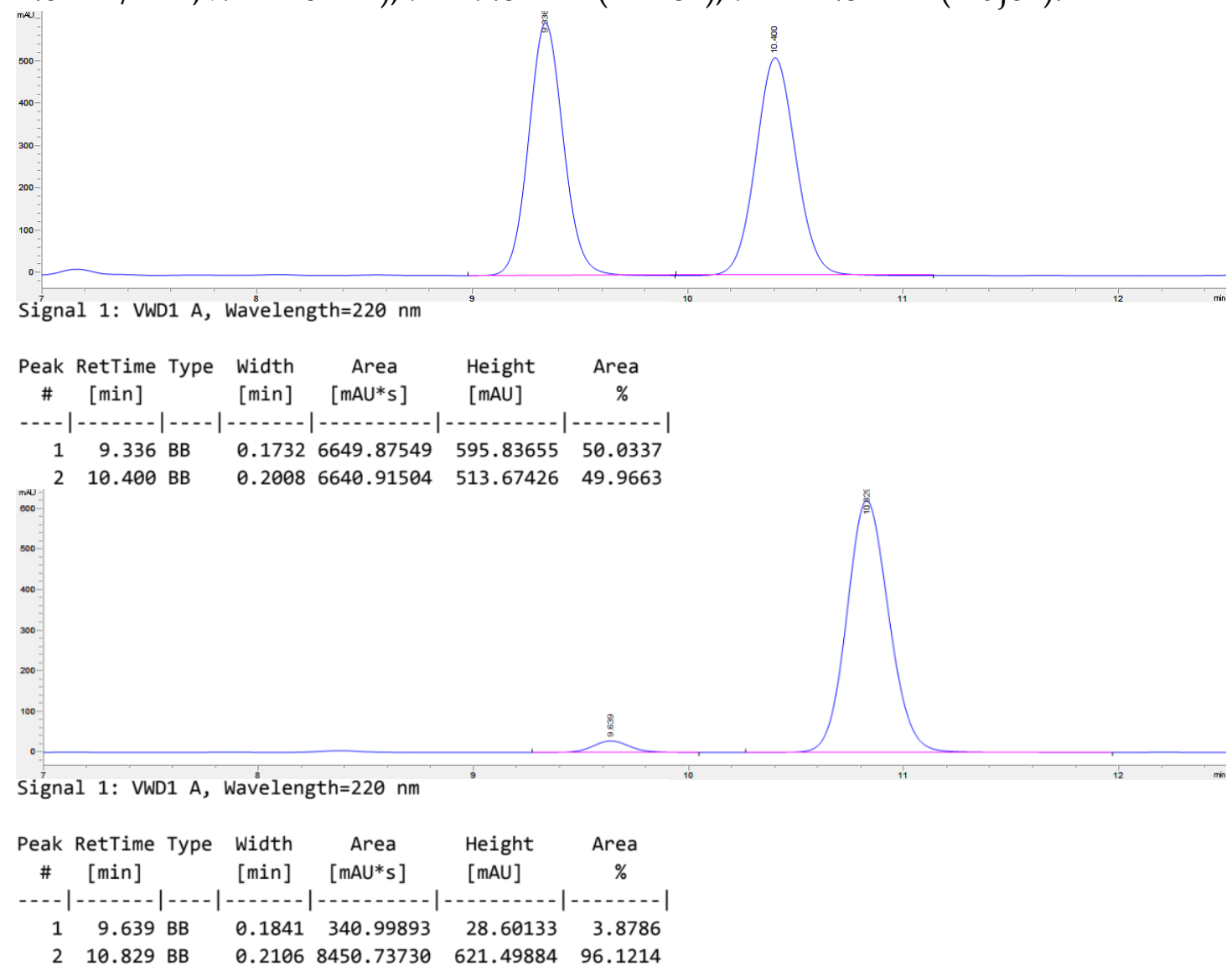

(S)-4-(1H-Indol-3-yl)-1-((triisopropylsilyl)oxy)butan-2-ol (3i) 


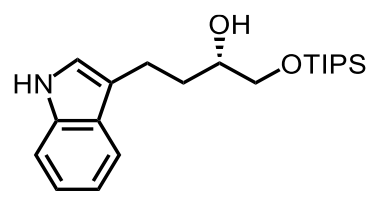

Compound $3 \mathbf{i}$ was prepared as a colorless oil in $65 \%$ yield $(46.9 \mathrm{mg}$, eluent: petroleum ether/EtOAc $=20 / 1)$, from $(Z)-3-(4-($ triisopropylsilyl)oxy $)$ but-3-en1-yl)- $1 \mathrm{H}$-indole $1 \mathrm{i}(68.6 \mathrm{mg}, 0.20 \mathrm{mmol})$, [ $\mathrm{RhCl}(\mathrm{cod})]_{2}(4.0 \mathrm{mg}, 0.008 \mathrm{mmol}),(S)$ L9 (7.0 mg, $0.016 \mathrm{mmol}), \mathrm{HBpin}(64.0 \mathrm{mg}, 0.50 \mathrm{mmol})$ and LiOAc $(4.0 \mathrm{mg}, 0.06$ $\mathrm{mmol}$ ) following the above general procedure $\mathrm{E}\left(30^{\circ} \mathrm{C}, 48 \mathrm{~h}\right)$.

$\mathbf{R}_{f}=0.35$ (petroleum ether/EtOAc $=5 / 1$ )

${ }^{1} \mathrm{H}$ NMR (400 MHz, CDCl $)$ ) $7.98(\mathrm{~s}, 1 \mathrm{H}), 7.62(\mathrm{~d}, J=7.9 \mathrm{~Hz}, 1 \mathrm{H}), 7.33(\mathrm{~d}, J=8.0$ $\mathrm{Hz}, 1 \mathrm{H}), 7.18(\mathrm{t}, J=7.5 \mathrm{~Hz}, 1 \mathrm{H}), 7.10(\mathrm{t}, J=7.4 \mathrm{~Hz}, 1 \mathrm{H}), 6.97(\mathrm{~s}, 1 \mathrm{H}), 3.84-3.68$ $(\mathrm{m}, 2 \mathrm{H}), 3.59-3.47(\mathrm{~m}, 1 \mathrm{H}), 3.04-2.92(\mathrm{~m}, 1 \mathrm{H}), 2.91-2.79(\mathrm{~m}, 1 \mathrm{H}), 2.63(\mathrm{~s}, 1 \mathrm{H})$, $1.93-1.77(\mathrm{~m}, 2 \mathrm{H}), 1.19-0.92(\mathrm{~m}, 21 \mathrm{H})$.

${ }^{13} \mathrm{C}$ NMR (101 MHz, $\left.\mathrm{CDCl}_{3}\right) \delta$ 136.5, 127.6, 122.0, 121.4, 119.2, 119.0, 116.3, 111.2, 71.7, 67.6, 33.3, 21.3, 18.1, 12.0.

HRMS (ESI') calcd for $\mathrm{C}_{21} \mathrm{H}_{36} \mathrm{O}_{2} \mathrm{NSi}^{+}[\mathrm{M}+\mathrm{H}]^{+}: 362.2510$, found: 362.2501 .

IR (neat, cm ${ }^{-1}$ ): 2942, 2866, 1458, 1118, 1067, 883, 740, 683

$[\alpha]_{\mathrm{D}^{23.3}}=-6.43\left(\mathrm{c}=0.97, \mathrm{CHCl}_{3}, 94 \%\right.$ ee); Enantiomeric excess was determined by chiral HPLC (Daicel Chiralpak IC, hexane/isopropyl alcohol =95/5, flow rate $=1.0 \mathrm{~mL} / \mathrm{min}, \lambda=220 \mathrm{~nm}$ ), $t^{1}=10.9 \min$ (major),$t^{2}=12.5 \mathrm{~min}$ (minor).

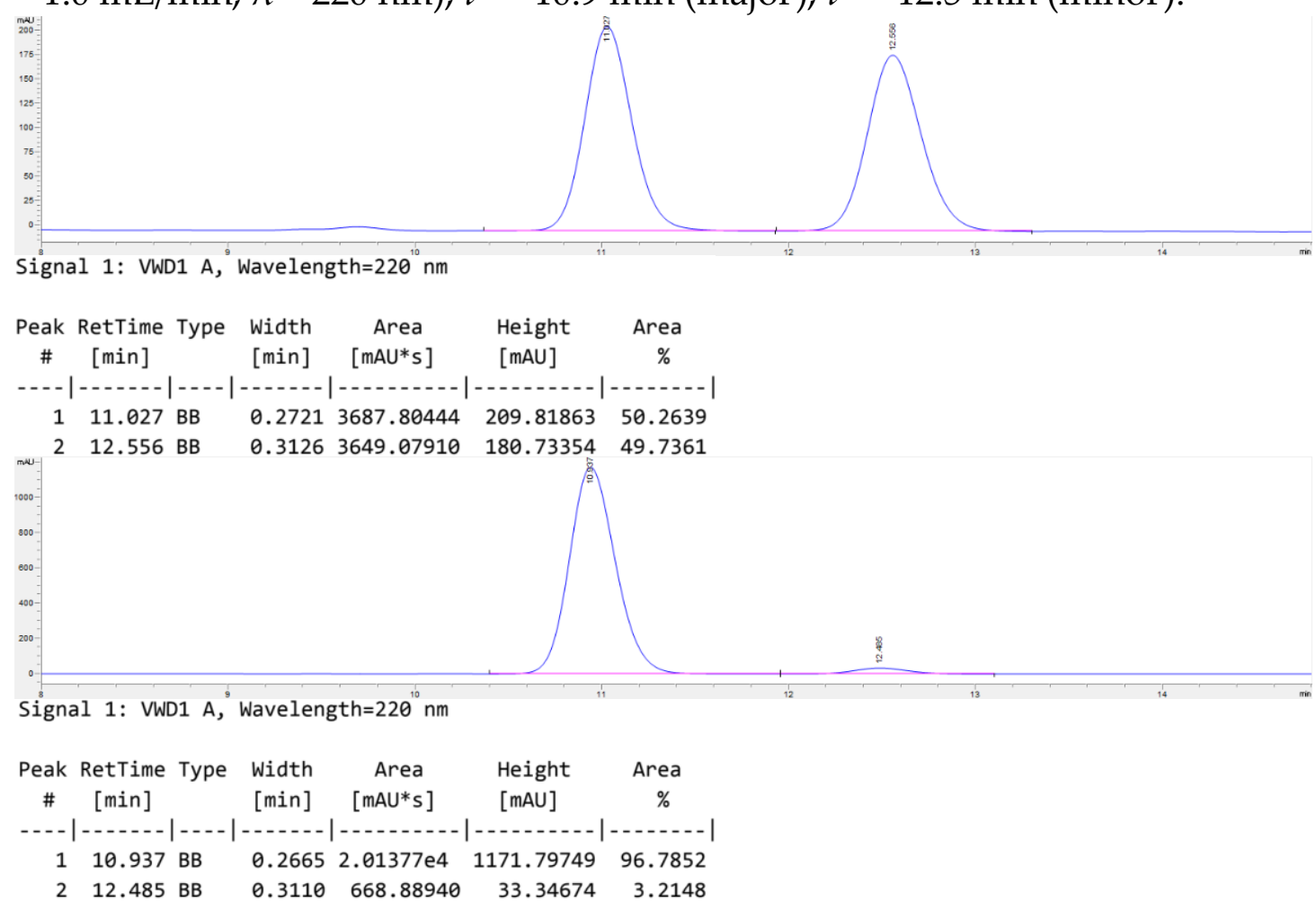


(S)-5-(Naphthalen-2-yl)-1-((triisopropylsilyl)oxy)pentan-2-ol (3j)<smiles>O[C@H](CCCc1ccc2ccccc2c1)CO[SnH3]</smiles>

Compound $3 \mathbf{j}$ was prepared as a colorless oil in $95 \%$ yield $(73.1 \mathrm{mg}$, eluent: petroleum ether/EtOAc $=50 / 1$ to $15 / 1)$, from $(Z)$-triisopropyl((5-(naphthalen-2yl)pent-1-en-1-yl)oxy)silane $\mathbf{1 j}$ (73.6 mg, $0.20 \mathrm{mmol})$, [RhCl(cod) ]2 (2.0 mg, 0.004 mmol), (S)-L9 (3.5 mg, $0.008 \mathrm{mmol})$, HBpin (38.4 mg, $0.30 \mathrm{mmol}$ ) and LiOAc $(4.0 \mathrm{mg}, 0.06 \mathrm{mmol})$ following the above general procedure $\mathrm{E}$.

$\mathbf{R}_{f}=0.65$ (petroleum ether $/ \mathrm{EtOAc}=10 / 1$ )

${ }^{1}$ H NMR (400 MHz, $\left.\mathrm{CDCl}_{3}\right) \delta$ 7.83-7.73 (m, 3H), 7.61 (s, 1H), 7.48-7.38 (m, 2H), 7.37-7.30 (m, 1H), 3.75-3.65 (m, 2H), 3.50-3.42 (m, 1H), 2.86-2.78 (m, 2H), 2.58 (s, $1 \mathrm{H}), 2.00-1.86(\mathrm{~m}, 1 \mathrm{H}), 1.84-1.70(\mathrm{~m}, 1 \mathrm{H}), 1.64-1.42(\mathrm{~m}, 2 \mathrm{H}), 1.18-0.98(\mathrm{~m}$, $21 \mathrm{H})$.

${ }^{13} \mathrm{C}$ NMR (100 MHz, $\left.\mathrm{CDCl}_{3}\right) \delta$ 140.0, 133.8, 132.1, 127.9, 127.7, 127.54, 127.48, 126.6, 125.9, 125.2, 72.0, 67.7, 36.2, 32.5, 27.4, 18.1, 12.0.

HRMS (ESI') calcd for $\mathrm{C}_{24} \mathrm{H}_{38} \mathrm{O}_{2} \mathrm{NaSi}^{+}[\mathrm{M}+\mathrm{Na}]^{+}:$409.2533, found: 409.2532 .

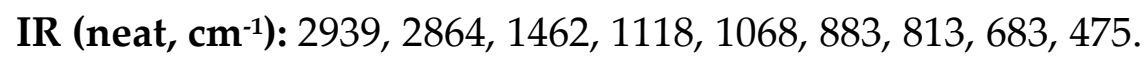

$[\alpha]_{D^{26.2}}=2.12\left(\mathrm{c}=1.02, \mathrm{CHCl}_{3}, 92 \%\right.$ ee); Enantiomeric excess was determined by chiral HPLC (Daicel Chiralcel OD-H, hexane/isopropyl alcohol =98/2, flow rate $=0.8 \mathrm{~mL} / \mathrm{min}, \lambda=254 \mathrm{~nm}$ ) $t^{1}=9.9 \mathrm{~min}$ (minor),$t^{2}=10.7 \mathrm{~min}$ (major).

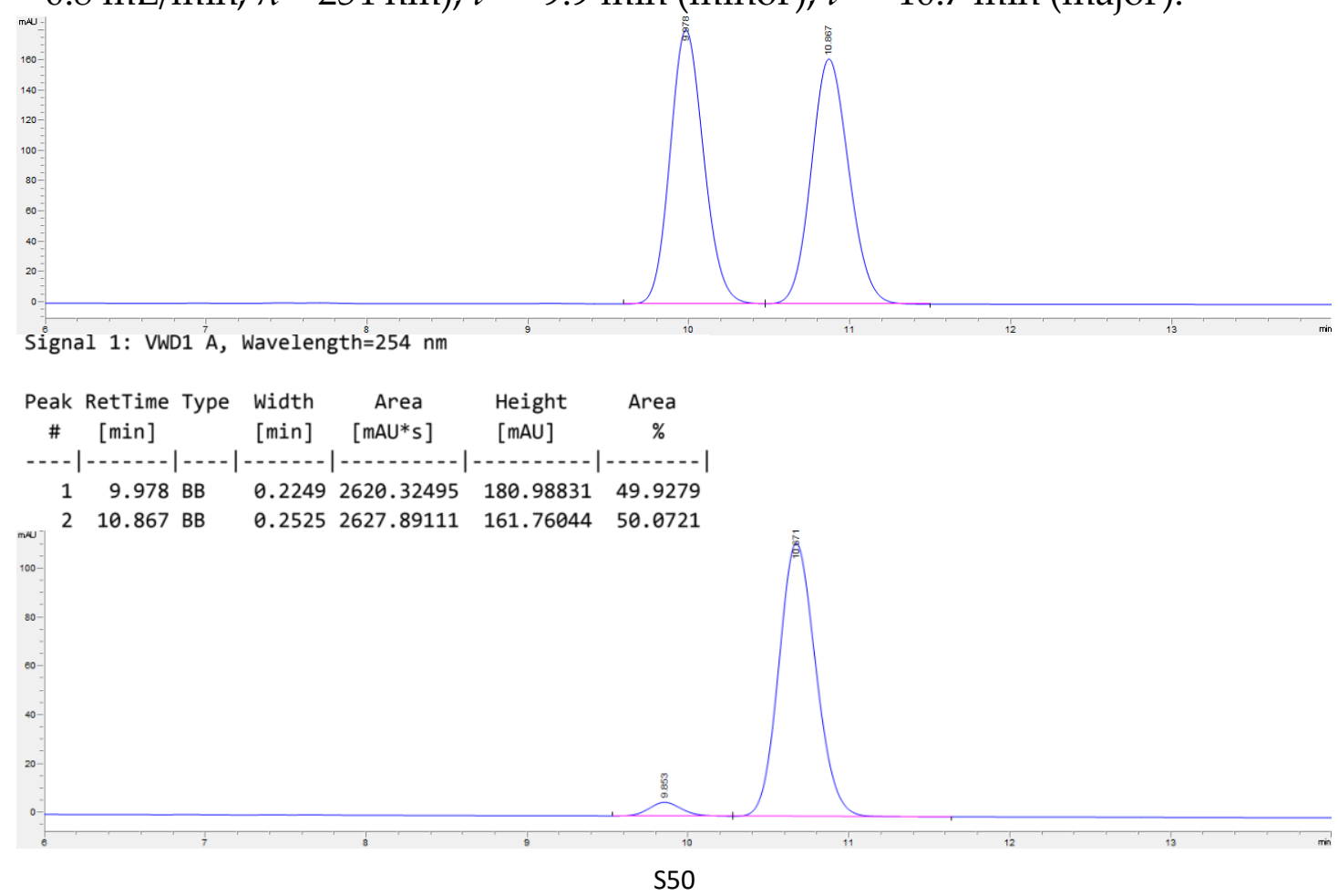




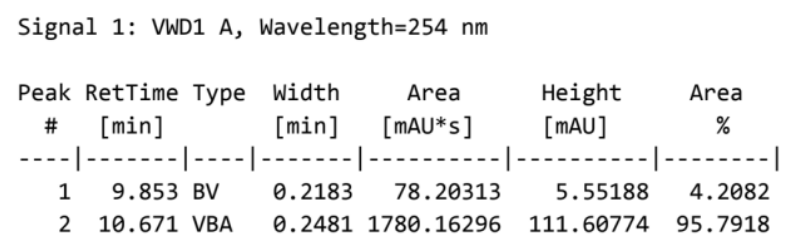

\section{(S)-1-Phenyl-3-((triisopropylsilyl)oxy)propan-2-ol (3k)}

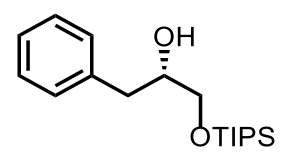

Compound 3k was prepared as a colorless oil in 83\% yield (51.2 mg, eluent: petroleum ether/EtOAc $=100 / 3$ to 20/1), from $(Z)$-triisopropyl((3-phenylprop1-en-1-yl)oxy)silane 1k (58.0 mg, $0.20 \mathrm{mmol})$, [RhCl(cod) ]2 (2.0 mg, $0.004 \mathrm{mmol})$, (S)-L9 (3.5 mg, $0.008 \mathrm{mmol}), \mathrm{HBpin}(38.4 \mathrm{mg}, 0.30 \mathrm{mmol})$ and $\mathrm{LiOAc}(4.0 \mathrm{mg}$, $0.06 \mathrm{mmol}$ ) following the above general procedure $\mathrm{E}$.

$\mathbf{R}_{f}=0.62$ (petroleum ether/EtOAc $=10 / 1$ )

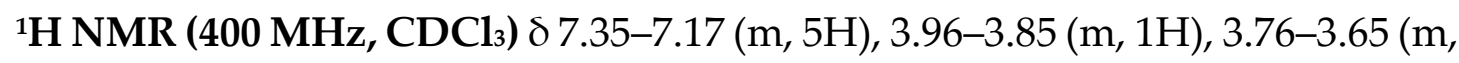
$1 \mathrm{H}), 3.63-3.53(\mathrm{~m}, 1 \mathrm{H}), 2.85-2.74(\mathrm{~m}, 2 \mathrm{H}), 2.51-2.11(\mathrm{~m}, 1 \mathrm{H}), 1.17-0.93(\mathrm{~m}, 21 \mathrm{H})$. ${ }^{13} \mathrm{C}$ NMR (100 MHz, $\left.\mathrm{CDCl}_{3}\right) \delta 138.5,129.4,128.5,126.4,73.1,66.8,39.7,18.1,12.1$. HRMS (ESI') calcd for $\mathrm{C}_{18} \mathrm{H}_{33} \mathrm{O}_{2} \mathrm{Si}^{+}[\mathrm{M}+\mathrm{H}]^{+}:$309.2244, found: 309.2243.

IR (neat, cm$^{-1}$ ): 2941, 2866, 1462, 1118, 1067, 883, 684.

$[\alpha]_{\mathrm{D}^{26.2}}=2.14\left(\mathrm{c}=0.83, \mathrm{CHCl}_{3}, 90 \%\right.$ ee); Enantiomeric excess was determined by chiral HPLC (Daicel Chiralcel OD-H, hexane/isopropyl alcohol =98/2, flow rate $=0.8 \mathrm{~mL} / \mathrm{min}, \lambda=220 \mathrm{~nm}$ ), $t^{1}=5.7 \mathrm{~min}$ (minor),$t^{2}=6.3 \mathrm{~min}$ (major).

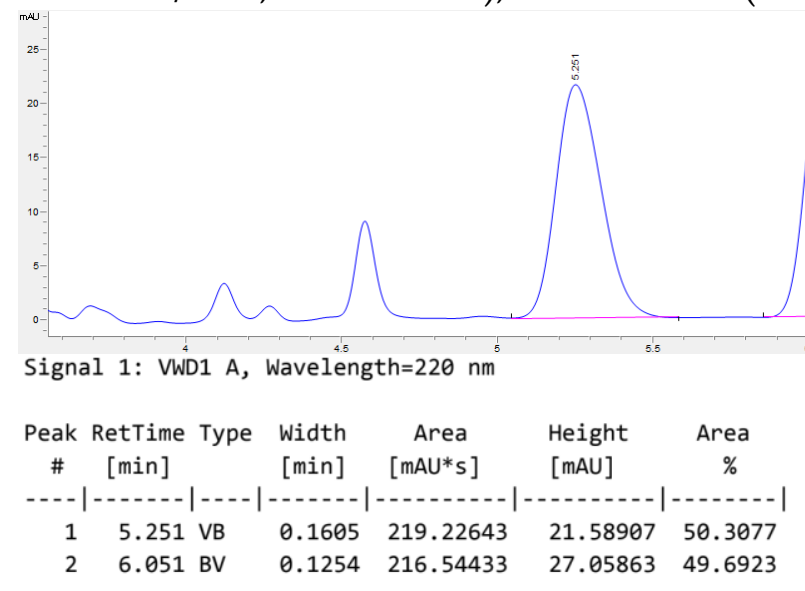




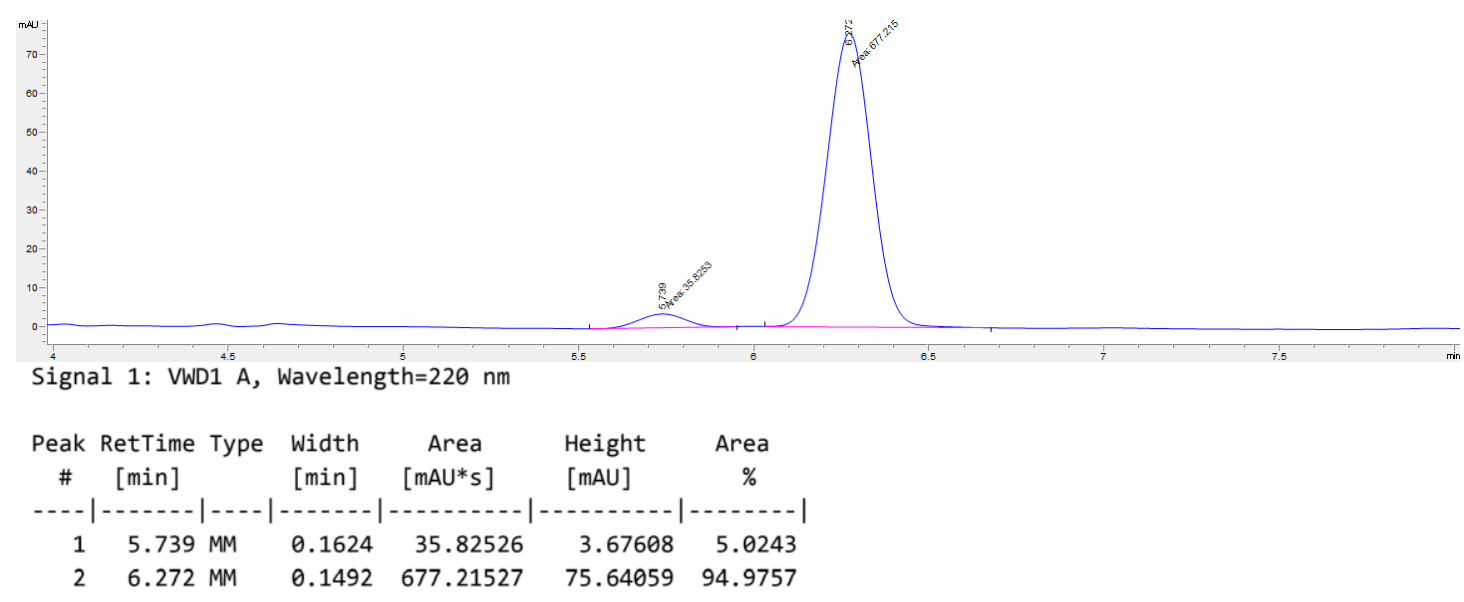

(S)-1-((Triisopropylsilyl)oxy)propan-2-ol (31)<smiles>[13CH3]C(O)C[18OH]</smiles>

Compound 31 was prepared as a colorless oil in $67 \%$ yield $(31.2 \mathrm{mg}$, eluent: petroleum ether/EtOAc $=100 / 3$ to $20 / 1$ ), from (Z)-triisopropyl(prop-1-en-1yloxy)silane 11 (42.8 mg, $0.20 \mathrm{mmol})$, [RhCl(cod)]2 (2.0 mg, $0.004 \mathrm{mmol}),(S)-\mathbf{L 9}$ (3.5 mg, $0.008 \mathrm{mmol})$, HBpin (38.4 mg, $0.30 \mathrm{mmol})$ and LiOAc (4.0 mg, 0.06 $\mathrm{mmol}$ ) following the above general procedure $\mathrm{E}$.

$\mathbf{R}_{f}=0.70$ (petroleum ether $/$ EtOAc $=10 / 1$ )

${ }^{1} \mathrm{H}$ NMR (400 MHz, $\mathrm{CDCl}_{3}$ ) 8 3.92-3.78 (m, 1H), 3.74-3.62 (m, 1H), 3.48-3.39 (m, $1 \mathrm{H}), 2.57$ (s, 1H), 1.18-0.99 (m, 24H).

${ }^{13} \mathrm{C}$ NMR (100 MHz, $\left.\mathrm{CDCl}_{3}\right) \delta$ 6 69.0, 68.2, 18.3, 18.1, 12.1.

HRMS (ESI ${ }^{+}$) calcd for $\mathrm{C}_{12} \mathrm{H}_{28} \mathrm{O}_{2} \mathrm{NaSi}^{+}[\mathrm{M}+\mathrm{Na}]^{+}: 255.1751$, found: 255.1750 .

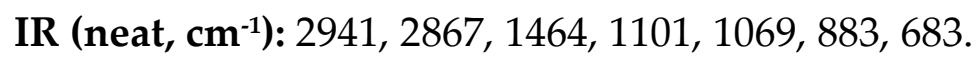

$[\alpha]^{26.2}=7.77\left(\mathrm{c}=0.86, \mathrm{CHCl}_{3}, 68 \%\right.$ ee $)$.

Compound 31 was subjected to general procedure J and the resulting ester was analyzed by chiral HPLC. Enantiomeric excess was determined by chiral HPLC (Daicel Chiralpak IA, hexane/isopropyl alcohol $=99.5 / 0.5$, flow rate $=0.5$ $\mathrm{mL} / \mathrm{min}, \lambda=220 \mathrm{~nm}$ ),$t^{1}=7.8 \mathrm{~min}$ (major),$t^{2}=8.4 \mathrm{~min}$ (minor).

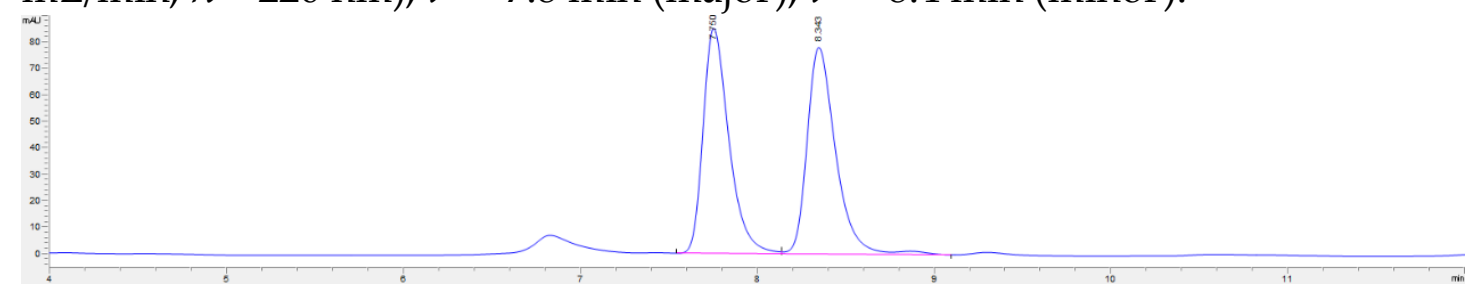




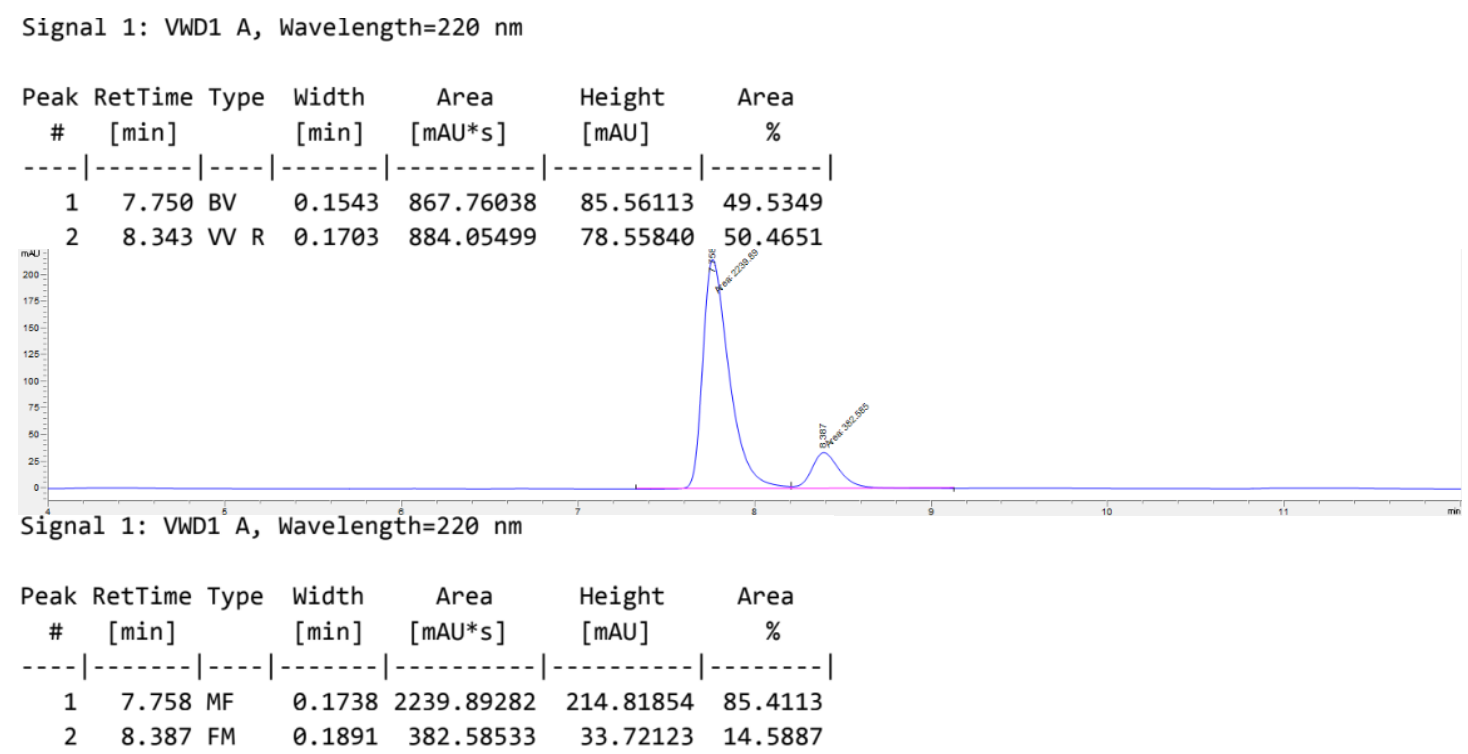

(S)-1-((Triisopropylsilyl)oxy)hexan-2-ol (3m)<smiles>CCCC[C@H](O)CO[Na]</smiles>

Compound 3m was prepared as a colorless oil in 91\% yield (50.0 mg, eluent: petroleum ether/EtOAc $=100 / 3$ to 20/1), from (Z)-(hex-1-en-1yloxy)triisopropylsilane $1 \mathrm{~m}(51.2 \mathrm{mg}, 0.20 \mathrm{mmol}),[\mathrm{RhCl}(\mathrm{cod})]_{2}(2.0 \mathrm{mg}, 0.004$ mmol), (S)-L9 (3.5 mg, $0.008 \mathrm{mmol})$, HBpin (38.4 mg, $0.30 \mathrm{mmol}$ ) and LiOAc $(4.0 \mathrm{mg}, 0.06 \mathrm{mmol})$ following the above general procedure E.

$\mathbf{R}_{f}=0.68$ (petroleum ether/EtOAc $=10 / 1$ )

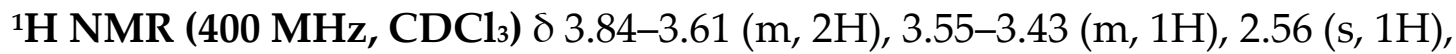
$1.49-1.30(\mathrm{~m}, 6 \mathrm{H}), 1.28-1.00(\mathrm{~m}, 21 \mathrm{H}), 0.91(\mathrm{t}, J=6.8 \mathrm{~Hz}, 3 \mathrm{H})$.

${ }^{13} \mathrm{C}$ NMR (100 MHz, $\left.\mathrm{CDCl}_{3}\right) \delta$ 72.1, 67.7, 32.6, 27.9, 23.0, 18.1, 14.2, 12.1.

HRMS (ESI') calcd for $\mathrm{C}_{15} \mathrm{H}_{34} \mathrm{O}_{2} \mathrm{NaSi}^{+}[\mathrm{M}+\mathrm{Na}]^{+}$: 297.2220, found: 297.2219.

IR (neat, cm-1): 2939, 2866, 1464, 1105, 883, 683.

$[\alpha]_{\mathrm{D}^{26.2}}=2.51\left(\mathrm{c}=0.41, \mathrm{CHCl}_{3}, 91 \%\right.$ ee $)$.

Compound $3 \mathrm{~m}$ was subjected to general procedure $\mathrm{J}$ and the resulting ester was analyzed by chiral HPLC. Enantiomeric excess was determined by chiral HPLC (Daicel Chiralcel AD-H, hexane/isopropyl alcohol $=99.7 / 0.3$, flow rate $=$ $0.5 \mathrm{~mL} / \mathrm{min}, \lambda=220 \mathrm{~nm}$ ), $t^{1}=10.4 \mathrm{~min}$ (major),$t^{2}=11.4 \mathrm{~min}$ (minor). 


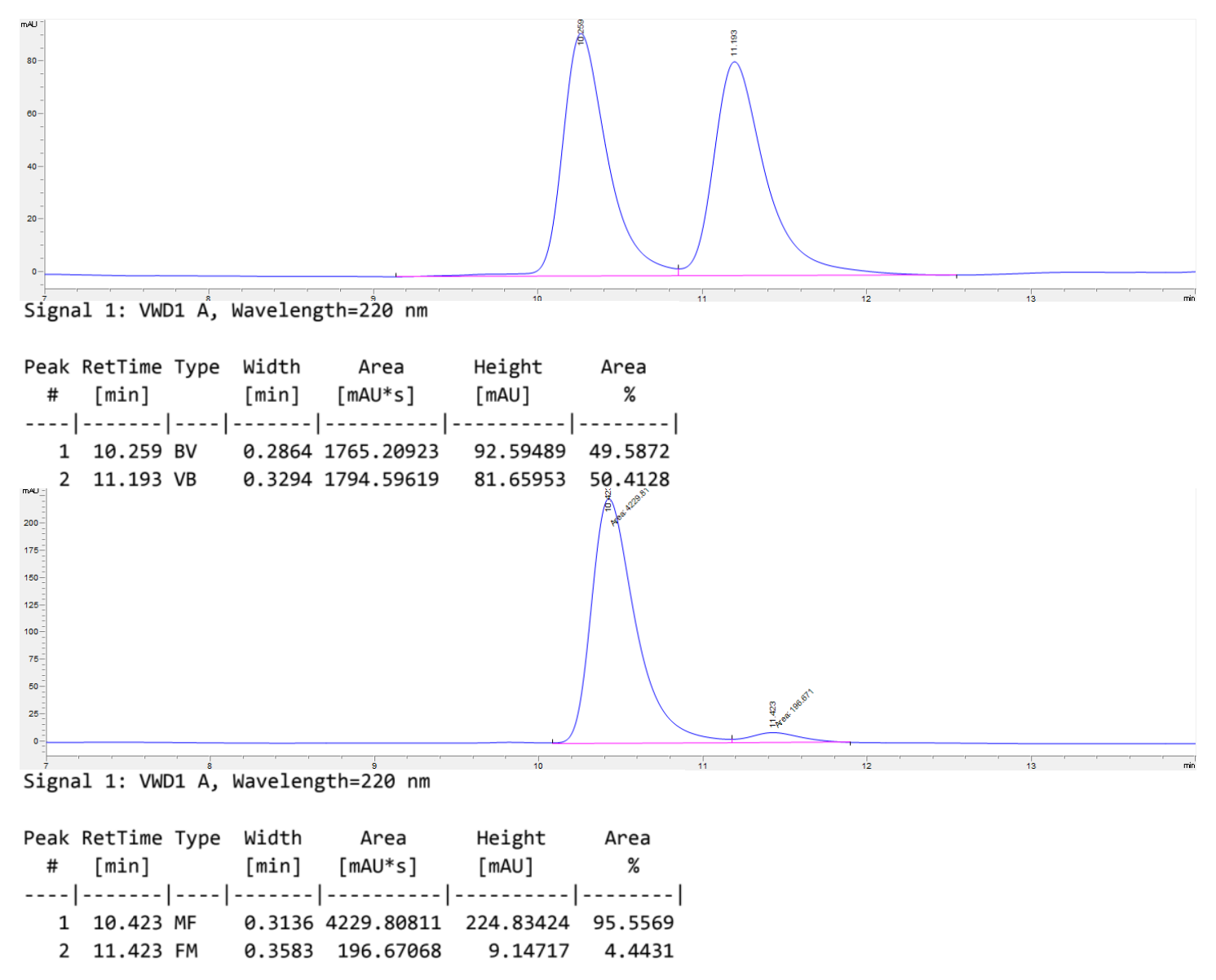

(S)-1-((Triisopropylsilyl)oxy)hexadecan-2-ol (3n)

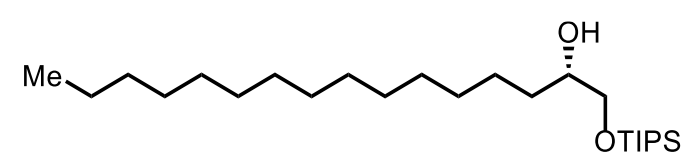

Compound 3n was prepared as a colorless oil in $71 \%$ yield $(59.2 \mathrm{mg}$, eluent: petroleum ether/EtOAc $=100 / 3$ to 20/1), from (Z)-(hexadec-1-en-1yloxy)triisopropylsilane $1 \mathrm{n}(79.2 \mathrm{mg}, 0.20 \mathrm{mmol})$, [ $\mathrm{RhCl}(\mathrm{cod})]_{2}(2.0 \mathrm{mg}, 0.004$ mmol), (S)-L9 (3.5 mg, $0.008 \mathrm{mmol})$, HBpin ( $38.4 \mathrm{mg}, 0.30 \mathrm{mmol})$ and LiOAc $(4.0 \mathrm{mg}, 0.06 \mathrm{mmol})$ following the above general procedure $\mathrm{E}$.

$\mathbf{R}_{f}=0.68$ (petroleum ether $/$ EtOAc $=10 / 1$ )

${ }^{1} \mathrm{H}$ NMR (400 MHz, CDCl $)$ 8 3.77-3.60 (m, 2H), 3.53-3.43 (m, 1H), $2.55(\mathrm{~s}, 1 \mathrm{H})$, $1.45-1.20(\mathrm{~m}, 26 \mathrm{H}), 1.19-0.98(\mathrm{~m}, 21 \mathrm{H}), 0.88(\mathrm{t}, J=6.7 \mathrm{~Hz}, 3 \mathrm{H})$.

${ }^{13} \mathrm{C}$ NMR (100 MHz, $\left.\mathrm{CDCl}_{3}\right) \delta$ 72.2, 67.8, 33.0, 32.1, 30.00-29.60 (m, 8C), 29.5, 25.8, 22.8, 18.1, 14.3, 12.1 .

HRMS (ESI ${ }^{+}$) calcd for $\mathrm{C}_{25} \mathrm{H}_{55} \mathrm{O}_{2} \mathrm{Si}^{+}[\mathrm{M}+\mathrm{H}]^{+}: 415.3966$, found: 415.3964 .

IR (neat, cm$^{-1}$ ): 2924, 2857, 1464, 1111, 883, 683.

$[\alpha]_{\mathrm{D}^{26.2}}=1.56\left(\mathrm{c}=1.01, \mathrm{CHCl}_{3}, 91 \%\right.$ ee $)$. 
Compound $3 n$ was subjected to general procedure J and the resulting ester was analyzed by chiral HPLC. Enantiomeric excess was determined by chiral HPLC (Daicel Chiralcel OD-H, hexane/isopropyl alcohol $=99.5 / 0.5$, flow rate $=0.5$ $\mathrm{mL} / \mathrm{min}, \lambda=220 \mathrm{~nm}$ ), $t^{1}=12.4 \mathrm{~min}$ (major),$t^{2}=14.4 \mathrm{~min}$ (minor).

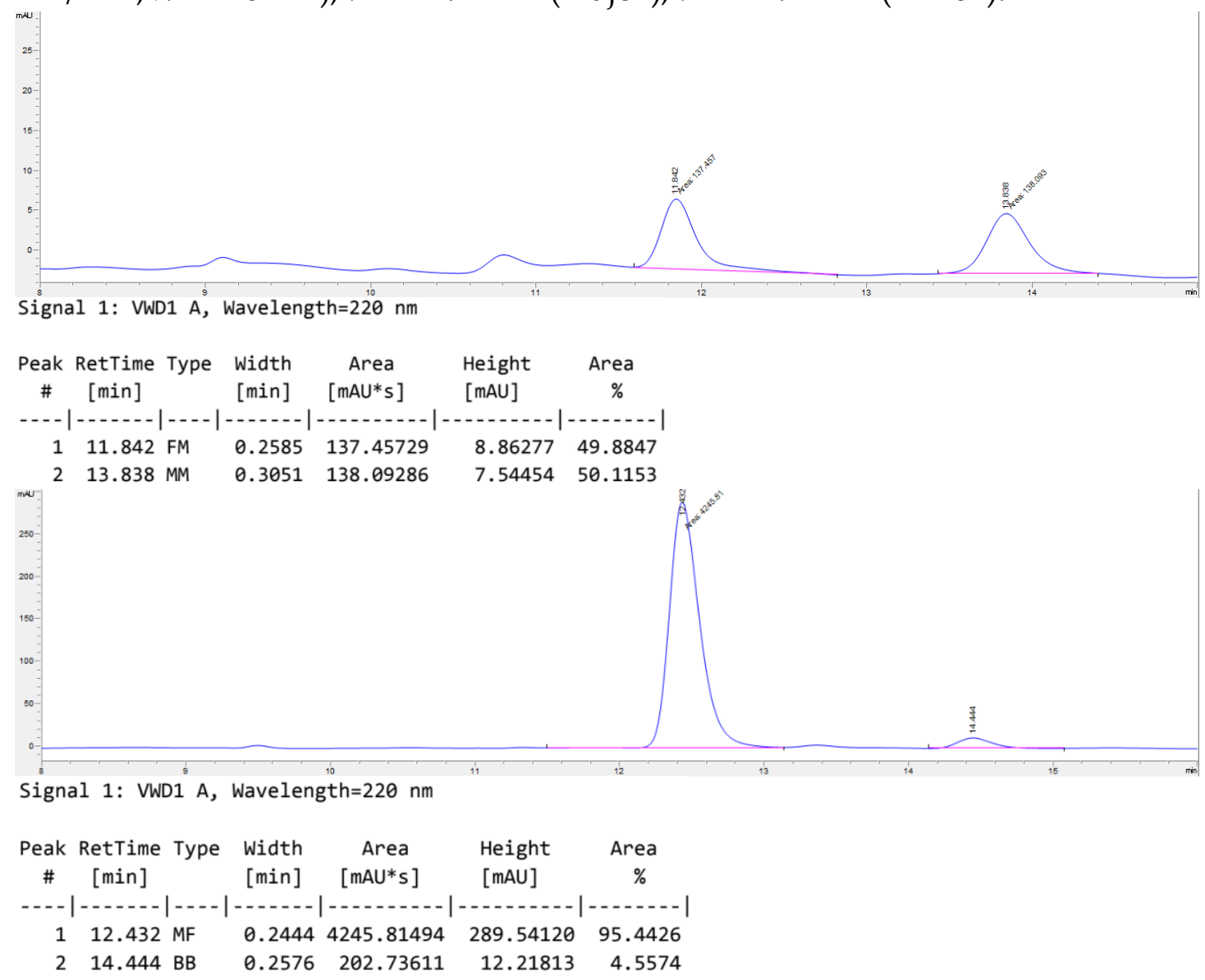

(S)-4-Cyclohexyl-1-((triisopropylsilyl)oxy)butan-2-ol (3o)<smiles>OC(CC[Hg])CC[Hg]</smiles>

Compound 30 was prepared as a colorless oil in $94 \%$ yield $(61.8 \mathrm{mg}$, eluent: petroleum ether/EtOAc $=100 / 3$ to $20 / 1)$, from $(Z)-((4-c y c l o h e x y l b u t-1-e n-1$ yl)oxy)triisopropylsilane 10 (62.0 mg, $0.20 \mathrm{mmol})$, [ RhCl(cod)]2 $(2.0 \mathrm{mg}, 0.004$ mmol), (S)-L9 (3.5 mg, $0.008 \mathrm{mmol})$, HBpin ( $38.4 \mathrm{mg}, 0.30 \mathrm{mmol})$ and LiOAc $(4.0 \mathrm{mg}, 0.06 \mathrm{mmol})$ following the above general procedure $\mathrm{E}$.

$\mathbf{R}_{f}=0.68$ (petroleum ether $/ \mathrm{EtOAc}=10 / 1$ )

${ }^{1} \mathrm{H}$ NMR (400 MHz, CDCl $)$ 8 3.74-3.68 (m, 1H), 3.67-3.59 (m, 1H), 3.50-3.43 (m, $1 \mathrm{H}), 2.57(\mathrm{~s}, 1 \mathrm{H}), 1.76-1.58(\mathrm{~m}, 5 \mathrm{H}), 1.49-1.32(\mathrm{~m}, 3 \mathrm{H}), 1.27-1.16(\mathrm{~m}, 5 \mathrm{H}), 1.14-$ 
$1.01(\mathrm{~m}, 21 \mathrm{H}), 0.94-0.80(\mathrm{~m}, 2 \mathrm{H})$.

${ }^{13} \mathrm{C}$ NMR (100 MHz, $\left.\mathrm{CDCl}_{3}\right) \delta$ 72.5, 67.7, 37.9, 33.5, 33.4, 30.2, 26.8, 26.5, 18.1, 12.0 .

HRMS (ESI ${ }^{+}$) calcd for $\mathrm{C}_{19} \mathrm{H}_{40} \mathrm{O}_{2} \mathrm{NaSi}^{+}[\mathrm{M}+\mathrm{Na}]^{+}:$351.2690, found: 351.2688.

IR (neat, cm¹): 2923, 2861, 1460, 1100, 1068, 883, 683.

$[\alpha]_{\mathrm{D}^{26.2}}=2.36\left(\mathrm{c}=0.66, \mathrm{CHCl}_{3}, 95 \%\right.$ ee $)$.

Compound 30 was subjected to general procedure J and the resulting ester was analyzed by chiral HPLC. Enantiomeric excess was determined by chiral HPLC (Daicel Chiralpak IA, hexane/isopropyl alcohol $=99.9 / 0.1$, flow rate $=0.5$ $\mathrm{mL} / \mathrm{min}, \lambda=220 \mathrm{~nm}$ ), $t^{1}=15.6 \mathrm{~min}$ (major),$t^{2}=17.7 \mathrm{~min}$ (minor).

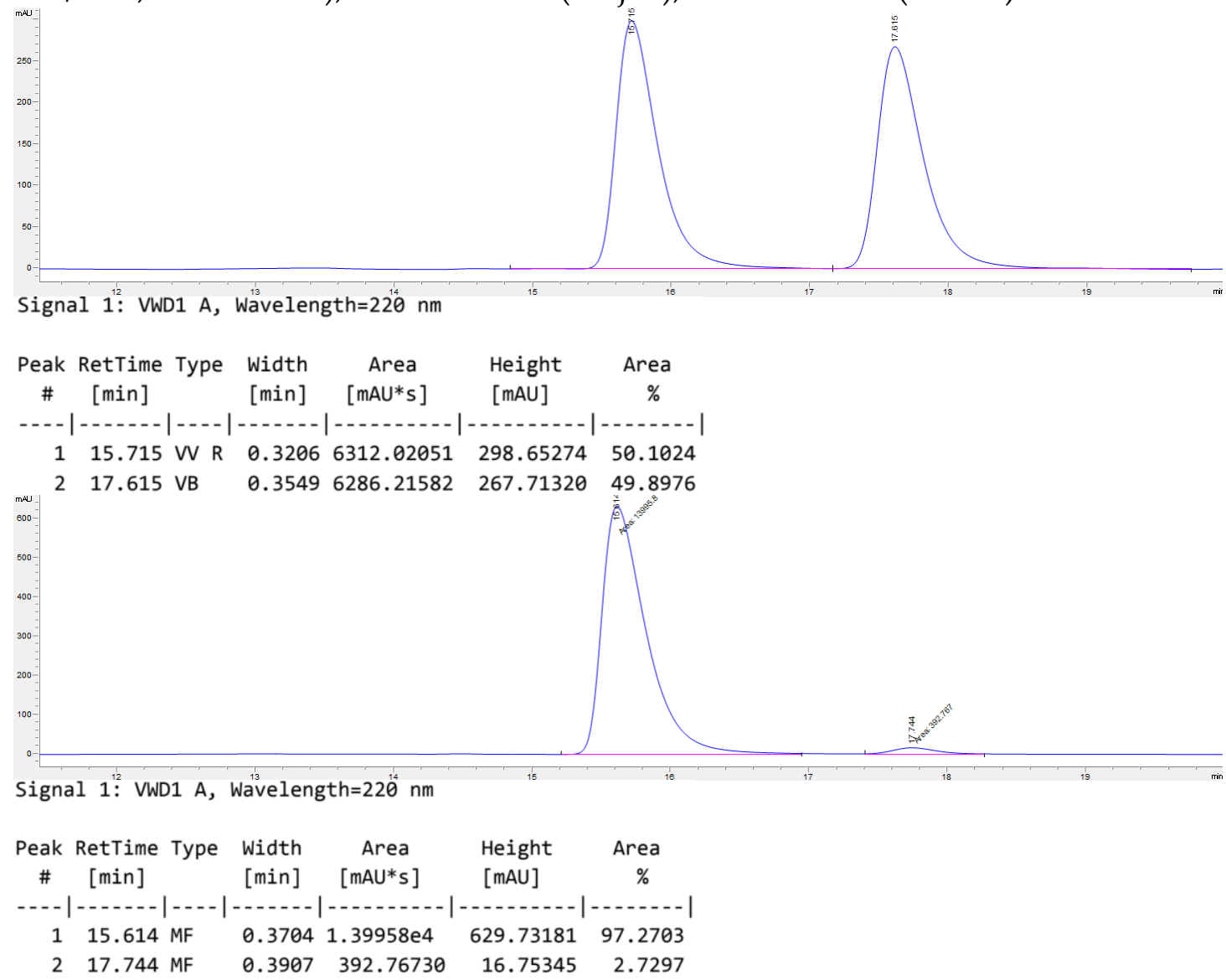

Compound 30 was prepared in $89 \%$ yield $(43.8 \mathrm{mg})$ and $94 \%$ ee from $1 \mathrm{~m}(E / Z$ $=1 / 8.9)(62.0 \mathrm{mg}, 0.20 \mathrm{mmol})$, [RhCl(cod) $]_{2}(2.0 \mathrm{mg}, 0.004 \mathrm{mmol}),(S)-\mathrm{L} 9$ (3.5 mg, $0.008 \mathrm{mmol}), \mathrm{HB}$ in $(38.4 \mathrm{mg}, 0.30 \mathrm{mmol})$ and $\mathrm{LiOAc}(4.0 \mathrm{mg}, 0.06 \mathrm{mmol})$ following the above general procedure E. 


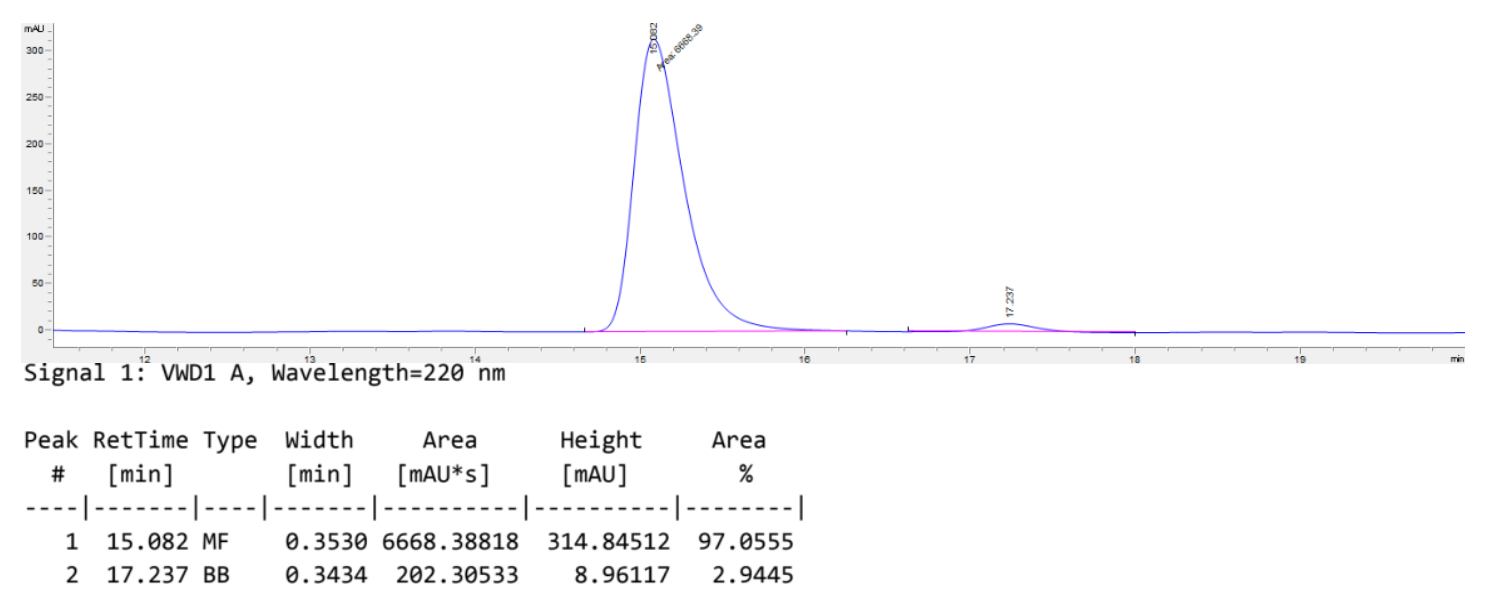

(S)-5,5-Dimethyl-1-((triisopropylsilyl)oxy)hexan-2-ol (3p)<smiles></smiles>

Compound $3 \mathbf{p}$ was prepared as a colorless oil in $97 \%$ yield $(59.1 \mathrm{mg}$, eluent: petroleum ether $/$ EtOAc $=100 / 3$ to 20/1), from (Z)-((5,5-dimethylhex-1-en-1yl)oxy)triisopropylsilane 1 p (57.0 mg, $0.20 \mathrm{mmol})$, [ RhCl(cod)]2 (2.0 mg, 0.004 mmol), (S)-L9 (3.5 mg, $0.008 \mathrm{mmol})$, HBpin ( $38.4 \mathrm{mg}, 0.30 \mathrm{mmol})$ and LiOAc $(4.0 \mathrm{mg}, 0.06 \mathrm{mmol}$ ) following the above general procedure $\mathrm{E}$.

$\mathbf{R}_{f}=0.68$ (petroleum ether $/$ EtOAc $=10 / 1$ )

${ }^{1} \mathrm{H}$ NMR (400 MHz, $\left.\mathrm{CDCl}_{3}\right)$ 8 3.77-3.69 (m, 1H), 3.66-3.56 (m, 1H), 3.54-3.44 (m, $1 \mathrm{H}), 2.58(\mathrm{~s}, 1 \mathrm{H}), 1.51-1.23(\mathrm{~m}, 4 \mathrm{H}), 1.21-0.98(\mathrm{~m}, 21 \mathrm{H}), 0.89(\mathrm{~s}, 9 \mathrm{H})$.

${ }^{13} \mathrm{C}$ NMR (100 MHz, $\left.\mathrm{CDCl}_{3}\right) \delta$ 8 72.9, 67.7, 39.9, 30.3, 29.4, 28.1, 18.1, 12.1.

HRMS (ESI') calcd for $\mathrm{C}_{17} \mathrm{H}_{38} \mathrm{O}_{2} \mathrm{NaSi}^{+}[\mathrm{M}+\mathrm{Na}]^{+}:$325.2533, found: 325.2532.

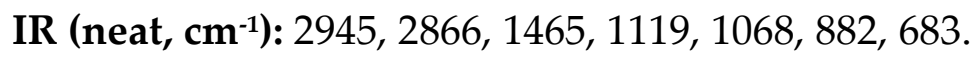

$[\alpha]_{\mathrm{D}^{26.2}}=2.00\left(\mathrm{c}=0.75, \mathrm{CHCl}_{3}, 97 \%\right.$ ee $)$.

Compound $3 p$ was subjected to general procedure $J$ and the resulting ester was analyzed by chiral HPLC. Enantiomeric excess was determined by chiral HPLC (Daicel Chiralcel OD-H, hexane/isopropyl alcohol $=99.5 / 0.5$, flow rate $=0.5$ $\mathrm{mL} / \mathrm{min}, \lambda=220 \mathrm{~nm}$ ), $t^{1}=10.1 \mathrm{~min}$ (major),$t^{2}=11.9 \mathrm{~min}$ (minor).

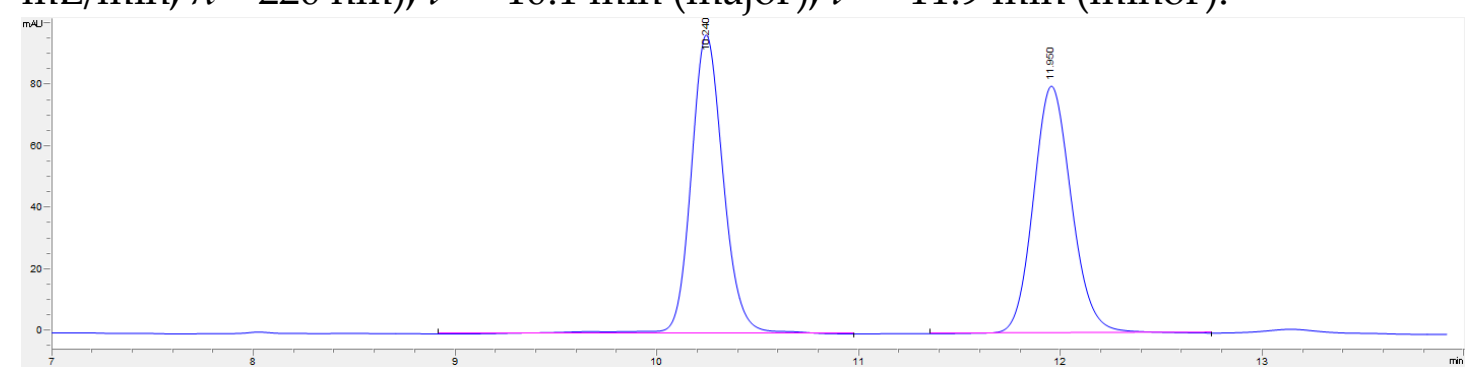




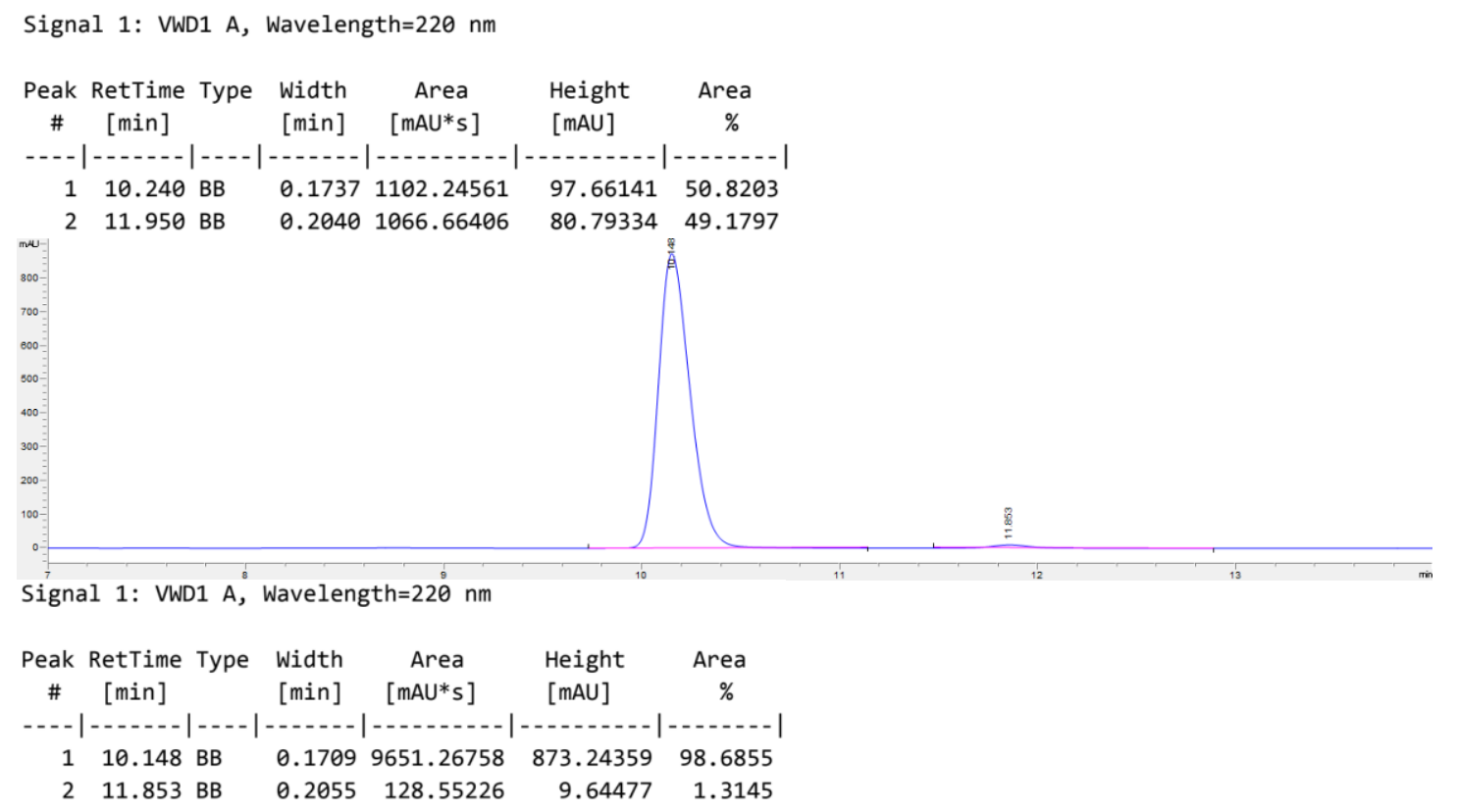

(S)-4-Hydroxy-5-((triisopropylsilyl)oxy)pentyl benzoate (3q)<smiles>OC(CCCC[18O])CO[18O]</smiles>

Compound 3q was prepared as a colorless oil in $66 \%$ yield $(50.0 \mathrm{mg}$, eluent: petroleum ether/EtOAc $=50 / 1$ to 20/1), from (Z)-5-((triisopropylsilyl)oxy)pent4-en-1-yl benzoate 1q (72.4 mg, $0.20 \mathrm{mmol})$, [ RhCl(cod)]2 (2.0 mg, $0.004 \mathrm{mmol})$, (S)-L9 (3.5 mg, $0.008 \mathrm{mmol})$, HBpin ( $38.4 \mathrm{mg}, 0.30 \mathrm{mmol}$ ) and LiOAc $(4.0 \mathrm{mg}$, $0.06 \mathrm{mmol}$ ) following the above general procedure $\mathrm{E}$.

$\mathbf{R}_{f}=0.56$ (petroleum ether $/$ EtOAc $=5 / 1$ )

${ }^{1} \mathrm{H}$ NMR (400 MHz, $\left.\mathrm{CDCl}_{3}\right)$ 8 8.10-8.00 (m, 2H), 7.59-7.52 (m, 1H), 7.47-7.39 (m, $2 \mathrm{H}), 4.45-4.29(\mathrm{~m}, 2 \mathrm{H}), 3.81-3.66(\mathrm{~m}, 2 \mathrm{H}), 3.59-3.47(\mathrm{~m}, 1 \mathrm{H}), 2.59(\mathrm{~s}, 1 \mathrm{H}), 2.06-$ $1.93(\mathrm{~m}, 1 \mathrm{H}), 1.93-1.79(\mathrm{~m}, 1 \mathrm{H}), 1.64-1.54(\mathrm{~m}, 2 \mathrm{H}), 1.17-0.95(\mathrm{~m}, 21 \mathrm{H})$.

${ }^{13} \mathrm{C}$ NMR (100 MHz, $\left.\mathrm{CDCl}_{3}\right) \delta$ 166.7, 133.0, 130.5, 129.7, 128.4, 71.7, 67.6, 65.1, 29.5, 25.2, 18.1, 12.0 .

HRMS (ESI') calcd for $\mathrm{C} 21 \mathrm{H} 36 \mathrm{O} 4 \mathrm{NaSi}^{+}[\mathrm{M}+\mathrm{Na}]^{+}: 403.2275$, found: 403.2273 .

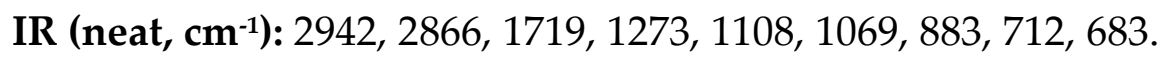

$[\alpha]_{\mathrm{D}^{26.2}}=-0.52\left(\mathrm{c}=0.91, \mathrm{CHCl}_{3}, 92 \%\right.$ ee); Enantiomeric excess was determined by chiral HPLC (Daicel Chiralcel OD-H, hexane/isopropyl alcohol $=98 / 2$, flow rate $=0.8 \mathrm{~mL} / \mathrm{min}, \lambda=220 \mathrm{~nm}$ ), $t^{1}=9.5 \mathrm{~min}$ (minor),$t^{2}=10.1 \mathrm{~min}$ (major) . 


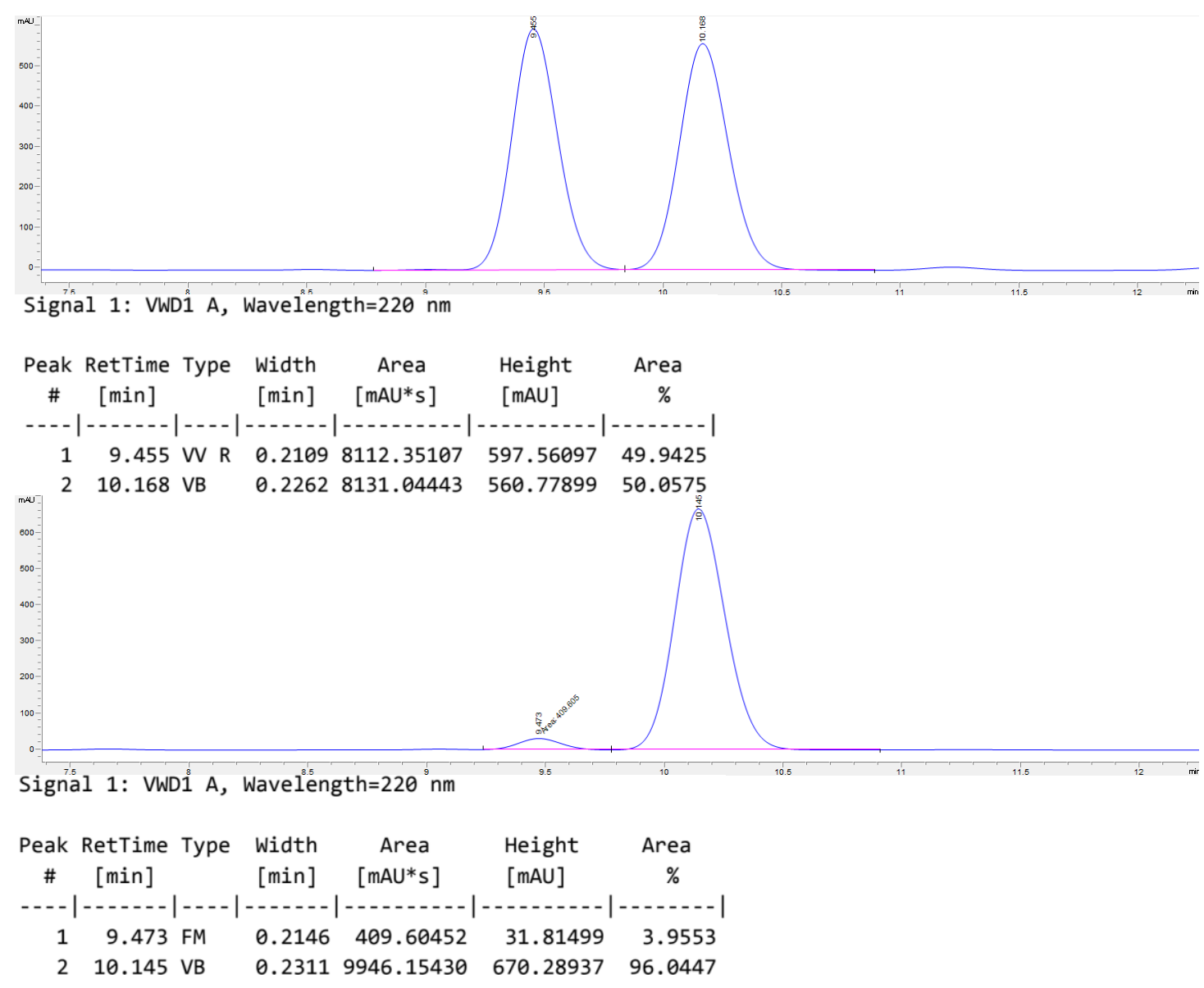

Compound $\mathbf{3 q}$ was prepared in $71 \%$ yield $(54.0 \mathrm{mg})$ and $92 \%$ ee from $\mathbf{1 q}(E / Z=$ 1/14.7) (72.4 mg, $0.20 \mathrm{mmol})$, [ RhCl(cod)]2 (2.0 mg, $0.004 \mathrm{mmol})$, (S)-L9 (3.5 mg, $0.008 \mathrm{mmol})$, HBpin $(38.4 \mathrm{mg}, 0.30 \mathrm{mmol})$ and LiOAc $(4.0 \mathrm{mg}, 0.06 \mathrm{mmol})$ following the above general procedure $\mathrm{E}$.

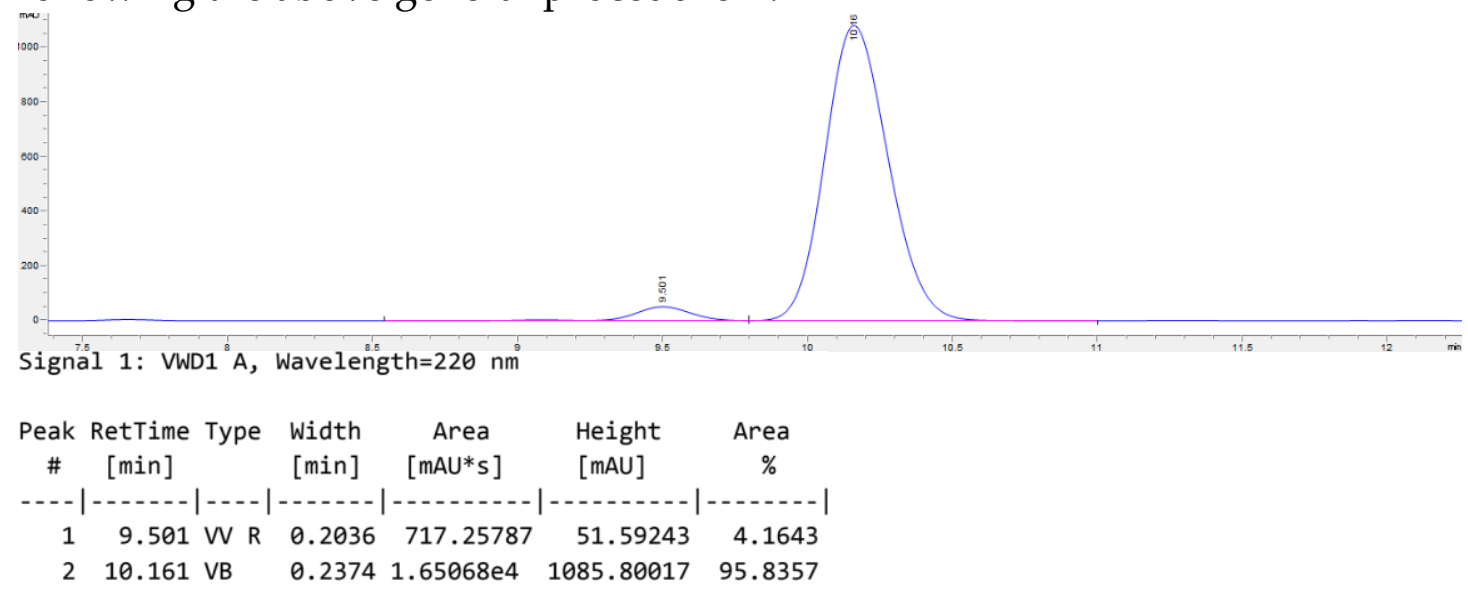

(S)-11-(Benzyloxy)-1-((triisopropylsilyl)oxy)undecan-2-ol (3r)<smiles>OCCCCCCCCCC(O)CO[18O]c1ccccc1</smiles> 
Compound 3r was prepared as a colorless oil in $69 \%$ yield $(62.1 \mathrm{mg}$, eluent: petroleum ether/EtOAc $=50 / 1$ to 20/1), from $(Z)$-triisopropyl((4-phenylbut-1en-1-yl)oxy)silane $1 \mathbf{r}(60.8 \mathrm{mg}, 0.20 \mathrm{mmol})$, [ $\mathrm{RhCl}(\mathrm{cod})]_{2}(2.0 \mathrm{mg}, 0.004 \mathrm{mmol})$, (S)-L9 (3.5 mg, $0.008 \mathrm{mmol})$, HBpin ( $38.4 \mathrm{mg}, 0.30 \mathrm{mmol})$ and LiOAc $(4.0 \mathrm{mg}$, $0.06 \mathrm{mmol}$ ) following the above general procedure $\mathrm{E}$.

$\mathbf{R}_{f}=0.37$ (petroleum ether $/$ EtOAc $=10 / 1$ )

${ }^{1} \mathrm{H}$ NMR (400 MHz, $\left.\mathrm{CDCl}_{3}\right)$ d 7.37-7.24 (m, 5H), $4.50(\mathrm{~s}, 2 \mathrm{H}), 3.74-3.60(\mathrm{~m}, 2 \mathrm{H})$, $3.51-3.42(\mathrm{~m}, 3 \mathrm{H}), 2.51(\mathrm{~s}, 1 \mathrm{H}), 1.66-1.57(\mathrm{~m}, 2 \mathrm{H}), 1.47-1.24(\mathrm{~m}, 14 \mathrm{H}), 1.17-1.02$ $(\mathrm{m}, 21 \mathrm{H})$.

${ }^{13} \mathrm{C}$ NMR (100 MHz, $\left.\mathrm{CDCl}_{3}\right) \delta$ 138.9, 128.5, 127.8, 127.6, 73.0, 72.2, 70.7, 67.8, 33.0, 29.92, 29.87, 29.68, 29.65, 29.6, 26.3, 25.8, 18.1, 12.1 .

HRMS (ESI') calcd for $\mathrm{C}_{27} \mathrm{H}_{50} \mathrm{O}_{3} \mathrm{NaSi}^{+}[\mathrm{M}+\mathrm{Na}]^{+}: 473.3421$, found: 473.3419 .

IR (neat, cm ${ }^{-1}$ ): 2926, 2860, 1461, 1104, 883, 686.

$[\alpha]_{D^{26.2}}=0.78\left(\mathrm{c}=0.44, \mathrm{CHCl}_{3}, 90 \%\right.$ ee); Enantiomeric excess was determined by chiral HPLC (Daicel Chiralpak IA, hexane/isopropyl alcohol $=97 / 3$, flow rate $=$ $1.0 \mathrm{~mL} / \mathrm{min}, \lambda=220 \mathrm{~nm}$ ), $t^{1}=8.7 \mathrm{~min}$ (minor),$t^{2}=8.9 \mathrm{~min}$ (major).

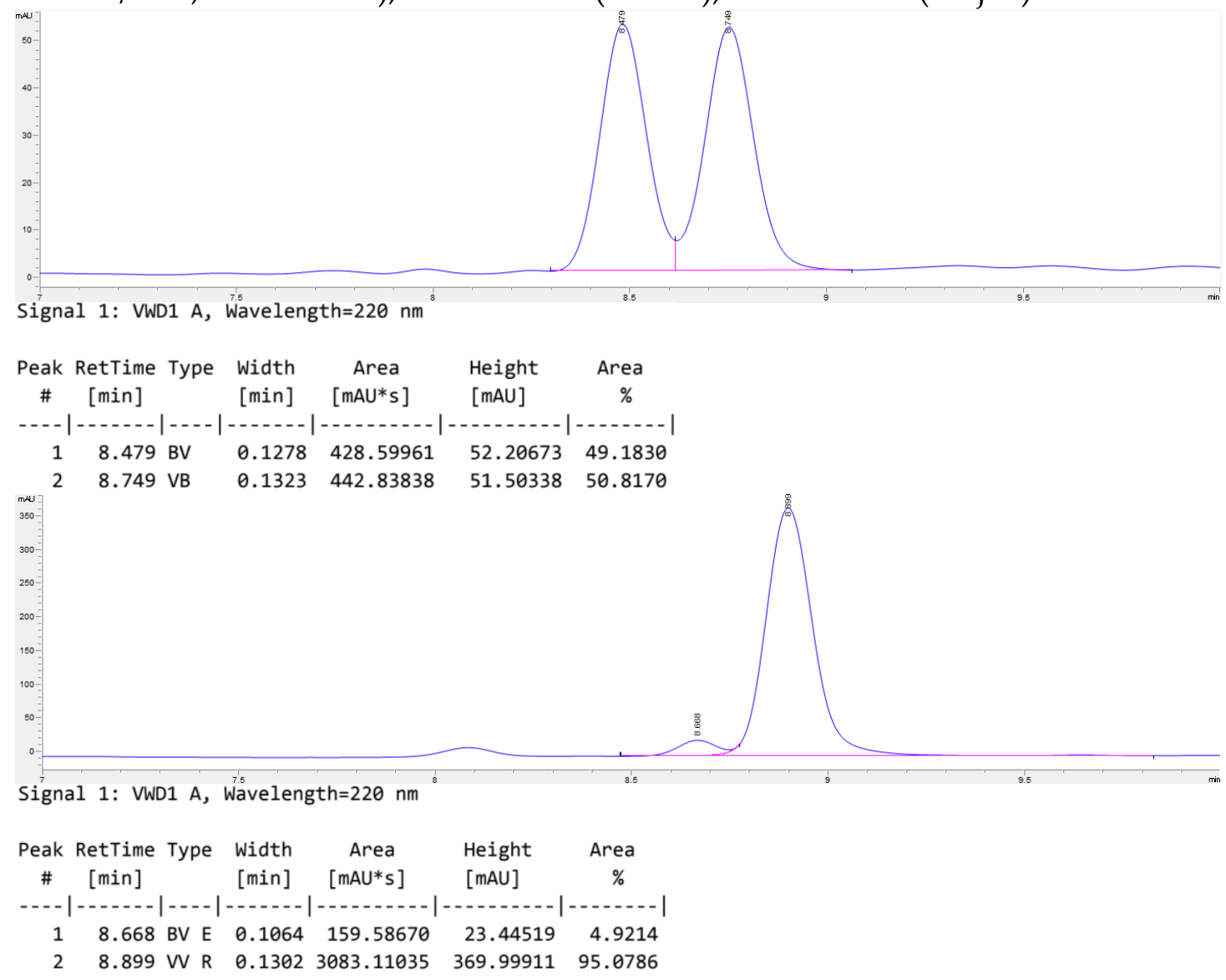

(S)-3,3-Diisopropyl-2,11,11,12,12-pentamethyl-4,10-dioxa-3,11-disilatridecan- 
6-ol (3s)

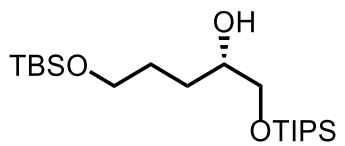

Compound 3s was prepared as a colorless oil in 61\% yield (47.6 mg, eluent: petroleum ether/EtOAc $=100 / 3$ to 20/1), from (Z)-3,3-diisopropyl-2,11,11,12,12pentamethyl-4,10-dioxa-3,11-disilatridec-5-ene $1 \mathrm{~s}$ (74.4 $\mathrm{mg}, 0.20 \mathrm{mmol})$, $[\mathrm{RhCl}(\mathrm{cod})]_{2}(2.0 \mathrm{mg}, 0.004 \mathrm{mmol}),(S)-\mathrm{L9}$ (3.5 mg, $\left.0.008 \mathrm{mmol}\right), \mathrm{HBpin}(38.4 \mathrm{mg}$, $0.30 \mathrm{mmol})$ and LiOAc $(4.0 \mathrm{mg}, 0.06 \mathrm{mmol})$ following the above general procedure E.

$\mathbf{R}_{f}=0.58$ (petroleum ether $/$ EtOAc $=10 / 1$ )

${ }^{1} \mathrm{H}$ NMR (400 MHz, $\left.\mathrm{CDCl}_{3}\right)$ ठ 3.76-3.59 (m, 4H), 3.59-3.49 (m, 1H), $2.81(\mathrm{~s}, 1 \mathrm{H})$, 1.75-1.39 (m, 4H), 1.19-0.99 (m, 21H), 0.89 (s, 9H), 0.05 (s, 6H).

${ }^{13} \mathrm{C}$ NMR (100 MHz, $\left.\mathrm{CDCl}_{3}\right) \delta$ 72.0, 67.7, 63.4, 29.8, 29.1, 26.1, 18.5, 18.1, 12.1, 5.2 .

HRMS (ESI ${ }^{+}$) calcd for $\mathrm{C}_{20} \mathrm{H}_{46} \mathrm{O}_{3} \mathrm{NaSi}_{2}{ }^{+}[\mathrm{M}+\mathrm{Na}]^{+}: 413.2878$, found: 413.2876 .

IR (neat, cm-1): 2931, 2864, 1254, 1097, 883, 835, 777, 683.

$[\alpha]_{D^{26.2}}=-0.68\left(\mathrm{c}=0.99, \mathrm{CHCl}_{3}, 90 \%\right.$ ee $)$.

Compound $3 \mathrm{~s}$ was subjected to general procedure J and the resulting ester was analyzed by chiral HPLC. Enantiomeric excess was determined by chiral HPLC (Daicel Chiralcel OD-H, hexane/isopropyl alcohol $=99.5 / 0.5$, flow rate $=0.5$ $\mathrm{mL} / \mathrm{min}, \lambda=220 \mathrm{~nm}$ ), $t^{1}=7.2 \mathrm{~min}$ (major),$t^{2}=8.2 \mathrm{~min}$ (minor).

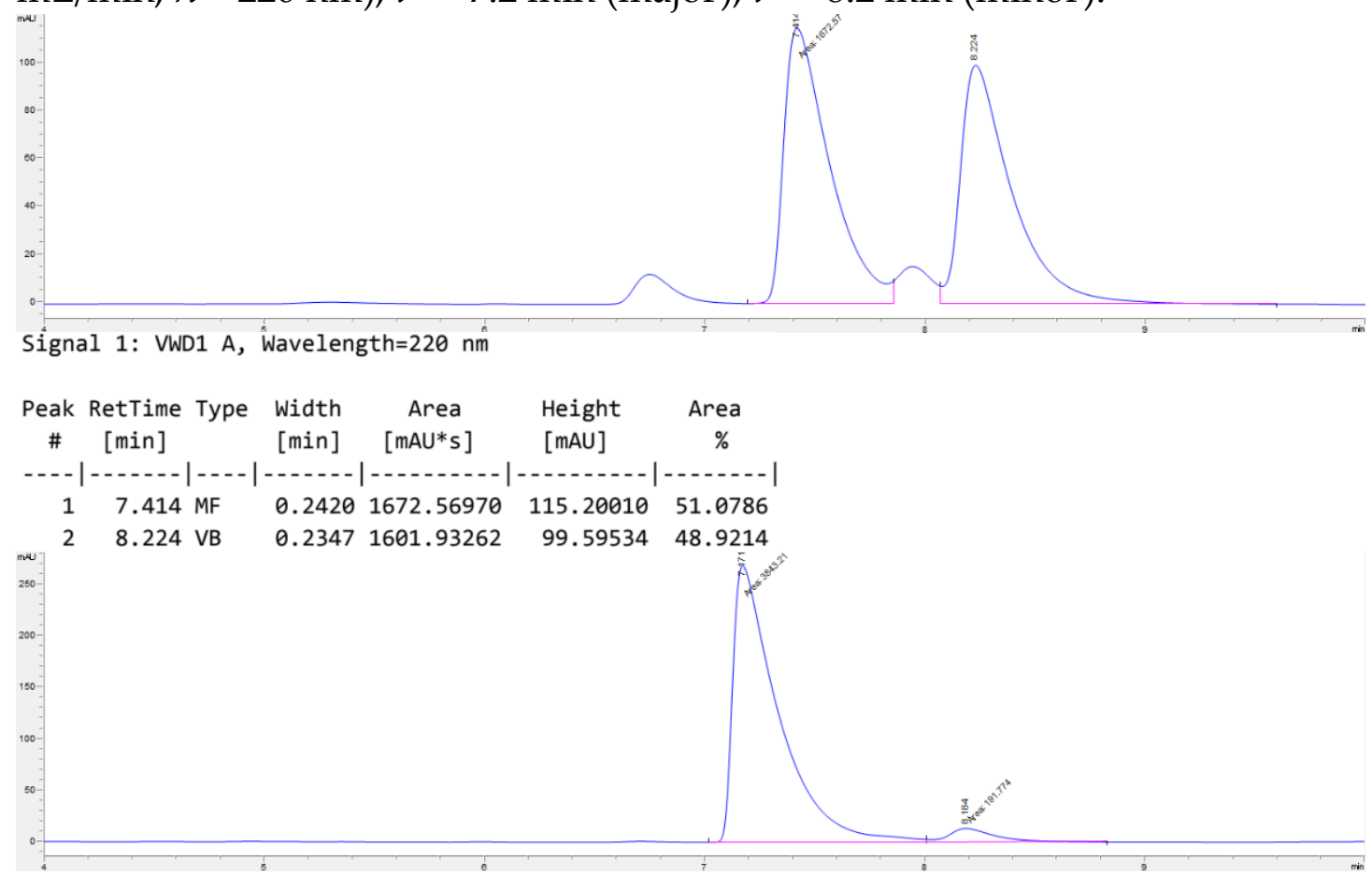


Signal 1: VWD1 A, Wavelength $=220 \mathrm{~nm}$

\begin{tabular}{cccccc}
$\begin{array}{c}\text { Peak RetTime Type } \\
\text { \# } \\
\text { [min] }\end{array}$ & $\begin{array}{c}\text { Width } \\
{[\mathrm{min}]}\end{array}$ & $\begin{array}{c}\text { Area } \\
{[\mathrm{mAU} \text { s }]}\end{array}$ & $\begin{array}{c}\text { Height } \\
{[\mathrm{mAU}]}\end{array}$ & $\begin{array}{c}\text { Area } \\
\%\end{array}$ \\
\hline 1 & $7.171 \mathrm{MF}$ & 0.2382 & 3843.20508 & 268.87515 & 95.2472 \\
2 & $8.184 \mathrm{FM}$ & 0.2428 & 191.77449 & 13.16280 & 4.7528
\end{tabular}

Compound 3s was prepared in $62 \%$ yield $(48.4 \mathrm{mg})$ and $90 \%$ ee from $1 \mathrm{~s}(E / \mathrm{Z}=$ 1/8.2) (74.4 mg, $0.20 \mathrm{mmol}),[\mathrm{RhCl}(\mathrm{cod})]_{2}(2.0 \mathrm{mg}, 0.004 \mathrm{mmol}),(S)-\mathrm{L} 9$ (3.5 mg, $0.008 \mathrm{mmol}), \mathrm{HBpin}(38.4 \mathrm{mg}, 0.30 \mathrm{mmol})$ and $\mathrm{LiOAc}(4.0 \mathrm{mg}, 0.06 \mathrm{mmol})$ following the above general procedure E.

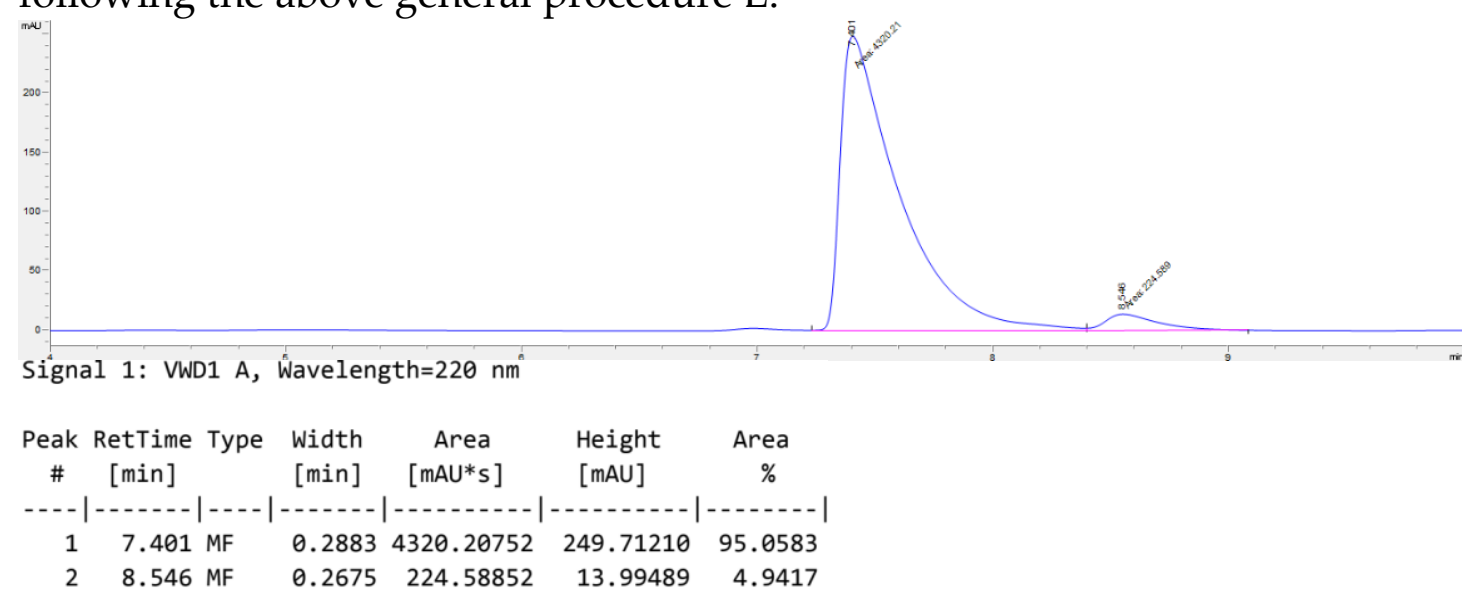

(S)-10-Hydroxy-11-((triisopropylsilyl)oxy)undecyl methylbenzenesulfonate ( $3 t)$<smiles>OCCO[C@@H](O)CC(O)CCCCCCCCOc1ccccc1</smiles>

Compound $3 \mathbf{t}$ was prepared as a colorless oil in $80 \%$ yield $(82.0 \mathrm{mg}$, eluent: petroleum ether/EtOAc $=10 / 1)$, from $(Z)-11-(($ triisopropylsilyl $)$ oxy $)$ undec-10en-1-yl 4-methylbenzenesulfonate $1 t$ (99.2 mg, $0.20 \mathrm{mmol}),[\mathrm{RhCl}(\mathrm{cod})]_{2}(2.0 \mathrm{mg}$, $0.004 \mathrm{mmol}$ ), (S)-L9 (3.5 mg, $0.008 \mathrm{mmol})$, HBpin (38.4 mg, $0.30 \mathrm{mmol}$ ) and LiOAc ( $4.0 \mathrm{mg}, 0.06 \mathrm{mmol})$ following the above general procedure E.

$\mathbf{R}_{f}=0.48$ (petroleum ether $/$ EtOAc $=5 / 1$ )

${ }^{1} \mathrm{H}$ NMR (400 MHz, $\left.\mathrm{CDCl}_{3}\right) \delta 7.79(\mathrm{~d}, J=7.8 \mathrm{~Hz}, 2 \mathrm{H}), 7.34(\mathrm{~d}, J=7.8 \mathrm{~Hz}, 2 \mathrm{H})$, 4.06-3.97 (m, 2H), 3.75-3.61 (m, 2H), 3.52-3.42 (m, 1H), 2.69-2.32 (m, 4H), 1.69$1.56(\mathrm{~m}, 2 \mathrm{H}), 1.46-1.19(\mathrm{~m}, 14 \mathrm{H}), 1.17-0.95(\mathrm{~m}, 21 \mathrm{H})$.

${ }^{13} \mathrm{C}$ NMR (100 MHz, $\left.\mathrm{CDCl}_{3}\right) \delta$ 144.7, 133.5, 129.9, 128.0, 72.1, 70.8, 67.7, 32.9, 29.8, 29.5, 29.4, 29.05, 28.96, 25.7, 25.5, 21.8, 18.1, 12.1 .

HRMS (ESI ${ }^{+}$) calcd for $\mathrm{C}_{27} \mathrm{H}_{50} \mathrm{O}_{5} \mathrm{NaSSi}^{+}[\mathrm{M}+\mathrm{Na}]^{+}: 537.3040$, found: 537.3037 . 
IR (neat, cm-1): 2927, 2862, 1362, 1178, 1101, 663, 555.

$[\alpha]_{\mathrm{D}^{26.2}}=1.08\left(\mathrm{c}=1.58, \mathrm{CHCl}_{3}, 90 \%\right.$ ee); Enantiomeric excess was determined by chiral HPLC (Daicel Chiralpak IA, hexane/isopropyl alcohol $=95 / 5$, flow rate $=$ $1.0 \mathrm{~mL} / \mathrm{min}, \lambda=220 \mathrm{~nm}$ ), $t^{1}=9.9 \mathrm{~min}$ (minor),$t^{2}=10.6 \mathrm{~min}$ (major).

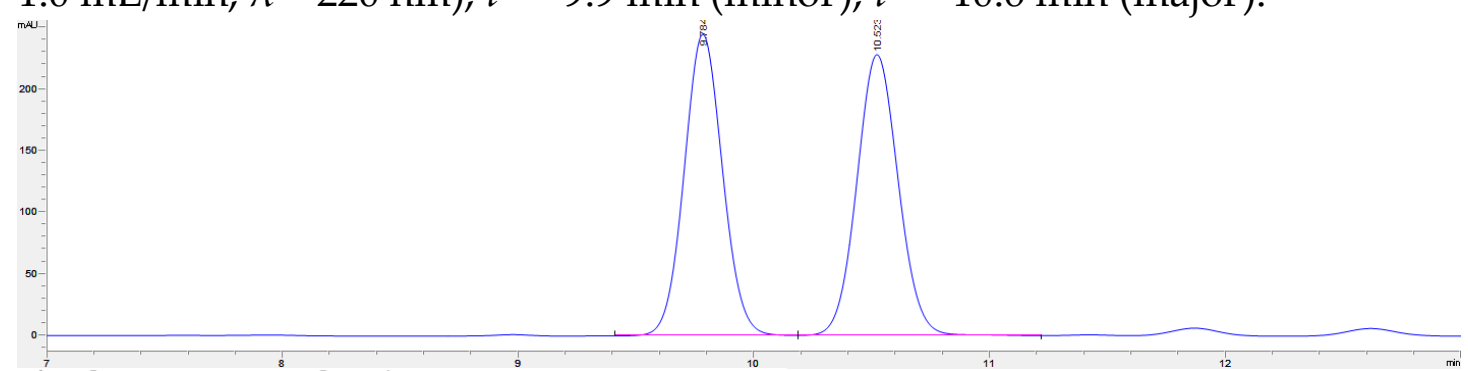

Signal 1: VWD1 A, Wavelength $=220 \mathrm{~nm}$
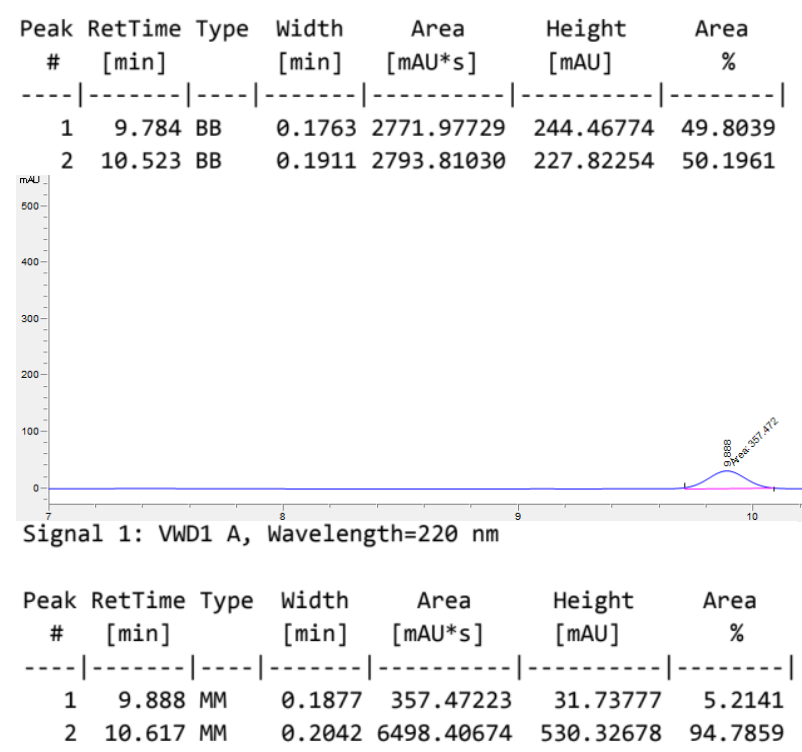

(S)-11-((Triisopropylsilyl)oxy)undecane-1,10-diol (3u)<smiles>OCCCCCCCCC[C@H](O)C[18OH]</smiles>

Compound $3 \mathbf{u}$ was prepared as a colorless oil in $78 \%$ yield $(56.2 \mathrm{mg}$, eluent: petroleum ether/EtOAc $=10 / 1)$, from $(Z)-11-(($ triisopropylsilyl $)$ oxy $)$ undec-10en-1-ol 1u (68.4 mg, $0.20 \mathrm{mmol})$, [RhCl(cod)]2 (2.0 mg, $0.004 \mathrm{mmol})$, (S)-L9 (3.5 $\mathrm{mg}, 0.008 \mathrm{mmol}), \mathrm{HB}$ in $(64.0 \mathrm{mg}, 0.50 \mathrm{mmol})$ and LiOAc $(4.0 \mathrm{mg}, 0.06 \mathrm{mmol})$ following the above general procedure E.

$\mathbf{R}_{f}=0.55$ (petroleum ether $/ \mathrm{EtOAc}=3 / 1$ )

${ }^{1} \mathrm{H}$ NMR (400 MHz, $\mathrm{CDCl}_{3}$ ) $\delta 3.74-3.60$ (m, 4H), 3.52 - 3.43 (m, 1H), 2.30 - 1.66 (br, 2H), $1.62-1.51(\mathrm{~m}, 2 \mathrm{H}), 1.49-1.21(\mathrm{~m}, 14 \mathrm{H}), 1.18-0.94(\mathrm{~m}, 21 \mathrm{H})$. ${ }^{13} \mathrm{C}$ NMR (100 MHz, $\left.\mathrm{CDCl}_{3}\right) \delta$ 72.1, 67.7, 63.1, 32.9, 32.9, 29.8, 29.65, 29.60, 29.5, 
25.9, 25.7, 18.1, 12.0 .

HRMS (ESI ${ }^{+}$) calcd for $\mathrm{C}_{20} \mathrm{H}_{44} \mathrm{O}_{3} \mathrm{NaSi}^{+}[\mathrm{M}+\mathrm{Na}]^{+}:$383.2952, found: 383.2943 .

IR (neat, cm$\left.^{-1}\right):$ 2927, 2866, 1464, 1106, 1067, 883, 682

$[\alpha]_{\mathrm{D}^{23.3}}=3.89$ ( $\mathrm{c}=0.62, \mathrm{CHCl}_{3}, 88 \%$ ee).

Compound $3 \mathbf{u}$ was subjected to general procedure J and the resulting ester was analyzed by chiral HPLC. Enantiomeric excess was determined by chiral HPLC (Daicel Chiralpak IA, hexane/isopropyl alcohol $=98 / 2$, flow rate $=1.0 \mathrm{~mL} / \mathrm{min}$, $\lambda=220 \mathrm{~nm}$ ), $t^{1}=5.3 \mathrm{~min}$ (major),$t^{2}=6.0 \mathrm{~min}$ (minor).

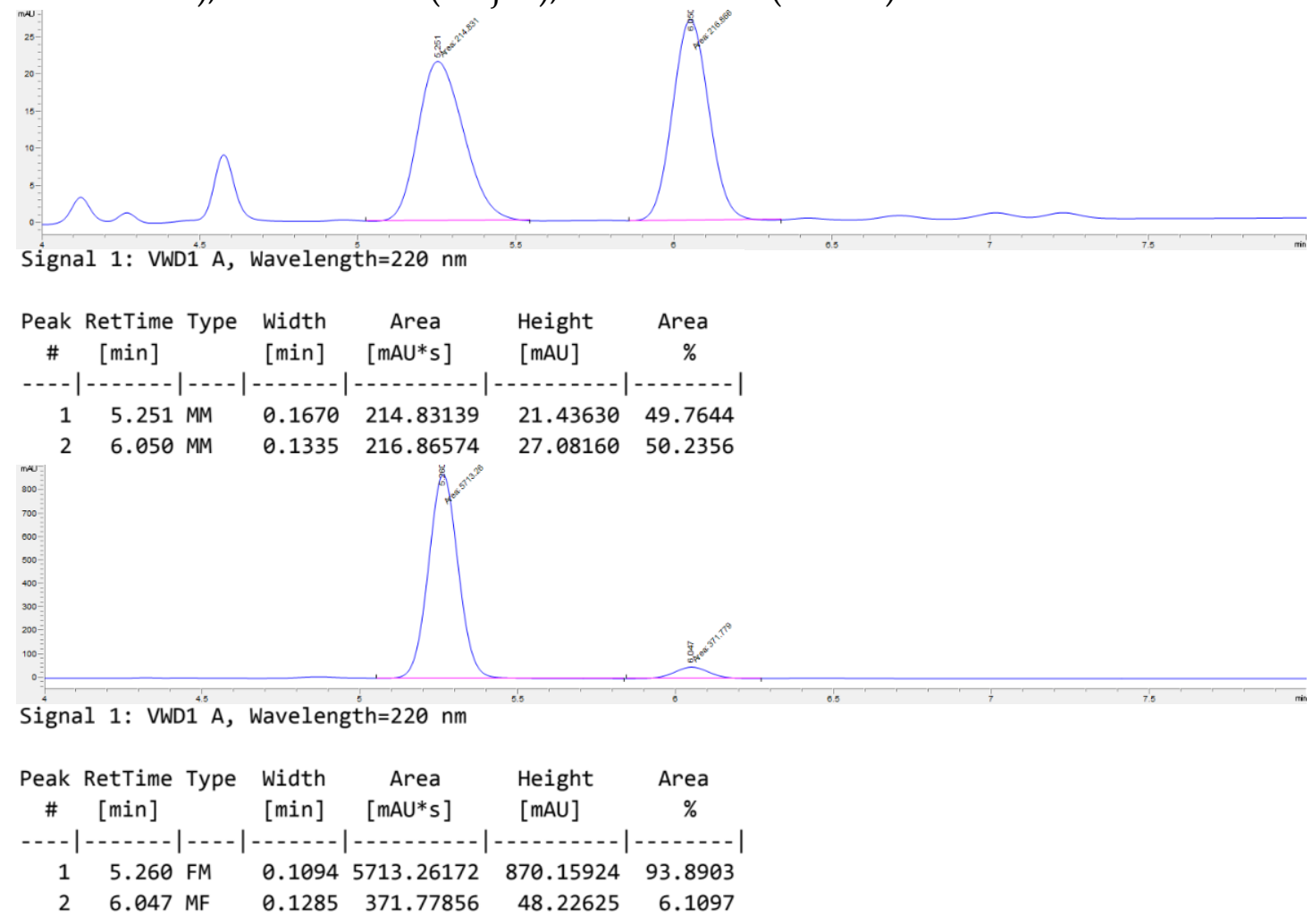

(S)-N-(10-Hydroxy-11-((triisopropylsilyl)oxy)undecyl)- $N$-(4methoxyphenyl)-4-methylbenzenesulfonamide (3v)<smiles>COc1ccc(NCCCCCCCCCC(O)CO[Na])cc1</smiles>

Compound $3 \mathbf{v}$ was prepared as a colorless oil in $78 \%$ yield $(97.3 \mathrm{mg}$, eluent: petroleum ether/EtOAc $=10 / 1$ to 5/1), from (Z)-N-(4-methoxyphenyl)-4-methylN-(11-((triisopropylsilyl)oxy)undec-10-en-1-yl)benzenesulfonamide 1v (121.4 mg, $0.20 \mathrm{mmol}$ ), [ RhCl(cod)]2 (4.0 mg, $0.008 \mathrm{mmol}),(S)-\mathrm{L9}$ (7.0 mg, $0.016 \mathrm{mmol})$, HBpin (38.4 mg, $0.30 \mathrm{mmol})$ and LiOAc $(4.0 \mathrm{mg}, 0.06 \mathrm{mmol})$ following the above general procedure $\mathrm{E}$. 
$\mathbf{R}_{f}=0.32$ (petroleum ether $/$ EtOAc $=5 / 1$ )

${ }^{1} \mathrm{H}$ NMR (400 MHz, $\left.\mathrm{CDCl}_{3}\right) \delta 7.47(\mathrm{~d}, J=7.7 \mathrm{~Hz}, 2 \mathrm{H}), 7.24(\mathrm{~d}, J=7.8 \mathrm{~Hz}, 2 \mathrm{H})$, $6.93(\mathrm{~d}, J=8.1 \mathrm{~Hz}, 2 \mathrm{H}), 6.80(\mathrm{~d}, J=8.3 \mathrm{~Hz}, 2 \mathrm{H}), 3.80(\mathrm{~s}, 3 \mathrm{H}), 3.73-3.60(\mathrm{~m}, 2 \mathrm{H})$, 3.52-3.41 (m, 3H), 2.72-2.27 (m, 4H), 1.43-1.19 (m, 16H), 1.15-1.01 (m, 21H). ${ }^{13} \mathrm{C}$ NMR (100 MHz, $\left.\mathrm{CDCl}_{3}\right) \delta$ 159.1, 143.2, 135.7, 131.8, 130.1, 129.4, 127.9, 114.2, 72.1, 67.7, 55.5, 50.8, 32.9, 29.8, 29.6, 29.5, 29.2, 28.3, 26.5, 25.7, 21.7, 18.1, 12.1 .

HRMS (ESI ${ }^{+}$) calcd for $\mathrm{C}_{34} \mathrm{H}_{57} \mathrm{NNaO}_{5} \mathrm{SSi}^{+}[\mathrm{M}+\mathrm{Na}]^{+}: 642.3619$, found: 642.3615 .

IR (neat, cm-1): 2927, 2861, 1508, 1248, 1162, 1092, 680.

$[\alpha]_{\mathrm{D}^{26.2}}=0.73\left(\mathrm{c}=0.77, \mathrm{CHCl}_{3}, 88 \%\right.$ ee); Enantiomeric excess was determined by chiral HPLC (Daicel Chiralpak IA, hexane/isopropyl alcohol $=95 / 5$, flow rate $=$ $1.0 \mathrm{~mL} / \mathrm{min}, \lambda=220 \mathrm{~nm}$ ), $t^{1}=19.5 \mathrm{~min}$ (minor),$t^{2}=20.0 \mathrm{~min}$ (major).

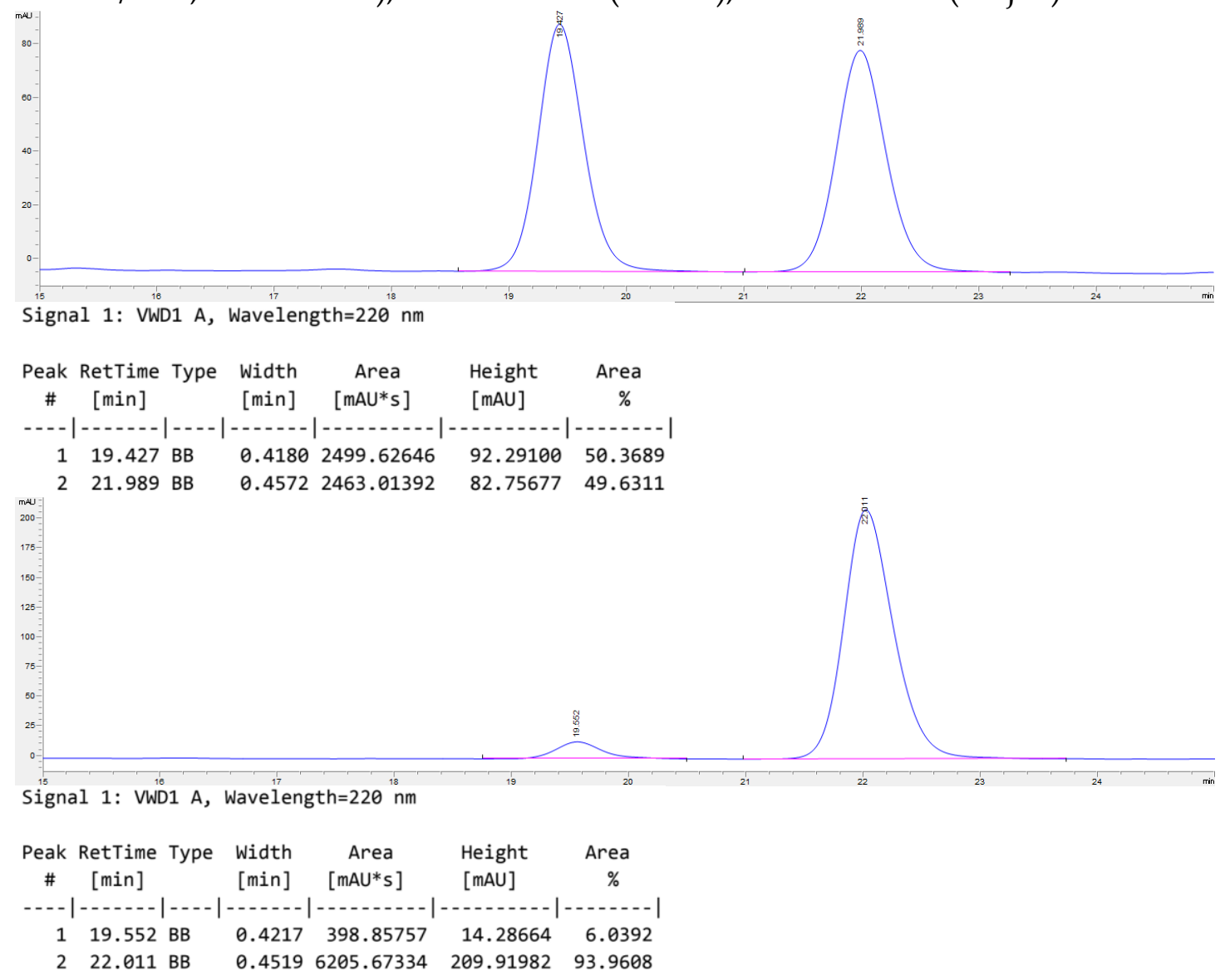

tert-Butyl (S)-(5-hydroxy-6-((triisopropylsilyl)oxy)hexyl)carbamate (3w)<smiles>O=C(NCCCCC(O)CO[Na])OC(=O)c1ccccc1</smiles>

Compound 3w was prepared as a colorless oil in 66\% yield (51.2 mg, eluent: petroleum ether/EtOAc = 10/1), from tert-butyl (Z)-(6- 
((triisopropylsilyl)oxy)hex-5-en-1-yl)carbamate $\mathbf{1 w}(74.2 \mathrm{mg}, 0.20 \mathrm{mmol})$, $[\mathrm{RhCl}(\mathrm{cod})]_{2}(4.0 \mathrm{mg}, 0.008 \mathrm{mmol}),(S)-\mathrm{L} 9(7.0 \mathrm{mg}, 0.016 \mathrm{mmol}), \mathrm{HBpin}(64.0 \mathrm{mg}$, $0.50 \mathrm{mmol})$ and $\mathrm{LiOAc}(4.0 \mathrm{mg}, 0.06 \mathrm{mmol})$ following the above general procedure $\mathrm{E}\left(30^{\circ} \mathrm{C}, 48 \mathrm{~h}\right)$.

$\mathbf{R}_{f}=0.43$ (petroleum ether/EtOAc $=5 / 1$ )

${ }^{1} \mathrm{H}$ NMR (400 MHz, $\left.\mathrm{CDCl}_{3}\right) \delta 4.57(\mathrm{~s}, 1 \mathrm{H}), 3.74-3.57(\mathrm{~m}, 2 \mathrm{H}), 3.45(\mathrm{dd}, J=9.2$, $7.2 \mathrm{~Hz}, 1 \mathrm{H}), 3.24-2.95(\mathrm{~m}, 2 \mathrm{H}), 2.56(\mathrm{br}, 1 \mathrm{H}), 1.57-1.31(\mathrm{~m}, 15 \mathrm{H}), 1.17-0.91$ $(\mathrm{m}, 21 \mathrm{H})$.

${ }^{13} \mathrm{C}$ NMR (100 MHz, $\left.\mathrm{CDCl}_{3}\right) \delta$ 156.1, 79.1, 71.9, 67.6, 40.5, 32.5, 30.2, 28.5, 22.9, 18.1, 12.0 .

HRMS (ESI ${ }^{+}$) calcd for $\mathrm{C}_{20} \mathrm{H}_{43} \mathrm{O}_{4} \mathrm{NNaSi}^{+}[\mathrm{M}+\mathrm{Na}]^{+}$: 412.2854 , found: 412.2844 .

IR (neat, cm$^{-1}$ ): 2941, 2866, 1693, 1366, 1250, 1173, 1102, 882

$[\alpha]_{\mathrm{D}^{23.3}}=2.11\left(\mathrm{c}=1.07, \mathrm{CHCl}_{3}, 86 \%\right.$ ee $)$.

Compound $3 \mathbf{w}$ was subjected to general procedure $\mathrm{J}$ and the resulting ester was analyzed by chiral HPLC. Enantiomeric excess was determined by chiral HPLC (Daicel Chiralpak IA, hexane/isopropyl alcohol $=95 / 5$, flow rate $=0.5 \mathrm{~mL} / \mathrm{min}$, $\lambda=220 \mathrm{~nm}$ ), $t^{1}=10.4 \mathrm{~min}$ (major),$t^{2}=10.9 \mathrm{~min}$ (minor).

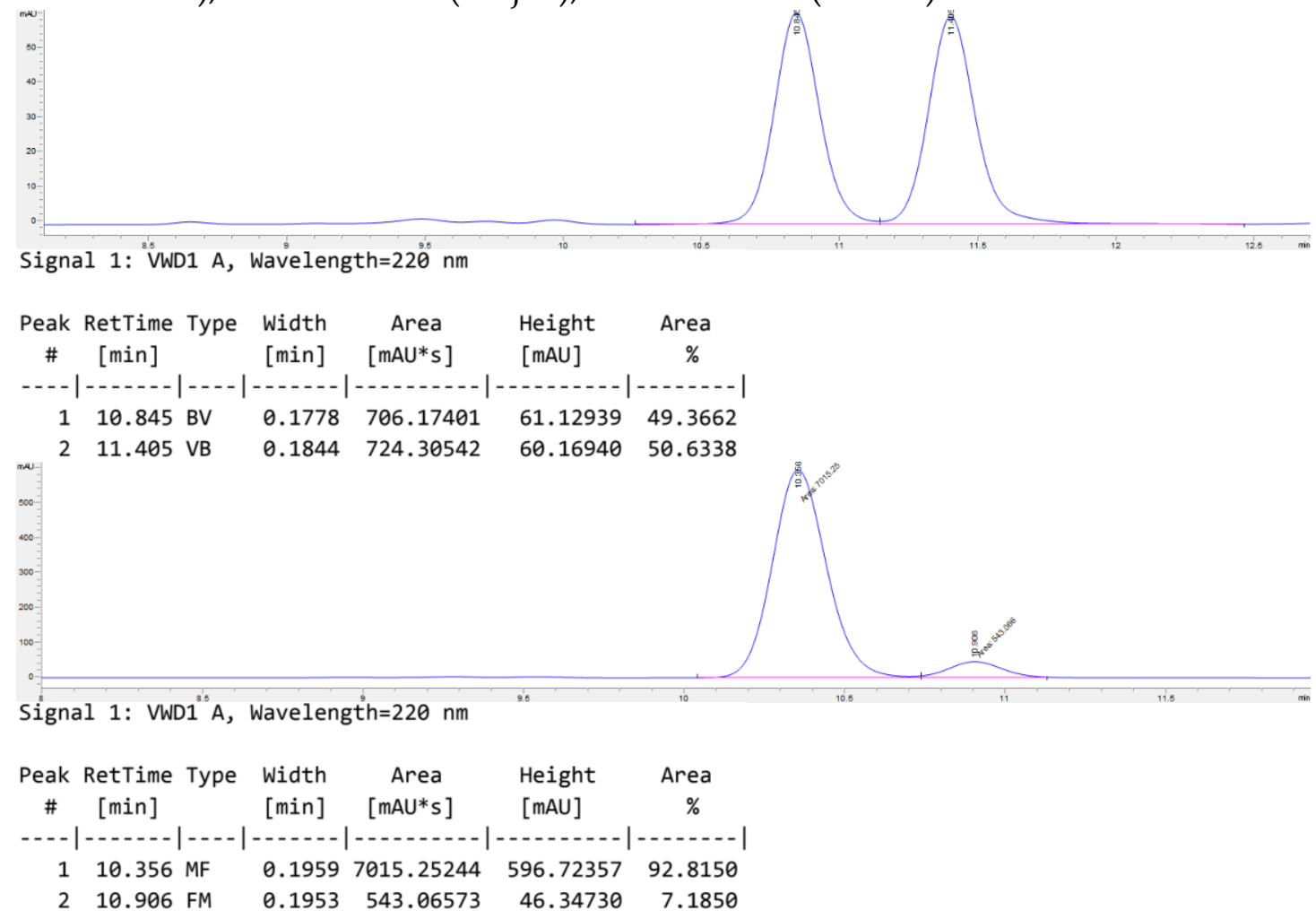

(S)-((2,5-Bis $(4,4,5,5-$ tetramethyl-1,3,2-dioxaborolan-2-

yl)pentyl)oxy)triisopropylsilane $(2 x)$ 


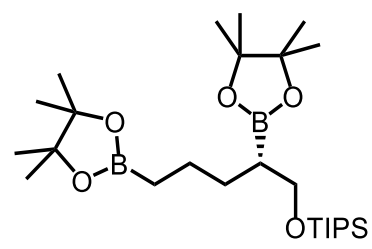

Compound 2x was prepared as a colorless oil in 73\% yield (72.5 mg, eluent: petroleum ether/EtOAc $=100 / 3$ to $20 / 1)$, from $(Z)$-triisopropyl((5-(4,4,5,5tetramethyl-1,3,2-dioxaborolan-2-yl)pent-1-en-1-yl)oxy)silane 1x (73.6 mg, 0.20 $\mathrm{mmol}),[\mathrm{RhCl}(\mathrm{cod})]_{2}(2.0 \mathrm{mg}, 0.004 \mathrm{mmol}),(S)-\mathrm{L9}$ (3.5 mg, $\left.0.008 \mathrm{mmol}\right), \mathrm{HBpin}$ (38.4 mg, $0.30 \mathrm{mmol})$ and LiOAc $(4.0 \mathrm{mg}, 0.06 \mathrm{mmol})$ following the above general procedure $\mathrm{E}$.

$\mathbf{R}_{f}=0.75$ (petroleum ether $/$ EtOAc $=10 / 1$ )

${ }^{1} \mathrm{H}$ NMR (400 MHz, $\left.\mathrm{CDCl}_{3}\right) \delta 3.74(\mathrm{~d}, J=6.9 \mathrm{~Hz}, 2 \mathrm{H}), 1.64-1.58$ (m, 1H), $1.47-$ $1.36(\mathrm{~m}, 4 \mathrm{H}), 1.28-1.18(\mathrm{~m}, 24 \mathrm{H}), 1.16-0.88(\mathrm{~m}, 21 \mathrm{H}), 0.83-0.68(\mathrm{~m}, 2 \mathrm{H})$.

${ }^{13} \mathrm{C}$ NMR (100 MHz, $\left.\mathrm{CDCl}_{3}\right) \delta$ 83.0, 82.9, 65.2, 30.5, 25.01, 24.97, 23.8, 18.2, 12.2.

The carbon signal attached to B atom was not observed due to low intensity.

HRMS (ESI') calcd for $\mathrm{C}_{26} \mathrm{H}_{54} \mathrm{~B}_{2} \mathrm{O}_{5} \mathrm{NaSi}^{+}[\mathrm{M}+\mathrm{Na}]^{+}: 519.3819$, found: 519.3817 .

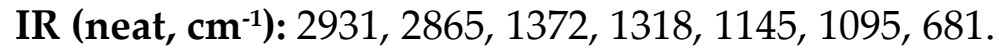

$[\alpha]_{\mathrm{D}^{26.2}}=0.74\left(\mathrm{c}=1.12, \mathrm{CHCl}_{3}, 92 \%\right.$ ee $)$.

Compound $2 \mathrm{x}$ was subjected to general procedure $\mathrm{J}$ after oxidation by $\mathrm{H}_{2} \mathrm{O}_{2} / \mathrm{NaOH}$ solution and the resulting ester was analyzed by chiral HPLC. Enantiomeric excess was determined by chiral HPLC (Daicel Chiralpak IA, hexane/isopropyl alcohol $=98 / 2$, flow rate $=1.0 \mathrm{~mL} / \mathrm{min}, \lambda=220 \mathrm{~nm}), t^{1}=6.6$ $\min$ (major),$t^{2}=7.2 \mathrm{~min}$ (minor).

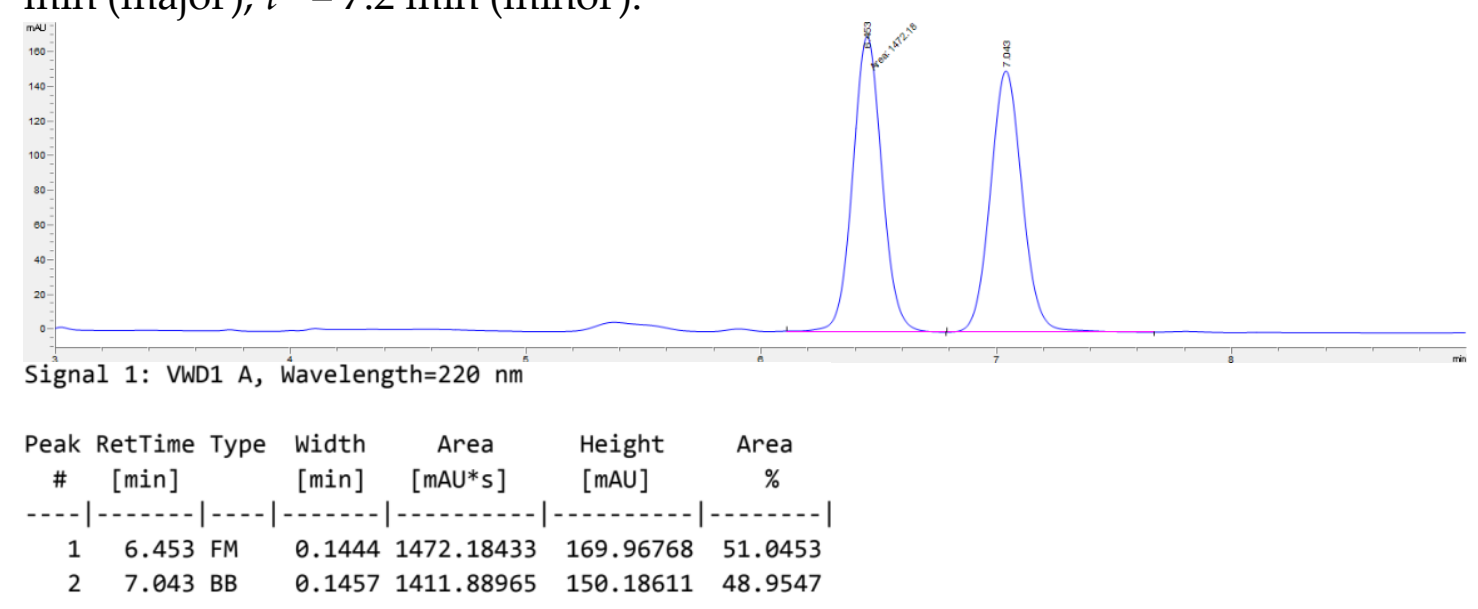




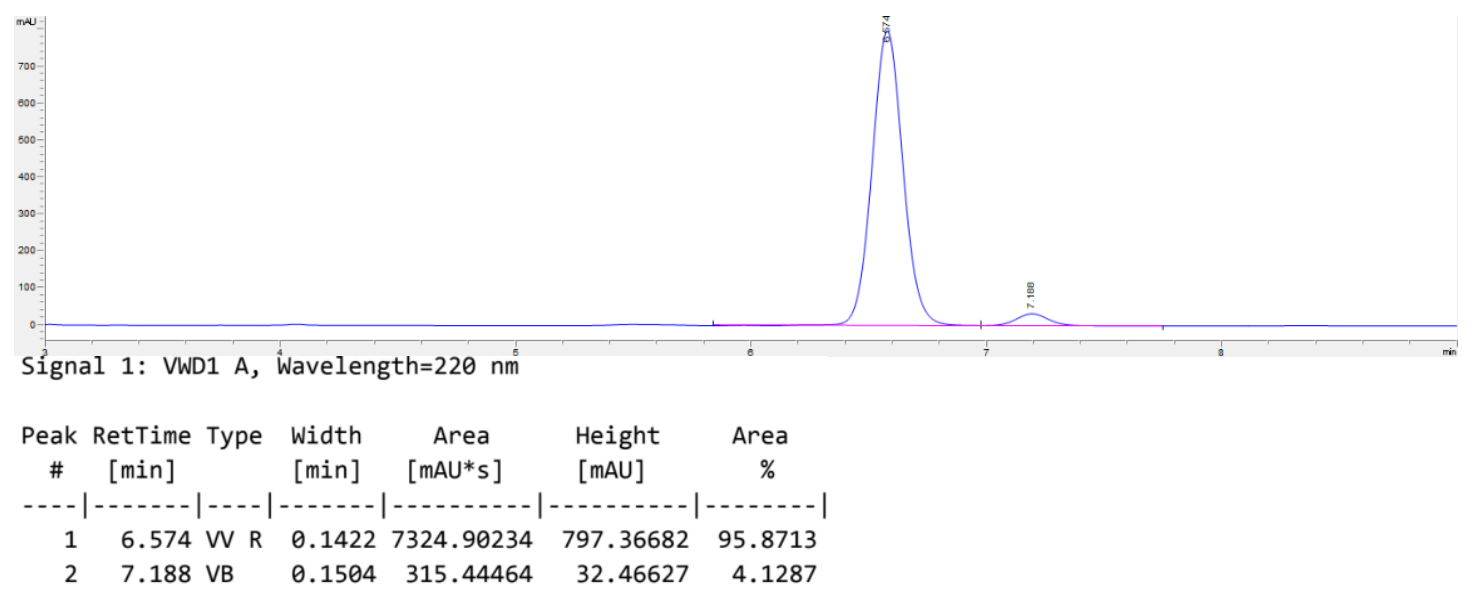

Compound $2 \mathbf{x}$ was prepared in $65 \%$ yield $(64.9 \mathrm{mg})$ and $92 \%$ ee from $1 \times \mathbf{x}(E / Z=$ 1/6.4) (73.6 mg, $0.20 \mathrm{mmol})$, [RhCl(cod)]2 (2.0 mg, $0.004 \mathrm{mmol}),(S)-\mathbf{L} 9$ (3.5 mg, $0.008 \mathrm{mmol}), \mathrm{HBpin}(38.4 \mathrm{mg}, 0.30 \mathrm{mmol})$ and LiOAc $(4.0 \mathrm{mg}, 0.06 \mathrm{mmol})$ following the above general procedure $\mathrm{E}$.

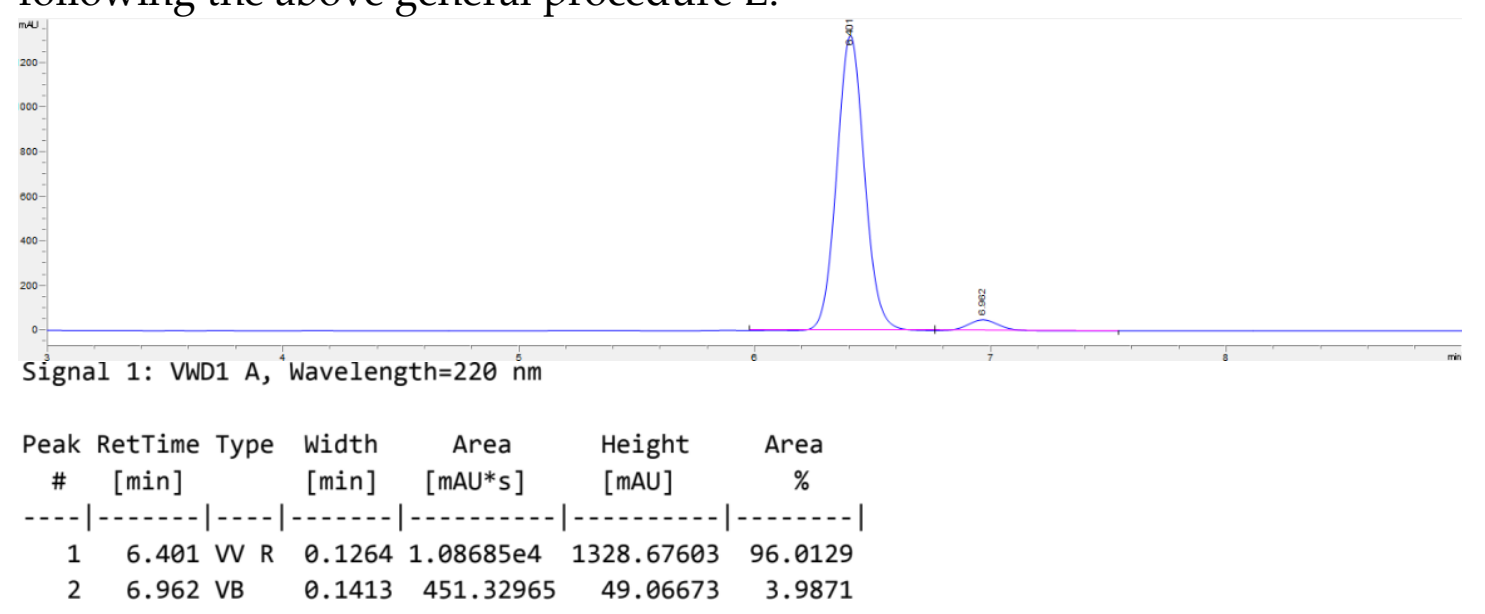

(S)-6-Chloro-1-((triisopropylsilyl)oxy)hexan-2-ol (3y)<smiles>OC(CCCCCCl)CO[Hg]</smiles>

Compound $3 y$ was prepared as a colorless oil in $84 \%$ yield $(52.0 \mathrm{mg}$, eluent: petroleum ether/EtOAc $=100 / 3$ to 20/1), from (Z)-((6-chlorohex-1-en-1yl)oxy)triisopropylsilane $1 \mathbf{y}(58.2 \mathrm{mg}, 0.20 \mathrm{mmol})$, [ RhCl(cod)]2 (2.0 mg, 0.004 mmol), (S)-L9 (3.5 mg, $0.008 \mathrm{mmol})$, HBpin ( $38.4 \mathrm{mg}, 0.30 \mathrm{mmol}$ ) and LiOAc $(4.0 \mathrm{mg}, 0.06 \mathrm{mmol})$ following the above general procedure $\mathrm{E}$.

$\mathbf{R}_{f}=0.65$ (petroleum ether $/$ EtOAc $=10 / 1$ )

${ }^{1} \mathrm{H}$ NMR (400 MHz, $\mathrm{CDCl}_{3}$ ) 8 3.76-3.62 (m, 2H), 3.58-3.52 (m, 2H), 3.52-3.45 (m, $1 \mathrm{H}), 2.40(\mathrm{~s}, 1 \mathrm{H}), 1.89-1.73(\mathrm{~m}, 2 \mathrm{H}), 1.69-1.57(\mathrm{~m}, 1 \mathrm{H}), 1.57-1.37(\mathrm{~m}, 3 \mathrm{H}), 1.19$ 
$0.95(\mathrm{~m}, 21 \mathrm{H})$.

${ }^{13} \mathrm{C}$ NMR (100 MHz, $\left.\mathrm{CDCl}_{3}\right) \delta$ 71.9, 67.6, 45.1, 32.8, 32.2, 23.2, 18.1, 12.1.

HRMS (ESI') calcd for $\mathrm{C}_{15} \mathrm{H}_{33} \mathrm{ClO}_{2} \mathrm{NaSi}^{+}[\mathrm{M}+\mathrm{Na}]^{+}$: 331.1831 , found: 331.1829 .

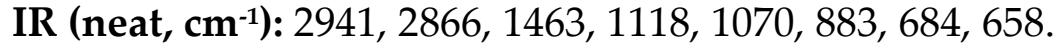

$[\alpha]_{\mathrm{D}^{26.2}}=0.89\left(\mathrm{c}=0.77, \mathrm{CHCl}_{3}, 92 \%\right.$ ee $)$.

Compound $3 y$ was subjected to general procedure $\mathrm{J}$ and the resulting ester was analyzed by chiral HPLC. Enantiomeric excess was determined by chiral HPLC (Daicel Chiralpak IA, hexane/isopropyl alcohol $=99.5 / 0.5$, flow rate $=0.5$ $\mathrm{mL} / \mathrm{min}, \lambda=220 \mathrm{~nm}$ ), $t^{1}=11.1 \mathrm{~min}$ (major),$t^{2}=14.1 \mathrm{~min}$ (minor).

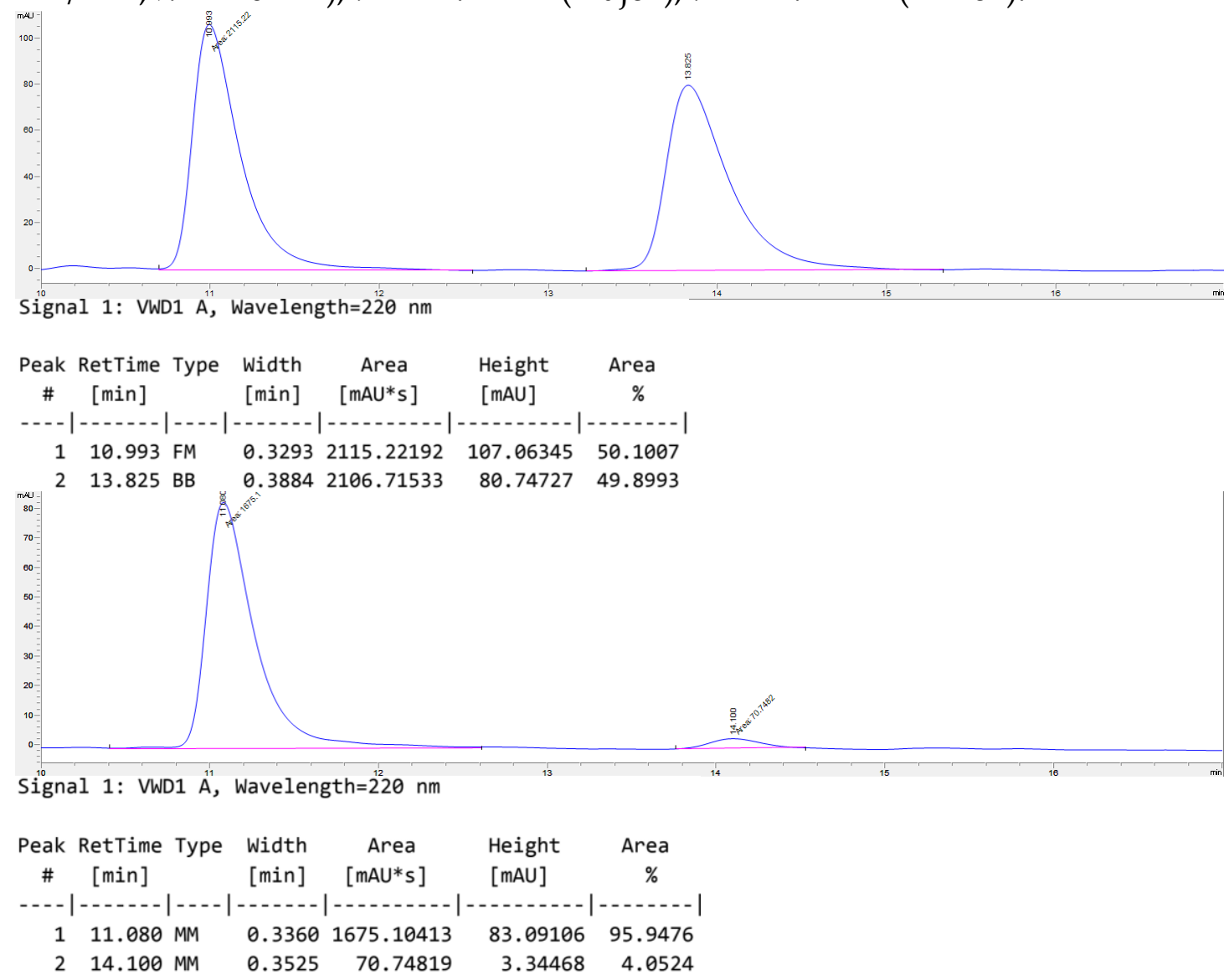

Compound $3 \mathbf{y}$ was prepared in $87 \%$ yield $(53.6 \mathrm{mg})$ and $89 \%$ ee from $1 \mathbf{y}(E / Z=$ 1/8.4) (58.2 mg, $0.20 \mathrm{mmol}),[\mathrm{RhCl}(\mathrm{cod})]_{2}(2.0 \mathrm{mg}, 0.004 \mathrm{mmol}),(S)-\mathbf{L 9}$ (3.5 mg, $0.008 \mathrm{mmol})$, HBpin (38.4 mg, $0.30 \mathrm{mmol})$ and LiOAc (4.0 mg, $0.06 \mathrm{mmol})$ following the above general procedure E. 


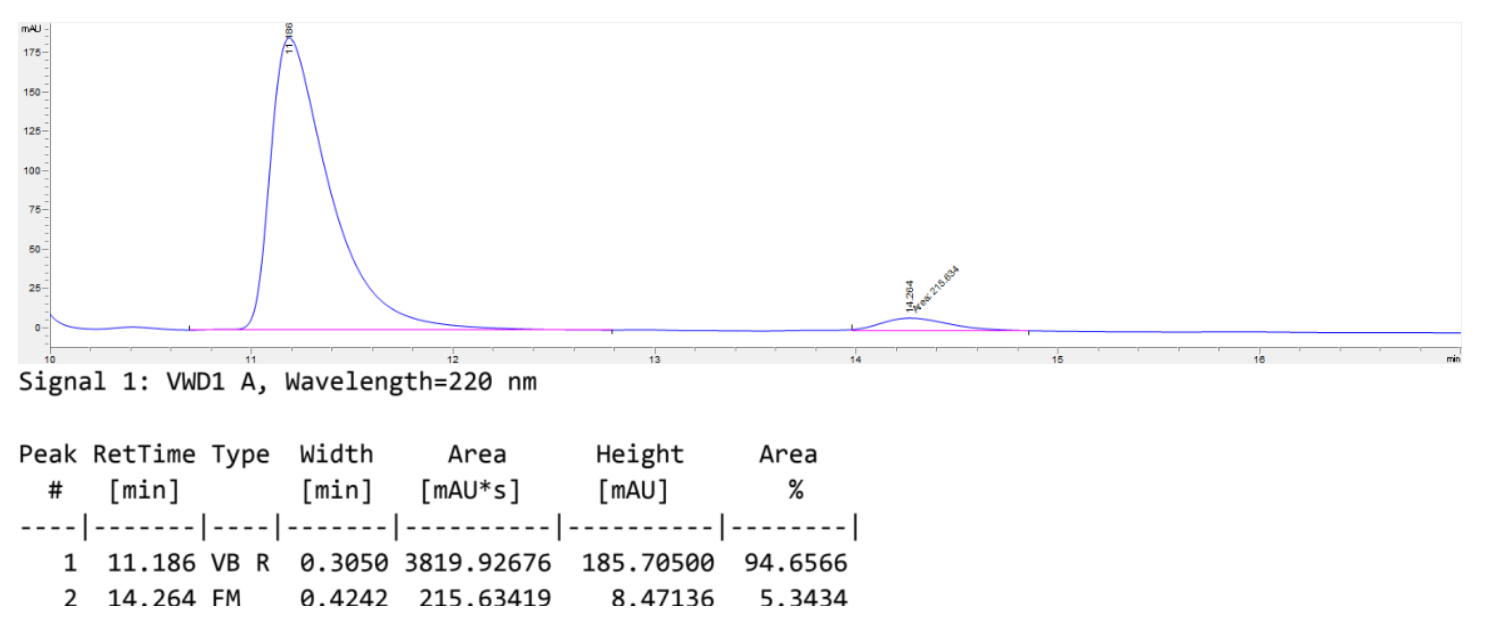

(S)-12,12,12-Trifluoro-1-((triisopropylsilyl)oxy)dodecan-2-ol (3z)

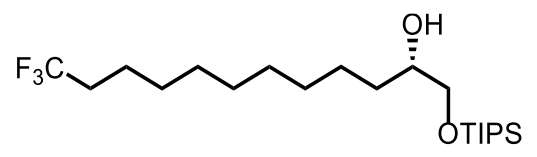

Compound $3 \mathbf{z}$ was prepared as a colorless oil in $83 \%$ yield $(68.0 \mathrm{mg}$, eluent: petroleum ether/EtOAc $=100 / 3$ to $20 / 1)$, from $(Z)$-triisopropyl((12,12,12trifluorododec-1-en-1-yl)oxy)silane $\mathbf{1 z}(69.6 \mathrm{mg}, 0.20 \mathrm{mmol}),[\mathrm{RhCl}(\mathrm{cod})]_{2}(2.0$ mg, $0.004 \mathrm{mmol})$, (S)-L9 (3.5 mg, $0.008 \mathrm{mmol})$, HBpin (38.4 mg, $0.30 \mathrm{mmol})$ and LiOAc ( $4.0 \mathrm{mg}, 0.06 \mathrm{mmol})$ following the above general procedure $\mathrm{E}$.

$\mathbf{R}_{f}=0.65$ (petroleum ether $/ \mathrm{EtOAc}=10 / 1$ )

${ }^{1} \mathrm{H}$ NMR (400 MHz, $\left.\mathrm{CDCl}_{3}\right) \delta$ 3.76-3.60 (m, 2H), 3.53-3.43 (m, 1H), $2.55(\mathrm{~s}, 1 \mathrm{H})$, 2.13-1.97 (m, 2H), 1.60-1.50 (m, 2H), 1.47-1.23 (m, 14H), 1.18-0.98 (m, 21H).

${ }^{13} \mathrm{C}$ NMR (100 MHz, $\left.\mathrm{CDCl}_{3}\right) \delta 127.4(\mathrm{q}, J=275 \mathrm{~Hz}), 72.1,67.8,33.9(\mathrm{q}, J=28.3$ $\mathrm{Hz}), 32.9,29.8,29.6,29.4,29.3,28.8,25.7,22.0$ (q, $J=2.8 \mathrm{~Hz}), 18.1,12.1$.

${ }^{19}$ F NMR (377 MHz, $\left.\mathrm{CDCl}_{3}\right) \delta-66.4(\mathrm{t}, J=10.9 \mathrm{~Hz})$.

HRMS (ESI') calcd for $\mathrm{C}_{21} \mathrm{H}_{43} \mathrm{~F}_{3} \mathrm{O}_{2} \mathrm{NaSi}^{+}[\mathrm{M}+\mathrm{Na}]^{+}:$435.2877, found: 435.2875 .

IR (neat, cm-1): 2928, 2863, 1254, 1137, 1104, 1067, 883, 683.

$[\alpha]_{\mathrm{D}^{26.2}}=1.38\left(\mathrm{c}=0.91, \mathrm{CHCl}_{3}, 91 \%\right.$ ee $)$.

Compound $3 \mathrm{z}$ was subjected to general procedure $\mathrm{J}$ and the resulting ester was analyzed by chiral HPLC. Enantiomeric excess was determined by chiral HPLC (Daicel Chiralpak IA, hexane/isopropyl alcohol $=99.5 / 0.5$, flow rate $=0.5$ $\mathrm{mL} / \mathrm{min}, \lambda=220 \mathrm{~nm}$ ), $t^{1}=14.6 \mathrm{~min}$ (major),$t^{2}=17.1 \mathrm{~min}$ (minor). 


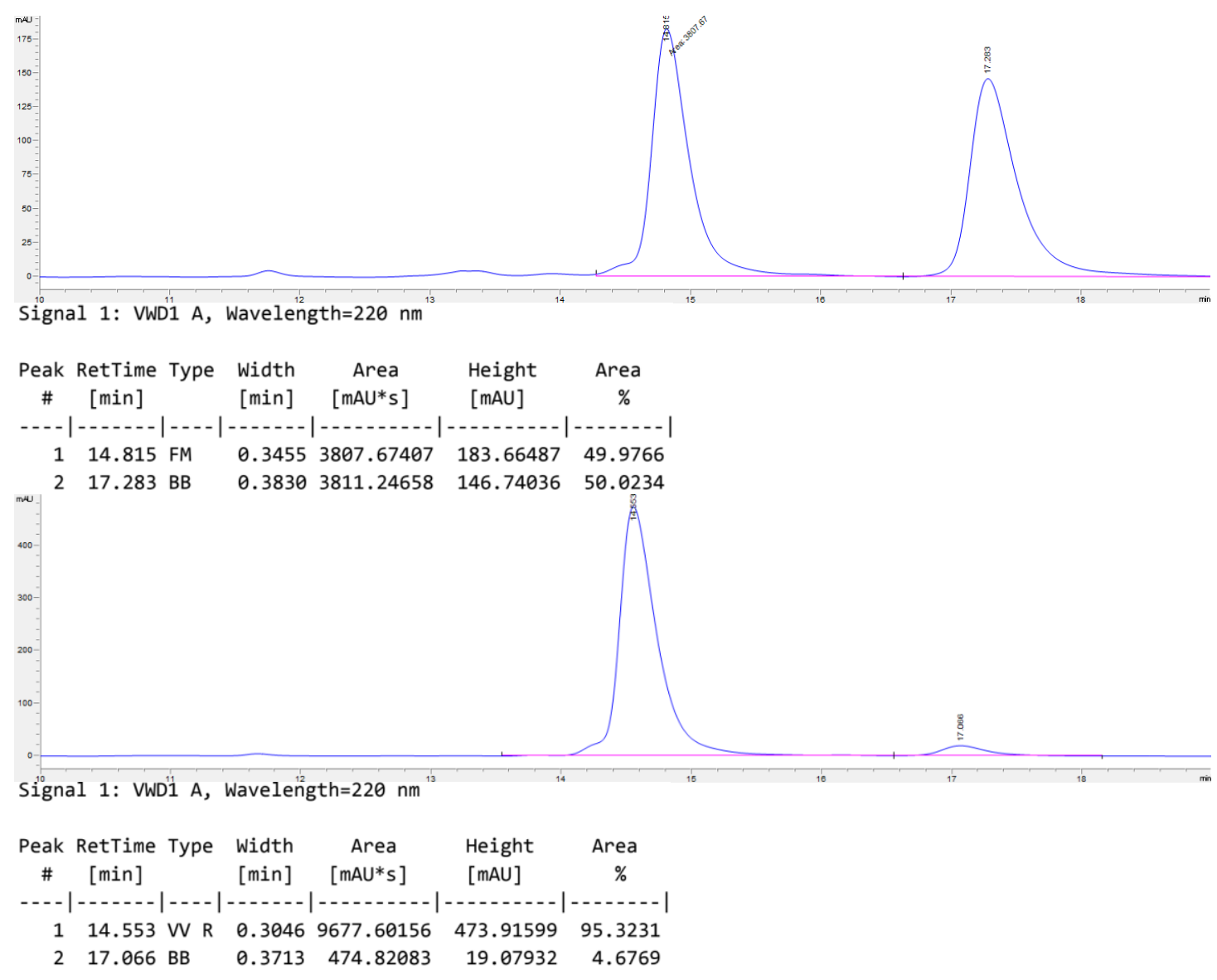

(S)-11-Fluoro-1-((triisopropylsilyl)oxy)undecan-2-ol (3aa)

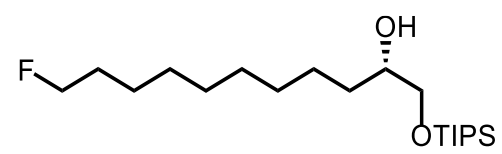

Compound 3aa was prepared as a colorless oil in $95 \%$ yield $(69.2 \mathrm{mg}$, eluent: petroleum ether/EtOAc $=100 / 3$ to 20/1), from (Z)-((11-fluoroundec-1-en-1yl)oxy)triisopropylsilane 1aa (69.0 mg, $0.20 \mathrm{mmol})$, [RhCl(cod)]2 $(2.0 \mathrm{mg}, 0.004$ mmol), (S)-L9 (3.5 mg, $0.008 \mathrm{mmol})$, HBpin ( $38.4 \mathrm{mg}, 0.30 \mathrm{mmol})$ and LiOAc $(4.0 \mathrm{mg}, 0.06 \mathrm{mmol})$ following the above general procedure $\mathrm{E}$.

$\mathbf{R}_{f}=0.65$ (petroleum ether $/ \mathrm{EtOAc}=10 / 1$ )

${ }^{1} \mathrm{H}$ NMR (400 MHz, $\left.\mathrm{CDCl}_{3}\right) \delta 4.49(\mathrm{t}, J=6.3 \mathrm{~Hz}, 1 \mathrm{H}), 4.37(\mathrm{t}, J=6.3 \mathrm{~Hz}, 1 \mathrm{H})$, $3.78-3.58(\mathrm{~m}, 2 \mathrm{H}), 3.53-3.41(\mathrm{~m}, 1 \mathrm{H}), 2.54(\mathrm{~s}, 1 \mathrm{H}), 1.76-1.58(\mathrm{~m}, 2 \mathrm{H}), 1.48-1.27$ (m, 14H), 1.18-0.95 (m, 21H).

${ }^{13} \mathrm{C}$ NMR (100 MHz, CDCl $) \delta 84.4(\mathrm{~d}, J=162 \mathrm{~Hz}), 72.1,67.8,32.9,30.7,30.5,29.8$, 29.6, 29.4, 25.7, $25.3(\mathrm{~d}, J=5.4 \mathrm{~Hz}), 18.1,12.1$.

${ }^{19} \mathrm{~F}$ NMR (377 MHz, $\left.\mathrm{CDCl}_{3}\right) \delta-218.0(\mathrm{~m})$.

HRMS (ESI+) calcd for $\mathrm{C}_{20} \mathrm{H}_{43} \mathrm{FO}_{2} \mathrm{NaSi}^{+}[\mathrm{M}+\mathrm{Na}]^{+}:$385.2909, found: 385.2907 . 
IR (neat, cm-1): 2927, 2862, 1463, 1105, 1066, 883, 683.

$[\alpha]_{\mathrm{D}^{26.2}}=1.87\left(\mathrm{c}=1.04, \mathrm{CHCl}_{3}, 92 \%\right.$ ee $)$.

Compound 3aa was subjected to general procedure J and the resulting ester was analyzed by chiral HPLC. Enantiomeric excess was determined by chiral HPLC (Daicel Chiralpak IA, hexane/isopropyl alcohol $=99 / 1$, flow rate $=1.0$ $\mathrm{mL} / \mathrm{min}, \lambda=220 \mathrm{~nm}$ ), $t^{1}=9.0 \mathrm{~min}$ (major),$t^{2}=11.1 \mathrm{~min}$ (minor).

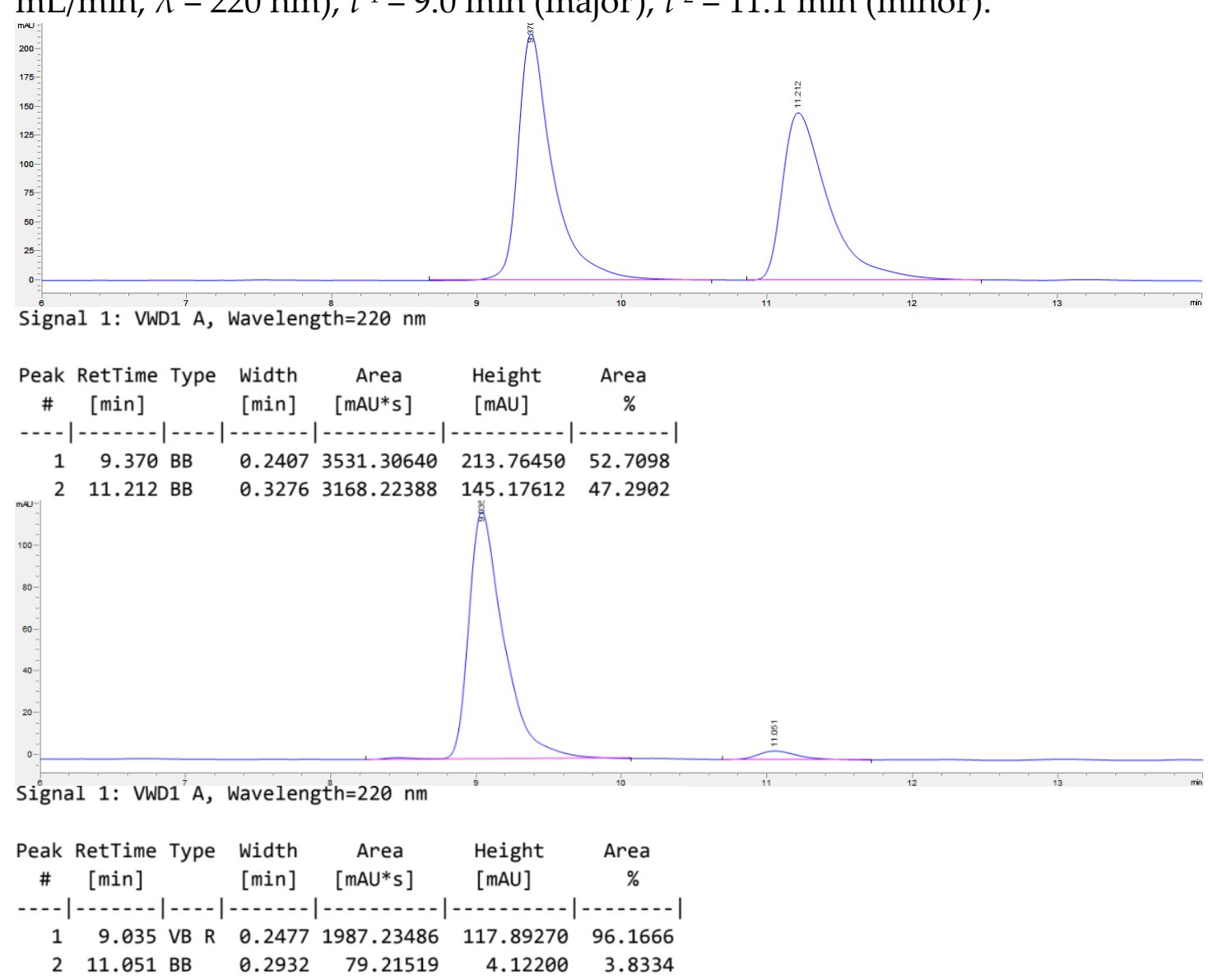

\section{Compound 3ab}

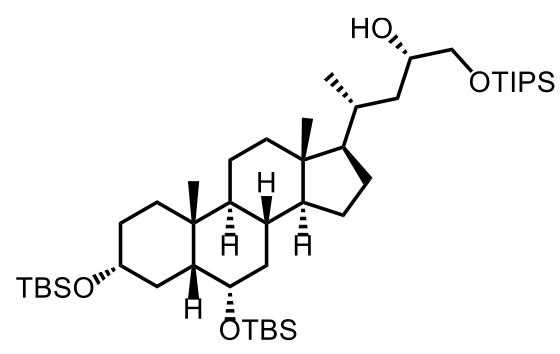

Compound 3ab was prepared as a colorless oil in $87 \%$ yield $(136.0 \mathrm{mg}$, eluent: petroleum ether/EtOAc $=20 / 1)$, from $1 \mathrm{ab}(152.3 \mathrm{mg}, 0.20 \mathrm{mmol}),[\mathrm{RhCl}(\operatorname{cod})]_{2}$ (4.0 mg, $0.008 \mathrm{mmol}),(S)-\mathrm{L} 9(7.0 \mathrm{mg}, 0.016 \mathrm{mmol}), \mathrm{HBpin}(38.4 \mathrm{mg}, 0.30 \mathrm{mmol})$ and $\mathrm{LiOAc}(4.0 \mathrm{mg}, 0.06 \mathrm{mmol})$ following the above general procedure $\mathrm{E}\left(30^{\circ} \mathrm{C}\right.$, 
$48 \mathrm{~h})$.

$\mathbf{R}_{f}=0.55$ (petroleum ether $/$ EtOAc $=10 / 1$ )

${ }^{1} \mathrm{H}$ NMR (400 MHz, $\left.\mathrm{CDCl}_{3}\right) \delta$ 4.03-3.93 (m, 1H), 3.79-3.68 (m, 2H), 3.58-3.49 (m, $1 \mathrm{H}), 3.48-3.40(\mathrm{~m}, 1 \mathrm{H}), 2.56(\mathrm{~s}, 1 \mathrm{H}), 2.03-1.80(\mathrm{~m}, 3 \mathrm{H}), 1.78-1.63(\mathrm{~m}, 2 \mathrm{H}), 1.61-$ $1.32(\mathrm{~m}, 11 \mathrm{H}), 1.30-1.03(\mathrm{~m}, 29 \mathrm{H}), 0.99(\mathrm{~d}, J=6.5 \mathrm{~Hz}, 3 \mathrm{H}), 0.95-0.78(\mathrm{~m}, 21 \mathrm{H})$, $0.63(\mathrm{~s}, 3 \mathrm{H}), 0.08--0.01(\mathrm{~m}, 12 \mathrm{H})$.

${ }^{13} \mathrm{C}$ NMR (100 MHz, $\left.\mathrm{CDCl}_{3}\right) \delta$ 73.1, 71.0, 68.8, 67.3, 57.1, 56.2, 49.7, 43.0, 40.1, 39.7, 39.3, 36.1, 36.0, 35.5, 35.0, 34.2, 31.1, 29.9, 28.6, 26.1, 26.0, 24.4, 23.7, 20.9, $19.6,18.6,18.3,18.1,12.1,12.0,-4.4,-4.5,-4.6,-4.7$.

HRMS (ESI ${ }^{+}$) calcd for $\mathrm{C}_{45} \mathrm{H}_{90} \mathrm{O}_{4} \mathrm{NaSi}_{3}{ }^{+}[\mathrm{M}+\mathrm{Na}]^{+}:$801.6039, found: 801.6032.

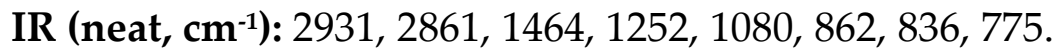

$[\alpha]_{\mathrm{D}^{26.2}}=5.05\left(\mathrm{c}=0.91, \mathrm{CHCl}_{3}, 95: 5 \mathrm{dr}\right)$.

Compound $3 \mathbf{a b}$ was subjected to general procedure $\mathrm{J}$ and the resulting ester was analyzed by chiral HPLC. Diastereomeric excess was determined by chiral HPLC (Daicel Chiralpak IA, hexane/isopropyl alcohol $=98.5 / 1.5$, flow rate $=0.5$ $\mathrm{mL} / \mathrm{min}, \lambda=220 \mathrm{~nm}$ ), $t^{1}=6.6 \mathrm{~min}$ (minor),$t^{2}=7.0 \mathrm{~min}$ (major).

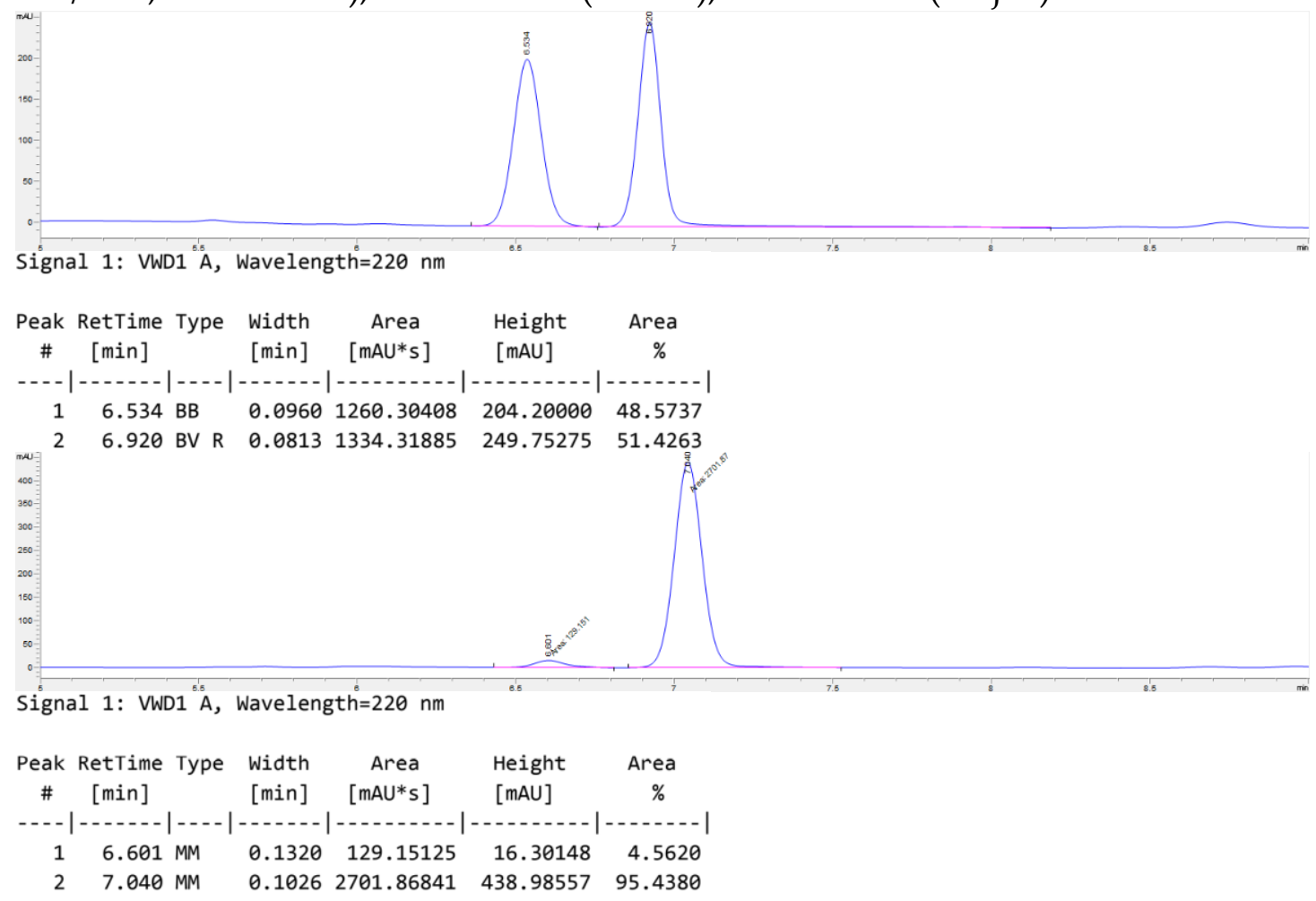

\section{Compound ent-3ab}




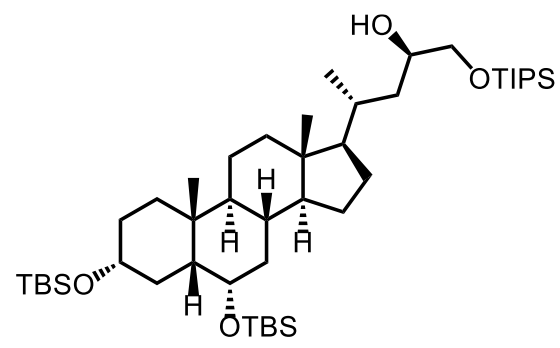

Compound 3ab was prepared as a colorless oil in 78\% yield (121.0 mg, eluent: petroleum ether/EtOAc $=20 / 1)$, from $1 \mathrm{ab}(152.3 \mathrm{mg}, 0.20 \mathrm{mmol}),[\mathrm{RhCl}(\operatorname{cod})]_{2}$ (4.0 mg, $0.008 \mathrm{mmol}),(R)-\mathrm{L} 9$ (7.0 mg, $0.016 \mathrm{mmol}), \mathrm{HBpin}(38.4 \mathrm{mg}$, $0.30 \mathrm{mmol})$ and $\mathrm{LiOAc}(4.0 \mathrm{mg}, 0.06 \mathrm{mmol})$ following the above general procedure $\mathrm{E}\left(30^{\circ} \mathrm{C}\right.$, $48 \mathrm{~h})$.

$\mathbf{R}_{f}=0.58$ (petroleum ether $/$ EtOAc $=10 / 1$ )

${ }^{1} \mathrm{H}$ NMR (400 MHz, $\left.\mathrm{CDCl}_{3}\right) \delta 4.04-3.92(\mathrm{~m}, 1 \mathrm{H}), 3.82-3.72(\mathrm{~m}, 1 \mathrm{H}), 3.66(\mathrm{dd}$, $J=9.6,3.4 \mathrm{~Hz}, 1 \mathrm{H}), 3.58-3.48(\mathrm{~m}, 1 \mathrm{H}), 3.44(\mathrm{dd}, J=9.6,7.7 \mathrm{~Hz}, 1 \mathrm{H}), 2.44(\mathrm{~s}, 1 \mathrm{H})$, $2.05-1.66(\mathrm{~m}, 5 \mathrm{H}), 1.65-1.49(\mathrm{~m}, 4 \mathrm{H}), 1.48-1.33(\mathrm{~m}, 7 \mathrm{H}), 1.31-1.21(\mathrm{~m}, 3 \mathrm{H})$, $1.20-1.00(\mathrm{~m}, 26 \mathrm{H}), 1.00-0.94(\mathrm{~m}, 3 \mathrm{H}), 0.91-0.84(\mathrm{~m}, 21 \mathrm{H}), 0.67(\mathrm{~s}, 3 \mathrm{H}), 0.08-$ $0.01(\mathrm{~m}, 12 \mathrm{H})$.

${ }^{13} \mathrm{C}$ NMR (100 MHz, $\left.\mathrm{CDCl}_{3}\right) \delta$ 73.1, 69.3, 68.8, 68.6, 57.1, 56.4, 49.7, 43.1, 40.2, 39.8, 39.1, 36.1, 36.1, 35.6, 35.0, 32.4, 31.2, 30.0, 28.5, 26.2, 26.0, 24.4, 23.7, 20.9, $18.7,18.6,18.3,18.1,17.9,12.3,12.1,-4.3,-4.5,-4.6,-4.6$.

$[\alpha]_{\mathrm{D}^{23.3}}=9.03\left(\mathrm{c}=0.50, \mathrm{CHCl}_{3}, 6: 94 \mathrm{dr}\right)$.

Compound ent-3ab was subjected to general procedure $\mathrm{J}$ and the resulting ester was analyzed by chiral HPLC. Diastereomeric excess was determined by chiral HPLC (Daicel Chiralpak IA, hexane/isopropyl alcohol $=98.5 / 1.5$, flow rate $=0.5 \mathrm{~mL} / \mathrm{min}, \lambda=220 \mathrm{~nm}$ ), $t^{1}=6.6 \mathrm{~min}$ (major),$t^{2}=7.0 \mathrm{~min}$ (minor).

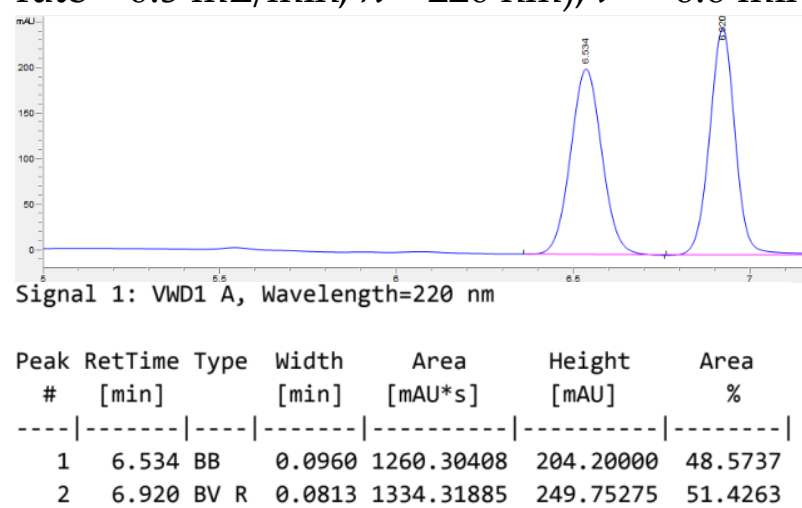




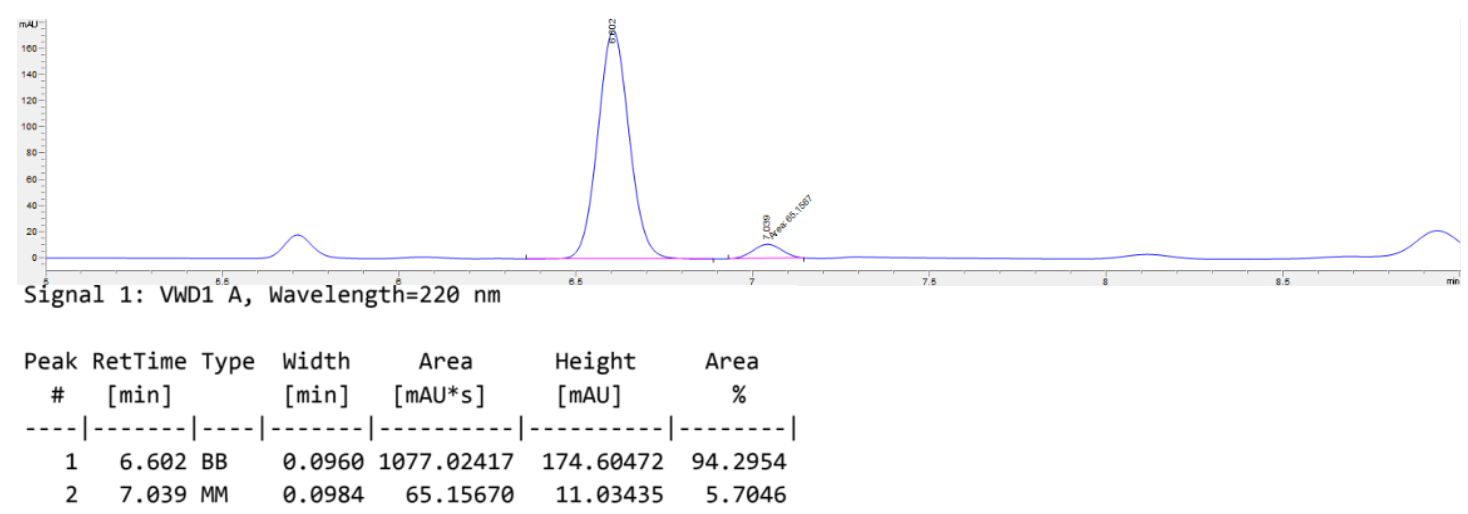

(R)-1-Phenyl-2-((triisopropylsilyl)oxy)ethan-1-ol (3ac)<smiles>O[C@H](CO[18OH])c1ccccc1</smiles>

Compound 3ac was prepared as a colorless oil in 95\% yield $(84.0 \mathrm{mg}$, eluent: petroleum ether/EtOAc $=100 / 3$ to $20 / 1)$, from $(Z)$-triisopropyl(styryloxy)silane 1ac $(82.9 \mathrm{mg}, 0.30 \mathrm{mmol}),[\mathrm{RhCl}(\mathrm{cod})]_{2}(3.0 \mathrm{mg}, 0.006 \mathrm{mmol}),(R, R)-3,5-i \mathrm{Pr}_{2}-$ DIOP (10.0 mg, $0.012 \mathrm{mmol})$, HBpin (57.6 mg, $0.45 \mathrm{mmol}$ ) and LiOAc (6.0 mg, $0.09 \mathrm{mmol}$ ) following the above general procedure $\mathrm{F}$.

$\mathbf{R}_{f}=0.42$ (petroleum ether $/$ EtOAc $=10 / 1$ )

${ }^{1}$ H NMR (400 MHz, $\left.\mathrm{CDCl}_{3}\right) \delta$ 7.42-7.21 (m, 5H), $4.77(\mathrm{dd}, J=8.9,3.5 \mathrm{~Hz}, 1 \mathrm{H})$, $3.84(\mathrm{dd}, J=9.9,3.6 \mathrm{~Hz}, 1 \mathrm{H}), 3.66-3.58(\mathrm{~m}, 1 \mathrm{H}), 3.12(\mathrm{~s}, 1 \mathrm{H}), 1.20-0.94(\mathrm{~m}, 21 \mathrm{H})$. ${ }^{13} \mathrm{C}$ NMR (100 MHz, $\left.\mathrm{CDCl}_{3}\right) \delta$ 140.4, 128.4, 127.8, 126.3, 74.6, 69.4, 18.0, 12.0.

HRMS (ESI+) calcd for $\mathrm{C}_{17} \mathrm{H}_{30} \mathrm{NaO}_{2} \mathrm{Si}^{+}[\mathrm{M}+\mathrm{Na}]^{+}$: 317.1907, found: 317.1906.

IR (neat, cm ${ }^{-1}$ ): 2941, 2866, 1461, 1197, 1110, 1061, 996, 883, 855, 759, 688, 536.

$[\alpha]_{\mathrm{D}^{24.3}}=-23.49\left(\mathrm{c}=0.65, \mathrm{CHCl}_{3}, 95 \%\right.$ ee $)$; Enantiomeric excess was determined by chiral HPLC (Daicel Chiralcel OD-H, hexane/isopropyl alcohol = 95/5, flow rate $=1.0 \mathrm{~mL} / \mathrm{min}, \lambda=220 \mathrm{~nm}$ ), $t^{1}=4.1 \mathrm{~min}$ (minor),$t^{2}=4.8 \mathrm{~min}$ (major).

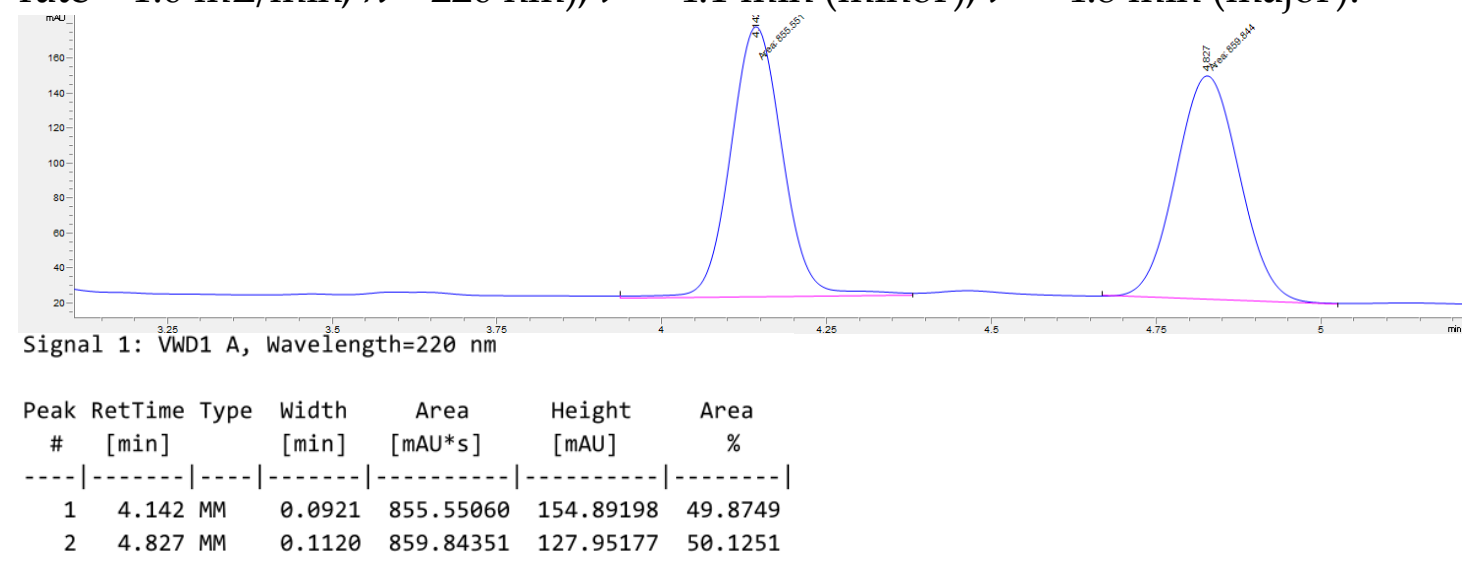




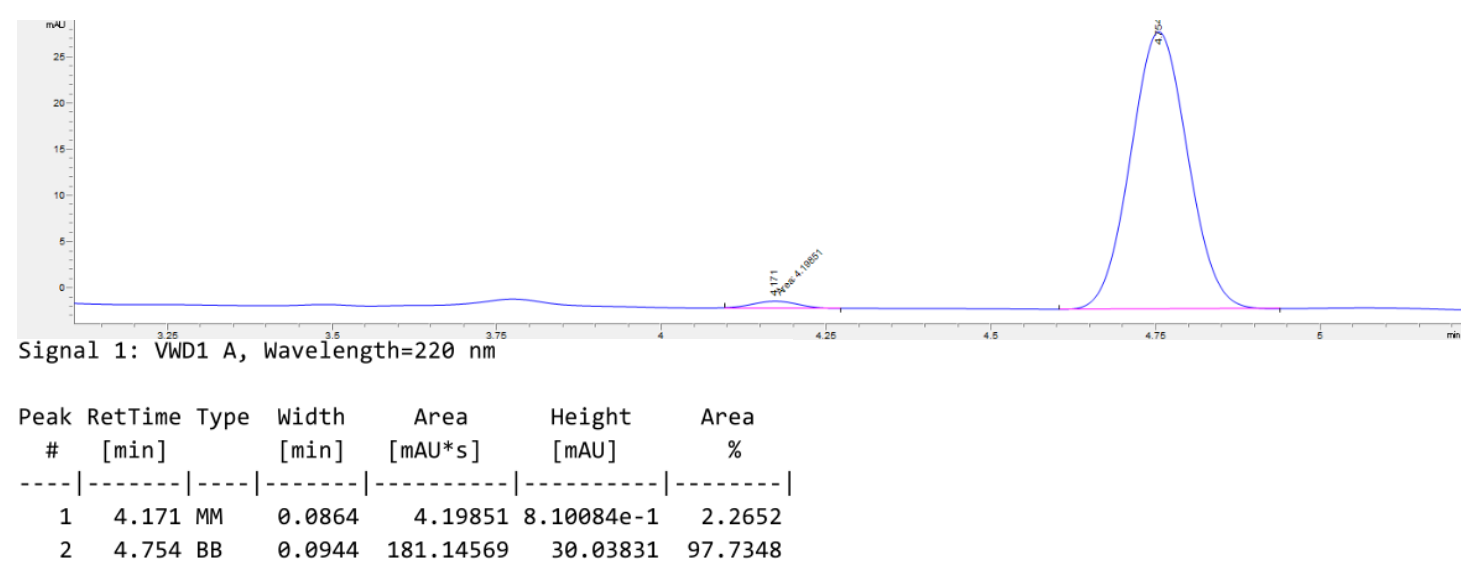

(R)-1-(p-Tolyl)-2-((triisopropylsilyl)oxy)ethan-1-ol (3ad)<smiles></smiles>

Compound 3ad was prepared as a colorless oil in $83 \%$ yield $(77.0 \mathrm{mg}$, eluent: petroleum ether/EtOAc $=100 / 3$ to 20/1), from (Z)-triisopropyl((4methylstyryl)oxy)silane 1ad (87.0 mg, $0.30 \mathrm{mmol}),[\mathrm{RhCl}(\mathrm{cod})]_{2}(3.0 \mathrm{mg}, 0.006$ $\mathrm{mmol}),(R, R)-3,5-i \mathrm{Pr}_{2}$-DIOP (10.0 mg, $\left.0.012 \mathrm{mmol}\right), \mathrm{HBpin}(57.6 \mathrm{mg}, 0.45 \mathrm{mmol})$ and $\mathrm{LiOAc}(6.0 \mathrm{mg}, 0.09 \mathrm{mmol})$ following the above general procedure $\mathrm{F}$.

$\mathbf{R}_{f}=0.42$ (petroleum ether/EtOAc $=10 / 1$ )

${ }^{1} \mathrm{H}$ NMR (400 MHz, $\left.\mathrm{CDCl}_{3}\right) \delta 7.27(\mathrm{~d}, J=7.8 \mathrm{~Hz}, 2 \mathrm{H}), 7.16(\mathrm{~d}, J=7.7 \mathrm{~Hz}, 2 \mathrm{H})$, $4.74(\mathrm{dd}, J=10.0,2.8 \mathrm{~Hz}, 1 \mathrm{H}), 3.82(\mathrm{dd}, J=9.9,3.4 \mathrm{~Hz}, 1 \mathrm{H}), 3.64-3.56(\mathrm{~m}, 1 \mathrm{H})$, $3.08(\mathrm{~s}, 1 \mathrm{H}), 2.34(\mathrm{~s}, 3 \mathrm{H}), 1.21-0.95(\mathrm{~m}, 21 \mathrm{H})$.

${ }^{13} \mathrm{C}$ NMR (100 MHz, $\left.\mathrm{CDCl}_{3}\right) \delta$ 137.5, 137.3, 129.1, 126.3, 74.5, 69.5, 21.3, 18.1, 12.1. HRMS (ESI+) calcd for $\mathrm{C}_{18} \mathrm{H}_{32} \mathrm{NaO}_{2} \mathrm{Si}^{+}[\mathrm{M}+\mathrm{Na}]^{+}$: 331.2064, found: 331.2063 .

IR (neat, cm-1): 2941, 2866, 1463, 1383, 1107, 1061, 1015, 883, 863, 811, 684, 535. $[\alpha]_{\mathrm{D}^{24.3}}=-23.07\left(\mathrm{c}=0.44, \mathrm{CHCl}_{3}, 96 \%\right.$ ee); Enantiomeric excess was determined by chiral HPLC (Daicel Chiralpak IA, hexane/isopropyl alcohol =99/1, flow rate $=1.0 \mathrm{~mL} / \mathrm{min}, \lambda=220 \mathrm{~nm}$ ), $t^{1}=11.6 \mathrm{~min}$ (minor),$t^{2}=12.0 \mathrm{~min}$ (major).

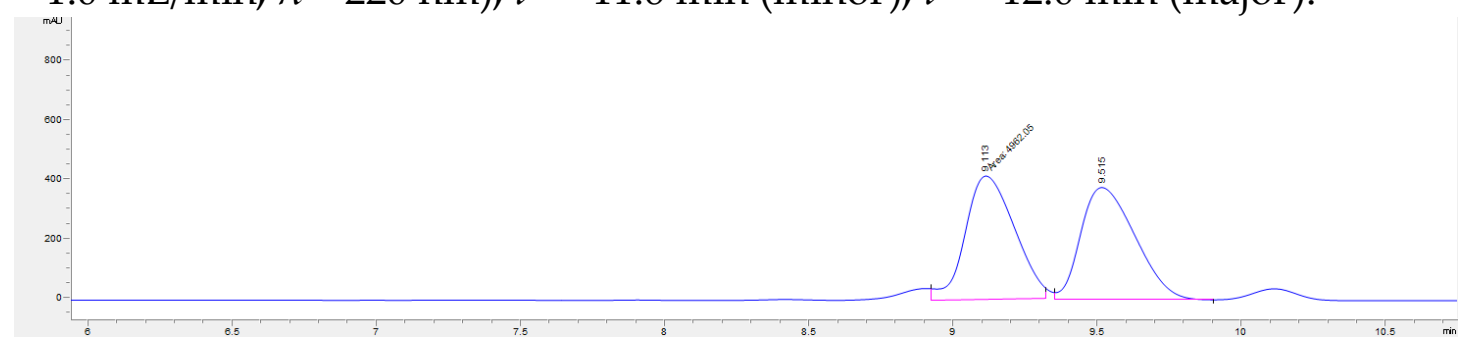




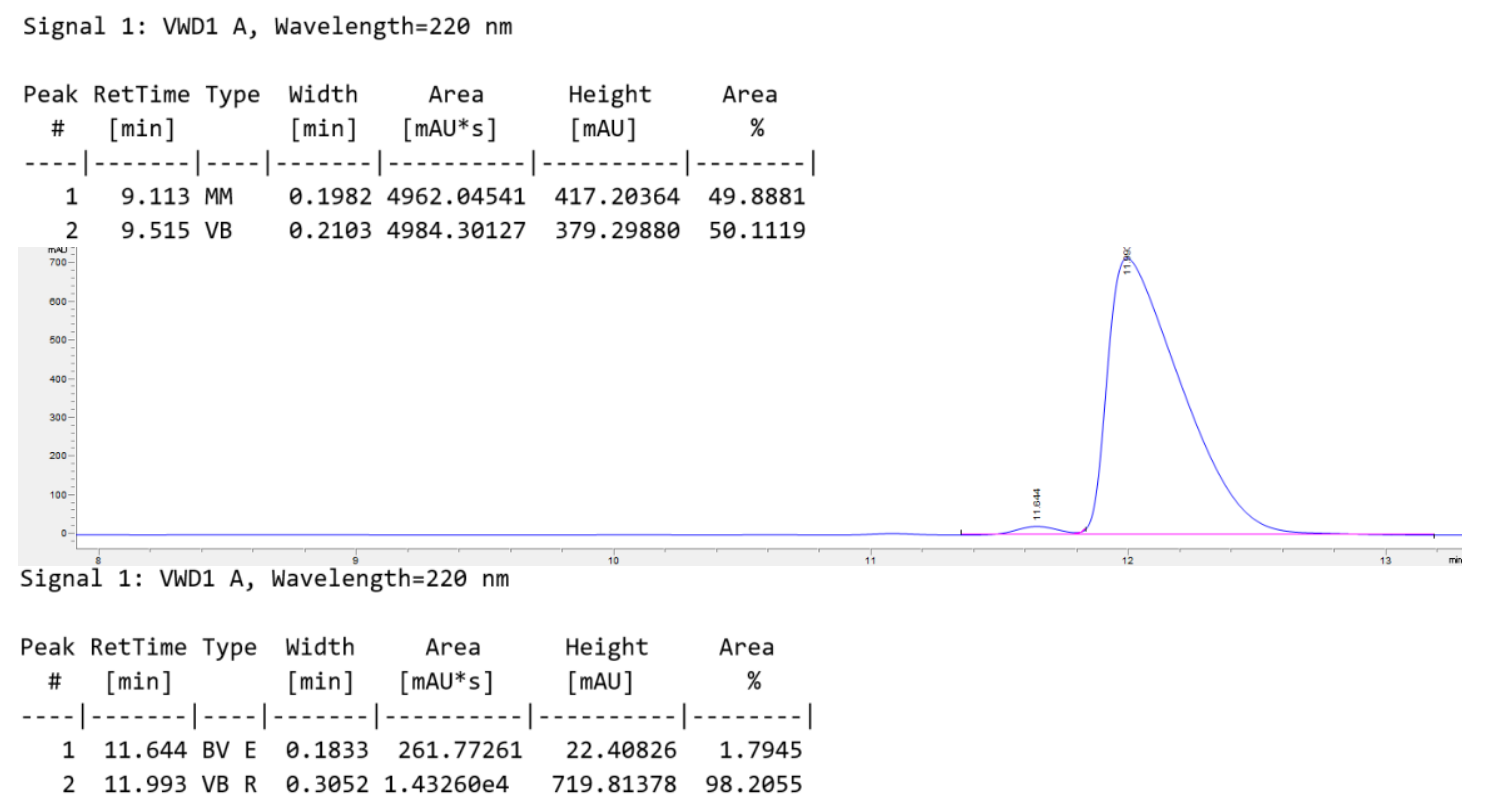

(R)-1-(o-Tolyl)-2-((triisopropylsilyl)oxy)ethan-1-ol (3ae)<smiles>Cc1ccccc1[C@@H](O)CO[Na]</smiles>

Compound 3ae was prepared as a colorless oil in $86 \%$ yield $(80.0 \mathrm{mg}$, eluent: petroleum ether/EtOAc $=100 / 3$ to 20/1), from (Z)-triisopropyl((2methylstyryl)oxy)silane 1ae (87.0 mg, $0.30 \mathrm{mmol}),[\mathrm{RhCl}(\mathrm{cod})]_{2}(3.0 \mathrm{mg}, 0.006$ mmol), (R,R)-3,5-iPr2-DIOP (10.0 mg, $0.012 \mathrm{mmol})$, HBpin (57.6 mg, $0.45 \mathrm{mmol}$ ) and $\mathrm{AgOAc}(15.0 \mathrm{mg}, 0.09 \mathrm{mmol})$ following the above general procedure $\mathrm{G}$.

$\mathbf{R}_{f}=0.40$ (petroleum ether/EtOAc $=10 / 1$ )

${ }^{1} \mathrm{H}$ NMR (400 MHz, $\left.\mathrm{CDCl}_{3}\right) \delta$ 7.59-7.50 (m, 1H), 7.27-7.07 (m, 3H), 5.01 (dd, $J=$ 9.9, $2.6 \mathrm{~Hz}, 1 \mathrm{H}), 3.83(\mathrm{dd}, J=10.0,3.3 \mathrm{~Hz}, 1 \mathrm{H}), 3.61-3.53(\mathrm{~m}, 1 \mathrm{H}), 3.10(\mathrm{~s}, 1 \mathrm{H})$, $2.34(\mathrm{~s}, 3 \mathrm{H}), 1.23-0.94(\mathrm{~m}, 21 \mathrm{H})$.

${ }^{13} \mathrm{C}$ NMR (100 MHz, $\left.\mathrm{CDCl}_{3}\right) \delta$ 138.2, 134.9, 130.3, 127.6, 126.3, 126.2, 71.4, 68.1, $19.2,18.1,12.1$.

HRMS (ESI') calcd for $\mathrm{C}_{18} \mathrm{H}_{32} \mathrm{NaO}_{2} \mathrm{Si}^{+}[\mathrm{M}+\mathrm{Na}]^{+}$: 331.2064, found: 331.2063.

IR (neat, cm-1): 2941, 2866, 1463, 1102, 1059, 995, 883, 804, 756, 724, 684.

$[\alpha]_{\mathrm{D}^{24.3}}=-38.98\left(\mathrm{c}=0.57, \mathrm{CHCl}_{3}, 92 \%\right.$ ee $)$; Enantiomeric excess was determined by chiral HPLC (Daicel Chiralpak IA, hexane/isopropyl alcohol =99/1, flow rate $=1.0 \mathrm{~mL} / \mathrm{min}, \lambda=220 \mathrm{~nm}$ ), $t^{1}=8.0 \mathrm{~min}$ (minor),$t^{2}=8.5 \mathrm{~min}$ (major). 


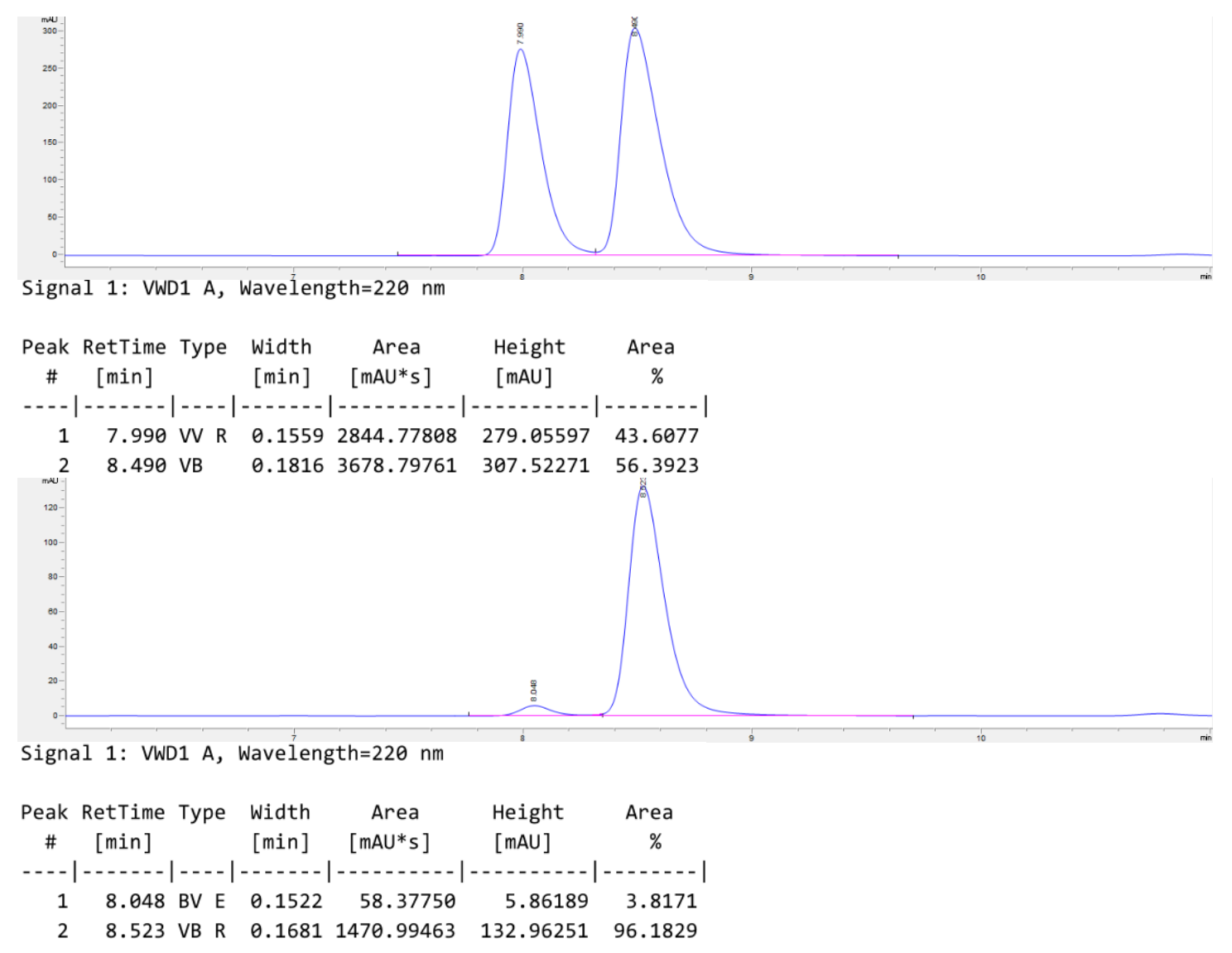

\section{(R)-1-(4-Methoxy-3,5-dimethylphenyl)-2-((triisopropylsilyl)oxy)ethan-1-ol} (3af)

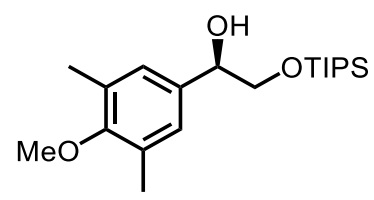

Compound 3af was prepared as a colorless oil in $62 \%$ yield $(65.2 \mathrm{mg}$, eluent: petroleum ether/EtOAc = 100/3 to 10/1), from (Z)-triisopropyl((4-methoxy-3,5dimethylstyryl)oxy)silane 1af $(100.2 \mathrm{mg}, 0.30 \mathrm{mmol})$, [RhCl(cod)]2 $(3.0 \mathrm{mg}$, $0.006 \mathrm{mmol}),(R, R)-3,5-\mathrm{iPr}$-DIOP (10.0 mg, $0.012 \mathrm{mmol})$, HBpin $(57.6 \mathrm{mg}, 0.45$ $\mathrm{mmol})$ and $\mathrm{LiOAc}(6.0 \mathrm{mg}, 0.09 \mathrm{mmol})$ following the above general procedure $\mathrm{F}$.

$\mathbf{R}_{f}=0.28$ (petroleum ether $/$ EtOAc $=10 / 1$ )

${ }^{1} \mathrm{H}$ NMR (400 MHz, CDCl $)$ ) 7.01 (s, 2H), $4.66(\mathrm{dd}, J=9.1,3.3 \mathrm{~Hz}, 1 \mathrm{H}), 3.80$ (dd, $J=9.9,3.5 \mathrm{~Hz}, 1 \mathrm{H}), 3.69(\mathrm{~s}, 3 \mathrm{H}), 3.64-3.56(\mathrm{~m}, 1 \mathrm{H}), 3.08(\mathrm{~s}, 1 \mathrm{H}), 2.28(\mathrm{~s}, 6 \mathrm{H}), 1.16-$ $1.00(\mathrm{~m}, 21 \mathrm{H})$.

${ }^{13} \mathrm{C}$ NMR (100 MHz, $\mathrm{CDCl}_{3}$ ) \& 156.7, 135.4, 130.8, 126.8, 74.4, 69.4, 59.7, 18.1, 16.2, 12.1. 
HRMS (ESI') calcd for $\mathrm{C}_{20} \mathrm{H}_{36} \mathrm{NaO}_{3} \mathrm{Si}^{+}$[M+Na] $]^{+}: 375.2326$, found: 375.2325.

IR (neat, cm-1): 2940, 2865, 1463, 1222, 1139, 1111, 1060, 1016, 882, 813, 683, 659. $[\alpha]_{\mathrm{D}^{24.3}}=-18.93\left(\mathrm{c}=0.47, \mathrm{CHCl}_{3}, 95 \%\right.$ ee); Enantiomeric excess was determined by chiral HPLC (Daicel Chiralpak AD-H, hexane/isopropyl alcohol = 98.5/1.5, flow rate $=0.6 \mathrm{~mL} / \mathrm{min}, \lambda=220 \mathrm{~nm}$ ), $t^{1}=8.1 \mathrm{~min}$ (major),$t^{2}=8.5 \mathrm{~min}$ (minor).

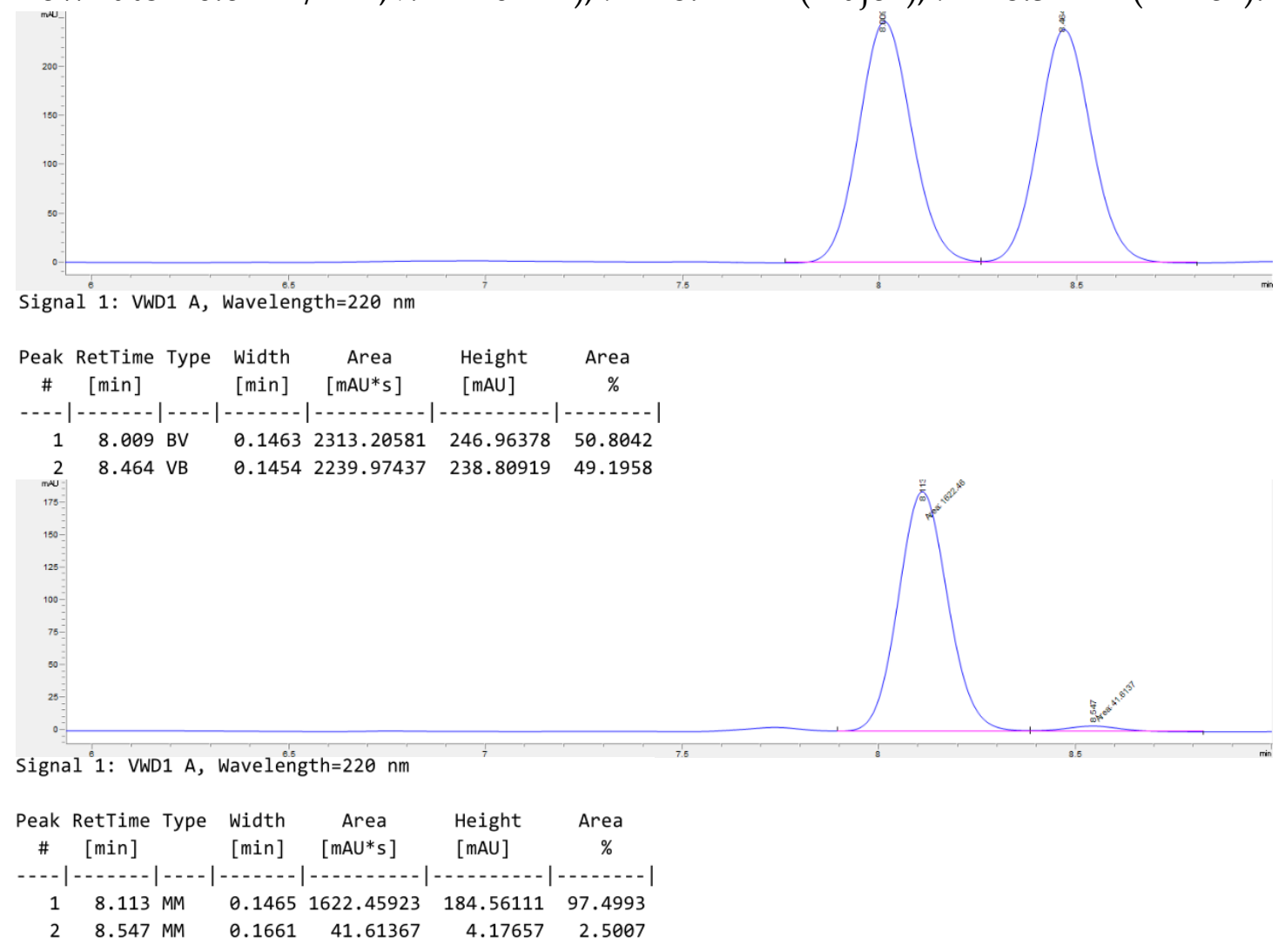

(R)-1-(4-(Dimethylamino)phenyl)-2-((triisopropylsilyl)oxy)ethan-1-ol (3ag)<smiles>O=[N+]([O-])c1ccc(C(O)COCCOCCO)cc1</smiles>

Compound 3ag was prepared as a colorless oil in $56 \%$ yield $(57.0 \mathrm{mg}$, eluent: petroleum ether/EtOAc $=100 / 3$ to $10 / 1)$, from (Z)-N,N-dimethyl-4-(2((triisopropylsilyl)oxy)vinyl)aniline 1ag $(95.8 \mathrm{mg}, 0.30 \mathrm{mmol})$, [ RhCl(cod) $]_{2}(3.0$ $\mathrm{mg}$, $0.006 \mathrm{mmol}),(R, R)-3,5-i \mathrm{Pr}_{2}-\mathrm{DIOP}(10.0 \mathrm{mg}, 0.012 \mathrm{mmol}), \mathrm{HBpin}(57.6 \mathrm{mg}$, $0.45 \mathrm{mmol})$ and LiOAc $(6.0 \mathrm{mg}, 0.09 \mathrm{mmol})$ following the above general procedure $\mathrm{F}(18 \mathrm{~h})$.

$\mathbf{R}_{f}=0.22$ (petroleum ether $/$ EtOAc $\left.=10 / 1\right)$ 
${ }^{1} \mathrm{H}$ NMR (400 MHz, $\left.\mathrm{CDCl}_{3}\right)$ \& $7.25(\mathrm{~d}, J=8.4 \mathrm{~Hz}, 2 \mathrm{H}), 6.72(\mathrm{~d}, J=8.3 \mathrm{~Hz}, 2 \mathrm{H})$, $4.69(\mathrm{dd}, J=9.3,3.4 \mathrm{~Hz}, 1 \mathrm{H}), 3.78(\mathrm{dd}, J=10.0,3.5 \mathrm{~Hz}, 1 \mathrm{H}), 3.65-3.57(\mathrm{~m}, 1 \mathrm{H})$, $3.04(\mathrm{~s}, 1 \mathrm{H}), 2.93(\mathrm{~s}, 6 \mathrm{H}), 1.20-0.96(\mathrm{~m}, 21 \mathrm{H})$.

${ }^{13} \mathrm{C}$ NMR (100 MHz, $\left.\mathrm{CDCl}_{3}\right) \delta$ 150.5, 128.1, 127.4, 112.7, 74.5, 69.5, 40.8, 18.1, 12.1. HRMS (ESI') calcd for $\mathrm{C}_{19} \mathrm{H}_{3} \mathrm{NNaO}_{2} \mathrm{Si}^{+}[\mathrm{M}+\mathrm{Na}]^{+}: 360.2329$, found: 360.2328 .

IR (neat, cm-1): 2940, 2866, 1616, 1524, 1463, 1348, 1105, 1059, 883, 813, 684.

$[\alpha]_{\mathrm{D}^{24.3}}=-22.19\left(\mathrm{c}=0.46, \mathrm{CHCl}_{3}, 88 \%\right.$ ee); Enantiomeric excess was determined by chiral HPLC (Daicel Chiralcel OD-H, hexane/isopropyl alcohol = 98/2, flow rate $=0.8 \mathrm{~mL} / \mathrm{min}, \lambda=254 \mathrm{~nm}$ ), $t^{1}=8.4 \mathrm{~min}$ (minor),$t^{2}=9.0 \mathrm{~min}$ (major).

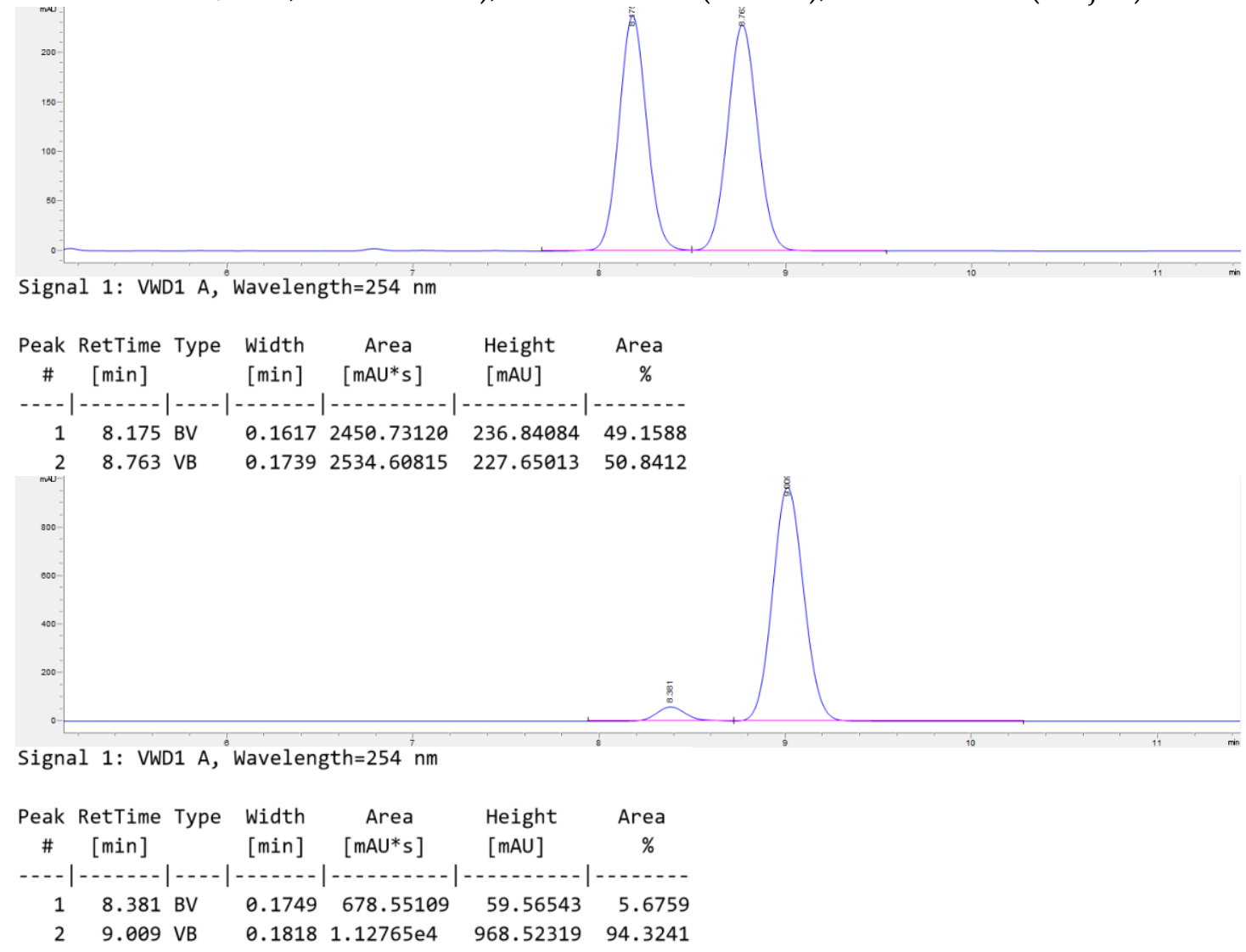

(R)-1-(4-Methoxyphenyl)-2-((triisopropylsilyl)oxy)ethan-1-ol (3ah)<smiles>COc1ccc(C(O)CO)cc1</smiles>

Compound 3ah was prepared as a colorless oil in 78\% yield $(76.0 \mathrm{mg}$, eluent: petroleum ether/EtOAc $=100 / 3$ to 10/1), from (Z)-triisopropyl((4methoxystyryl)oxy)silane 1ah ( $91.9 \mathrm{mg}, 0.30 \mathrm{mmol})$, [RhCl(cod)]2 (3.0 mg, 0.006 $\mathrm{mmol}),(R, R)-3,5-i \mathrm{Pr}_{2}$-DIOP (10.0 mg, $\left.0.012 \mathrm{mmol}\right), \mathrm{HBpin}(57.6 \mathrm{mg}, 0.45 \mathrm{mmol})$ 
and $\mathrm{LiOAc}(6.0 \mathrm{mg}, 0.09 \mathrm{mmol})$ following the above general procedure $\mathrm{F}$.

$\mathbf{R}_{f}=0.28$ (petroleum ether $/$ EtOAc $=10 / 1$ )

${ }^{1} \mathrm{H}$ NMR (400 MHz, $\left.\mathrm{CDCl}_{3}\right) \delta 7.30(\mathrm{~d}, J=8.2 \mathrm{~Hz}, 2 \mathrm{H}), 6.88(\mathrm{~d}, J=8.2 \mathrm{~Hz}, 2 \mathrm{H})$, $4.72(\mathrm{dd}, J=9.1,3.3 \mathrm{~Hz}, 1 \mathrm{H}), 3.87-3.74(\mathrm{~m}, 4 \mathrm{H}), 3.64-3.56(\mathrm{~m}, 1 \mathrm{H}), 3.09(\mathrm{~s}, 1 \mathrm{H})$, $1.19-1.02(\mathrm{~m}, 21 \mathrm{H})$.

${ }^{13} \mathrm{C}$ NMR (100 MHz, $\left.\mathrm{CDCl}_{3}\right) \delta$ 159.4, 132.5, 127.6, 113.9, 74.2, 69.4, 55.4, 18.1, 12.0. HRMS (ESI') calcd for $\mathrm{C}_{18} \mathrm{H}_{32} \mathrm{NaO}_{3} \mathrm{Si}^{+}[\mathrm{M}+\mathrm{Na}]^{+}$: 347.2013, found: 347.2012.

IR (neat, cm-1): 2941, 2866, 1514, 1463, 1247, 1104, 1060, 1039, 882, 828, 683.

$[\alpha]_{\mathrm{D}^{24.3}}=-22.78\left(\mathrm{c}=0.63, \mathrm{CHCl}_{3}, 96 \%\right.$ ee $)$; Enantiomeric excess was determined by chiral HPLC (Daicel Chiralcel OD-H, hexane/isopropyl alcohol =95/5, flow rate $=1.0 \mathrm{~mL} / \mathrm{min}, \lambda=220 \mathrm{~nm}$ ),$t^{1}=4.7 \mathrm{~min}$ (minor),$t^{2}=5.1 \mathrm{~min}$ (major).

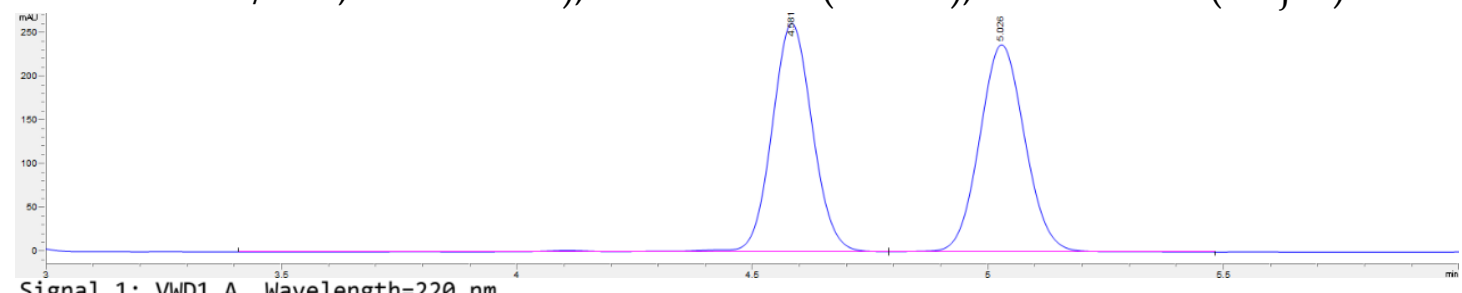

Signal 1: VWD1 A, Wavelength $=220 \mathrm{~nm}$

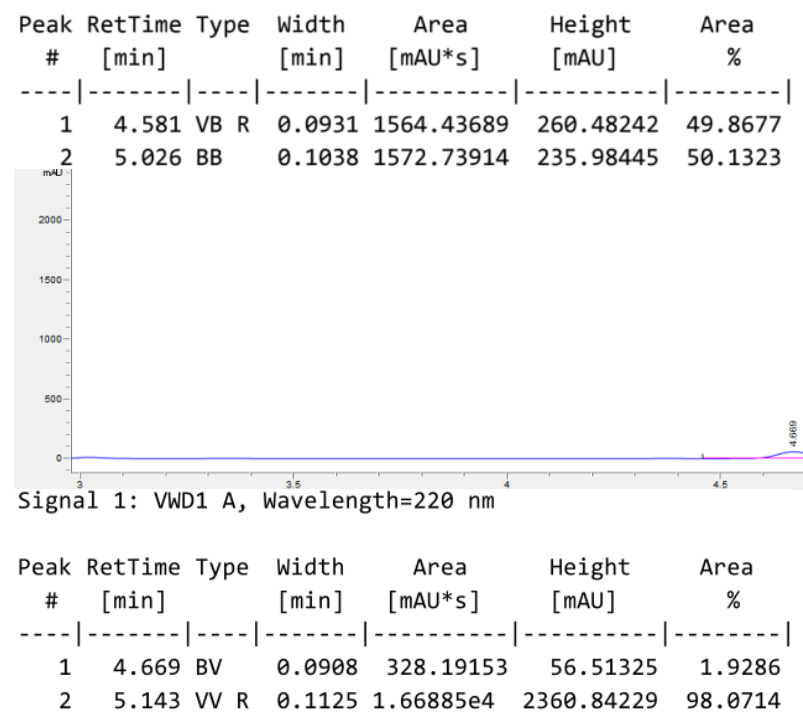

(R)-1-(4-(Benzyloxy)phenyl)-2-((triisopropylsilyl)oxy)ethan-1-ol (3ai)<smiles>O=C(Oc1ccc([C@H](O)CO[18OH])cc1)c1ccccc1</smiles>

Compound 3ai was prepared as a colorless oil in 91\% yield (109.2 mg, eluent: petroleum ether/EtOAc $=100 / 3$ to 20/1), from (Z)-((4(benzyloxy)styryl)oxy)triisopropylsilane $\mathbf{1 a i}(114.7 \mathrm{mg}, \quad 0.30 \mathrm{mmol})$, $[\mathrm{RhCl}(\mathrm{cod})]_{2}(3.0 \mathrm{mg}, 0.006 \mathrm{mmol}),(R, R)-3,5-i \operatorname{Pr}_{2}-\mathrm{DIOP}(10.0 \mathrm{mg}, 0.012 \mathrm{mmol})$, 
HBpin (57.6 mg, $0.45 \mathrm{mmol})$ and AgOAc $(15.0 \mathrm{mg}, 0.09 \mathrm{mmol})$ following the above general procedure $\mathrm{G}(18 \mathrm{~h})$.

$\mathbf{R}_{f}=0.35$ (petroleum ether $/$ EtOAc $=10 / 1$ )

${ }^{1} \mathrm{H}$ NMR (400 MHz, $\left.\mathrm{CDCl}_{3}\right) \delta$ 8.45-7.39 (m, 2H), 7.39-7.33 (m, 2H), 7.33-7.25 (m, $3 \mathrm{H}), 7.01-6.89(\mathrm{~m}, 2 \mathrm{H}), 5.04(\mathrm{~s}, 2 \mathrm{H}), 4.72(\mathrm{dd}, J=9.1,3.4 \mathrm{~Hz}, 1 \mathrm{H}), 3.80(\mathrm{dd}, J=9.9$, $3.6 \mathrm{~Hz}, 1 \mathrm{H}), 3.64-3.56(\mathrm{~m}, 1 \mathrm{H}), 3.08(\mathrm{~s}, 1 \mathrm{H}), 1.18-0.99(\mathrm{~m}, 21 \mathrm{H})$.

${ }^{13} \mathrm{C}$ NMR (100 MHz, $\left.\mathrm{CDCl}_{3}\right) \delta$ 158.6, 137.1, 132.8, 128.7, 128.0, 127.6, 127.5, 114.8, 74.2, 70.1, 69.4, 18.1, 12.0.

HRMS (ESI') calcd for $\mathrm{C}_{24} \mathrm{H}_{36} \mathrm{NaO}_{3} \mathrm{Si}^{+}[\mathrm{M}+\mathrm{Na}]^{+}$: 423.2326, found: 423.2325 .

IR (neat, cm ${ }^{-1}$ ): 2941, 2866, 1512, 1461, 1241, 1172, 1105, 1060, 1017, 882, 827, 736, 688.

$[\alpha]_{\mathrm{D}^{24.3}}=-17.34\left(\mathrm{c}=0.60, \mathrm{CHCl}_{3}, 96 \%\right.$ ee $)$; Enantiomeric excess was determined by chiral HPLC (Daicel Chiralcel OD-H, hexane/isopropyl alcohol =95/5, flow rate $=1.0 \mathrm{~mL} / \mathrm{min}, \lambda=220 \mathrm{~nm}$ ),$t^{1}=6.5 \mathrm{~min}$ (minor),$t^{2}=7.2 \mathrm{~min}$ (major).

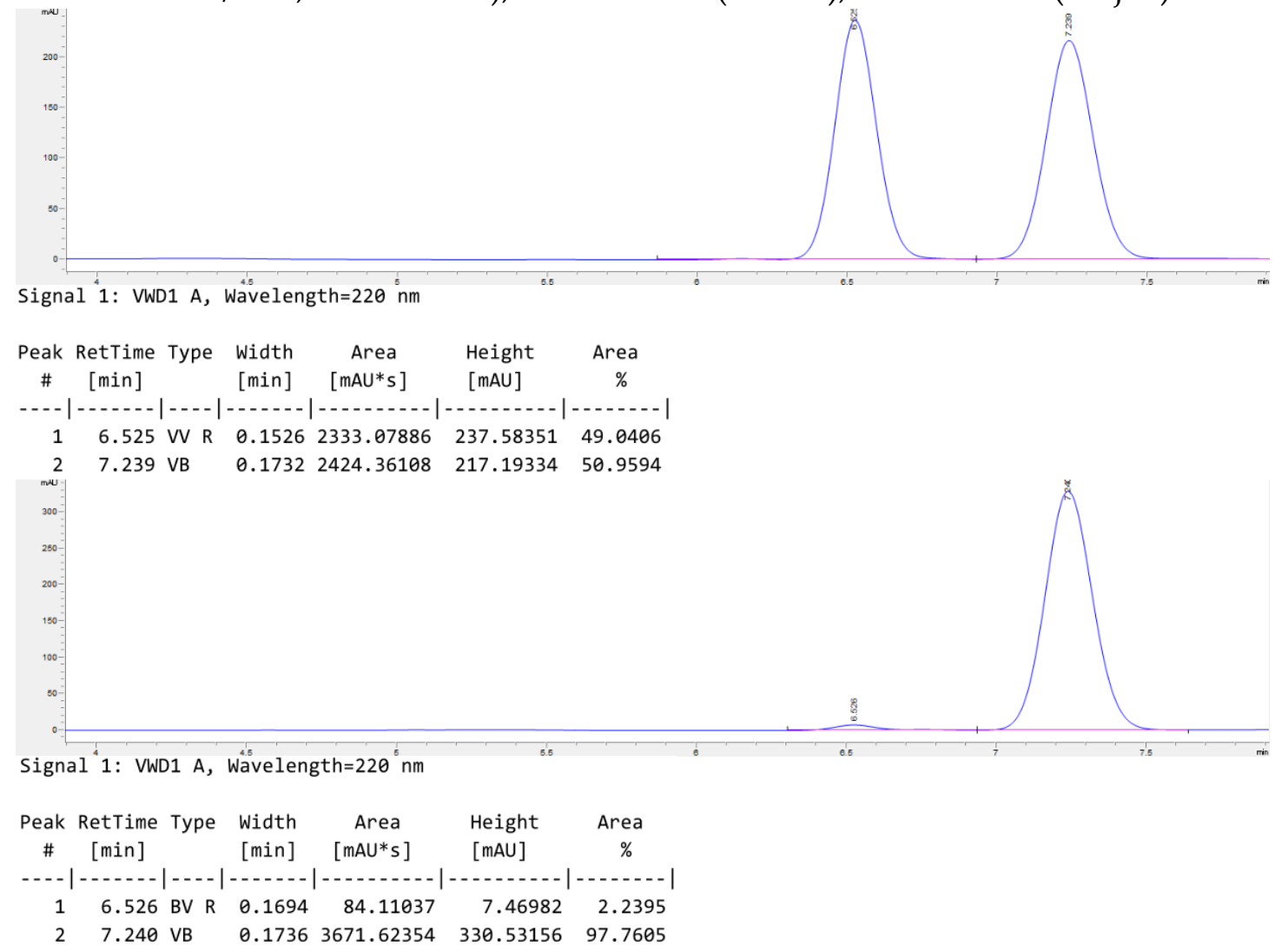

(R)-1-(3-(1,3-Dioxolan-2-yl)phenyl)-2-((triisopropylsilyl)oxy)ethan-1-ol (3aj)<smiles>OC(C[Po+])c1cccc(C2OCCO2)c1</smiles> 
Compound 3aj was prepared as a colorless oil in 79\% yield (86.7 mg, eluent: petroleum ether/EtOAc $=100 / 3$ to $10 / 1)$, from (Z)-((3-(1,3-dioxolan-2yl)styryl)oxy)triisopropylsilane 1aj (104.4 mg, $0.30 \mathrm{mmol}$ ), [ RhCl(cod)]2 (3.0 mg, $0.006 \mathrm{mmol}),(R, R)-3,5-\mathrm{PPr}$-DIOP (10.0 mg, $0.012 \mathrm{mmol})$, HBpin (57.6 mg, 0.45 $\mathrm{mmol})$ and $\mathrm{AgOAc}(15.0 \mathrm{mg}, 0.09 \mathrm{mmol})$ following the above general procedure $\mathrm{G}(18 \mathrm{~h})$.

$\mathbf{R}_{f}=0.33$ (petroleum ether $/$ EtOAc $=10 / 1$ )

${ }^{1} \mathrm{H}$ NMR (400 MHz, $\mathrm{CDCl}_{3}$ ) $\delta 7.49$ (s, 1H), 7.45-7.32 (m, 3H), 5.82 (s, 1H), 4.80 $(\mathrm{dd}, J=8.8,3.5 \mathrm{~Hz}, 1 \mathrm{H}), 4.19-4.08(\mathrm{~m}, 2 \mathrm{H}), 4.08-3.99(\mathrm{~m}, 2 \mathrm{H}), 3.85(\mathrm{dd}, J=9.9$, $3.6 \mathrm{~Hz}, 1 \mathrm{H}), 3.68-3.56(\mathrm{~m}, 1 \mathrm{H}), 3.10(\mathrm{~s}, 1 \mathrm{H}), 1.18-0.96(\mathrm{~m}, 21 \mathrm{H})$.

${ }^{13} \mathrm{C}$ NMR (100 MHz, $\left.\mathrm{CDCl}_{3}\right) \delta$ 140.6, 138.1, 128.5, 127.2, 126.0, 124.5, 103.8, 74.5, $69.3,65.5,18.1,12.1$.

HRMS (ESI') calcd for $\mathrm{C}_{20} \mathrm{H}_{34} \mathrm{NaO}_{4} \mathrm{Si}^{+}[\mathrm{M}+\mathrm{Na}]^{+}: 389.2119$, found: 389.2119 .

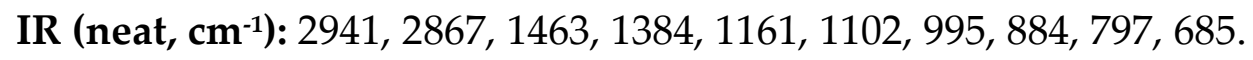

$[\alpha]_{\mathrm{D}^{24.3}}=+5.61\left(\mathrm{c}=0.50, \mathrm{CHCl}_{3}, 94 \%\right.$ ee $)$; Enantiomeric excess was determined by chiral HPLC (Daicel Chiralcel OD-H, hexane/isopropyl alcohol $=95 / 5$, flow rate $=1.0 \mathrm{~mL} / \mathrm{min}, \lambda=220 \mathrm{~nm}$ ), $t^{1}=10.7 \mathrm{~min}$ (minor),$t^{2}=11.9 \mathrm{~min}$ (major).

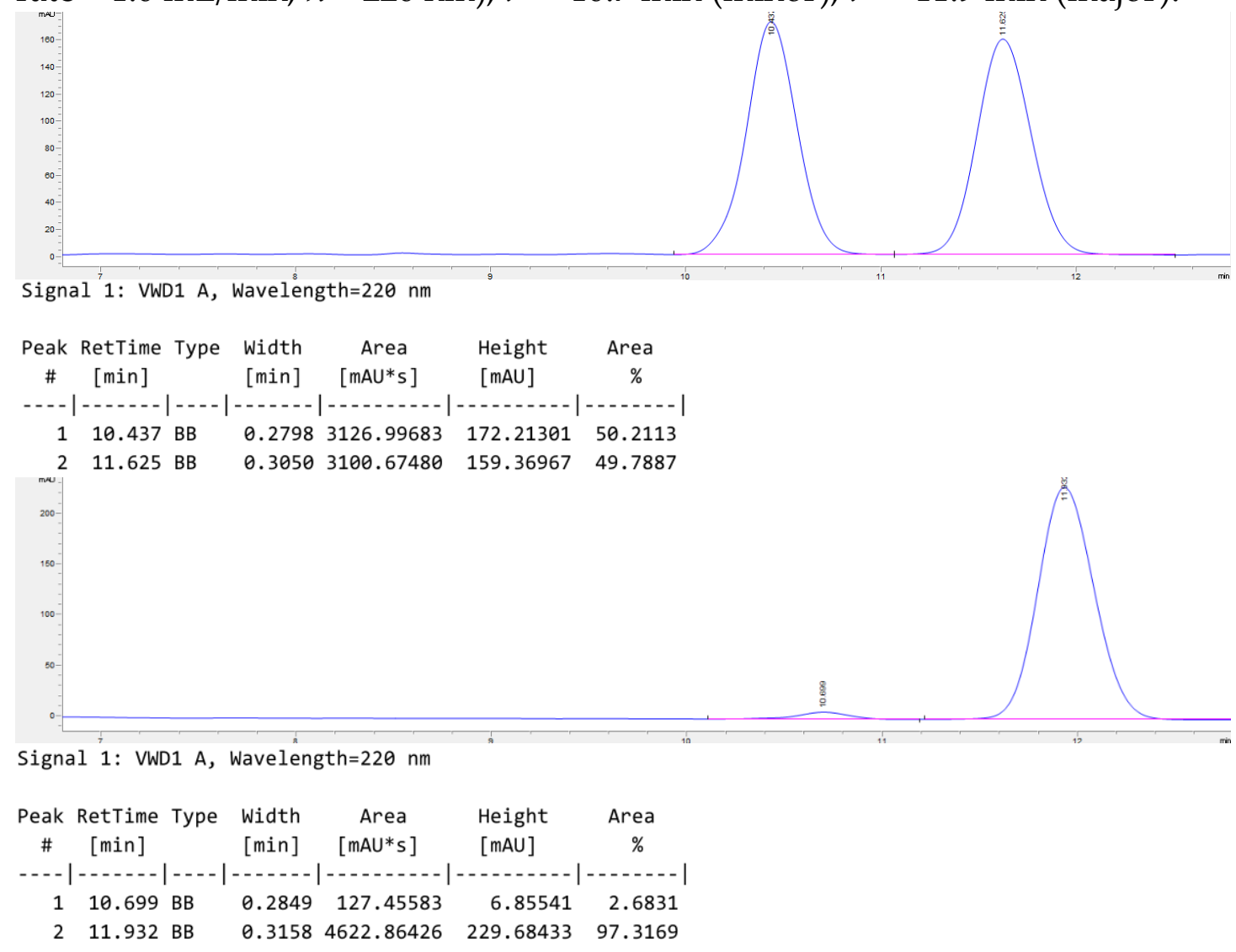




\section{(R)-1-(3'-Methoxy-[1,1'-biphenyl]-4-yl)-2-((triisopropylsilyl)oxy)ethan-1-ol}

(3ak)<smiles>COc1cccc(-c2ccc([C@@H](O)CO[Na])cc2)c1</smiles>

Compound 3ak was prepared as a colorless oil in 91\% yield (109.0 mg, eluent: petroleum ether/EtOAc $=100 / 3$ to 10/1), from $(Z)$-triisopropyl((2-(3'-methoxy[1,1'-biphenyl]-4-yl)vinyl)oxy)silane 1ak (114.6 mg, $0.30 \mathrm{mmol}),[\mathrm{RhCl}(\mathrm{cod})]_{2}$ (3.0 mg, $0.006 \mathrm{mmol}),(R, R)-3,5-i \mathrm{Pr}_{2}$-DIOP (10.0 mg, $\left.0.012 \mathrm{mmol}\right)$, HBpin (57.6 $\mathrm{mg}, 0.45 \mathrm{mmol})$ and AgOAc (15.0 mg, $0.09 \mathrm{mmol})$ following the above general procedure G $(18 \mathrm{~h})$.

$\mathbf{R}_{f}=0.28$ (petroleum ether $/$ EtOAc $=10 / 1$ )

${ }^{1}$ H NMR (400 MHz, $\left.\mathrm{CDCl}_{3}\right) \delta 7.57(\mathrm{~d}, J=8.2 \mathrm{~Hz}, 2 \mathrm{H}), 7.44(\mathrm{~d}, J=8.2 \mathrm{~Hz}, 2 \mathrm{H})$, 7.39-7.29 (m, 1H), 7.20-7.14 (m, 1H), 7.14-7.06 (m, 1H), 6.95-6.82 (m, 1H), 4.82 $(\mathrm{dd}, J=9.0,3.4 \mathrm{~Hz}, 1 \mathrm{H}), 3.96-3.77(\mathrm{~m}, 4 \mathrm{H}), 3.70-3.62(\mathrm{~m}, 1 \mathrm{H}), 3.16(\mathrm{~s}, 1 \mathrm{H}), 1.20$ $1.02(\mathrm{~m}, 21 \mathrm{H})$.

${ }^{13} \mathrm{C}$ NMR (100 MHz, $\left.\mathrm{CDCl}_{3}\right) \delta$ 160.0, 142.6, 140.7, 139.6, 129.8, 127.2, 126.7, 119.7, 113.0, 112.7, 74.4, 69.3, 55.4, 18.1, 12.1 .

HRMS (ESI ${ }^{+}$) calcd for $\mathrm{C}_{24} \mathrm{H}_{36} \mathrm{NaO}_{3} \mathrm{Si}^{+}[\mathrm{M}+\mathrm{Na}]^{+}:$423.2326, found: 423.2325 .

IR (neat, cm$^{-1}$ ): 2941, 2866, 1603, 1464, 1216, 1105, 1057, 882, 851, 780, 688.

$[\alpha]_{\mathrm{D}^{24.3}}=-11.43\left(\mathrm{c}=0.66, \mathrm{CHCl}_{3}, 96 \%\right.$ ee $)$; Enantiomeric excess was determined by chiral HPLC (Daicel Chiralcel OD-H, hexane/isopropyl alcohol = 95/5, flow rate $=1.0 \mathrm{~mL} / \mathrm{min}, \lambda=220 \mathrm{~nm}$ ), $t^{1}=6.6 \mathrm{~min}$ (minor),$t^{2}=7.1 \mathrm{~min}$ (major).

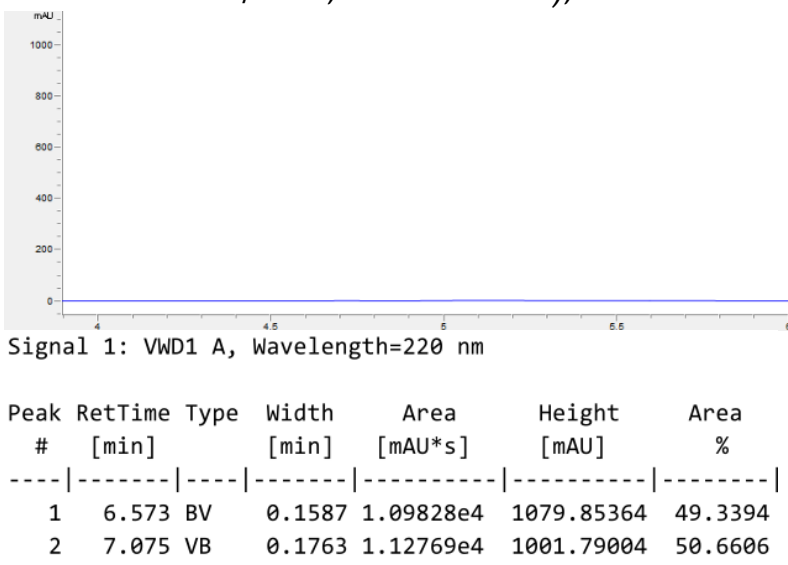




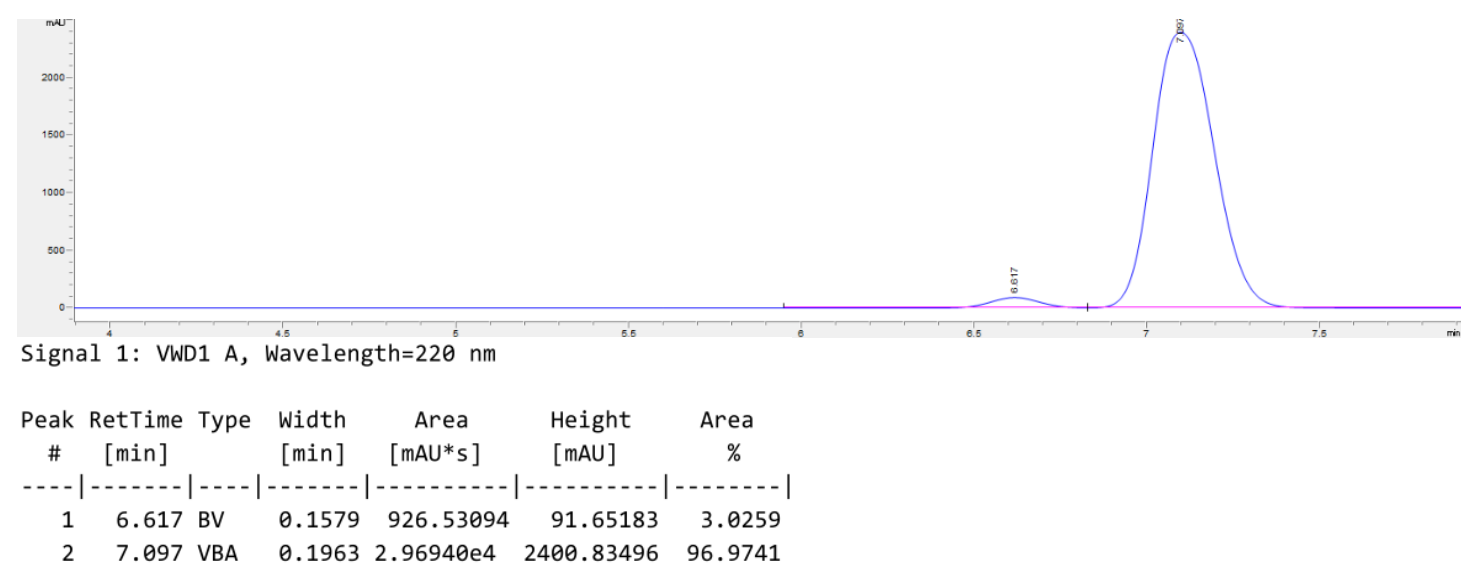

(R)-1-(4-Fluorophenyl)-2-((triisopropylsilyl)oxy)ethan-1-ol (3al)<smiles>OCC(O)c1ccc(F)cc1</smiles>

Compound 3al was prepared as a colorless oil in $89 \%$ yield $(83.0 \mathrm{mg}$, eluent: petroleum ether/EtOAc $=100 / 3$ to 20/1), from (Z)-((4fluorostyryl)oxy)triisopropylsilane $1 \mathrm{al}(88.3 \mathrm{mg}, 0.30 \mathrm{mmol})$, [ $\mathrm{RhCl}(\operatorname{cod})]_{2}(3.0$ $\mathrm{mg}, 0.006 \mathrm{mmol}),(R, R)-3,5-i \mathrm{Pr}_{2}$-DIOP (10.0 mg, $\left.0.012 \mathrm{mmol}\right)$, HBpin (57.6 mg, $0.45 \mathrm{mmol})$ and LiOAc $(6.0 \mathrm{mg}, 0.09 \mathrm{mmol})$ following the above general procedure $\mathrm{F}$.

$\mathbf{R}_{f}=0.42$ (petroleum ether $/$ EtOAc $=10 / 1$ )

${ }^{1} \mathrm{H}$ NMR (400 MHz, CDCl $)$ ) 7.42-7.28 (m, 2H), 7.08-6.98 (m, 2H), 4.84-4.69 (m, $1 \mathrm{H}), 3.90-3.76(\mathrm{~m}, 1 \mathrm{H}), 3.63-3.55(\mathrm{~m}, 1 \mathrm{H}), 3.12(\mathrm{~s}, 1 \mathrm{H}), 1.25-0.95(\mathrm{~m}, 21 \mathrm{H})$.

${ }^{13} \mathrm{C}$ NMR (100 MHz, $\left.\mathrm{CDCl}_{3}\right) \delta 162.5(\mathrm{~d}, J=245.4 \mathrm{~Hz}), 136.2(\mathrm{~d}, J=3.1 \mathrm{~Hz}), 128.0$ $(\mathrm{d}, J=8.1 \mathrm{~Hz}), 115.2(\mathrm{~d}, J=21.4 \mathrm{~Hz}), 74.0,69.3,18.0,12.0$.

${ }^{19} \mathrm{~F}$ NMR (377 MHz, $\left.\mathrm{CDCl}_{3}\right) \delta-114.9(\mathrm{~m})$.

HRMS (ESI ${ }^{+}$) calcd for $\mathrm{C}_{17} \mathrm{H}_{2} \mathrm{FNaO}_{2} \mathrm{Si}^{+}[\mathrm{M}+\mathrm{Na}]^{+}: 335.1813$, found: 335.1812 .

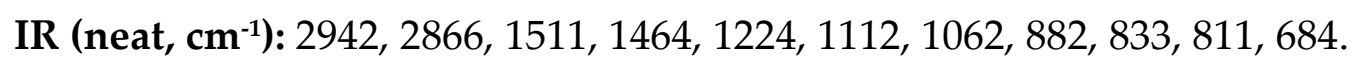

$[\alpha]_{\mathrm{D}^{24.3}}=-20.25\left(\mathrm{c}=0.66, \mathrm{CHCl}_{3}, 93 \%\right.$ ee $)$; Enantiomeric excess was determined by chiral HPLC (Daicel Chiralpak IA, hexane/isopropyl alcohol = 99/1, flow rate $=1.0 \mathrm{~mL} / \mathrm{min}, \lambda=220 \mathrm{~nm}$ ), $t^{1}=13.5 \mathrm{~min}$ (minor),$t^{2}=14.2 \mathrm{~min}$ (major). 


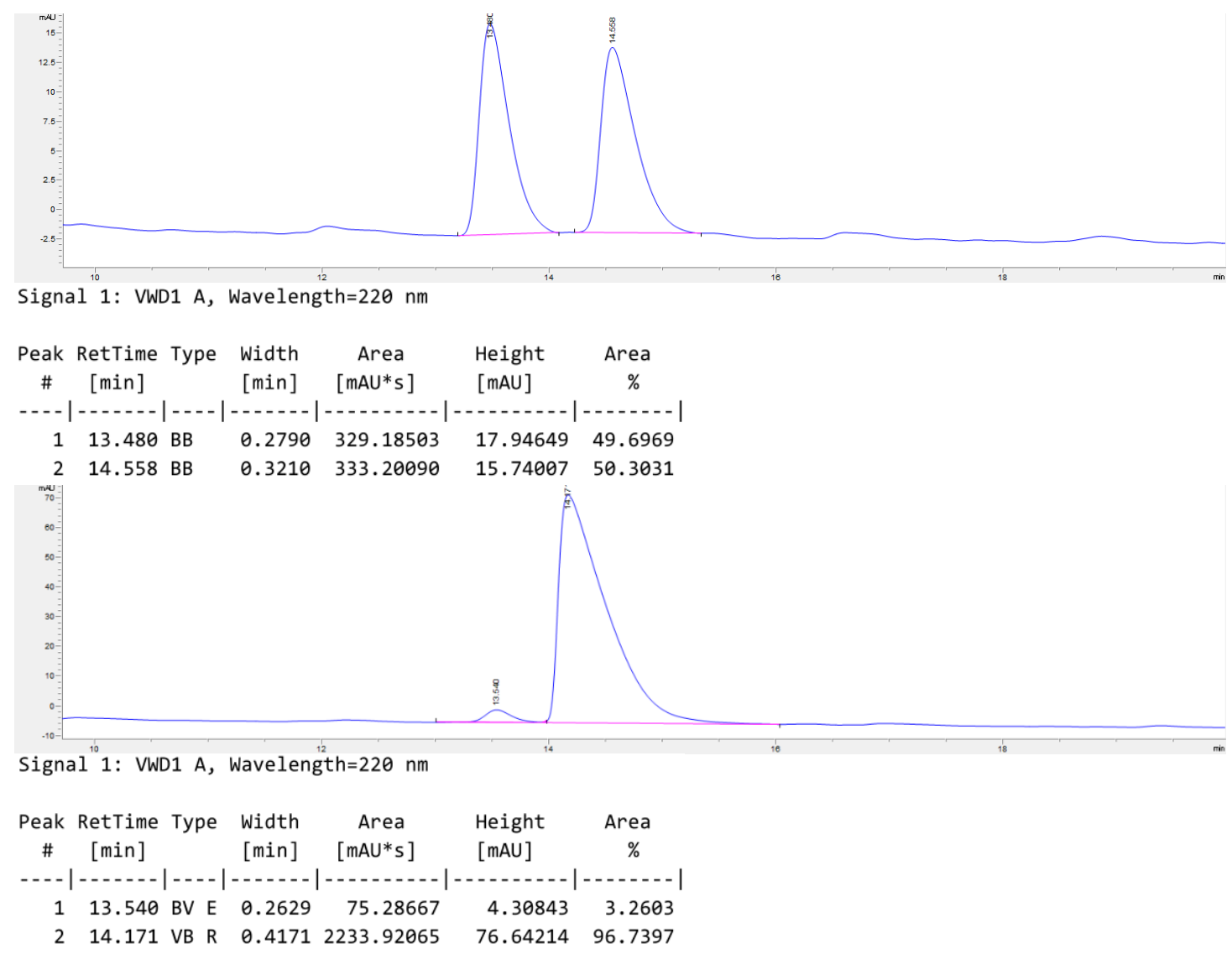

(R)-1-(4-Chlorophenyl)-2-((triisopropylsilyl)oxy)ethan-1-ol (3am)<smiles>OCC(O)c1ccc(Cl)cc1</smiles>

Compound 3am was prepared as a colorless oil in $88 \%$ yield $(87.0 \mathrm{mg}$, eluent: petroleum ether/EtOAc $=100 / 3$ to 20/1), from (Z)- $((4-$ chlorostyryl)oxy)triisopropylsilane 1am $(93.0 \mathrm{mg}, 0.30 \mathrm{mmol}),[\mathrm{RhCl}(\operatorname{cod})]_{2}(3.0$ $\mathrm{mg}, 0.006 \mathrm{mmol}),(R, R)-3,5-\mathrm{P} \mathrm{Pr}_{2}$-DIOP $(10.0 \mathrm{mg}, 0.012 \mathrm{mmol})$, HBpin $(57.6 \mathrm{mg}$, $0.45 \mathrm{mmol})$ and LiOAc $(6.0 \mathrm{mg}, 0.09 \mathrm{mmol})$ following the above general procedure $\mathrm{F}$.

$\mathbf{R}_{f}=0.42$ (petroleum ether $/$ EtOAc $\left.=10 / 1\right)$

${ }^{1} \mathrm{H}$ NMR (400 MHz, $\left.\mathrm{CDCl}_{3}\right) \delta$ 7.41-7.27 (m, 4H), $4.74(\mathrm{dd}, J=8.6,1.6 \mathrm{~Hz}, 1 \mathrm{H})$, $3.82(\mathrm{dd}, J=9.9,3.6 \mathrm{~Hz}, 1 \mathrm{H}), 3.70-3.50(\mathrm{~m}, 1 \mathrm{H}), 3.12(\mathrm{~s}, 1 \mathrm{H}), 1.21-0.99(\mathrm{~m}, 21 \mathrm{H})$. ${ }^{13} \mathrm{C}$ NMR (100 MHz, $\left.\mathrm{CDCl}_{3}\right) \delta$ d 139.0, 133.5, 128.6, 127.7, 73.9, 69.2, 18.1, 12.0.

HRMS (ESI') calcd for $\mathrm{C}_{17} \mathrm{H}_{29} \mathrm{ClNaO}_{2} \mathrm{Si}^{+}[\mathrm{M}+\mathrm{Na}]^{+}: 351.1518$, found: 351.1517 .

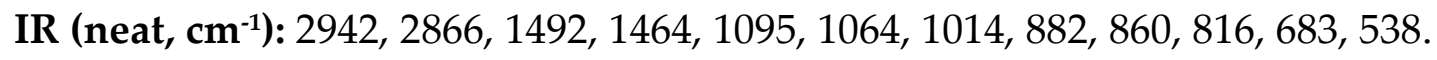
$[\alpha]_{\mathrm{D}^{24.3}}=-19.29\left(\mathrm{c}=0.73, \mathrm{CHCl}_{3}, 94 \%\right.$ ee$)$; Enantiomeric excess was determined 
by chiral HPLC (Daicel Chiralpak IA, hexane/isopropyl alcohol =99/1, flow rate $=1.0 \mathrm{~mL} / \mathrm{min}, \lambda=220 \mathrm{~nm}$ ), $t^{1}=11.8 \mathrm{~min}$ (minor),$t^{2}=12.6 \mathrm{~min}$ (major).

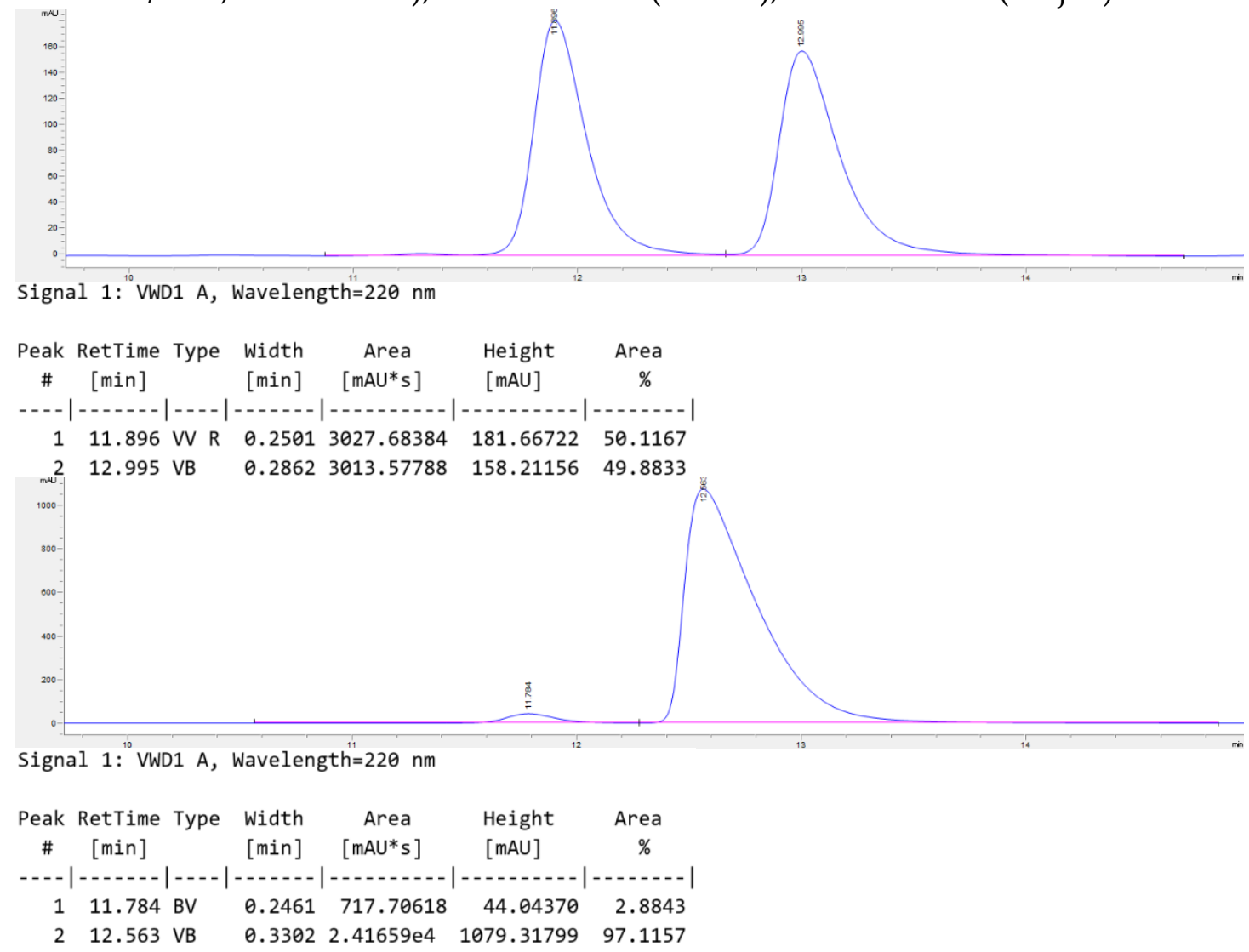

(R)-1-(4-Bromophenyl)-2-((triisopropylsilyl)oxy)ethan-1-ol (3an)<smiles>O[C@H](CO[PbH])c1ccc(Br)cc1</smiles>

Compound 3an was prepared as a colorless oil in $78 \%$ yield $(87.0 \mathrm{mg}$, eluent: petroleum ether/EtOAc $=100 / 3$ to 20/1), from (Z)-((4bromostyryl)oxy)triisopropylsilane 1an (106.2 mg, $0.30 \mathrm{mmol}),[\mathrm{RhCl}(\mathrm{cod})]_{2}$ (3.0 mg, $0.006 \mathrm{mmol}),(R, R)-3,5-\mathrm{PPr}_{2}$-DIOP (10.0 mg, $\left.0.012 \mathrm{mmol}\right)$, HBpin (57.6 $\mathrm{mg}, 0.45 \mathrm{mmol})$ and AgOAc (15.0 mg, $0.09 \mathrm{mmol})$ following the above general procedure G.

$\mathbf{R}_{f}=0.45$ (petroleum ether $/$ EtOAc $=10 / 1$ )

${ }^{1} \mathrm{H}$ NMR (400 MHz, $\left.\mathrm{CDCl}_{3}\right) \delta$ 7.50-7.44 (m, 2H), 7.28-7.22 (m, 2H), 4.73 (dd, J = 8.7, $2.0 \mathrm{~Hz}, 1 \mathrm{H}), 3.82(\mathrm{dd}, J=9.9,3.7 \mathrm{~Hz}, 1 \mathrm{H}), 3.62-3.53(\mathrm{~m}, 1 \mathrm{H}), 3.10(\mathrm{~s}, 1 \mathrm{H})$, $1.18-0.96(\mathrm{~m}, 21 \mathrm{H})$.

${ }^{13} \mathrm{C}$ NMR (100 MHz, $\left.\mathrm{CDCl}_{3}\right) \delta 139.5,131.5,128.1,121.7,74.0,69.1,18.1,12.0$. 
HRMS (ESI') calcd for $\mathrm{C}_{17} \mathrm{H}_{29} \mathrm{BrNaO}_{2} \mathrm{Si}^{+}[\mathrm{M}+\mathrm{Na}]^{+}: 395.1012$, found: 395.1011 .

IR (neat, $\mathbf{c m}^{-1}$ ): 2943, 2866, 1464, 1112, 1068, 1010, 882, 761, 684.

$[\alpha]_{D^{24.3}}=-16.91\left(\mathrm{c}=0.54, \mathrm{CHCl}_{3}, 94 \%\right.$ ee$)$; Enantiomeric excess was determined by chiral HPLC (Daicel Chiralpak IA, hexane/isopropyl alcohol = 99/1, flow rate $=1.0 \mathrm{~mL} / \mathrm{min}, \lambda=220 \mathrm{~nm}$ ), $t^{1}=14.3 \mathrm{~min}$ (minor),$t^{2}=15.6 \mathrm{~min}$ (major).

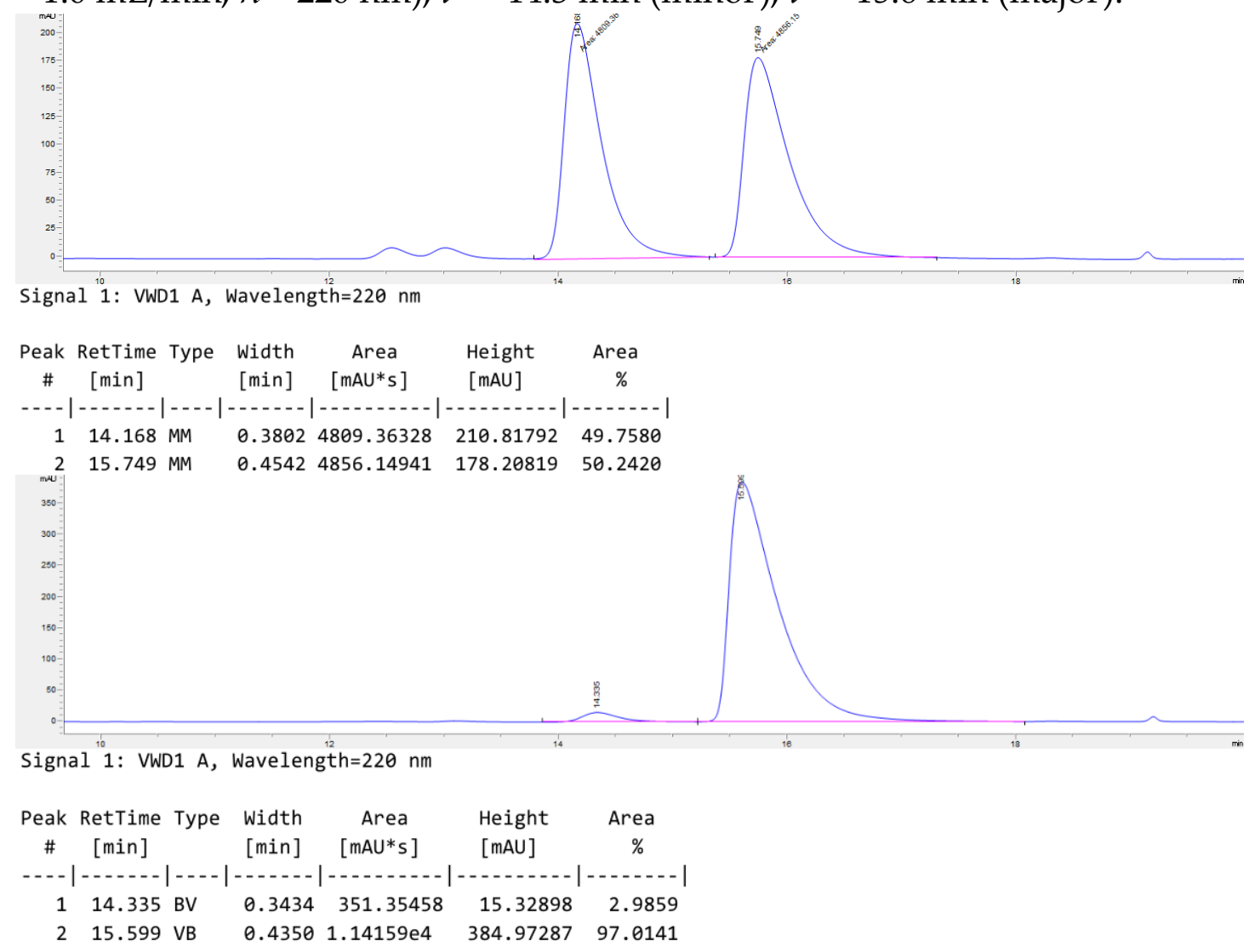

(R)-4-(1-Hydroxy-2-((triisopropylsilyl)oxy)ethyl)phenol (3ao)<smiles>OCC(O)c1ccc(O)cc1</smiles>

Compound 3ao was prepared as a colorless solid in 74\% yield $(69.0 \mathrm{mg}$, eluent: petroleum ether $/ \mathrm{EtOAc}=20 / 1$ to $5 / 1)$, from (Z)-triisopropyl $((4-(4,4,5,5-$ tetramethyl-1,3,2-dioxaborolan-2-yl)styryl)oxy)silane $\mathbf{1 a o}(120.7 \mathrm{mg}, \quad 0.30$ $\mathrm{mmol})$, $[\mathrm{RhCl}(\mathrm{cod})]_{2}(3.0 \mathrm{mg}, 0.006 \mathrm{mmol}),(R, R)-3,5-i \mathrm{Pr}_{2}-\mathrm{DIOP}$ (10.0 mg, 0.012 $\mathrm{mmol})$, HBpin ( $57.6 \mathrm{mg}, 0.45 \mathrm{mmol})$ and LiOAc $(6.0 \mathrm{mg}, 0.09 \mathrm{mmol})$ following the above general procedure $\mathrm{F}$. $\mathbf{R}_{f}=0.45$ (petroleum ether $/ \mathrm{EtOAc}=5 / 1$ ) 
${ }^{1} \mathrm{H}$ NMR (400 MHz, $\left.\mathrm{CDCl}_{3}\right) \delta 7.18(\mathrm{~d}, J=8.1 \mathrm{~Hz}, 2 \mathrm{H}), 6.72(\mathrm{~d}, J=8.1 \mathrm{~Hz}, 2 \mathrm{H})$, $6.42(\mathrm{~s}, 1 \mathrm{H}), 4.72(\mathrm{dd}, J=9.2,3.3 \mathrm{~Hz}, 1 \mathrm{H}), 3.79(\mathrm{dd}, J=10.0,3.5 \mathrm{~Hz}, 1 \mathrm{H}), 3.67-3.59$ (m, 1H), $3.34(\mathrm{~s}, 1 \mathrm{H}), 1.20-0.98(\mathrm{~m}, 21 \mathrm{H})$.

${ }^{13} \mathrm{C}$ NMR (100 MHz, $\left.\mathrm{CDCl}_{3}\right) \delta$ 155.8, 131.6, 127.9, 115.5, 74.5, 69.1, 18.1, 12.3, 12.0, 11.7.

HRMS (ESI+) calcd for $\mathrm{C}_{17} \mathrm{H}_{30} \mathrm{NaO}_{3} \mathrm{Si}^{+}[\mathrm{M}+\mathrm{Na}]^{+}$: 333.1856, found: 333.1856.

IR (neat, cm-1): 2940, 2866, 1516, 1459, 1238, 1201, 1104, 1057, 881, 832, 685, 645, 542.

$[\alpha]_{\mathrm{D}^{24.3}}=-23.25\left(\mathrm{c}=0.25, \mathrm{CHCl}_{3}, 96 \%\right.$ ee); Enantiomeric excess was determined by chiral HPLC (Daicel Chiralcel OD-H, hexane/isopropyl alcohol = 95/5, flow rate $=1.0 \mathrm{~mL} / \mathrm{min}, \lambda=220 \mathrm{~nm}$ ), $t^{1}=9.8 \mathrm{~min}$ (minor), $t^{2}=12.5 \mathrm{~min}$ (major).

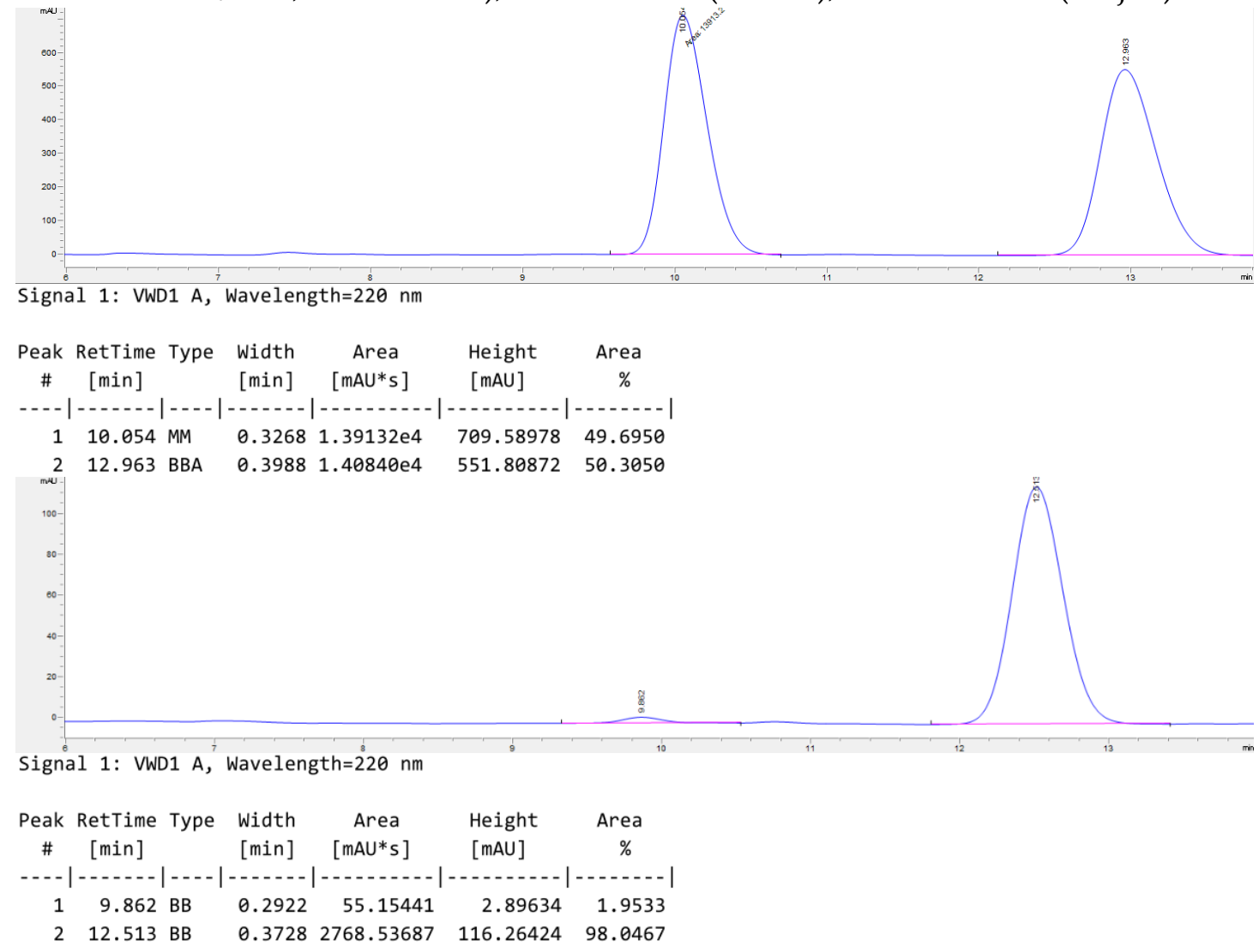

(R)-1-(4-(Trifluoromethyl)phenyl)-2-((triisopropylsilyl)oxy)ethan-1-ol (3ap)<smiles>O[C@H](COc1ccc(C(F)(F)F)cc1)c1ccc(F)cc1</smiles>

Compound 3ap was prepared as a colorless oil in $86 \%$ yield $(93.4 \mathrm{mg}$, eluent: petroleum ether/EtOAc $=100 / 3$ to $20 / 1)$, from (Z)-triisopropyl((4- 
(trifluoromethyl)styryl)oxy)silane 1ap (103.2 mg, $0.30 \mathrm{mmol})$, [ RhCl(cod) ]2 (3.0 $\mathrm{mg}, 0.006 \mathrm{mmol}),(R, R)-3,5-\mathrm{iPr}$-DIOP (10.0 mg, $0.012 \mathrm{mmol})$, HBpin (57.6 mg, $0.45 \mathrm{mmol})$ and AgOAc $(15.0 \mathrm{mg}, 0.09 \mathrm{mmol})$ following the above general procedure $\mathrm{G}$.

$\mathbf{R}_{f}=0.42$ (petroleum ether $/ \mathrm{EtOAc}=10 / 1$ )

${ }^{1} \mathrm{H}$ NMR $\left(400 \mathrm{MHz}, \mathrm{CDCl}_{3}\right) \delta 7.60(\mathrm{~d}, J=8.1 \mathrm{~Hz}, 2 \mathrm{H}), 7.50(\mathrm{~d}, J=8.1 \mathrm{~Hz}, 2 \mathrm{H})$, $4.83(\mathrm{dd}, J=8.4,3.6 \mathrm{~Hz}, 1 \mathrm{H}), 3.88(\mathrm{dd}, J=9.9,3.7 \mathrm{~Hz}, 1 \mathrm{H}), 3.67-3.57(\mathrm{~m}, 1 \mathrm{H}), 3.14$ $(\mathrm{s}, 1 \mathrm{H}), 1.19-0.97(\mathrm{~m}, 21 \mathrm{H})$.

${ }^{13} \mathrm{C}$ NMR (100 MHz, $\left.\mathrm{CDCl}_{3}\right) \delta 144.5(\mathrm{~d}, J=0.9 \mathrm{~Hz}), 130.1(\mathrm{q}, J=32.4 \mathrm{~Hz}), 126.6$, $125.4(\mathrm{q}, J=3.8 \mathrm{~Hz}), 74.0,69.1,18.0,12.0$.

${ }^{19} \mathrm{~F}$ NMR $\left(377 \mathrm{MHz}, \mathrm{CDCl}_{3}\right) \delta-62.5$.

HRMS (ESI') calcd for $\mathrm{C}_{18} \mathrm{H}_{29} \mathrm{~F}_{3} \mathrm{NaO}_{2} \mathrm{Si}^{+}$[M+Na] $]^{+}: 385.1781$, found: 385.1780 .

IR (neat, cm-1): 2925, 2867, 1463, 1325, 1165, 1126, 1068, 1017, 882, 685.

$[\alpha]_{\mathrm{D}^{24.3}}=-17.95\left(\mathrm{c}=0.40, \mathrm{CHCl}_{3}, 90 \%\right.$ ee); Enantiomeric excess was determined by chiral HPLC (Daicel Chiralpak IA, hexane/isopropyl alcohol = 99/1, flow rate $=0.5 \mathrm{~mL} / \mathrm{min}, \lambda=220 \mathrm{~nm}$ ), $t^{1}=12.5 \mathrm{~min}$ (minor),$t^{2}=13.1 \mathrm{~min}$ (major).

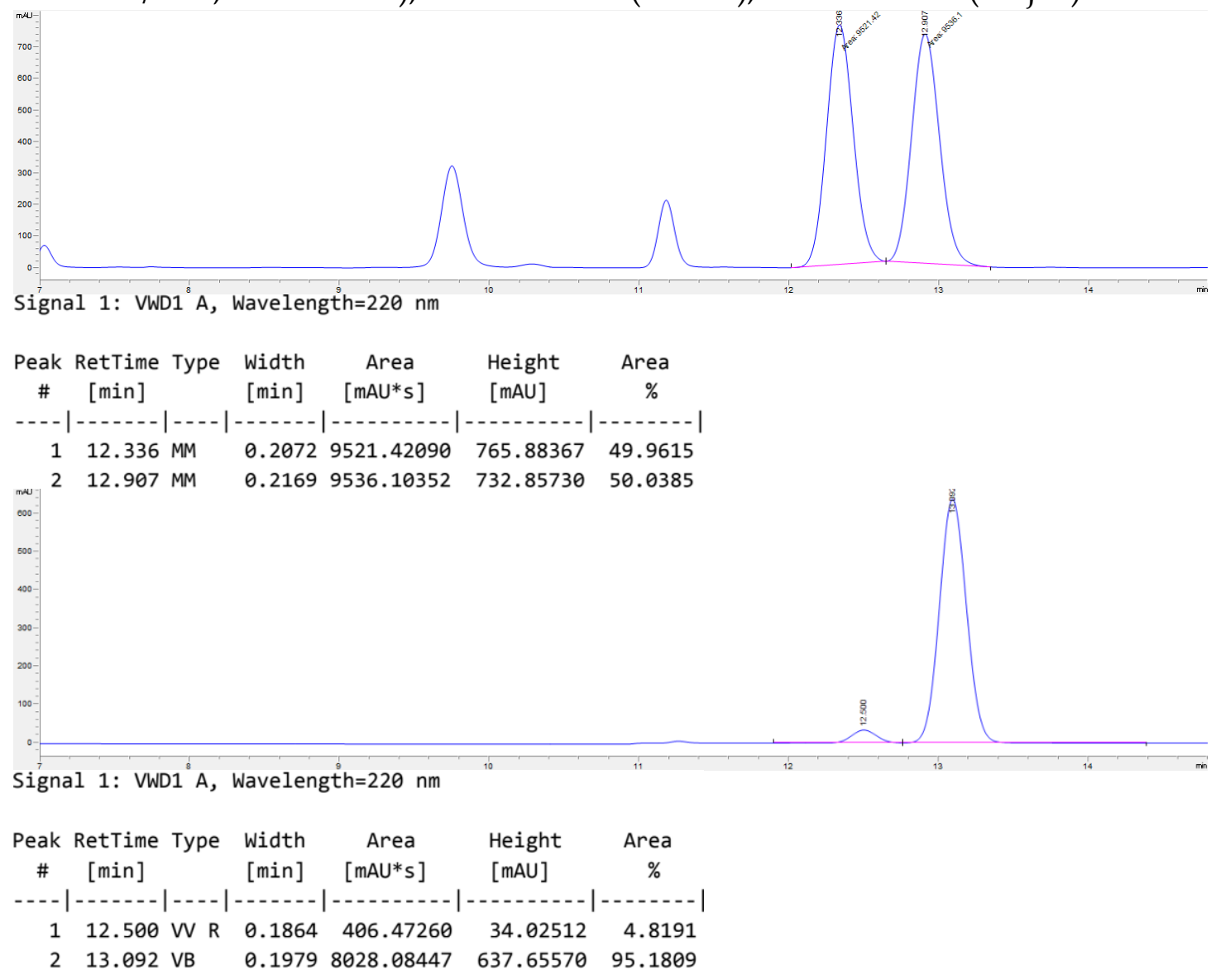

(R)-4-(1-Hydroxy-2-((triisopropylsilyl)oxy)ethyl)benzonitrile (3aq) 
<smiles>N#Cc1ccc(C(O)COP)cc1</smiles>

Compound 3aq was prepared as a colorless oil in $74 \%$ yield $(71.9 \mathrm{mg}$, eluent: petroleum ether/EtOAc $=100 / 3$ to 10/1), from (Z)-4-(2((triisopropylsilyl)oxy)vinyl)benzonitrile $\mathbf{1 a q}(93.3 \mathrm{mg}, \quad 0.30 \mathrm{mmol})$, $[\mathrm{RhCl}(\mathrm{cod})]_{2}(3.0 \mathrm{mg}, 0.006 \mathrm{mmol}),(R, R)-3,5-i \mathrm{Pr}_{2}$-DIOP (10.0 mg, $\left.0.012 \mathrm{mmol}\right)$, HBpin (57.6 mg, $0.45 \mathrm{mmol}$ ) and AgOAc (15.0 mg, $0.09 \mathrm{mmol})$ following the above general procedure $\mathrm{G}$.

$\mathbf{R}_{f}=0.28$ (petroleum ether $/$ EtOAc $=10 / 1$ )

${ }^{1}$ H NMR (400 MHz, $\left.\mathrm{CDCl}_{3}\right) \delta 7.64(\mathrm{~d}, J=8.2 \mathrm{~Hz}, 2 \mathrm{H}), 7.50(\mathrm{~d}, J=8.2 \mathrm{~Hz}, 2 \mathrm{H})$, $4.86-4.79(\mathrm{~m}, 1 \mathrm{H}), 3.88(\mathrm{dd}, J=9.9,3.8 \mathrm{~Hz}, 1 \mathrm{H}), 3.60(\mathrm{dd}, J=9.9,8.1 \mathrm{~Hz}, 1 \mathrm{H})$, 3.19-3.10 (m, 1H), 1.17-1.01 (m, 21H).

${ }^{13} \mathrm{C}$ NMR (100 MHz, $\left.\mathrm{CDCl}_{3}\right) \delta$ 146.0, 132.3, 127.0, 119.0, 111.6, 73.9, 68.8, 18.0, $18.0,12.0$.

HRMS (ESI') calcd for $\mathrm{C}_{18} \mathrm{H}_{29} \mathrm{NNaO}_{2} \mathrm{Si}^{+}[\mathrm{M}+\mathrm{Na}]^{+}: 342.1860$, found: 342.1859.

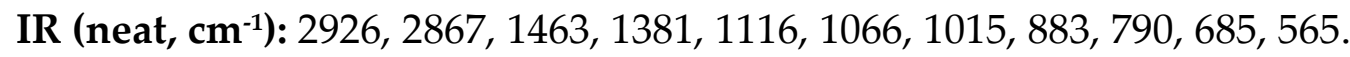

$[\alpha]_{\mathrm{D}^{24.3}}=-7.44\left(\mathrm{c}=0.36, \mathrm{CHCl}_{3}, 85 \%\right.$ ee); Enantiomeric excess was determined by chiral HPLC (Daicel Chiralpak AS-H, hexane/isopropyl alcohol =98/2, flow rate $=0.5 \mathrm{~mL} / \mathrm{min}, \lambda=220 \mathrm{~nm}$ ) $t^{1}=10.5 \mathrm{~min}$ (major),$t^{2}=11.2 \mathrm{~min}$ (minor).

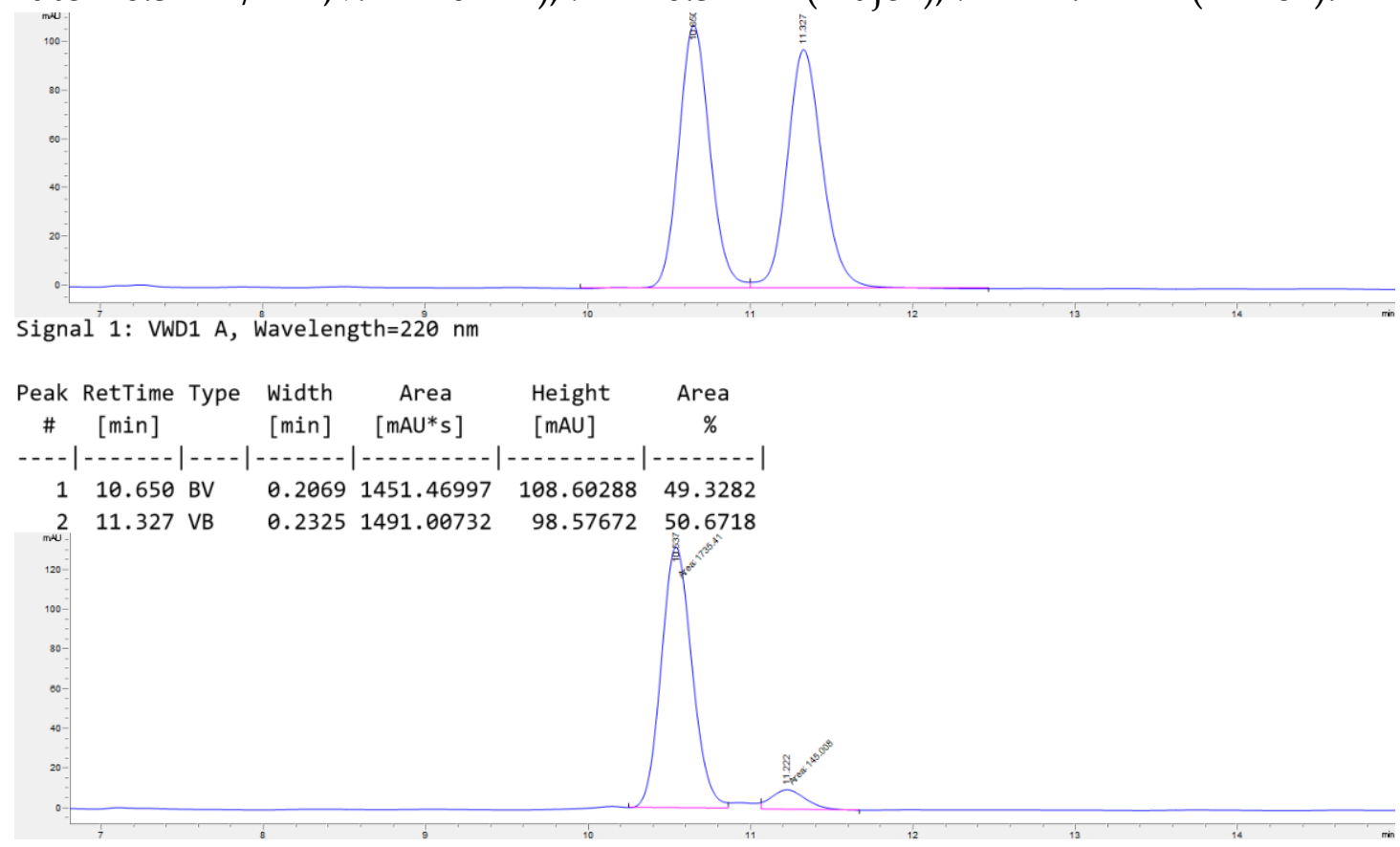




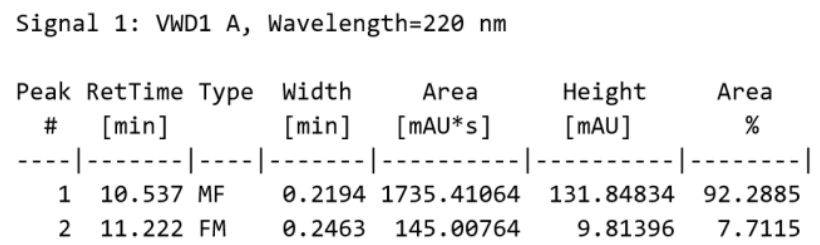

(R)-1-(5-Fluoro-2-methoxyphenyl)-2-((triisopropylsilyl)oxy)ethan-1-ol (3ar)<smiles>COc1ccc(F)cc1[C@@H](O)CO[Na]</smiles>

Compound 3ar was prepared as a white solid in $85 \%$ yield $(87.0 \mathrm{mg}$, eluent: petroleum ether/EtOAc $=100 / 3$ to 20/1), from (Z)-((5-fluoro-2methoxystyryl)oxy)triisopropylsilane 1 ar $(97.2 \mathrm{mg}, 0.30 \mathrm{mmol}),[\mathrm{RhCl}(\mathrm{cod})]_{2}$ (3.0 mg, $0.006 \mathrm{mmol}),(R, R)-3,5-i P r 2-D I O P ~(10.0 \mathrm{mg}, 0.012 \mathrm{mmol})$, HBpin (57.6 $\mathrm{mg}, 0.45 \mathrm{mmol})$ and AgOAc (15.0 mg, $0.09 \mathrm{mmol})$ following the above general procedure $\mathrm{G}$.

$\mathbf{R}_{f}=0.42$ (petroleum ether $/$ EtOAc $=10 / 1$ )

${ }^{1} \mathrm{H}$ NMR (400 MHz, $\left.\mathrm{CDCl}_{3}\right)$ ठ 7.29-7.23 (m, 1H), 6.96-6.86 (m, 1H), 6.80-6.72 (m, $1 \mathrm{H}), 5.13-5.01(\mathrm{~m}, 1 \mathrm{H}), 4.07-3.91(\mathrm{~m}, 1 \mathrm{H}), 3.79(\mathrm{~s}, 3 \mathrm{H}), 3.56-3.43(\mathrm{~m}, 1 \mathrm{H}), 3.21-$ $3.05(\mathrm{~m}, 1 \mathrm{H}), 1.21-0.94(\mathrm{~m}, 21 \mathrm{H})$.

${ }^{13} \mathrm{C}$ NMR (100 MHz, $\left.\mathrm{CDCl}_{3}\right) \delta 157.5(\mathrm{~d}, J=238.1 \mathrm{~Hz}), 152.4(\mathrm{~d}, J=2.1 \mathrm{~Hz}), 130.7$ $(\mathrm{d}, J=7.0 \mathrm{~Hz}), 114.3(\mathrm{~d}, J=14.7 \mathrm{~Hz}), 114.0(\mathrm{~d}, J=13.2 \mathrm{~Hz}), 111.0(\mathrm{~d}, J=8.2 \mathrm{~Hz})$, $69.5,67.4,55.9,18.0,12.1$.

${ }^{19} \mathrm{~F}$ NMR (377 $\left.\mathrm{MHz}, \mathrm{CDCl}_{3}\right) \delta-123.6$.

HRMS (ESI') calcd for $\mathrm{C}_{18} \mathrm{H}_{31} \mathrm{FNaO}_{3} \mathrm{Si}^{+}[\mathrm{M}+\mathrm{Na}]^{+}:$365.1919, found: 365.1918.

IR (neat, cm-1): 2944, 2867, 1496, 1465, 1255, 1143, 1124, 1097, 1063, 884, 685.

$[\alpha]_{\mathrm{D}^{24.3}}=-22.79\left(\mathrm{c}=0.40, \mathrm{CHCl}_{3}, 88 \%\right.$ ee $)$; Enantiomeric excess was determined by chiral HPLC (Daicel Chiralcel OD-H, hexane/isopropyl alcohol = 99/1, flow rate $=0.5 \mathrm{~mL} / \mathrm{min}, \lambda=220 \mathrm{~nm}$ ),$t^{1}=9.2 \mathrm{~min}$ (major),$t^{2}=9.8 \mathrm{~min}$ (minor).

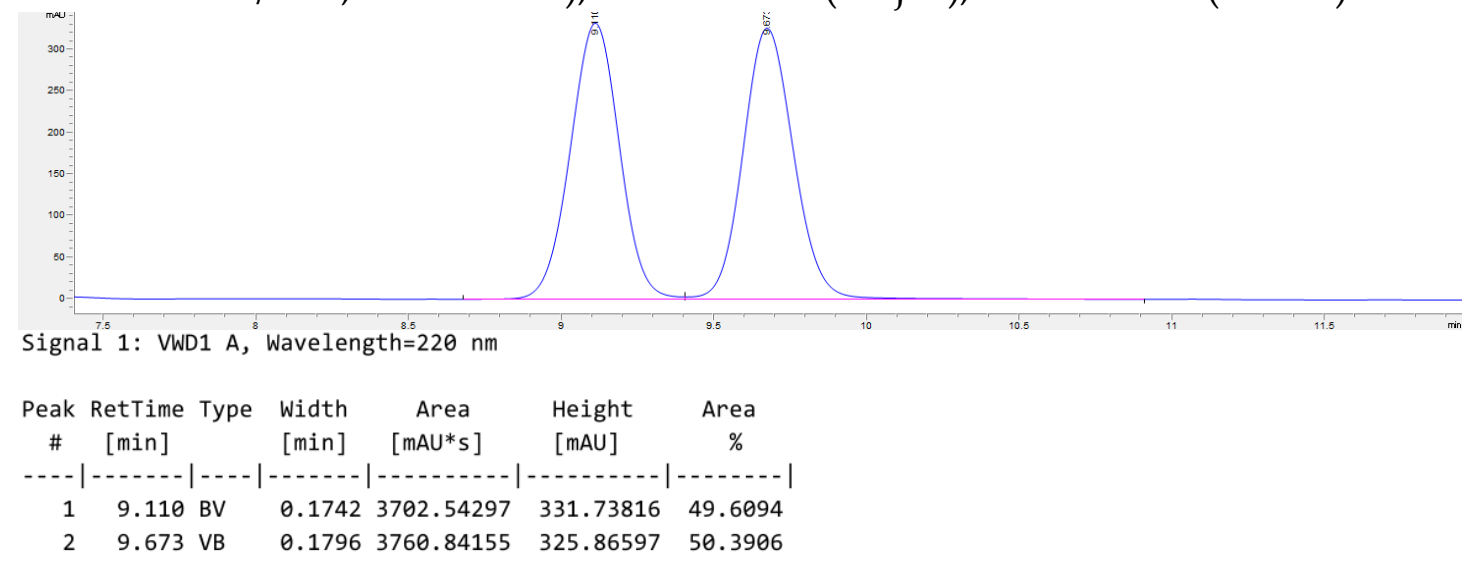




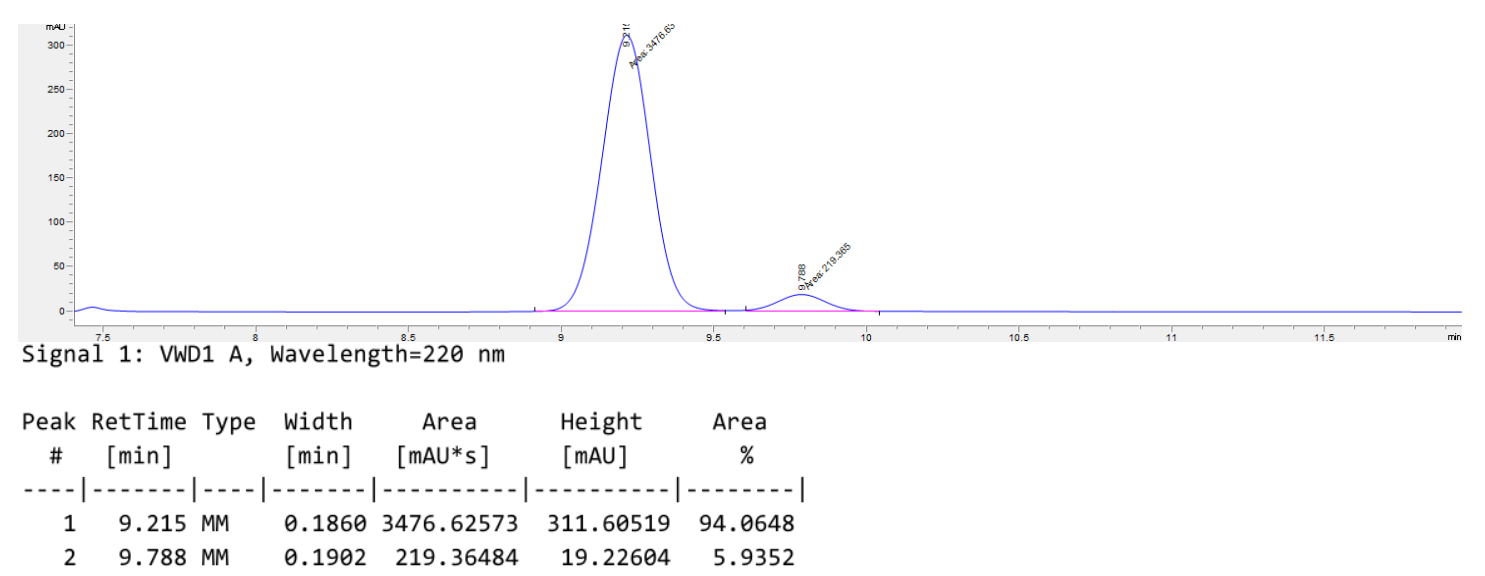

(R)-1-(1-Methyl-1H-indol-5-yl)-2-((triisopropylsilyl)oxy)ethan-1-ol (3as)<smiles>Cn1ccc2cc(C(O)CO)ccc21</smiles>

Compound 3as was prepared as a colorless oil in $90 \%$ yield $(93.7 \mathrm{mg}$, eluent: petroleum ether/EtOAc $=100 / 3$ to 20/1), from (Z)-1-methyl-5-(2((triisopropylsilyl)oxy)vinyl)- $1 H$-indole 1 as $(98.8 \mathrm{mg}, 0.30 \mathrm{mmol})$, [RhCl(cod) $]_{2}$ (3.0 mg, $0.006 \mathrm{mmol}),(R, R)-3,5-i \mathrm{Pr}_{2}$-DIOP (10.0 mg, $\left.0.012 \mathrm{mmol}\right)$, HBpin (57.6 $\mathrm{mg}, 0.45 \mathrm{mmol})$ and $\mathrm{AgOAc}(15.0 \mathrm{mg}, 0.09 \mathrm{mmol})$ following the above general procedure G.

$\mathrm{R}_{f}=0.22$ (petroleum ether $\left./ \mathrm{EtOAc}=10 / 1\right)$

${ }^{1} \mathrm{H}$ NMR $\left(400 \mathrm{MHz}, \mathrm{CDCl}_{3}\right)$ \& 7.71-7.58 (m, 1H), 7.37-7.16 (m, 2H), 7.10-6.94 (m, 1H), 6.52-6.38 (m, 1H), $4.88(\mathrm{dd}, J=9.4,3.4 \mathrm{~Hz}, 1 \mathrm{H}), 3.85(\mathrm{dd}, J=10.0,3.5 \mathrm{~Hz}$, $1 \mathrm{H}), 3.75(\mathrm{~s}, 3 \mathrm{H}), 3.72-3.64(\mathrm{~m}, 1 \mathrm{H}), 3.15(\mathrm{~s}, 1 \mathrm{H}), 1.22-1.01(\mathrm{~m}, 21 \mathrm{H})$.

${ }^{13} \mathrm{C}$ NMR (100 MHz, $\mathrm{CDCl}_{3}$ ) $\delta$ 136.6, 131.1, 129.3, 128.5, 120.2, 118.8, 109.1, 101.1, 75.3, 69.9, 32.9, 18.1, 12.1.

HRMS (ESI') calcd for $\mathrm{C}_{20} \mathrm{H}_{33} \mathrm{NNaO}_{2} \mathrm{Si}^{+}[\mathrm{M}+\mathrm{Na}]^{+}: 370.2173$, found: 370.2172 .

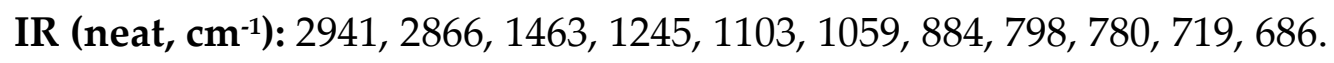

$[\alpha]_{\mathrm{D}^{2.3}}=-16.39\left(\mathrm{c}=0.51, \mathrm{CHCl}_{3}, 96 \%\right.$ ee); Enantiomeric excess was determined by chiral HPLC (Daicel Chiralcel OD-H, hexane/isopropyl alcohol $=95 / 5$, flow rate $=1.0 \mathrm{~mL} / \mathrm{min}, \lambda=220 \mathrm{~nm}$ ), $t^{1}=11.1 \mathrm{~min}$ (minor),$t^{2}=11.9 \mathrm{~min}$ (major).

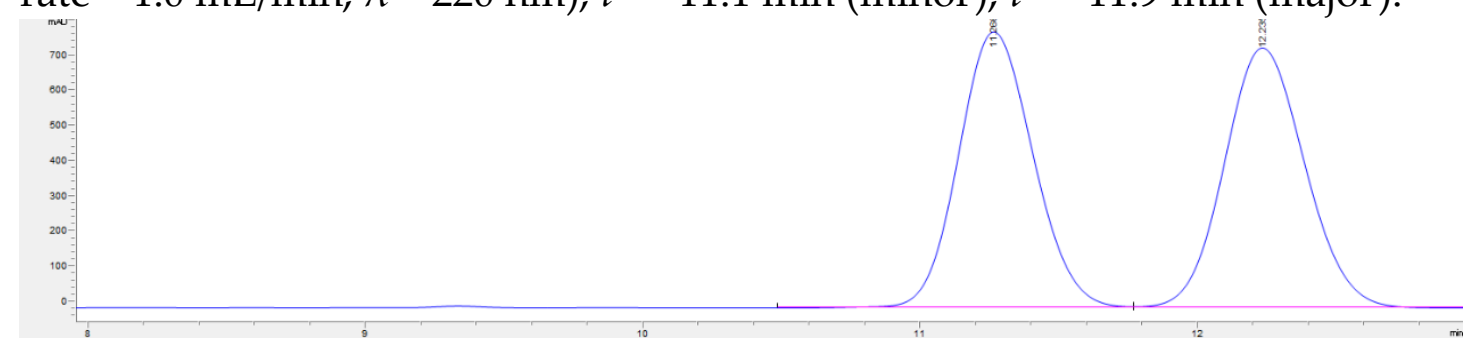




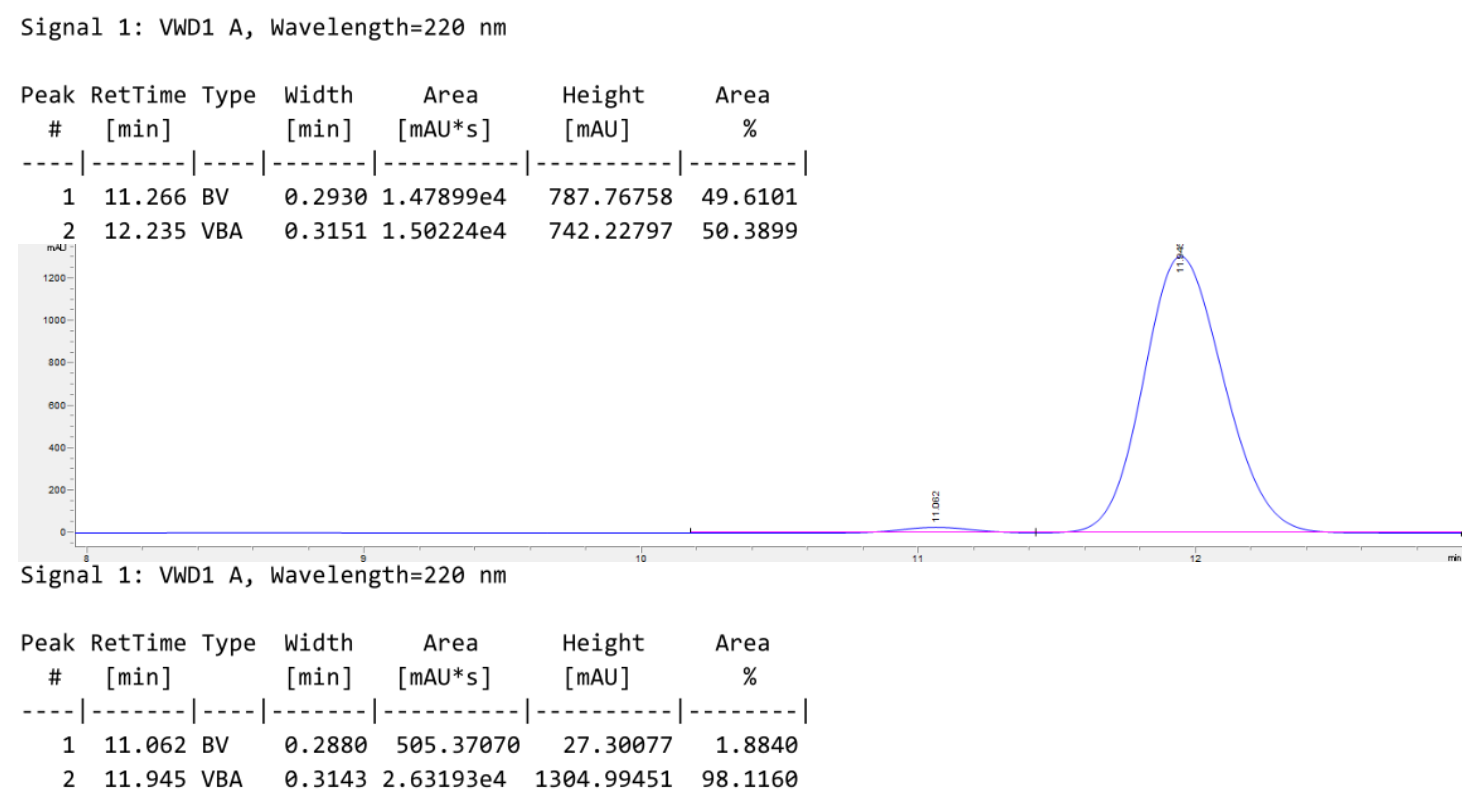

(R)-1-(Furan-3-yl)-2-((triisopropylsilyl)oxy)ethan-1-ol (3at)<smiles>OC(COc1ccccc1)c1ccoc1</smiles>

Compound 3at was prepared as a colorless oil in $66 \%$ yield $(56.0 \mathrm{mg}$, eluent: petroleum ether/EtOAc $=100 / 3$ to 20/1), from (Z)-((2-(furan-3yl)vinyl)oxy)triisopropylsilane 1at $(79.9 \mathrm{mg}, 0.30 \mathrm{mmol}),[\mathrm{RhCl}(\mathrm{cod})]_{2}(3.0 \mathrm{mg}$, $0.006 \mathrm{mmol}),(R, R)-3,5-i \mathrm{Pr}_{2}$-DIOP (10.0 mg, $0.012 \mathrm{mmol}$ ), HBpin (57.6 mg, 0.45 $\mathrm{mmol})$ and $\mathrm{LiOAc}(6.0 \mathrm{mg}, 0.09 \mathrm{mmol})$ following the above general procedure $\mathrm{F}(18 \mathrm{~h})$.

$\mathbf{R}_{f}=0.53$ (petroleum ether $/$ EtOAc $=10 / 1$ )

${ }^{1} \mathrm{H}$ NMR (400 MHz, $\left.\mathrm{CDCl}_{3}\right) \delta$ 7.53-7.31 (m, 2H), 6.47-6.32 (m, 1H), 4.73 (dd, J = 8.3, 3.5 Hz, 1H), $3.85(\mathrm{dd}, J=9.8,3.6 \mathrm{~Hz}, 1 \mathrm{H}), 3.75-3.66(\mathrm{~m}, 1 \mathrm{H}), 2.96(\mathrm{~s}, 1 \mathrm{H})$, $1.38-1.00(\mathrm{~m}, 21 \mathrm{H})$.

${ }^{13} \mathrm{C}$ NMR (100 MHz, $\left.\mathrm{CDCl}_{3}\right) \delta$ 143.2, 139.8, 125.0, 108.9, 68.2, 67.8, 18.1, 12.0.

HRMS (ESI') calcd for $\mathrm{C}_{15} \mathrm{H}_{28} \mathrm{NaO}_{3} \mathrm{Si}^{+}[\mathrm{M}+\mathrm{Na}]^{+}$: 307.1700, found: 307.1699.

IR (neat, cm-1): 2943, 2867, 1464, 1160, 1116, 1061, 1021, 881, 828, 788, 684.

$[\alpha]_{\mathrm{D}^{24.3}}=-10.40\left(\mathrm{c}=0.57, \mathrm{CHCl}_{3}, 93 \%\right.$ ee $)$; Enantiomeric excess was determined by chiral HPLC (Daicel Chiralpak IC, hexane/isopropyl alcohol =99/1, flow rate $=0.5 \mathrm{~mL} / \mathrm{min}, \lambda=220 \mathrm{~nm}$ ), $t^{1}=15.9 \mathrm{~min}$ (minor),$t^{2}=17.3 \mathrm{~min}$ (major). 


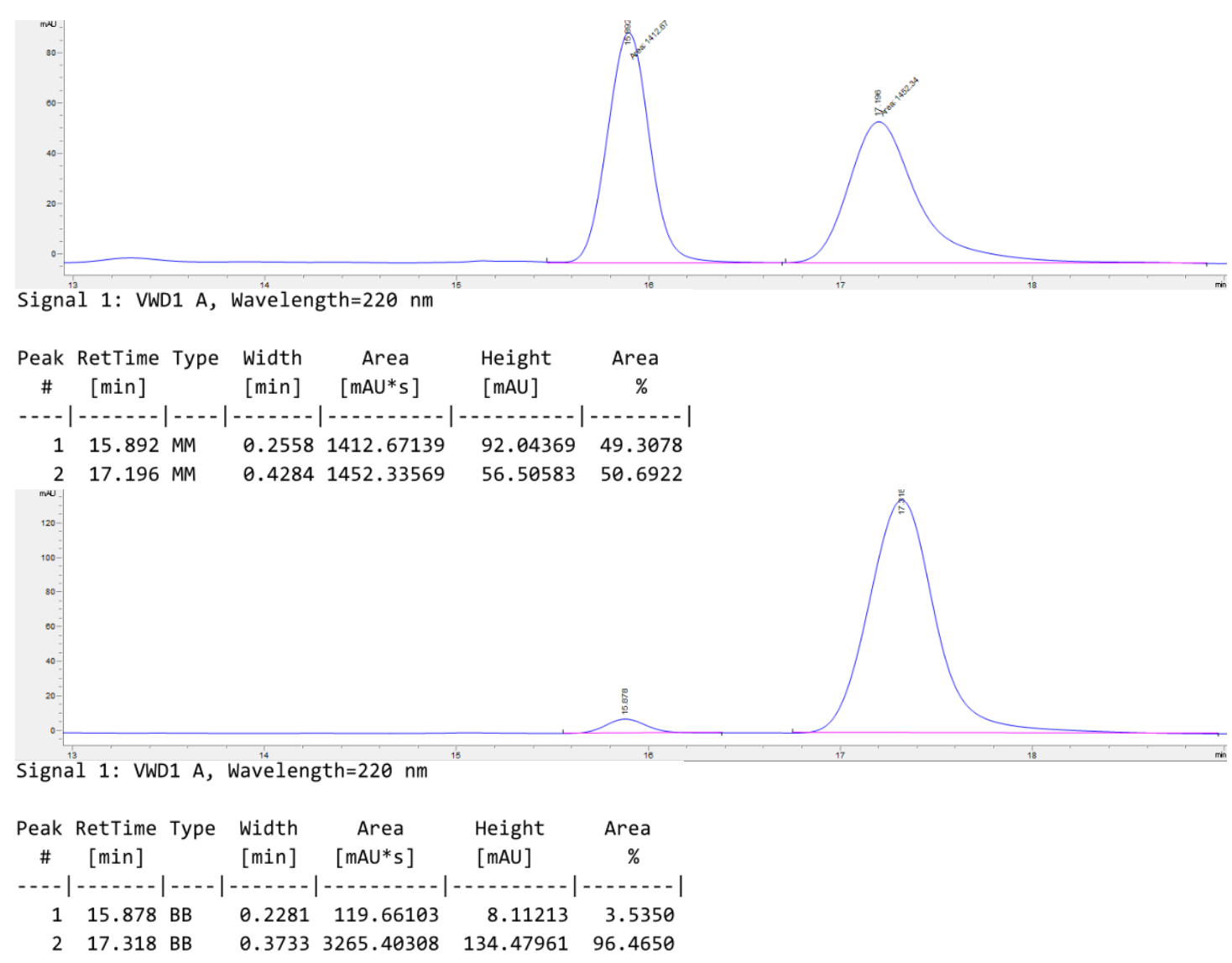

(R)-1-(4-(Pyridin-2-yl)phenyl)-2-((triisopropylsilyl)oxy)ethan-1-ol (3au)<smiles>OCC(O)c1ccc(-c2ccccn2)cc1</smiles>

Compound 3au was prepared as a colorless oil in $64 \%$ yield $(74.2 \mathrm{mg}$, eluent: petroleum ether/EtOAc $=20 / 1)$, from $(Z)-2-(4-(2-(($ triisopropylsilyl $)$ oxy $)$ vinyl $)$ phenyl)pyridine 1au (105.9 mg, $0.30 \mathrm{mmol})$, $[\mathrm{RhCl}(\mathrm{cod})]_{2}(3.0 \mathrm{mg}, 0.006 \mathrm{mmol})$, $(R, R)-3,5-i$ Pr2$_{2}$ DIOP $(10.0 \mathrm{mg}, 0.012 \mathrm{mmol}), \mathrm{HBpin}(57.6 \mathrm{mg}, 0.45 \mathrm{mmol})$ and AgOAc ( $15.0 \mathrm{mg}, 0.09 \mathrm{mmol})$ following the above general procedure $\mathrm{G}$.

$\mathbf{R}_{f}=0.38$ (petroleum ether/EtOAc $=5 / 1$ )

${ }^{1} \mathrm{H}$ NMR (400 MHz, $\left.\mathrm{CDCl}_{3}\right) \delta 8.73-8.64(\mathrm{~m}, 1 \mathrm{H}), 8.02-7.94(\mathrm{~m}, 2 \mathrm{H}), 7.79-7.68$ $(\mathrm{m}, 2 \mathrm{H}), 7.49(\mathrm{~d}, J=8.1 \mathrm{~Hz}, 2 \mathrm{H}), 7.25-7.18(\mathrm{~m}, 1 \mathrm{H}), 4.84(\mathrm{dd}, J=8.8,3.5 \mathrm{~Hz}, 1 \mathrm{H})$, $3.89(\mathrm{dd}, J=9.9,3.6 \mathrm{~Hz}, 1 \mathrm{H}), 3.71-3.61(\mathrm{~m}, 1 \mathrm{H}), 3.17(\mathrm{~s}, 1 \mathrm{H}), 1.20-0.98(\mathrm{~m}, 21 \mathrm{H})$. ${ }^{13} \mathrm{C}$ NMR (100 MHz, CDCl $) \delta$ d 157.3, 149.8, 141.2, 139.0, 136.9, 127.0, 126.7, 122.2, 120.6, 74.4, 69.3, 18.1, 12.1 .

HRMS (ESI+) calcd for $\mathrm{C}_{22} \mathrm{H}_{34} \mathrm{NO}_{2} \mathrm{Si}^{+}[\mathrm{M}+\mathrm{H}]^{+}: 372.2353$, found: 372.2356 . 
IR (neat, cm$^{-1}$ ): 2941, 2866, 1466, 1436, 1118, 882, 781, 684.

$[\alpha]_{\mathrm{D}^{24.3}}=-9.52\left(\mathrm{c}=0.50, \mathrm{CHCl}_{3}, 94 \%\right.$ ee); Enantiomeric excess was determined by chiral HPLC (Daicel Chiralcel OD-H, hexane/isopropyl alcohol =90/10, flow rate $=1.0 \mathrm{~mL} / \mathrm{min}, \lambda=220 \mathrm{~nm}$ ), $t^{1}=8.8 \mathrm{~min}$ (major),$t^{2}=11.5 \mathrm{~min}$ (minor).

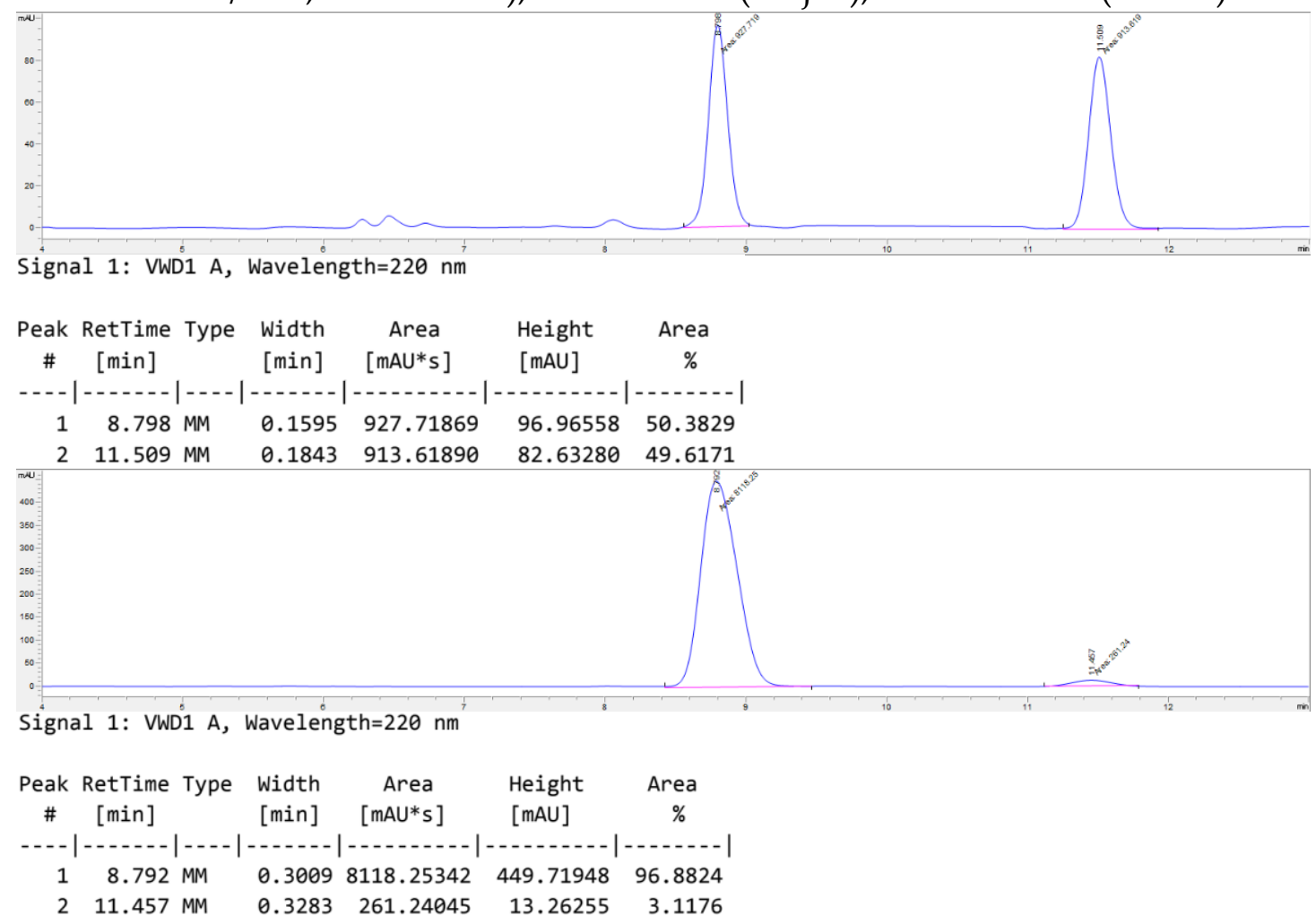

(R)-1-(6-Methoxynaphthalen-2-yl)-2-((triisopropylsilyl)oxy)ethan-1-ol (3av)<smiles>COc1ccc2cc([C@@H](O)CO[Na])ccc2c1</smiles>

Compound 3av was prepared as a white solid in $83 \%$ yield $(92.0 \mathrm{mg}$, eluent: petroleum ether/EtOAc $=100 / 3$ to $10 / 1)$, from (Z)-triisopropyl((2-(6methoxynaphthalen-2-yl)vinyl)oxy)silane 1 av (106.8 $\mathrm{mg}, 0.30 \mathrm{mmol}$ ), $[\mathrm{RhCl}(\mathrm{cod})]_{2}(3.0 \mathrm{mg}, 0.006 \mathrm{mmol}),(R, R)-3,5-i \operatorname{Pr}_{2}-\mathrm{DIOP}(10.0 \mathrm{mg}, 0.012 \mathrm{mmol})$, HBpin (57.6 mg, $0.45 \mathrm{mmol})$ and AgOAc $(15.0 \mathrm{mg}, 0.09 \mathrm{mmol})$ following the above general procedure G.

$\mathbf{R}_{f}=0.28$ (petroleum ether $/$ EtOAc $=10 / 1$ )

${ }^{1} \mathrm{H}$ NMR (400 MHz, $\left.\mathrm{CDCl}_{3}\right) \delta$ 7.81-7.75 (m, 1H), 7.75-7.68 (m, 2H), 7.48-7.41 (m, $1 \mathrm{H})$, 7.17-7.07 (m, 2H), 4.95-4.85 (m, 1H), 3.97-3.85 (m, 4H), 3.77-3.61 (m, 1H), $3.19(\mathrm{~s}, 1 \mathrm{H}), 1.20-0.99(\mathrm{~m}, 21 \mathrm{H})$. 
${ }^{13} \mathrm{C}$ NMR (100 MHz, $\left.\mathrm{CDCl}_{3}\right) \delta$ 157.8, 135.5, 134.4, 129.5, 128.9, 127.0, 125.2, 124.9, $119.0,105.8,74.7,69.4,55.4,18.1,12.1$.

HRMS (ESI') calcd for $\mathrm{C}_{22} \mathrm{H}_{34} \mathrm{NaO}_{3} \mathrm{Si}^{+}[\mathrm{M}+\mathrm{Na}]^{+}$: 397.2169, found: 397.2168.

IR (neat, cm-1): 2940, 2865, 1609, 1463, 1264, 1168, 1107, 1061, 1036, 885, 852, 684. $[\alpha]_{\mathrm{D}^{24.3}}=-17.14\left(\mathrm{c}=0.40, \mathrm{CHCl}_{3}, 96 \%\right.$ ee $)$; Enantiomeric excess was determined by chiral HPLC (Daicel Chiralcel OD-H, hexane/isopropyl alcohol =95/5, flow rate $=1.0 \mathrm{~mL} / \mathrm{min}, \lambda=254 \mathrm{~nm}$ ),$t^{1}=6.2 \mathrm{~min}$ (major),$t^{2}=6.8 \mathrm{~min}$ (minor).

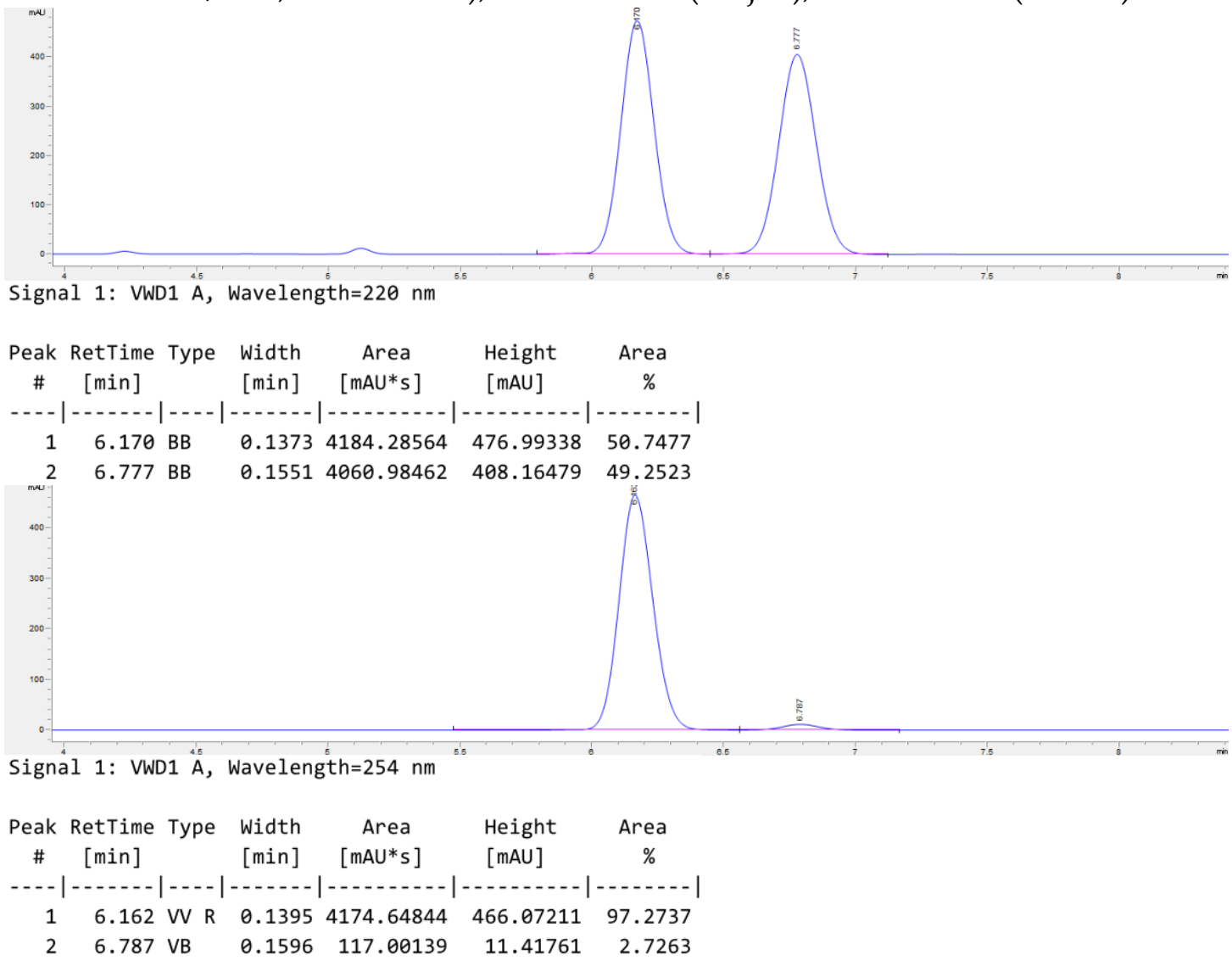

\section{Compound 3aw}

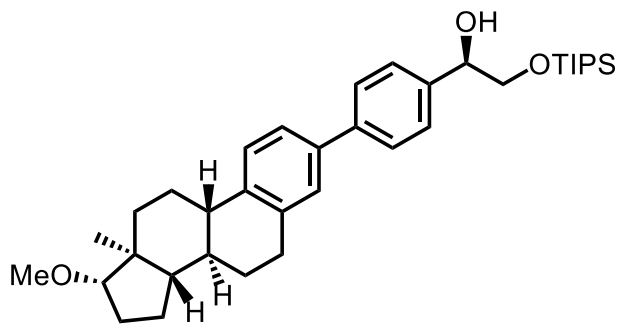

Compound 3aw was prepared as a colorless oil in $84 \%$ yield (142.0 mg, eluent: petroleum ether/EtOAc $=100 / 3$ to $10 / 1)$, from triisopropyl $(((Z)-4-$ ((8R,9S,13S,14S,17S)-17-methoxy-13-methyl-7,8,9,11,12,13,14,15,16,17decahydro-6H-cyclopenta[a]phenanthren-3-yl)styryl)oxy)silane 1aw (163.2 mg, 
$0.30 \mathrm{mmol}),[\mathrm{RhCl}(\mathrm{cod})]_{2}(3.0 \mathrm{mg}, 0.006 \mathrm{mmol}),(R, R)-3,5-i \operatorname{Pr}_{2}-\mathrm{DIOP}(10.0 \mathrm{mg}$, $0.012 \mathrm{mmol}), \mathrm{HB}$ in $(57.6 \mathrm{mg}, 0.45 \mathrm{mmol})$ and AgOAc (15.0 mg, $0.09 \mathrm{mmol})$ following the above general procedure $\mathrm{G}$.

$\mathbf{R}_{f}=0.28$ (petroleum ether $/$ EtOAc $=10 / 1$ )

${ }^{1} \mathrm{H}$ NMR $\left(400 \mathrm{MHz}, \mathrm{CDCl}_{3}\right) \delta 7.55(\mathrm{~d}, J=8.2 \mathrm{~Hz}, 2 \mathrm{H}), 7.42(\mathrm{~d}, J=8.1 \mathrm{~Hz}, 2 \mathrm{H})$, 7.38-7.34 (m, 2H), $7.30(\mathrm{~s}, 1 \mathrm{H}), 4.81(\mathrm{dd}, J=8.9,1.8 \mathrm{~Hz}, 1 \mathrm{H}), 3.88(\mathrm{dd}, J=10.0,3.6$ $\mathrm{Hz}, 1 \mathrm{H}), 3.71-3.62(\mathrm{~m}, 1 \mathrm{H}), 3.38(\mathrm{~s}, 3 \mathrm{H}), 3.36-3.28(\mathrm{~m}, 1 \mathrm{H}), 3.13(\mathrm{~s}, 1 \mathrm{H}), 2.98-2.88$ (m, 2H), 2.42-2.19 (m, 2H), 2.14-2.01 (m, 2H), 1.97-1.87 (m, 1H), 1.77-1.67 (m, 1H), 1.64-1.19 (m, 7H), 1.17-1.00 (m, 21H), $0.80(\mathrm{~s}, 3 \mathrm{H})$.

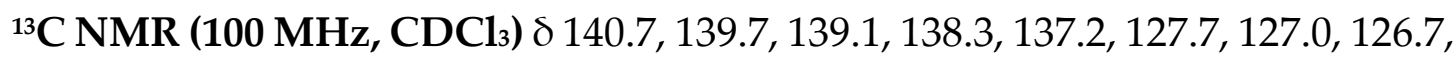
125.9, 124.5, 90.9, 74.4, 69.4, 58.0, 50.6, 44.5, 43.3, 38.5, 38.2, 29.8, 27.9, 27.4, 26.4, 23.2, 18.1, 12.1, 11.7 .

HRMS (ESI ${ }^{+}$) calcd for $\mathrm{C}_{36} \mathrm{H}_{54} \mathrm{NaO}_{3} \mathrm{Si}^{+}[\mathrm{M}+\mathrm{Na}]^{+}: 585.3734$, found: 585.3732 .

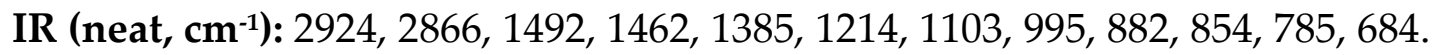

$[\alpha]_{D^{24.3}}=+23.68\left(\mathrm{c}=0.65, \mathrm{CHCl}_{3}, 97: 3 \mathrm{dr}\right)$; Diastereomeric excess was determined by chiral HPLC (Daicel Chiralpak IA, hexane/isopropyl alcohol =97/3, flow rate $=1.0 \mathrm{~mL} / \mathrm{min}, \lambda=220 \mathrm{~nm}$ ), $t^{1}=6.7 \mathrm{~min}$ (major),$t^{2}=7.7 \mathrm{~min}$ (minor).
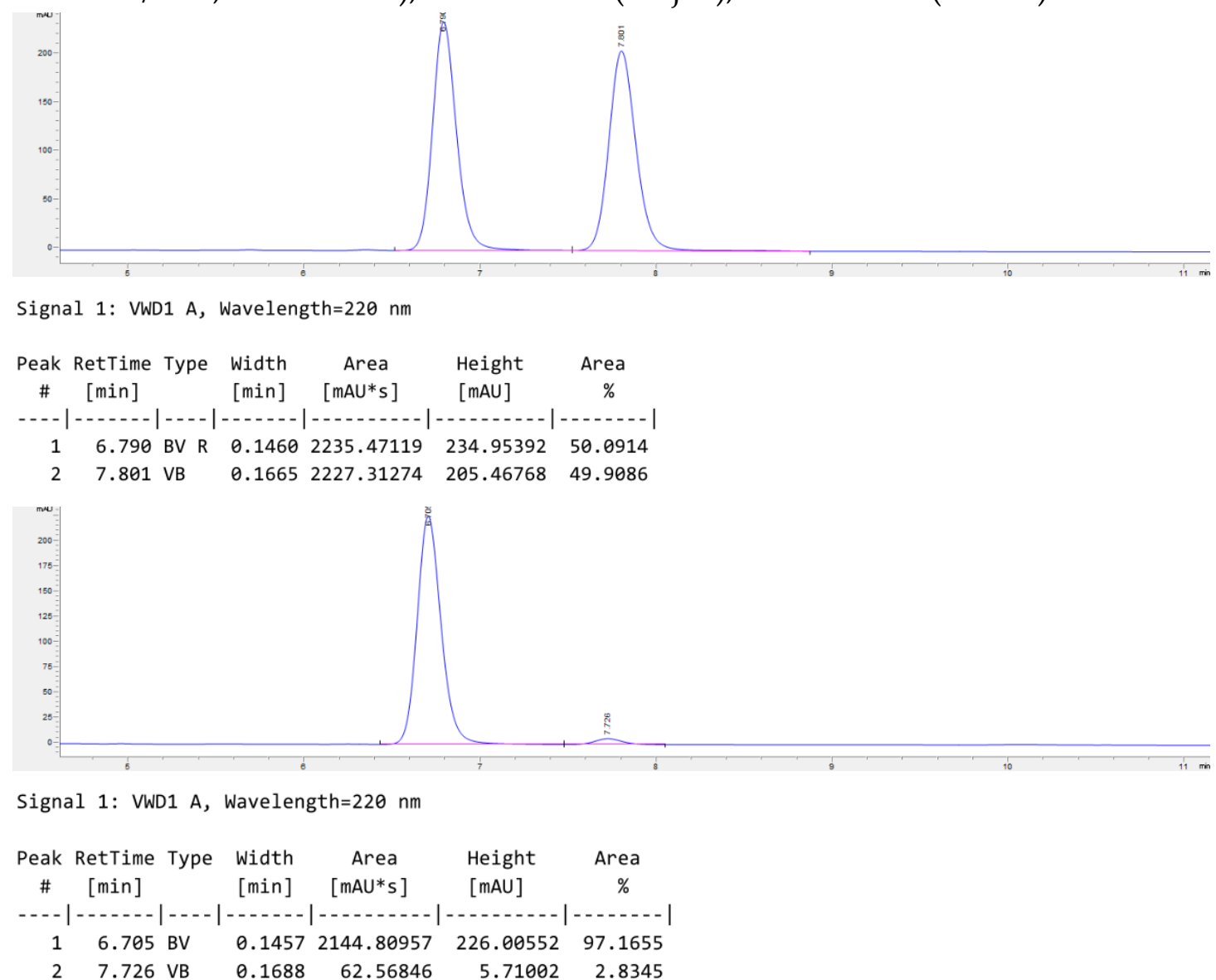


\section{Compound ent-3aw}

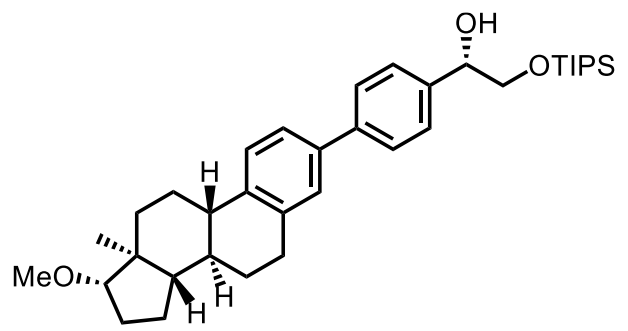

Compound ent-3aw was prepared as a colorless oil in $81 \%$ yield (136.9 mg, eluent: petroleum ether/EtOAc $=100 / 3$ to $10 / 1)$, from triisopropyl $((Z)-4-$ ((8R,9S,13S,14S,17S)-17-methoxy-13-methyl-7,8,9,11,12,13,14,15,16,17-

decahydro-6H-cyclopenta[a]phenanthren-3-yl)styryl)oxy)silane 1aw (163.2 mg, $0.30 \mathrm{mmol}$ ), [RhCl(cod)]2 (3.0 mg, $0.006 \mathrm{mmol}),(S, S)-3,5-i \operatorname{Pr}_{2}$-DIOP (10.0 mg, $0.012 \mathrm{mmol})$, HBpin (57.6 mg, $0.45 \mathrm{mmol})$ and AgOAc (15.0 mg, $0.09 \mathrm{mmol})$ following the above general procedure G.

$\mathbf{R}_{f}=0.28$ (petroleum ether/EtOAc $=10 / 1$ )

${ }^{1}$ H NMR (400 MHz, $\left.\mathrm{CDCl}_{3}\right) \delta 7.60-7.51(\mathrm{~m}, 2 \mathrm{H}), 7.42(\mathrm{~d}, J=8.1 \mathrm{~Hz}, 2 \mathrm{H}), 7.36$ $(\mathrm{d}, J=1.3 \mathrm{~Hz}, 2 \mathrm{H}), 7.32-7.28(\mathrm{~m}, 1 \mathrm{H}), 4.81(\mathrm{dd}, J=8.8,3.5 \mathrm{~Hz}, 1 \mathrm{H}), 3.88(\mathrm{dd}, J=$ 9.9, 3.6 Hz, 1H), $3.66(\mathrm{dd}, J=9.9,8.9 \mathrm{~Hz}, 1 \mathrm{H}), 3.38(\mathrm{~s}, 3 \mathrm{H}), 3.32(\mathrm{t}, J=8.3 \mathrm{~Hz}, 1 \mathrm{H})$, $3.12(\mathrm{~s}, 1 \mathrm{H}), 2.99-2.85(\mathrm{~m}, 2 \mathrm{H}), 2.41-2.22(\mathrm{~m}, 2 \mathrm{H}), 2.14-2.02(\mathrm{~m}, 2 \mathrm{H}), 1.97$ $1.87(\mathrm{~m}, 1 \mathrm{H}), 1.78-1.65(\mathrm{~m}, 1 \mathrm{H}), 1.63-1.21(\mathrm{~m}, 7 \mathrm{H}), 1.20-0.98(\mathrm{~m}, 21 \mathrm{H}), 0.80$ $(\mathrm{s}, 3 \mathrm{H})$.

${ }^{13} \mathrm{C}$ NMR (100 MHz, $\left.\mathrm{CDCl}_{3}\right) \delta$ 140.8, 139.7, 139.1, 138.4, 137.2, 127.7, 127.0, 126.7, 126.0, 124.5, 90.9, 76.8, 74.4, 69.4, 58.0, 50.6, 44.5, 43.4, 38.6, 38.2, 29.8, 27.9, 27.4, 26.4, 23.2, 18.1, 12.1, 11.7.

$[\alpha]_{\mathrm{D}^{23.3}}=+48.25\left(\mathrm{c}=0.97, \mathrm{CHCl}_{3}, 2: 98 \mathrm{dr}\right)$; Diastereomeric excess was determined by chiral HPLC (Daicel Chiralpak IA, hexane/isopropyl alcohol $=97 / 3$, flow rate $=1.0 \mathrm{~mL} / \mathrm{min}, \lambda=220 \mathrm{~nm}$ ), $t^{1}=6.7 \mathrm{~min}$ (major),$t^{2}=7.7 \mathrm{~min}$ (minor).

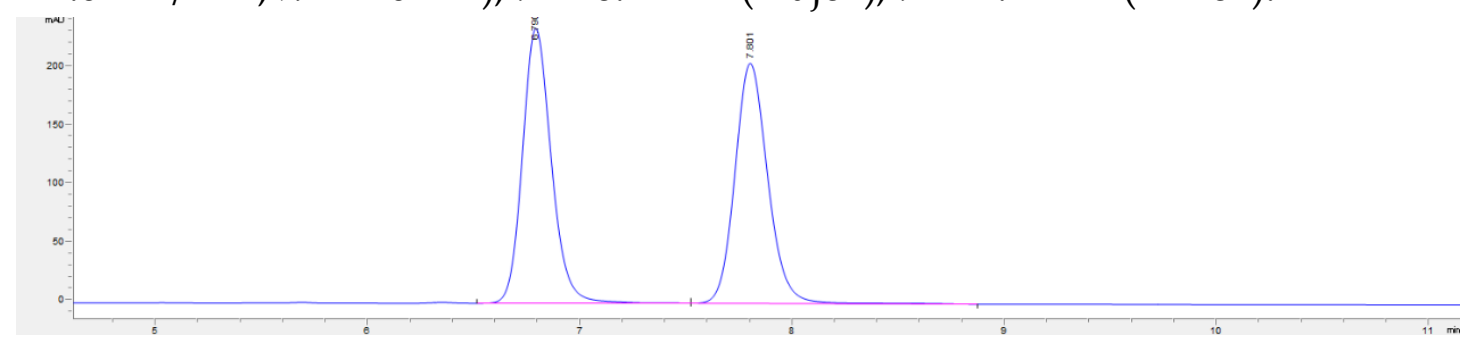



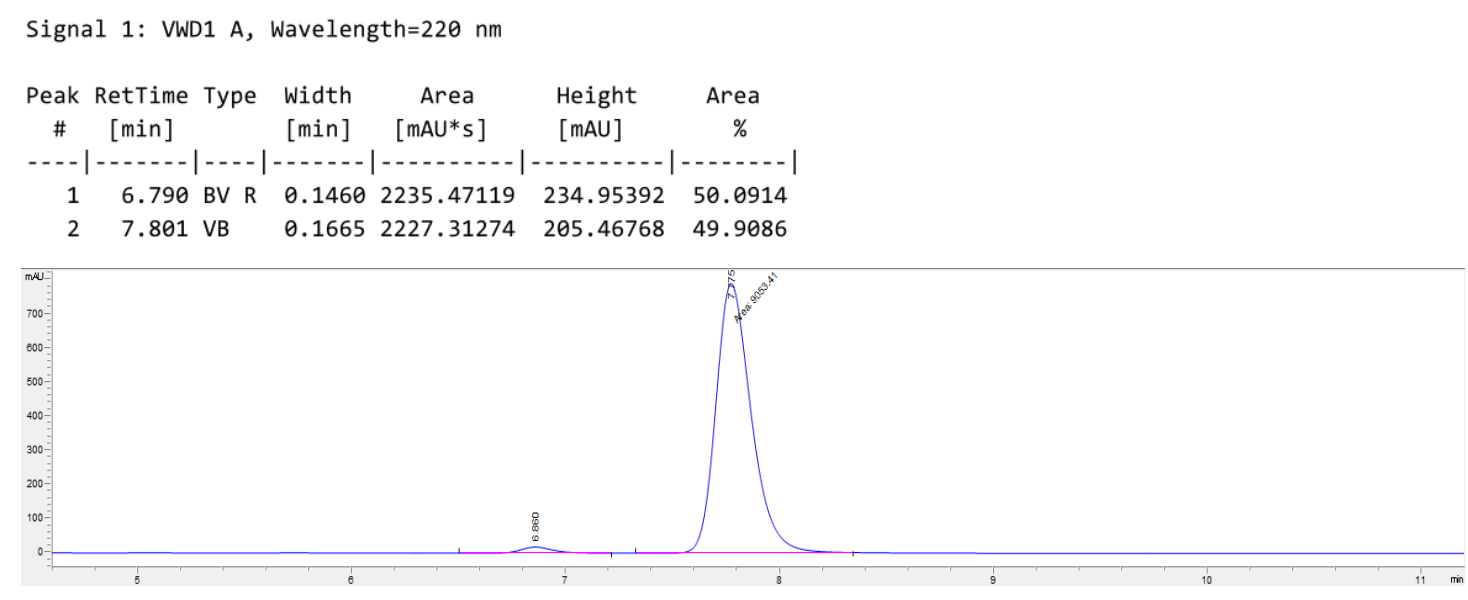

Signal 1: VWD1 A, Wavelength $=220 \mathrm{~nm}$

\begin{tabular}{|c|c|c|c|c|c|c|}
\hline $\begin{array}{c}\text { Peak } \\
\#\end{array}$ & $\begin{array}{c}\text { RetTime } \\
\text { [min] }\end{array}$ & Type & $\begin{array}{l}\text { Width } \\
\text { [min] }\end{array}$ & $\begin{array}{c}\text { Area } \\
{\left[\mathrm{mAU}^{*} \mathrm{~s}\right]}\end{array}$ & $\begin{array}{l}\text { Height } \\
\text { [mAU] }\end{array}$ & $\begin{array}{c}\text { Area } \\
\%\end{array}$ \\
\hline & & & & 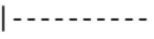 & & \\
\hline 1 & 6.8 & & & 182.99240 & 17.62560 & 1.9812 \\
\hline 2 & 7.77 & $\mathrm{~N}$ & 915 & 9053.40625 & 787.75751 & 98.0188 \\
\hline
\end{tabular}

(S)-7-(Pyridin-2-yl)-1-((triisopropylsilyl)oxy)heptan-2-ol (3ax)

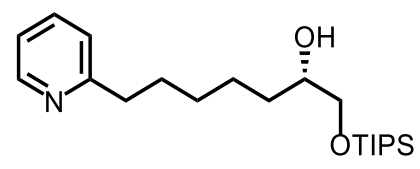

Compound 3ax was prepared as a colorless oil in $76 \%$ yield $(55.5 \mathrm{mg}$, eluent: petroleum ether/EtOAc = 10/1), from 2-(7-((triisopropylsilyl)oxy)hept-6-en-1yl)pyridine 1ax $(E / Z=1 / 1.1)(69.5 \mathrm{mg}, 0.20 \mathrm{mmol})$, [RhCl(cod) $]_{2}(4.0 \mathrm{mg}, 0.004$ mmol), (S)-L9 (7.0 mg, $0.008 \mathrm{mmol})$, HBpin (38.4 mg, $0.30 \mathrm{mmol})$ and LiOAc $(4.0 \mathrm{mg}, 0.06 \mathrm{mmol})$ following the above general procedure $\mathrm{E}\left(30^{\circ} \mathrm{C}, 48 \mathrm{~h}\right)$.

$\mathbf{R}_{f}=0.65$ (petroleum ether/EtOAc $=10 / 1$ )

${ }^{1} \mathrm{H}$ NMR (400 MHz, $\left.\mathrm{CDCl}_{3}\right) \delta 8.51(\mathrm{~d}, J=4.0 \mathrm{~Hz}, 1 \mathrm{H}), 7.62-7.54(\mathrm{~m}, 1 \mathrm{H}), 7.17$ $7.05(\mathrm{~m}, 2 \mathrm{H}), 3.76-3.60(\mathrm{~m}, 2 \mathrm{H}), 3.53-3.40(\mathrm{~m}, 1 \mathrm{H}), 2.88-2.75(\mathrm{~m}, 2 \mathrm{H}), 2.65(\mathrm{~s}$, $1 \mathrm{H}), 1.83-1.66(\mathrm{~m}, 2 \mathrm{H}), 1.57-1.48(\mathrm{~m}, 1 \mathrm{H}), 1.48-1.35(\mathrm{~m}, 5 \mathrm{H}), 1.10-1.02(\mathrm{~m}$, $21 \mathrm{H})$.

${ }^{13} \mathrm{C}$ NMR (100 MHz, $\left.\mathrm{CDCl}_{3}\right) \delta$ 162.5, 149.2, 136.3, 122.8, 121.0, 72.0, 67.7, 38.4, $32.8,29.9,29.5,25.5,18.1,12.0$.

HRMS (ESI') calcd for $\mathrm{C}_{21} \mathrm{H}_{40} \mathrm{O}_{2} \mathrm{NSi}^{+}[\mathrm{M}+\mathrm{H}]^{+}$: 366.2823, found: 366.2817.

IR (neat, cm$^{-1}$ ): 2940, 2865, 1464, 1123, 1101, 883, 681

$[\alpha]_{\mathrm{D}^{23.3}}=2.83\left(\mathrm{c}=1.21, \mathrm{CHCl}_{3}, 88 \%\right.$ ee $)$. Enantiomeric excess was determined by chiral HPLC (Daicel Chiralpak IC, hexane/isopropyl alcohol $=95 / 5$, flow rate $=$ $1.0 \mathrm{~mL} / \mathrm{min}, \lambda=220 \mathrm{~nm}$ ) $t^{1}=10.6 \mathrm{~min}$ (major), $t^{2}=11.4 \mathrm{~min}$ (minor). 


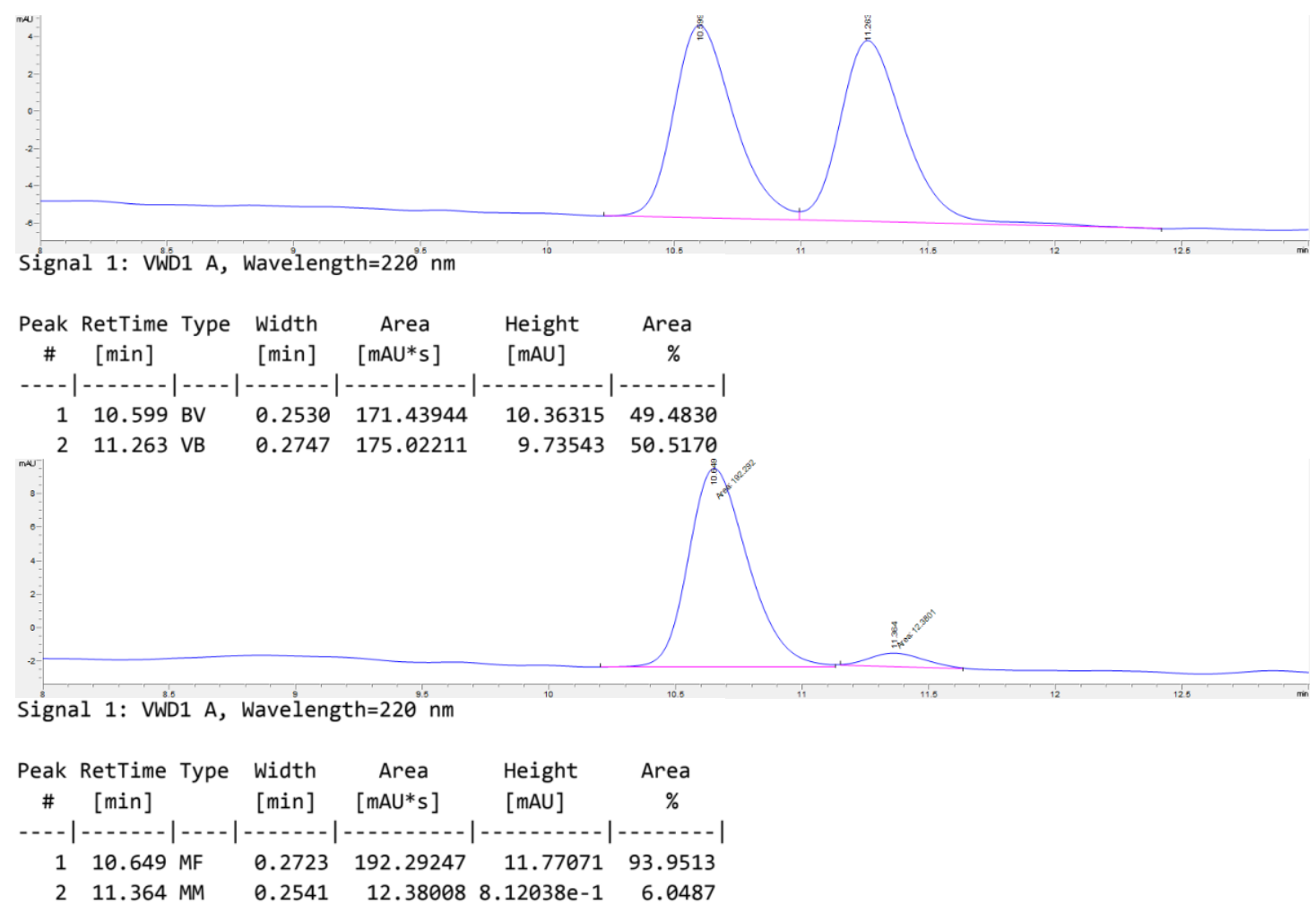

\section{Control Experiments}

\section{Hydroboration of aryl-substituted silyl enol ether 1ac}

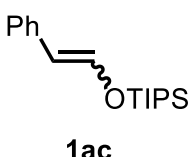

1 ac

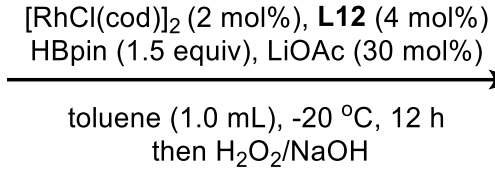
then $\mathrm{H}_{2} \mathrm{O}_{2} / \mathrm{NaOH}$

$\frac{1 \text { ac }}{(Z \text { only })}$
$(E / Z=1.9 / 1)$
(E only $)$

$[\mathrm{RhCl}(\mathrm{cod})]_{2}(2 \mathrm{~mol} \%)$, L9 (4 mol\%)

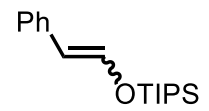

1 ac

\section{HBpin (1.5 equiv), LiOAc (30 mol\%)}

DME (0.5 mL), $40^{\circ} \mathrm{C}, 24 \mathrm{~h}$ then $\mathrm{H}_{2} \mathrm{O}_{2} / \mathrm{NaOH}$<smiles>OC(C[OH+])c1ccccc1</smiles>

(R)-3ac
L12: $A r^{\prime}=3,5-i \mathrm{Pr}_{2} \mathrm{C}_{6} \mathrm{H}_{3}$

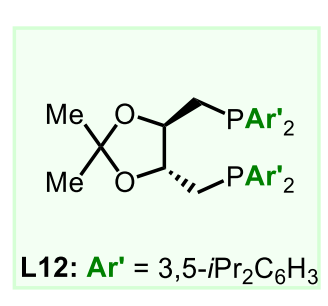

(R)-3ac

$95 \%, 95 \%$ ee

$72 \%, 46 \%$ ee

$58 \%, 45 \%$ ee

\begin{tabular}{cc} 
1ac & $\frac{(R)-3 a c}{\text { (Z only) }}$ \\
\cline { 3 - 3 }$(E$ only) & $45 \%, 50 \%$ ee \\
$64 \%, 12 \%$ ee
\end{tabular}

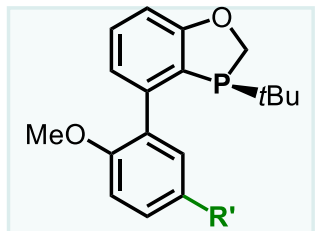

L9: $\mathrm{R}^{\prime}=3,5-\mathrm{OMe}_{2} \mathrm{C}_{6} \mathrm{H}_{3}$

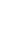




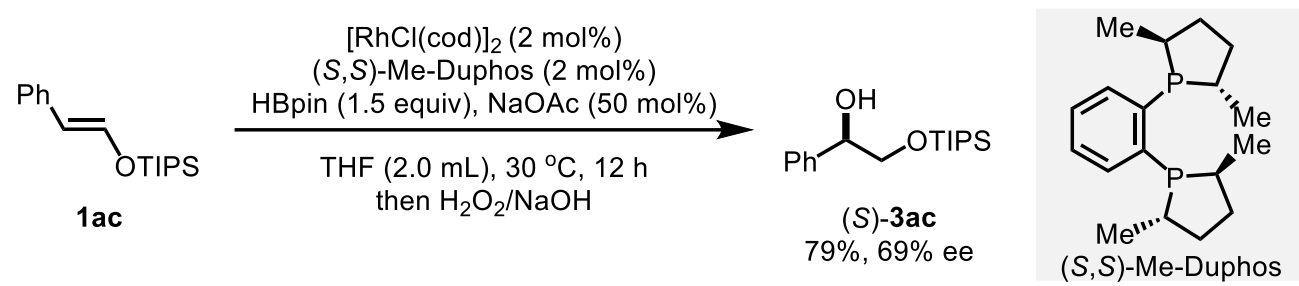

$(S, S)-M e-D u p h o s$ as ligand: In a glove box, to an oven-dried $10-\mathrm{mL}$ vial were added $[\mathrm{RhCl}(\mathrm{cod})]_{2}(3.0 \mathrm{mg}, 0.006 \mathrm{mmol}),(S, S)-\mathrm{Me}-\mathrm{Duphos}(1.8 \mathrm{mg}, 0.006$ $\mathrm{mmol})$ and anhydrous DME $(2.0 \mathrm{~mL})$. The resulting solution was stirred for 10 min at room temperature, at which time HBpin $(57.6 \mathrm{mg}, 0.45 \mathrm{mmol})$ was added. After the resulting mixture was stirred for an additional $10 \mathrm{~min}, \mathrm{NaOAc}$ (12.3 $\mathrm{mg}, 0.15 \mathrm{mmol}$ ) was added and stirred for $30 \mathrm{~min}$. (E)-1ac $(82.9 \mathrm{mg}, 0.30 \mathrm{mmol})$ was then added at room temperature and stirred for $12 \mathrm{~h}$ at $30{ }^{\circ} \mathrm{C}$. The resulting suspension was quenched with $\mathrm{H}_{2} \mathrm{O}$ and cooled to room temperature, filtered through a silica gel pad and washed with EtOAc, concentrated.The residue was dissolved in THF $(1.0 \mathrm{~mL})$ and cooled to $0{ }^{\circ} \mathrm{C}$, then $\mathrm{NaOH}(1.5$ equiv, $3.0 \mathrm{M})$, $\mathrm{H}_{2} \mathrm{O}_{2}$ (1.5 equiv, 30\%) was added. The resulting mixture was stirred for $0.5 \mathrm{~h}$, then extracted with EtOAc, dried with $\mathrm{Na}_{2} \mathrm{SO}_{4}$, filtered and concentrated. The residue was purified by silica gel flash chromatography (eluent: petroleum ether/EtOAc $=20 / 1)$ to afford compound 3ac as a colorless oil in 79\% yield $(69.7$ $\mathrm{mg}, 69 \%$ ee).

\section{Control Experiments for the hydroboration of $1 \mathrm{a}^{a}$}

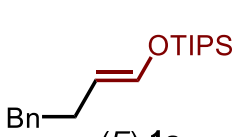

(E)-1a

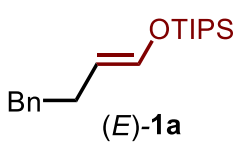

(E)-1a

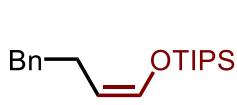

(Z)-1a

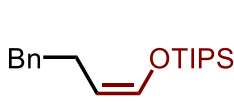

(Z)-1a
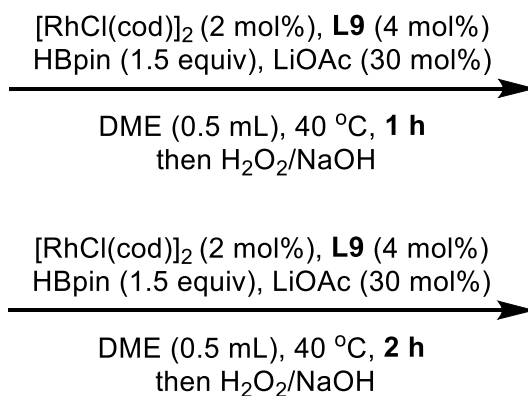

[RhCl(cod) $]_{2}$ (2 mol\%), L9 (4 mol\%) HBpin (1.5 equiv), LiOAc (30 mol\%)

DME $(0.5 \mathrm{~mL}), 40^{\circ} \mathrm{C}, 15 \mathrm{~min}$ then $\mathrm{H}_{2} \mathrm{O}_{2} / \mathrm{NaOH}$

$[\mathrm{RhCl}(\mathrm{cod})]_{2}$ (2 mol\%), L9 (4 mol\%)

HBpin (1.5 equiv), LiOAc (30 mol\%)

DME $(0.5 \mathrm{~mL}), 40^{\circ} \mathrm{C}, 1.5 \mathrm{~h}$ then $\mathrm{H}_{2} \mathrm{O}_{2} / \mathrm{NaOH}$

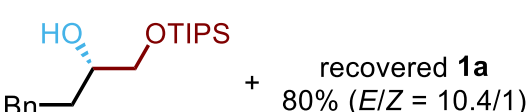

$3 a, 12 \%$

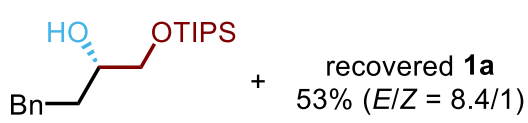

$3 a, 23 \%$
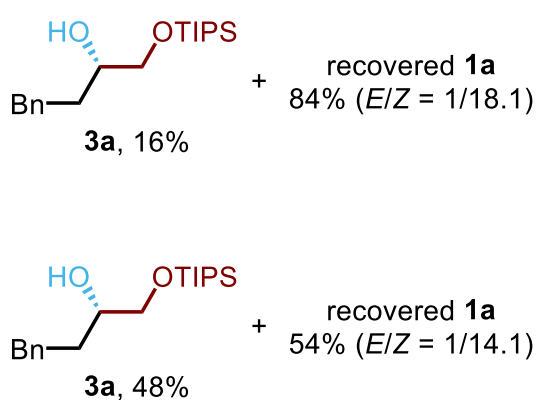


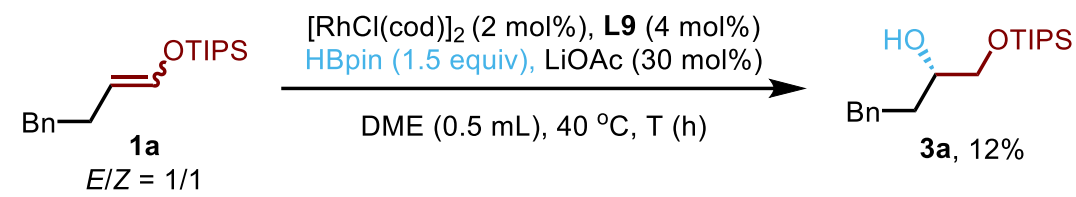

\begin{tabular}{ccc}
$\mathrm{T}(\mathrm{h})$ & conv. $(\%)$ & $E / Z$ \\
\hline 0.5 & 16 & $1.3 / 1$ \\
1.5 & 38 & $1.8 / 1$ \\
3.0 & 52 & $2.0 / 1$ \\
8.0 & 83 & $2.4 / 1$
\end{tabular}

a 1a $(0.1 \mathrm{mmol}),{ }^{1} \mathrm{H}$ NMR yield.

\section{Gram-scale Reaction and Applications}

\section{Gram-scale Reaction}
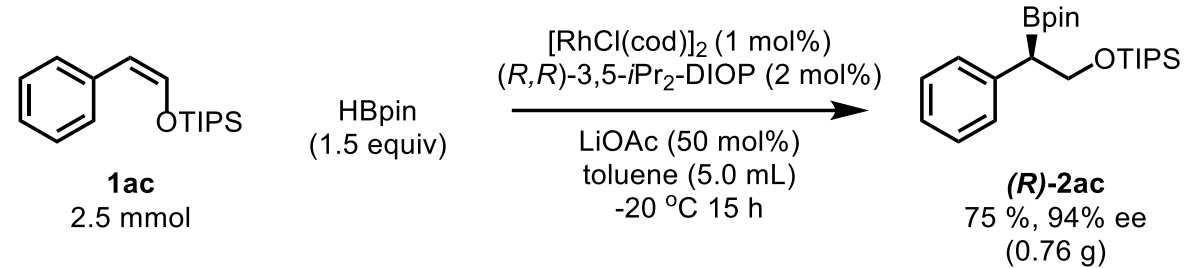

In a glove box, to an oven-dried 10-mL vial were added $[\mathrm{RhCl}(\mathrm{cod})]_{2}(12.3$ $\mathrm{mg}, 0.025 \mathrm{mmol}),(R, R)-3,5-i \operatorname{Pr}_{2}$-DIOP $(41.7 \mathrm{mg}, 0.050 \mathrm{mmol})$ and anhydrous toluene $(5.0 \mathrm{~mL})$. The resulting solution was stirred for $10 \mathrm{~min}$ at room temperature, at which time HBpin $(0.48 \mathrm{~g}$, $3.75 \mathrm{mmol})$ was added. After stirred for an additional $10 \mathrm{~min}$, LiOAc $(82.5 \mathrm{mg}, 1.25 \mathrm{mmol})$ was added and stirred for $30 \mathrm{~min}$. (Z)-1ac was then added at $-20^{\circ} \mathrm{C}$ and stirred for $15 \mathrm{~h}$. The resulting suspension was quenched with $\mathrm{H}_{2} \mathrm{O}$ and warmed up to room temperature, filtered through a silica gel pad and washed with EtOAc, concentrated. The residue was purified by silica gel chromatography to afford compound 2ac as a colorless oil in $75 \%$ yield $(0.76 \mathrm{~g}$, petroleum ether $/ \mathrm{EtOAc}=30 / 1)$.

$\mathbf{R}_{f}=0.72$ (petroleum ether $/$ EtOAc $=20 / 1$ )

${ }^{1} \mathrm{H}$ NMR (400 MHz, $\left.\mathrm{CDCl}_{3}\right) \delta$ 7.29-7.20 (m, 4H), 7.17-7.10 (m, 1H), 4.09-4.01 (m, $1 \mathrm{H}), 3.91(\mathrm{dd}, J=9.1,6.9 \mathrm{~Hz}, 1 \mathrm{H}), 2.64(\mathrm{dd}, J=8.6,6.8 \mathrm{~Hz}, 1 \mathrm{H}), 1.28-1.18(\mathrm{~m}$, $12 \mathrm{H}), 1.07-0.99(\mathrm{~m}, 21 \mathrm{H})$.

${ }^{13} \mathrm{C}$ NMR (100 MHz, $\left.\mathrm{CDCl}_{3}\right) \delta$ 140.3, 129.2, 128.3, 125.7, 83.5, 66.7, 24.9, 24.8, 18.1, 12.1. 
HRMS (ESI') calcd for $\mathrm{C}_{23} \mathrm{H}_{41} \mathrm{BNaO}_{3} \mathrm{Si}^{+}$[M+Na] ${ }^{+}$: 427.2810, found: 427.2809.

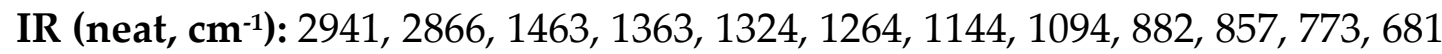

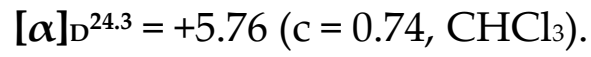

Compound 2ac was subjected to oxidation using $\mathrm{H}_{2} \mathrm{O}_{2}$ and the resulting alcohol was analyzed by chiral HPLC. Enantiomeric excess was determined by chiral HPLC (Daicel Chiraldel OD-H, hexane/isopropyl alcohol $=95 / 5$, flow rate $=0.5$ $\mathrm{mL} / \mathrm{min}, \lambda=220 \mathrm{~nm}$ ),$t^{1}=8.4 \mathrm{~min}$ (minor),$t^{2}=9.7 \mathrm{~min}$ (major).

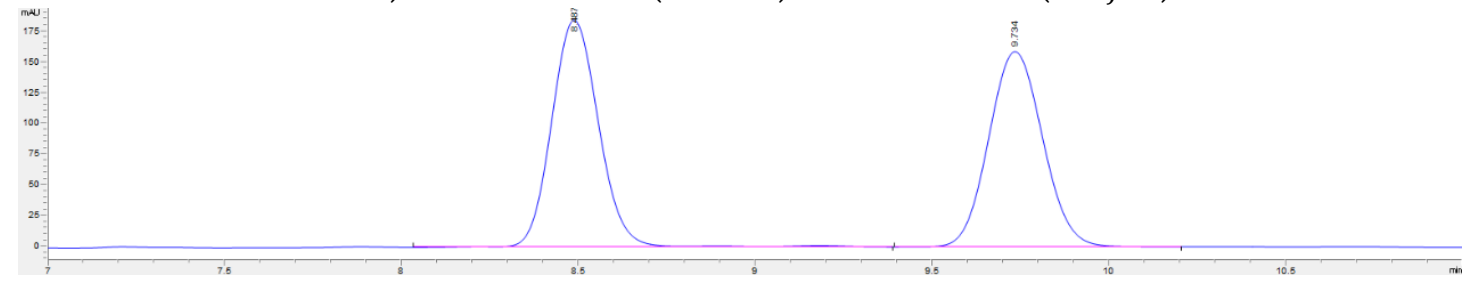

Signal 1: VWD1 A, Wavelength=220 nm
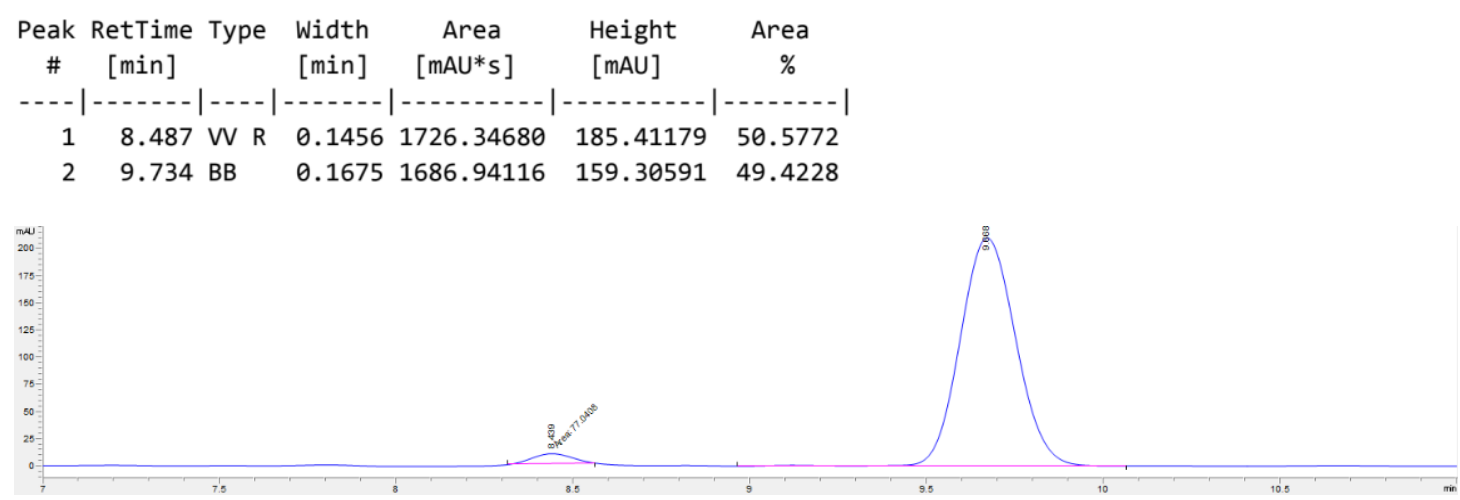

Signal 1: VWD1 A, Wavelength $=220 \mathrm{~nm}$

\begin{tabular}{cccccc}
$\begin{array}{c}\text { Peak RetTime Type } \\
\text { Width } \\
\text { [min] }\end{array}$ & $\begin{array}{c}\text { Area } \\
\text { [min] }\end{array}$ & $\begin{array}{c}\text { Height } \\
{[\text { mAU*s }]}\end{array}$ & $\begin{array}{c}\text { Area } \\
{[\mathrm{mAU}]}\end{array}$ & $\%$ \\
\hline 1 & 8.439 MM & 0.1328 & 77.04077 & 9.66584 & 3.2502 \\
2 & 9.668 VB R & 0.1725 & 2293.31519 & 209.83167 & 96.7498
\end{tabular}

\section{Synthesis of 4}

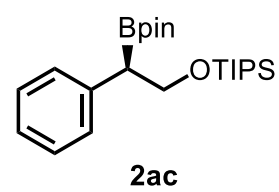

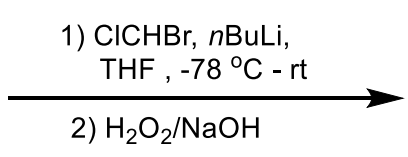<smiles>OC[C@H](CO[Na])c1ccccc1</smiles>

Following a modified procedure ${ }^{32}$, to a solution of $2 \mathrm{ac}(80.8 \mathrm{mg}, 0.20 \mathrm{mmol})$ and bromochloromethane $(64.7 \mathrm{mg}, 0.50 \mathrm{mmol})$ in THF was added $n \mathrm{BuLi}(2.5$ $\mathrm{M}$ in hexane, 2.3 equiv) at $-78^{\circ} \mathrm{C}$. The resulting mixture was then warmed up to room temperature and stirred for $2 \mathrm{~h}$, then quenched with saturated $\mathrm{NH}_{4} \mathrm{Cl}$, extracted with EtOAc, dried with $\mathrm{Na}_{2} \mathrm{SO}_{4}$, filtered and concentrated. The crude 
residue was dissolved in THF $(1.0 \mathrm{~mL})$ and cooled to $0{ }^{\circ} \mathrm{C}$, then $\mathrm{NaOH}$ (1.5 equiv, $3.0 \mathrm{M})$ and $\mathrm{H}_{2} \mathrm{O}_{2}(1.5$ equiv, $30 \%)$ was added. The resulting mixture was stirred for $0.5 \mathrm{~h}$, then extracted with EtOAc, dried with $\mathrm{Na}_{2} \mathrm{SO}_{4}$, filtered and concentrated. The residue was purified by silica gel chromatography to afford compound 4 as a colorless oil in $76 \%$ yield (46.8 $\mathrm{mg}$, eluent: petroleum ether/EtOAc $=100 / 5)$.

$\mathbf{R}_{f}=0.28$ (petroleum ether $/$ EtOAc $=10 / 1$ )

${ }^{1} \mathrm{H}$ NMR (400 MHz, $\mathrm{CDCl}_{3}$ ) $\delta$ 7.36-7.17 (m, 5H), 4.17-4.07 (m, 1H), 4.06-3.99 (m, 2H), 3.96-3.83 (m, 1H), 3.21-3.07 (m, 1H), 2.98-2.85 (m, 1H), 1.19-0.96 (m, $21 \mathrm{H})$.

${ }^{13} \mathrm{C}$ NMR (100 MHz, $\left.\mathrm{CDCl}_{3}\right) \delta$ 139.8, 128.7, 128.1, 127.2, 68.3, 67.1, 49.8, 18.1, 11.9.

HRMS (ESI') calcd for $\mathrm{C}_{18} \mathrm{H}_{32} \mathrm{NaO}_{2} \mathrm{Si}^{+}[\mathrm{M}+\mathrm{Na}]^{+}$: 331.2064, found: 331.2063 .

IR (neat, cm-1): 2941, 2866, 1463, 1384, 1107, 1017, 883, 811, 758, 695.

$[\alpha]_{\mathrm{D}^{24.3}}=+7.02\left(\mathrm{c}=0.20, \mathrm{CHCl}_{3}, 91 \%\right.$ ee); Enantiomeric excess was determined by chiral HPLC (Daicel Chiralcel OD-H, hexane/isopropyl alcohol =98/2, flow rate $=0.8 \mathrm{~mL} / \mathrm{min}, \lambda=220 \mathrm{~nm}$ ),$t^{1}=7.7 \mathrm{~min}$ (major),$t^{2}=8.1 \mathrm{~min}$ (minor).

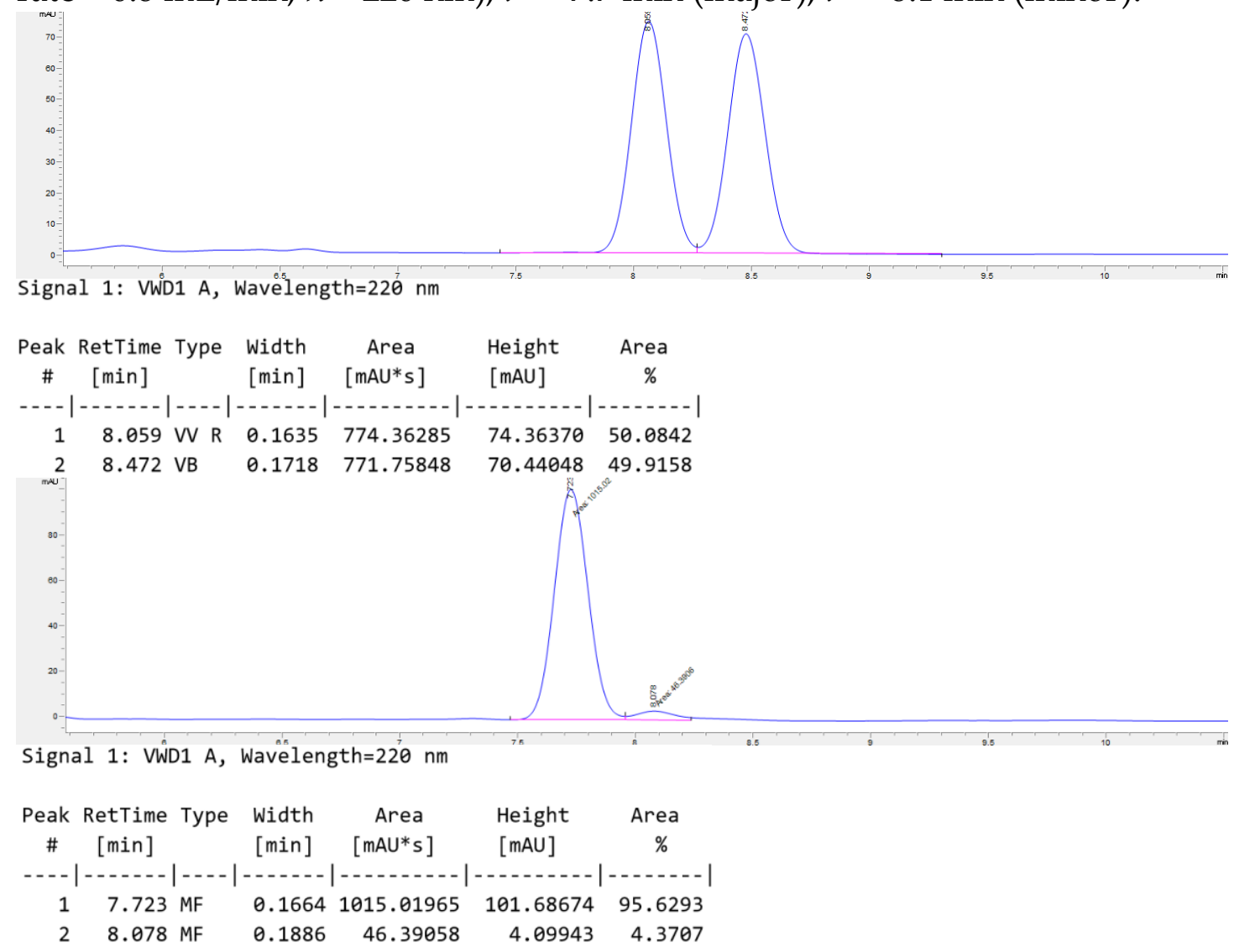

\section{Synthesis of 5}




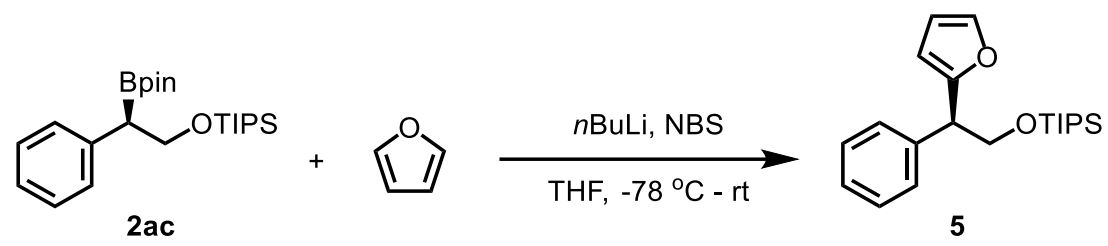

Following a modified procedure ${ }^{32}$, to a solution of furan $(20.4 \mathrm{mg}, 0.30$ $\mathrm{mmol})$ in THF was added $n \mathrm{BuLi}\left(2.5 \mathrm{M}\right.$ in hexane, 1.5 equiv) at $-78^{\circ} \mathrm{C}$. After stirred for $1 \mathrm{~h}$ at room temperature, the reaction mixture was cooled back to -78 ${ }^{\circ} \mathrm{C}$ and $2 \mathrm{ac}(80.8 \mathrm{mg}, 0.20 \mathrm{mmol})$ was added. The reaction was vigorously stirred at $-78{ }^{\circ} \mathrm{C}$ for $5 \mathrm{~h}$ and a solution of NBS $(53.4 \mathrm{mg}, 0.30 \mathrm{mmol})$ in THF was added. The resulting mixture was then warmed up to room temperature and stirred for $5 \mathrm{~h}$, then quenched with saturated $\mathrm{NH}_{4} \mathrm{Cl}$, extracted with EtOAc, dried with $\mathrm{Na}_{2} \mathrm{SO}_{4}$, filtered and concentrated. The residue was purified by silica gel flash chromatography to afford compound 5 as a colorless oil in $67 \%$ yield (46.1 mg, eluent: petroleum ether).

$\mathbf{R}_{f}=0.62$ (petroleum ether)

${ }^{1} \mathrm{H}$ NMR (400 MHz, $\mathrm{CDCl}_{3}$ ) $\delta$ 7.36-7.17 (m, 6H), 6.32-6.28 (m, 1H), 6.18-6.14 (m, 1H), 4.25-4.14 (m, 2H), 4.12-4.05 (m, 1H), 1.11-0.83 (m, 21H). ${ }^{13} \mathrm{C}$ NMR (100 MHz, $\left.\mathrm{CDCl}_{3}\right) \delta$ 155.7, 141.4, 140.4, 128.6, 128.4, 126.9, 110.2, 106.6, $66.5,48.7,18.0,12.1$.

HRMS (ESI') calcd for $\mathrm{C}_{21} \mathrm{H}_{32} \mathrm{NaO}_{2} \mathrm{Si}^{+}[\mathrm{M}+\mathrm{Na}]^{+}$: 367.2064, found: 367.2063.

IR (neat, cm-1): 2941, 2866, 1462, 1113, 1010, 882, 799, 769, 730, 688.

$[\alpha]_{\mathrm{D}^{24.3}}=+18.20\left(\mathrm{c}=0.51, \mathrm{CHCl}_{3}, 94 \%\right.$ ee $)$.

Compound 5 was subjected to general procedure I and the resulting alcohol was analyzed by chiral HPLC. Enantiomeric excess was determined by chiral HPLC (Daicel Chiralpak IC, hexane/isopropyl alcohol $=98 / 2$, flow rate $=1.0$ $\mathrm{mL} / \mathrm{min}, \lambda=220 \mathrm{~nm}$ ), $t^{1}=11.6 \mathrm{~min}$ (major),$t^{2}=12.3 \mathrm{~min}$ (minor).

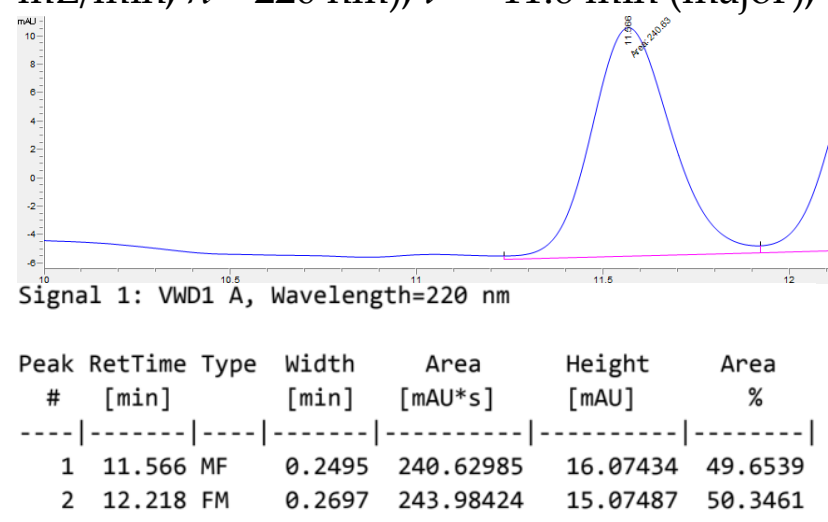




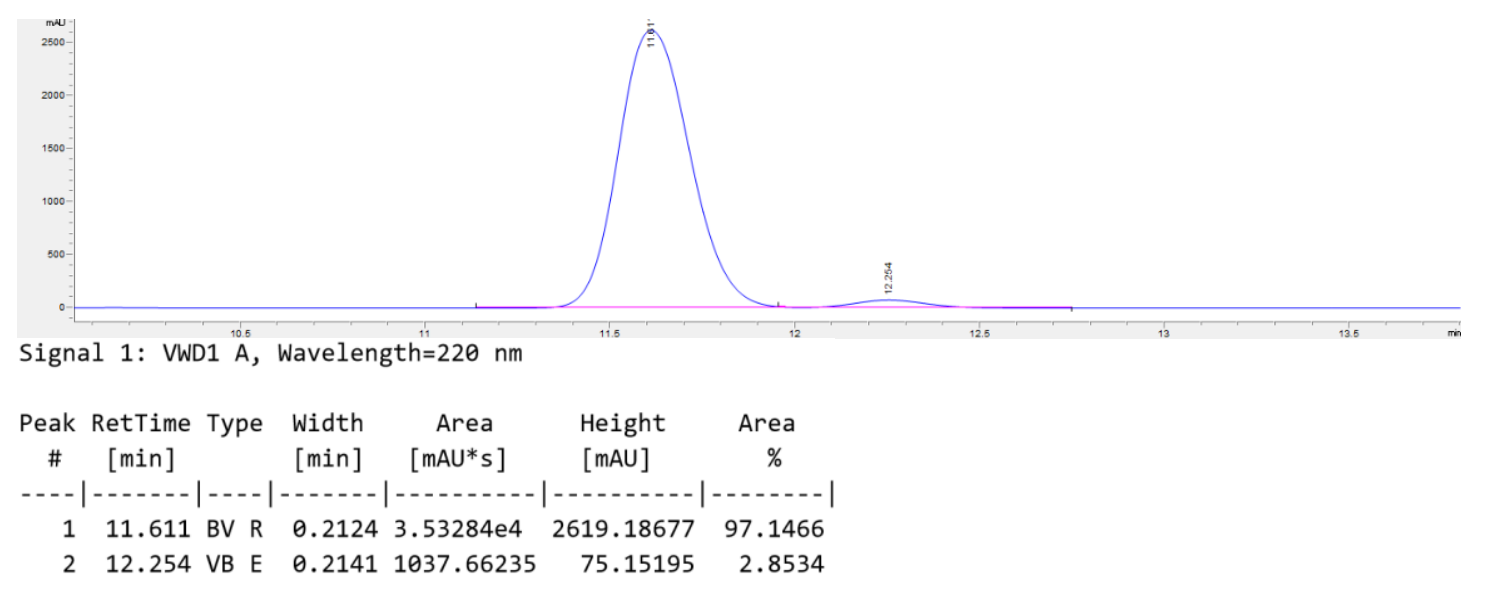

\section{Synthesis of 6}

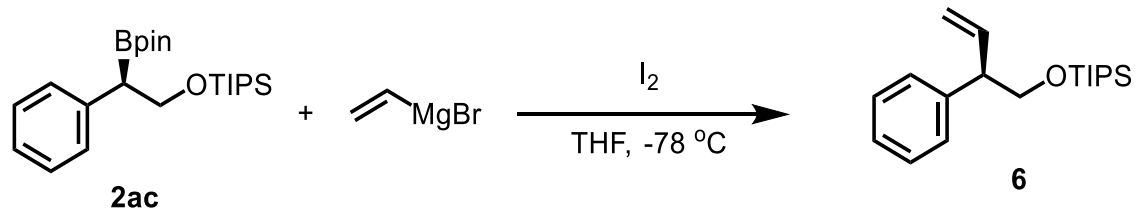

Following a modified procedure ${ }^{33}$, to a solution of $2 \mathrm{ac}(80.8 \mathrm{mg}, 0.20 \mathrm{mmol})$ in THF was slowly added vinylmagnesium bromide (1.0 M in THF, 4.0 equiv) over $10 \mathrm{~min}$ at $-78^{\circ} \mathrm{C}$. After stirred for $0.5 \mathrm{~h}$ at $-78^{\circ} \mathrm{C}$, a solution of $\mathrm{I}_{2}(203.1 \mathrm{mg}$, $0.8 \mathrm{mmol}$ ) in THF was added and stirred for $0.5 \mathrm{~h}$, then allowed to warm to $0{ }^{\circ} \mathrm{C}$, quenched with $10 \%$ aqueous solution of $\mathrm{Na}_{2} \mathrm{~S}_{2} \mathrm{O}_{3}$, extracted with EtOAc, dried with $\mathrm{Na}_{2} \mathrm{SO}_{4}$, filtered and concentrated. The residue was purified by silica gel flash chromatography to afford 6 as a colorless oil in $75 \%$ yield ( $45.6 \mathrm{mg}$, eluent: petroleum ether).

$\mathbf{R}_{f}=0.86$ (petroleum ether)

${ }^{1} \mathrm{H}$ NMR (400 MHz, $\left.\mathrm{CDCl}_{3}\right)$ \& 7.33-7.18 (m, 5H), 6.21-6.02 (m, 1H), 5.22-5.01 $(\mathrm{m}, 2 \mathrm{H}), 3.99-3.83(\mathrm{~m}, 2 \mathrm{H}), 3.58-3.43(\mathrm{~m}, 1 \mathrm{H}), 1.18-0.87(\mathrm{~m}, 21 \mathrm{H})$. ${ }^{13} \mathrm{C}$ NMR (100 MHz, $\left.\mathrm{CDCl}_{3}\right) \delta$ d 142.0, 139.3, 128.41, 128.39, 126.5, 116.0, 67.6, 52.7, 18.1, 12.1 .

HRMS (ESI') calcd for $\mathrm{C}_{19} \mathrm{H}_{32} \mathrm{NaOSi}^{+}[\mathrm{M}+\mathrm{Na}]^{+}: 327.2115$, found: 327.2113 .

IR (neat, cm-1): 2941, 2865, 1463, 1109, 1071, 995, 916, 883, 780, 695. $[\alpha]_{\mathrm{D}^{24.3}}=-22.15\left(\mathrm{c}=0.40, \mathrm{CHCl}_{3}, 93 \%\right.$ ee $)$.

Compound 6a was subjected to general procedure I and the resulting alcohol was analyzed by chiral HPLC. Enantiomeric excess was determined by chiral HPLC (Daicel Chiralpak AD-H, hexane/isopropyl alcohol $=97 / 3$, flow rate $=0.5$ $\mathrm{mL} / \mathrm{min}, \lambda=220 \mathrm{~nm}$ ), $t^{1}=15.5 \mathrm{~min}$ (major),$t^{2}=16.1 \mathrm{~min}$ (minor). 


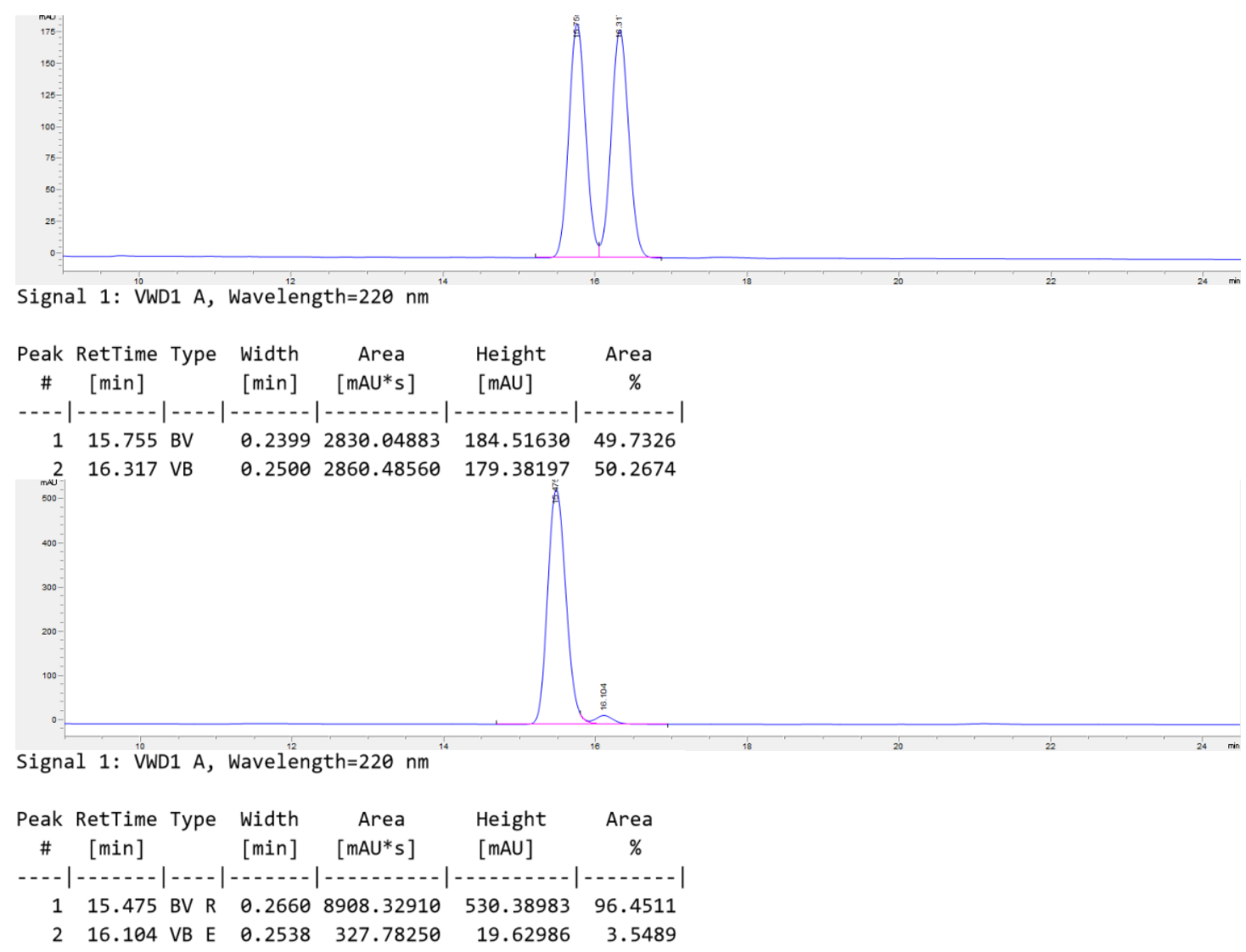

\section{Synthesis of 7}<smiles>O[PbH2]OC[C@H](Br)c1ccccc1</smiles>

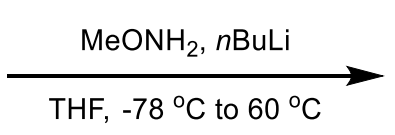

THF, $-78^{\circ} \mathrm{C}$ to $60^{\circ} \mathrm{C}$<smiles>N[C@@H](CO[PbH])c1ccccc1</smiles>

7

Following a modified procedure ${ }^{34}$, to a solution of $\mathrm{MeONH}_{2}(4.0 \mathrm{M}$ in THF, 3.0 equiv) in THF was added $n \operatorname{BuLi}\left(2.5 \mathrm{M}\right.$ in hexane, 3.0 equiv) at $-78{ }^{\circ} \mathrm{C}$. After stirred for $0.5 \mathrm{~h}$ at room temperature, 2ac $(159.0 \mathrm{mg}, 0.40 \mathrm{mmol})$ was added. The resulting mixture was then heated to $60{ }^{\circ} \mathrm{C}$ and stirred for $15 \mathrm{~h}$, then quenched with $\mathrm{H}_{2} \mathrm{O}$, extracted with EtOAc, dried with $\mathrm{Na}_{2} \mathrm{SO}_{4}$, filtered and concentrated. The residue was purified by silica gel chromatography to afford compound 7 as a colorless oil in $81 \%$ yield (94.9 mg, eluent: DCM/MeOH =20/1), which was previously reported ${ }^{35}$.

$\mathbf{R}_{f}=0.38(\mathrm{DCM} / \mathrm{MeOH}=10 / 1)$

${ }^{1} \mathrm{H}$ NMR (400 MHz, $\left.\mathrm{CDCl}_{3}\right) \delta 7.42-7.36(\mathrm{~m}, 2 \mathrm{H}), 7.36-7.29(\mathrm{~m}, 2 \mathrm{H}), 7.29$ $7.22(\mathrm{~m}, 1 \mathrm{H}), 4.10(\mathrm{dd}, J=8.5,3.9 \mathrm{~Hz}, 1 \mathrm{H}), 3.81(\mathrm{dd}, J=9.6,3.9 \mathrm{~Hz}, 1 \mathrm{H}), 3.67-$ $3.54(\mathrm{~m}, 1 \mathrm{H}), 1.88(\mathrm{~s}, 2 \mathrm{H}), 1.17-0.87(\mathrm{~m}, 21 \mathrm{H})$.

${ }^{13} \mathrm{C}$ NMR (100 MHz, $\left.\mathrm{CDCl}_{3}\right) \delta$ 142.7, 128.4, 127.4, 127.1, 70.0, 58.0, 18.1, 12.1. 
$[\alpha]_{\mathrm{D}^{24.3}}=-7.18\left(\mathrm{c}=1.34, \mathrm{CHCl}_{3}, 94 \%\right.$ ee $)$; Enantiomeric excess was determined by chiral HPLC (Daicel Chiralcel OD-H, hexane/isopropyl alcohol $=95 / 5$, flow rate $=0.5 \mathrm{~mL} / \mathrm{min}, \lambda=220 \mathrm{~nm}$ ), $t^{1}=8.2 \mathrm{~min}$ (minor),$t^{2}=10.4 \mathrm{~min}$ (major).

Enantiomeric excess was determined by chiral HPLC (Daicel Chiralcel OD-H, hexane/isopropyl alcohol $=95 / 5$, flow rate $=0.5 \mathrm{~mL} / \mathrm{min}, \lambda=220 \mathrm{~nm}), t^{1}=8.2$ $\min$ (minor), $t^{2}=10.4 \mathrm{~min}$ (major).

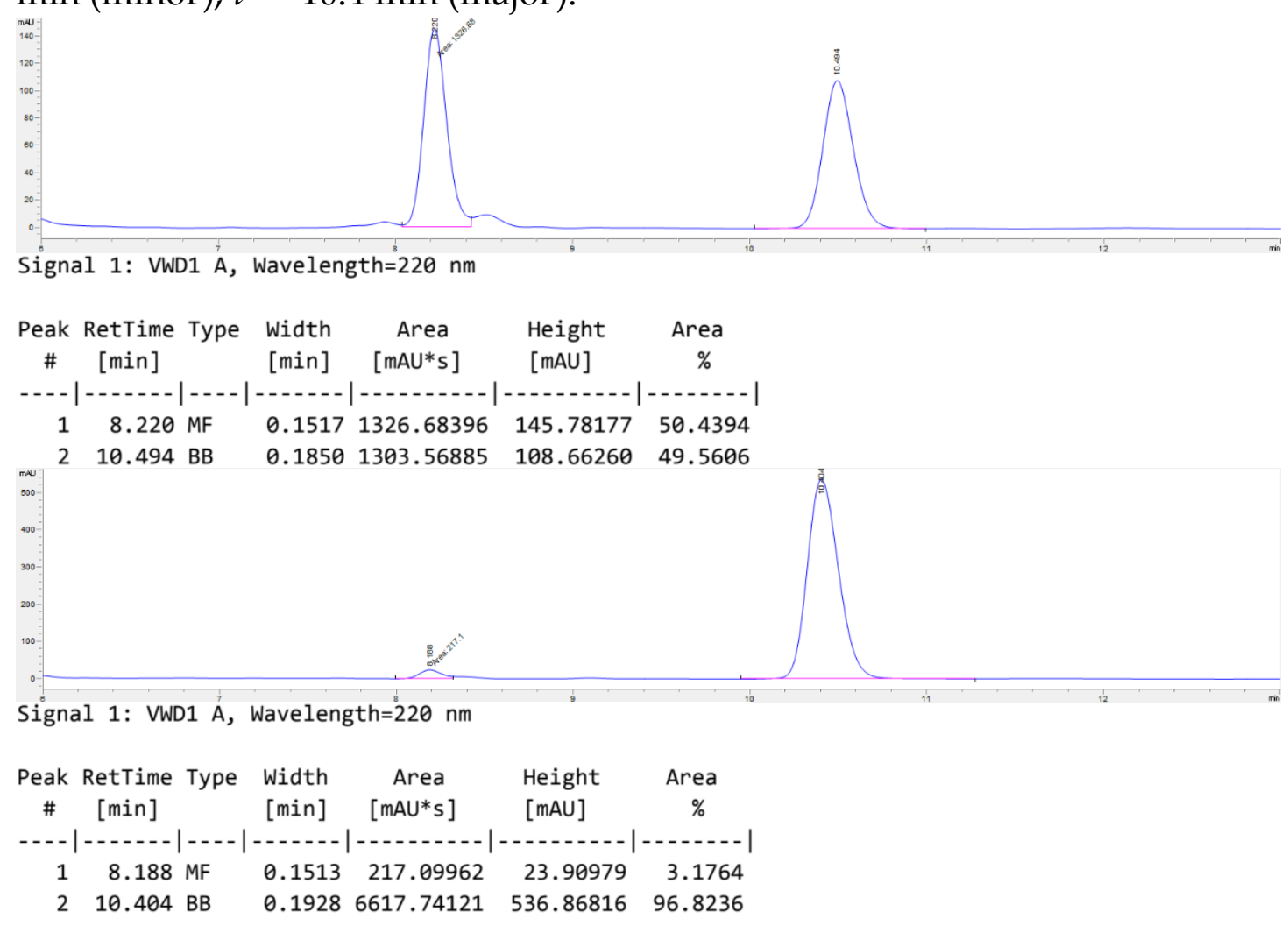

\section{Synthesis of 8}

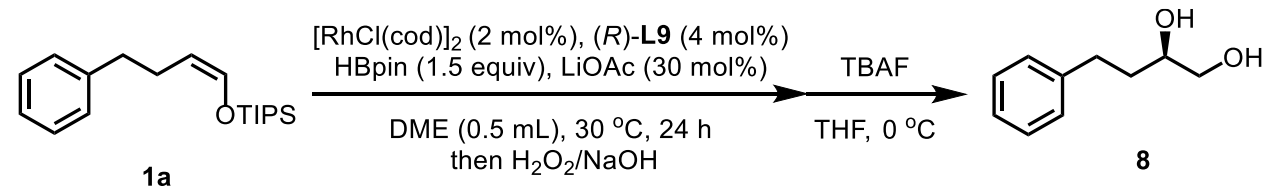

In a glove box, to an oven-dried $10 \mathrm{~mL}$ vial were added $[\mathrm{RhCl}(\mathrm{cod})]_{2}(2.0$ $\mathrm{mg}, 0.004 \mathrm{mmol}),(R)-\mathbf{L} 9(3.5 \mathrm{mg}, 0.008 \mathrm{mmol})$ and anhydrous DME $(0.5 \mathrm{~mL})$. The resulting solution was stirred for $10 \mathrm{~min}$ at room temperature, at which time HBpin ( $38.4 \mathrm{mg}, 0.30 \mathrm{mmol}$ ) was added. After the resulting mixture was stirred for an additional $10 \mathrm{~min}$, LiOAc $(4.0 \mathrm{mg}, 0.06 \mathrm{mmol})$ was added and stirred for $30 \mathrm{~min}$. (Z)-1a $(60.8 \mathrm{mg}, 0.20 \mathrm{mmol})$ was then added at room temperature and stirred for $24 \mathrm{~h}$ at $30{ }^{\circ} \mathrm{C}$. The resulting suspension was quenched with $\mathrm{H}_{2} \mathrm{O}$ and cooled to room temperature, filtered through a silica gel pad and washed with EtOAc, concentrated. The residue was dissolved in 
THF (1.0 mL) and cooled to $0{ }^{\circ} \mathrm{C}$, then $\mathrm{NaOH}(1.5$ equiv, $3.0 \mathrm{M}), \mathrm{H}_{2} \mathrm{O}_{2}$ (1.5 equiv, $30 \%)$ was added. The resulting mixture was stirred for $0.5 \mathrm{~h}$, then extracted with EtOAc, dried with $\mathrm{Na}_{2} \mathrm{SO}_{4}$, filtered and concentrated. The residue was dissolved in THF $(1.0 \mathrm{~mL})$ and cooled to $0{ }^{\circ} \mathrm{C}$, then TBAF $(1.0 \mathrm{M}$ in THF, 1.5 equiv) was added. The resulting mixture was stirred for $0.5 \mathrm{~h}$, then extracted with EtOAc, dried with $\mathrm{Na}_{2} \mathrm{SO}_{4}$, filtered and concentrated. The residue was purified by silica gel flash chromatography to afford compound 8 as a colorless oil in 70\% yield (3 steps, $23.2 \mathrm{mg}$, eluent: petroleum ether $/ \mathrm{EtOAc}=2 / 1$ ), which was previously reported ${ }^{36}$.

$\mathbf{R}_{f}=0.28$ (petroleum ether $/$ EtOAc $=1 / 1$ )

${ }^{1} \mathrm{H}$ NMR (400 MHz, $\left.\mathrm{CDCl}_{3}\right) \delta 8.31$ - $7.23(\mathrm{~m}, 2 \mathrm{H}), 7.22-7.12(\mathrm{~m}, 3 \mathrm{H}), 3.74-$

$3.57(\mathrm{~m}, 2 \mathrm{H}), 3.56-2.89(\mathrm{br}, 3 \mathrm{H}), 2.86-2.73(\mathrm{~m}, 1 \mathrm{H}), 2.72-2.59(\mathrm{~m}, 1 \mathrm{H}), 1.81-$ $1.64(\mathrm{~m}, 2 \mathrm{H})$.

${ }^{13} \mathrm{C}$ NMR (100 MHz, $\left.\mathrm{CDCl}_{3}\right) \delta$ 141.8, 128.5, 128.5, 126.0, 71.7, 66.8, 34.7, 31.9.

$[\alpha]_{\mathrm{D}^{24.3}}=+12.83\left(\mathrm{c}=0.94, \mathrm{CHCl}_{3}, 94 \%\right.$ ee $)$; Enantiomeric excess was determined by chiral HPLC (Daicel Chiralcel OD-H, hexane/isopropyl alcohol $=80 / 20$, flow rate $=1.0 \mathrm{~mL} / \mathrm{min}, \lambda=220 \mathrm{~nm}$ ),$t^{1}=6.6 \mathrm{~min}$ (major),$t^{2}=8.3 \mathrm{~min}$ (minor).

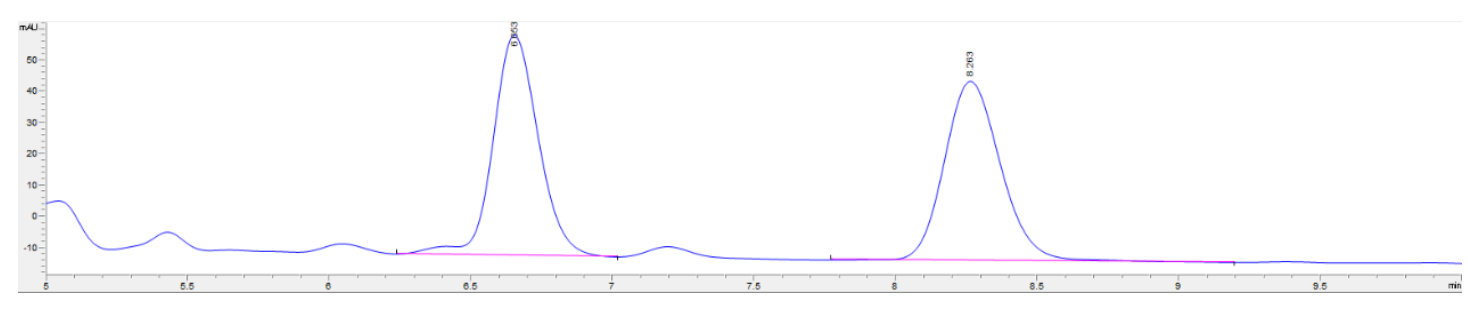

Signal 1: VWD1 A, Wavelength $=220 \mathrm{~nm}$
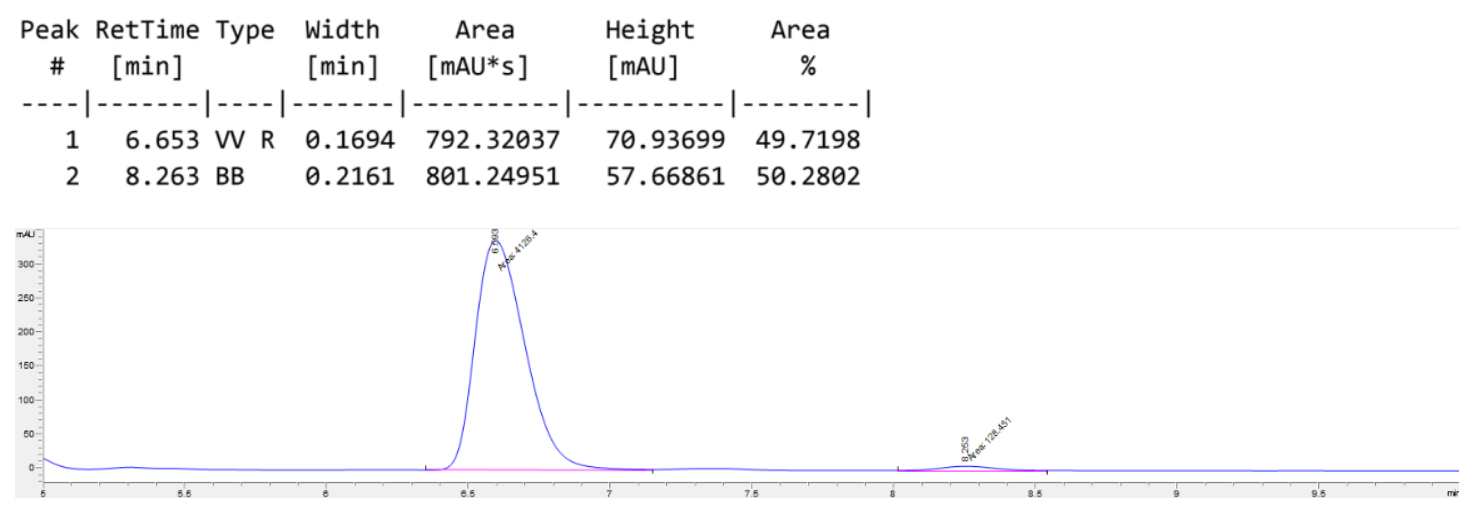

Signal 1: VWD1 A, Wavelength $=220 \mathrm{~nm}$

\begin{tabular}{cccccc}
$\begin{array}{c}\text { Peak RetTime Type } \\
\text { \# }\end{array}$ & $\begin{array}{c}\text { Width } \\
{[\mathrm{min}]}\end{array}$ & $\begin{array}{c}\text { Area } \\
{[\mathrm{min}]}\end{array}$ & $\begin{array}{c}\text { Height } \\
{[\mathrm{mAU} \text { s }]}\end{array}$ & $\begin{array}{c}\text { Area } \\
{[\mathrm{mAU}]}\end{array}$ & $\%$ \\
\hline 1 & 6.593 FM & 0.2041 & 4126.40332 & 337.02975 & 96.9811 \\
2 & 8.253 MM & 0.2755 & 128.45137 & 7.77073 & 3.0189
\end{tabular}




\section{Synthesis of 9}

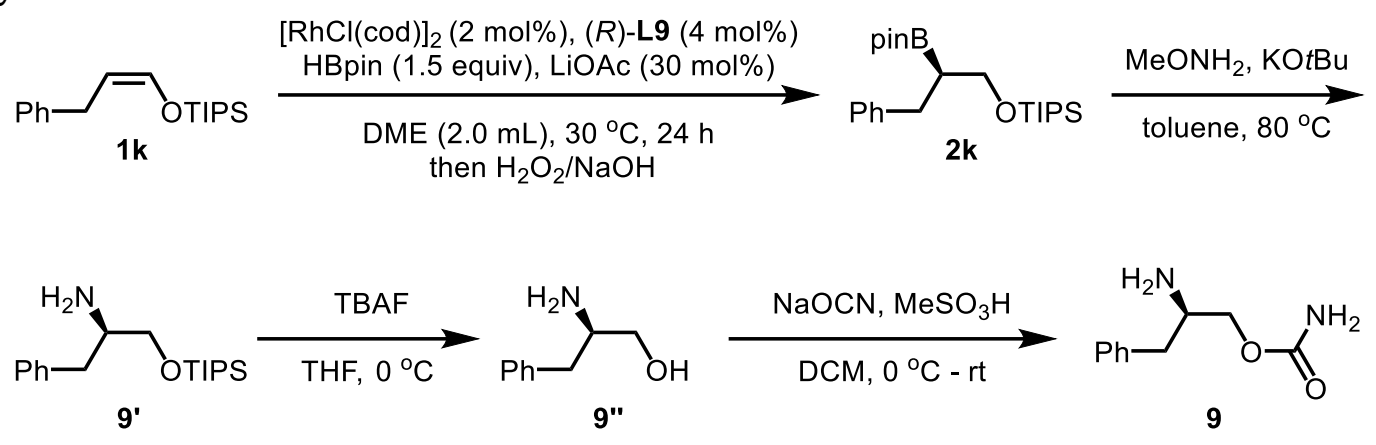

In a glove box, to an oven-dried $10 \mathrm{~mL}$ vial were added $[\mathrm{RhCl}(\operatorname{cod})]_{2}(4.9$ $\mathrm{mg}, 0.01 \mathrm{mmol}),(R)-\mathbf{L} 9(8.8 \mathrm{mg}, 0.02 \mathrm{mmol})$ and anhydrous DME $(2.0 \mathrm{~mL})$. The resulting solution was stirred for $10 \mathrm{~min}$ at room temperature, at which time HBpin (96.2 mg, $0.75 \mathrm{mmol}$ ) was added. After the resulting mixture was stirred for an additional $10 \mathrm{~min}$, LiOAc $(10.0 \mathrm{mg}, 0.15 \mathrm{mmol})$ was added and stirred for $30 \mathrm{~min}$. (Z)-1k (145.0 $\mathrm{mg}, 0.50 \mathrm{mmol})$ was then added at room temperature and stirred for $24 \mathrm{~h}$ at $30^{\circ} \mathrm{C}$. The resulting suspension was quenched with $\mathrm{H}_{2} \mathrm{O}$ and cooled to room temperature, filtered through a silica gel pad and washed with EtOAc, concentrated. The residue was purified by silica gel flash chromatography to afford compound $2 \mathbf{k}$ as a colorless oil in $78 \%$ yield (163.0 mg, eluent: petroleum ether $/ \mathrm{Et}_{2} \mathrm{O}=50 / 1$ ).

Following a modified procedure ${ }^{37}$, to a solution of $\mathbf{2 k}(83.6 \mathrm{mg}, 0.20 \mathrm{mmol})$ and $\mathrm{KO} t \mathrm{Bu}(44.9 \mathrm{mg}, 0.40 \mathrm{mmol})$ in toluene was added $\mathrm{MeONH}_{2}(4.0 \mathrm{M}$ in THF, 2.0 equiv) under $\mathrm{N}_{2}$. The resulting mixture was then heated to $80^{\circ} \mathrm{C}$ and stirred for $15 \mathrm{~h}$, then quenched with $\mathrm{H}_{2} \mathrm{O}$, extracted with EtOAc, dried with $\mathrm{Na}_{2} \mathrm{SO}_{4}$, filtered and concentrated. The residue was purified by silica gel chromatography to afford compound $\mathbf{9}^{\prime}$ as a colorless oil in $95 \%$ yield (58.4 $\mathrm{mg}$, eluent: $\mathrm{DCM} / \mathrm{MeOH}=20 / 1)$.

To a solution of 9' $^{\prime}(61.4 \mathrm{mg}, 0.20 \mathrm{mmol})$ in THF was added TBAF (1.0 M in THF, 1.5 equiv) at $0{ }^{\circ} \mathrm{C}$. The resulting mixture was stirred for $0.5 \mathrm{~h}$, then extracted with EtOAc, dried with $\mathrm{Na}_{2} \mathrm{SO}_{4}$, filtered and concentrated. The residue was purified by silica gel flash chromatography to afford compound 9" as a colorless oil in $81 \%$ yield $(24.5 \mathrm{mg}$, eluent: $\mathrm{DCM} / \mathrm{MeOH}=3 / 1)$.

Following a modified procedure ${ }^{38}$, To a solution of 9" $^{\prime \prime}(24.5 \mathrm{mg}, 0.16 \mathrm{mmol})$ and $\mathrm{NaOCN}(21.1 \mathrm{mg}, 0.32 \mathrm{mmol})$ in DCM was slowly added a solution of $\mathrm{MeSO}_{3} \mathrm{H}(62.2 \mathrm{mg}, 0.65 \mathrm{mmol})$ in $\mathrm{DCM}$ at $0{ }^{\circ} \mathrm{C}$. The resulting mixture was then 
warmed up to room temperature and stirred for $3 \mathrm{~h}$. After the completion, the mixture was cooled in an ice bath followed by the addition of $20 \% \mathrm{NaOH}$ aqueous solution to bring $\mathrm{pH}$ between 10 and 11. The aqueous layer was extracted with DCM and the combined organic phase was washed with brine and dried over $\mathrm{Na}_{2} \mathrm{SO}_{4}$. After the filtration, the solution was concentrated to afford 9 as a yellow solid in $79 \%$ yield $(24.8 \mathrm{mg})$.

\section{(R)-Triisopropyl(3-phenyl-2-(4,4,5,5-tetramethyl-1,3,2-dioxaborolan-2-} yl)propoxy)silane (2k)

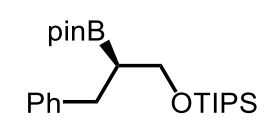

$\mathbf{R}_{f}=0.70$ (petroleum ether $/$ EtOAc $=20 / 1$ )

${ }^{1} \mathrm{H}$ NMR (400 MHz, $\left.\mathrm{CDCl}_{3}\right) \delta 7.25$ - $7.20(\mathrm{~m}, 4 \mathrm{H}), 7.18-7.10(\mathrm{~m}, 1 \mathrm{H}), 3.83-$

$3.68(\mathrm{~m}, 2 \mathrm{H}), 2.87-2.67(\mathrm{~m}, 2 \mathrm{H}), 1.70-1.59(\mathrm{~m}, 1 \mathrm{H}), 1.20-1.12(\mathrm{~m}, 12 \mathrm{H}), 1.10$

$-1.02(\mathrm{~m}, 21 \mathrm{H})$.

${ }^{13} \mathrm{C}$ NMR (100 MHz, $\left.\mathrm{CDCl}_{3}\right) \delta$ 142.2, 129.1, 128.1, 125.7, 83.2, 64.5, 33.5, 25.0, 24.9, $18.2,12.2$.

HRMS (ESI ${ }^{+}$) calcd for $\mathrm{C}_{24} \mathrm{H}_{44} \mathrm{O}_{3} \mathrm{BSi}^{+}[\mathrm{M}+\mathrm{H}]^{+}: 419.3147$, found: 419.3140 .

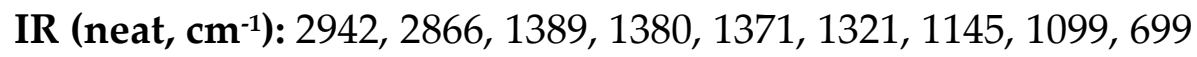

$[\alpha]_{\mathrm{D}^{23.3}}=+5.73\left(\mathrm{c}=0.98, \mathrm{CHCl}_{3}, 90 \%\right.$ ee $)$.

Compound 2k was subjected to general procedure I and the resulting alcohol was analyzed by chiral HPLC. Enantiomeric excess was determined by chiral HPLC (Daicel Chiralcel OD-H, hexane/isopropyl alcohol $=98 / 2$, flow rate $=1.0$ $\mathrm{mL} / \mathrm{min}, \lambda=220 \mathrm{~nm}$ ), $t^{1}=5.6 \mathrm{~min}$ (major),$t^{2}=6.2 \mathrm{~min}$ (minor).

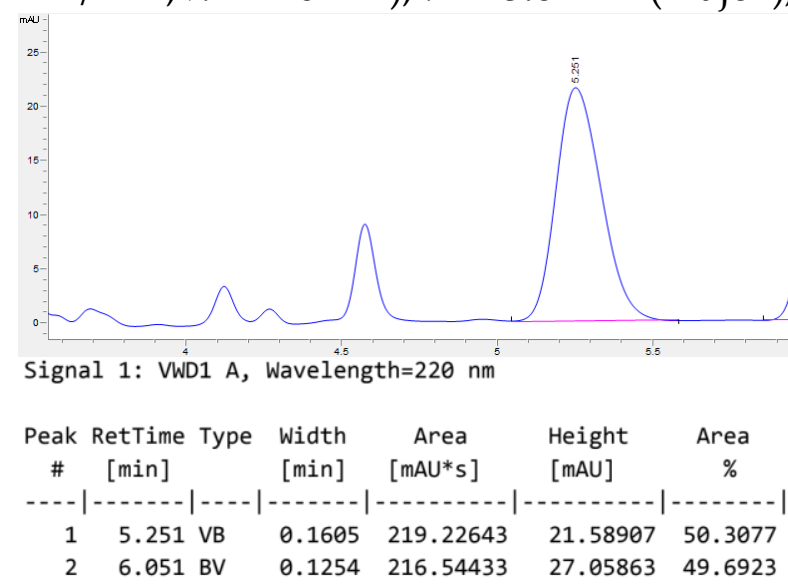




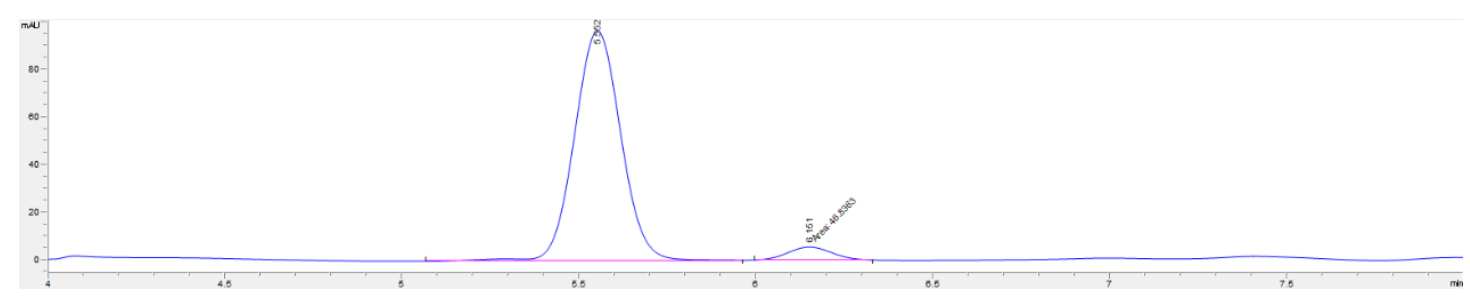

Signal 1: VWD1 A, Wavelength $=220 \mathrm{~nm}$

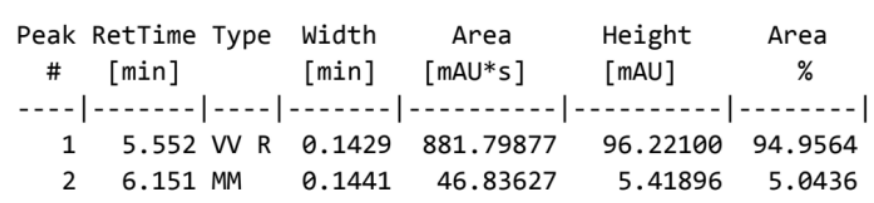

(R)-1-Phenyl-3-((triisopropylsilyl)oxy)propan-2-amine $\left(9^{\prime}\right)$

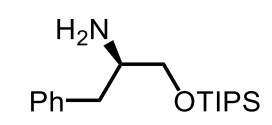

$\mathbf{R}_{f}=0.48(\mathrm{DCM} / \mathrm{MeOH}=10 / 1)$

${ }^{1} \mathrm{H}$ NMR (400 MHz, $\left.\mathrm{CDCl}_{3}\right) \delta 7.33$ - 7.27 (m, 2H), 7.25 - 7.18 (m, 3H), 3.67 (dd, $J=9.6,4.5 \mathrm{~Hz}, 1 \mathrm{H}), 3.55(\mathrm{dd}, J=9.6,6.6 \mathrm{~Hz}, 1 \mathrm{H}), 3.19-3.03(\mathrm{~m}, 1 \mathrm{H}), 2.82(\mathrm{dd}, J$ $=13.3,5.2 \mathrm{~Hz}, 1 \mathrm{H}), 2.54(\mathrm{dd}, J=13.3,8.4 \mathrm{~Hz}, 1 \mathrm{H}), 1.66(\mathrm{~s}, 2 \mathrm{H}), 1.18-0.94(\mathrm{~m}$, $21 \mathrm{H})$.

${ }^{13} \mathrm{C}$ NMR (100 MHz, $\left.\mathrm{CDCl}_{3}\right) \delta$ 139.4, 129.4, 128.5, 126.3, 68.0, 54.8, 40.6, 18.1, 12.1. HRMS (ESI') calcd for $\mathrm{C}_{18} \mathrm{H}_{34} \mathrm{NOSi}^{+}[\mathrm{M}+\mathrm{H}]^{+}:$367.2404, found: 308.2396 .

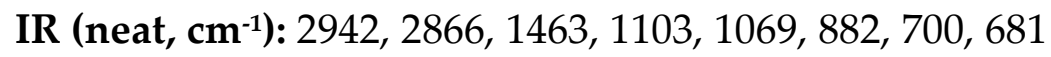

$[\alpha]_{\mathrm{D}^{23.3}}=+4.06\left(\mathrm{c}=1.03, \mathrm{CHCl}_{3}, 90 \%\right.$ ee) . Enantiomeric excess was determined by chiral HPLC (Daicel Chiralpak IA, hexane/isopropyl alcohol =95/5, flow rate $=0.5 \mathrm{~mL} / \mathrm{min}, \lambda=220 \mathrm{~nm}$ ), $t^{1}=8.2 \mathrm{~min}$ (major),$t^{2}=8.9 \mathrm{~min}$ (minor).

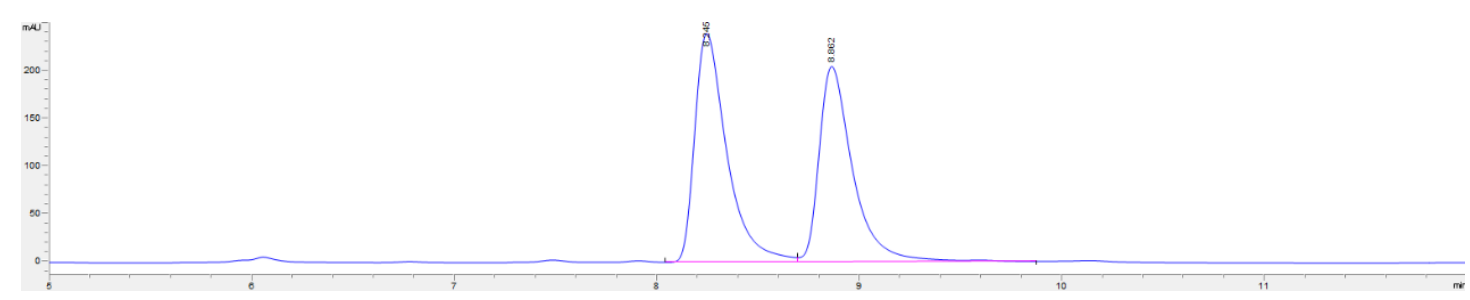

Signal 1: VWD1 A, Wavelength $=220 \mathrm{~nm}$

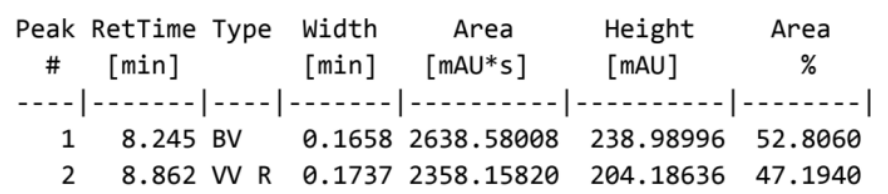




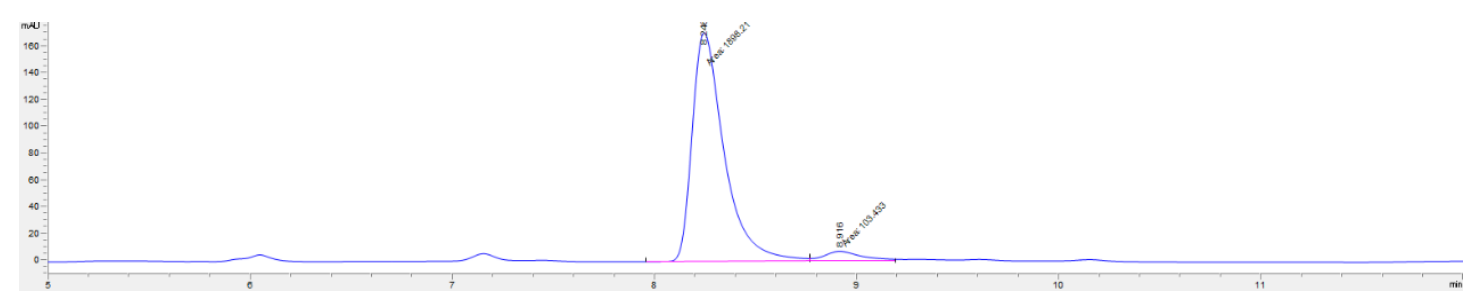

Signal 1: VWD1 A, Wavelength $=220 \mathrm{~nm}$

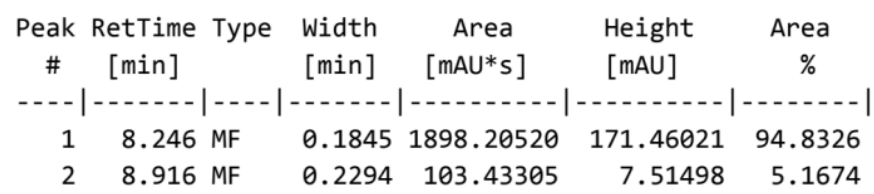

(R)-2-Amino-3-phenylpropan-1-ol (9')

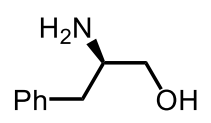

${ }^{1} \mathrm{H}$ NMR (400 MHz, $\left.\mathrm{CDCl}_{3}\right) \delta 7.35-7.28(\mathrm{~m}, 2 \mathrm{H}), 7.25-7.16(\mathrm{~m}, 3 \mathrm{H}), 3.63(\mathrm{dd}$, $J=10.7,3.9 \mathrm{~Hz}, 1 \mathrm{H}), 3.39(\mathrm{dd}, J=10.7,7.2 \mathrm{~Hz}, 1 \mathrm{H}), 3.17-3.06(\mathrm{~m}, 1 \mathrm{H}), 2.79$ $(\mathrm{dd}, J=13.5,5.2 \mathrm{~Hz}, 1 \mathrm{H}), 2.52(\mathrm{dd}, J=13.5,8.7 \mathrm{~Hz}, 1 \mathrm{H}), 2.24-1.68$ (br, 3H). ${ }^{13} \mathrm{C}$ NMR (100 MHz, $\left.\mathrm{CDCl}_{3}\right) \delta$ 138.8, 129.3, 128.7, 126.5, 66.4, 54.3, 41.0.

$[\alpha]_{\mathrm{D}^{24.3}}=+22.45\left(\mathrm{c}=0.89, \mathrm{CHCl}_{3}\right)$.

(R)-2-Amino-3-phenylpropyl carbamate (9)

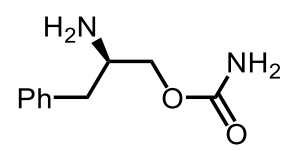

${ }^{1} \mathrm{H}$ NMR (400 MHz, $\left.\mathrm{CDCl}_{3}\right) \delta 7.35-7.28(\mathrm{~m}, 2 \mathrm{H}), 7.26-7.14(\mathrm{~m}, 3 \mathrm{H}), 5.07$ (s, 2H), $4.10(\mathrm{dd}, J=10.7,4.2 \mathrm{~Hz}, 1 \mathrm{H}), 3.90(\mathrm{dd}, J=10.7,7.1 \mathrm{~Hz}, 1 \mathrm{H}), 3.36-3.23$ $(\mathrm{m}, 1 \mathrm{H}), 2.81(\mathrm{dd}, J=13.5,5.2 \mathrm{~Hz}, 1 \mathrm{H}), 2.56(\mathrm{dd}, J=13.5,8.5 \mathrm{~Hz}, 1 \mathrm{H}), 1.41(\mathrm{br}$, $2 \mathrm{H})$.

${ }^{13} \mathrm{C}$ NMR (100 MHz, $\left.\mathrm{CDCl}_{3}\right) \delta$ 157.1, 138.2, 129.3, 128.7, 126.6, 69.4, 51.8, 40.6. $[\alpha]_{\mathrm{D}^{23.3}}=+2.67\left(\mathrm{c}=0.73, \mathrm{CHCl}_{3}, 90 \%\right.$ ee$)$. Enantiomeric excess was determined by chiral HPLC (Daicel Chiralcel AD-H, hexane/isopropyl alcohol =90/10, flow rate $=1.0 \mathrm{~mL} / \mathrm{min}, \lambda=220 \mathrm{~nm}$ ),$t^{1}=17.6 \mathrm{~min}$ (minor),$t^{2}=18.5 \mathrm{~min}$ (major).

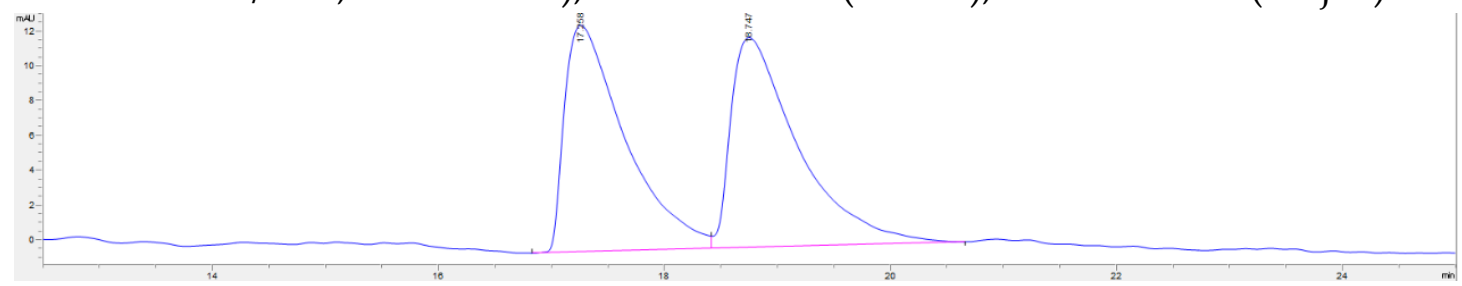




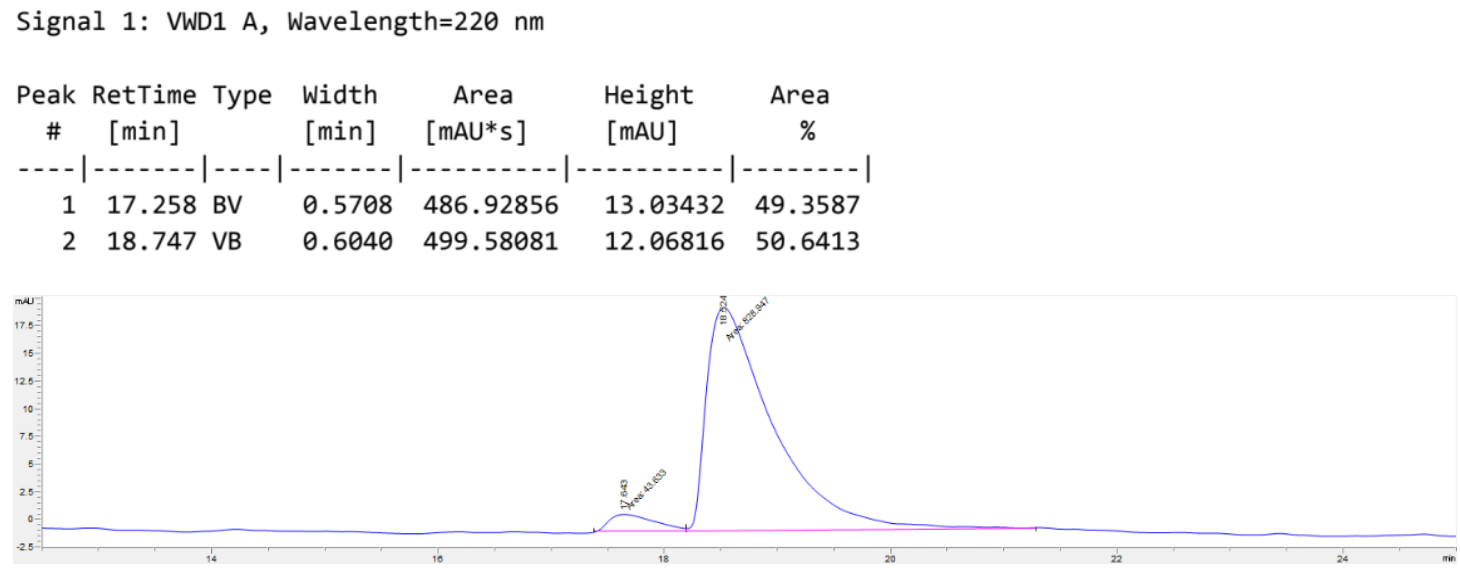

Signal 1: VWD1 A, Wavelength $=220 \mathrm{~nm}$

\begin{tabular}{|c|c|c|c|c|c|}
\hline $\begin{array}{c}\text { Peak } \\
\#\end{array}$ & $\begin{array}{l}\text { RetTime Type } \\
\text { [min] }\end{array}$ & $\begin{array}{l}\text { Width } \\
\text { [min] }\end{array}$ & $\begin{array}{c}\text { Area } \\
{\left[\mathrm{mAU}^{*} \mathrm{~s}\right]}\end{array}$ & $\begin{array}{l}\text { Height } \\
\text { [mAU] }\end{array}$ & $\begin{array}{c}\text { Area } \\
\%\end{array}$ \\
\hline & & & & & \\
\hline 1 & $17.643 \mathrm{MF}$ & 0.4706 & 43.63303 & 1.54527 & 5.0005 \\
\hline 2 & $18.524 \mathrm{FM}$ & 0.6853 & 828.94745 & 20.16059 & 94.9995 \\
\hline
\end{tabular}

\section{Computational Details}

All geometries of the intermediates and transition state were optimized using $\omega B 97 X-D$ via DFT calculations. ${ }^{39}$, 40 Frequency calculations were performed at the same level to ensure all of the optimized structures are local minima (zero imaginary frequency) or transition state (one imaginary frequency). The $6-31 \mathrm{G}(\mathrm{d}, \mathrm{p})^{41,42}$ basis set was used for $\mathrm{C}, \mathrm{H}, \mathrm{O}, \mathrm{Si}$ atoms and Lanl2DZ ${ }^{43}$ basis set was used for $\mathrm{Rh}$ and $\mathrm{P}$ atoms. Polarization functions were added for $\mathrm{Rh}(\xi \mathrm{f}=1.350)^{44}$ and $\mathrm{P}(\xi \mathrm{d}=0.387)^{45}$. All calculations were carried out using Gaussian 09. ${ }^{46}$
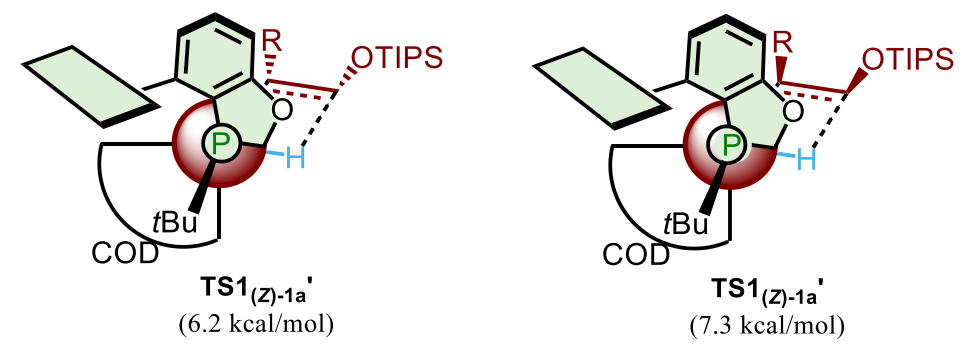

$$
\begin{aligned}
& O=R h \\
& P=P \\
& R=C_{2} B n
\end{aligned}
$$

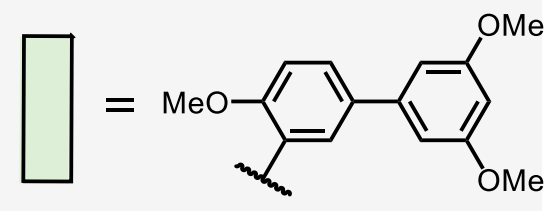

Figure S1. The structures calculated for the pair of transition states that would 
eventually lead to the $S$ - products for the insertion reactions of the (Z)-1a with diastereomer of $[(\mathrm{COD}) \mathbf{L} \mathbf{R h H}]$. The relative electronic energies are given in $\mathrm{kcal} / \mathrm{mol}$. Please note that TS1(z)-1a discussed in the main text is taken as the energy reference.
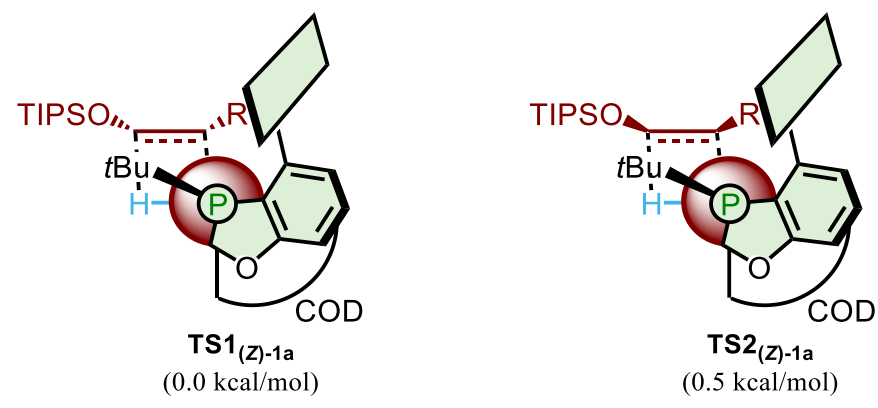

$$
\begin{aligned}
& Q=R h \\
& P=P \\
& R=\mathrm{CH}_{2} \mathrm{Bn}
\end{aligned}
$$

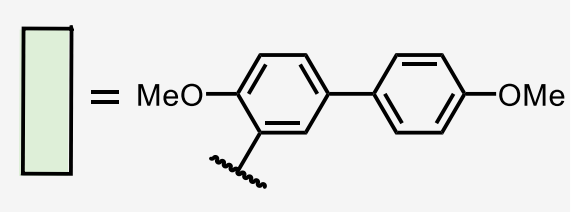

Figure S2. The structures calculated the pair of transition states that would eventually lead to the $S$ - products for the insertion reactions of the (Z)-1a with [(COD)L11RhH]. A modified L25 was used in the calculations, in which the 3,5dimethoxy substituents were replaced with two hydrogen atoms. The relative electronic energies are given in $\mathrm{kcal} / \mathrm{mol}$

\section{Cartesian coordinates}

135

TS1(Z)-1a; $E=-2845.235755$

$\begin{array}{lrrr}\mathrm{P} & -2.92475400 & -1.19587500 & 0.21878200 \\ \mathrm{O} & -5.22542700 & -1.80315700 & 1.55893700 \\ \mathrm{O} & -4.94721900 & 1.60178600 & -2.88338700 \\ \mathrm{C} & -3.91220900 & -2.32431400 & 1.35749100 \\ \mathrm{H} & -4.00391500 & -3.33800100 & 0.95878300 \\ \mathrm{H} & -3.40572100 & -2.37808400 & 2.32304500 \\ \mathrm{C} & -5.35899600 & -0.53871900 & 1.09146900 \\ \mathrm{C} & -6.52784500 & 0.16302500 & 1.36256900 \\ \mathrm{H} & -7.30436600 & -0.29791600 & 1.96215900 \\ \mathrm{C} & -6.65758900 & 1.44379500 & 0.84230900 \\ \mathrm{H} & -7.55916600 & 2.01435700 & 1.04241700\end{array}$




\begin{tabular}{lrrr}
$\mathrm{C}$ & -5.64856800 & 1.99852700 & 0.06252600 \\
$\mathrm{H}$ & -5.76949400 & 2.99109100 & -0.35819000 \\
$\mathrm{C}$ & -4.47298500 & 1.29009600 & -0.20922600 \\
$\mathrm{C}$ & -4.31656600 & 0.00400000 & 0.32650100 \\
$\mathrm{C}$ & -3.46952200 & 1.95096800 & -1.09561500 \\
$\mathrm{C}$ & -1.36814900 & 3.15337700 & -1.41846500 \\
$\mathrm{C}$ & -1.71364900 & 3.35393600 & -2.75324700 \\
$\mathrm{H}$ & -1.05858400 & 3.92978600 & -3.39934100 \\
$\mathrm{C}$ & -2.90085100 & 2.85127500 & -3.27659300 \\
$\mathrm{H}$ & -3.13059900 & 3.02173600 & -4.32139600 \\
$\mathrm{C}$ & -3.78095900 & 2.15229000 & -2.45386600 \\
$\mathrm{C}$ & -5.26452600 & 1.69884000 & -4.25256200 \\
$\mathrm{H}$ & -5.38936900 & 2.74269000 & -4.56650400 \\
$\mathrm{H}$ & -6.21039500 & 1.17253500 & -4.38066100 \\
$\mathrm{H}$ & -4.49782500 & 1.22354200 & -4.87765100 \\
$\mathrm{C}$ & -3.18100400 & -2.03155800 & -1.47433800 \\
$\mathrm{C}$ & -4.66276300 & -2.25199700 & -1.80180800 \\
$\mathrm{H}$ & -4.75508800 & -2.76700800 & -2.76608400 \\
$\mathrm{H}$ & -5.16864900 & -2.86476300 & -1.04891400 \\
$\mathrm{H}$ & -5.19224900 & -1.29798700 & -1.87579900 \\
$\mathrm{C}$ & -2.44218900 & -3.37905600 & -1.44965100 \\
$\mathrm{H}$ & -2.51396500 & -3.85154700 & -2.43637700 \\
$\mathrm{H}$ & -1.38205500 & -3.26111000 & -1.20914800 \\
$\mathrm{H}$ & -2.86811400 & -4.07832700 & -0.72329600 \\
$\mathrm{C}$ & -2.55676800 & -1.13288400 & -2.55172600 \\
$\mathrm{H}$ & -2.53640500 & -1.66571700 & -3.51035400 \\
$\mathrm{H}$ & -3.14100900 & -0.22267300 & -2.68949000 \\
$\mathrm{H}$ & -1.53310200 & -0.83617900 & -2.30618800 \\
$\mathrm{H}$ & -0.24686200 & -2.05892800 & 0.24345200 \\
$\mathrm{C}$ & 4.23843800 & 1.30978800 & -0.41593900 \\
$\mathrm{C}$ & 5.37311900 & 1.17263300 & -1.22429900 \\
$\mathrm{C}$ & 6.62754400 & 1.58024300 & -0.78569400 \\
$\mathrm{C}$ & 6.77826400 & 2.13557200 & 0.48424700 \\
$\mathrm{C}$ & 5.66218900 & 2.27074600 & 1.30341500 \\
\hline
\end{tabular}




\begin{tabular}{lccc}
$\mathrm{C}$ & 4.40606300 & 1.86192500 & 0.85671300 \\
$\mathrm{H}$ & 5.26207500 & 0.73885400 & -2.21537400 \\
$\mathrm{H}$ & 7.49086300 & 1.46285100 & -1.43408600 \\
$\mathrm{H}$ & 7.75665700 & 2.45206500 & 0.83235900 \\
$\mathrm{H}$ & 5.76703800 & 2.69478600 & 2.29824700 \\
$\mathrm{H}$ & 3.54777400 & 1.98134900 & 1.50970500 \\
$\mathrm{C}$ & 2.89394500 & 0.85918400 & -0.94288400 \\
$\mathrm{H}$ & 2.59183400 & 1.53226500 & -1.75330600 \\
$\mathrm{H}$ & 3.02413600 & -0.13070600 & -1.39164900 \\
$\mathrm{C}$ & 1.78123700 & 0.78647000 & 0.10904800 \\
$\mathrm{H}$ & 2.16437000 & 0.25160400 & 0.98096500 \\
$\mathrm{H}$ & 1.52338100 & 1.79657100 & 0.43487600 \\
$\mathrm{C}$ & 0.54457100 & 0.07228900 & -0.38685100 \\
$\mathrm{H}$ & -0.07120100 & 0.61354600 & -1.10210700 \\
$\mathrm{C}$ & 0.66102000 & -1.33778500 & -0.58925900 \\
$\mathrm{H}$ & 0.18789800 & -1.75253100 & -1.48281400 \\
$\mathrm{O}$ & 1.86440400 & -1.91603900 & -0.26789200 \\
$\mathrm{Si}$ & 2.56907100 & -3.35891600 & -0.76511900 \\
$\mathrm{C}$ & 1.81905400 & -3.80496900 & -2.44812600 \\
$\mathrm{H}$ & 0.72850900 & -3.74569700 & -2.30944000 \\
$\mathrm{C}$ & 2.12835900 & -5.23040700 & -2.92833300 \\
$\mathrm{H}$ & 1.74444300 & -5.98558400 & -2.23600700 \\
$\mathrm{H}$ & 1.66836400 & -5.41653300 & -3.90616400 \\
$\mathrm{H}$ & 3.20473900 & -5.40034100 & -3.03971400 \\
$\mathrm{C}$ & 2.21261900 & -2.76317300 & -3.50744600 \\
$\mathrm{H}$ & 1.97470300 & -1.74118700 & -3.19165800 \\
$\mathrm{H}$ & 3.28888600 & -2.79968400 & -3.71212400 \\
$\mathrm{H}$ & 1.69346300 & -2.94844400 & -4.45504000 \\
$\mathrm{C}$ & 4.38529800 & -2.84996700 & -0.87539200 \\
$\mathrm{H}$ & 4.36543900 & -1.97942000 & -1.54862000 \\
$\mathrm{C}$ & 4.88844300 & -2.34706200 & 0.48685300 \\
$\mathrm{H}$ & 5.89011600 & -1.91181700 & 0.39516200 \\
$\mathrm{H}$ & 4.22952000 & -1.57242900 & 0.89120300 \\
& & & \\
$\mathrm{H}$ & & -3.16301800 & 1.21773700500 \\
\hline
\end{tabular}




$\begin{array}{lrrr}\mathrm{C} & 5.33919500 & -3.88111500 & -1.49225700 \\ \mathrm{H} & 5.04219800 & -4.15531600 & -2.51003600 \\ \mathrm{H} & 6.35694800 & -3.47693300 & -1.54696200 \\ \mathrm{H} & 5.38830600 & -4.80277400 & -0.90329700 \\ \mathrm{C} & 2.18936700 & -4.63619500 & 0.57776400 \\ \mathrm{H} & 2.41444900 & -4.10982500 & 1.51694700 \\ \mathrm{C} & 3.06213400 & -5.89940000 & 0.54490400 \\ \mathrm{H} & 2.76100900 & -6.59564200 & 1.33664900 \\ \mathrm{H} & 2.97849100 & -6.43566600 & -0.40630400 \\ \mathrm{H} & 4.11941700 & -5.66521900 & 0.70132400 \\ \mathrm{C} & 0.69168100 & -4.98573700 & 0.59550000 \\ \mathrm{H} & 0.38371700 & -5.48779000 & -0.32973800 \\ \mathrm{H} & 0.45927000 & -5.66332500 & 1.42559900 \\ \mathrm{H} & 0.06618100 & -4.09391200 & 0.71592600 \\ \mathrm{Rh} & -0.73394300 & -0.83330400 & 1.20042200 \\ \mathrm{C} & 0.50431400 & -1.71294500 & 2.71262400 \\ \mathrm{C} & -0.82388300 & -2.06833700 & 3.03612600 \\ \mathrm{C} & -1.57809800 & -1.39846600 & 4.17893700 \\ \mathrm{C} & -2.34845300 & -0.13817500 & 3.74302100 \\ \mathrm{C} & -1.71610100 & 0.59740700 & 2.57910200 \\ \mathrm{C} & -0.37400700 & 0.95295000 & 2.47950500 \\ \mathrm{C} & 0.64860000 & 0.68677300 & 3.57694600 \\ \mathrm{C} & 1.33409200 & -0.68544400 & 3.46038200 \\ \mathrm{H} & -1.13090800 & -3.08525900 & 2.79929300 \\ \mathrm{H} & -0.12989900 & 1.76188600 & 1.79876700 \\ \mathrm{H} & -0.87375100 & -1.14841100 & 4.98136500 \\ \mathrm{H} & -2.28405200 & -2.10988700 & 4.62125100 \\ \mathrm{H} & -2.47457700 & 0.55195400 & 4.59029200 \\ \mathrm{H} & -3.36202900 & -0.42075400 & 3.43726500 \\ \mathrm{H} & 0.15518400 & 0.78700200 & 4.55089900 \\ \mathrm{H} & 1.40399100 & 1.47884700 & 3.54359900 \\ \mathrm{H} & 1.60484300 & -1.06383700 & 4.45814500 \\ \mathrm{H} & 2.27754400 & -0.57065500 & 2.91682800 \\ & & & \\ & 1.10604900 & -2.47403200 & 2.21763500 \\ & & & \\ \mathrm{H} & & \end{array}$




$\begin{array}{cccc}\mathrm{H} & -2.43005500 & 1.17092500 & 1.99372300 \\ \mathrm{C} & -2.26462800 & 2.44093800 & -0.61104400 \\ \mathrm{H} & -2.01227900 & 2.25604000 & 0.42563000 \\ \mathrm{C} & -0.09056500 & 3.67061200 & -0.86730300 \\ \mathrm{C} & -0.00078600 & 4.06818000 & 0.46853600 \\ \mathrm{C} & 1.04403500 & 3.73482000 & -1.67661300 \\ \mathrm{C} & 1.22173400 & 4.48690500 & 0.99084600 \\ \mathrm{H} & -0.86353100 & 4.05943200 & 1.12460100 \\ \mathrm{C} & 2.25709700 & 4.18161700 & -1.15490500 \\ \mathrm{H} & 1.02359100 & 3.39790700 & -2.70672600 \\ \mathrm{C} & 2.36219600 & 4.55790300 & 0.18624700 \\ \mathrm{H} & 3.31333900 & 4.86538400 & 0.59587600 \\ \mathrm{O} & 1.21817300 & 4.80358000 & 2.31176200 \\ \mathrm{O} & 3.30063800 & 4.18029900 & -2.01886300 \\ \mathrm{C} & 2.44143400 & 5.15832600 & 2.91328000 \\ \mathrm{H} & 2.22357100 & 5.32849700 & 3.96777900 \\ \mathrm{H} & 3.18466200 & 4.35499100 & 2.82412800 \\ \mathrm{H} & 2.85673200 & 6.07710500 & 2.47970600 \\ \mathrm{C} & 4.55426900 & 4.64508000 & -1.56066300 \\ \mathrm{H} & 4.93292000 & 4.03852100 & -0.73146300 \\ \mathrm{H} & 5.23614000 & 4.55190200 & -2.40581300 \\ \mathrm{H} & 4.49772000 & 5.69798600 & -1.25551200\end{array}$

135

TS2(Z)-1a; E=-2845.229384

$\begin{array}{lrrr}\mathrm{P} & 0.17144100 & 2.64802800 & 0.01543900 \\ \mathrm{O} & -0.52256900 & 5.27145500 & 0.09601400 \\ \mathrm{O} & -0.32294200 & 1.24756400 & 4.31849700 \\ \mathrm{C} & -0.05851200 & 4.29465400 & -0.83597300 \\ \mathrm{H} & 0.85525100 & 4.66696700 & -1.30150900 \\ \mathrm{H} & -0.81914100 & 4.16098400 & -1.60871500 \\ \mathrm{C} & -1.15519900 & 4.67737800 & 1.13644100 \\ \mathrm{C} & -1.86255700 & 5.46547300 & 2.03530200 \\ \mathrm{H} & -1.91847900 & 6.53699800 & 1.88124200\end{array}$




\begin{tabular}{|c|c|c|c|}
\hline $\mathrm{C}$ & -2.47730500 & 4.83898600 & 3.11113900 \\
\hline $\mathrm{H}$ & -3.03704800 & 5.43042900 & 3.82907900 \\
\hline $\mathrm{C}$ & -2.40624200 & 3.45869400 & 3.25946900 \\
\hline $\mathrm{H}$ & -2.91713300 & 2.97244600 & 4.08378700 \\
\hline C & -1.70080200 & 2.66642400 & 2.34570900 \\
\hline C & -1.03566900 & 3.28344300 & 1.27684400 \\
\hline C & -1.81948500 & 1.18978800 & 2.51910600 \\
\hline $\mathrm{C}$ & -2.95089600 & -0.87631100 & 1.88518900 \\
\hline $\mathrm{C}$ & -2.32785200 & -1.52215400 & 2.94961100 \\
\hline $\mathrm{H}$ & -2.50243700 & -2.57969800 & 3.11768900 \\
\hline $\mathrm{C}$ & -1.44291200 & -0.84542300 & 3.78288700 \\
\hline $\mathrm{H}$ & -0.94782700 & -1.39531600 & 4.57484600 \\
\hline $\mathrm{C}$ & -1.17928100 & 0.50777800 & 3.56703800 \\
\hline $\mathrm{C}$ & 0.45367700 & 0.57696700 & 5.28722700 \\
\hline $\mathrm{H}$ & -0.17112800 & 0.16568000 & 6.09001900 \\
\hline $\mathrm{H}$ & 1.12740600 & 1.32649000 & 5.70315300 \\
\hline $\mathrm{H}$ & 1.03660900 & -0.23465300 & 4.83707400 \\
\hline $\mathrm{C}$ & 1.83340700 & 2.91173000 & 0.92122700 \\
\hline $\mathrm{C}$ & 1.88951500 & 4.19311200 & 1.76271800 \\
\hline $\mathrm{H}$ & 2.88130900 & 4.27149500 & 2.22466200 \\
\hline $\mathrm{H}$ & 1.72606900 & 5.09374700 & 1.16524300 \\
\hline $\mathrm{H}$ & 1.14877500 & 4.17750700 & 2.56647700 \\
\hline $\mathrm{C}$ & 2.92904200 & 2.95169400 & -0.15108500 \\
\hline $\mathrm{H}$ & 3.90598200 & 3.06834500 & 0.33413900 \\
\hline $\mathrm{H}$ & 2.94220800 & 2.02542900 & -0.72919400 \\
\hline $\mathrm{H}$ & 2.81170300 & 3.79008800 & -0.84530200 \\
\hline C & 2.07004000 & 1.70281600 & 1.83467300 \\
\hline $\mathrm{H}$ & 3.02008400 & 1.83416000 & 2.36913600 \\
\hline $\mathrm{H}$ & 1.27757100 & 1.60047800 & 2.58295300 \\
\hline $\mathrm{H}$ & 2.14239500 & 0.78455000 & 1.24865700 \\
\hline $\mathrm{H}$ & 1.51405300 & 0.72396000 & -1.83259700 \\
\hline $\mathrm{C}$ & 0.79626000 & -3.19628900 & 2.32957800 \\
\hline $\mathrm{C}$ & 1.72966300 & -2.60662200 & 3.18745700 \\
\hline $\mathrm{C}$ & 1.72899600 & -2.88778000 & 4.54977500 \\
\hline
\end{tabular}




\begin{tabular}{lccc}
$\mathrm{C}$ & 0.78276800 & -3.76139700 & 5.08406900 \\
$\mathrm{C}$ & -0.14582600 & -4.36267700 & 4.23914500 \\
$\mathrm{C}$ & -0.13094200 & -4.08572800 & 2.87395800 \\
$\mathrm{H}$ & 2.46425100 & -1.91812400 & 2.77633800 \\
$\mathrm{H}$ & 2.47044100 & -2.42779500 & 5.19713300 \\
$\mathrm{H}$ & 0.77852400 & -3.98007300 & 6.14741200 \\
$\mathrm{H}$ & -0.88013000 & -5.05464200 & 4.64082500 \\
$\mathrm{H}$ & -0.85556300 & -4.56457000 & 2.21954200 \\
$\mathrm{C}$ & 0.78550800 & -2.84286200 & 0.86521400 \\
$\mathrm{H}$ & 1.80049700 & -2.92848000 & 0.46283400 \\
$\mathrm{H}$ & 0.16656100 & -3.55891900 & 0.31061000 \\
$\mathrm{C}$ & 0.27425600 & -1.41331700 & 0.59205300 \\
$\mathrm{H}$ & -0.75790200 & -1.32754100 & 0.92657500 \\
$\mathrm{H}$ & 0.84816800 & -0.70361900 & 1.18953000 \\
$\mathrm{C}$ & 0.36135000 & -1.07628700 & -0.86941800 \\
$\mathrm{H}$ & -0.22705800 & -1.72432500 & -1.51581800 \\
$\mathrm{C}$ & 1.59592800 & -0.65456400 & -1.44208600 \\
$\mathrm{H}$ & 1.82074100 & -1.02804800 & -2.44275500 \\
$\mathrm{O}$ & 2.70157300 & -0.53351600 & -0.61907400 \\
$\mathrm{Si}$ & 4.28460900 & -0.97612200 & -1.02077300 \\
$\mathrm{C}$ & 4.57442200 & -0.50061500 & -2.83497800 \\
$\mathrm{H}$ & 3.77255400 & -0.99875400 & -3.40029000 \\
$\mathrm{C}$ & 5.90029700 & -1.02152600 & -3.41149900 \\
$\mathrm{H}$ & 5.95728500 & -2.11366700 & -3.37909000 \\
$\mathrm{H}$ & 6.00633000 & -0.72068900 & -4.46040300 \\
$\mathrm{H}$ & 6.76882200 & -0.62877400 & -2.87348100 \\
$\mathrm{C}$ & 4.41656500 & 1.01133200 & -3.06013000 \\
$\mathrm{H}$ & 3.40903800 & 1.35823200 & -2.80747600 \\
$\mathrm{H}$ & 5.12607700 & 1.58898400 & -2.45619700 \\
$\mathrm{H}$ & 4.59695300 & 1.26643800 & -4.11103000 \\
$\mathrm{C}$ & 5.30103900 & 0.01738800 & 0.22278400 \\
$\mathrm{H}$ & 4.86432900 & 1.02280900 & 0.17755400 \\
& 5.08012900 & -0.50702200 & 1.65025700 \\
$\mathrm{H}$ & 56500100 & 0.14753800 & 2.38421800 \\
\hline
\end{tabular}




$\begin{array}{lrrr}\mathrm{H} & 4.01497700 & -0.54830900 & 1.89697100 \\ \mathrm{H} & 5.49690300 & -1.51186200 & 1.78314600 \\ \mathrm{C} & 6.79501200 & 0.14547300 & -0.10558900 \\ \mathrm{H} & 6.95760400 & 0.66652500 & -1.05406800 \\ \mathrm{H} & 7.31244000 & 0.71638200 & 0.67453900 \\ \mathrm{H} & 7.28925700 & -0.82944300 & -0.17490600 \\ \mathrm{C} & 4.42035500 & -2.84561900 & -0.73905700 \\ \mathrm{H} & 3.94533100 & -2.99830400 & 0.24183600 \\ \mathrm{C} & 5.85352900 & -3.38862400 & -0.63388700 \\ \mathrm{H} & 5.84110500 & -4.46821000 & -0.44270400 \\ \mathrm{H} & 6.42255000 & -3.23070900 & -1.55582600 \\ \mathrm{H} & 6.41033100 & -2.91902200 & 0.18251200 \\ \mathrm{C} & 3.60506300 & -3.63237100 & -1.77758800 \\ \mathrm{H} & 4.01984400 & -3.51003400 & -2.78529200 \\ \mathrm{H} & 3.61353100 & -4.70487800 & -1.55059700 \\ \mathrm{H} & 2.55718600 & -3.31402900 & -1.80587100 \\ \mathrm{Rh} & -0.10881500 & 0.93043300 & -1.71116300 \\ \mathrm{C} & -0.18833200 & 0.58256100 & -3.81624900 \\ \mathrm{C} & -0.12626700 & 1.98978000 & -3.64089700 \\ \mathrm{C} & -1.34719100 & 2.86736600 & -3.83726100 \\ \mathrm{C} & -2.56878700 & 2.41044500 & -3.00945800 \\ \mathrm{C} & -2.22952600 & 1.54576900 & -1.79825100 \\ \mathrm{C} & -2.16494300 & 0.15329600 & -1.82981500 \\ \mathrm{C} & -2.28803600 & -0.68172700 & -3.08717000 \\ \mathrm{C} & -1.45663300 & -0.14431800 & -4.26550100 \\ \mathrm{H} & -2.06305500 & 0.52599500 & -4.88567400 \\ \mathrm{H} & -2.36029200 & -0.38315000 & -0.90867800 \\ \mathrm{H} & -1.62140200 & 2.90923500 & -4.90288300 \\ \mathrm{H} & -1.08337200 & 3.89504100 & -3.56985900 \\ \mathrm{H} & -3.26673900 & 1.85510200 & -3.64578000 \\ \mathrm{H} & -3.12267200 & 3.28852400 & -2.66128300 \\ \mathrm{H} & -3.34926000 & -0.77953500 & -3.36258100 \\ \mathrm{H} & -1.95597100 & -1.69632600 & -2.84005900 \\ & & & \\ \mathrm{H} & -47855100 & -3.85484400 \\ & & \end{array}$




$\begin{array}{lrrr}\mathrm{H} & -1.18138200 & -0.97777000 & -4.92055100 \\ \mathrm{H} & 0.74028100 & 0.09548300 & -4.11834000 \\ \mathrm{H} & -2.46409300 & 1.99809800 & -0.83793000 \\ \mathrm{C} & -2.69554400 & 0.48615800 & 1.70439800 \\ \mathrm{H} & -3.17332900 & 1.01157700 & 0.88388700 \\ \mathrm{C} & -3.72093200 & -1.60440400 & 0.84810900 \\ \mathrm{C} & -4.87694000 & -1.05337800 & 0.29730000 \\ \mathrm{C} & -3.18229000 & -2.77241700 & 0.30509100 \\ \mathrm{C} & -5.47183900 & -1.65306300 & -0.81063200 \\ \mathrm{H} & -5.31526900 & -0.14722700 & 0.69957300 \\ \mathrm{C} & -3.77808100 & -3.36141000 & -0.80736900 \\ \mathrm{H} & -2.25975400 & -3.19305900 & 0.68868700 \\ \mathrm{C} & -4.92913500 & -2.81076200 & -1.37801400 \\ \mathrm{H} & -5.38756200 & -3.26705300 & -2.24362000 \\ \mathrm{O} & -6.58162600 & -1.03456300 & -1.28777800 \\ \mathrm{O} & -3.14862800 & -4.46172400 & -1.29645000 \\ \mathrm{C} & -7.17442600 & -1.53474700 & -2.46295700 \\ \mathrm{H} & -8.00232700 & -0.86412300 & -2.69422800 \\ \mathrm{H} & -6.46740200 & -1.53493800 & -3.30265800 \\ \mathrm{H} & -7.56574700 & -2.55052600 & -2.32144800 \\ \mathrm{C} & -3.62465700 & -5.02238200 & -2.49789900 \\ \mathrm{H} & -3.62028600 & -4.28773100 & -3.31331800 \\ \mathrm{H} & -2.94183600 & -5.83560300 & -2.74516000 \\ \mathrm{H} & -4.63784800 & -5.42891200 & -2.38396800\end{array}$

135

TS1(E)-1a; $E=-2845.226564$

$\begin{array}{lrrr}\mathrm{P} & 2.30211000 & -1.97912300 & 0.17457500 \\ \mathrm{O} & 3.49224600 & -4.28466800 & 1.01282300 \\ \mathrm{O} & 0.72963400 & -3.28616200 & -3.39129300 \\ \mathrm{C} & 3.55402200 & -2.87940600 & 1.25194300 \\ \mathrm{H} & 4.57669800 & -2.54930800 & 1.06526300 \\ \mathrm{H} & 3.32017300 & -2.69556900 & 2.30197000 \\ \mathrm{C} & 2.33089300 & -4.64205900 & 0.41329000\end{array}$




$\begin{array}{lrrr}\mathrm{C} & 1.98389500 & -5.98802100 & 0.36548900 \\ \mathrm{H} & 2.63968500 & -6.72588000 & 0.81326400 \\ \mathrm{C} & 0.79214100 & -6.33574600 & -0.25344000 \\ \mathrm{H} & 0.49313800 & -7.37836700 & -0.29757000 \\ \mathrm{C} & -0.02188800 & -5.35848000 & -0.81788800 \\ \mathrm{H} & -0.94751100 & -5.63701700 & -1.31063800 \\ \mathrm{C} & 0.33819900 & -4.00792900 & -0.78077900 \\ \mathrm{C} & 1.53342200 & -3.63293000 & -0.14669500 \\ \mathrm{C} & -0.57984900 & -3.04895000 & -1.46391200 \\ \mathrm{C} & -2.65604000 & -1.75984600 & -1.49859700 \\ \mathrm{C} & -2.35160200 & -1.36367900 & -2.80098400 \\ \mathrm{H} & -3.00569000 & -0.67063600 & -3.32033500 \\ \mathrm{C} & -1.22226400 & -1.83780600 & -3.46128500 \\ \mathrm{H} & -1.03804500 & -1.53009800 & -4.48348200 \\ \mathrm{C} & -0.35328900 & -2.70976400 & -2.80890200 \\ \mathrm{C} & 1.01975200 & -2.96701300 & -4.73292700 \\ \mathrm{H} & 0.20848100 & -3.27539400 & -5.40381900 \\ \mathrm{H} & 1.92599900 & -3.51925100 & -4.98220600 \\ \mathrm{H} & 1.20928200 & -1.89394000 & -4.86073600 \\ \mathrm{C} & 3.35741300 & -1.61903300 & -1.37622100 \\ \mathrm{C} & 2.51476400 & -0.79473900 & -2.35817300 \\ \mathrm{H} & 3.05409000 & -0.70388200 & -3.31031000 \\ \mathrm{H} & 1.55069500 & -1.25875200 & -2.56026000 \\ \mathrm{H} & 2.34045700 & 0.20948600 & -1.96940800 \\ \mathrm{C} & 3.86202600 & -2.90306500 & -2.04264600 \\ \mathrm{H} & 4.50817100 & -2.64359700 & -2.89104100 \\ \mathrm{H} & 4.44821800 & -3.52039700 & -1.35410700 \\ \mathrm{H} & 3.02890300 & -3.50352800 & -2.41611900 \\ \mathrm{C} & 4.54213400 & -0.74228900 & -0.94214500 \\ \mathrm{H} & 5.03356200 & -0.34048700 & -1.83634000 \\ \mathrm{H} & 4.20256000 & 0.10518400 & -0.33769500 \\ \mathrm{H} & 5.30055000 & -1.29413000 & -0.37929200 \\ \mathrm{C} & 2.18944000 & 0.87599200 & 0.94268200 \\ & -3356300 & 2.57170700 & -0.67292800\end{array}$




$\begin{array}{lccc}\mathrm{C} & -4.32481400 & 2.44025900 & -1.64824400 \\ \mathrm{C} & -5.62378900 & 2.88988700 & -1.42622000 \\ \mathrm{C} & -5.96251200 & 3.46866800 & -0.20790100 \\ \mathrm{C} & -4.99043100 & 3.59402300 & 0.78296100 \\ \mathrm{C} & -3.69250000 & 3.15195900 & 0.54978800 \\ \mathrm{H} & -4.07540000 & 1.97503700 & -2.59796900 \\ \mathrm{H} & -6.37227000 & 2.77039800 & -2.20352700 \\ \mathrm{H} & -6.97269700 & 3.82771500 & -0.03220100 \\ \mathrm{H} & -5.23954200 & 4.05191300 & 1.73624900 \\ \mathrm{H} & -2.94237400 & 3.27042500 & 1.32691100 \\ \mathrm{C} & -1.91390500 & 2.13104500 & -0.94463700 \\ \mathrm{H} & -1.87325900 & 1.61151700 & -1.90933000 \\ \mathrm{H} & -1.27209100 & 3.01690300 & -1.04721900 \\ \mathrm{C} & -1.33206400 & 1.20613700 & 0.13682600 \\ \mathrm{H} & -1.34984300 & 1.71197900 & 1.10987400 \\ \mathrm{H} & -1.99704500 & 0.34820000 & 0.21972000 \\ \mathrm{C} & 0.07866800 & 0.76811600 & -0.17567300 \\ \mathrm{H} & 0.18378800 & 0.25302600 & -1.12852100 \\ \mathrm{C} & 1.13292200 & 1.65588600 & 0.16571200 \\ \mathrm{H} & 0.87496200 & 2.45556300 & 0.86292000 \\ \mathrm{O} & 2.07691600 & 2.02091200 & -0.78444400 \\ \mathrm{Si} & 3.02985400 & 3.41578600 & -0.71025500 \\ \mathrm{C} & 3.63149600 & 3.63681600 & 1.07591000 \\ \mathrm{H} & 2.73075200 & 3.55641100 & 1.70370600 \\ \mathrm{C} & 4.25090800 & 5.01646600 & 1.35000100 \\ \mathrm{H} & 3.53702800 & 5.82755000 & 1.17894300 \\ \mathrm{H} & 4.58514700 & 5.08864800 & 2.39171600 \\ \mathrm{H} & 5.12388800 & 5.20633400 & 0.71666600 \\ \mathrm{C} & 4.58613300 & 2.50925200 & 1.50085000 \\ \mathrm{H} & 4.11983500 & 1.52312800 & 1.40563200 \\ \mathrm{H} & 5.50094800 & 2.50708200 & 0.89700000 \\ \mathrm{H} & 4.88578100 & 2.63082500 & 2.548666000 \\ & 4.41903200 & 2.99404400 & -1.91822400 \\ \mathrm{H} & 4.75879900 & 2.00554500 & -1.58024300\end{array}$




$\begin{array}{lrrr}\mathrm{C} & 3.87334400 & 2.82371800 & -3.34455700 \\ \mathrm{H} & 4.65716700 & 2.46194200 & -4.02093900 \\ \mathrm{H} & 3.04812300 & 2.10594600 & -3.37468200 \\ \mathrm{H} & 3.50689500 & 3.77338900 & -3.75190600 \\ \mathrm{C} & 5.63619900 & 3.92929700 & -1.88695900 \\ \mathrm{H} & 6.11448600 & 3.94407000 & -0.90270300 \\ \mathrm{H} & 6.39059200 & 3.59932200 & -2.61103100 \\ \mathrm{H} & 5.37460700 & 4.96162600 & -2.14121200 \\ \mathrm{C} & 1.93567000 & 4.85024100 & -1.30121700 \\ \mathrm{H} & 1.41237300 & 4.42941400 & -2.17246400 \\ \mathrm{C} & 2.70795200 & 6.08815500 & -1.78302100 \\ \mathrm{H} & 2.01386400 & 6.86334000 & -2.12898700 \\ \mathrm{H} & 3.31593400 & 6.53024500 & -0.98656500 \\ \mathrm{H} & 3.37655800 & 5.85233700 & -2.61616800 \\ \mathrm{C} & 0.86572000 & 5.24100600 & -0.26904200 \\ \mathrm{H} & 1.31425100 & 5.64483500 & 0.64572400 \\ \mathrm{H} & 0.19967200 & 6.01342300 & -0.67143500 \\ \mathrm{H} & 0.23717400 & 4.39188700 & 0.01740000 \\ \mathrm{Rh} & 1.17709800 & -0.22666500 & 1.49173100 \\ \mathrm{C} & 1.64050400 & 0.73579900 & 3.36156500 \\ \mathrm{C} & 2.27573700 & -0.52495100 & 3.37818600 \\ \mathrm{C} & 1.77236000 & -1.71259400 & 4.19502300 \\ \mathrm{C} & 0.75385500 & -2.59759400 & 3.44490700 \\ \mathrm{C} & -0.06300900 & -1.84948600 & 2.41311800 \\ \mathrm{C} & -0.70410300 & -0.64039700 & 2.64985300 \\ \mathrm{C} & -0.72382500 & 0.05757100 & 4.00004600 \\ \mathrm{C} & 0.38234600 & 1.11188900 & 4.12069900 \\ \mathrm{H} & 3.34763900 & -0.51486800 & 3.18907300 \\ \mathrm{H} & -1.50596000 & -0.36503500 & 1.97975300 \\ \mathrm{H} & 1.33924700 & -1.34974700 & 5.13364000 \\ \mathrm{H} & 2.62599700 & -2.33114200 & 4.49393300 \\ \mathrm{H} & 0.08411600 & -3.09590100 & 4.16091100 \\ \mathrm{H} & 1.28227500 & -3.40382600 & 2.92540000 \\ \mathrm{H} & -0.68658800 & 4.79835200\end{array}$




$\begin{array}{lrrr}\mathrm{H} & -1.70201200 & 0.53067900 & 4.13443900 \\ \mathrm{H} & 0.61273500 & 1.32011800 & 5.17727700 \\ \mathrm{H} & 0.01551100 & 2.05309700 & 3.69434400 \\ \mathrm{H} & 2.28916700 & 1.58891300 & 3.15815300 \\ \mathrm{H} & -0.42001400 & -2.45632200 & 1.58655700 \\ \mathrm{C} & -1.74001700 & -2.59713000 & -0.84859600 \\ \mathrm{H} & -1.94789400 & -2.93873600 & 0.16039400 \\ \mathrm{C} & -3.88848000 & -1.27765100 & -0.82428000 \\ \mathrm{C} & -3.90990400 & -1.10192100 & 0.55974500 \\ \mathrm{C} & -5.01541400 & -0.92931400 & -1.57018200 \\ \mathrm{C} & -5.00242200 & -0.49824600 & 1.17433700 \\ \mathrm{H} & -3.06329300 & -1.36515200 & 1.18122200 \\ \mathrm{C} & -6.11944700 & -0.35876600 & -0.94235700 \\ \mathrm{H} & -5.06319600 & -1.08846500 & -2.64101500 \\ \mathrm{C} & -6.12419700 & -0.12410300 & 0.43335700 \\ \mathrm{H} & -6.96499300 & 0.35912600 & 0.90863200 \\ \mathrm{O} & -4.88072100 & -0.29123100 & 2.51092100 \\ \mathrm{O} & -7.15577500 & -0.03433500 & -1.75999300 \\ \mathrm{C} & -5.85653000 & 0.50146100 & 3.14749100 \\ \mathrm{H} & -5.51525800 & 0.63569000 & 4.17446200 \\ \mathrm{H} & -5.95039000 & 1.48135800 & 2.66242900 \\ \mathrm{H} & -6.83705500 & 0.00786800 & 3.16079700 \\ \mathrm{C} & -8.31658700 & 0.50845900 & -1.17540600 \\ \mathrm{H} & -8.11072400 & 1.46985000 & -0.68799700 \\ \mathrm{H} & -9.02401400 & 0.66417900 & -1.99065000 \\ \mathrm{H} & -8.75876100 & -0.17959400 & -0.44336500\end{array}$

135

TS2(E)-1a; $E=-2845.224775$

$\begin{array}{lrrr}\mathrm{P} & 0.91231900 & 1.91138500 & -1.41809200 \\ \mathrm{O} & 1.20250800 & 3.44426700 & -3.64198100 \\ \mathrm{O} & -1.84493700 & 4.03832400 & 0.76451800 \\ \mathrm{C} & 1.81246100 & 2.31971700 & -3.00914700 \\ \mathrm{H} & 2.87178100 & 2.53310300 & -2.86569100\end{array}$




\begin{tabular}{lrrr}
$\mathrm{H}$ & 1.71761700 & 1.44925400 & -3.66395600 \\
$\mathrm{C}$ & -0.09935700 & 3.54704700 & -3.27387400 \\
$\mathrm{C}$ & -0.95272100 & 4.35050500 & -4.02037800 \\
$\mathrm{H}$ & -0.57127900 & 4.87474900 & -4.88908900 \\
$\mathrm{C}$ & -2.28106400 & 4.43627500 & -3.62770400 \\
$\mathrm{H}$ & -2.97140500 & 5.04850400 & -4.19953700 \\
$\mathrm{C}$ & -2.73641300 & 3.73441900 & -2.51610000 \\
$\mathrm{H}$ & -3.77760400 & 3.80165900 & -2.21852800 \\
$\mathrm{C}$ & -1.87160300 & 2.93871500 & -1.75743600 \\
$\mathrm{C}$ & -0.52367000 & 2.84166000 & -2.13592400 \\
$\mathrm{C}$ & -2.46229900 & 2.22737800 & -0.58495700 \\
$\mathrm{C}$ & -3.79673400 & 0.37887300 & 0.30144300 \\
$\mathrm{C}$ & -3.68630400 & 0.95465600 & 1.56757500 \\
$\mathrm{H}$ & -4.10200100 & 0.45350600 & 2.43491300 \\
$\mathrm{C}$ & -3.02997800 & 2.16413500 & 1.76784100 \\
$\mathrm{H}$ & -2.97319100 & 2.56942400 & 2.77126100 \\
$\mathrm{C}$ & -2.44323700 & 2.81985700 & 0.68775900 \\
$\mathrm{C}$ & -1.85435400 & 4.70494300 & 2.00839600 \\
$\mathrm{H}$ & -2.87863600 & 4.87515700 & 2.36180700 \\
$\mathrm{H}$ & -1.36303900 & 5.66320900 & 1.83827600 \\
$\mathrm{H}$ & -1.30392900 & 4.14136800 & 2.77043000 \\
$\mathrm{C}$ & 1.70874200 & 3.16668300 & -0.19929800 \\
$\mathrm{C}$ & 1.43070400 & 4.62303300 & -0.59260200 \\
$\mathrm{H}$ & 1.96317000 & 5.29249300 & 0.09474900 \\
$\mathrm{H}$ & 1.77226900 & 4.84276200 & -1.60787200 \\
$\mathrm{H}$ & 0.36250500 & 4.84708000 & -0.53018500 \\
$\mathrm{C}$ & 3.22375800 & 2.91212700 & -0.16988700 \\
$\mathrm{H}$ & 3.66717500 & 3.46264500 & 0.66839100 \\
$\mathrm{H}$ & 3.45385900 & 1.85153300 & -0.03039600 \\
$\mathrm{H}$ & 3.72656400 & 3.25041900 & -1.07996100 \\
$\mathrm{H}$ & 1.16340400 & 2.91791600 & 1.21282700 \\
$\mathrm{H}$ & 1.52752200 & 3.70540600 & 1.88520400 \\
$\mathrm{H}$ & 0.07448100 & 2.93649700 & 1.23814300 \\
$\mathrm{H}$ & 1.49848900 & 1.96001100 & 1.61592200 \\
\hline
\end{tabular}




$\begin{array}{lrrr}\mathrm{H} & 2.43138400 & -0.18536900 & -0.38771100 \\ \mathrm{C} & -0.85819200 & 0.61823200 & 4.12261500 \\ \mathrm{C} & -2.09989000 & 0.13441200 & 4.54307000 \\ \mathrm{C} & -2.98155600 & 0.94388100 & 5.25272900 \\ \mathrm{C} & -2.63267700 & 2.25768100 & 5.56126000 \\ \mathrm{C} & -1.39452700 & 2.74955000 & 5.15562100 \\ \mathrm{C} & -0.52019500 & 1.93618800 & 4.43836600 \\ \mathrm{H} & -2.37749800 & -0.88972600 & 4.30605100 \\ \mathrm{H} & -3.94055500 & 0.54669500 & 5.57200900 \\ \mathrm{H} & -3.31472800 & 2.88752100 & 6.12364500 \\ \mathrm{H} & -1.10751800 & 3.76837500 & 5.40023100 \\ \mathrm{H} & 0.44308100 & 2.32787100 & 4.11952600 \\ \mathrm{C} & 0.07390100 & -0.24209300 & 3.30915700 \\ \mathrm{H} & 1.09959300 & 0.12729100 & 3.43201500 \\ \mathrm{H} & 0.06744200 & -1.27146700 & 3.68704900 \\ \mathrm{C} & -0.27671800 & -0.25067900 & 1.80628300 \\ \mathrm{H} & -1.27777900 & -0.67506900 & 1.67346500 \\ \mathrm{H} & -0.35395800 & 0.77624500 & 1.44752200 \\ \mathrm{C} & 0.70727900 & -1.05469300 & 0.98678600 \\ \mathrm{H} & 0.54135600 & -2.13059800 & 1.00623900 \\ \mathrm{C} & 2.09015000 & -0.72382300 & 0.91554500 \\ \mathrm{H} & 2.40623100 & 0.19894800 & 1.41045200 \\ \mathrm{O} & 2.99945600 & -1.75249100 & 1.02280300 \\ \mathrm{Rh} & 1.01372500 & -0.49611200 & -1.13672500 \\ \mathrm{C} & 1.74641900 & -2.35042300 & -1.91482600 \\ \mathrm{C} & 2.02130600 & -1.36785700 & -2.89632600 \\ \mathrm{C} & 1.19882700 & -1.23024700 & -4.16361700 \\ \mathrm{C} & -0.31860900 & -1.18119300 & -3.89867500 \\ \mathrm{C} & -0.68692200 & -0.67880900 & -2.50676000 \\ \mathrm{C} & -0.90117800 & -1.51435500 & -1.406688200 \\ \mathrm{C} & -0.67988300 & -3.01229500 & -1.42848700 \\ \mathrm{C} & 0.65935300 & -3.41382700 & -2.07698500 \\ \mathrm{H} & 3.04902500 & -1.01974200 & -2.98585500 \\ \mathrm{H} & -1.15201600 & -0.62522100\end{array}$




\begin{tabular}{|c|c|c|c|}
\hline $\mathrm{H}$ & 1.42811400 & -2.04437100 & -4.86843200 \\
\hline $\mathrm{H}$ & 1.50338900 & -0.31062900 & -4.67451000 \\
\hline $\mathrm{H}$ & -0.76529600 & -2.17033800 & -4.04605600 \\
\hline $\mathrm{H}$ & -0.79548000 & -0.52886900 & -4.63751200 \\
\hline $\mathrm{H}$ & -1.52765700 & -3.50260300 & -1.92988000 \\
\hline $\mathrm{H}$ & -0.69944600 & -3.36535400 & -0.39275600 \\
\hline $\mathrm{H}$ & 0.51655200 & -3.63266600 & -3.14104000 \\
\hline $\mathrm{H}$ & 1.00765300 & -4.34968300 & -1.62780200 \\
\hline $\mathrm{H}$ & 2.56798100 & -2.64071200 & -1.26240800 \\
\hline $\mathrm{H}$ & -1.19182800 & 0.28157700 & -2.48996400 \\
\hline $\mathrm{Si}$ & 4.68751300 & -1.64158800 & 1.04974200 \\
\hline $\mathrm{C}$ & 5.37384800 & -1.50279500 & -0.71668100 \\
\hline $\mathrm{H}$ & 4.75640600 & -2.18740100 & -1.31619400 \\
\hline $\mathrm{C}$ & 6.83266200 & -1.97872100 & -0.82993700 \\
\hline $\mathrm{H}$ & 6.95000500 & -3.02838600 & -0.54664700 \\
\hline $\mathrm{H}$ & 7.18677700 & -1.87766700 & -1.86266400 \\
\hline $\mathrm{H}$ & 7.50682700 & -1.38814300 & -0.19955300 \\
\hline $\mathrm{C}$ & 5.22238200 & -0.09981600 & -1.32633400 \\
\hline $\mathrm{H}$ & 4.17307000 & 0.20008200 & -1.39733400 \\
\hline $\mathrm{H}$ & 5.75357100 & 0.66013700 & -0.74151200 \\
\hline $\mathrm{H}$ & 5.63924200 & -0.07620200 & -2.34068900 \\
\hline $\mathrm{C}$ & 5.12392200 & -0.12653700 & 2.10429000 \\
\hline $\mathrm{H}$ & 4.60914800 & 0.72187900 & 1.62678300 \\
\hline C & 4.56133800 & -0.27212100 & 3.52695400 \\
\hline $\mathrm{H}$ & 4.71297900 & 0.64661000 & 4.10555700 \\
\hline $\mathrm{H}$ & 3.48907700 & -0.49532100 & 3.52398000 \\
\hline $\mathrm{H}$ & 5.06085900 & -1.08515700 & 4.06631700 \\
\hline $\mathrm{C}$ & 6.61949100 & 0.22266300 & 2.13250000 \\
\hline $\mathrm{H}$ & 7.01433600 & 0.42231300 & 1.13205500 \\
\hline $\mathrm{H}$ & 6.79558500 & 1.11886900 & 2.73914500 \\
\hline $\mathrm{H}$ & 7.21770000 & -0.58496500 & 2.56682100 \\
\hline $\mathrm{C}$ & 5.09932900 & -3.28967100 & 1.87879300 \\
\hline $\mathrm{H}$ & 4.40992600 & -3.31161000 & 2.73525800 \\
\hline C & 6.52321500 & -3.43839400 & 2.43151900 \\
\hline
\end{tabular}




\begin{tabular}{lrrr}
$\mathrm{H}$ & 6.65219700 & -4.42169100 & 2.89955700 \\
$\mathrm{H}$ & 7.28413400 & -3.35046700 & 1.64943500 \\
$\mathrm{H}$ & 6.74424300 & -2.68449600 & 3.19360700 \\
$\mathrm{C}$ & 4.71771200 & -4.46456900 & 0.96310300 \\
$\mathrm{H}$ & 5.36158300 & -4.51189900 & 0.07732500 \\
$\mathrm{H}$ & 4.82202400 & -5.42007500 & 1.49055000 \\
$\mathrm{H}$ & 3.68218100 & -4.38158100 & 0.61869800 \\
$\mathrm{C}$ & -3.16797100 & 1.04326900 & -0.75994200 \\
$\mathrm{H}$ & -3.24736200 & 0.64438600 & -1.76559900 \\
$\mathrm{C}$ & -4.53671200 & -0.89426800 & 0.08742300 \\
$\mathrm{C}$ & -4.22335500 & -1.72728400 & -0.98924900 \\
$\mathrm{C}$ & -5.55253500 & -1.28165300 & 0.96573300 \\
$\mathrm{C}$ & -4.88836900 & -2.93733200 & -1.16233000 \\
$\mathrm{H}$ & -3.44347100 & -1.48077400 & -1.69766000 \\
$\mathrm{C}$ & -6.21738600 & -2.49182800 & 0.78318400 \\
$\mathrm{H}$ & -5.86708300 & -0.64965600 & 1.78768300 \\
$\mathrm{C}$ & -5.89150400 & -3.34097200 & -0.27832700 \\
$\mathrm{H}$ & -6.40327900 & -4.28350300 & -0.41166300 \\
$\mathrm{O}$ & -4.48061600 & -3.67704500 & -2.22773200 \\
$\mathrm{O}$ & -7.18659800 & -2.76920000 & 1.69507000 \\
$\mathrm{C}$ & -5.11185600 & -4.91298000 & -2.46459800 \\
$\mathrm{H}$ & -4.64407500 & -5.32676600 & -3.35817700 \\
$\mathrm{H}$ & -4.96712400 & -5.61066500 & -1.62956500 \\
$\mathrm{H}$ & -6.18705400 & -4.78934800 & -2.64724000 \\
$\mathrm{C}$ & -7.90458900 & -3.97278000 & 1.56175000 \\
$\mathrm{H}$ & -7.24907600 & -4.84896600 & 1.64997500 \\
$\mathrm{H}$ & -8.62874900 & -3.98642000 & 2.37669700 \\
$\mathrm{H}$ & -8.44139100 & -4.02232100 & 0.60554500 \\
& & & \\
\hline & & & \\
& &
\end{tabular}

135

TS1(Z)-1a'; E=-2845.225880
$\mathrm{O}$
$-3.57973300 \quad-1.68776600$
3.16640200
$\mathrm{O}$
$-4.96314300 \quad 2.53587400$
$-0.03701400$
C
$\begin{array}{lll}-2.97206800 & -2.20684900 & 1.98294500\end{array}$ 


$\begin{array}{lrrr}\mathrm{H} & -3.41412300 & -3.17733600 & 1.76178800 \\ \mathrm{H} & -1.90168800 & -2.34751800 & 2.17467500 \\ \mathrm{C} & -3.51958500 & -0.33094500 & 3.15798300 \\ \mathrm{C} & -3.73474500 & 0.37681500 & 4.33387600 \\ \mathrm{H} & -3.94345700 & -0.15618000 & 5.25443700 \\ \mathrm{C} & -3.65638800 & 1.76278500 & 4.28481900 \\ \mathrm{H} & -3.81294900 & 2.33999300 & 5.19077200 \\ \mathrm{C} & -3.35438100 & 2.41510900 & 3.09315400 \\ \mathrm{H} & -3.26571300 & 3.49628200 & 3.06932700 \\ \mathrm{C} & -3.15007700 & 1.69692700 & 1.91073500 \\ \mathrm{C} & -3.25356400 & 0.30075100 & 1.93360200 \\ \mathrm{C} & -2.74137300 & 2.45826200 & 0.69651800 \\ \mathrm{C} & -0.95838900 & 3.55656400 & -0.55089500 \\ \mathrm{C} & -1.88412400 & 3.90866000 & -1.52918200 \\ \mathrm{H} & -1.55165800 & 4.45163100 & -2.40851100 \\ \mathrm{C} & -3.23125100 & 3.57886600 & -1.39494700 \\ \mathrm{H} & -3.93103700 & 3.88875400 & -2.16166400 \\ \mathrm{C} & -3.66534900 & 2.86568200 & -0.27720900 \\ \mathrm{C} & -5.94410600 & 2.96633600 & -0.95334500 \\ \mathrm{H} & -5.96617700 & 4.06000700 & -1.03340200 \\ \mathrm{H} & -6.89632200 & 2.61029000 & -0.55965400 \\ \mathrm{H} & -5.78632900 & 2.53316400 & -1.94954100 \\ \mathrm{C} & -4.97787300 & -1.21693900 & 0.07846300 \\ \mathrm{C} & -5.98002100 & -0.64803100 & 1.08966800 \\ \mathrm{H} & -6.99904700 & -0.83816400 & 0.72917500 \\ \mathrm{H} & -5.87786900 & -1.12146600 & 2.06983700 \\ \mathrm{H} & -5.85456800 & 0.43104200 & 1.20958200 \\ \mathrm{C} & -5.23192900 & -2.72176300 & -0.10622600 \\ \mathrm{H} & -6.15448300 & -2.86634800 & -0.68010400 \\ \mathrm{H} & -4.41917700 & -3.21431800 & -0.65087500 \\ \mathrm{H} & -5.36266700 & -3.23171600 & 0.85260200 \\ \mathrm{C} & -5.16591200 & -0.51843100 & -1.27270800 \\ \mathrm{H} & -6.22260800 & -0.55831100 & -1.56539000 \\ & -4.86725600 & 0.52929700 & -1.21709600\end{array}$




$\begin{array}{lccc}\mathrm{H} & -4.57684400 & -1.00395400 & -2.05081500 \\ \mathrm{H} & -0.97409400 & -2.67787600 & -0.05516400 \\ \mathrm{Rh} & -1.17708300 & -1.33179800 & -0.92082900 \\ \mathrm{P} & -3.14295300 & -0.97111100 & 0.58841000 \\ \mathrm{C} & 3.25278300 & 1.41290700 & 1.59067800 \\ \mathrm{C} & 4.21681000 & 0.92471900 & 0.70114800 \\ \mathrm{C} & 5.46673000 & 1.52537300 & 0.59641100 \\ \mathrm{C} & 5.78056900 & 2.63214400 & 1.38271200 \\ \mathrm{C} & 4.82911900 & 3.12996300 & 2.26770000 \\ \mathrm{C} & 3.57668500 & 2.52974600 & 2.36161200 \\ \mathrm{H} & 3.99293400 & 0.05752500 & 0.08699000 \\ \mathrm{H} & 6.20225400 & 1.11960500 & -0.09301800 \\ \mathrm{H} & 6.75989400 & 3.09631400 & 1.31097000 \\ \mathrm{H} & 5.05706300 & 3.99182200 & 2.88751200 \\ \mathrm{H} & 2.83847400 & 2.93604200 & 3.04735800 \\ \mathrm{C} & 1.92725400 & 0.70738700 & 1.76478700 \\ \mathrm{H} & 2.10905400 & -0.23205700 & 2.30236400 \\ \mathrm{H} & 1.27733600 & 1.30508800 & 2.41551200 \\ \mathrm{C} & 1.19149700 & 0.38278300 & 0.45593100 \\ \mathrm{H} & 0.82740200 & 1.31078200 & 0.01053200 \\ \mathrm{H} & 1.89878800 & -0.05030700 & -0.25307600 \\ \mathrm{C} & 0.07220300 & -0.60623400 & 0.68938800 \\ \mathrm{H} & -0.55759800 & -0.33989200 & 1.54030700 \\ \mathrm{C} & 0.36938100 & -2.00473800 & 0.59160700 \\ \mathrm{H} & 0.09801800 & -2.64859800 & 1.43115700 \\ \mathrm{O} & 1.52386300 & -2.39043500 & -0.04196300 \\ \mathrm{Si} & 2.65945200 & -3.52837200 & 0.45443500 \\ \mathrm{C} & 1.71413800 & -5.10361000 & 0.91574100 \\ \mathrm{H} & 0.96648500 & -4.78430100 & 1.65819100 \\ \mathrm{H} & 2.56380200 & -6.19883700 & 1.57587100 \\ \mathrm{H} & 3.03552700 & -5.85015800 & 2.49960800 \\ \mathrm{C} & 1.94580000 & -7.06809200 & 1.83050900 \\ \mathrm{H} & 3.36037400 & -6.55232300 & 0.91272600 \\ \mathrm{H} & & -5.63599800 & -0.30535800\end{array}$




$\begin{array}{lrrr}\mathrm{H} & 0.32433100 & -4.85578400 & -0.75081300 \\ \mathrm{H} & 1.63884400 & -5.99326300 & -1.07950500 \\ \mathrm{H} & 0.30051800 & -6.47575300 & -0.03115700 \\ \mathrm{C} & 3.68564200 & -3.70266200 & -1.12112600 \\ \mathrm{H} & 2.93138600 & -3.90640600 & -1.89524500 \\ \mathrm{C} & 4.35455600 & -2.36801600 & -1.48527600 \\ \mathrm{H} & 4.87602200 & -2.44164800 & -2.44698000 \\ \mathrm{H} & 3.61192400 & -1.56759000 & -1.56790900 \\ \mathrm{H} & 5.09590000 & -2.06852900 & -0.73409300 \\ \mathrm{C} & 4.68475200 & -4.86706800 & -1.13840500 \\ \mathrm{H} & 4.18433300 & -5.83256500 & -1.01507800 \\ \mathrm{H} & 5.22172500 & -4.89872300 & -2.09393700 \\ \mathrm{H} & 5.43653400 & -4.78098200 & -0.34709200 \\ \mathrm{C} & 3.60820700 & -2.79905100 & 1.92435600 \\ \mathrm{H} & 3.77265500 & -1.74626200 & 1.65256500 \\ \mathrm{C} & 4.98846300 & -3.42551800 & 2.17244100 \\ \mathrm{H} & 5.46702500 & -2.96766600 & 3.04621800 \\ \mathrm{H} & 4.92327100 & -4.50231300 & 2.36470000 \\ \mathrm{H} & 5.65934100 & -3.28094000 & 1.32035500 \\ \mathrm{C} & 2.75960000 & -2.81778800 & 3.20648500 \\ \mathrm{H} & 2.58061400 & -3.84158100 & 3.55401800 \\ \mathrm{H} & 3.26744700 & -2.28261500 & 4.01743600 \\ \mathrm{H} & 1.78245200 & -2.34277000 & 3.06612700 \\ \mathrm{C} & -0.00441800 & -0.17325400 & -2.41175300 \\ \mathrm{C} & -1.26065500 & 0.42365500 & -2.29336800 \\ \mathrm{C} & -2.35659400 & 0.26796500 & -3.32350900 \\ \mathrm{C} & -2.60854300 & -1.19614500 & -3.73779600 \\ \mathrm{C} & -2.26140400 & -2.21571200 & -2.65832200 \\ \mathrm{C} & -1.00127700 & -2.81150400 & -2.51569100 \\ \mathrm{C} & 0.23525900 & -2.51315700 & -3.33716200 \\ \mathrm{C} & 0.43385300 & -1.01691700 & -3.59897900 \\ \mathrm{H} & -1.33873100 & 1.30354600 & -1.66521300 \\ & -0.99309300 & -3.80110000 & -2.06276700 \\ \mathrm{H} & & & \\ \mathrm{H} & & & \end{array}$




\begin{tabular}{|c|c|c|c|}
\hline $\mathrm{H}$ & -3.27209600 & 0.69052900 & -2.90120100 \\
\hline $\mathrm{H}$ & -2.04993200 & -1.43592500 & -4.64870200 \\
\hline $\mathrm{H}$ & -3.66305300 & -1.31612600 & -4.00952000 \\
\hline [ & 0.21564100 & -3.08151100 & -4.28010200 \\
\hline$H$ & 1.09315400 & -2.87979700 & -2.76383400 \\
\hline$H$ & -0.10088400 & -0.70246700 & -4.50097900 \\
\hline $\mathrm{H}$ & 1.49298300 & -0.82281000 & -3.79773000 \\
\hline$\Pi$ & 0.80877500 & 0.28850400 & -1.86943300 \\
\hline $\mathrm{H}$ & -3.10121800 & -2.79674100 & -2.28752500 \\
\hline C & -1.41248900 & 2.83793700 & 0.55820400 \\
\hline $\mathrm{H}$ & -0.70377000 & 2.53418600 & 1.32309100 \\
\hline C & 0.49104400 & 3.84985200 & -0.69452800 \\
\hline $\mathrm{C}$ & 1.15419100 & 4.61748300 & 0.26161800 \\
\hline $\mathrm{C}$ & 1.19366700 & 3.29827200 & -1.76439500 \\
\hline $\mathrm{C}$ & 2.52192400 & 4.84946800 & 0.12846700 \\
\hline $\mathrm{H}$ & 0.61998500 & 5.06775200 & 1.09062800 \\
\hline C & 2.57298000 & 3.48179100 & -1.84946600 \\
\hline $\mathrm{H}$ & 0.69604900 & 2.66728500 & -2.49241200 \\
\hline $\mathrm{C}$ & 3.24726400 & 4.27203600 & -0.91611900 \\
\hline$H$ & 4.31720000 & 4.40949000 & -0.97895700 \\
\hline $\mathrm{O}$ & 3.08099700 & 5.65841800 & 1.06681600 \\
\hline $\mathrm{O}$ & 3.18885400 & 2.84365400 & -2.87739600 \\
\hline C & 4.36624900 & 6.17704800 & 0.80951400 \\
\hline $\mathrm{H}$ & 4.56799500 & 6.89616700 & 1.60466500 \\
\hline $\mathrm{H}$ & 5.13396100 & 5.39505100 & 0.83242700 \\
\hline $\mathrm{H}$ & 4.40253100 & 6.68909400 & -0.16036500 \\
\hline C & 4.59640600 & 2.88262000 & -2.93340300 \\
\hline $\mathrm{H}$ & 5.04311700 & 2.49022100 & -2.01152900 \\
\hline $\mathrm{H}$ & 4.88371000 & 2.24999200 & -3.77395900 \\
\hline $\mathrm{H}$ & 4.96906300 & 3.90029800 & -3.10971200 \\
\hline
\end{tabular}

135

TS2(Z)-1a'; $E=-2845.224058$ 


$\begin{array}{lrrr}\mathrm{O} & -2.97240600 & 1.61957300 & 2.29514800 \\ \mathrm{O} & 1.87259200 & 4.13096700 & 2.29382200 \\ \mathrm{C} & -2.81597000 & 1.53876300 & 0.87395400 \\ \mathrm{H} & -3.62513400 & 2.09721300 & 0.40480100 \\ \mathrm{H} & -2.87941500 & 0.49243000 & 0.57591600 \\ \mathrm{C} & -1.76402800 & 1.53150200 & 2.90407200 \\ \mathrm{C} & -1.69951700 & 1.25441600 & 4.26506800 \\ \mathrm{H} & -2.61495500 & 1.09262800 & 4.82299300 \\ \mathrm{C} & -0.44728400 & 1.19385300 & 4.86183700 \\ \mathrm{H} & -0.36701800 & 0.97683000 & 5.92269700 \\ \mathrm{C} & 0.70714100 & 1.39256600 & 4.11051800 \\ \mathrm{H} & 1.68260900 & 1.31726300 & 4.57878000 \\ \mathrm{C} & 0.63378100 & 1.67383600 & 2.74392100 \\ \mathrm{C} & -0.61962700 & 1.75067300 & 2.12263600 \\ \mathrm{C} & 1.90898300 & 1.81029700 & 1.98519500 \\ \mathrm{C} & 3.70848800 & 0.72033000 & 0.75508200 \\ \mathrm{C} & 4.27005900 & 1.97198600 & 0.51072200 \\ \mathrm{H} & 5.18176300 & 2.04683700 & -0.07423900 \\ \mathrm{C} & 3.69073900 & 3.13376600 & 1.01606500 \\ \mathrm{H} & 4.16939600 & 4.08757400 & 0.82959500 \\ \mathrm{C} & 2.50821500 & 3.05842200 & 1.75356700 \\ \mathrm{C} & 2.42235700 & 5.41157000 & 2.08350700 \\ \mathrm{H} & 3.41703800 & 5.50111400 & 2.53729600 \\ \mathrm{H} & 1.73975600 & 6.11283100 & 2.56367100 \\ \mathrm{H} & 2.48856400 & 5.65204400 & 1.01484400 \\ \mathrm{C} & -1.37457400 & 4.03865100 & 0.41437300 \\ \mathrm{C} & -1.55418600 & 4.59921900 & 1.82950500 \\ \mathrm{H} & -1.74081900 & 5.67890100 & 1.76539000 \\ \mathrm{H} & -2.40593600 & 4.14224300 & 2.34127100 \\ \mathrm{H} & -0.65852400 & 4.44198500 & 2.43561800 \\ & -2.60774000 & 4.37292400 & -0.44098600 \\ \mathrm{H} & -2.59980300 & 5.44269500 & -0.68077200 \\ \mathrm{H} & -2.60105100 & 3.81724100 & -1.38552700 \\ \mathrm{H} & & & \\ \mathrm{H} & & & \\ \mathrm{H} & & & \end{array}$




\begin{tabular}{lrrc} 
C & -0.15994700 & 4.68452800 & -0.25630400 \\
$\mathrm{H}$ & -0.18864900 & 5.77183600 & -0.11147700 \\
$\mathrm{H}$ & 0.77988500 & 4.30912400 & 0.15344700 \\
$\mathrm{H}$ & -0.17583600 & 4.48073200 & -1.32732100 \\
$\mathrm{H}$ & -2.03833000 & 0.45202700 & -1.73319400 \\
$\mathrm{Rh}$ & -0.48162100 & 1.00708500 & -1.76916100 \\
$\mathrm{C}$ & 1.61111500 & 0.62998000 & -2.44029600 \\
$\mathrm{C}$ & 1.58689800 & 1.87801300 & -1.83392000 \\
$\mathrm{C}$ & 1.68787400 & 3.15621400 & -2.63824000 \\
$\mathrm{C}$ & 0.55044800 & 3.35613800 & -3.66778600 \\
$\mathrm{C}$ & -0.68570500 & 2.49296600 & -3.44010500 \\
$\mathrm{C}$ & -0.77330500 & 1.15655100 & -3.88550500 \\
$\mathrm{C}$ & 0.30762700 & 0.37789500 & -4.61675800 \\
$\mathrm{C}$ & 1.69007000 & 0.46901500 & -3.95134800 \\
$\mathrm{H}$ & 1.89873000 & 1.94279800 & -0.79827300 \\
$\mathrm{H}$ & -1.78021500 & 0.79583700 & -4.10551200 \\
$\mathrm{H}$ & 2.66161600 & 3.16872800 & -3.15013200 \\
$\mathrm{H}$ & 1.71620500 & 3.99636400 & -1.94412800 \\
$\mathrm{H}$ & 0.93419200 & 3.16943800 & -4.67928600 \\
$\mathrm{H}$ & 0.24914000 & 4.40929400 & -3.66218000 \\
$\mathrm{H}$ & 0.36915600 & 0.68677800 & -5.67187300 \\
$\mathrm{H}$ & -0.00693000 & -0.67258200 & -4.62542600 \\
$\mathrm{H}$ & 2.25420800 & 1.30934500 & -4.37185600 \\
$\mathrm{H}$ & 2.27328100 & -0.42742400 & -4.18550000 \\
$\mathrm{H}$ & 1.96210300 & -0.20632000 & -1.84502500 \\
$\mathrm{H}$ & -1.62267400 & 3.03938700 & -3.36876600 \\
$\mathrm{C}$ & 0.47604400 & -2.91610100 & 2.07525200 \\
$\mathrm{C}$ & 1.71481600 & -3.51339200 & 2.30998900 \\
$\mathrm{C}$ & 2.29749300 & -3.48016800 & 3.57459700 \\
$\mathrm{C}$ & 1.64267600 & -2.84765900 & 4.62725700 \\
$\mathrm{C}$ & 0.40402300 & -2.24877800 & 4.40548900 \\
$\mathrm{C}$ & -0.17151400 & -2.27992400 & 3.14008800 \\
$\mathrm{H}$ & -3.98807600 & 1.49114900 \\
& -3.93782400 & 3.72357600 \\
\hline
\end{tabular}




$\begin{array}{lccc}\mathrm{H} & 2.09663700 & -2.81494200 & 5.61301600 \\ \mathrm{H} & -0.11084900 & -1.74204900 & 5.21565000 \\ \mathrm{H} & -1.12540400 & -1.78455700 & 2.96962500 \\ \mathrm{C} & -0.09569400 & -2.82769500 & 0.68306600 \\ \mathrm{H} & 0.36351100 & -3.57804000 & 0.02888300 \\ \mathrm{H} & -1.17369400 & -3.01508800 & 0.69537900 \\ \mathrm{C} & 0.15973000 & -1.41285900 & 0.13289700 \\ \mathrm{H} & -0.25312700 & -0.69593600 & 0.84859200 \\ \mathrm{H} & 1.24128800 & -1.24571000 & 0.12026300 \\ \mathrm{C} & -0.36200500 & -1.11950500 & -1.24462300 \\ \mathrm{H} & 0.19623000 & -1.56396600 & -2.06551700 \\ \mathrm{C} & -1.75091700 & -0.96188800 & -1.50947900 \\ \mathrm{H} & -2.10309300 & -1.28333900 & -2.49195900 \\ \mathrm{P} & -1.11990300 & 2.13692600 & 0.37409900 \\ \mathrm{O} & -2.62866000 & -1.21692200 & -0.47603200 \\ \mathrm{Si} & -4.14604100 & -1.96167500 & -0.52582100 \\ \mathrm{C} & -4.10546600 & -3.20660300 & -1.95314500 \\ \mathrm{H} & -3.78602300 & -2.63362300 & -2.83678700 \\ \mathrm{C} & -5.46744000 & -3.82533300 & -2.30246200 \\ \mathrm{H} & -6.17707700 & -3.07026800 & -2.65396600 \\ \mathrm{H} & -5.35840000 & -4.56823300 & -3.10153900 \\ \mathrm{H} & -5.92324900 & -4.33449000 & -1.44722100 \\ \mathrm{C} & -3.04054000 & -4.28517100 & -1.69528900 \\ \mathrm{H} & -2.05304100 & -3.84622700 & -1.51734500 \\ \mathrm{H} & -3.29254000 & -4.89434600 & -0.81939000 \\ \mathrm{H} & -2.95687000 & -4.96452600 & -2.55157300 \\ \mathrm{C} & -4.23020400 & -2.76542700 & 1.18284600 \\ \mathrm{H} & -3.26213400 & -3.27661300 & 1.28573300 \\ \mathrm{C} & -4.30766500 & -1.70500600 & 2.29295000 \\ \mathrm{H} & -4.23308800 & -2.17507600 & 3.28051400 \\ \mathrm{H} & -3.50622200 & -0.96367200 & 2.21969100 \\ \mathrm{H} & -5.25889900 & -1.16095200 & 2.26158300 \\ \mathrm{C} & -5.32760100 & -3.82647500 & 1.34976800 \\ \mathrm{H} & -20606700 & -4.65377700 & 0.64392400\end{array}$




$\begin{array}{lccc}\mathrm{H} & -5.29994400 & -4.25145600 & 2.35983900 \\ \mathrm{H} & -6.32929900 & -3.40875700 & 1.20125900 \\ \mathrm{C} & -5.41842100 & -0.58299300 & -0.76846900 \\ \mathrm{H} & -5.14975300 & 0.16400900 & -0.00862100 \\ \mathrm{C} & -6.87559600 & -0.98847800 & -0.50518500 \\ \mathrm{H} & -7.54348100 & -0.12774800 & -0.62856900 \\ \mathrm{H} & -7.21963800 & -1.76516000 & -1.19582400 \\ \mathrm{H} & -7.01430300 & -1.36617400 & 0.51251700 \\ \mathrm{C} & -5.25374500 & 0.07883100 & -2.14575200 \\ \mathrm{H} & -5.47243200 & -0.62528100 & -2.95758900 \\ \mathrm{H} & -5.94132300 & 0.92546300 & -2.25751600 \\ \mathrm{H} & -4.23721000 & 0.46104000 & -2.29604400 \\ \mathrm{C} & 2.54306600 & 0.66499300 & 1.52603300 \\ \mathrm{H} & 2.08446300 & -0.29244700 & 1.74839300 \\ \mathrm{C} & 4.26036600 & -0.52003300 & 0.15777700 \\ \mathrm{C} & 4.31380100 & -1.70008400 & 0.89998600 \\ \mathrm{C} & 4.66613100 & -0.52006400 & -1.17762500 \\ \mathrm{C} & 4.76736900 & -2.87297500 & 0.30149300 \\ \mathrm{H} & 4.00839800 & -1.72804900 & 1.93950100 \\ \mathrm{C} & 5.11110500 & -1.70000100 & -1.76892800 \\ \mathrm{H} & 4.58718900 & 0.37327900 & -1.78676400 \\ \mathrm{C} & 5.16855300 & -2.89021500 & -1.03772100 \\ \mathrm{H} & 5.51350200 & -3.80477900 & -1.49873200 \\ \mathrm{O} & 4.78596000 & -3.97346200 & 1.10225300 \\ \mathrm{O} & 5.45466600 & -1.60548000 & -3.08029600 \\ \mathrm{C} & 5.21641700 & -5.19638400 & 0.55173000 \\ \mathrm{H} & 5.14731200 & -5.93168100 & 1.35359400 \\ \mathrm{H} & 4.57551000 & -5.51365000 & -0.28091900 \\ \mathrm{H} & 6.25592300 & -5.14031700 & 0.20431300 \\ \mathrm{H} & 5.87252700 & -2.77245500 & -3.74772000 \\ \mathrm{H} & 5.09038400 & -3.54257200 & -3.74614400 \\ \mathrm{H} & -2.07863300 & -2.47734500 & -4.776868000 \\ \mathrm{H} & -3.18944900 & -3.30514900\end{array}$


TS1(Z)-1a_L25; $E=-2730.739114$

\begin{tabular}{|c|c|c|c|}
\hline $\mathrm{P}$ & 2.75582200 & -1.29121500 & -0.34252500 \\
\hline $\mathrm{O}$ & 5.00880100 & -1.97845600 & -1.71885600 \\
\hline $\mathrm{O}$ & 4.88076200 & 1.08174100 & 2.96531100 \\
\hline C & 3.64927300 & -2.39115900 & -1.57974400 \\
\hline $\mathrm{H}$ & 3.63815400 & -3.44303700 & -1.28284700 \\
\hline $\mathrm{H}$ & 3.15355300 & -2.30209500 & -2.54869700 \\
\hline $\mathrm{C}$ & 5.23900800 & -0.76475800 & -1.16183500 \\
\hline $\mathrm{C}$ & 6.46375200 & -0.14213200 & -1.37308400 \\
\hline $\mathrm{H}$ & 7.20920700 & -0.62041600 & -1.99800100 \\
\hline $\mathrm{C}$ & 6.68677400 & 1.08567000 & -0.76387500 \\
\hline $\mathrm{H}$ & 7.63315500 & 1.59509400 & -0.91633400 \\
\hline $\mathrm{C}$ & 5.71342600 & 1.66579000 & 0.04255700 \\
\hline $\mathrm{H}$ & 5.90413100 & 2.61755900 & 0.52695700 \\
\hline $\mathrm{C}$ & 4.48178300 & 1.03666500 & 0.25530200 \\
\hline $\mathrm{C}$ & 4.23381600 & -0.19485300 & -0.36690600 \\
\hline $\mathrm{C}$ & 3.51955600 & 1.72594500 & 1.16716300 \\
\hline $\mathrm{C}$ & 1.55724000 & 3.14461800 & 1.51288500 \\
\hline $\mathrm{C}$ & 1.86934200 & 3.19942500 & 2.87040500 \\
\hline $\mathrm{H}$ & 1.26324300 & 3.79925900 & 3.54223400 \\
\hline $\mathrm{C}$ & 2.96589200 & 2.51839000 & 3.39102100 \\
\hline $\mathrm{H}$ & 3.17013000 & 2.58275600 & 4.45303800 \\
\hline $\mathrm{C}$ & 3.79675900 & 1.78751300 & 2.54535200 \\
\hline $\mathrm{C}$ & 5.11968700 & 0.99135500 & 4.35113900 \\
\hline $\mathrm{H}$ & 5.33902300 & 1.97172700 & 4.79183600 \\
\hline $\mathrm{H}$ & 5.99039600 & 0.34556500 & 4.46467000 \\
\hline $\mathrm{H}$ & 4.26524400 & 0.54360300 & 4.87473700 \\
\hline $\mathrm{C}$ & 2.96501000 & -2.28350800 & 1.27084800 \\
\hline $\mathrm{C}$ & 4.42557400 & -2.66750900 & 1.53958400 \\
\hline $\mathrm{H}$ & 4.48674100 & -3.26296300 & 2.45900500 \\
\hline $\mathrm{H}$ & 4.85436300 & -3.26356200 & 0.72803300 \\
\hline $\mathrm{H}$ & 5.04688200 & -1.77746600 & 1.67243800 \\
\hline
\end{tabular}




$\begin{array}{lrrc}\mathrm{C} & 2.10616600 & -3.55289800 & 1.15819500 \\ \mathrm{H} & 2.14466500 & -4.10290900 & 2.10577200 \\ \mathrm{H} & 1.05873300 & -3.32522000 & 0.94307400 \\ \mathrm{H} & 2.46216000 & -4.22962300 & 0.37495400 \\ \mathrm{C} & 2.45694600 & -1.42156500 & 2.43599600 \\ \mathrm{H} & 2.38686000 & -2.03310100 & 3.34409000 \\ \mathrm{H} & 3.14992300 & -0.60440600 & 2.63902800 \\ \mathrm{H} & 1.47205300 & -0.98725600 & 2.24275800 \\ \mathrm{H} & 0.02482400 & -1.92546100 & -0.35511600 \\ \mathrm{C} & -4.10404700 & 1.71389400 & 0.81336400 \\ \mathrm{C} & -5.22269700 & 1.38917400 & 1.58848300 \\ \mathrm{C} & -6.49646700 & 1.81964400 & 1.23449500 \\ \mathrm{C} & -6.67800900 & 2.59226100 & 0.08935200 \\ \mathrm{C} & -5.57476300 & 2.92767200 & -0.68820100 \\ \mathrm{C} & -4.30185300 & 2.49041200 & -0.33065800 \\ \mathrm{H} & -5.08828000 & 0.78383600 & 2.48227700 \\ \mathrm{H} & -7.34914200 & 1.54844500 & 1.85019500 \\ \mathrm{H} & -7.67097900 & 2.92885400 & -0.19242000 \\ \mathrm{H} & -5.70290200 & 3.52997300 & -1.58291500 \\ \mathrm{H} & -3.45239200 & 2.75872200 & -0.95056000 \\ \mathrm{C} & -2.73798300 & 1.21405900 & 1.22574600 \\ \mathrm{H} & -2.32901000 & 1.87287100 & 2.00160400 \\ \mathrm{H} & -2.86517800 & 0.23143100 & 1.69055200 \\ \mathrm{C} & -1.72184500 & 1.09675600 & 0.07921700 \\ \mathrm{H} & -2.22059200 & 0.65441600 & -0.78611300 \\ \mathrm{H} & -1.38841800 & 2.09440300 & -0.21560000 \\ \mathrm{C} & -0.53700800 & 0.23401400 & 0.45120800 \\ \mathrm{H} & 0.16586100 & 0.65912700 & 1.16424000 \\ \mathrm{C} & -0.79100000 & -1.16635200 & 0.56735000 \\ \mathrm{H} & -0.32770300 & -1.69303600 & 1.40497400 \\ \mathrm{C} & -2.05551100 & -1.59568700 & 0.25835600 \\ \mathrm{H} & -2.89568600 & -2.99021000 & 0.68035100 \\ & -2.12591300 & -3.65796100 & 2.27934000 \\ \mathrm{H} & -04371800 & -3.72148200 & 2.08574000\end{array}$




$\begin{array}{lrrr}\text { C } & -2.58945900 & -5.07061100 & 2.66475800 \\ \mathrm{H} & -2.32706300 & -5.80767100 & 1.90007900 \\ \mathrm{H} & -2.11852600 & -5.38937100 & 3.60230800 \\ \mathrm{H} & -3.67332500 & -5.12083300 & 2.81374300 \\ \mathrm{C} & -2.33749600 & -2.66667800 & 3.43498500 \\ \mathrm{H} & -1.97012000 & -1.66321200 & 3.19220000 \\ \mathrm{H} & -3.40091500 & -2.57286900 & 3.68323100 \\ \mathrm{H} & -1.81813900 & -3.00075300 & 4.34083200 \\ \mathrm{C} & -4.63765000 & -2.30334300 & 0.92277000 \\ \mathrm{H} & -4.49100400 & -1.48055200 & 1.63794900 \\ \mathrm{C} & -5.15524500 & -1.67297700 & -0.37973900 \\ \mathrm{H} & -6.09945000 & -1.14447000 & -0.20638700 \\ \mathrm{H} & -4.44262300 & -0.94374900 & -0.77742500 \\ \mathrm{H} & -5.33370400 & -2.43423000 & -1.14931900 \\ \mathrm{C} & -5.66190100 & -3.26642700 & 1.53697100 \\ \mathrm{H} & -5.34768900 & -3.62353300 & 2.52328000 \\ \mathrm{H} & -6.63009400 & -2.76746800 & 1.66280500 \\ \mathrm{H} & -5.83113900 & -4.14571200 & 0.90710000 \\ \mathrm{C} & -2.72694900 & -4.18299600 & -0.77731200 \\ \mathrm{H} & -2.91741100 & -3.55025600 & -1.65649400 \\ \mathrm{C} & -3.75496100 & -5.32327000 & -0.81018900 \\ \mathrm{H} & -3.57131700 & -5.98258100 & -1.66689200 \\ \mathrm{H} & -3.71597400 & -5.94454200 & 0.09104400 \\ \mathrm{H} & -4.77577600 & -4.94084400 & -0.90512500 \\ \mathrm{C} & -1.28857800 & -4.71361800 & -0.89653800 \\ \mathrm{H} & -1.02490900 & -5.35801500 & -0.04919200 \\ \mathrm{H} & -1.16768100 & -5.30919200 & -1.80902900 \\ \mathrm{H} & -0.55440600 & -3.90065600 & -0.93801600 \\ \mathrm{Rh} & 0.58531500 & -0.68468000 & -1.24059400 \\ \mathrm{C} & -0.76172800 & -1.35893400 & -2.76290800 \\ \mathrm{C} & 0.52393700 & -1.79107700 & -3.15835000 \\ \mathrm{C} & 1.29144100 & -1.10338400 & -4.28138500 \\ \mathrm{C} & 2.17405300 & 0.05993100 & -3.79244800 \\ \mathrm{C} & & & \\ & & & \end{array}$




\begin{tabular}{|c|c|c|c|}
\hline $\mathrm{C}$ & 0.33908600 & 1.20805600 & -2.38104700 \\
\hline $\mathrm{C}$ & -0.73418200 & 1.09925600 & -3.45587400 \\
\hline $\mathrm{C}$ & -1.53105000 & -0.21465900 & -3.39702900 \\
\hline $\mathrm{H}$ & 0.75815600 & -2.84183200 & -2.99992100 \\
\hline $\mathrm{H}$ & 0.18202100 & 1.97967800 & -1.63538300 \\
\hline $\mathrm{H}$ & 0.58210800 & -0.74390100 & -5.03667100 \\
\hline $\mathrm{H}$ & 1.92271600 & -1.83414600 & -4.79845200 \\
\hline $\mathrm{H}$ & 2.32365600 & 0.79668500 & -4.59581800 \\
\hline $\mathrm{H}$ & 3.17269800 & -0.31988500 & -3.54973300 \\
\hline $\mathrm{H}$ & -0.26368500 & 1.21884600 & -4.43886800 \\
\hline $\mathrm{H}$ & -1.42135100 & 1.94561700 & -3.34756700 \\
\hline $\mathrm{H}$ & -1.88235200 & -0.49409000 & -4.40233400 \\
\hline $\mathrm{H}$ & -2.43196700 & -0.05901000 & -2.79512900 \\
\hline $\mathrm{H}$ & -1.40713100 & -2.10611700 & -2.30382500 \\
\hline $\mathrm{H}$ & 2.42333800 & 1.22253400 & -1.95710100 \\
\hline $\mathrm{C}$ & 2.40004700 & 2.38939200 & 0.68536600 \\
\hline $\mathrm{H}$ & 2.17412900 & 2.30779400 & -0.37046800 \\
\hline $\mathrm{C}$ & 0.37635300 & 3.84546600 & 0.95320400 \\
\hline $\mathrm{C}$ & 0.35490100 & 4.29204600 & -0.36923500 \\
\hline $\mathrm{C}$ & -0.78109200 & 4.05001600 & 1.71997300 \\
\hline $\mathrm{H}$ & 1.24090600 & 4.18805800 & -0.98792700 \\
\hline $\mathrm{H}$ & -0.81563600 & 3.69575900 & 2.74586600 \\
\hline $\mathrm{C}$ & -1.92562800 & 5.04347300 & -0.15475400 \\
\hline $\mathrm{C}$ & -1.91202600 & 4.63957200 & 1.18384100 \\
\hline $\mathrm{H}$ & -2.81870600 & 4.75682300 & 1.76686800 \\
\hline $\mathrm{C}$ & -0.78006100 & 4.87227700 & -0.93231900 \\
\hline $\mathrm{H}$ & -0.75051300 & 5.18981600 & -1.96793000 \\
\hline $\mathrm{O}$ & -3.08815400 & 5.58079000 & -0.60014900 \\
\hline $\mathrm{C}$ & -3.22274400 & 5.80286800 & -1.98446800 \\
\hline $\mathrm{H}$ & -4.24790800 & 6.13962400 & -2.13993500 \\
\hline $\mathrm{H}$ & -2.53313800 & 6.57711500 & -2.34393900 \\
\hline $\mathrm{H}$ & -3.05349600 & 4.87974000 & -2.55490700 \\
\hline
\end{tabular}


TS2(Z)-1a_L25; E= -2730.738323

$\begin{array}{lrrc}\mathrm{P} & 0.00274200 & -2.48022100 & 0.54962900 \\ \mathrm{O} & 0.73124600 & -5.03621300 & 1.11747300 \\ \mathrm{O} & 0.94080300 & -0.30906300 & 4.36974400 \\ \mathrm{C} & 0.12975200 & -4.28170300 & 0.06523900 \\ \mathrm{H} & -0.83423700 & -4.73240000 & -0.17361600 \\ \mathrm{H} & 0.77249900 & -4.33697600 & -0.81585200 \\ \mathrm{C} & 1.49784400 & -4.23891800 & 1.89995500 \\ \mathrm{C} & 2.34842800 & -4.82194300 & 2.83051000 \\ \mathrm{H} & 2.40325700 & -5.90201100 & 2.90498500 \\ \mathrm{C} & 3.10505300 & -3.98563500 & 3.64031700 \\ \mathrm{H} & 3.77976200 & -4.41374300 & 4.37521600 \\ \mathrm{C} & 3.02169800 & -2.60515800 & 3.50171100 \\ \mathrm{H} & 3.63374500 & -1.95748700 & 4.12044400 \\ \mathrm{C} & 2.16544400 & -2.02106000 & 2.56057000 \\ \mathrm{C} & 1.36922700 & -2.84605900 & 1.75447700 \\ \mathrm{C} & 2.23736200 & -0.53656300 & 2.43434200 \\ \mathrm{C} & 3.18997200 & 1.40951400 & 1.31037200 \\ \mathrm{C} & 2.63967100 & 2.22067300 & 2.30069000 \\ \mathrm{H} & 2.77884900 & 3.29607200 & 2.25867200 \\ \mathrm{C} & 1.87672500 & 1.68501800 & 3.33321100 \\ \mathrm{H} & 1.43600200 & 2.35709400 & 4.06019800 \\ \mathrm{C} & 1.66972700 & 0.30774200 & 3.40258100 \\ \mathrm{C} & 0.23665300 & 0.50249700 & 5.28369500 \\ \mathrm{H} & 0.91807800 & 1.09124700 & 5.91070400 \\ \mathrm{H} & -0.33383300 & -0.17932400 & 5.91480600 \\ \mathrm{H} & -0.44698900 & 1.18316000 & 4.76380000 \\ \mathrm{C} & -1.53579900 & -2.49857300 & 1.68680900 \\ \mathrm{C} & -1.44610100 & -3.52432400 & 2.82359500 \\ \mathrm{H} & -2.38198800 & -3.50322400 & 3.39555800 \\ \mathrm{H} & -1.30132400 & -4.54328100 & 2.45562500 \\ \mathrm{C} & -0.62945100 & -3.28706300 & 3.51068000 \\ & -2.74679700 & -2.81076800 & 0.79893900\end{array}$




\begin{tabular}{|c|c|c|c|}
\hline $\mathrm{H}$ & -3.66078300 & -2.75921300 & 1.40340200 \\
\hline $\mathrm{H}$ & -2.83200100 & -2.08296800 & -0.01147800 \\
\hline $\mathrm{H}$ & -2.70509500 & -3.81365900 & 0.36307400 \\
\hline C & -1.71075600 & -1.09837100 & 2.28780200 \\
\hline $\mathrm{H}$ & -2.56438100 & -1.10567600 & 2.97837800 \\
\hline $\mathrm{H}$ & -0.82751900 & -0.79069500 & 2.85584600 \\
\hline $\mathrm{H}$ & -1.92008700 & -0.36582500 & 1.50575900 \\
\hline $\mathrm{H}$ & -1.50826400 & -1.04941700 & -1.58828500 \\
\hline C & -0.65426300 & 3.58801300 & 1.67314300 \\
\hline C & -1.42721700 & 3.12216800 & 2.74107100 \\
\hline C & -1.29178300 & 3.66540600 & 4.01449700 \\
\hline C & -0.36330400 & 4.67851900 & 4.24901100 \\
\hline C & 0.41059900 & 5.15362700 & 3.19388000 \\
\hline C & 0.25705400 & 4.61607300 & 1.91805300 \\
\hline $\mathrm{H}$ & -2.14497800 & 2.32458800 & 2.56522300 \\
\hline $\mathrm{H}$ & -1.91541500 & 3.30095400 & 4.82609700 \\
\hline $\mathrm{H}$ & -0.25430400 & 5.10372700 & 5.24185800 \\
\hline $\mathrm{H}$ & 1.13071500 & 5.94896800 & 3.36163000 \\
\hline $\mathrm{H}$ & 0.85670100 & 5.00191000 & 1.09692900 \\
\hline C & -0.79053400 & 2.96099400 & 0.31020300 \\
\hline $\mathrm{H}$ & -1.85141100 & 2.85283200 & 0.06493800 \\
\hline $\mathrm{H}$ & -0.35317900 & 3.61964700 & -0.45036300 \\
\hline C & -0.13032100 & 1.56941200 & 0.22056000 \\
\hline $\mathrm{H}$ & 0.93771300 & 1.66595700 & 0.40996600 \\
\hline $\mathrm{H}$ & -0.51849800 & 0.93236700 & 1.01571200 \\
\hline C & -0.33932200 & 0.93472800 & -1.12542300 \\
\hline $\mathrm{H}$ & 0.17456800 & 1.43296800 & -1.94515100 \\
\hline C & -1.59945600 & 0.38110000 & -1.49442500 \\
\hline $\mathrm{H}$ & -1.90349100 & 0.53113800 & -2.53178200 \\
\hline $\mathrm{O}$ & -2.64546500 & 0.42116900 & -0.58751700 \\
\hline $\mathrm{Si}$ & -4.26035300 & 0.73581900 & -0.99272100 \\
\hline C & -4.63031200 & -0.17591900 & -2.61720800 \\
\hline $\mathrm{H}$ & -3.86474500 & 0.16378400 & -3.33049200 \\
\hline C & -5.99015900 & 0.17387900 & -3.24145000 \\
\hline
\end{tabular}




$\begin{array}{lrrr}\mathrm{H} & -6.06232600 & 1.23653900 & -3.49108200 \\ \mathrm{H} & -6.14109200 & -0.38948000 & -4.16971200 \\ \mathrm{H} & -6.82517700 & -0.06743700 & -2.57609500 \\ \mathrm{C} & -4.45825100 & -1.69514800 & -2.45829200 \\ \mathrm{H} & -3.44137900 & -1.95868500 & -2.14923000 \\ \mathrm{H} & -5.14968300 & -2.10411300 & -1.71222900 \\ \mathrm{H} & -4.65711000 & -2.20868400 & -3.40619400 \\ \mathrm{C} & -5.19076400 & 0.05908800 & 0.50464900 \\ \mathrm{H} & -4.74045600 & -0.92677300 & 0.67374000 \\ \mathrm{C} & -4.90535700 & 0.90804900 & 1.75310100 \\ \mathrm{H} & -5.33859900 & 0.44428200 & 2.64708100 \\ \mathrm{H} & -3.83016600 & 1.01601700 & 1.92335000 \\ \mathrm{H} & -5.33474200 & 1.91262900 & 1.66552100 \\ \mathrm{C} & -6.69766900 & -0.15214700 & 0.30024200 \\ \mathrm{H} & -6.90163900 & -0.88314100 & -0.48810900 \\ \mathrm{H} & -7.15976400 & -0.52865200 & 1.22053800 \\ \mathrm{H} & -7.21524900 & 0.77568900 & 0.03456000 \\ \mathrm{C} & -4.43294700 & 2.61618000 & -1.16057500 \\ \mathrm{H} & -3.94315600 & 3.01185500 & -0.25852400 \\ \mathrm{C} & -5.87790900 & 3.13825300 & -1.14600700 \\ \mathrm{H} & -5.89031900 & 4.23157500 & -1.22835600 \\ \mathrm{H} & -6.46933400 & 2.74597500 & -1.97907600 \\ \mathrm{H} & -6.39805600 & 2.87552700 & -0.21999500 \\ \mathrm{C} & -3.65897300 & 3.14716100 & -2.37728400 \\ \mathrm{H} & -4.06502100 & 2.74952400 & -3.31503200 \\ \mathrm{H} & -3.72212300 & 4.24021600 & -2.43386400 \\ \mathrm{H} & -2.59671300 & 2.88359200 & -2.33199100 \\ \mathrm{Rh} & 0.12312500 & -1.20307500 & -1.54246500 \\ \mathrm{C} & 0.05951900 & -1.33685800 & -3.67383700 \\ \mathrm{C} & 0.03962000 & -2.66892400 & -3.18537400 \\ \mathrm{C} & 1.26544300 & -3.55798900 & -3.26503200 \\ \mathrm{C} & 2.52487200 & -2.92066900 & -2.63921300 \\ \mathrm{C} & 2.24501100 & -1.80403400 & -1.63694200 \\ \mathrm{C} & & -0.45402600 & -1.97520400\end{array}$




$\begin{array}{lrrr}\mathrm{C} & 2.16679400 & 0.08131200 & -3.39135700 \\ \mathrm{C} & 1.28081700 & -0.72250900 & -4.35904100 \\ \mathrm{H} & -0.90996100 & -3.19972400 & -3.22079700 \\ \mathrm{H} & 2.39132300 & 0.27721600 & -1.21279100 \\ \mathrm{H} & 1.47289400 & -3.82801000 & -4.31202500 \\ \mathrm{H} & 1.04362500 & -4.50510100 & -2.76425100 \\ \mathrm{H} & 3.17921200 & -2.52753200 & -3.42527500 \\ \mathrm{H} & 3.10934100 & -3.69555200 & -2.13206100 \\ \mathrm{H} & 3.20409400 & 0.13367600 & -3.75525800 \\ \mathrm{H} & 1.81689400 & 1.11850300 & -3.35433600 \\ \mathrm{H} & 1.86446600 & -1.51381600 & -4.84306100 \\ \mathrm{H} & 0.94878600 & -0.06562400 & -5.16971600 \\ \mathrm{H} & -0.89658300 & -0.93645100 & -4.01450400 \\ \mathrm{H} & 2.55046100 & -2.02702000 & -0.61782400 \\ \mathrm{C} & 2.98963900 & 0.02891800 & 1.41427700 \\ \mathrm{H} & 3.41193300 & -0.62926900 & 0.66199000 \\ \mathrm{C} & 3.83884100 & 1.97250300 & 0.10297900 \\ \mathrm{C} & 4.94336800 & 1.36493600 & -0.49312600 \\ \mathrm{C} & 3.26823200 & 3.07452700 & -0.55155200 \\ \mathrm{H} & 5.41137400 & 0.51370300 & -0.00724300 \\ \mathrm{H} & 2.38917200 & 3.54890200 & -0.12440400 \\ \mathrm{C} & 4.86922000 & 2.90570600 & -2.34643200 \\ \mathrm{C} & 3.77169300 & 3.53881600 & -1.75445100 \\ \mathrm{H} & 3.31979500 & 4.38118500 & -2.26691700 \\ \mathrm{C} & 5.46176700 & 1.81716200 & -1.70460700 \\ \mathrm{H} & 6.32103400 & 1.31389400 & -2.13149000 \\ \mathrm{O} & 5.27862800 & 3.41813900 & -3.53495800 \\ \mathrm{C} & 6.34150900 & 2.77457700 & -4.19882600 \\ \mathrm{H} & 6.48145500 & 3.30788900 & -5.13933900 \\ \mathrm{H} & 7.27163600 & 2.82194500 & -3.61804400 \\ & 6.10737400 & 1.72370200 & -4.41275000 \\ & & & \\ \mathrm{H} & & \end{array}$




\section{References}

1. Giordano, G.; Crabtree, R. H.; Heintz, R. M.; Forster, D.; Morris, D. E. Inorg. Synth. 1990, 28, 88-90.

2. Xiong, W.; Xu, G.; Yu, X.; Tang, W. Organometallics 2019, 38, 4003-4013.

3. Noguchi, H.; Hojo, K.; Suginome, M. J. Am. Chem. Soc. 2007, 129, 758-759.

4. Ohmaru, Y.; Sato, N.; Mizutani, M.; Kotani, S.; Sugiura, M.; Nakajima, M. Org. Biomol. Chem. 2012, 10, 4562-4570.

5. Li, Y.; Lu, L.-Q.; Das, S.; Pisiewicz, S.; Junge, K.; Beller, M. J. Am. Chem. Soc. 2012, 134, 18325-18329.

6. Koschker, P.; Kähny, M.; Breit, B. J. Am. Chem. Soc. 2015, 137, 3131-3137.

7. Chen, B.; Fang, C.; Liu, P.; Ready, J. M. Angew. Chem. Int. Ed. 2017, 56, 87808784.

8. Nguyen, T. Q.; Chai, W.; Gu, J.; Cook, K.; Kim, E.; Goetz, S.; Farni, Z.; Chepuru, M.; Cox, M.; Nguyen, P.; Raja, H.; Magistrado, P.; Michael, F.; Oelschlaeger, P.; Buynak, J. D. Tetrahedron Lett. 2015, 56, 3385-3389.

9. Danheiser, R. L.; Nishida, A.; Savariar, S.; Trova, M. P. Tetrahedron Lett. 1988, 29, 4917-4920.

10. Yamamoto, Y.; Fujikawa, R.; Umemoto, T.; Miyaura, N. Tetrahedron 2004, 60, 10695-10700.

11. Yang, C.-T.; Zhang, Z.-Q.; Tajuddin, H.; Wu, C.-C.; Liang, J.; Liu, J.-H.; Fu, Y.; Czyzewska, M.; Steel, P. G.; Marder, T. B.; Liu, L. Angew. Chem. Int. Ed. 2012, $51,528-532$.

12. Li, J.; Qu, S.; Zhao, W. Angew. Chem. Int. Ed. 2020, 59, 2360-2364.

13. Yamasaki, Y.; Kumagai, T.; Kanno, S.; Kakiuchi, F.; Kochi, T. J. Org. Chem. 2018, 83, 9322-9333.

14. Dong, W.; Yang, H.; Yang, W.; Zhao, W. Org. Lett. 2020, 22, 1265-1269.

15. Sandford, C.; Aggarwal, V. K. Chem. Commun. 2017, 53, 5481-5494.

16. Nyasse, B.; Grehn, L.; Ragnarsson, U. Chem. Commun. 1997, 1017-1018.

17. Chong, P. Y.; Shotwell, J. B.; Miller, J.; Price, D. J.; Maynard, A.; Voitenleitner, C.; Mathis, A.; Williams, S.; Pouliot, J. J.; Creech, K.; Wang, F.; Fang, J.; Zhang, H.; Tai, V. W. F.; Turner, E.; Kahler, K. M.; Crosby, R.; Peat, A. J. J. Med. Chem. 2019, 62, 3254-3267.

18. Shekhar, S.; Dunn, T. B.; Kotecki, B. J.; Montavon, D. K.; Cullen, S. C. J. Org. 
Chem. 2011, 76, 4552-4563.

19. Guha, S. K.; Shibayama, A.; Abe, D.; Sakaguchi, M.; Ukaji, Y.; Inomata, K. Bull. Chem. Soc. Jpn. 2004, 77, 2147-2157.

20. Kondoh, A.; Odaira, K.; Terada, M. Angew. Chem. Int. Ed. 2015, 54, 1124011244.

21. Preshlock, S. M.; Ghaffari, B.; Maligres, P. E.; Krska, S. W.; Maleczka, R. E.; Smith, M. R. J. Am. Chem. Soc. 2013, 135, 7572-7582.

22. Okamoto, A.; Tainaka, K.; Nishiza, K.-i.; Saito, I. J. Am. Chem. Soc. 2005, 127, 13128-13129.

23. Claudel, S.; Gosmini, C.; Paris, J. M.; Périchon, J. Chem. Commun. 2007, 36673669.

24. Ebisawa, M.; Ueno, M.; Oshima, Y.; Kondo, Y. Tetrahedron Lett. 2007, 48, 8918-8921.

25. Kolonko, K. J.; Reich, H. J. J. Am. Chem. Soc. 2008, 130, 9668-9669.

26. Chen, G.-Q.; Xu, Z.-J.; Zhou, C.-Y.; Che, C.-M. Chem. Commun. 2011, 47, 10963-10965.

27. Kouser, F.; Sharma, V. K.; Rizvi, M.; Sultan, S.; Chalotra, N.; Gupta, V. K.; Nandi, U.; Shah, B. A. Tetrahedron Lett. 2018, 59, 2161-2166.

28. Stadlwieser, J. F.; Dambaur, M. E. Helv. Chim. Acta 2006, 89, 936-946.

29. Iwamoto, T.; Okuzono, C.; Adak, L.; Jin, M.; Nakamura, M. Chem. Commun. 2019, 55, 1128-1131.

30. Zhang, X.; Xia, A.; Chen, H.; Liu, Y. Org. Lett. 2017, 19, 2118-2121.

31. Hostetler, E. D.; Jonson, S. D.; Welch, M. J.; Katzenellenbogen, J. A. J. Org. Chem. 1999, 64, 178-185.

32. Jarava-Barrera, C.; Parra, A.; López, A.; Cruz-Acosta, F.; Collado-Sanz, D.; Cárdenas, D. J.; Tortosa, M. ACS Catal. 2016, 6, 442-446.

33. Sun, S.-Z.; Martin, R. Angew. Chem. Int. Ed. 2018, 57, 3622-3625.

34. Mlynarski, S. N.; Karns, A. S.; Morken, J. P. J. Am. Chem. Soc. 2012, 134, 16449-16451.

35. Sato, Y.; Kayaki, Y.; Ikariya, T. Chem Asian J 2016, 11, 2924-2931.

36. Tian, B.; Chen, P.; Leng, X.; Liu, G. Nat. Catal. 2021, 4, 172-179.

37. Edelstein, E. K.; Grote, A. C.; Palkowitz, M. D.; Morken, J. P. Synlett 2018, 29, 1749-1752.

38. Hu, Y.; Chen, J.; Li, B.; Zhang, Z.; Gridnev, I. D.; Zhang, W. Angew. Chem. 
Int. Ed. 2020, 59, 5371-5375.

39. Stephens, P. J.; Devlin, F. J.; Chabalowski, C. F.; Frisch, M. J. J. Phys. Chem. 1994, 98, 11623-11627.

40. Chai, J.-D.; Head-Gordon, M. Phys. Chem. Chem. Phys. 2008, 10, 6615-6620.

41. Hehre, W. J.; Ditchfield, R.; Pople, J. A. J. Chem. Phys. 1972, 56, 2257-2261.

42. Hariharan, P. C.; Pople, J. A. Theor. Chim. Acta 1973, 28, 213-222.

43. Hay, P. J.; Wadt, W. R. J. Chem. Phys. 1985, 82, 270-283.

44. Ehlers, A. W.; Böhme, M.; Dapprich, S.; Gobbi, A.; Höllwarth, A.; Jonas, V.; Köhler, K. F.; Stegmann, R.; Veldkamp, A.; Frenking, G. Chem. Phys. Lett. 1993, $208,111-114$.

45. Höllwarth, A.; Böhme, M.; Dapprich, S.; Ehlers, A. W.; Gobbi, A.; Jonas, V.; Köhler, K. F.; Stegmann, R.; Veldkamp, A.; Frenking, G. Chem. Phys. Lett. 1993, $208,237-240$.

46. Fukui, K. Acc. Chem. Res. 1981, 14, 363-368. Frisch, M. J.; Trucks, G. W.; Schlegel, H. B.; Scuseria, G. E.; Robb, M. A.; Cheeseman, J. R.;Scalmani, G.; Barone, V.; Mennucci, B.; Petersson, G. A.; Nakatsuji, H.; Caricato, M.; Li, X.;Hratchian, H. P.; Izmaylov, A. F.; Bloino, J.; Zheng, G.; Sonnenberg, J. L.; Hada, M.; Ehara, M.;Toyota, K.; Fukuda, R.; Hasegawa, J.; Ishida, M.; Nakajima, T.; Honda, Y.; Kitao, O.; Nakai, H.;Vreven, T.; Montgomery, Jr., J. A.; Peralta, J. E.; Ogliaro, F.; Bearpark, M.; Heyd, J. J.; Brothers, E.;Kudin, K. N.; Staroverov, V. N.; Kobayashi, R.; Normand, J.; Raghavachari, K.; Rendell, A.; Burant,J. C.; Iyengar, S. S.; Tomasi, J.; Cossi, M.; Rega, N.; Millam, J. M.; Klene, M.; Knox, J. E.; Cross, J.B.; Bakken, V.; Adamo, C.; Jaramillo, J.; Gomperts, R.; Stratmann, R. E.; Yazyev, O.; Austin, A. J.;Cammi, R.; Pomelli, C.; Ochterski, J. W.; Martin, R. L.; Morokuma, K.; Zakrzewski, V. G.; Voth, G.A.; Salvador, P.; Dannenberg, J. J.; Dapprich, S.; Daniels, A. D.; Farkas, O “; Foresman, J. B.; Ortiz,J. V.; Cioslowski, J.; Fox, D. J. Gaussian 09, Revision D.01; Gaussian, Inc.: Wallingford, CT, 2009. 


\section{NMR Spectra}

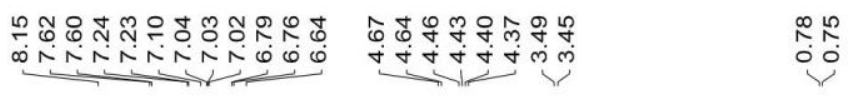

${ }^{1} \mathrm{H} \mathrm{NMR}\left(400 \mathrm{MHz}, \mathrm{C}_{6} \mathrm{D}_{6}\right)$

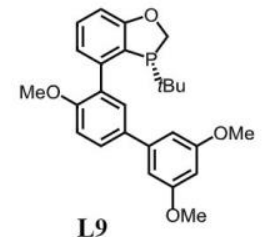

L9

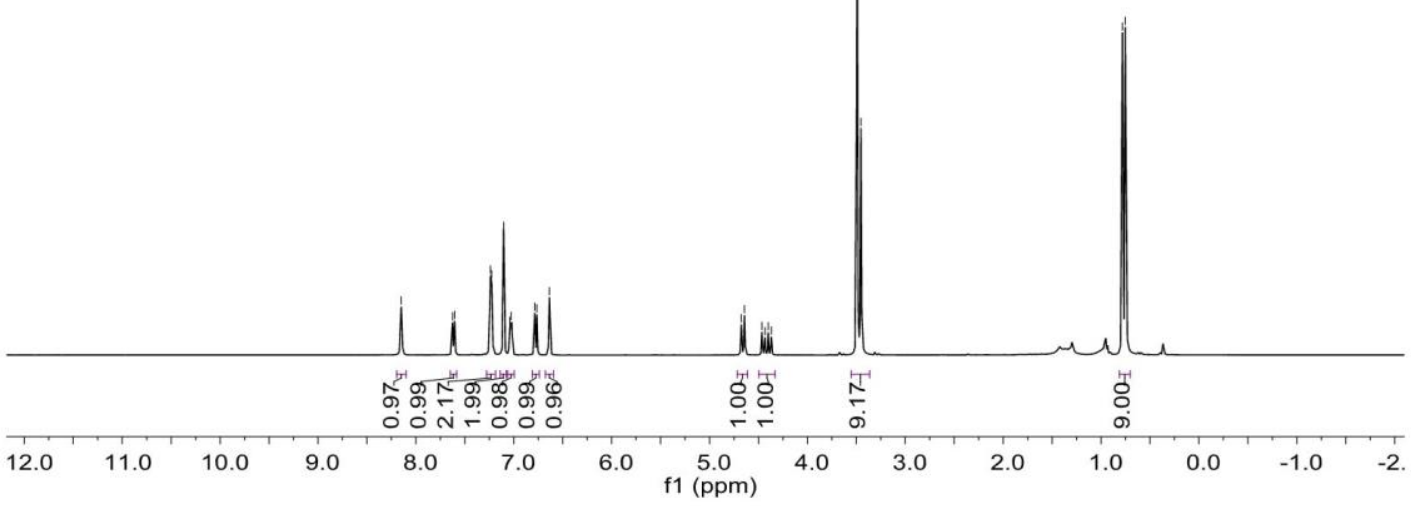

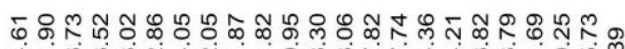

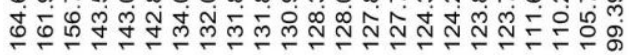

ผุ๊

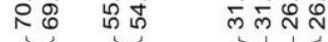
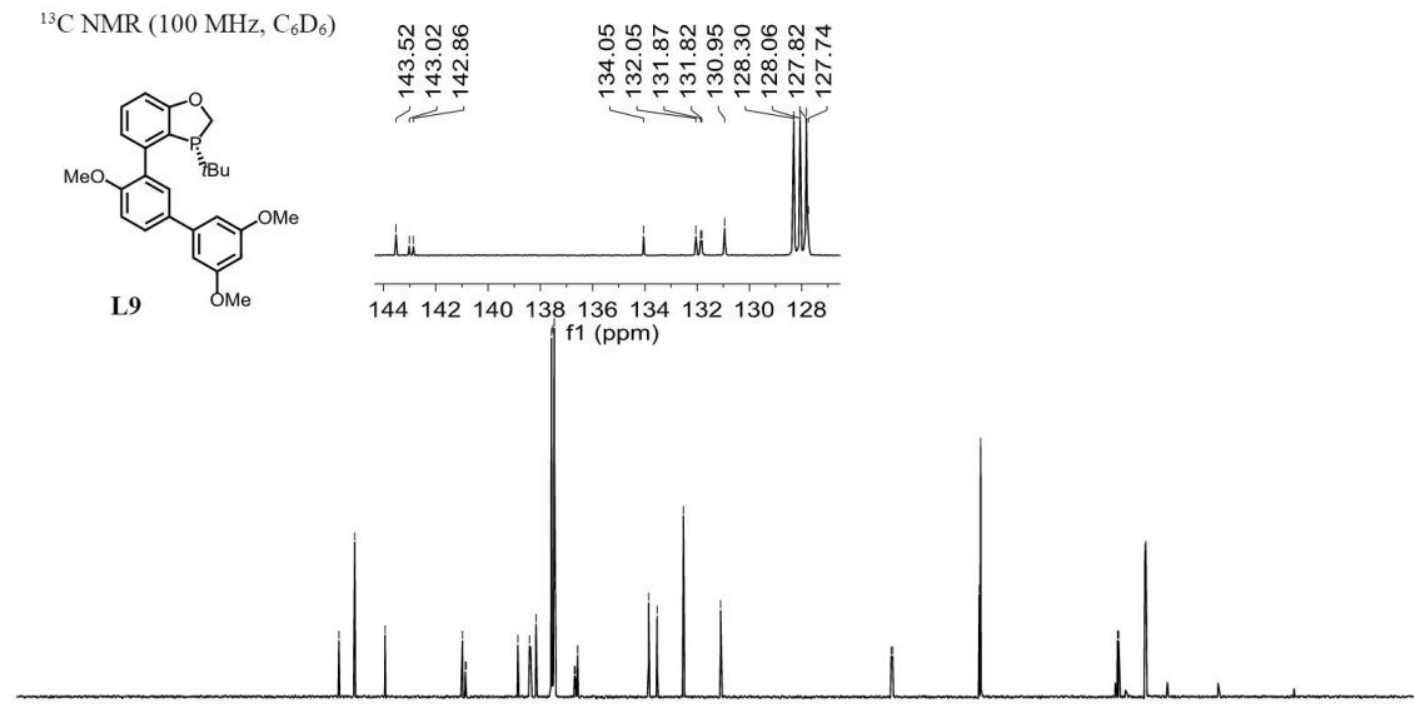

$\begin{array}{llllllllllllllllllllllll}210 & 200 & 190 & 180 & 170 & 160 & 150 & 140 & 130 & 120 & 110 & 100 & 90 & 80 & 70 & 60 & 50 & 40 & 30 & 20 & 10 & 0 & -10\end{array}$ f1 (ppm) 
${ }^{31} \mathrm{P}$ NMR $\left(162 \mathrm{MHz}, \mathrm{C}_{6} \mathrm{D}_{6}\right)$
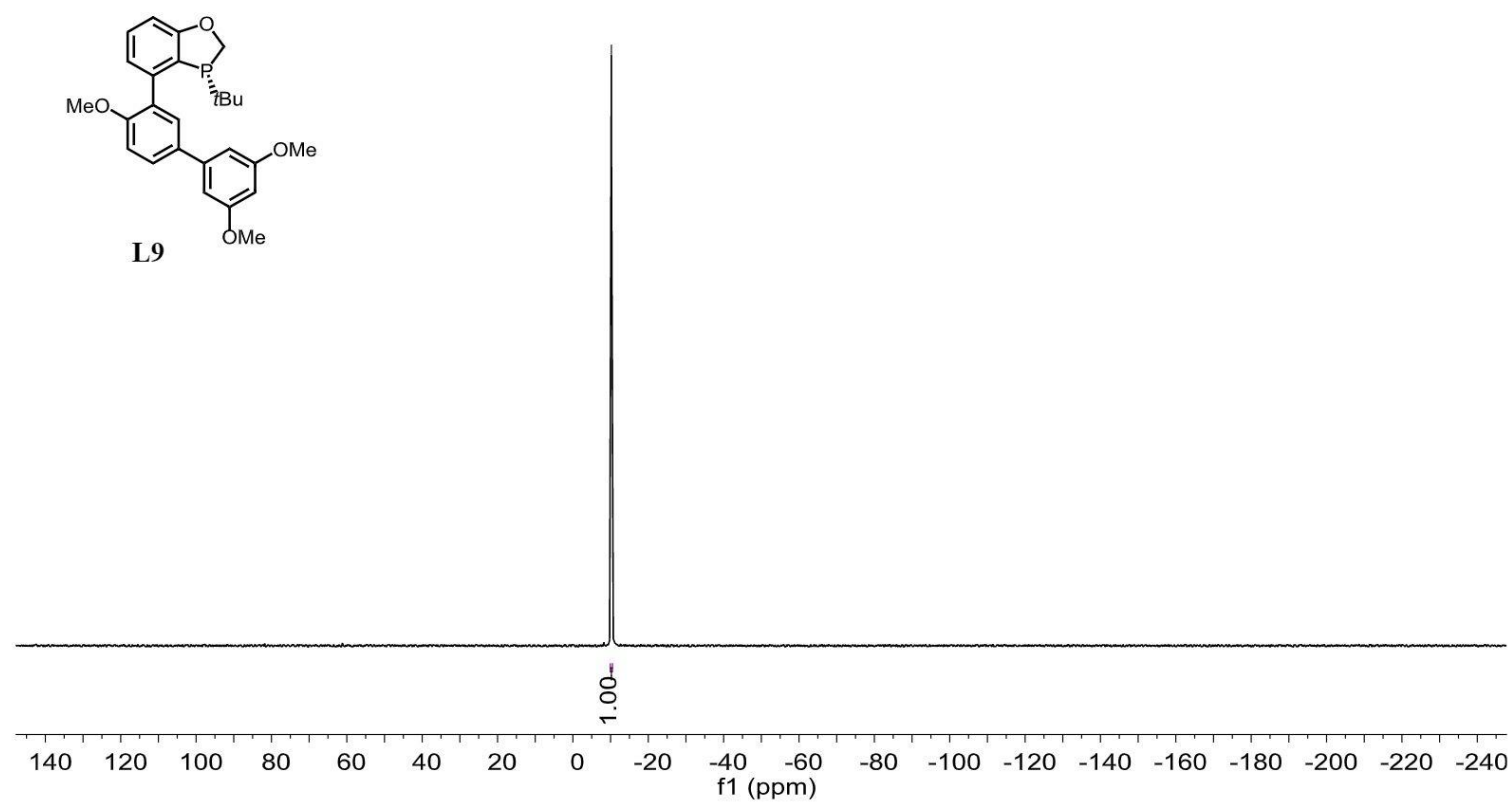

뚠둔은두응ㅇํㅇ

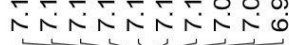

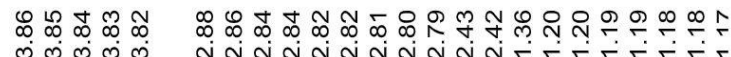
$\underbrace{m m m}$

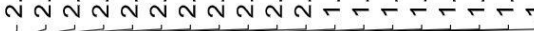

${ }^{1} \mathrm{H} \mathrm{NMR}\left(400 \mathrm{MHz}, \mathrm{CDCl}_{3}\right)$

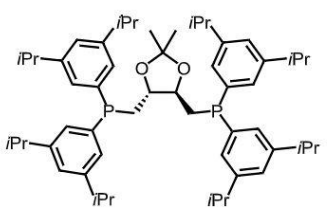

L12

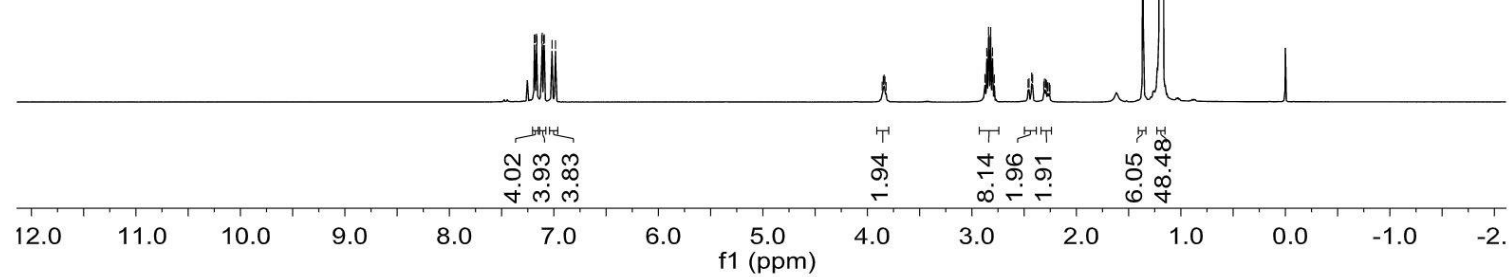




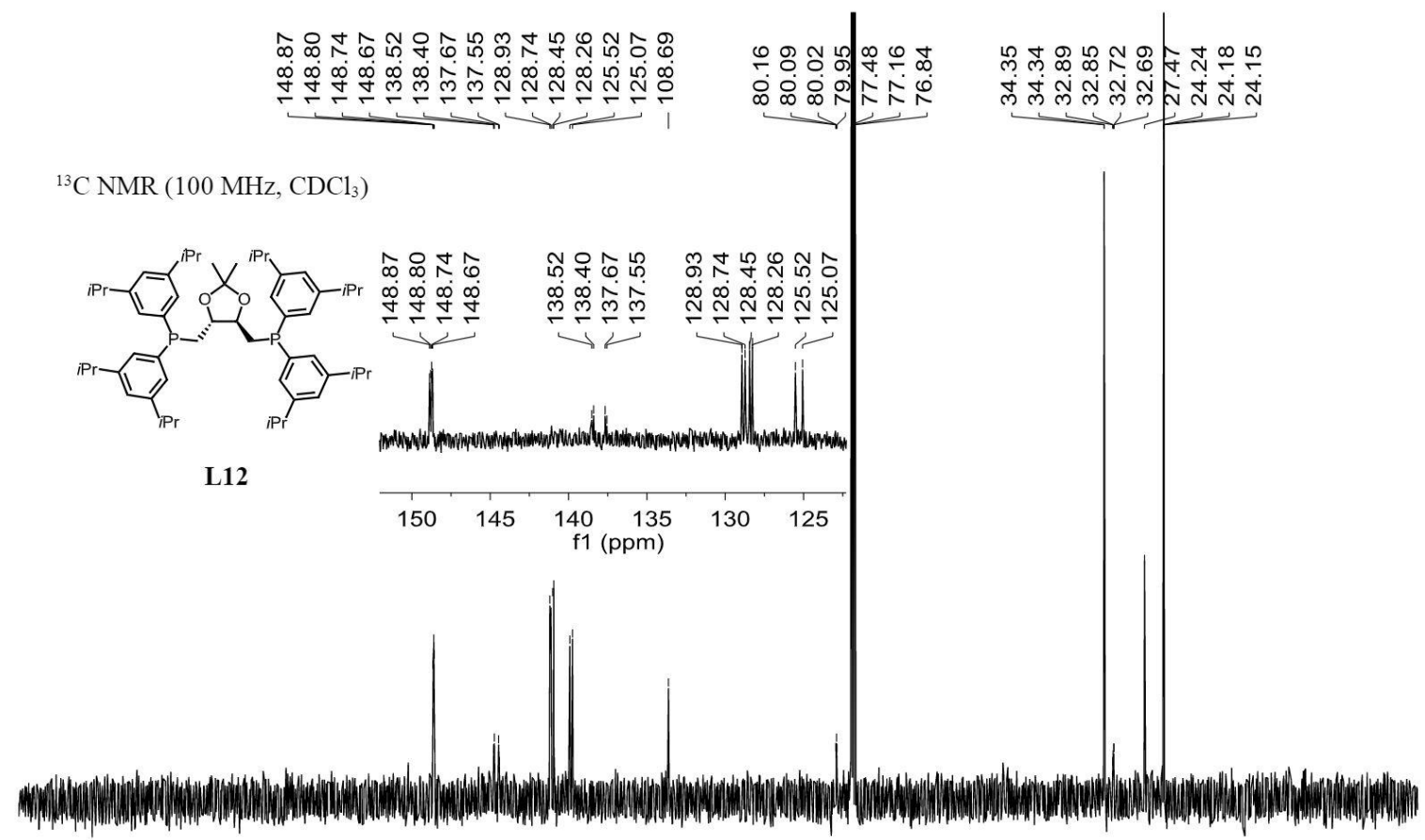

$\begin{array}{lllllllllllllllllllllll}210 & 200 & 190 & 180 & 170 & 160 & 150 & 140 & 130 & 120 & \begin{array}{c}110 \\ \mathrm{f} 1\end{array}(\mathrm{ppm}) & 100 & 90 & 70 & 60 & 50 & 40 & 30 & 20 & 10 & 0 & -10\end{array}$ $\bar{\infty} \infty$ \&

సุ సฺָ

${ }^{31} \mathrm{P}$ NMR (162 MHz, $\mathrm{CDCl}_{3}$ )

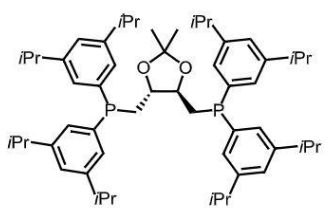

L12

$\begin{array}{lllllllllll}140 & 120 & 100 & 80 & 60 & 40 & 20 & 0 & -20 & -40 & -60 \\ & & & \end{array}$ $\begin{array}{lllllllll}-80 & -100 & -120 & -140 & -160 & -180 & -200 & -220 & -240\end{array}$ 
${ }^{1} \mathrm{H} \mathrm{NMR}\left(400 \mathrm{MHz}, \mathrm{CDCl}_{3}\right)$

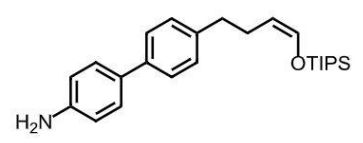

1e

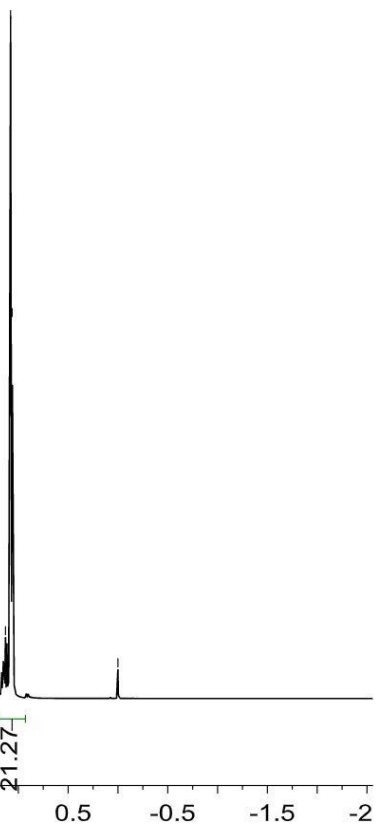

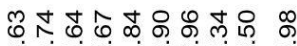

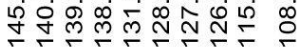

${ }^{13} \mathrm{C} \mathrm{NMR}\left(100 \mathrm{MHz}, \mathrm{CDCl}_{3}\right)$

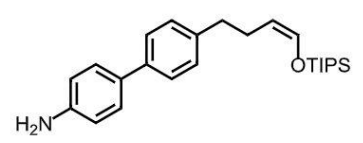

1e

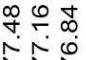

ベド

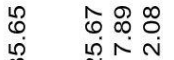

1
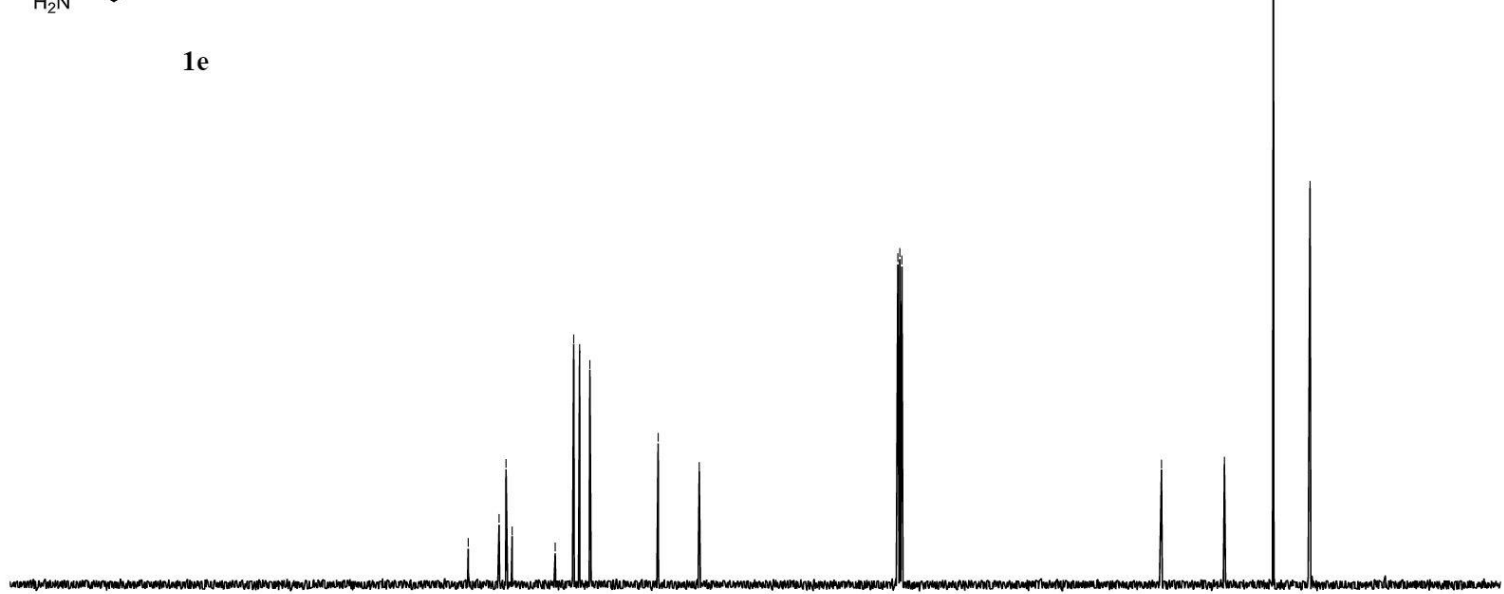

$\begin{array}{llllllllllllllllllllll}210 & 200 & 190 & 180 & 170 & 160 & 150 & 140 & 130 & 120 & \begin{array}{c}110 \\ \mathrm{f} 1\end{array} \begin{array}{l}100 \\ (\mathrm{ppm})\end{array} & 90 & 80 & 70 & 60 & 50 & 40 & 30 & 20 & 10 & 0 & -10\end{array}$ 


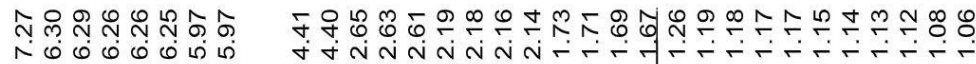

${ }^{1} \mathrm{H}$ NMR $\left(400 \mathrm{MHz}, \mathrm{CDCl}_{3}\right)$

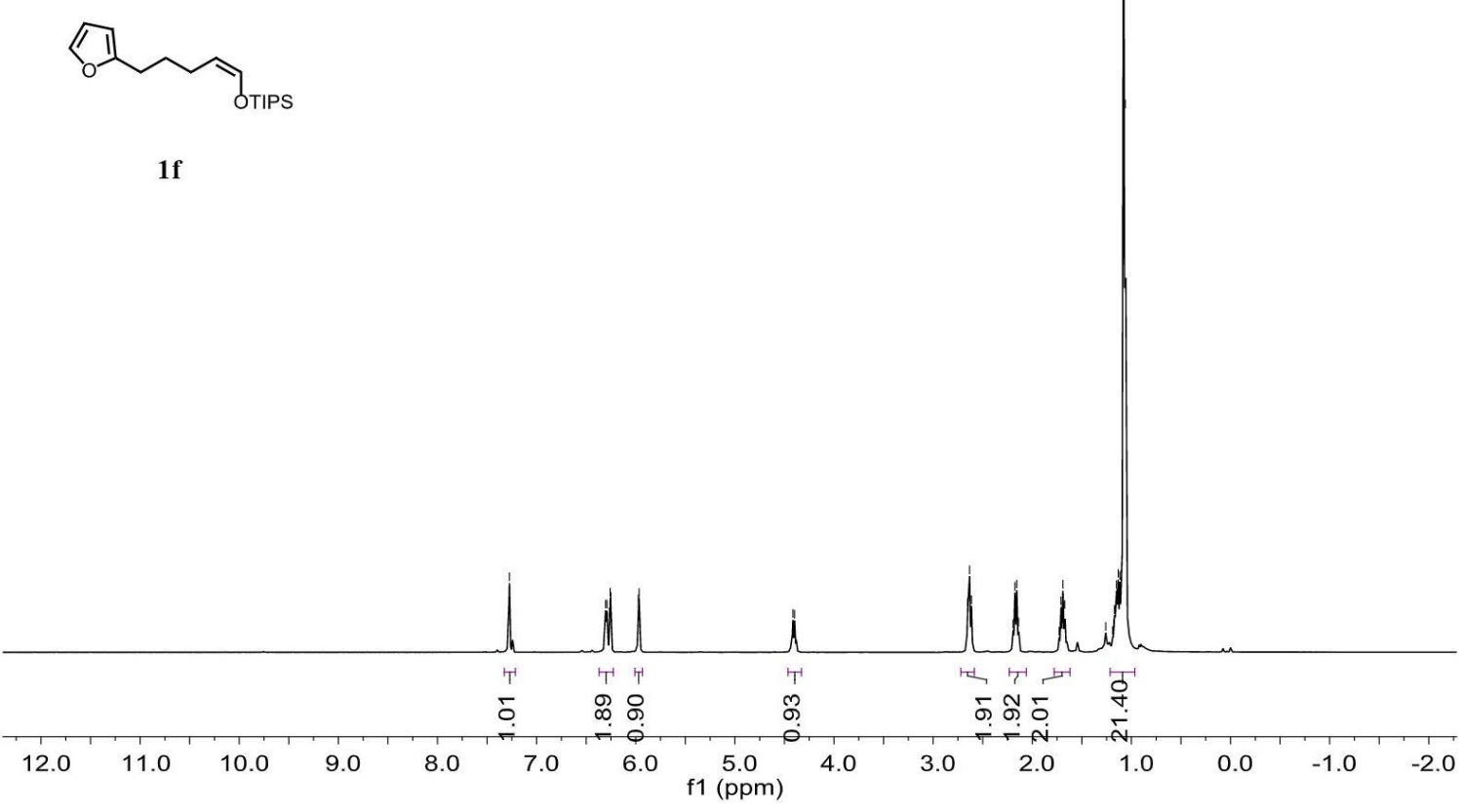

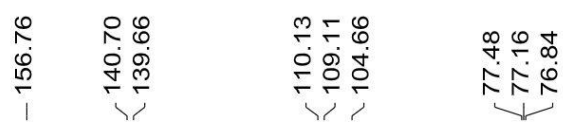

$\stackrel{\infty}{\infty} \infty \prod_{\infty}^{\infty} \infty$

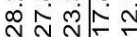

${ }^{13} \mathrm{C} \mathrm{NMR}\left(100 \mathrm{MHz}, \mathrm{CDCl}_{3}\right)$<smiles>C/C=C\O[Na]</smiles>

1f

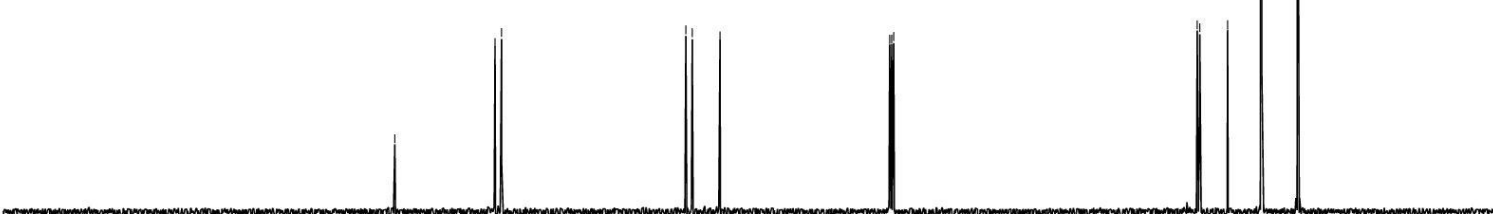

$\begin{array}{lllllllllllllllllllllll}210 & 200 & 190 & 180 & 170 & 160 & 150 & 140 & 130 & 120 & 110 & 100 & 90 & 80 & 70 & 60 & 50 & 40 & 30 & 20 & 10 & 0 & -10\end{array}$ f1 (ppm) 
${ }^{1} \mathrm{H}$ NMR $\left(400 \mathrm{MHz}, \mathrm{CDCl}_{3}\right)$

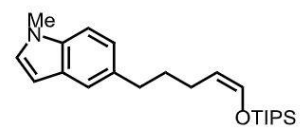

1h

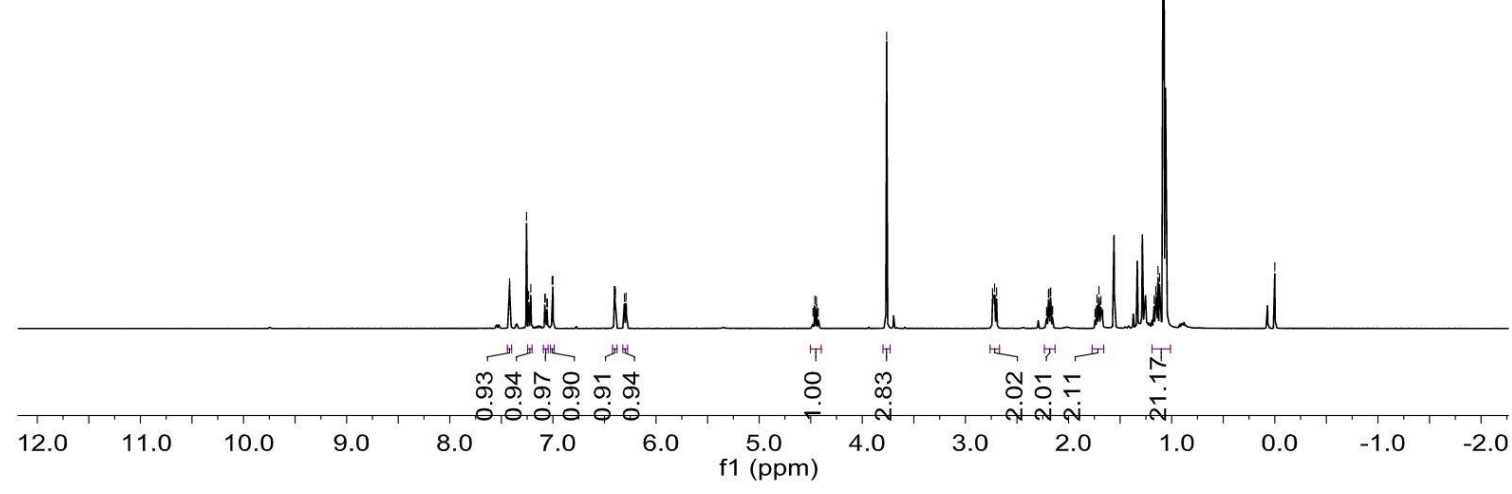

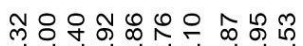

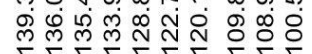

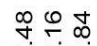

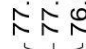

후 ᄋ

岗

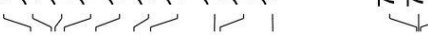

mुए।

${ }^{13} \mathrm{C}$ NMR $\left(100 \mathrm{MHz}, \mathrm{CDCl}_{3}\right)$

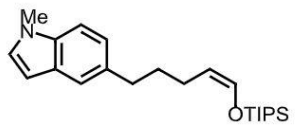

1h

$\begin{array}{lllllllllllllllllllllll}210 & 200 & 190 & 180 & 170 & 160 & 150 & 140 & 130 & 120 & 110 & 100 & 90 & 80 & 70 & 60 & 50 & 40 & 30 & 20 & 10 & 0 & -10\end{array}$ 
${ }^{1} \mathrm{H}$ NMR $\left(400 \mathrm{MHz}, \mathrm{CDCl}_{3}\right)$

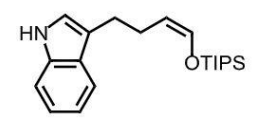

1i

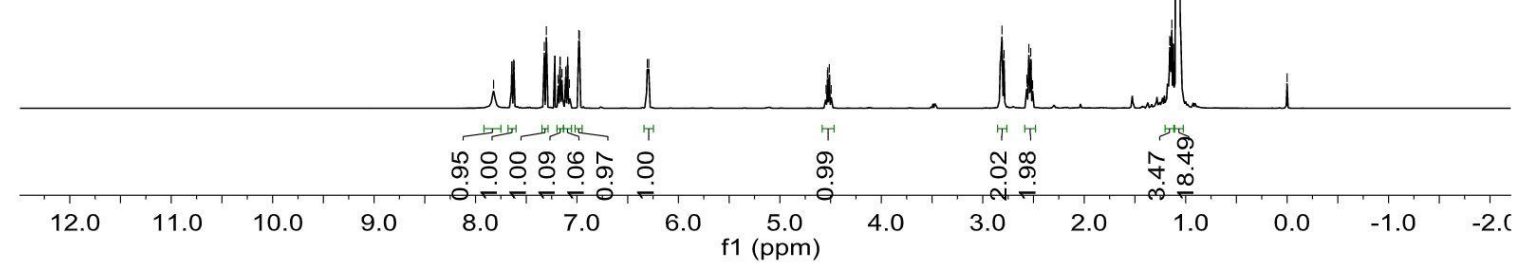

कร

Mำ

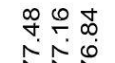

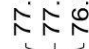

ๆ ำำ

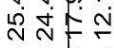

${ }^{13} \mathrm{C} \mathrm{NMR}\left(100 \mathrm{MHz}, \mathrm{CDCl}_{3}\right)$

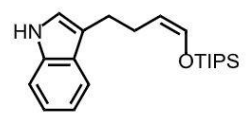

$1 i$

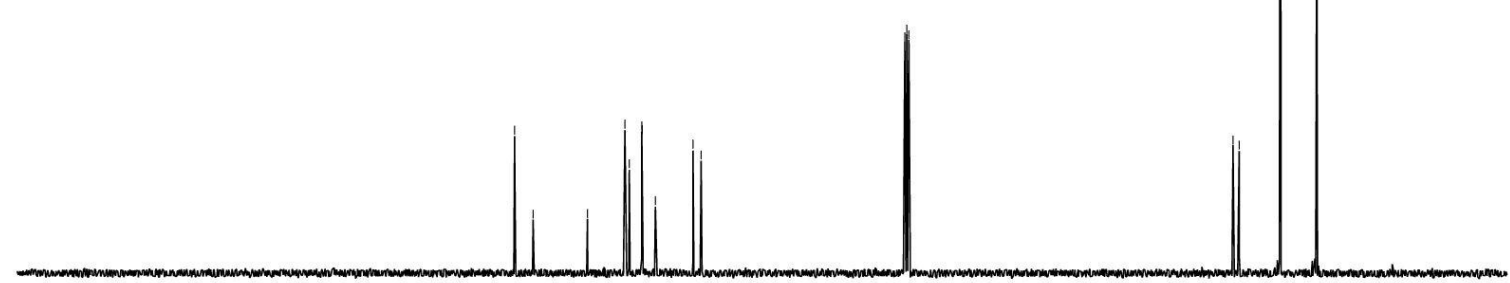

$\begin{array}{lllllllllllllllllllllll}210 & 200 & 190 & 180 & 170 & 160 & 150 & 140 & 130 & 120 & \begin{array}{c}110 \\ \mathrm{f} 1\end{array}(\mathrm{ppm}) & 90 & 80 & 70 & 60 & 50 & 40 & 30 & 20 & 10 & 0 & -10\end{array}$ 
${ }^{1} \mathrm{H}$ NMR $\left(400 \mathrm{MHz}, \mathrm{CDCl}_{3}\right)$

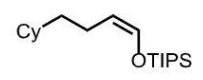

10

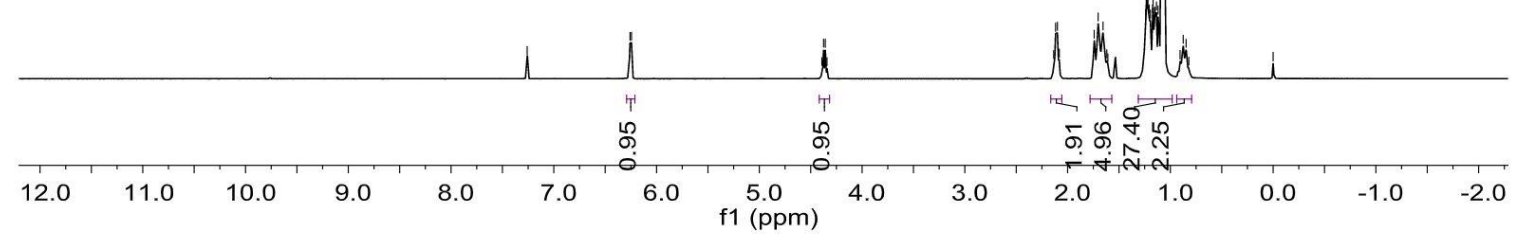

\begin{tabular}{|c|c|c|c|}
\hline$N$ & $\begin{array}{l}\stackrel{m}{+} \\
\stackrel{0}{\leftarrow} \\
i\end{array}$ & 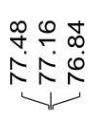 & 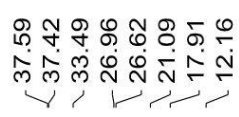 \\
\hline
\end{tabular}

${ }^{13} \mathrm{C}$ NMR $\left(100 \mathrm{MHz}, \mathrm{CDCl}_{3}\right)$<smiles>[SiH3]CCCC=CO[SbH3]</smiles>

10

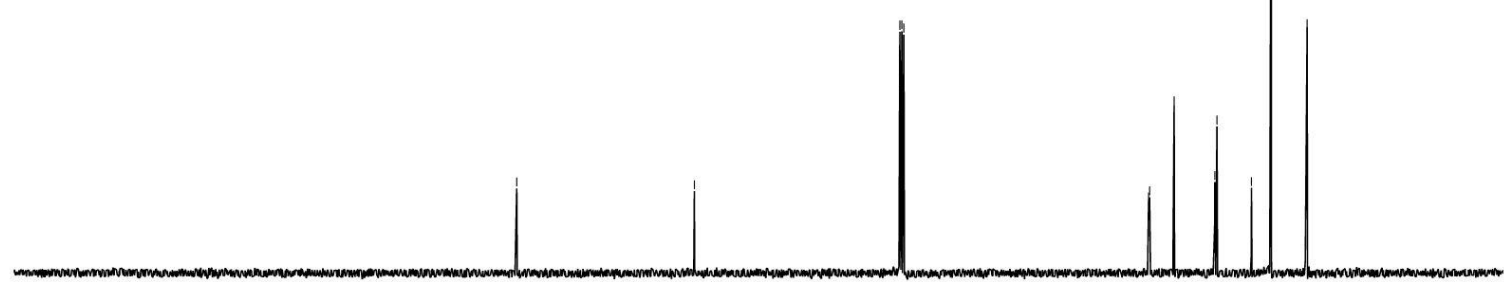

$\begin{array}{llllllllllllllllllllllllllllll}210 & 200 & 190 & 180 & 170 & 160 & 150 & 140 & 130 & 120 & 110 & 100 & 90 & 80 & 70 & 60 & 50 & 40 & 30 & 20 & 10 & 0 & -10\end{array}$ f1 (ppm) 
${ }^{1} \mathrm{H}$ NMR $\left(400 \mathrm{MHz}, \mathrm{CDCl}_{3}\right)$<smiles>CC(C)(C)CC/C=C\O[PH-]</smiles>

$1 \mathbf{p}$

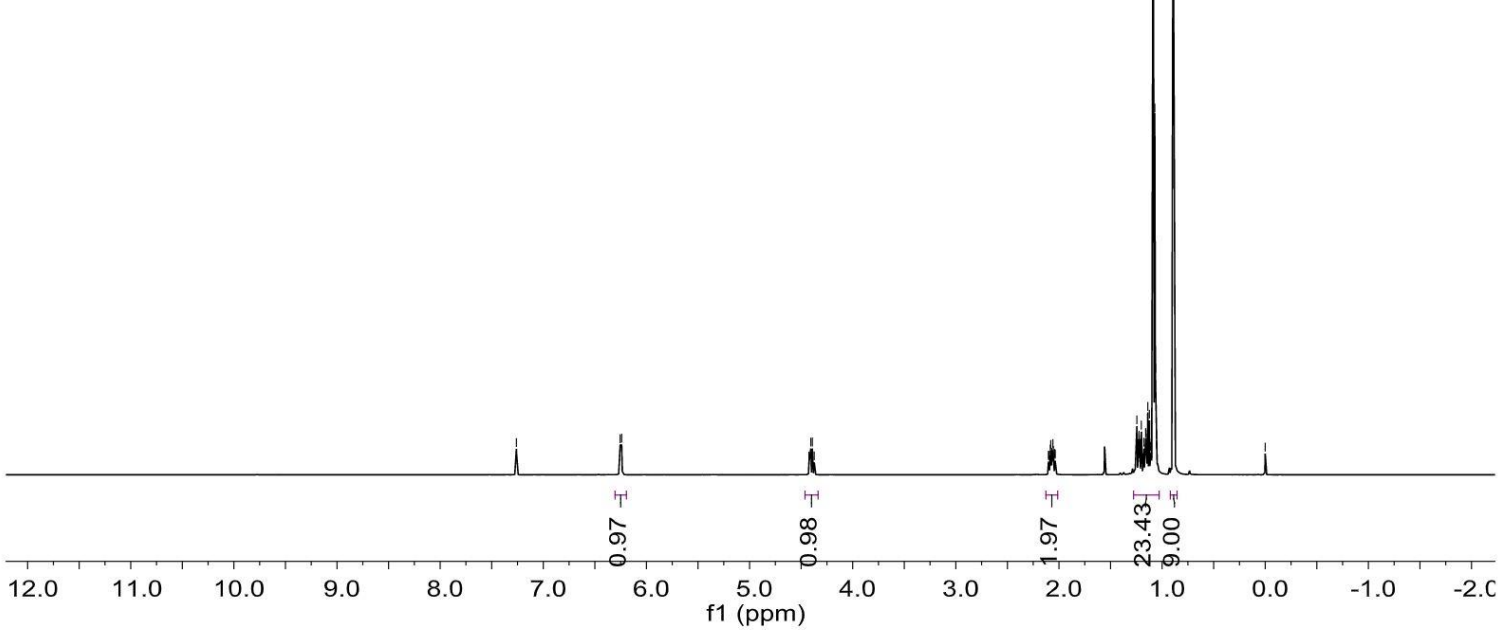

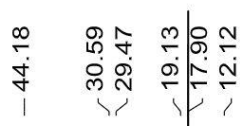

${ }^{13} \mathrm{C} \mathrm{NMR}\left(100 \mathrm{MHz}, \mathrm{CDCl}_{3}\right)$<smiles>[Mg][Mg]CC/C=C\O[Hg]</smiles>

$1 \mathbf{p}$

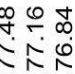

N 
${ }^{1} \mathrm{H}$ NMR $\left(400 \mathrm{MHz}, \mathrm{CDCl}_{3}\right)$<smiles>O=C(O)OCCC/C=C\O[Pb]</smiles>

1q

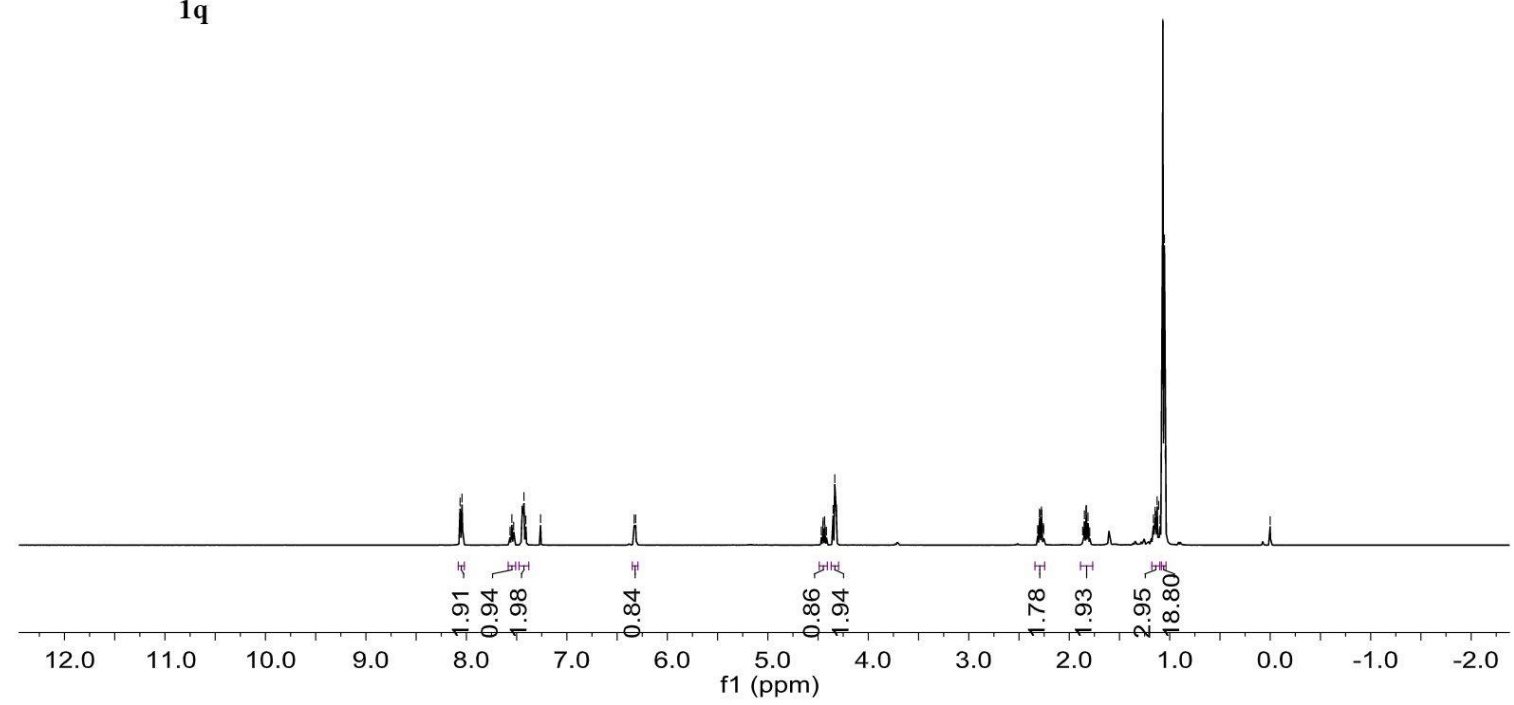

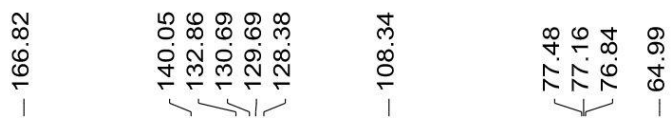

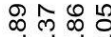

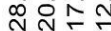

${ }^{13} \mathrm{C} \mathrm{NMR}\left(100 \mathrm{MHz}, \mathrm{CDCl}_{3}\right)$

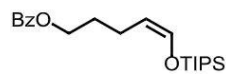

$1 \mathrm{q}$

$\begin{array}{lllllllllllllllllllllll}210 & 200 & 190 & 180 & 170 & 160 & 150 & 140 & 130 & 120 & 110 & 100 & 90 & 80 & 70 & 60 & 50 & 40 & 30 & 20 & 10 & 0 & -10\end{array}$ f1 (ppm) 
${ }^{1} \mathrm{H}$ NMR $\left(400 \mathrm{MHz}, \mathrm{CDCl}_{3}\right)$

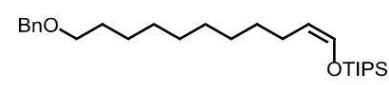

$1 \mathbf{r}$
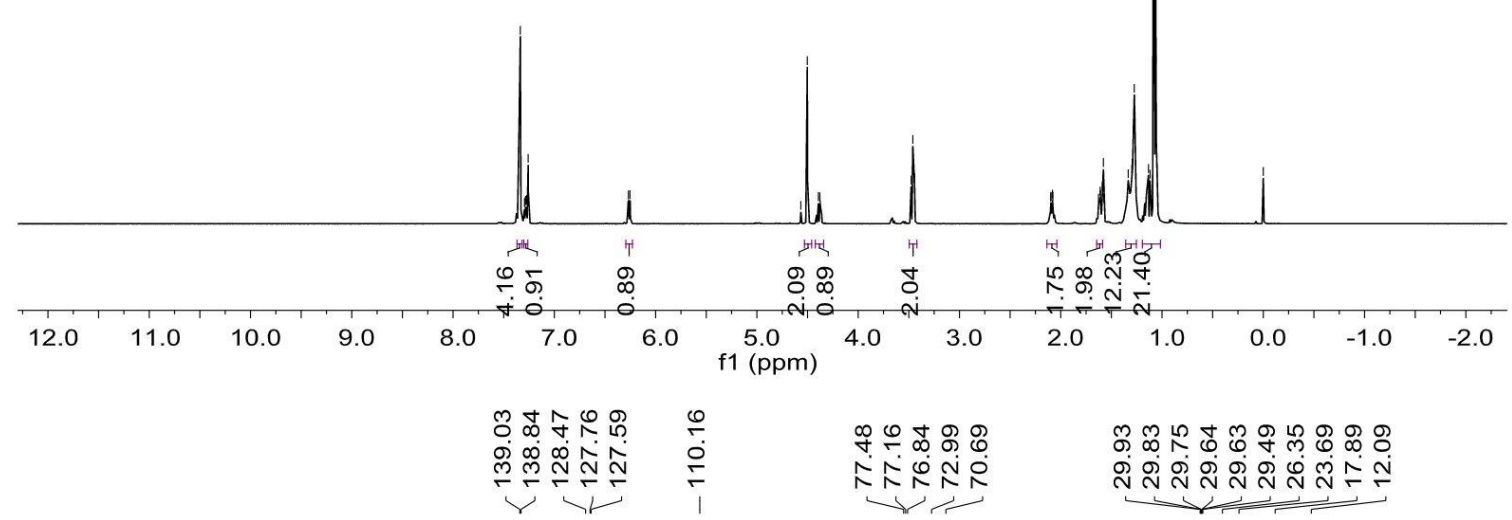

${ }^{13} \mathrm{C}$ NMR (100 MHz, $\left.\mathrm{CDCl}_{3}\right)$

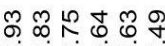

(N)

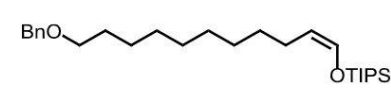

$1 \mathbf{r}$
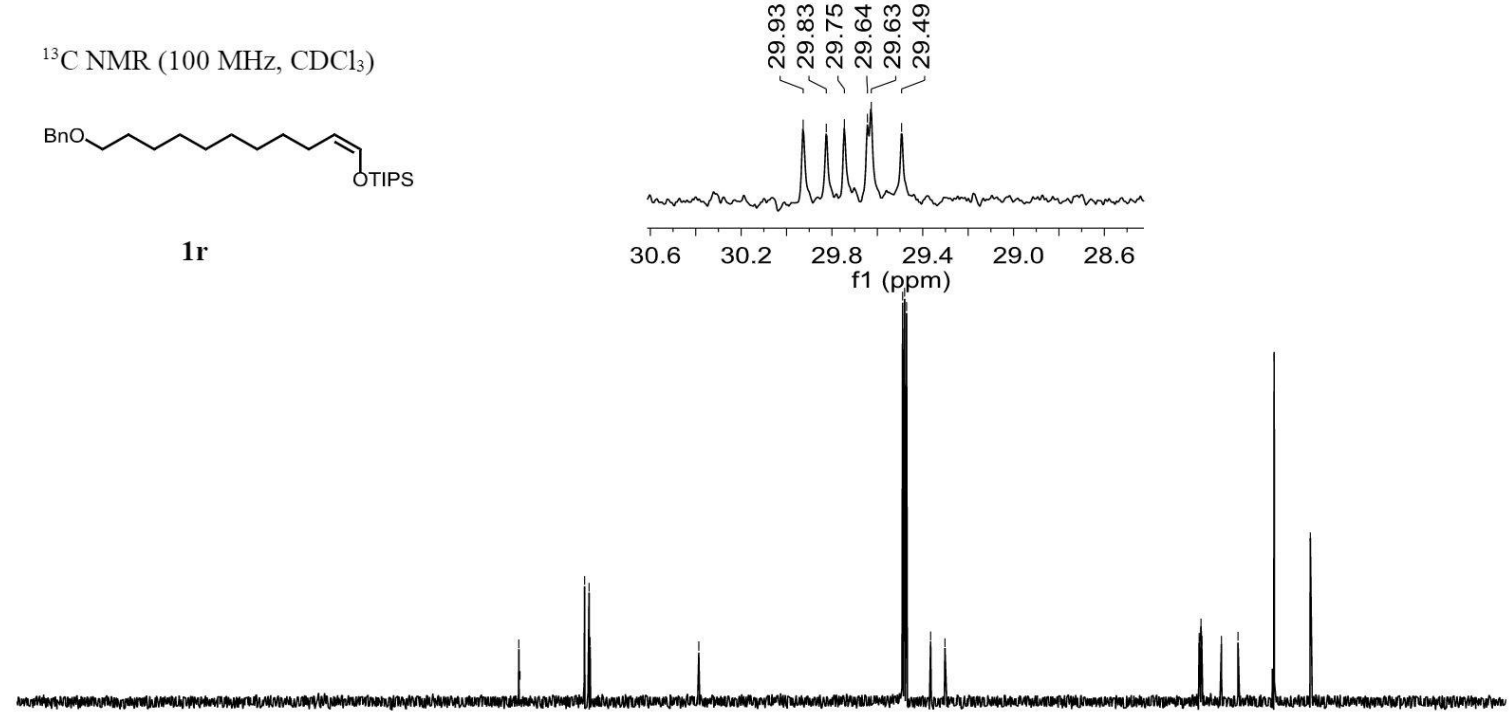

$\begin{array}{llllllllllllllllllllllllll}210 & 200 & 190 & 180 & 170 & 160 & 150 & 140 & 130 & 120 & 110 & 100 & 90 & 80 & 70 & 60 & 50 & 40 & 30 & 20 & 10 & 0 & -10\end{array}$ 
${ }^{1} \mathrm{H}$ NMR $\left(400 \mathrm{MHz}, \mathrm{CDCl}_{3}\right)$

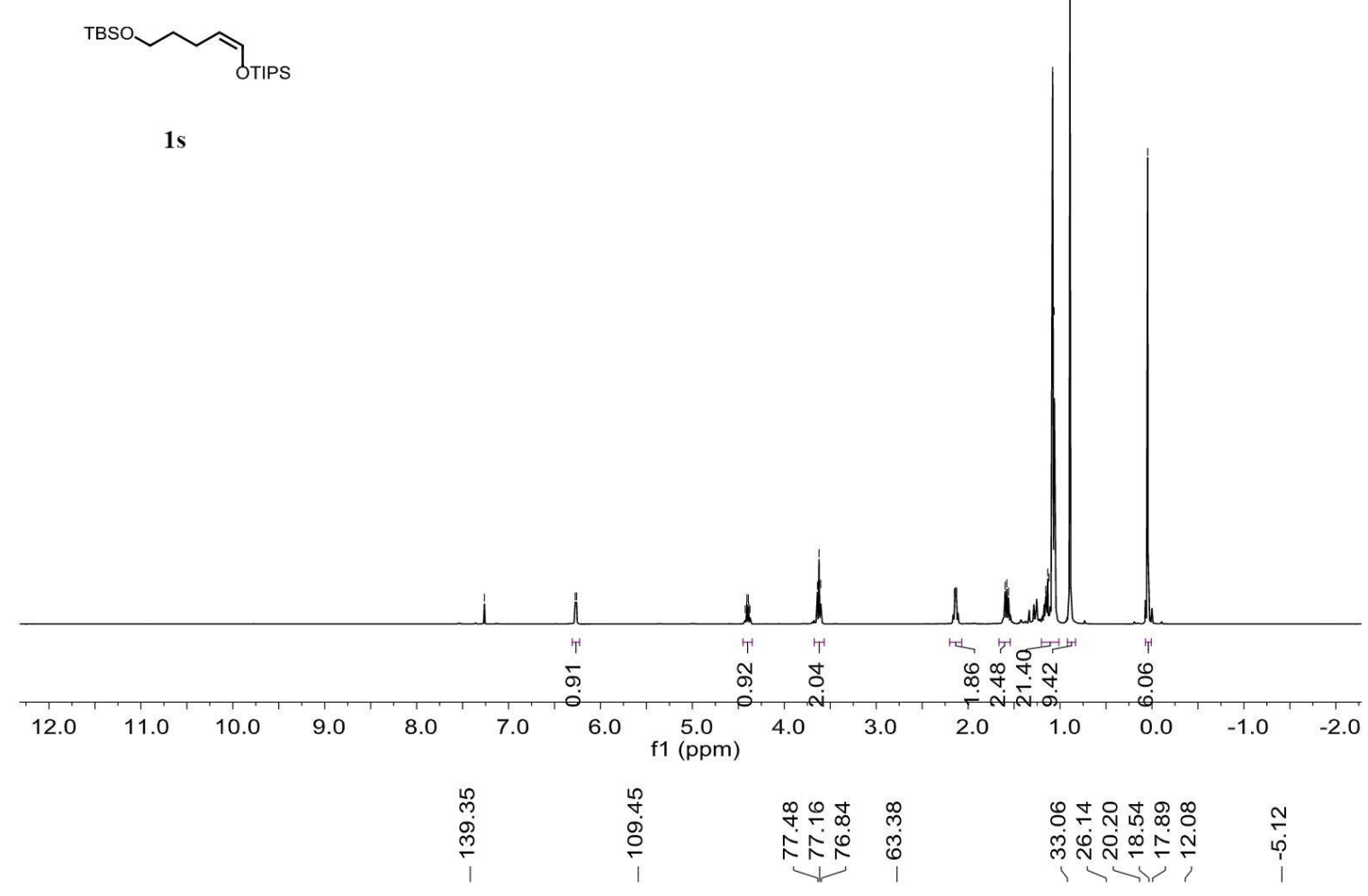

${ }^{13} \mathrm{C} \mathrm{NMR}\left(100 \mathrm{MHz}, \mathrm{CDCl}_{3}\right)$

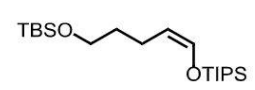

1s

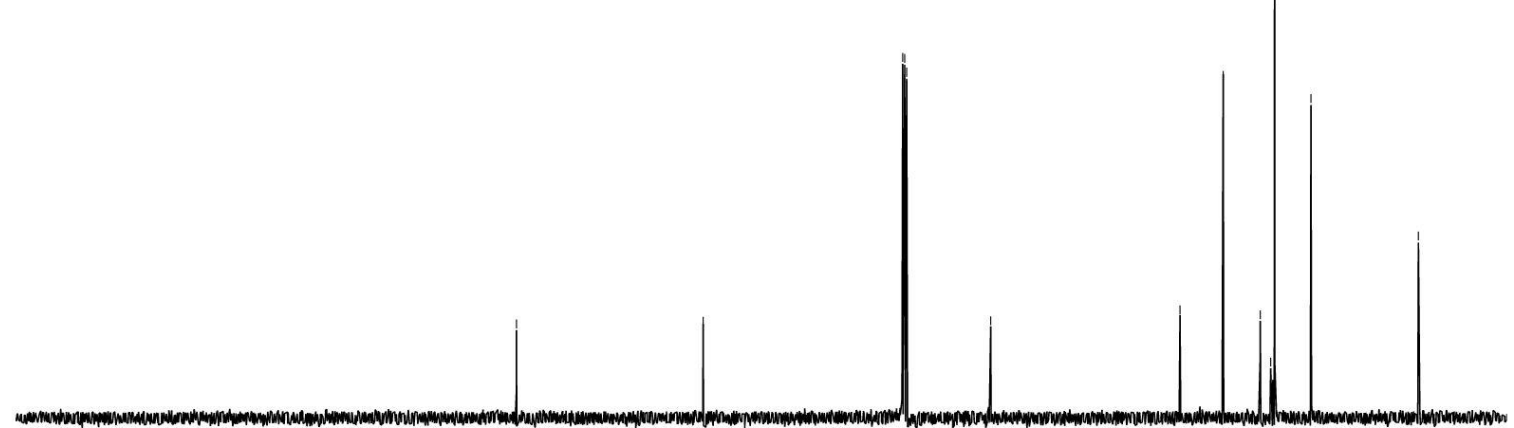

$\begin{array}{lllllllllllllllllllllll}210 & 200 & 190 & 180 & 170 & 160 & 150 & 140 & 130 & 120 & 110 & 100 & 90 & 80 & 70 & 60 & 50 & 40 & 30 & 20 & 10 & 0 & -10\end{array}$ 
${ }^{1} \mathrm{H}$ NMR (400 MHz, $\mathrm{CDCl}_{3}$ )

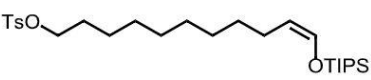

$1 \mathrm{t}$
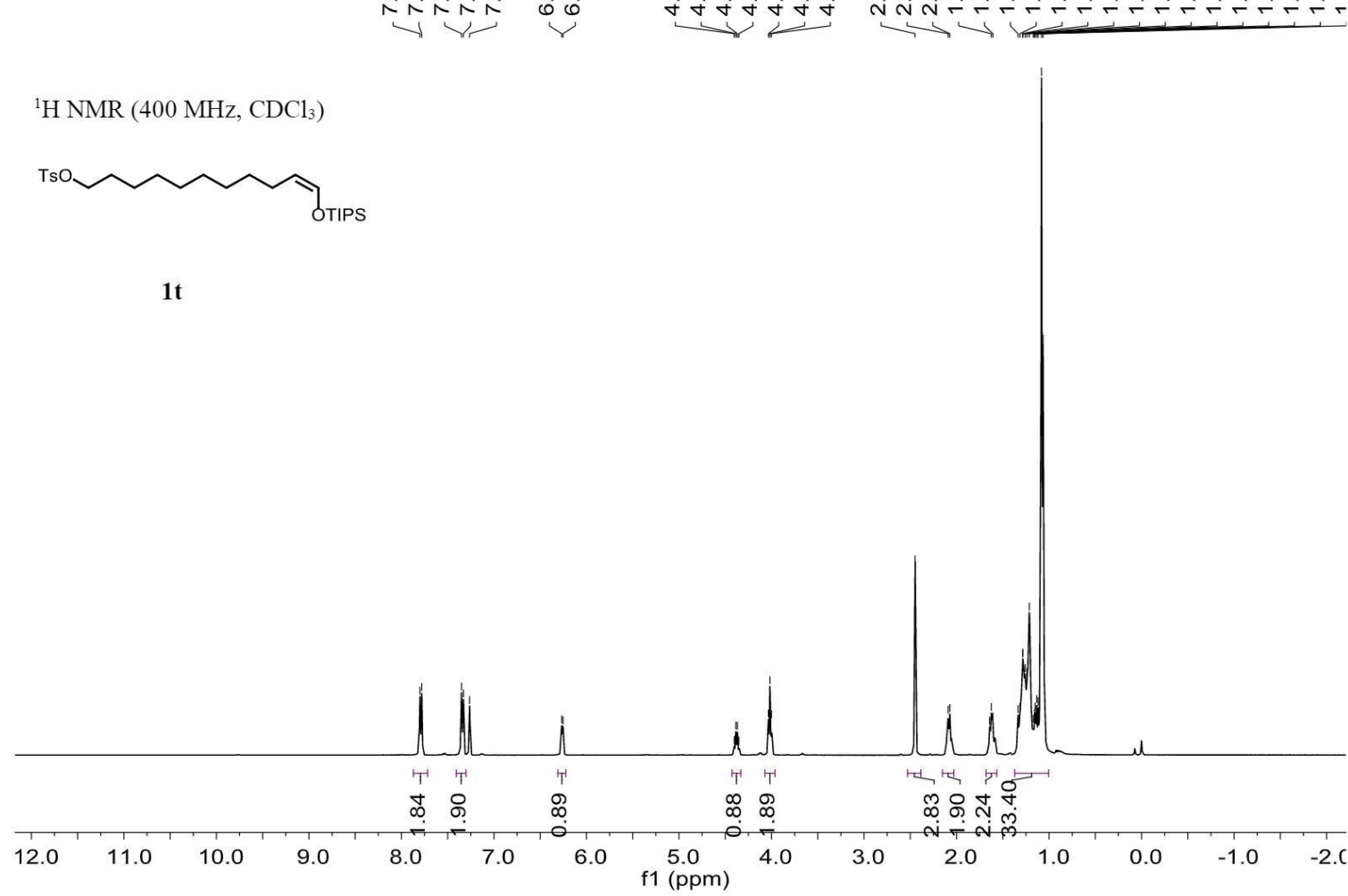

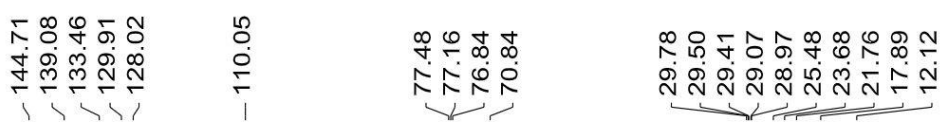

${ }^{13} \mathrm{C}$ NMR (100 MHz, $\mathrm{CDCl}_{3}$ )

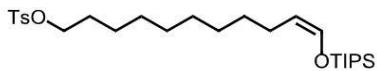

1t
象㲅司合

กิ่

$\backslash \backslash 1$ ।?

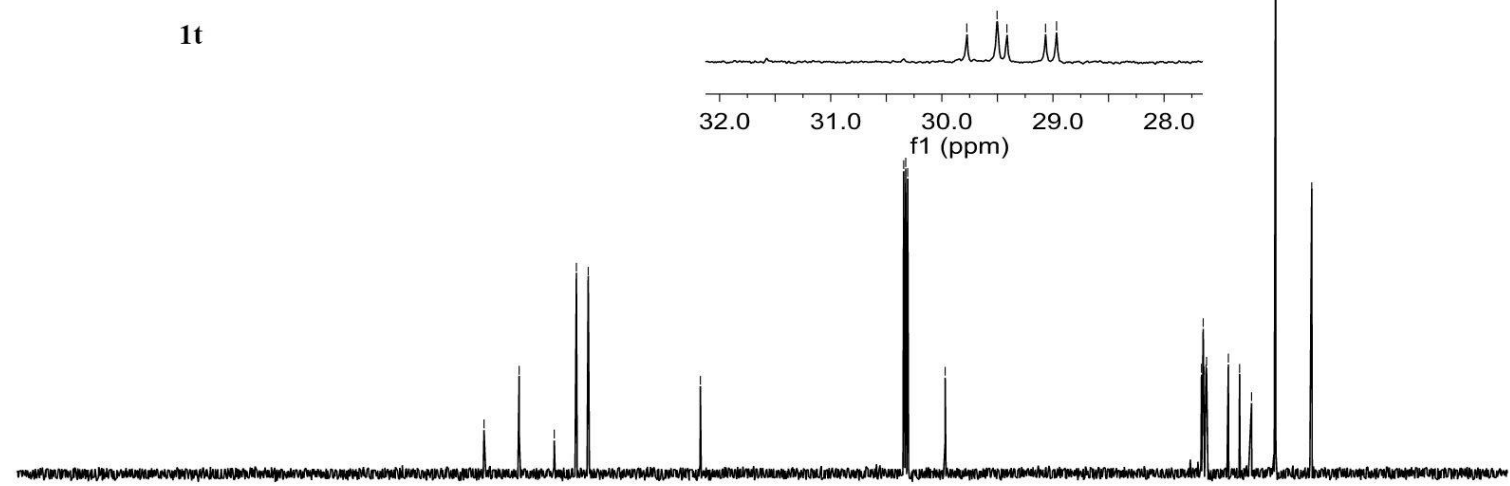

$\begin{array}{llllllllllllllllllllllll}210 & 200 & 190 & 180 & 170 & 160 & 150 & 140 & 130 & 120 & 110 & 100 & 90 & 80 & 70 & 60 & 50 & 40 & 30 & 20 & 10 & 0 & -10\end{array}$ f1 (ppm) 
${ }^{1} \mathrm{H}$ NMR $\left(400 \mathrm{MHz}, \mathrm{CDCl}_{3}\right)$

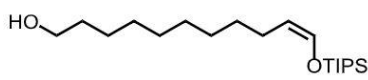

1u
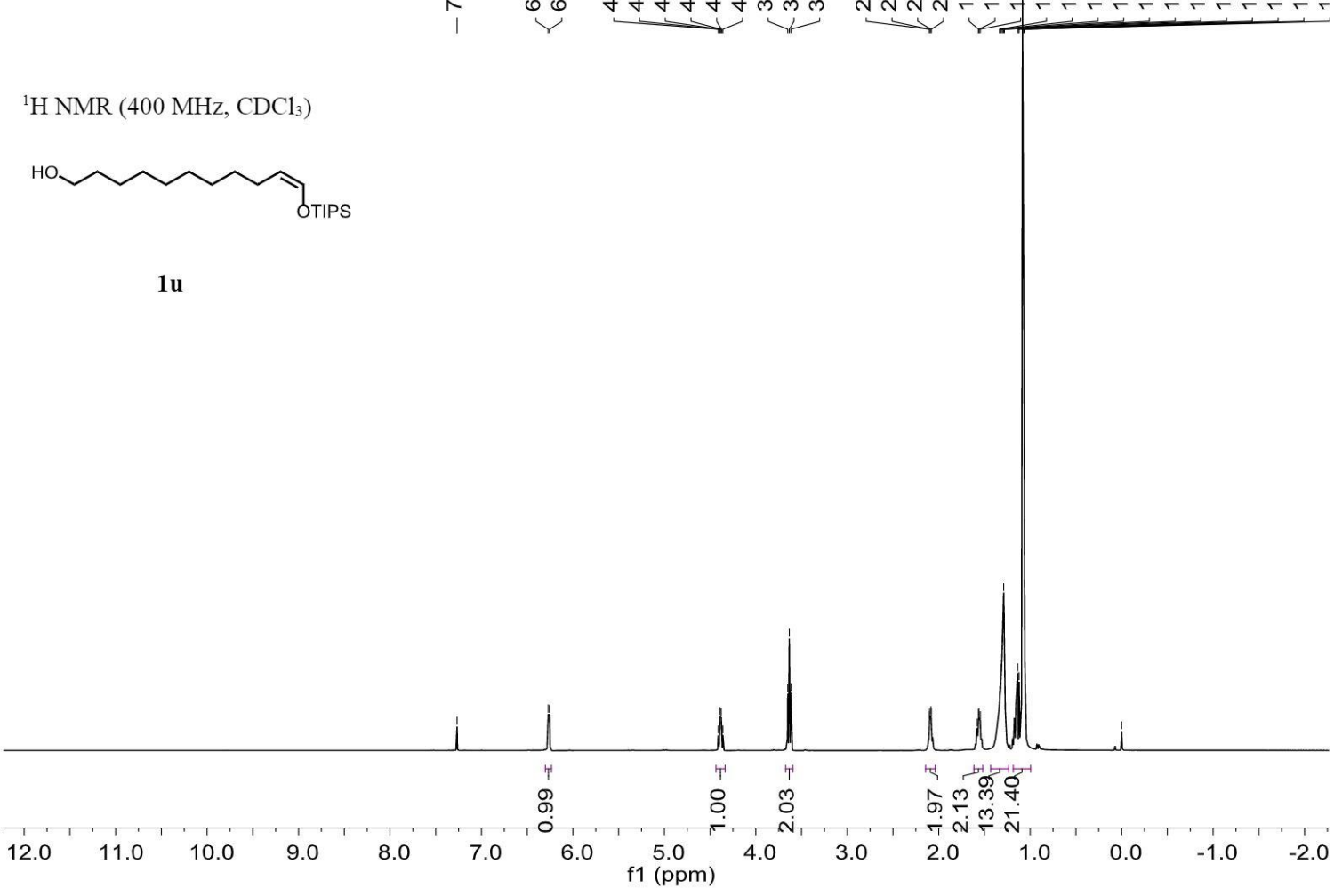

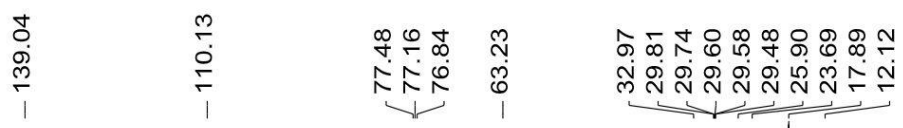

${ }^{13} \mathrm{C}$ NMR (100 MHz, $\left.\mathrm{CDCl}_{3}\right)$<smiles>CO/C=C\CCCCCCCCCO</smiles>

1u

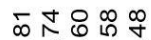

กิ

$\lambda$,
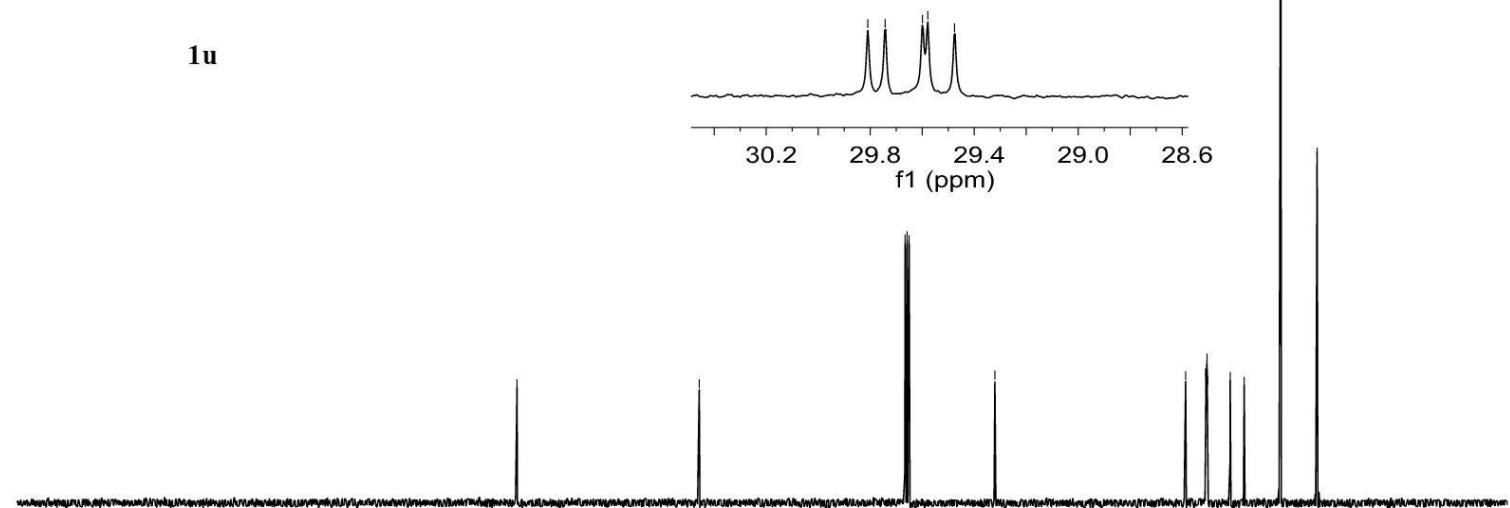

$\begin{array}{llllllllllllllllllllllllllllll}210 & 200 & 190 & 180 & 170 & 160 & 150 & 140 & 130 & 120 & 110 & 100 & 90 & 80 & 70 & 60 & 50 & 40 & 30 & 20 & 10 & 0 & -10\end{array}$ 


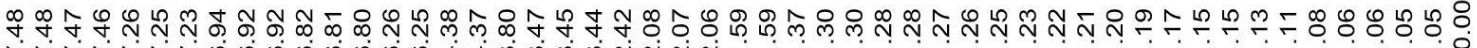

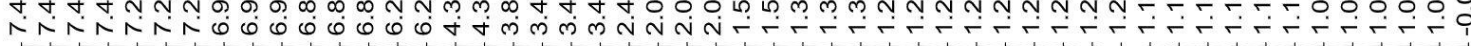

${ }^{1} \mathrm{H}$ NMR $\left(400 \mathrm{MHz}, \mathrm{CDCl}_{3}\right)$

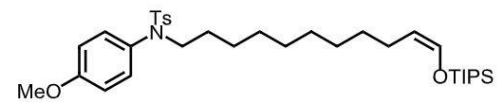

1v

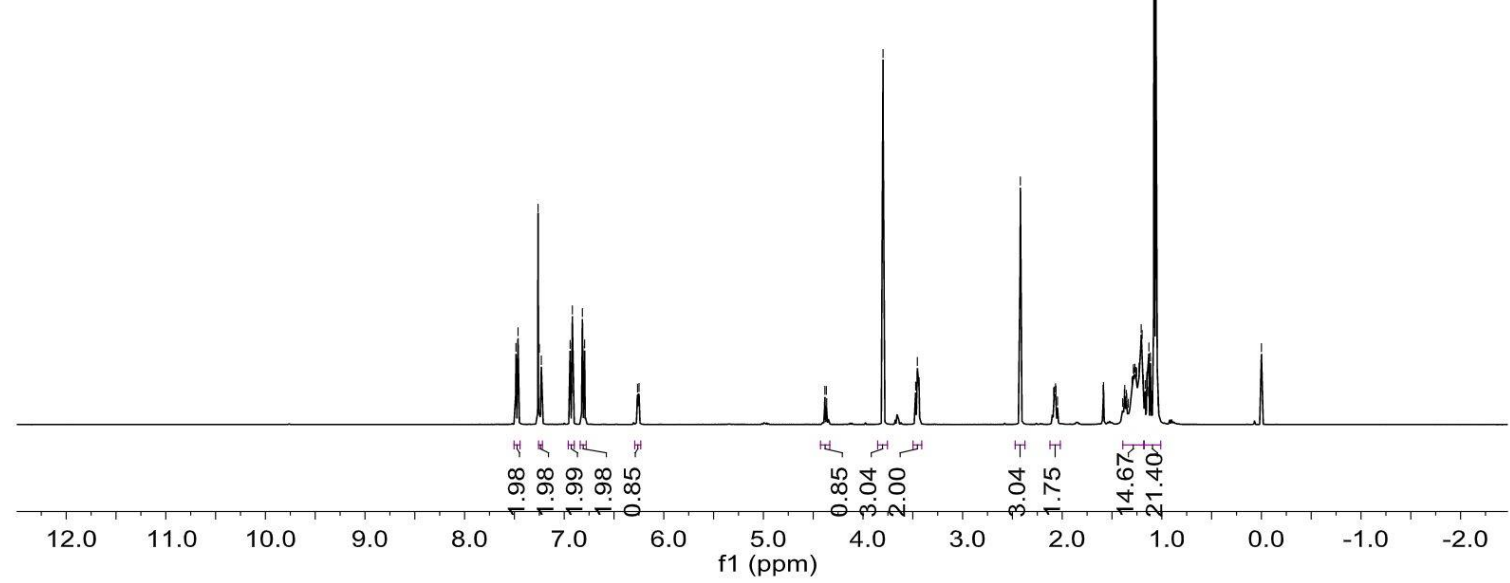

O)

官

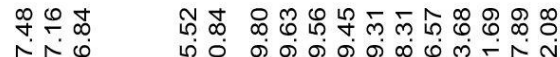

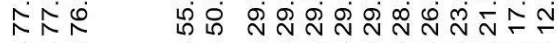

${ }^{13} \mathrm{C}$ NMR (100 MHz, $\left.\mathrm{CDCl}_{3}\right)$<smiles>COc1ccc(NCCCCCCCCC/C=C\O[Na])cc1</smiles>

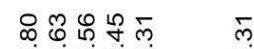

กิ่

$\bar{m}$
$\infty$
$\infty$

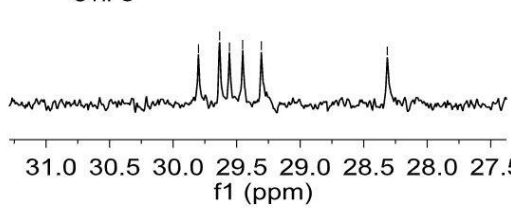
f1 (ppm)

$\begin{array}{llllllllllll}210 & 200 & 190 & 180 & 170 & 160 & 150 & 140 & 130 & 120 & 110 & 100 \\ \mathrm{f} 1 & (\mathrm{ppm})\end{array}$ 


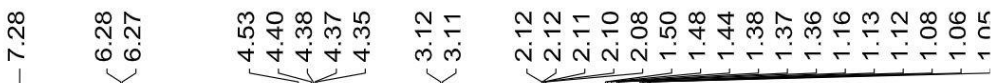

${ }^{1} \mathrm{H}$ NMR $\left(400 \mathrm{MHz}, \mathrm{CDCl}_{3}\right)$

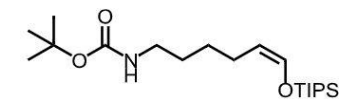

1w

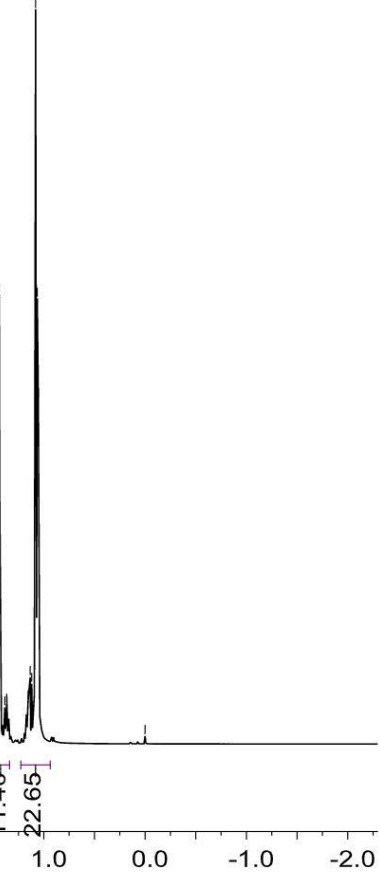

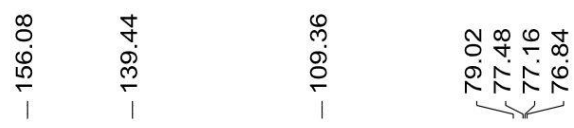

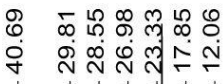

${ }^{13} \mathrm{C}$ NMR (100 MHz, $\left.\mathrm{CDCl}_{3}\right)$

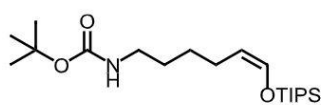

$1 w$
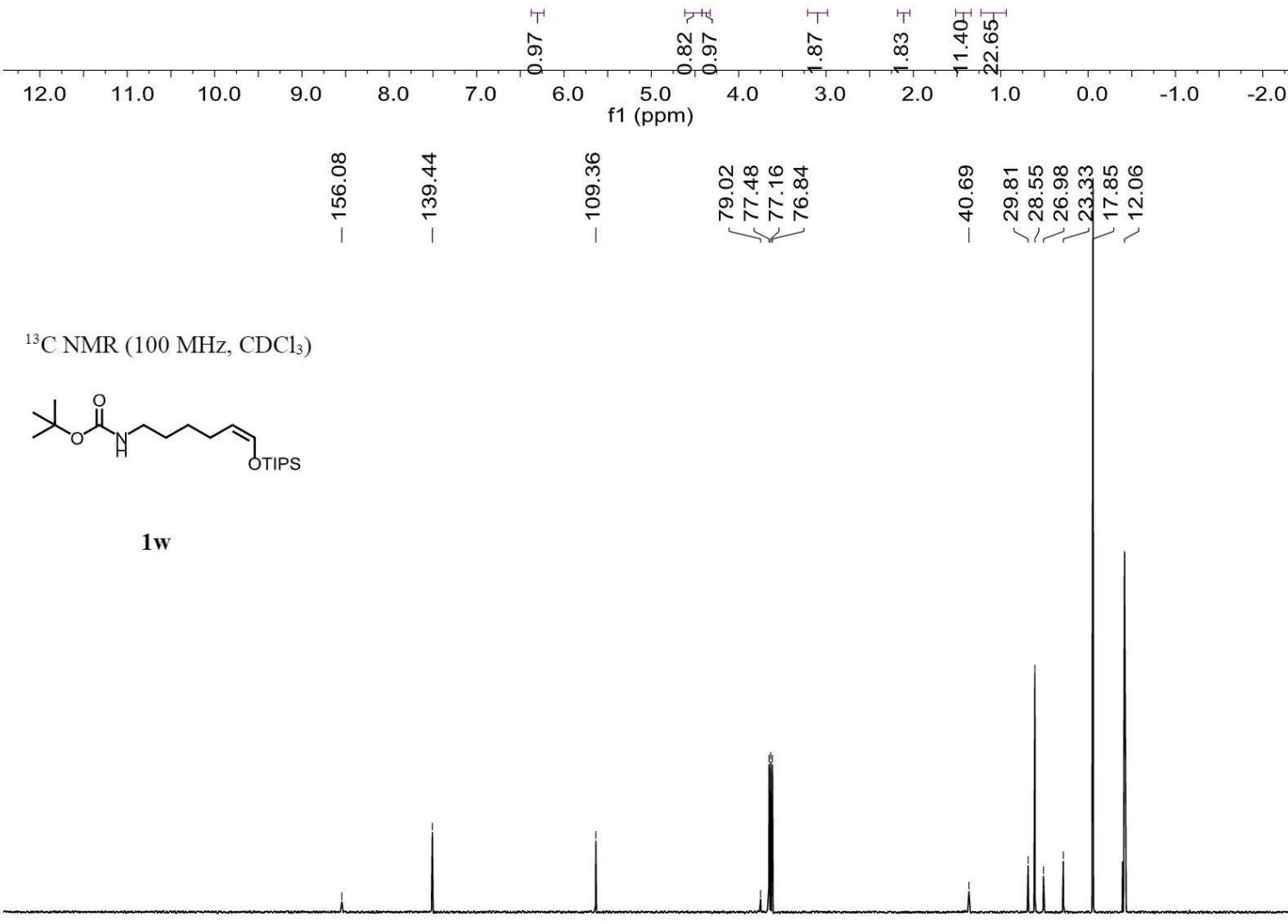

$\begin{array}{lllllllllllllllllllllll}210 & 200 & 190 & 180 & 170 & 160 & 150 & 140 & 130 & 120 & 110 & 100 & 90 & 80 & 70 & 60 & 50 & 40 & 30 & 20 & 10 & 0 & -10\end{array}$ 
${ }^{1} \mathrm{H} \operatorname{NMR}\left(400 \mathrm{MHz}, \mathrm{CDCl}_{3}\right)$

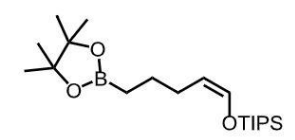

$\mathbf{1 x}$

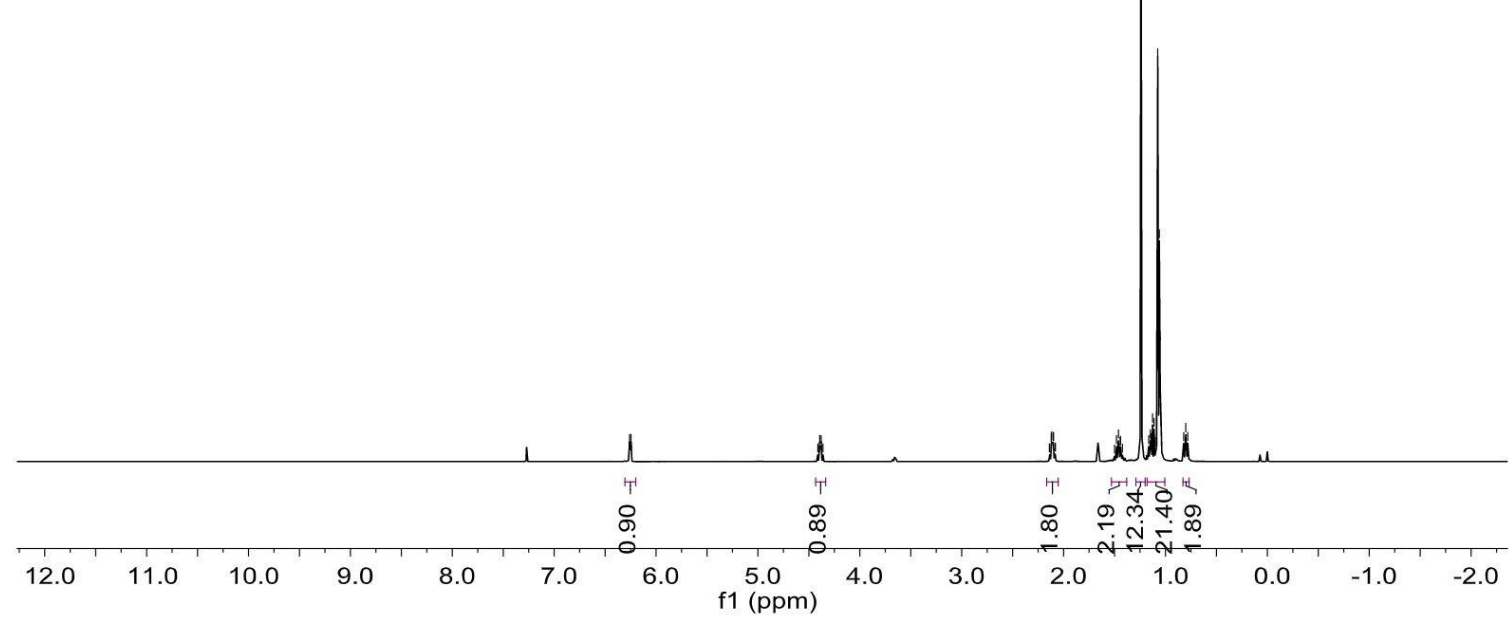

సิ

ஸे

${ }^{13} \mathrm{C}$ NMR (100 MHz, $\left.\mathrm{CDCl}_{3}\right)$

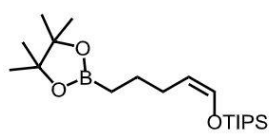

1x

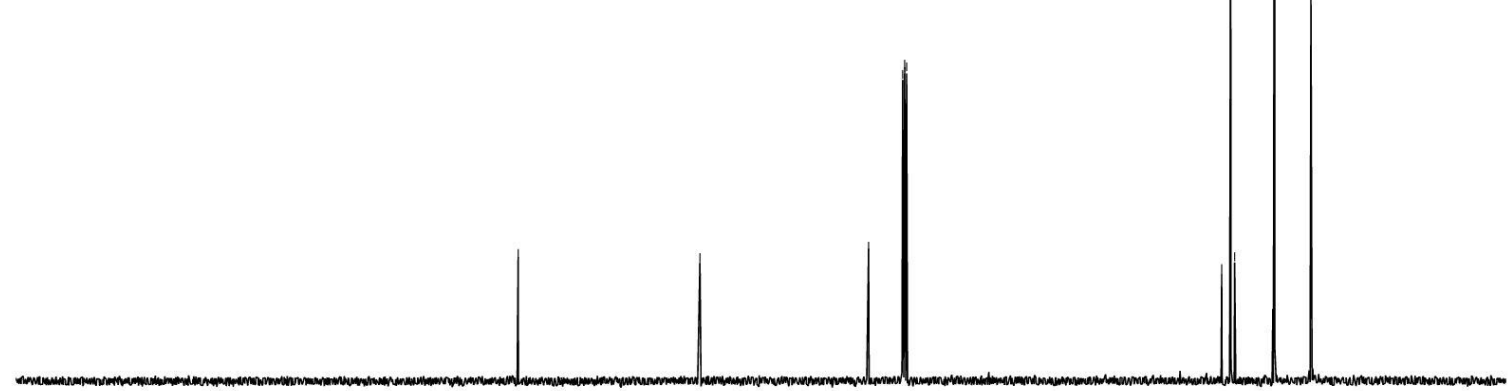

$\begin{array}{lllllllllllllllllllllll}210 & 200 & 190 & 180 & 170 & 160 & 150 & 140 & 130 & 120 & 110 & 100 & 90 & 80 & 70 & 60 & 50 & 40 & 30 & 20 & 10 & 0 & -10\end{array}$ 


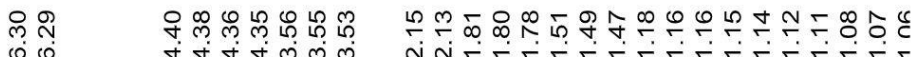

in

${ }^{1} \mathrm{H} \mathrm{NMR}\left(400 \mathrm{MHz}, \mathrm{CDCl}_{3}\right)$

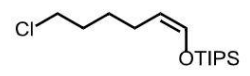

$1 \mathbf{y}$

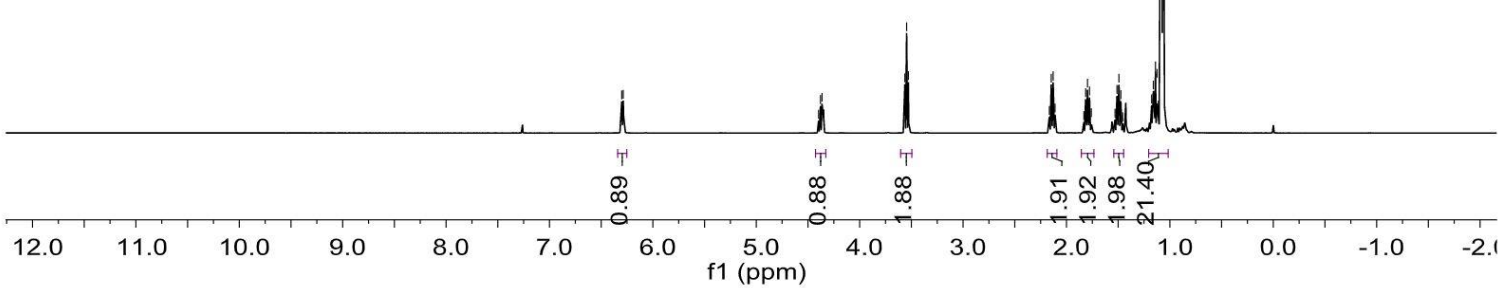

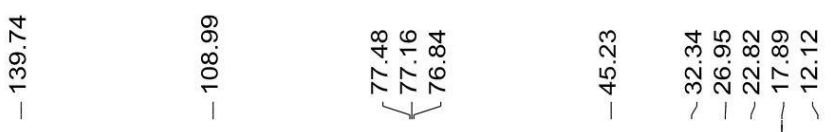

${ }^{13} \mathrm{C}$ NMR $\left(100 \mathrm{MHz}, \mathrm{CDCl}_{3}\right)$<smiles>ClCCCC/C=C\[TeH2]</smiles>

$1 \mathbf{y}$

$\begin{array}{lllllllllllllllllllllll}210 & 200 & 190 & 180 & 170 & 160 & 150 & 140 & 130 & 120 & 110 & 100 & 90 & 80 & 70 & 60 & 50 & 40 & 30 & 20 & 10 & 0 & -10\end{array}$ f1 (ppm) 
${ }^{1} \mathrm{H}$ NMR $\left(400 \mathrm{MHz}, \mathrm{CDCl}_{3}\right)$

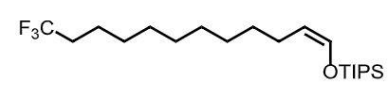

$1 \mathrm{z}$

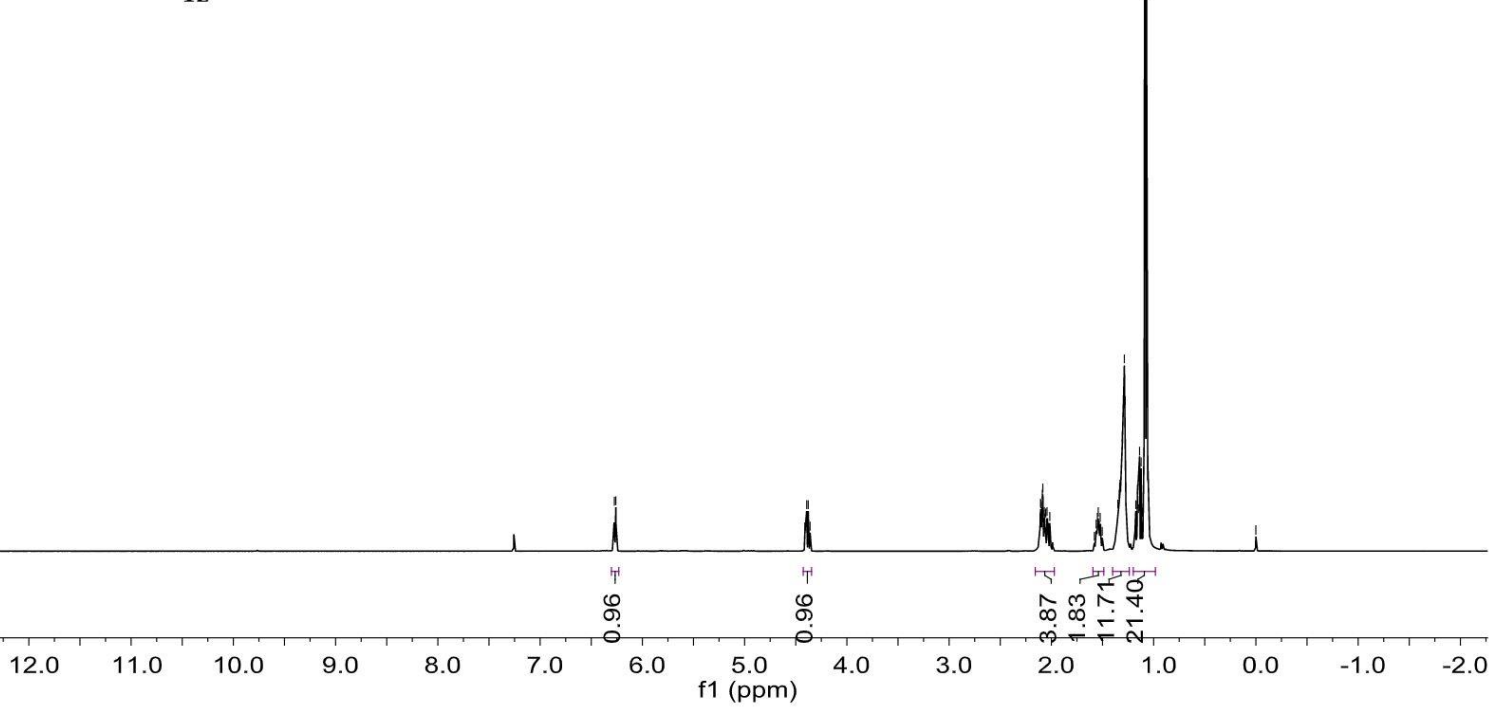

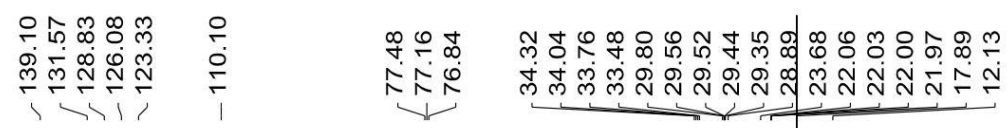

${ }^{13} \mathrm{C} \mathrm{NMR}\left(100 \mathrm{MHz}, \mathrm{CDCl}_{3}\right)$

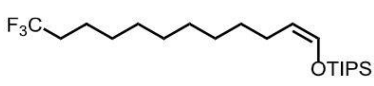

$1 z$

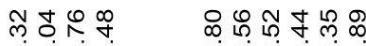

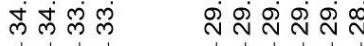

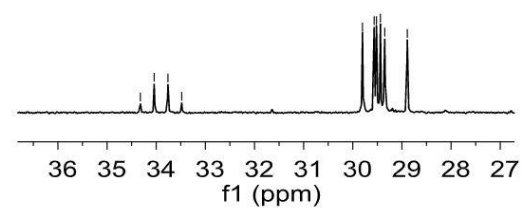

) /1

$$
\sqrt{20}
$$

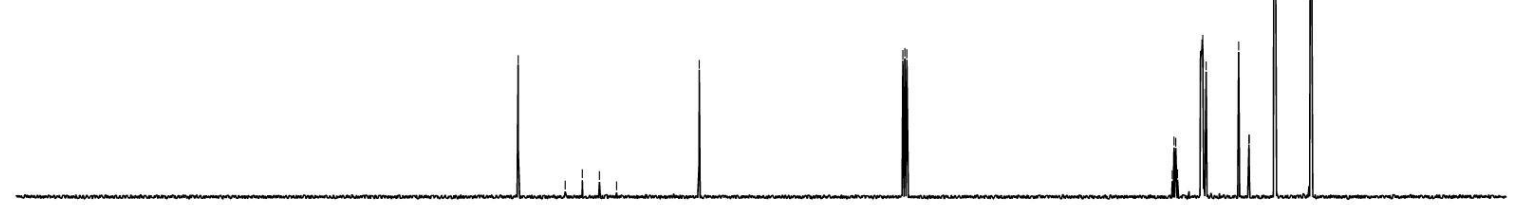

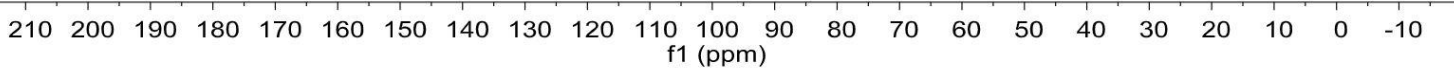




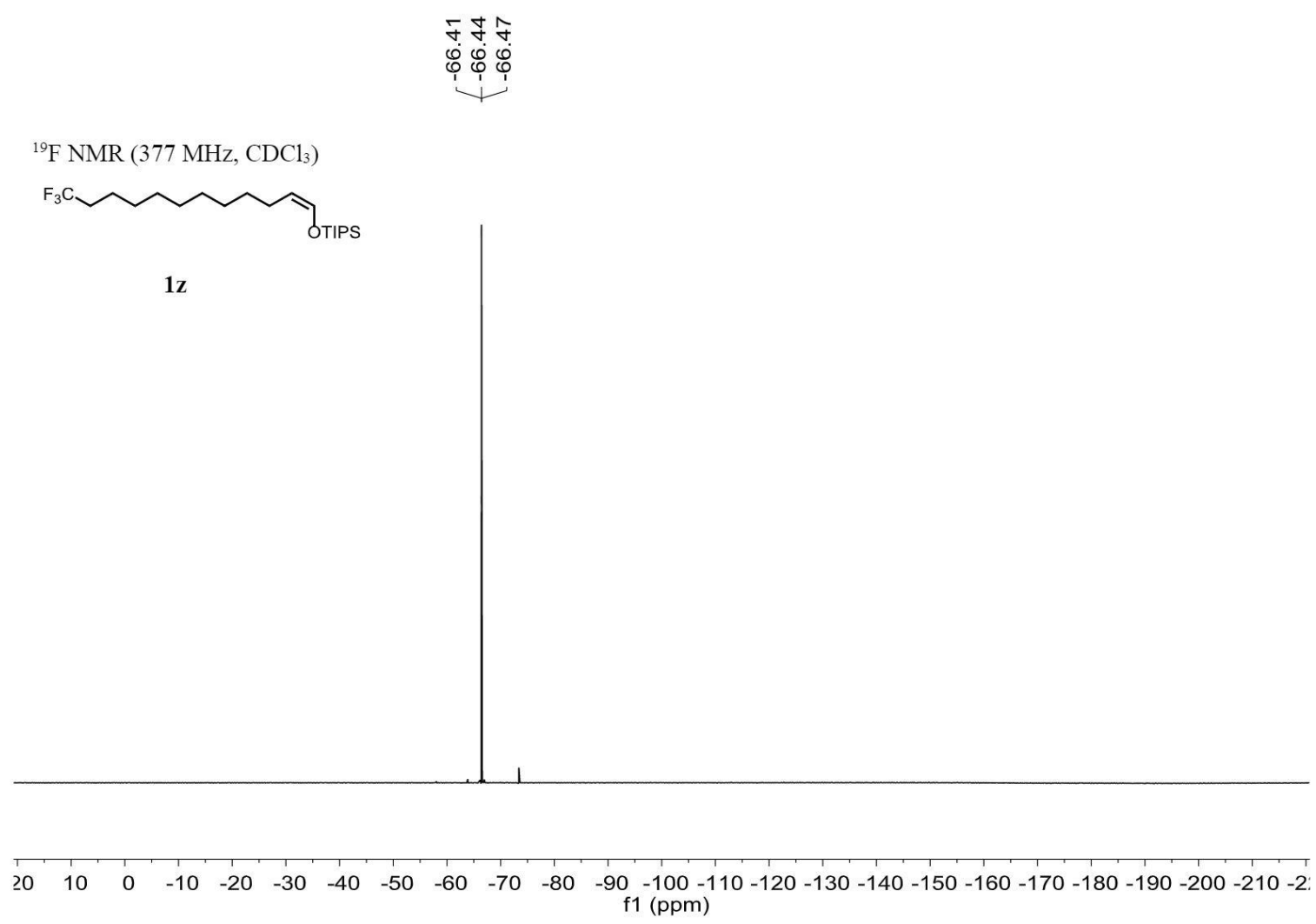

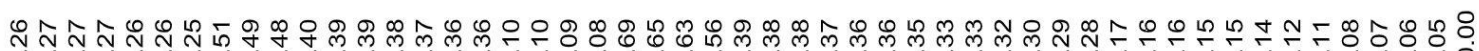

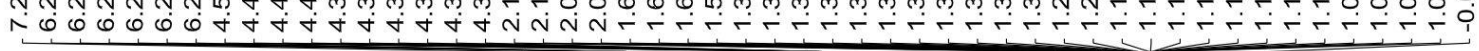

${ }^{1} \mathrm{H} \mathrm{NMR}\left(400 \mathrm{MHz}, \mathrm{CDCl}_{3}\right)$

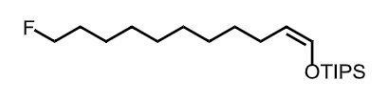

1aa

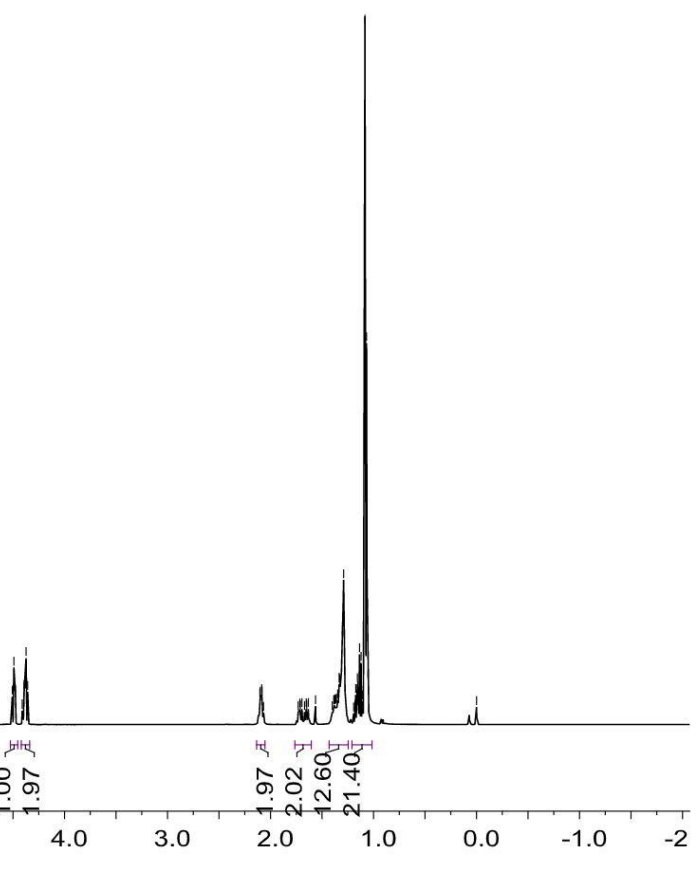




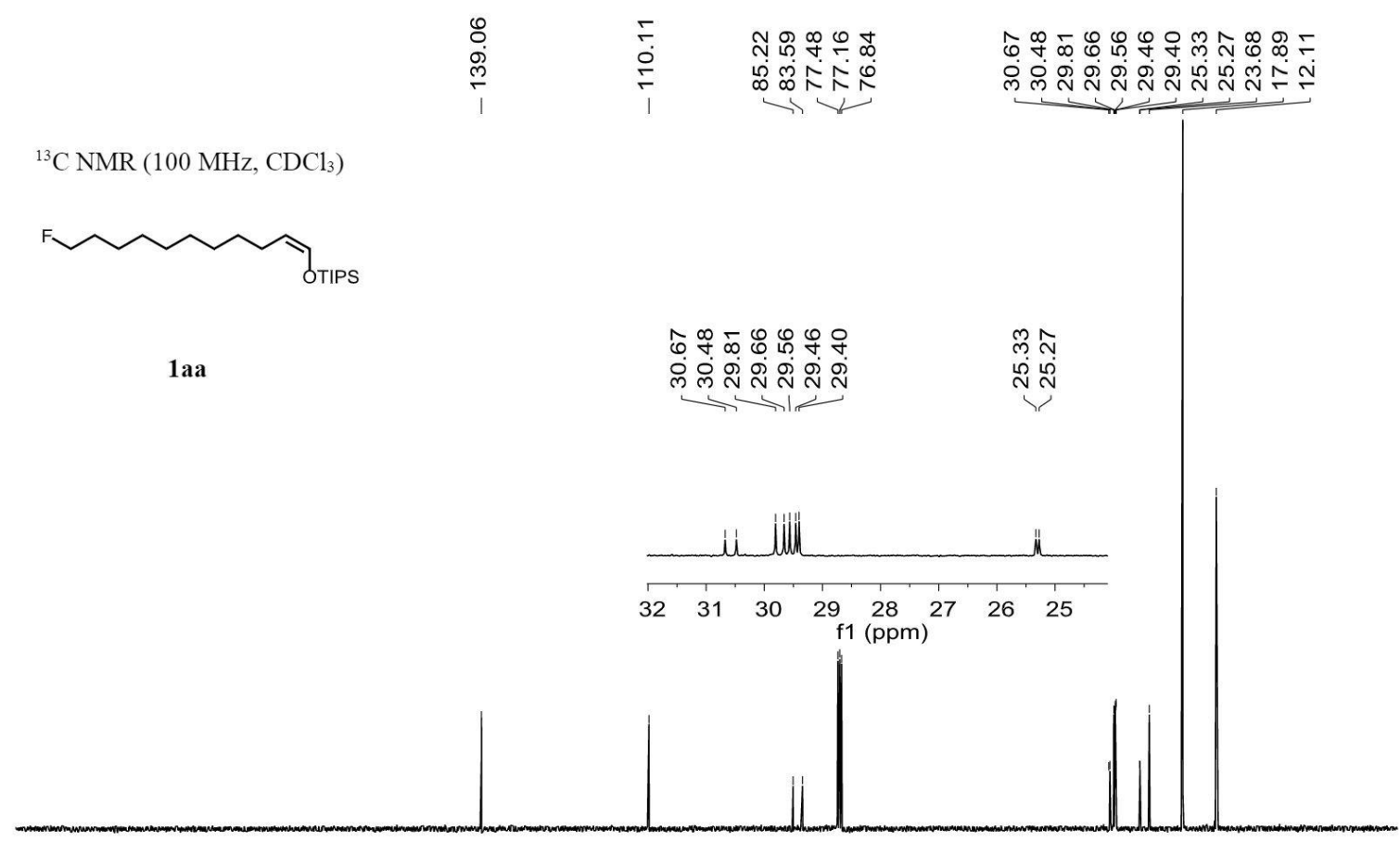

$\begin{array}{lllllllllllllllllllllllllll}210 & 200 & 190 & 180 & 170 & 160 & 150 & 140 & 130 & 120 & 110 & 100 & 90 & 80 & 70 & 60 & 50 & 40 & 30 & 20 & 10 & 0 & -10\end{array}$ f1 (ppm)

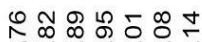

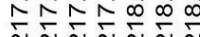

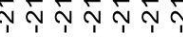

${ }^{19} \mathrm{~F}$ NMR $\left(377 \mathrm{MHz}, \mathrm{CDCl}_{3}\right)$

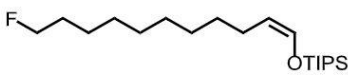

1 aa

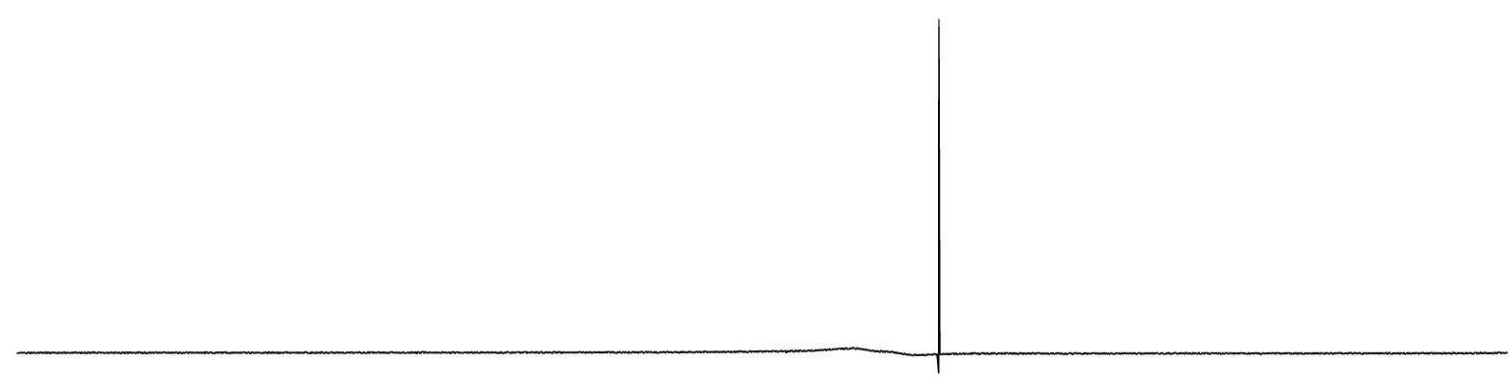

$\begin{array}{lllllllllllllllllll}350 & 300 & 250 & 200 & 150 & 100 & 50 & 0 & -50 & -100 & -150 & -200 & -250 & -300 & -350 & -400 & -450 & -500 & -550\end{array}$ 


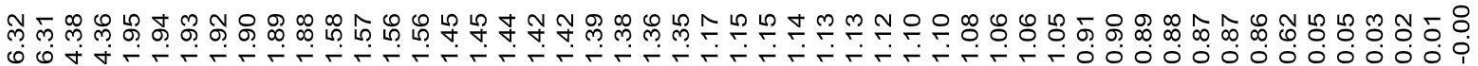

${ }^{1} \mathrm{H} \mathrm{NMR}\left(400 \mathrm{MHz}, \mathrm{CDCl}_{3}\right)$

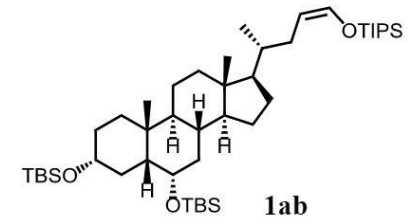
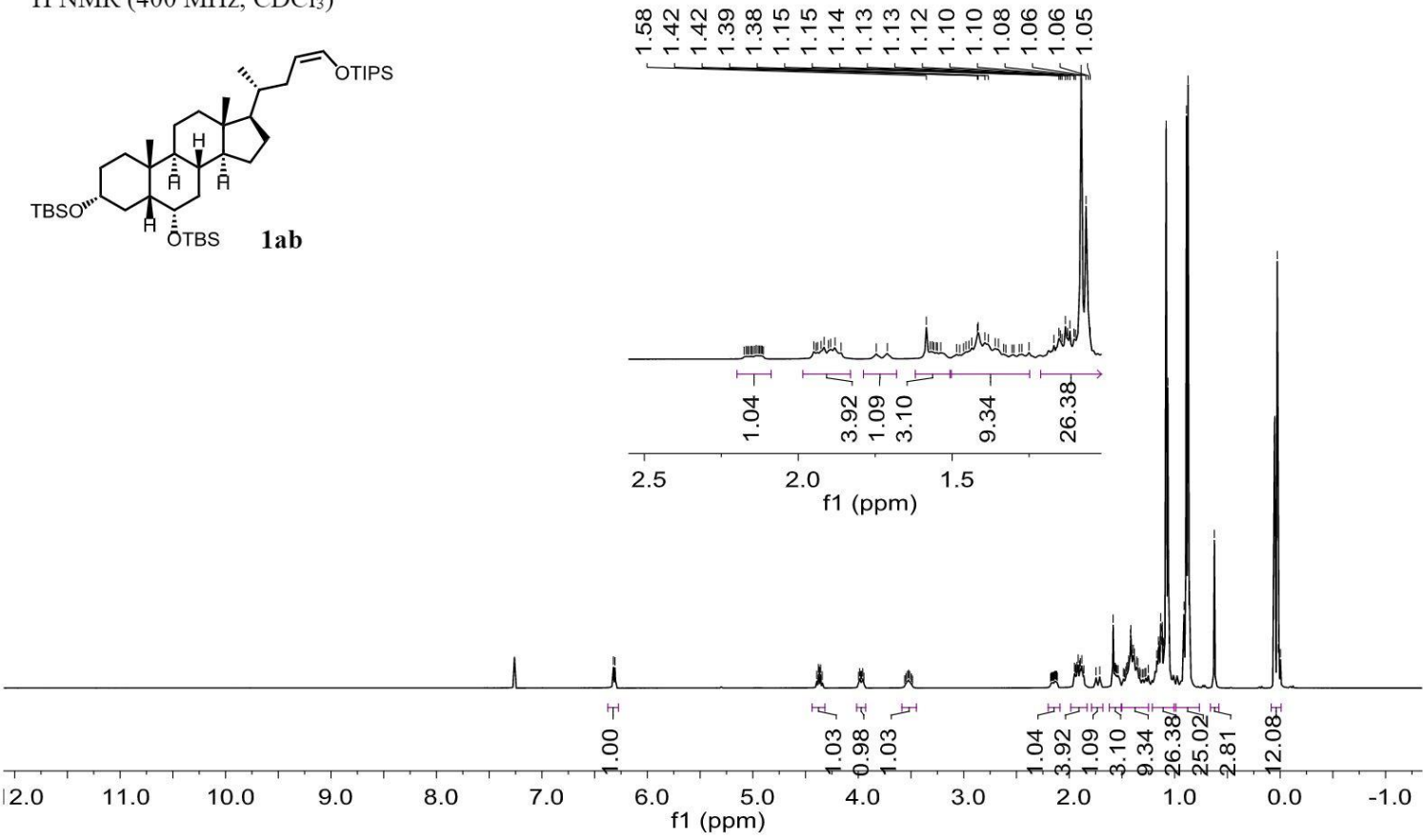

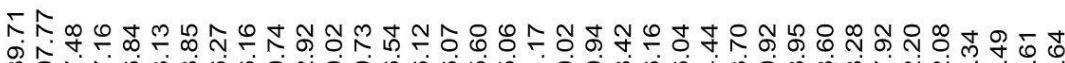

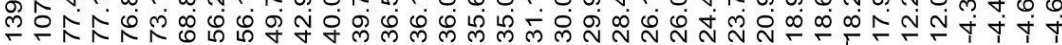

${ }^{13} \mathrm{C} \mathrm{NMR}\left(100 \mathrm{MHz}, \mathrm{CDCl}_{3}\right)$
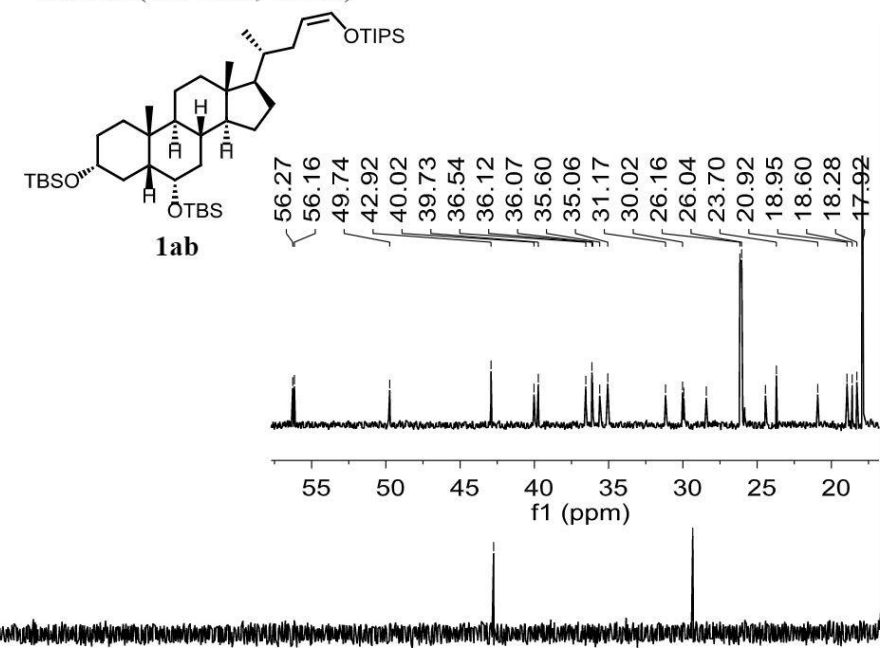

$\begin{array}{lllllllllllll}210 & 200 & 190 & 180 & 170 & 160 & 150 & 140 & 130 & 120 & 110 & 100 & 9\end{array}$

f1 (ppm) 
${ }^{1} \mathrm{H}$ NMR (400 MHz, $\mathrm{CDCl}_{3}$ )

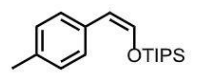

1ad

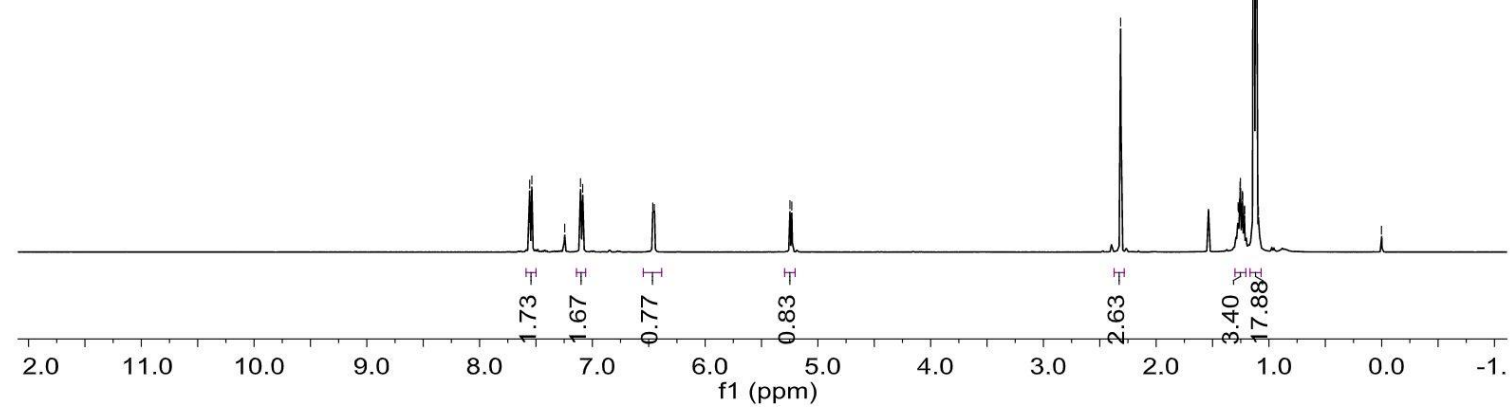

वृ

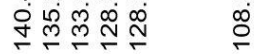

Nָळ

กัก

ये

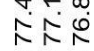

1)

${ }^{13} \mathrm{C} \mathrm{NMR}\left(100 \mathrm{MHz}, \mathrm{CDCl}_{3}\right)$

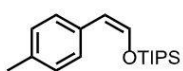

1 ad

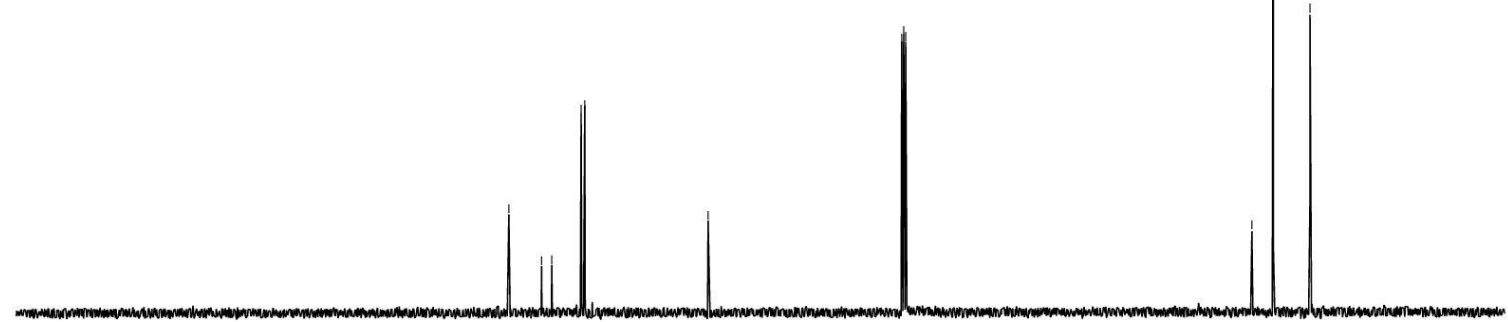

$\begin{array}{lllllllllllllllllllllll}210 & 200 & 190 & 180 & 170 & 160 & 150 & 140 & 130 & 120 & 110 & 100 & 90 & 80 & 70 & 60 & 50 & 40 & 30 & 20 & 10 & 0 & -10\end{array}$ $\mathrm{f} 1(\mathrm{ppm})$ 
${ }^{1} \mathrm{H}$ NMR $\left(400 \mathrm{MHz}, \mathrm{CDCl}_{3}\right)$

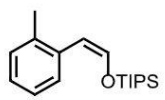

1 ae

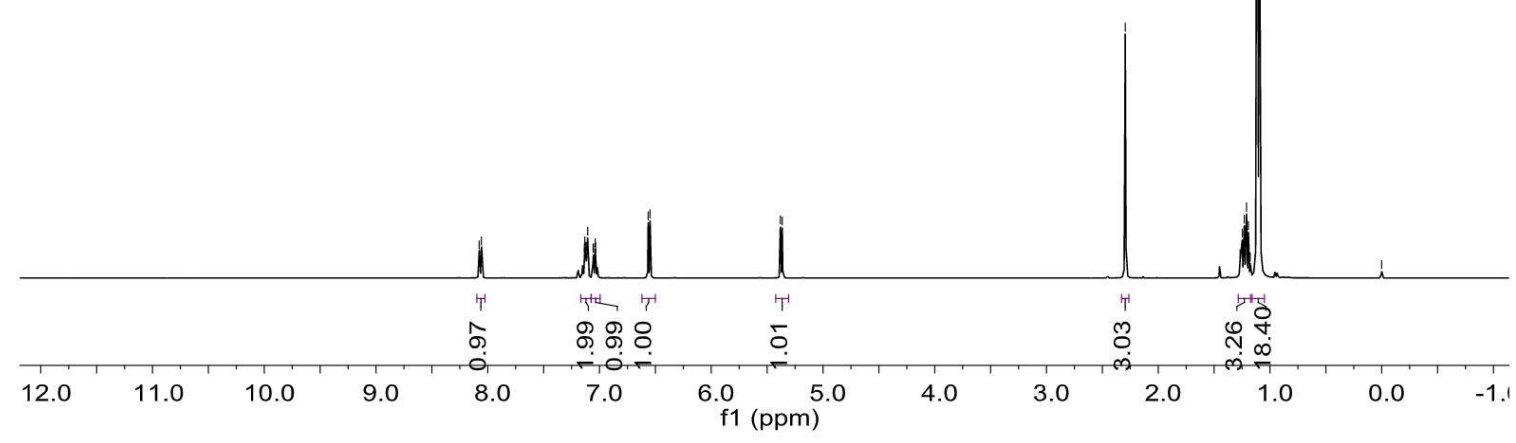

\begin{tabular}{|c|c|c|}
\hline 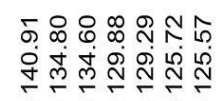 & 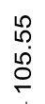 & 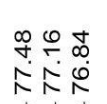 \\
\hline
\end{tabular}

${ }^{13} \mathrm{C} \mathrm{NMR}\left(100 \mathrm{MHz}, \mathrm{CDCl}_{3}\right)$

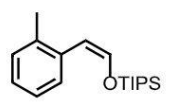

1 ae

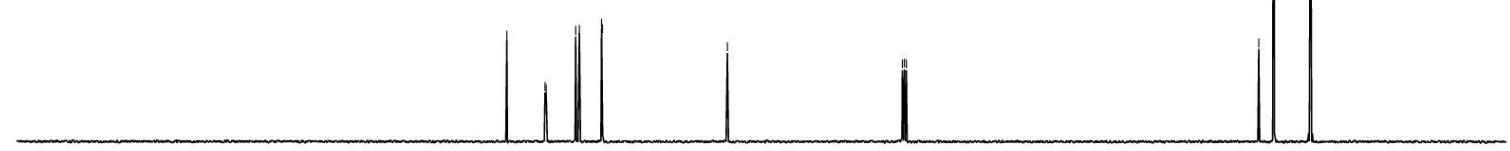

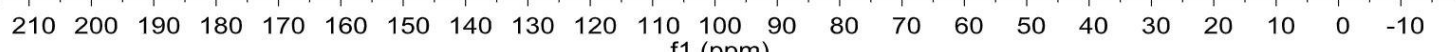




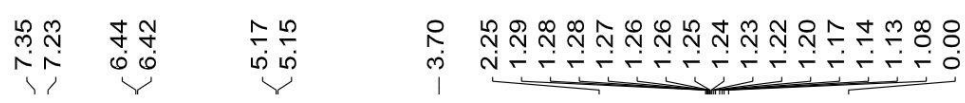

${ }^{1} \mathrm{H}$ NMR $\left(400 \mathrm{MHz}, \mathrm{CDCl}_{3}\right)$

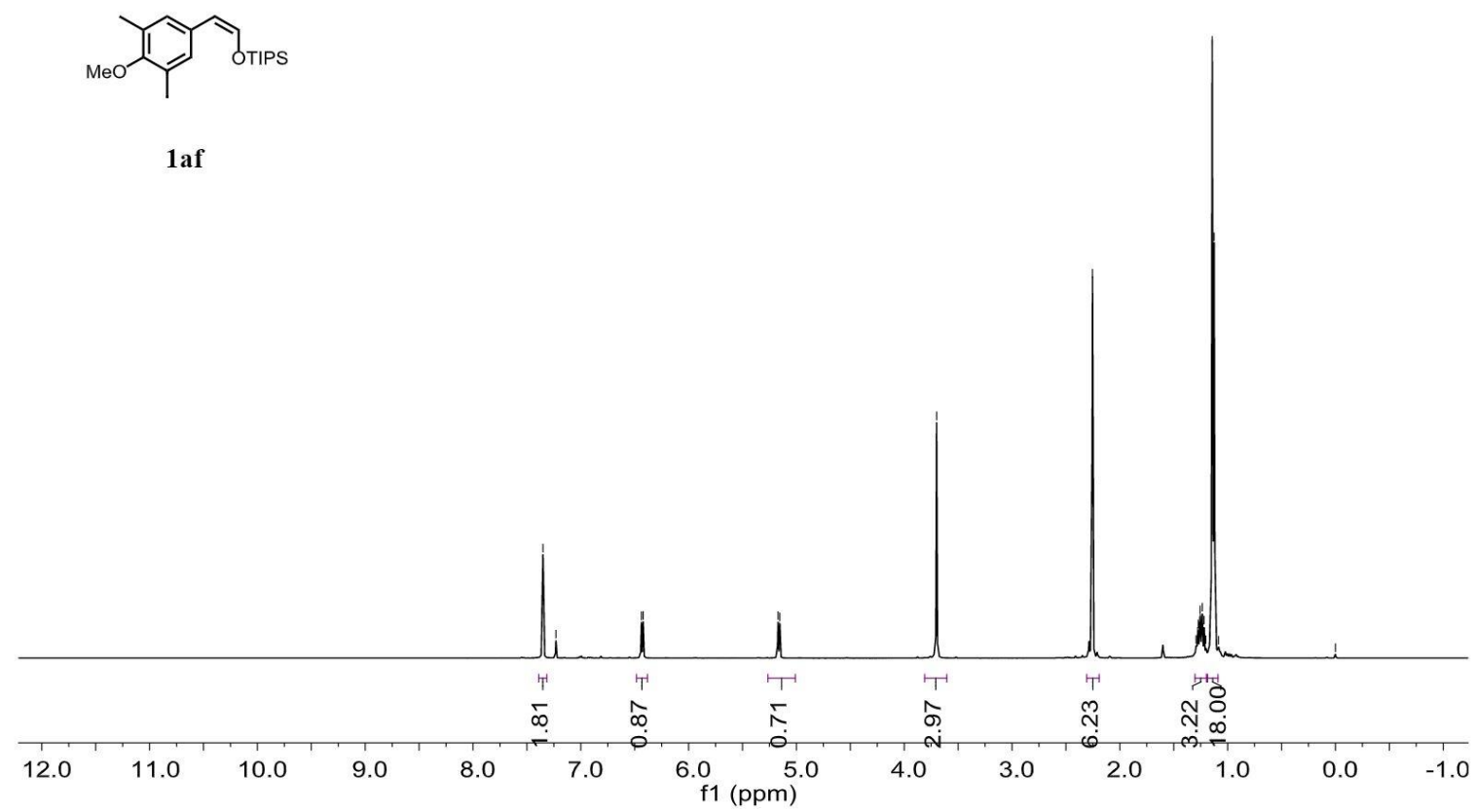

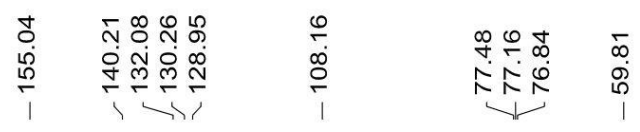

${ }^{13} \mathrm{C} \mathrm{NMR}\left(100 \mathrm{MHz}, \mathrm{CDCl}_{3}\right)$

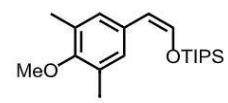

1af

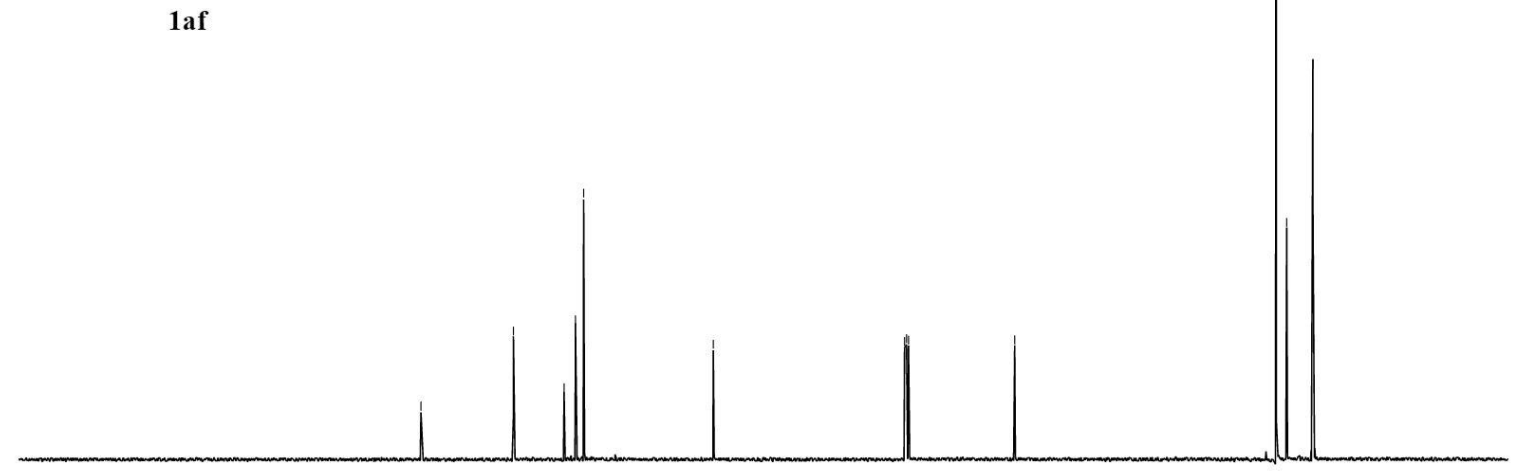

$\begin{array}{llllllllllllllllllllllllllllllll}210 & 200 & 190 & 180 & 170 & 160 & 150 & 140 & 130 & 120 & 110 & 100 & 90 & 80 & 70 & 60 & 50 & 40 & 30 & 20 & 10 & 0 & -10\end{array}$ 


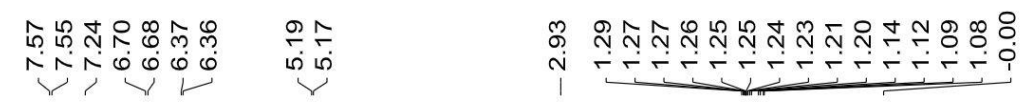

${ }^{1} \mathrm{H}$ NMR $\left(400 \mathrm{MHz}, \mathrm{CDCl}_{3}\right)$

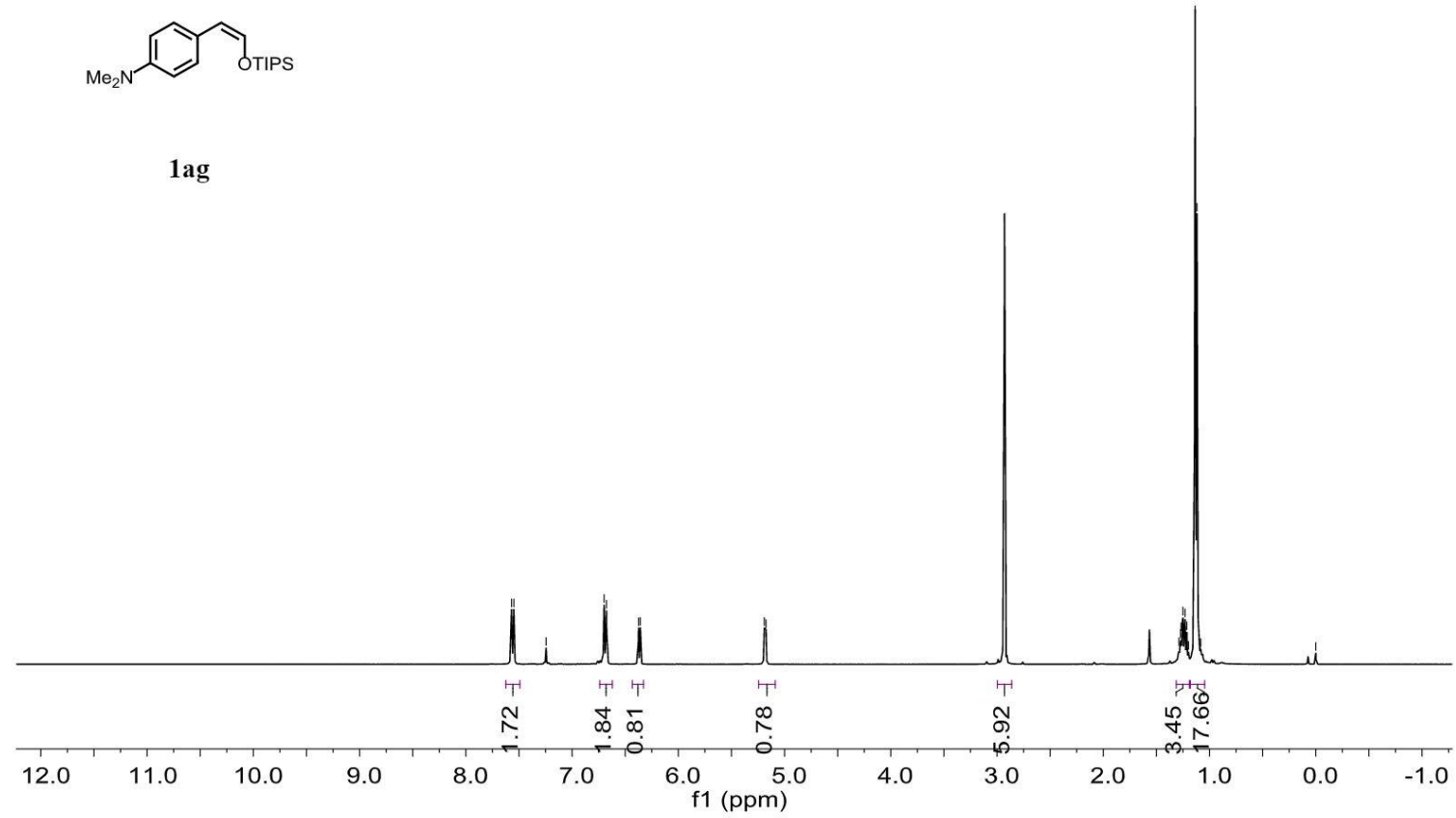

\begin{tabular}{|c|c|c|c|}
\hline 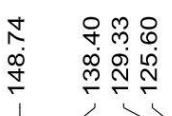 & 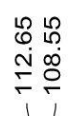 & 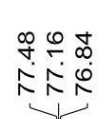 & $\begin{array}{l}0 \\
\infty \\
0 \\
+\end{array}$ \\
\hline
\end{tabular}

${ }^{13} \mathrm{C}$ NMR (100 MHz, $\mathrm{CDCl}_{3}$ )

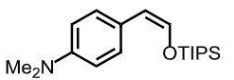

1 ag

$\begin{array}{lllllllllllllllllllllll}210 & 200 & 190 & 180 & 170 & 160 & 150 & 140 & 130 & 120 & 110 & 100 & 90 & 80 & 70 & 60 & 50 & 40 & 30 & 20 & 10 & 0 & -10\end{array}$ 
${ }^{1} \mathrm{H}$ NMR $\left(400 \mathrm{MHz}, \mathrm{CDCl}_{3}\right)$

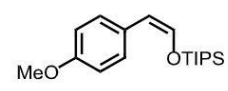

$1 \mathbf{a h}$

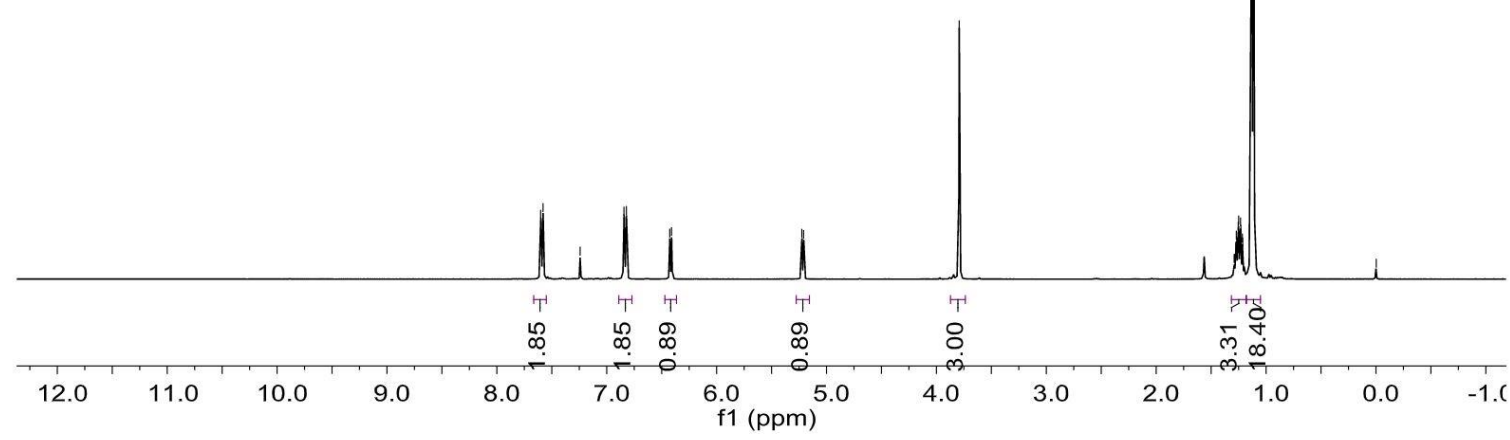

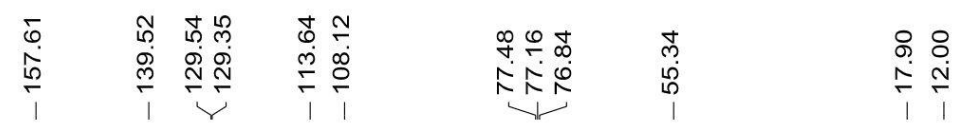

${ }^{13} \mathrm{C}$ NMR (100 MHz, $\left.\mathrm{CDCl}_{3}\right)$

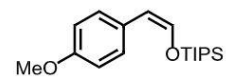

1ah

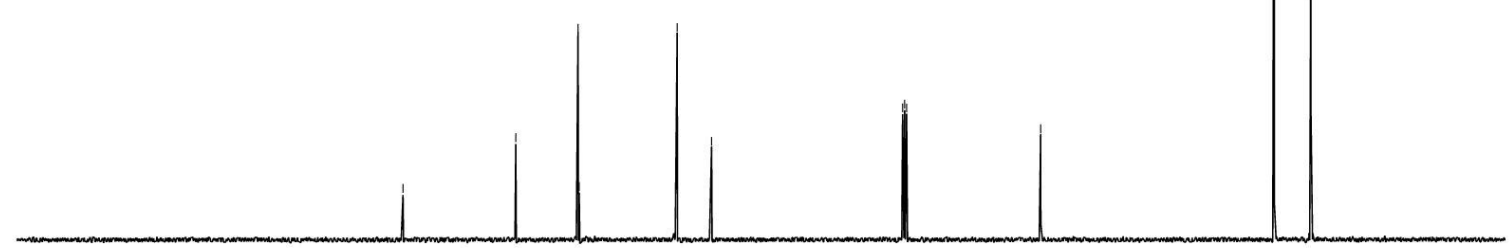
$\begin{array}{lllllllllllllllllllllllllll}210 & 200 & 190 & 180 & 170 & 160 & 150 & 140 & 130 & 120 & \begin{array}{c}110 \\ \mathrm{f} 1\end{array}\left(\begin{array}{ll}100 \\ (\mathrm{ppm})\end{array}\right. & 90 & 80 & 70 & 60 & 50 & 40 & 30 & 20 & 10 & 0 & -10\end{array}$ 
${ }^{1} \mathrm{H}$ NMR $\left(400 \mathrm{MHz}, \mathrm{CDCl}_{3}\right)$<smiles>[OH2+]/C=C\c1ccc([18O])cc1</smiles>

1ai
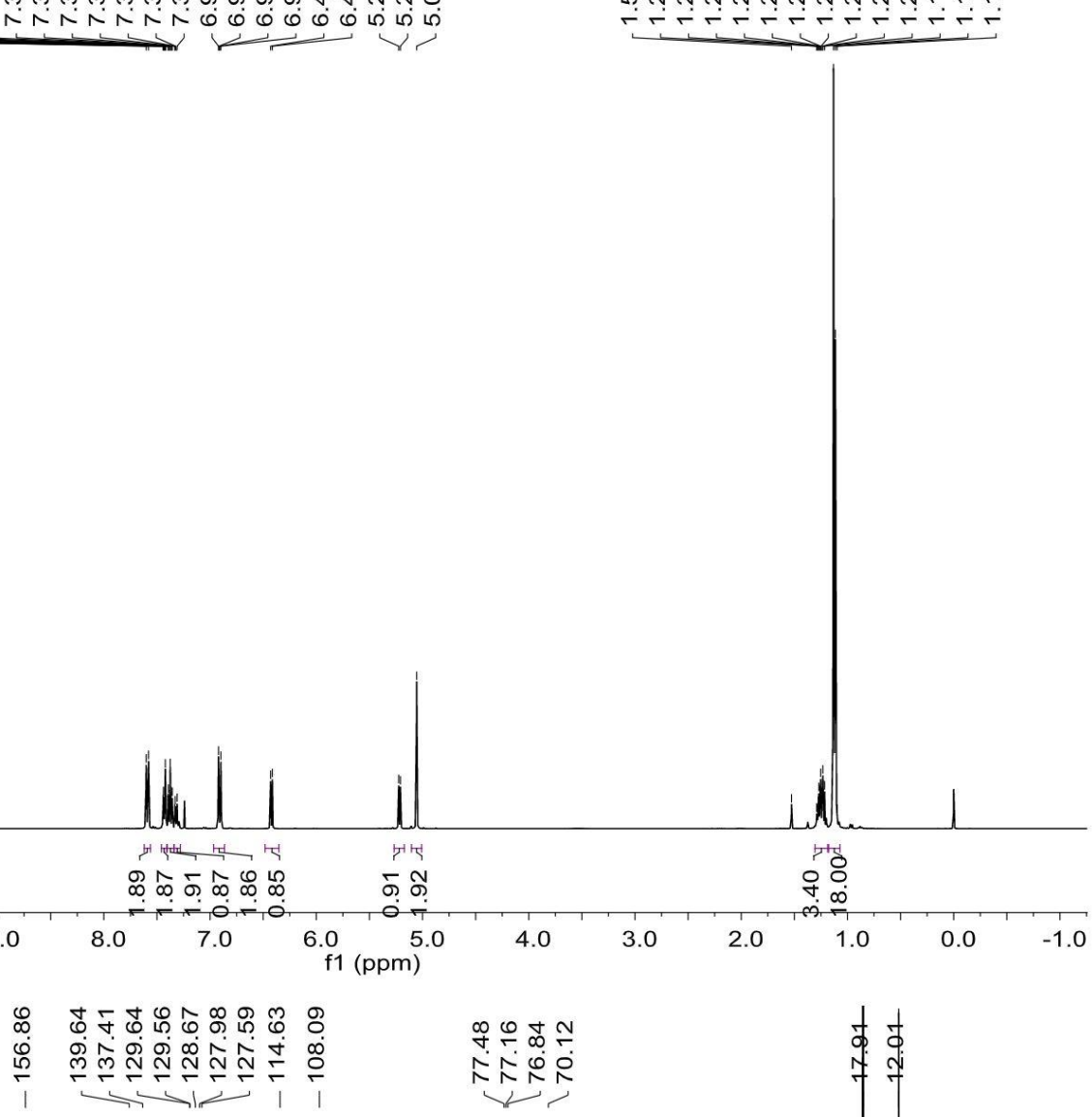

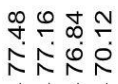

$\$$

${ }^{13} \mathrm{C}$ NMR $\left(100 \mathrm{MHz}, \mathrm{CDCl}_{3}\right)$

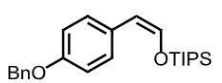

1ai

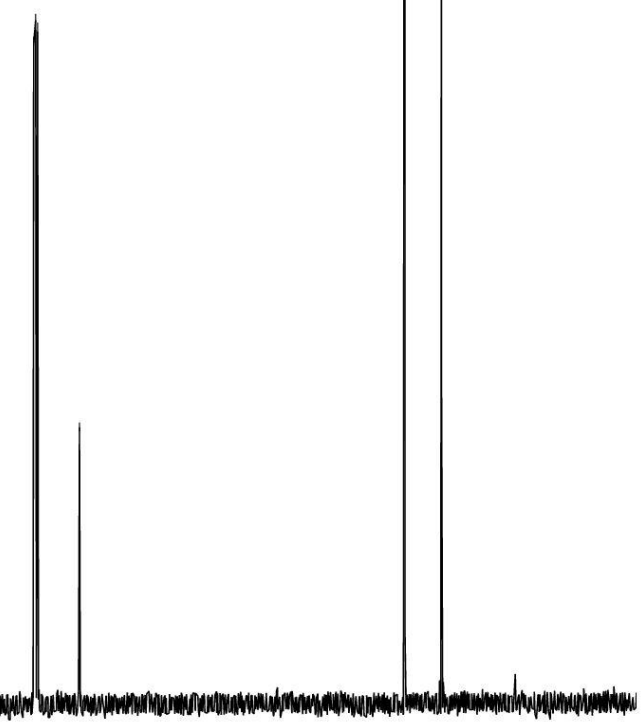
$\begin{array}{llllllllllll}210 & 200 & 190 & 180 & 170 & 160 & 150 & 140 & 130 & 120 & 110 & 100 \\ \mathrm{f} 1(\mathrm{ppm})\end{array}$ 
${ }^{1} \mathrm{H}$ NMR $\left(400 \mathrm{MHz}, \mathrm{C}_{6} \mathrm{D}_{6}\right)$

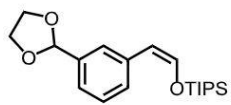

1 aj

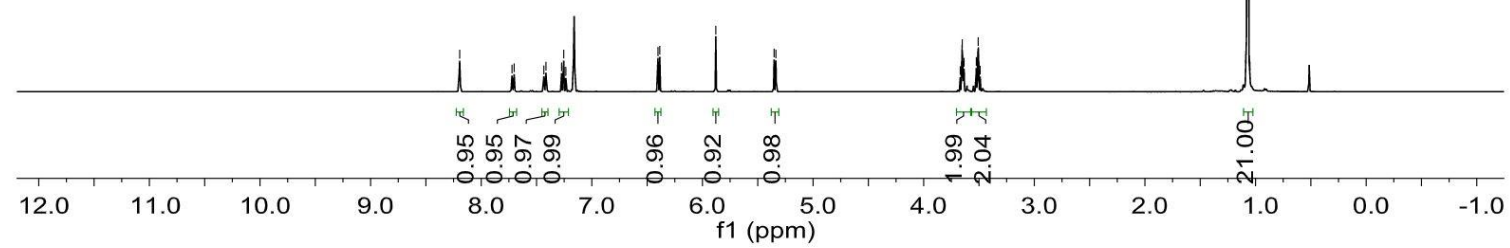

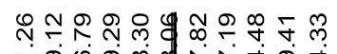

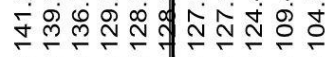

:

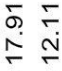

${ }^{13} \mathrm{C}$ NMR (100 MHz, $\mathrm{C}_{6} \mathrm{D}_{6}$ )

(1)

1aj

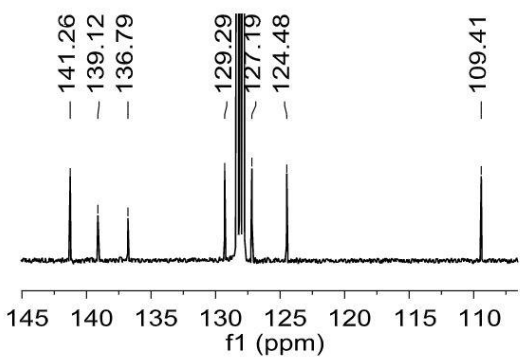

$\begin{array}{lllllllllllllllllllllll}210 & 200 & 190 & 180 & 170 & 160 & 150 & 140 & 130 & 120 & 110 & 100 & 90 & 80 & 70 & 60 & 50 & 40 & 30 & 20 & 10 & 0 & -10\end{array}$ $\mathrm{f1}(\mathrm{ppm})$ 
${ }^{1} \mathrm{H}$ NMR $\left(400 \mathrm{MHz}, \mathrm{CDCl}_{3}\right)$

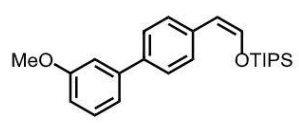

$1 \mathrm{ak}$

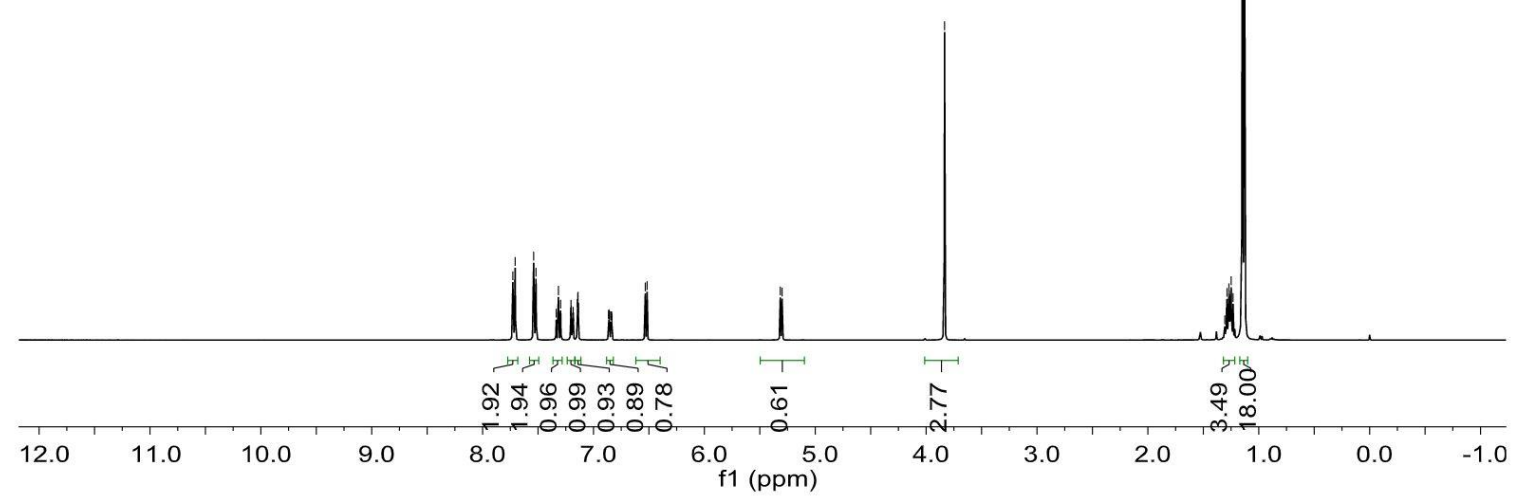

s. $\quad$ c.

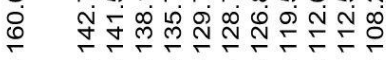

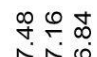

トペ是

is

${ }^{13} \mathrm{C}$ NMR $\left(100 \mathrm{MHz}, \mathrm{CDCl}_{3}\right)$

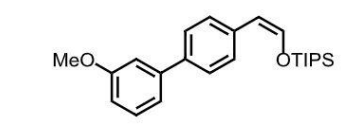

$1 \mathrm{ak}$

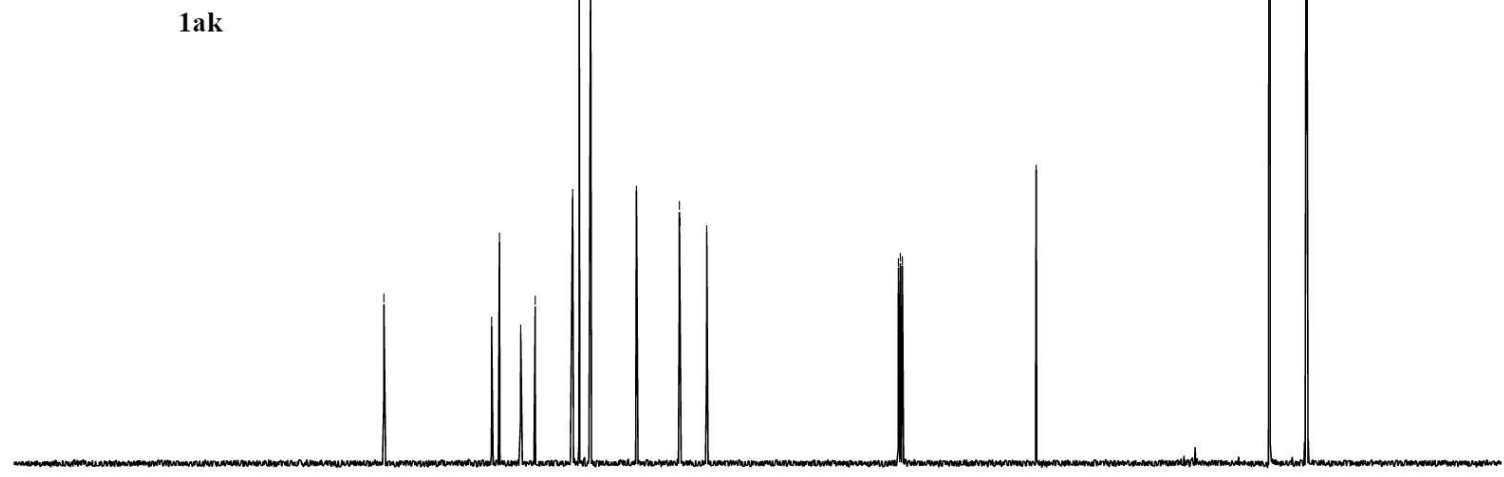

$\begin{array}{lllllllllllllllllllllll}210 & 200 & 190 & 180 & 170 & 160 & 150 & 140 & 130 & 120 & 110 & 100 & 90 & 80 & 70 & 60 & 50 & 40 & 30 & 20 & 10 & 0 & -10\end{array}$ 
${ }^{1} \mathrm{H}$ NMR (400 MHz, $\left.\mathrm{CDCl}_{3}\right)$<smiles>[OH2+]/C=C\c1ccc(F)cc1</smiles>

1al

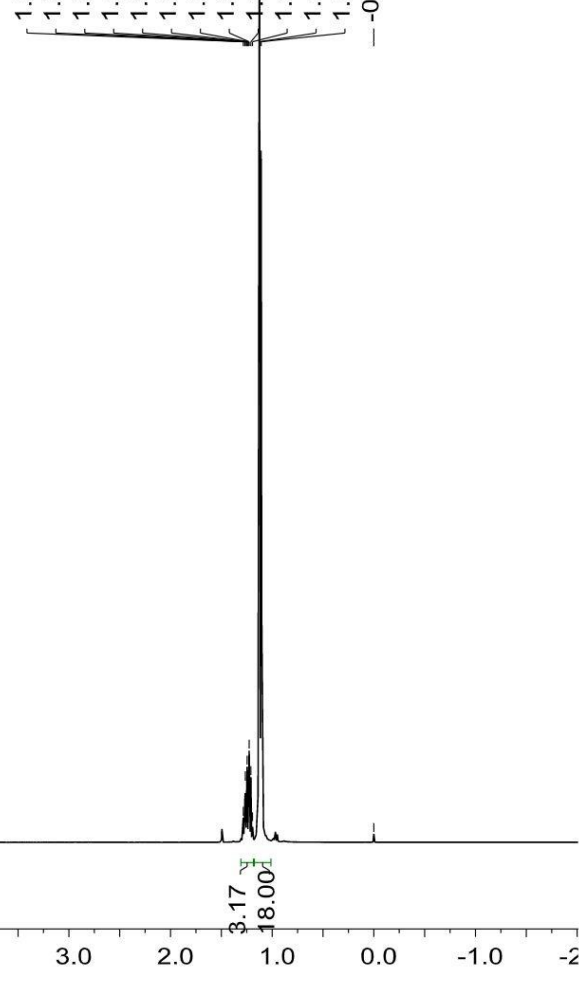

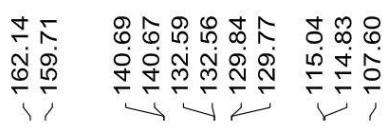

dị

定余

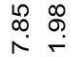

倘

${ }^{13} \mathrm{C}$ NMR (100 MHz, $\left.\mathrm{CDCl}_{3}\right)$

Oेtips

1al

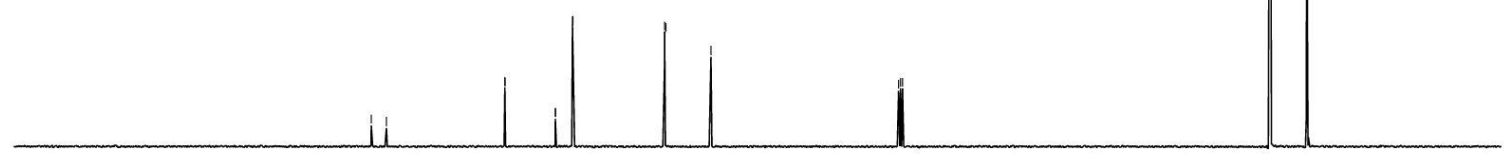

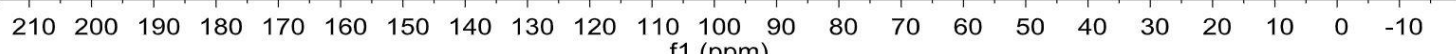


$\operatorname{cis}$

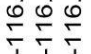

i

${ }^{19} \mathrm{~F} \mathrm{NMR}\left(377 \mathrm{MHz}, \mathrm{CDCl}_{3}\right)$

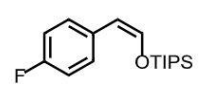

1al

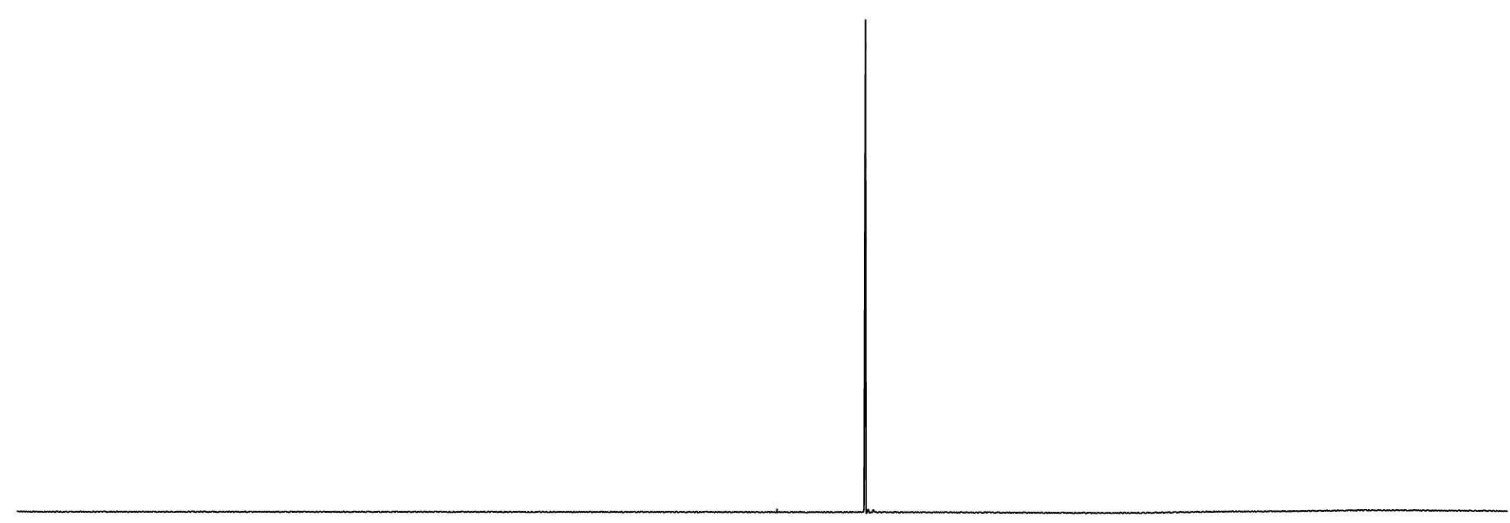

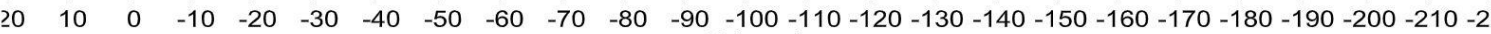
f1 (ppm) 


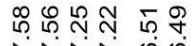

NNÑo

กูก

เा เค

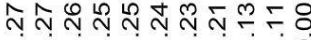

-5i-r.

${ }^{1} \mathrm{H}$ NMR $\left(400 \mathrm{MHz}, \mathrm{CDCl}_{3}\right)$

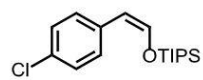

$1 \mathrm{am}$

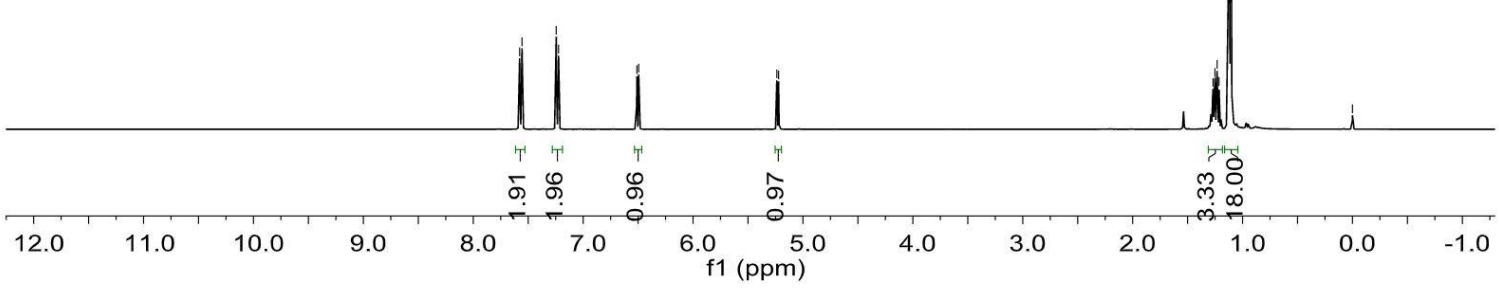

\begin{tabular}{|c|c|c|}
\hline 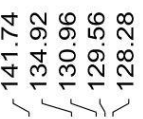 & $\begin{array}{c}0 \\
\stackrel{0}{0} \\
\stackrel{0}{\circ} \\
1\end{array}$ & 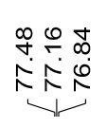 \\
\hline
\end{tabular}

${ }^{13} \mathrm{C} \mathrm{NMR}\left(100 \mathrm{MHz}, \mathrm{CDCl}_{3}\right)$

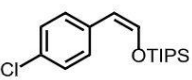

1 am

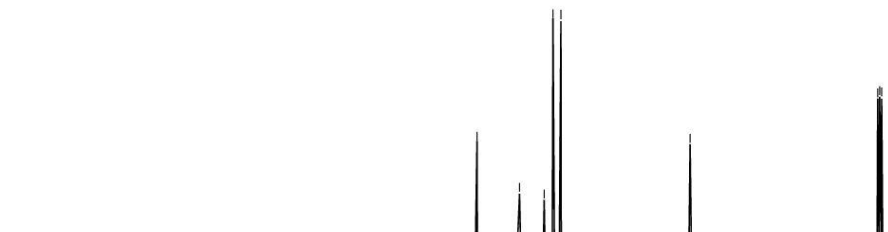

$\begin{array}{lllllllllllllllllllllll}210 & 200 & 190 & 180 & 170 & 160 & 150 & 140 & 130 & 120 & 110 & 100 & 90 & 80 & 70 & 60 & 50 & 40 & 30 & 20 & 10 & 0 & -10\end{array}$ $\mathrm{f} 1(\mathrm{ppm})$ 
${ }^{1} \mathrm{H}$ NMR $\left(400 \mathrm{MHz}, \mathrm{CDCl}_{3}\right)$

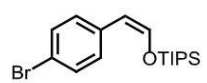

1 an
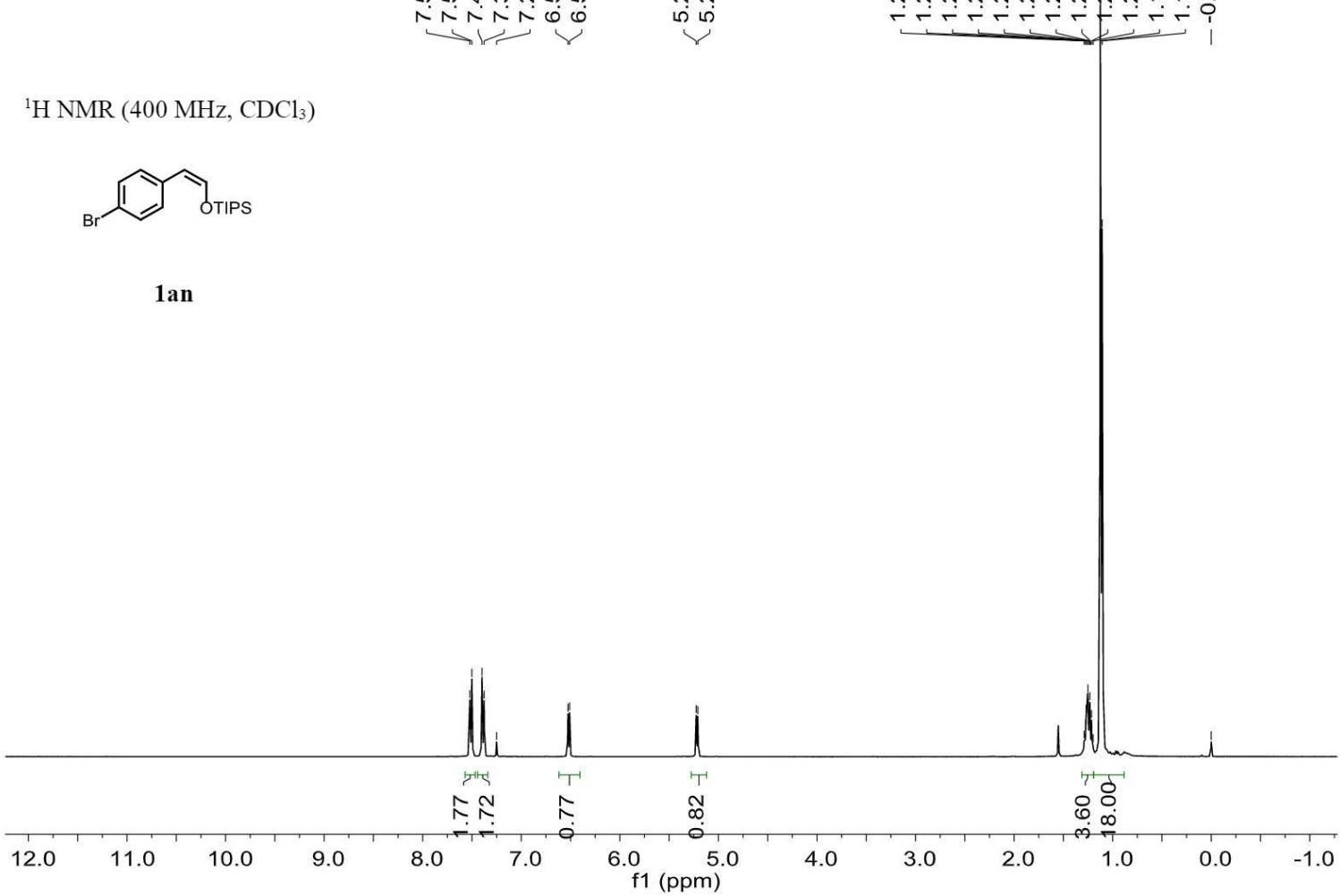

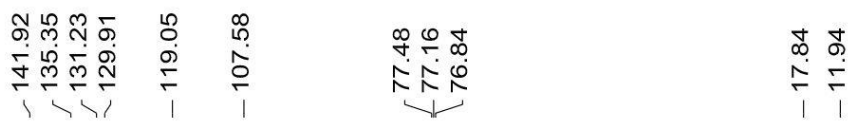

${ }^{13} \mathrm{C} \mathrm{NMR}\left(100 \mathrm{MHz}, \mathrm{CDCl}_{3}\right)$

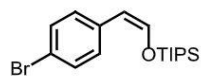

1 an

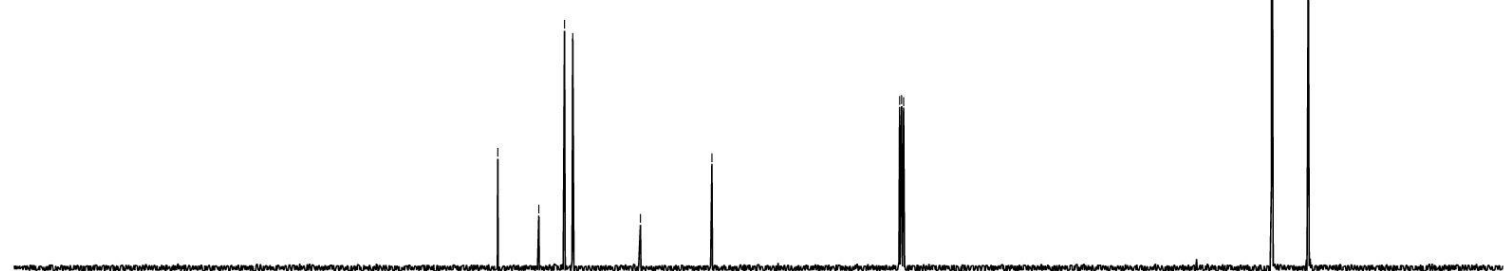

$\begin{array}{lllllllllllllllllllllll}210 & 200 & 190 & 180 & 170 & 160 & 150 & 140 & 130 & 120 & 110 & 100 & 90 & 80 & 70 & 60 & 50 & 40 & 30 & 20 & 10 & 0 & -10\end{array}$ $\mathrm{f} 1(\mathrm{ppm})$ 
${ }^{1} \mathrm{H}$ NMR $\left(400 \mathrm{MHz}, \mathrm{CDCl}_{3}\right)$

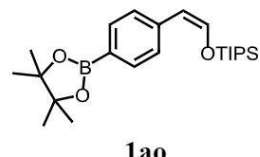

$1 \mathrm{ao}$
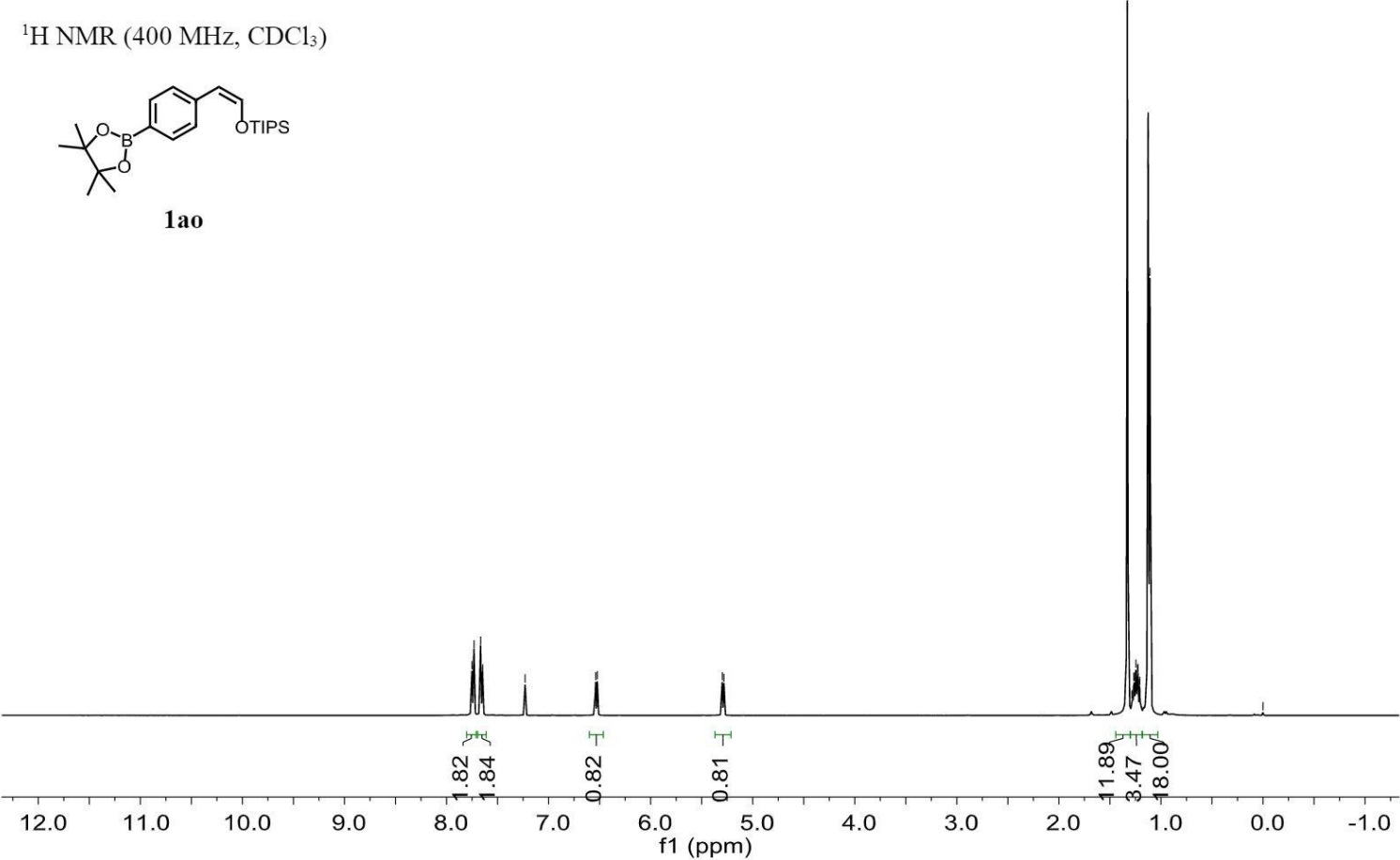

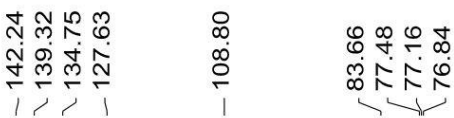

סִ ח

aำ

$\left.{ }^{13} \mathrm{C} \mathrm{NMR} \mathrm{(100} \mathrm{MHz,} \mathrm{CDCl}_{3}\right)$

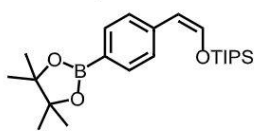

1 ao

$\begin{array}{lllllllllllllllllllllll}210 & 200 & 190 & 180 & 170 & 160 & 150 & 140 & 130 & 120 & 110 & 100 & 90 & 80 & 70 & 60 & 50 & 40 & 30 & 20 & 10 & 0 & -10\end{array}$ 


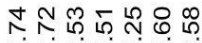

NNNNTOO

$\Rightarrow 1+$

${ }^{1} \mathrm{H}$ NMR $\left(400 \mathrm{MHz}, \mathrm{CDCl}_{3}\right)$

1ap

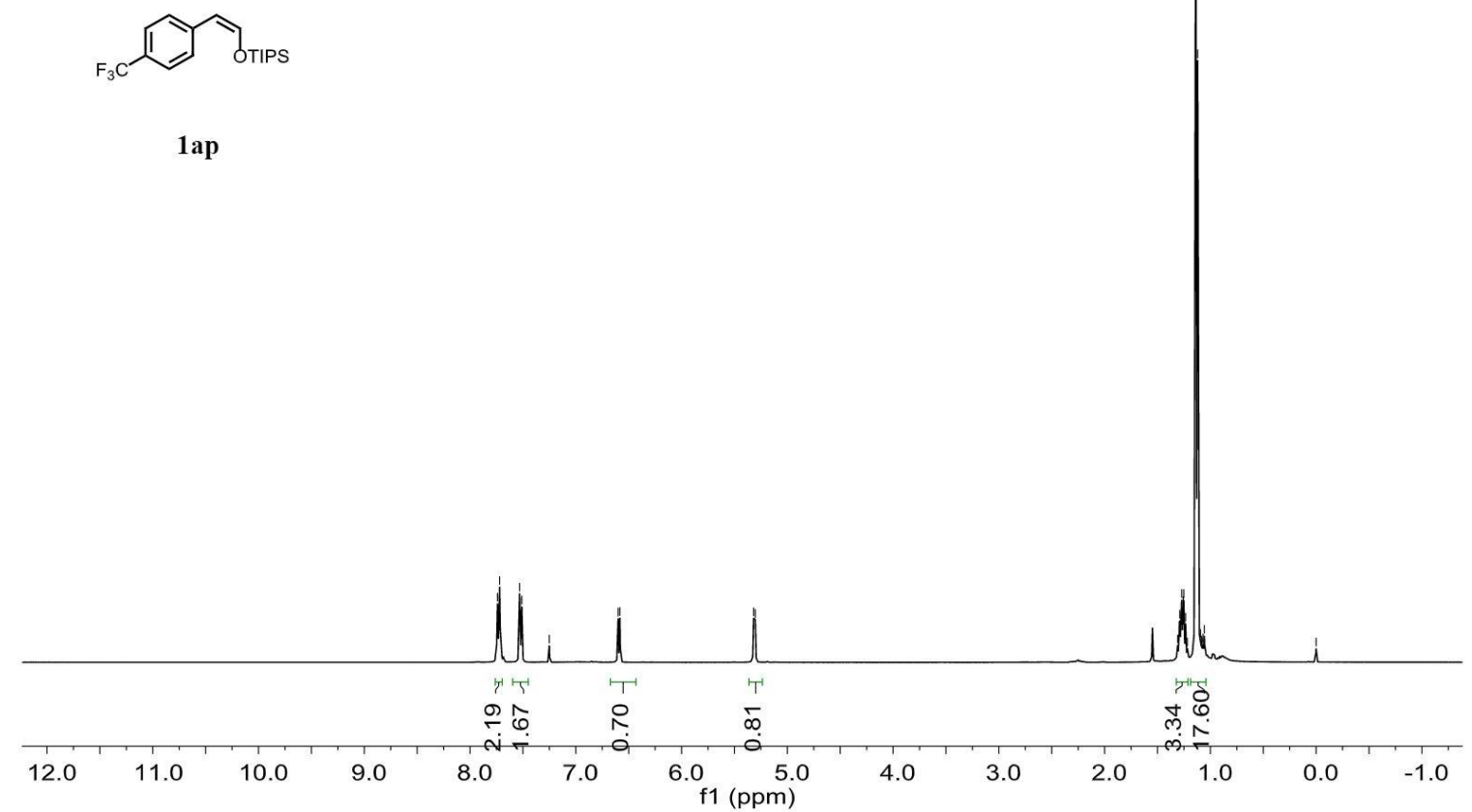

ల్లె

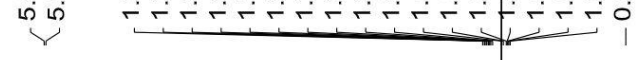

ผ

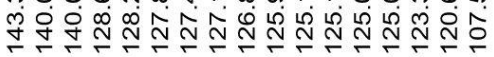

我

र下保

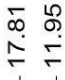

${ }^{13} \mathrm{C}$ NMR $\left(100 \mathrm{MHz}, \mathrm{CDCl}_{3}\right)$<smiles>FC(F)(F)c1ccc(C=CO[Mg])cc1</smiles>

1ap

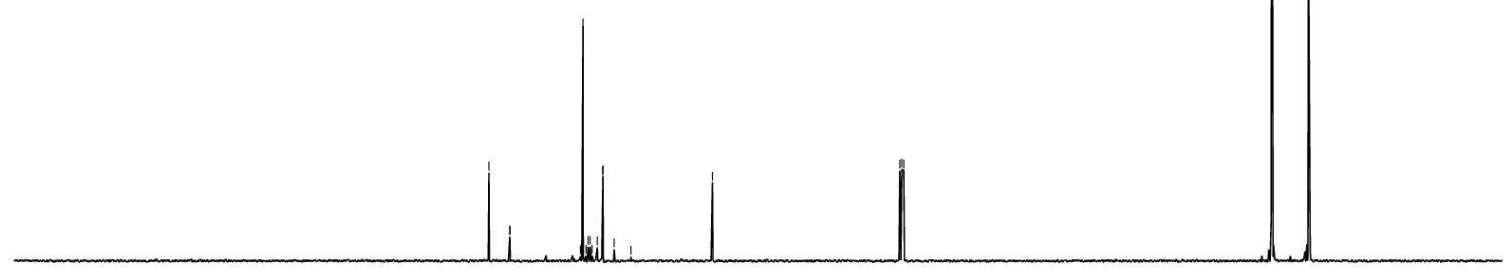

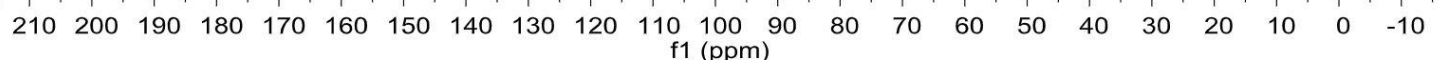


${ }^{19} \mathrm{~F}$ NMR (377 MHz, $\left.\mathrm{CDCl}_{3}\right)$

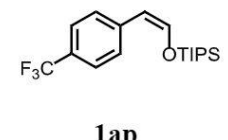

1 ap

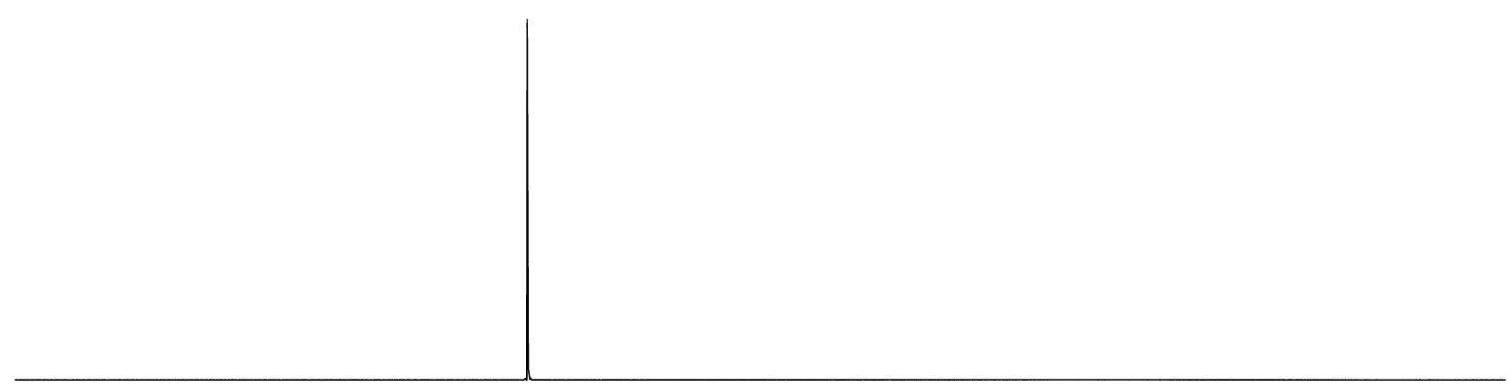

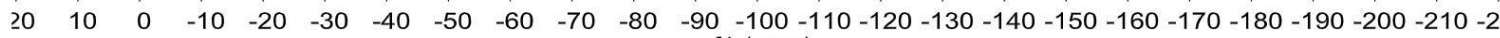
f1 $(\mathrm{ppm})$ 


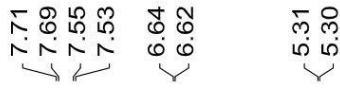

${ }^{1} \mathrm{H}$ NMR (400 MHz, $\mathrm{CDCl}_{3}$ )

1 aq

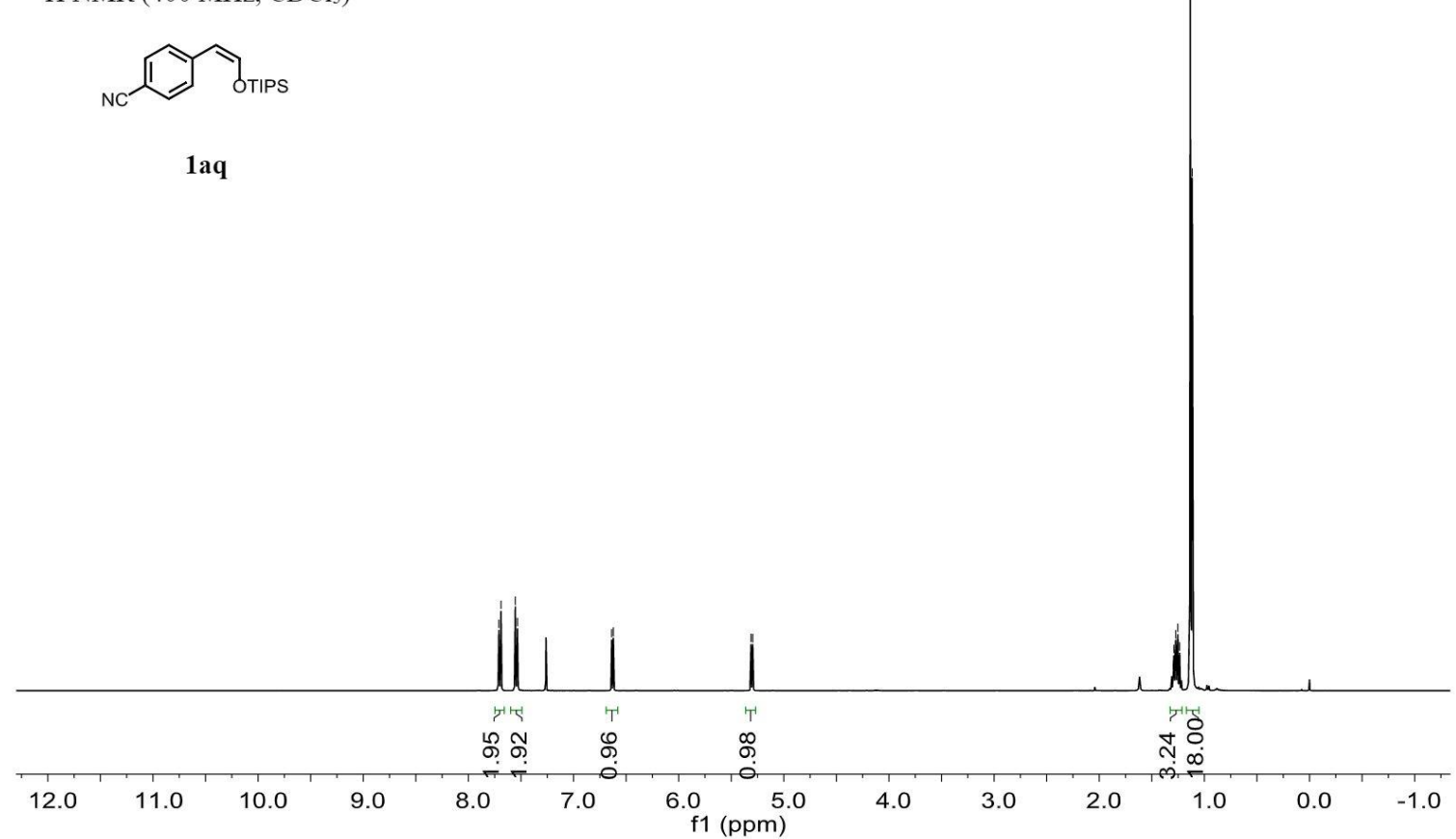

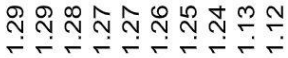

\begin{tabular}{|c|c|c|c|}
\hline 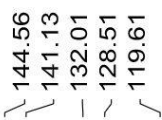 & 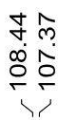 & 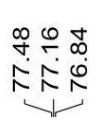 & 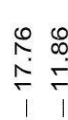 \\
\hline
\end{tabular}

${ }^{13} \mathrm{C}$ NMR (100 MHz, $\left.\mathrm{CDCl}_{3}\right)$

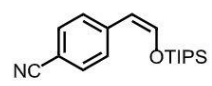

$1 a q$

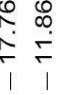

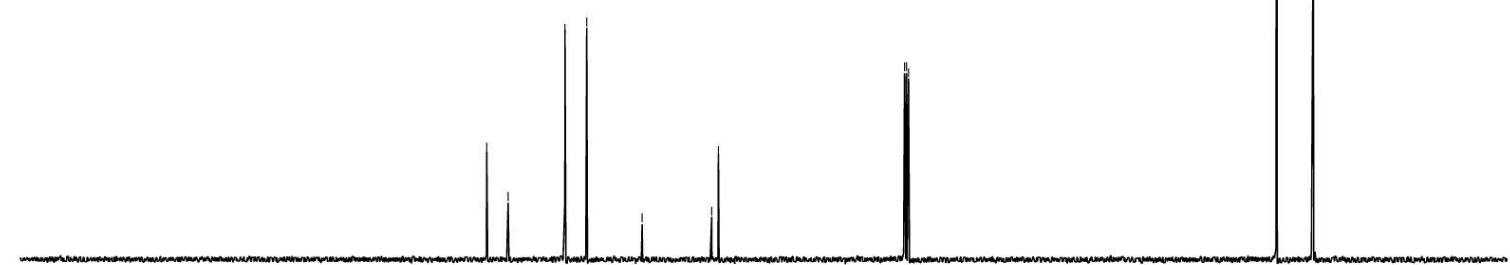

$\begin{array}{lllllllllllllllllllllll}210 & 200 & 190 & 180 & 170 & 160 & 150 & 140 & 130 & 120 & 110 & 100 & 90 & 80 & 70 & 60 & 50 & 40 & 30 & 20 & 10 & 0 & -10\end{array}$ 
${ }^{1} \mathrm{H}$ NMR (400 MHz, $\left.\mathrm{CDCl}_{3}\right)$

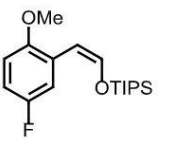

1ar

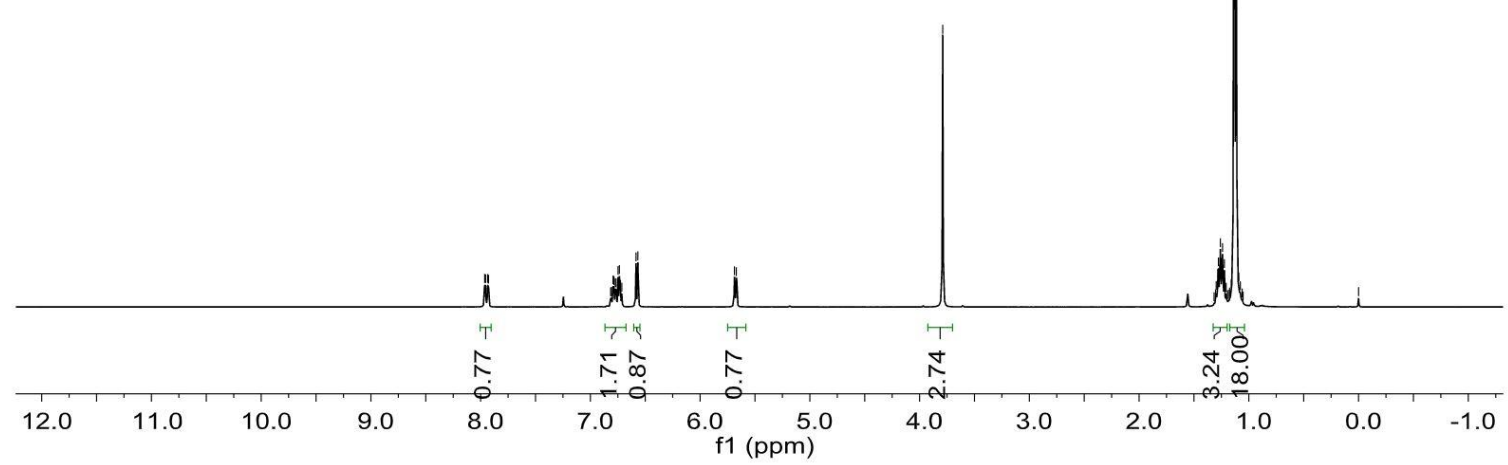

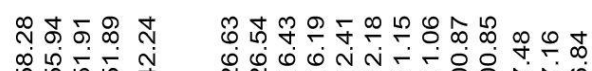

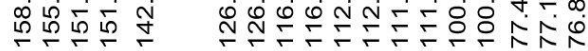

या

${ }^{13} \mathrm{C} \mathrm{NMR}\left(100 \mathrm{MHz}, \mathrm{CDCl}_{3}\right)$
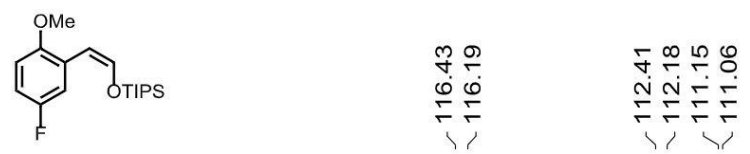

1ar

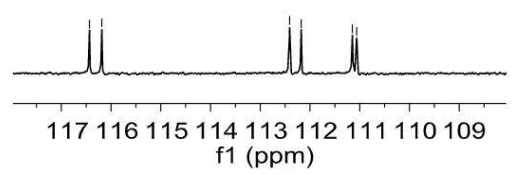

$$
\text { f1 (ppm) }
$$

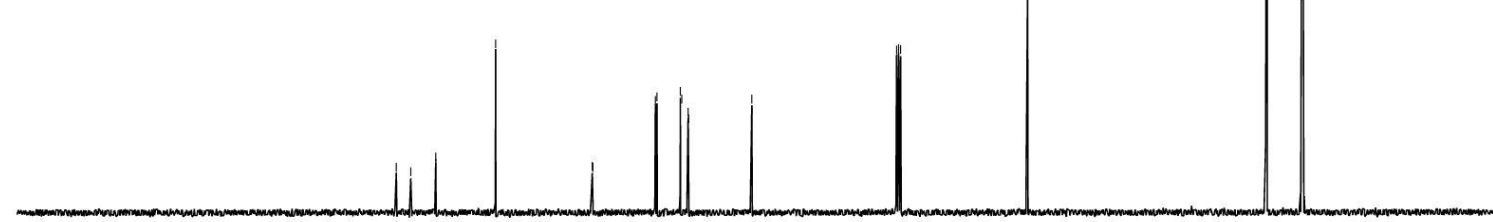

$\begin{array}{llllllllllllllllllllllllllll}210 & 200 & 190 & 180 & 170 & 160 & 150 & 140 & 130 & 120 & 110 & 100 & 90 & 80 & 70 & 60 & 50 & 40 & 30 & 20 & 10 & 0 & -10\end{array}$ 
${ }^{19} \mathrm{~F}$ NMR (377 MHz, $\left.\mathrm{CDCl}_{3}\right)$

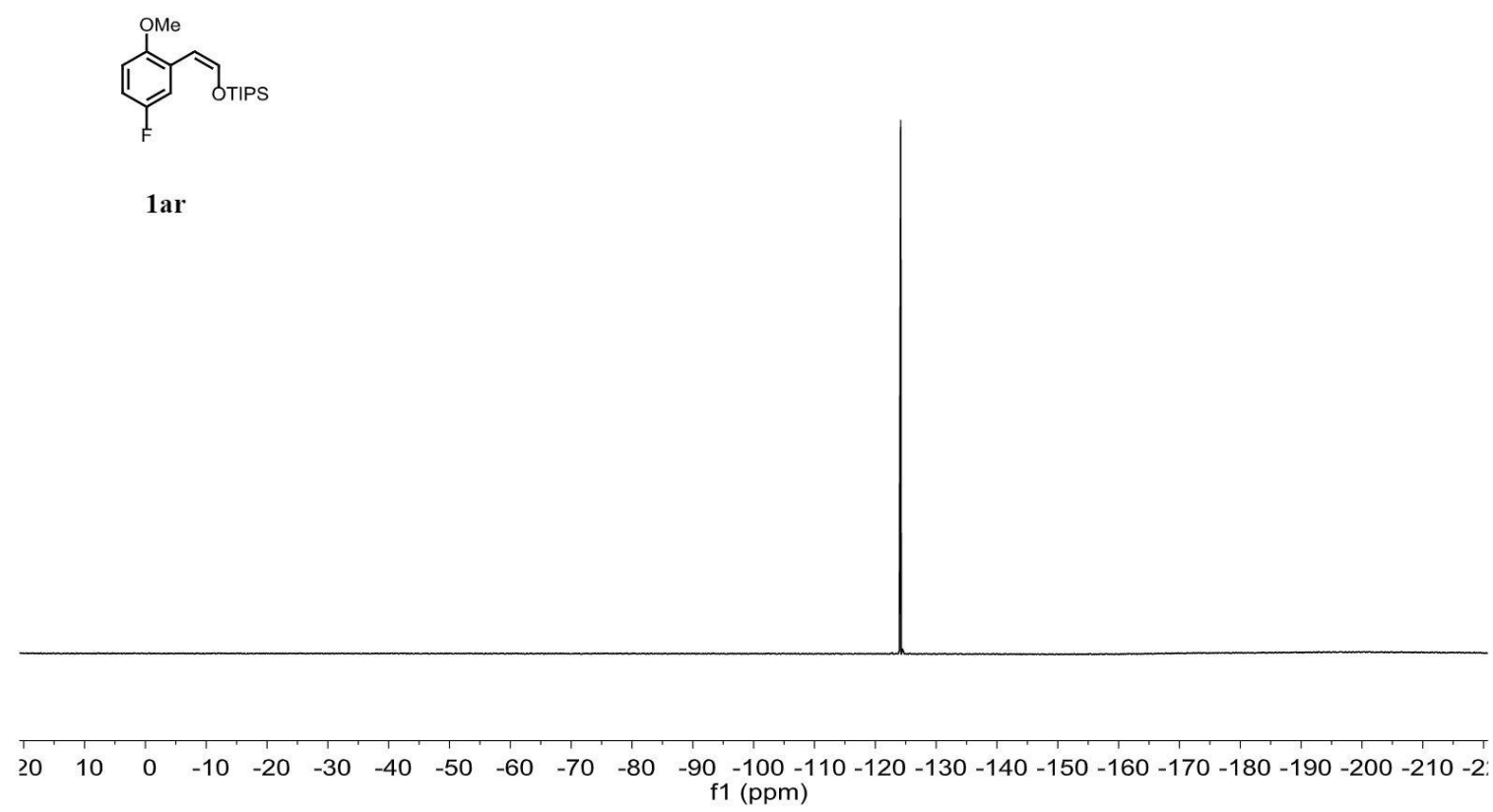


${ }^{1} \mathrm{H}$ NMR (400 MHz, $\mathrm{CDCl}_{3}$ )

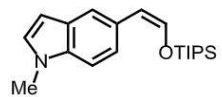

1 as

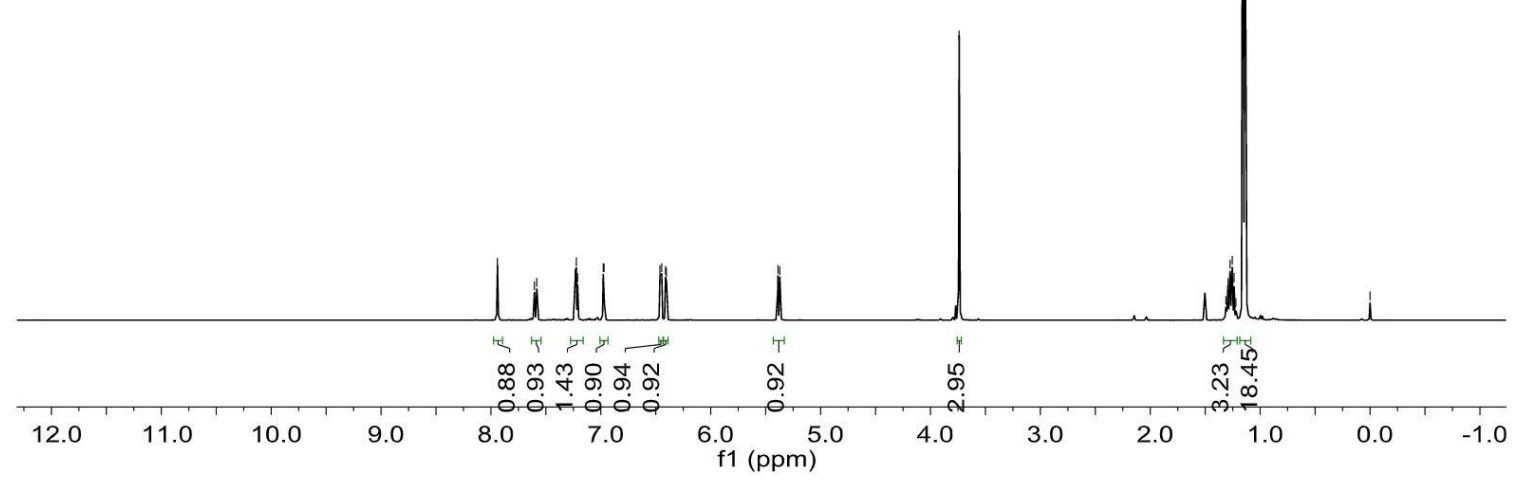

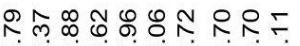

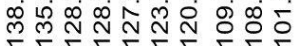

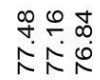

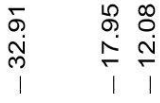

${ }^{13} \mathrm{C} \mathrm{NMR}\left(100 \mathrm{MHz}, \mathrm{CDCl}_{3}\right)$

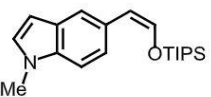

1as

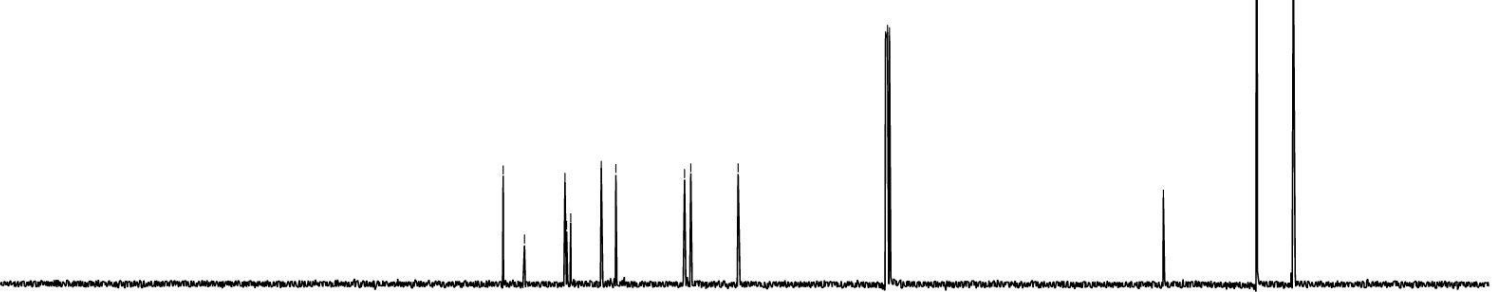

$\begin{array}{llllllllllllllllllllllll}210 & 200 & 190 & 180 & 170 & 160 & 150 & 140 & 130 & 120 & 110 & 100 & 90 & 80 & 70 & 60 & 50 & 40 & 30 & 20 & 10 & 0 & -10\end{array}$ $\mathrm{f} 1(\mathrm{ppm})$ 
${ }^{1} \mathrm{H}$ NMR $\left(400 \mathrm{MHz}, \mathrm{CDCl}_{3}\right)$

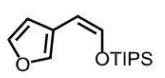

1at

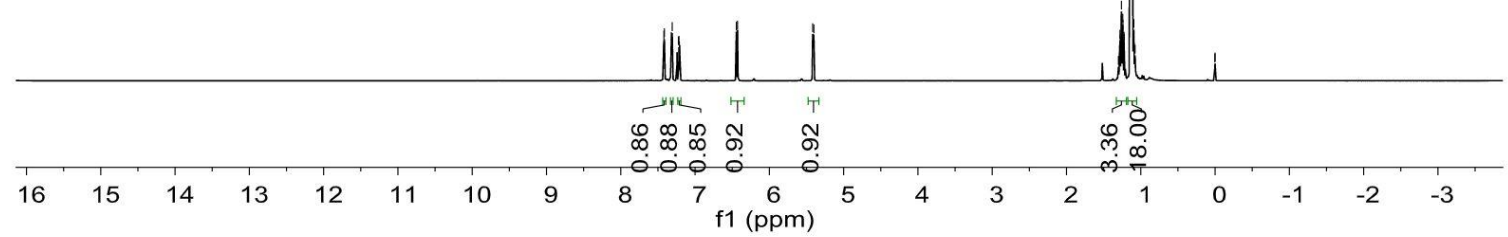

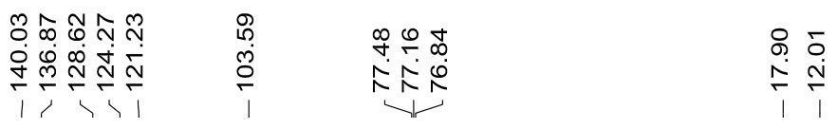

${ }^{13} \mathrm{C} \mathrm{NMR}\left(100 \mathrm{MHz}, \mathrm{CDCl}_{3}\right)$

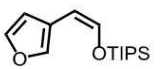

1at

\section{$\stackrel{\text { ó }}{\stackrel{i}{i}}$}

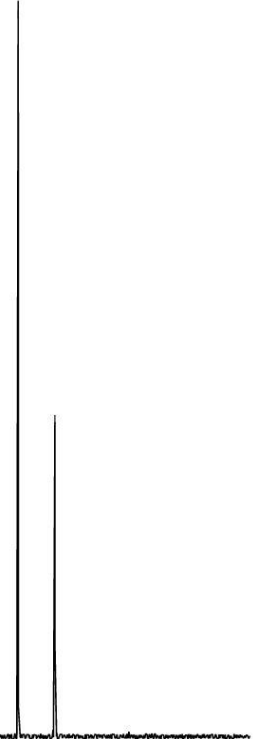

$\begin{array}{llllllllllllllllllllllllll}210 & 200 & 190 & 180 & 170 & 160 & 150 & 140 & 130 & 120 & 110 & 100 & 90 & 80 & 70 & 60 & 50 & 40 & 30 & 20 & 10 & 0 & -10\end{array}$ $\mathrm{f1}(\mathrm{ppm})$ 
${ }^{1} \mathrm{H}$ NMR (400 MHz, $\left.\mathrm{CDCl}_{3}\right)$

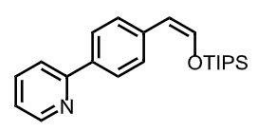

1au

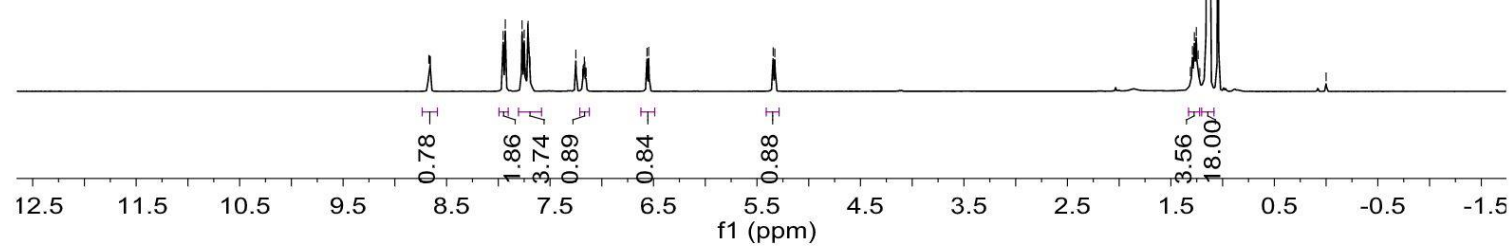

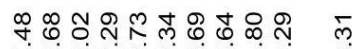

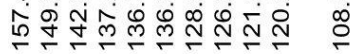

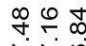

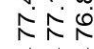

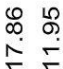

${ }^{13} \mathrm{C} \mathrm{NMR}\left(100 \mathrm{MHz}, \mathrm{CDCl}_{3}\right)$<smiles>COc1ccc(-c2ccccn2)cc1</smiles>

1 au

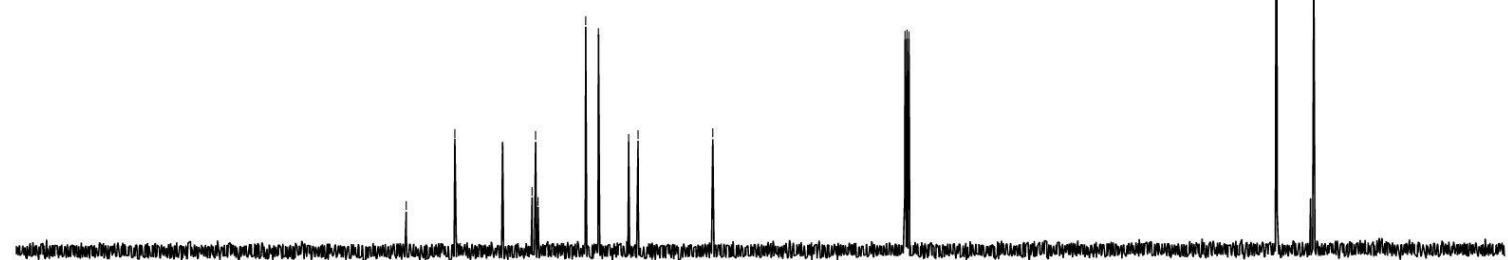

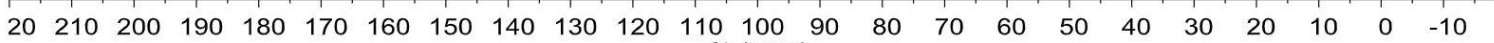
$\mathrm{f} 1(\mathrm{ppm})$ 
${ }^{1} \mathrm{H}$ NMR (400 MHz, $\mathrm{CDCl}_{3}$ )

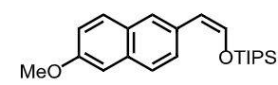

1av

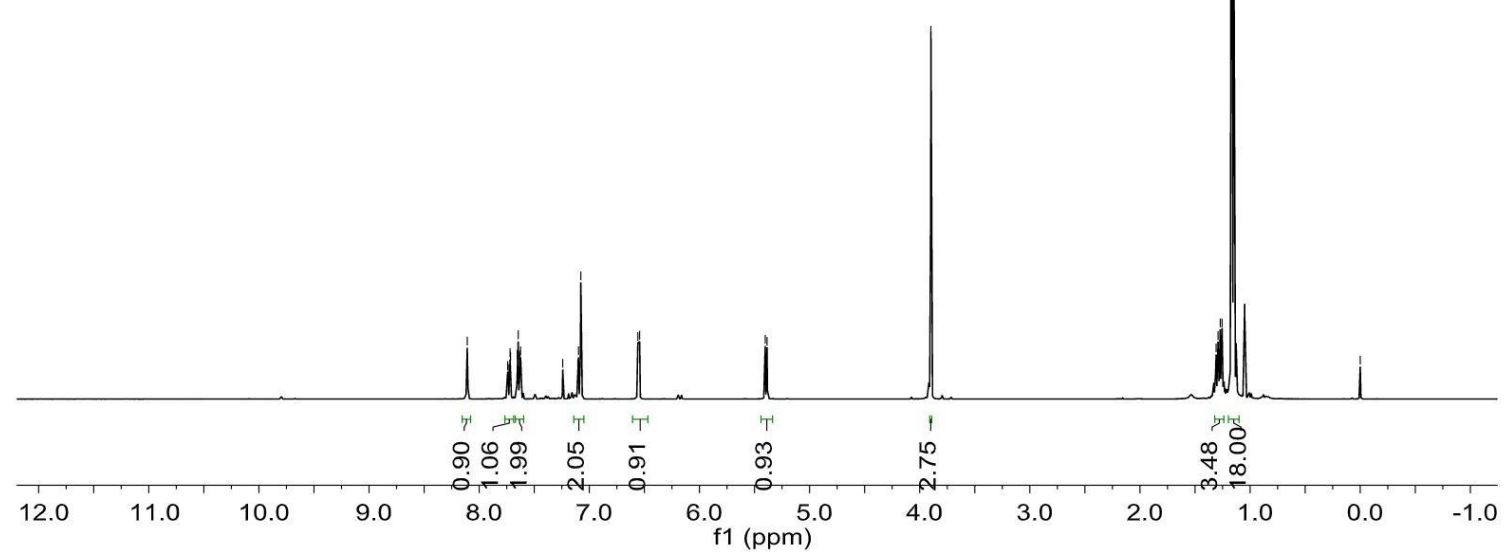

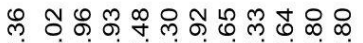

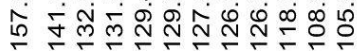

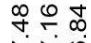

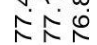

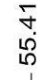

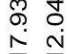

।

${ }^{13} \mathrm{C}$ NMR (100 MHz, $\mathrm{CDCl}_{3}$ )

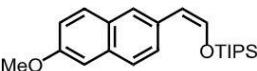

1av

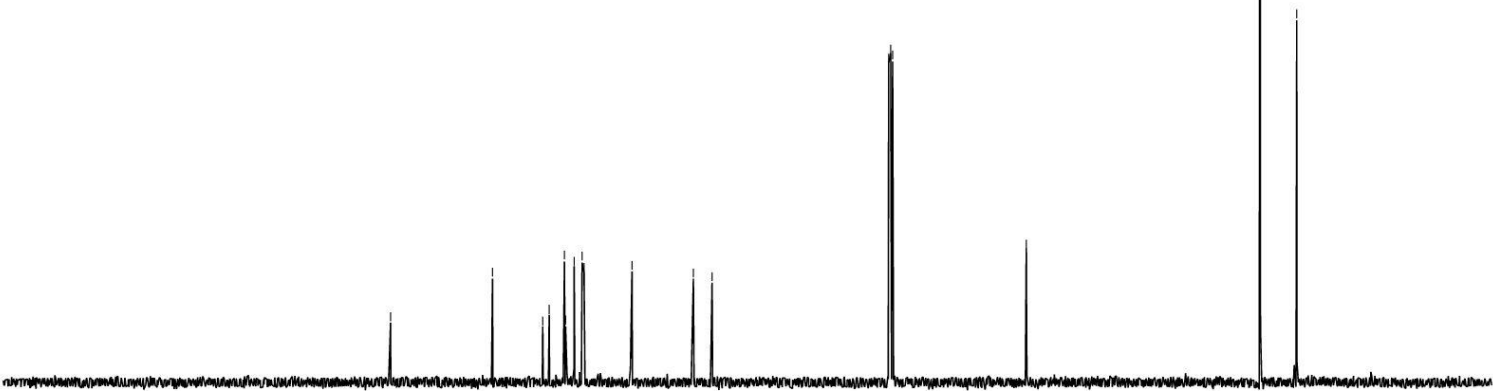

$\begin{array}{lllllllllllllllllllllll}210 & 200 & 190 & 180 & 170 & 160 & 150 & 140 & 130 & 120 & 110 & 100 & 90 & 80 & 70 & 60 & 50 & 40 & 30 & 20 & 10 & 0 & -10\end{array}$ $\mathrm{f} 1(\mathrm{ppm})$ 


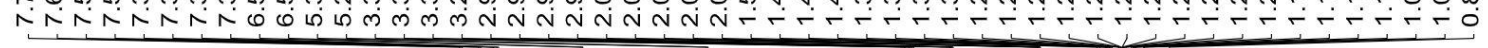
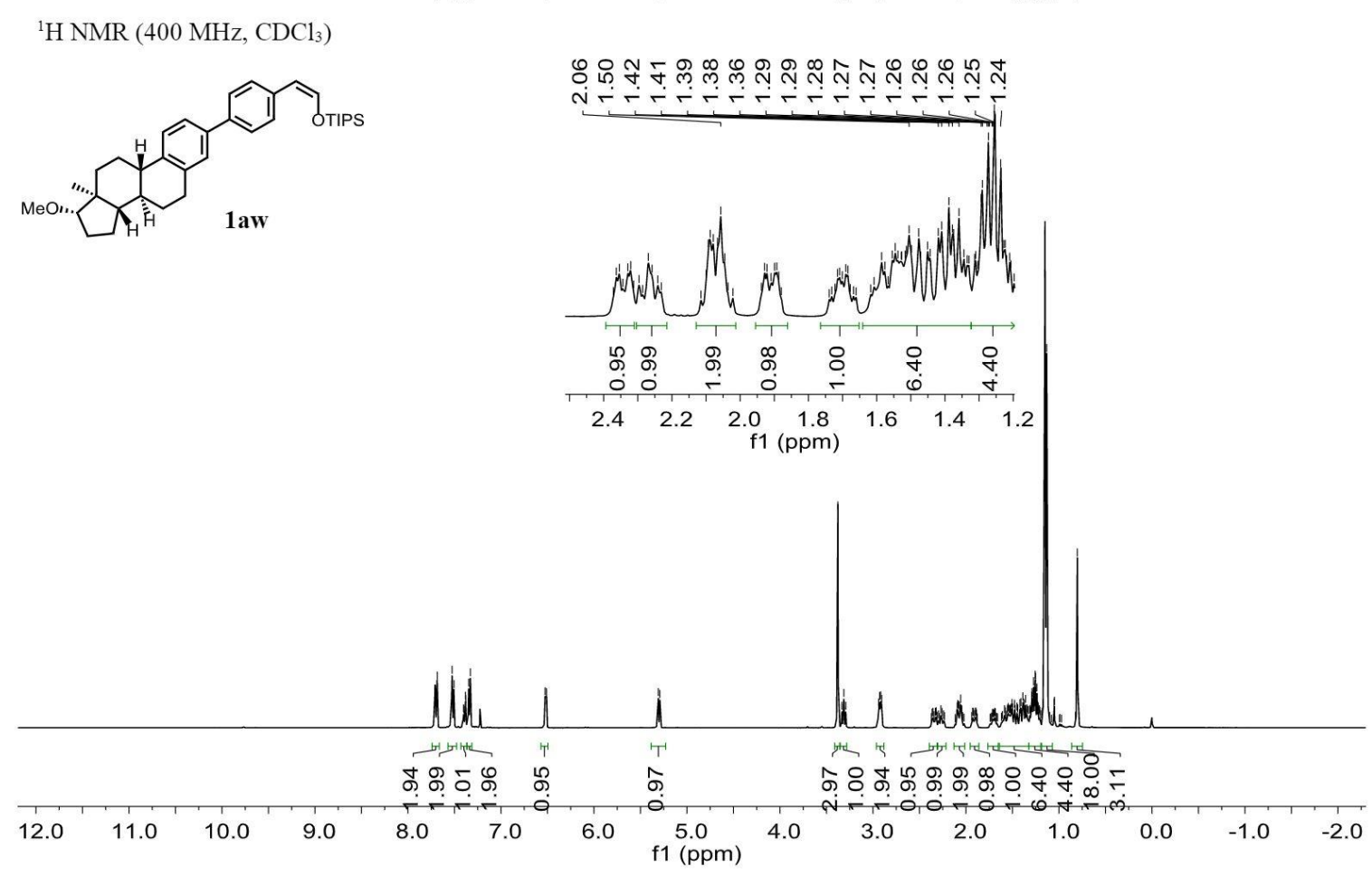

우

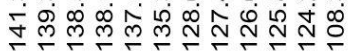

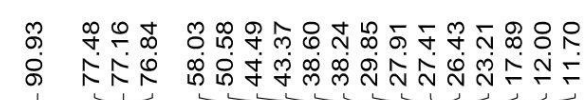

${ }^{13} \mathrm{C} \mathrm{NMR}\left(100 \mathrm{MHz}, \mathrm{CDCl}_{3}\right)$
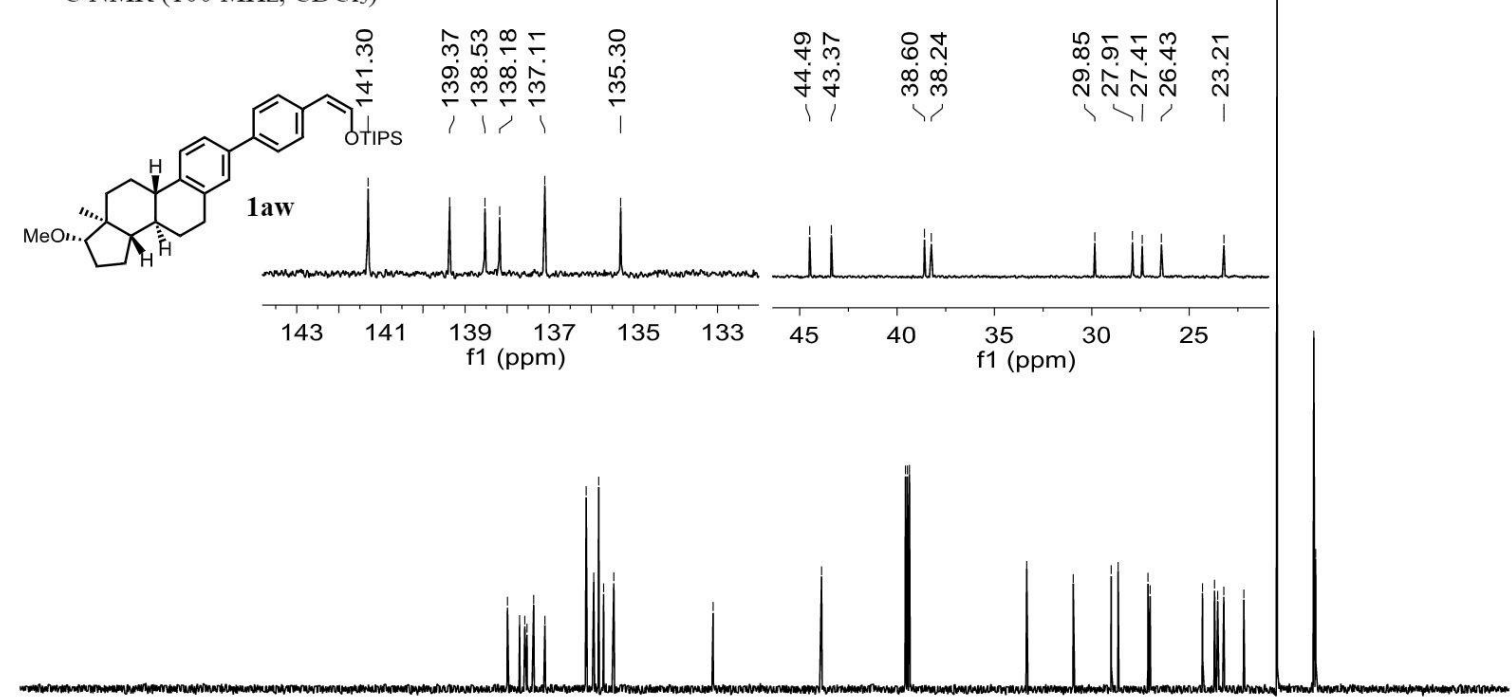

$\begin{array}{lllllllllllllllllllllll}210 & 200 & 190 & 180 & 170 & 160 & 150 & 140 & 130 & 120 & 110 & 100 & 90 & 80 & 70 & 60 & 50 & 40 & 30 & 20 & 10 & 0 & -10\end{array}$ f1 (ppm) 
负

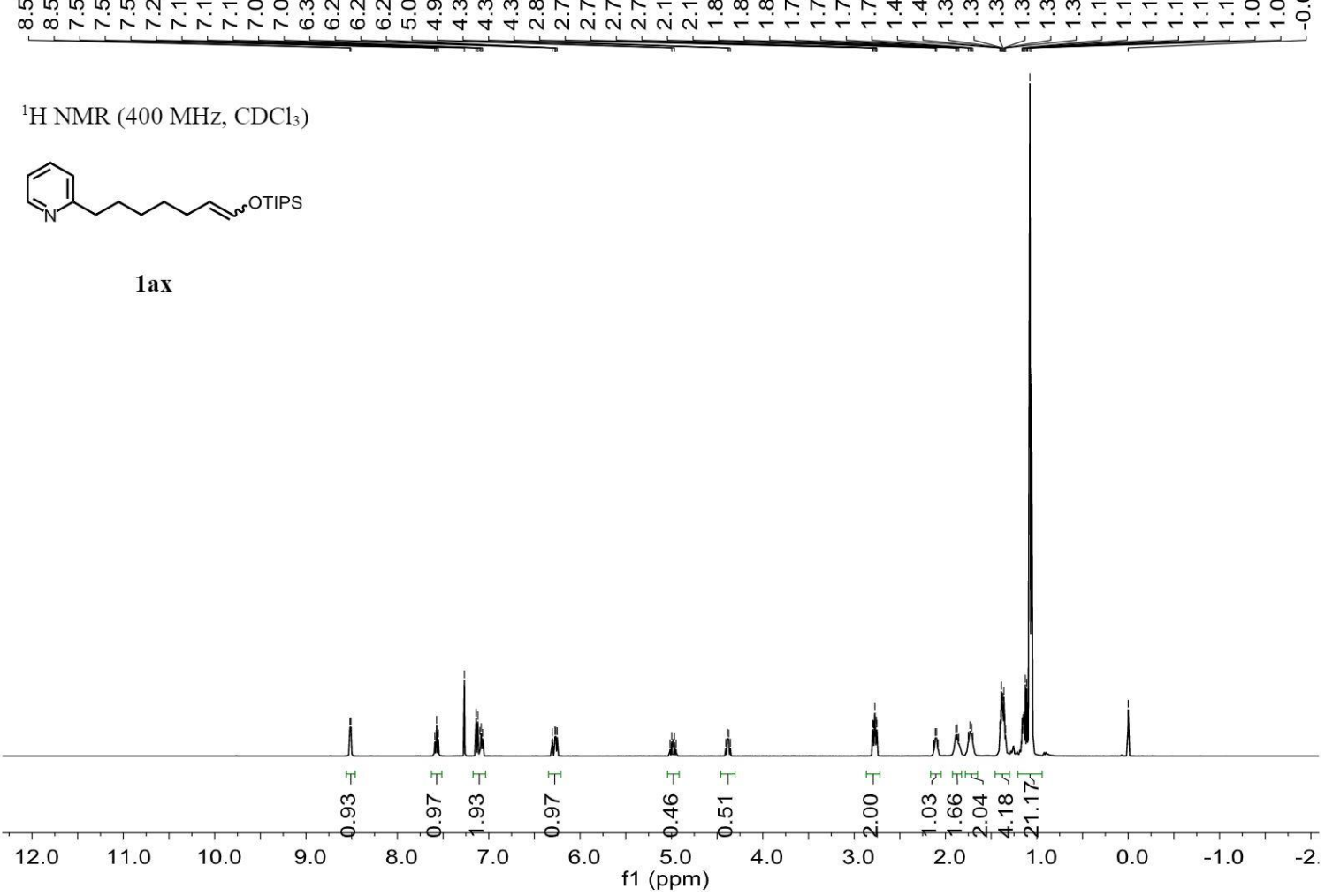

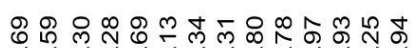

ปั่

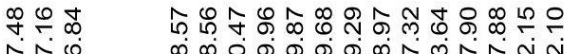

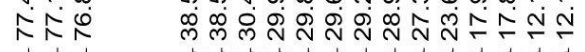

${ }^{13} \mathrm{H}$ NMR (100 MHz, $\left.\mathrm{CDCl}_{3}\right)$

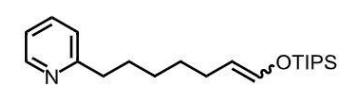

$1 \mathrm{ax}$

广ษ

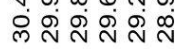

परों।
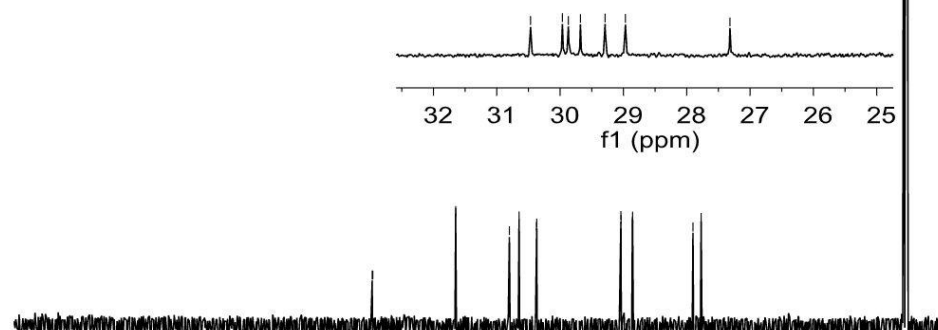

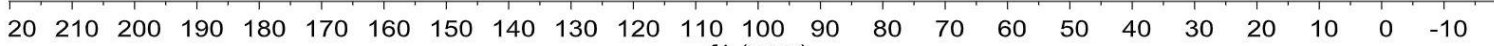
f1 (ppm) 


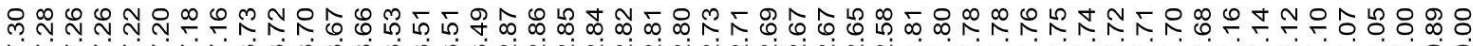

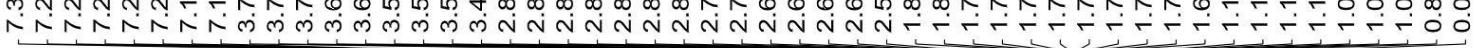

${ }^{1} \mathrm{H}$ NMR $\left(400 \mathrm{MHz}, \mathrm{CDCl}_{3}\right)$

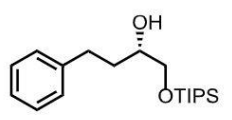

$3 \mathbf{a}$

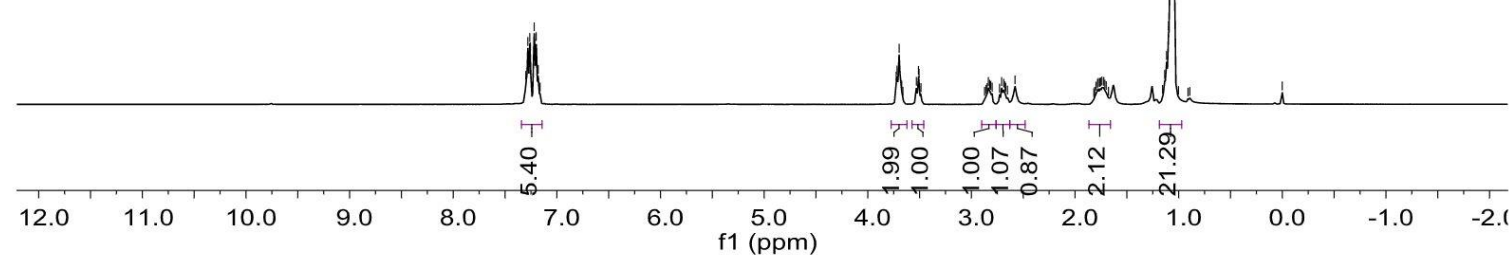

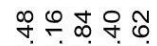

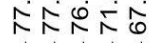

Y⿻

${ }^{13} \mathrm{C}$ NMR (100 MHz, $\left.\mathrm{CDCl}_{3}\right)$<smiles>O[Hg]CC(O)CCc1ccccc1</smiles>

3a

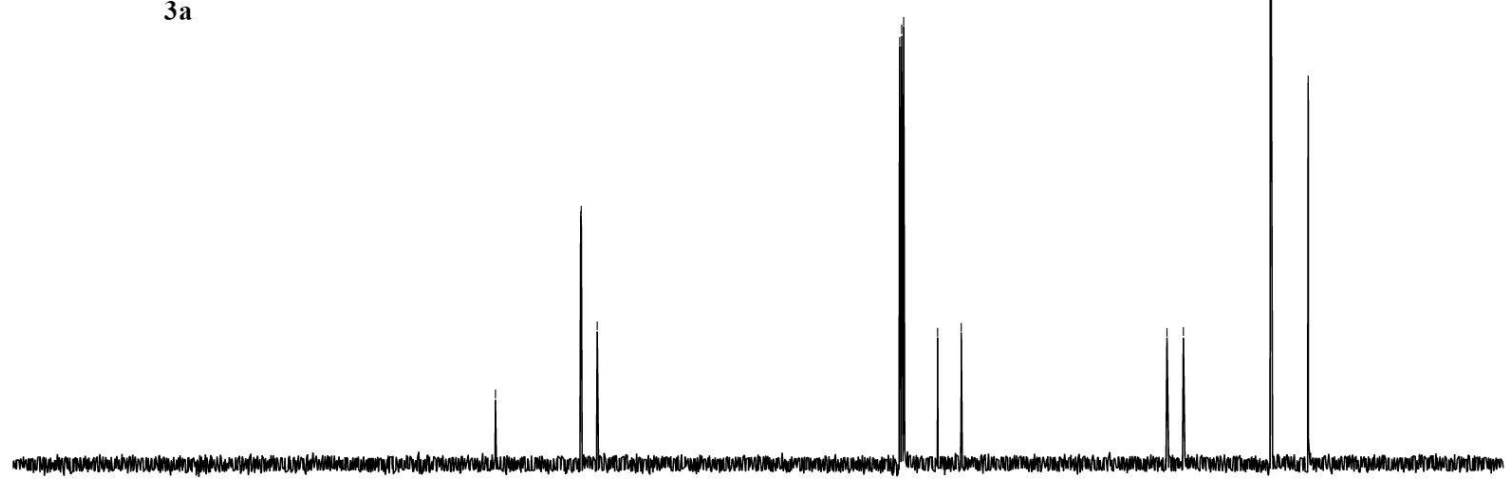

$\begin{array}{lllllllllllllllllllllll}210 & 200 & 190 & 180 & 170 & 160 & 150 & 140 & 130 & 120 & 110 & 100 & 90 & 80 & 70 & 60 & 50 & 40 & 30 & 20 & 10 & 0 & -10\end{array}$ f1 (ppm) 
${ }^{1} \mathrm{H}$ NMR $\left(400 \mathrm{MHz}, \mathrm{CDCl}_{3}\right)$

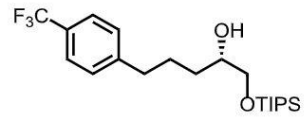

$3 b$

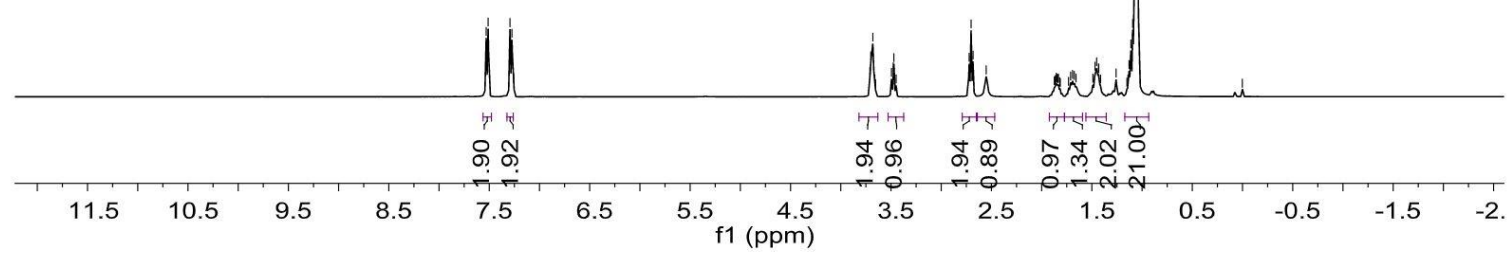

\section{ใน}

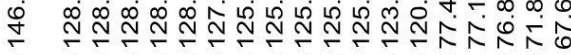

\section{œ \\ 色}

${ }^{13} \mathrm{C} \mathrm{NMR}\left(100 \mathrm{MHz}, \mathrm{CDCl}_{3}\right)$

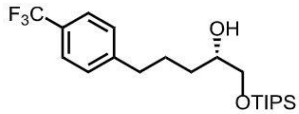

3b

DN

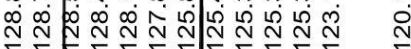
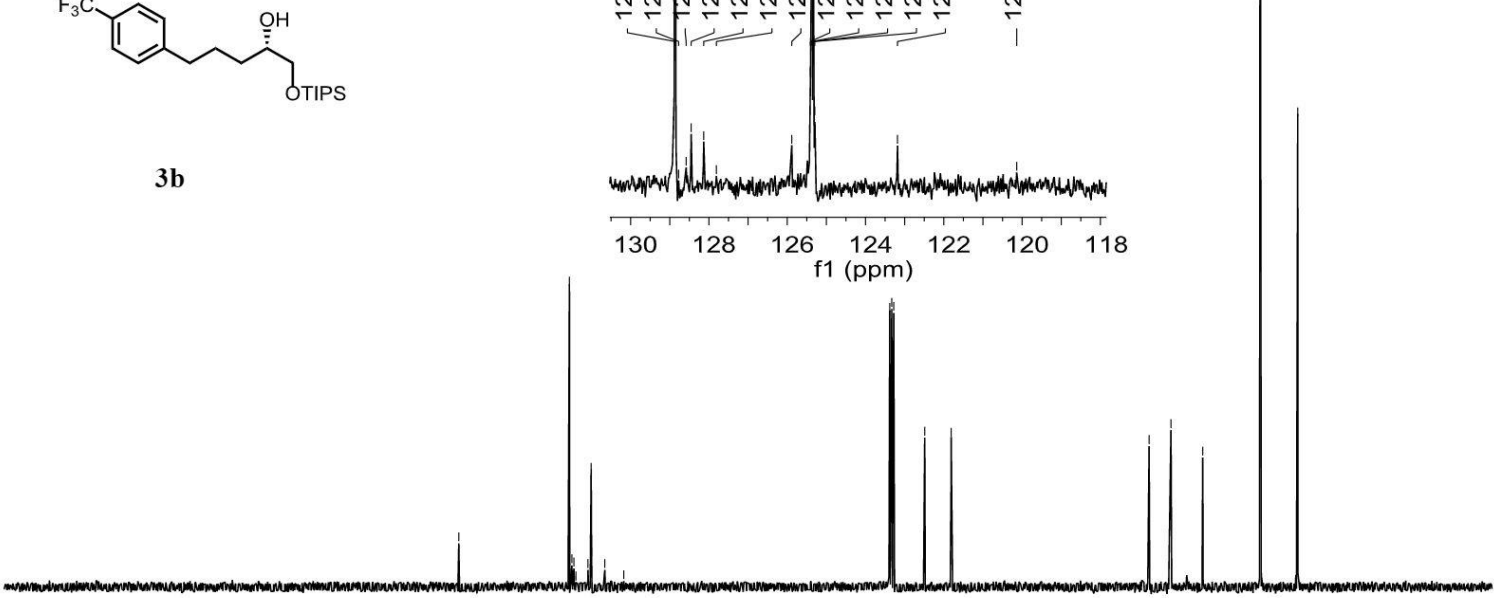

$\begin{array}{lllllllllllllllllllllll}210 & 200 & 190 & 180 & 170 & 160 & 150 & 140 & 130 & 120 & 110 & 100 & 90 & 80 & 70 & 60 & 50 & 40 & 30 & 20 & 10 & 0 & -10\end{array}$ f1 $(\mathrm{ppm})$ 
${ }^{19} \mathrm{~F}$ NMR (377 MHz, $\left.\mathrm{CDCl}_{3}\right)$

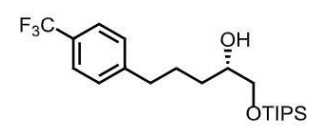

$3 \mathbf{b}$

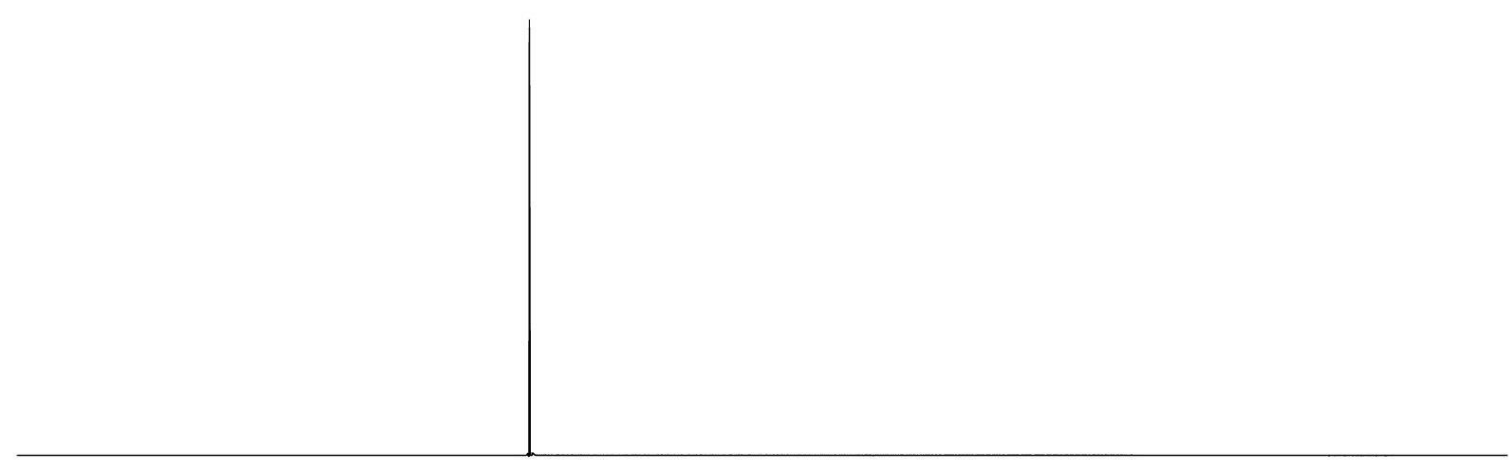

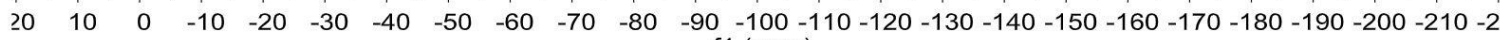
f1 (ppm) 


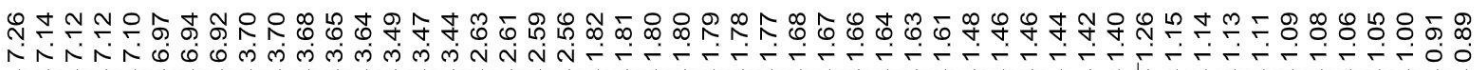

${ }^{1} \mathrm{H}$ NMR $\left(400 \mathrm{MHz}, \mathrm{CDCl}_{3}\right)$

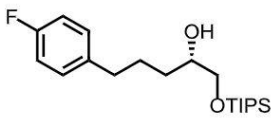

$3 c$

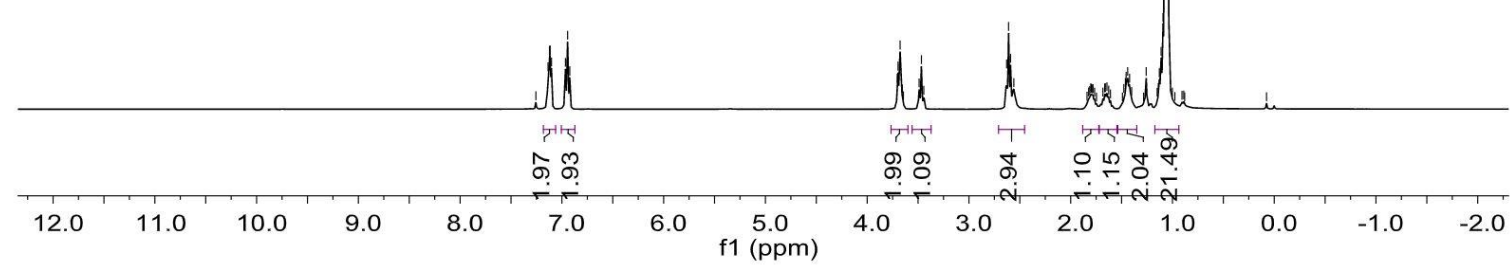

\begin{tabular}{|c|c|c|c|}
\hline 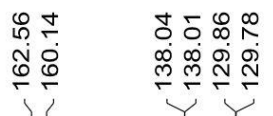 & 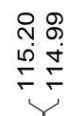 & 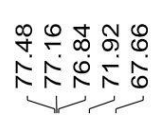 & 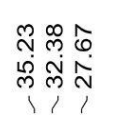 \\
\hline
\end{tabular}

${ }^{13} \mathrm{C}$ NMR $\left(100 \mathrm{MHz}, \mathrm{CDCl}_{3}\right)$

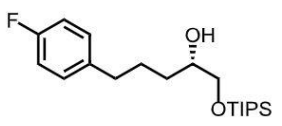

$3 c$

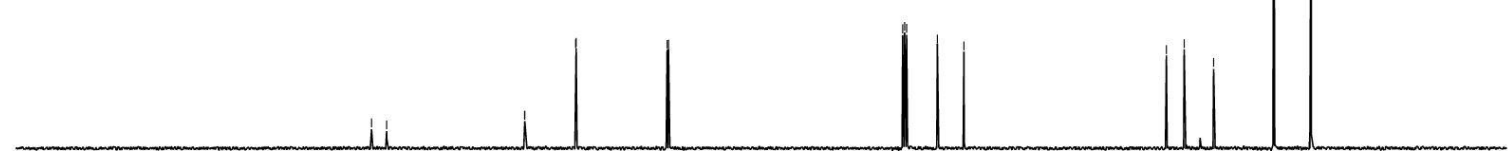

$\begin{array}{lllllllllllllllllllllll}210 & 200 & 190 & 180 & 170 & 160 & 150 & 140 & 130 & 120 & 110 & 100 & 90 & 80 & 70 & 60 & 50 & 40 & 30 & 20 & 10 & 0 & -10\end{array}$ f1 (ppm) 
${ }^{19} \mathrm{~F}$ NMR $\left(377 \mathrm{MHz}, \mathrm{CDCl}_{3}\right)$

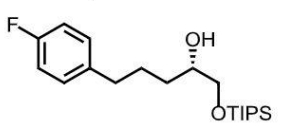

$3 c$

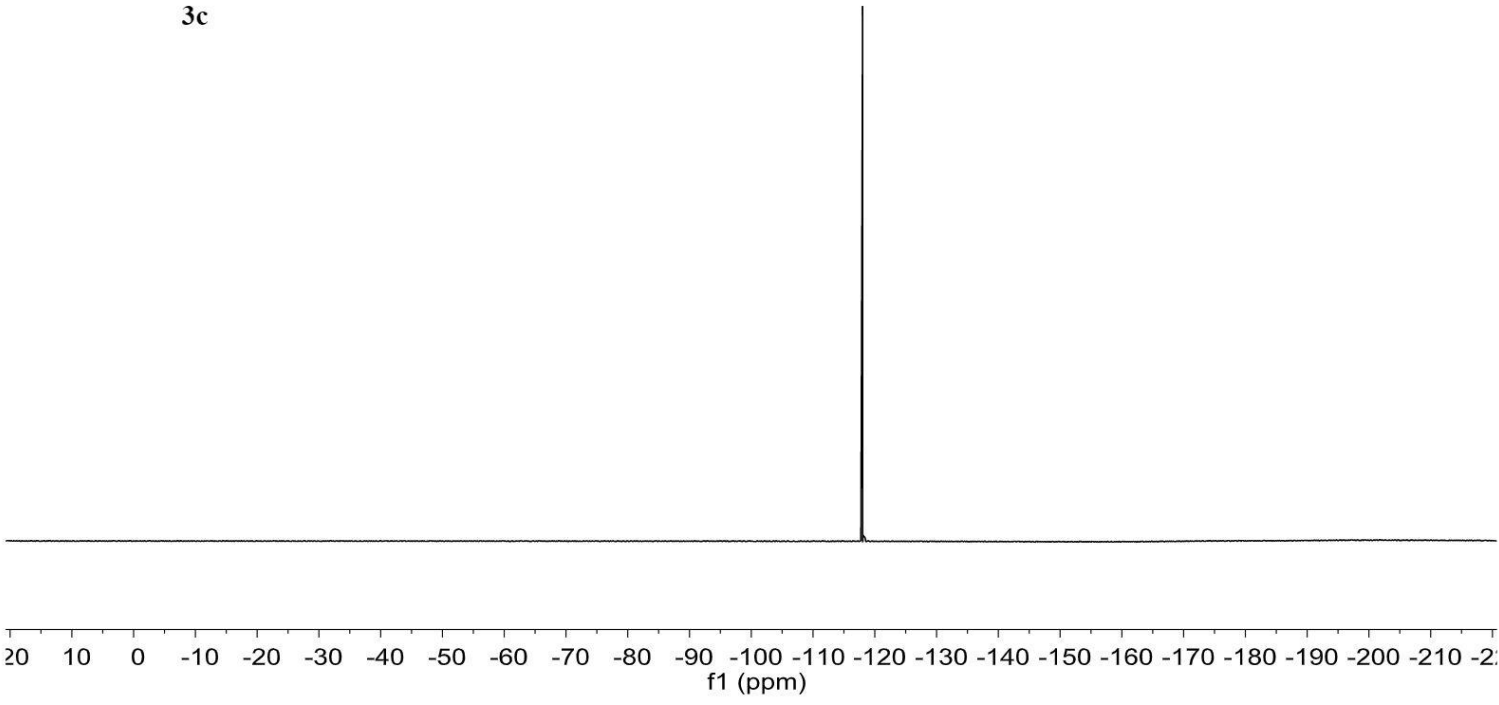




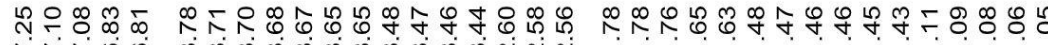
तिं

${ }^{1} \mathrm{H}$ NMR $\left(400 \mathrm{MHz}, \mathrm{CDCl}_{3}\right)$

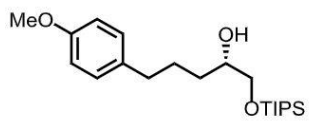

$3 d$

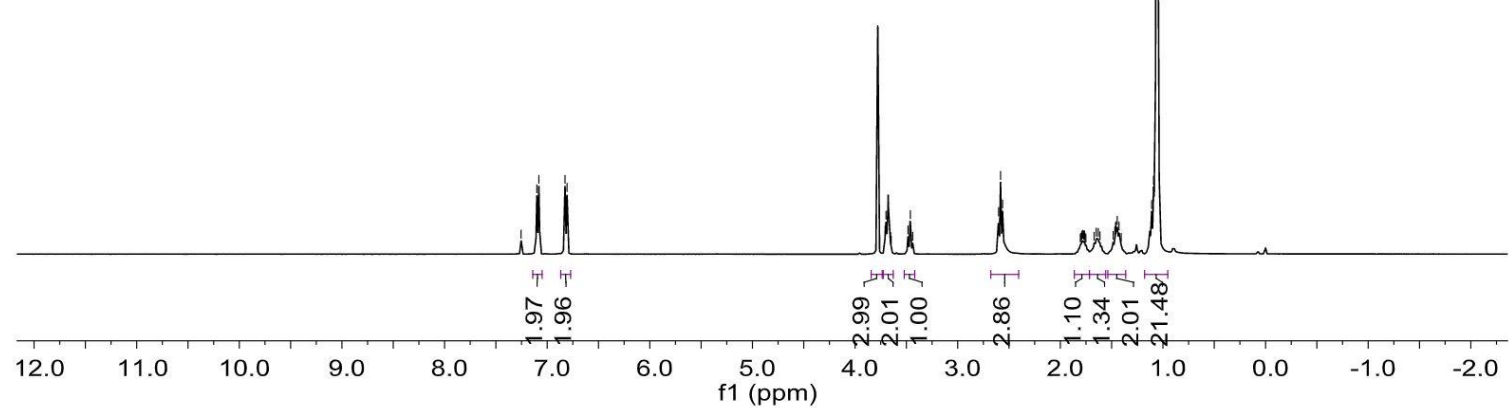

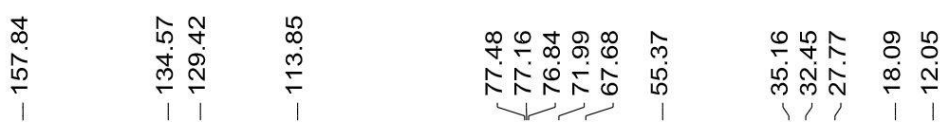

${ }^{13} \mathrm{C}$ NMR $\left(100 \mathrm{MHz}, \mathrm{CDCl}_{3}\right)$

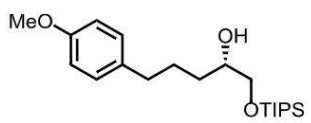

$3 d$

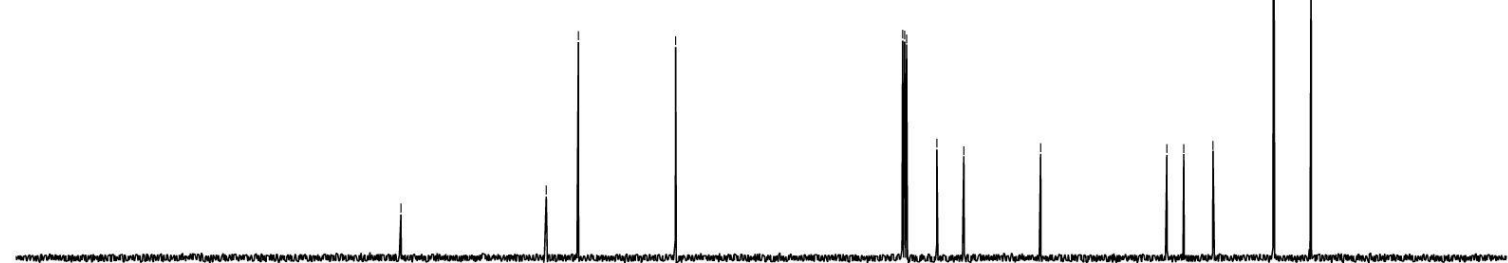

$\begin{array}{lllllllllllllllllllllll}210 & 200 & 190 & 180 & 170 & 160 & 150 & 140 & 130 & 120 & 110 & 100 & 90 & 80 & 70 & 60 & 50 & 40 & 30 & 20 & 10 & 0 & -10\end{array}$ f1 $(\mathrm{ppm})$ 


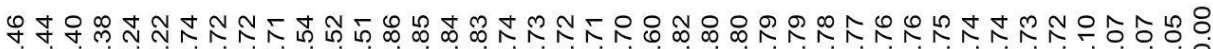

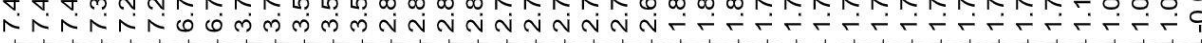

${ }^{1} \mathrm{H}$ NMR (400 MHz, $\left.\mathrm{CDCl}_{3}\right)$

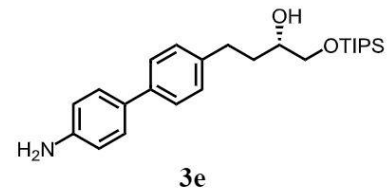

$3 e$

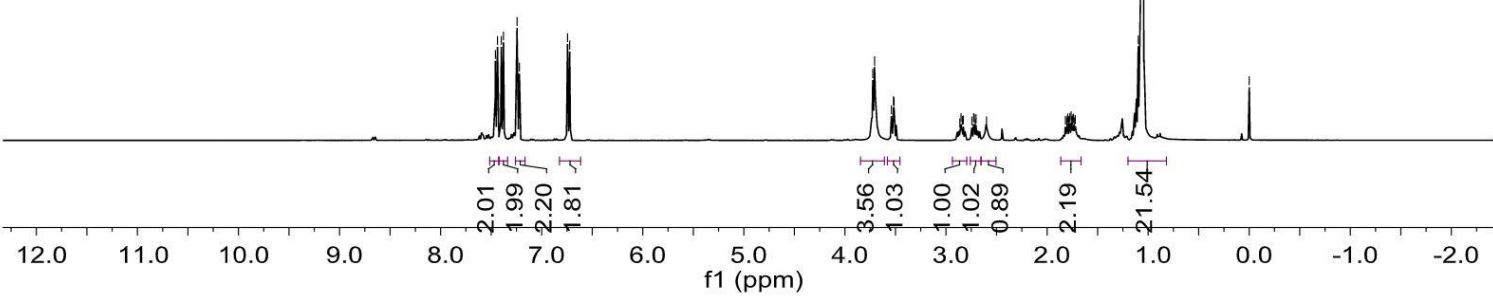

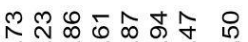

b

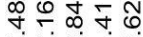

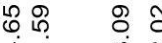

-

रト்̃

लेल क्ष

${ }^{13} \mathrm{C} \mathrm{NMR}\left(100 \mathrm{MHz}, \mathrm{CDCl}_{3}\right)$

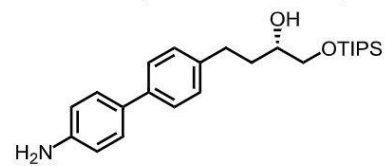

$3 \mathrm{e}$

$\begin{array}{lllllllllllllllllllllll}210 & 200 & 190 & 180 & 170 & 160 & 150 & 140 & 130 & 120 & \begin{array}{c}110 \\ \mathrm{f} 1\end{array}(\mathrm{ppm}) & 100 & 90 & 80 & 70 & 60 & 50 & 40 & 30 & 20 & 10 & 0 & -10\end{array}$ 


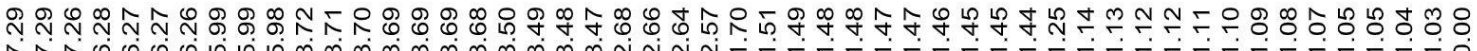

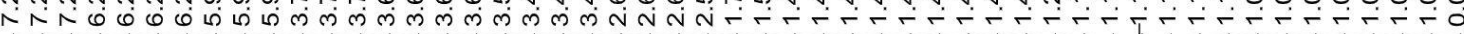

${ }^{1} \mathrm{H}$ NMR $\left(400 \mathrm{MHz}, \mathrm{CDCl}_{3}\right)$

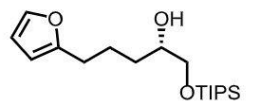

3f

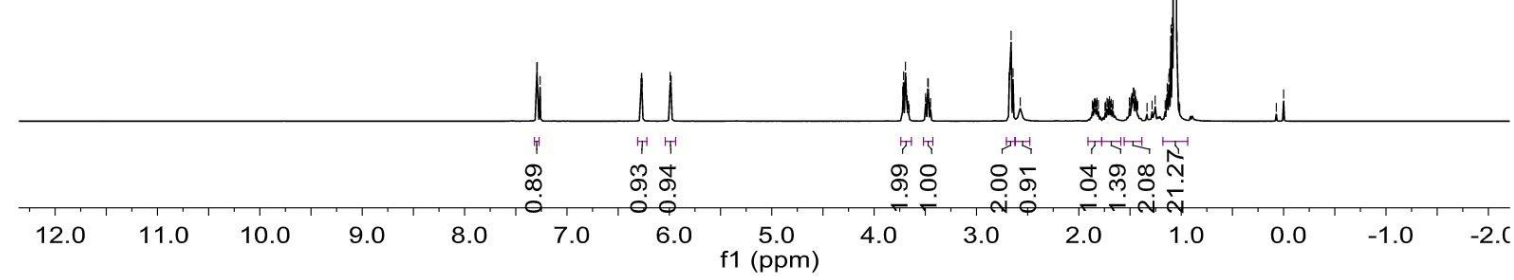

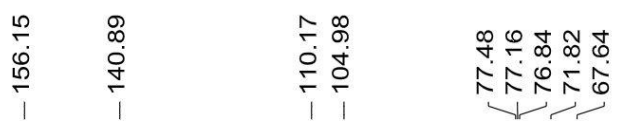

${ }^{13} \mathrm{C}$ NMR $\left(100 \mathrm{MHz}, \mathrm{CDCl}_{3}\right)$

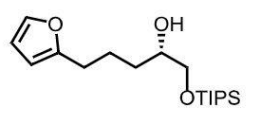

3f

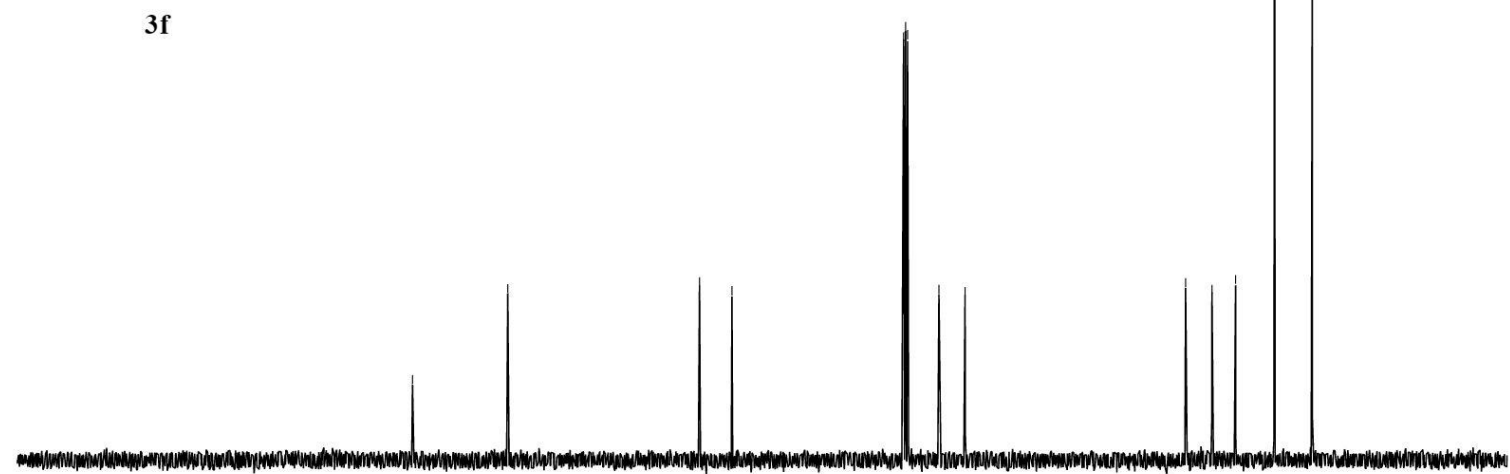

$\begin{array}{lllllllllllllllllllllll}210 & 200 & 190 & 180 & 170 & 160 & 150 & 140 & 130 & 120 & 110 & 100 & 90 & 80 & 70 & 60 & 50 & 40 & 30 & 20 & 10 & 0 & -10\end{array}$ 


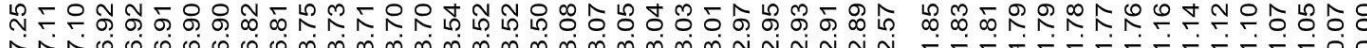

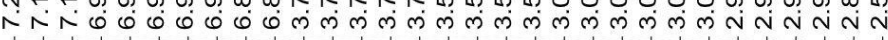

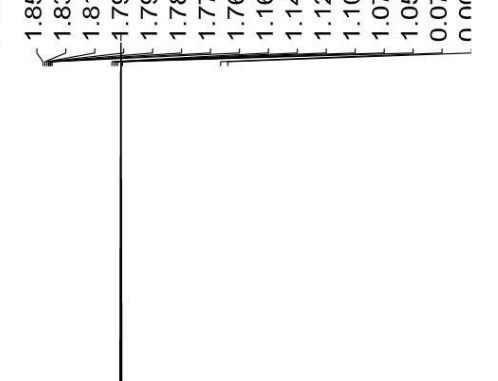

${ }^{1} \mathrm{H} \mathrm{NMR}\left(400 \mathrm{MHz}, \mathrm{CDCl}_{3}\right)$

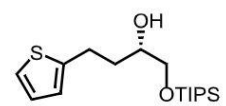

$3 g$

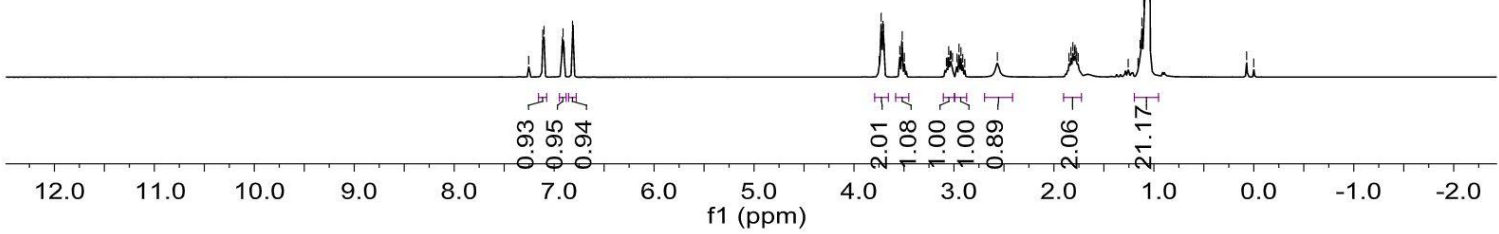

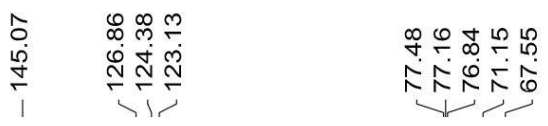

${ }^{13} \mathrm{C}$ NMR $\left(100 \mathrm{MHz}, \mathrm{CDCl}_{3}\right)$<smiles>OCC(O)CCc1cccs1</smiles>

$3 g$

$\begin{array}{llllllllllll}210 & 200 & 190 & 180 & 170 & 160 & 150 & 140 & 130 & 120 & \begin{array}{c}110 \\ \mathrm{f} 1(\mathrm{ppm})\end{array}\end{array}$ 


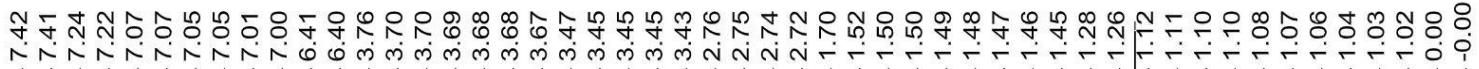

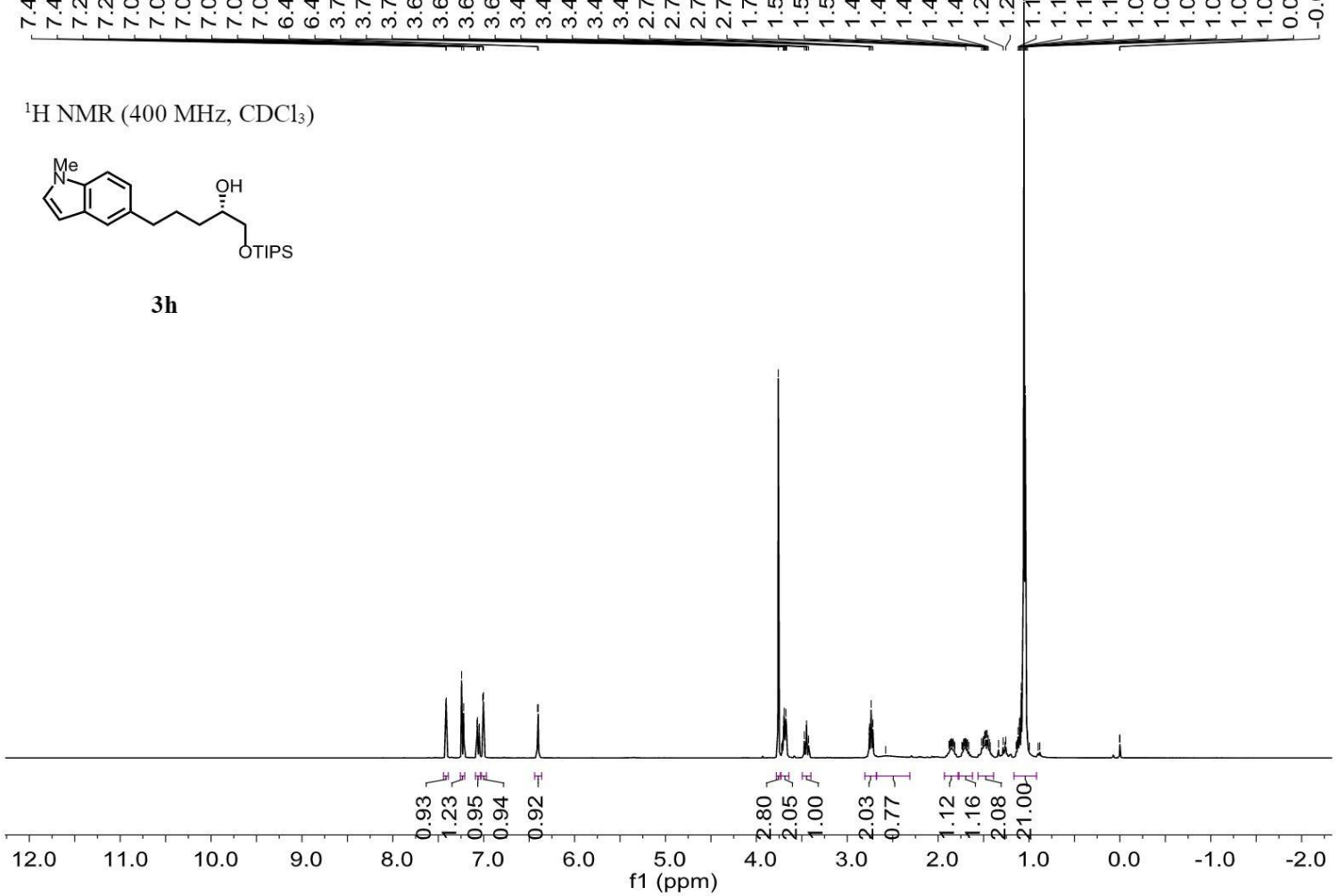

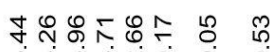

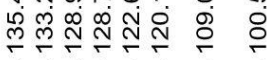

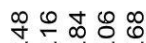

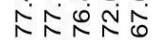

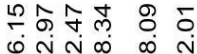

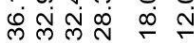

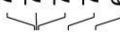

${ }^{13} \mathrm{C} \mathrm{NMR}\left(100 \mathrm{MHz}, \mathrm{CDCl}_{3}\right)$

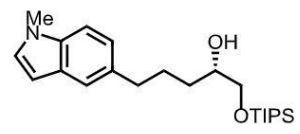

$3 \mathrm{~h}$

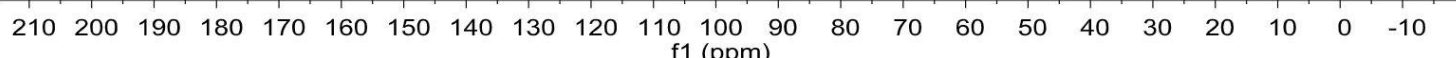


${ }^{1} \mathrm{H}$ NMR $\left(400 \mathrm{MHz}, \mathrm{CDCl}_{3}\right)$

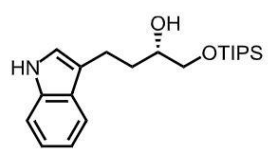

$3 \mathrm{i}$

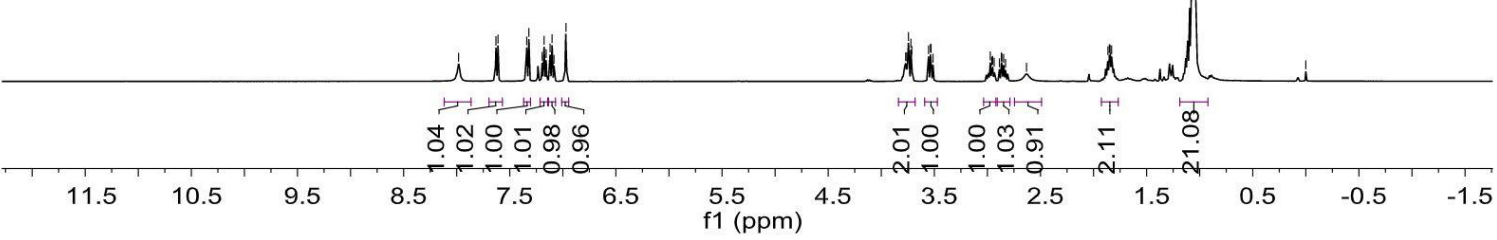

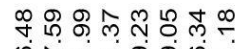

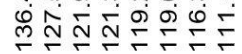

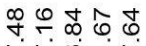

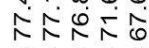

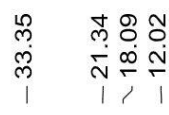

${ }^{13} \mathrm{C}$ NMR $\left(100 \mathrm{MHz}, \mathrm{CDCl}_{3}\right)$

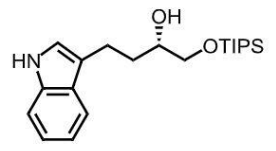

$3 \mathbf{i}$

$\begin{array}{llllllllllllllllllllll}210 & 200 & 190 & 180 & 170 & 160 & 150 & 140 & 130 & 120 & \begin{array}{c}110 \\ \mathrm{f} 1\end{array}(\mathrm{ppm}) & 90 & 80 & 70 & 60 & 50 & 40 & 30 & 20 & 10 & 0 & -10\end{array}$ 
${ }^{1} \mathrm{H}$ NMR $\left(400 \mathrm{MHz}, \mathrm{CDCl}_{3}\right)$

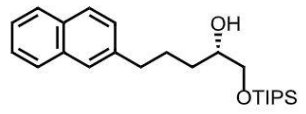

$3 \mathbf{j}$

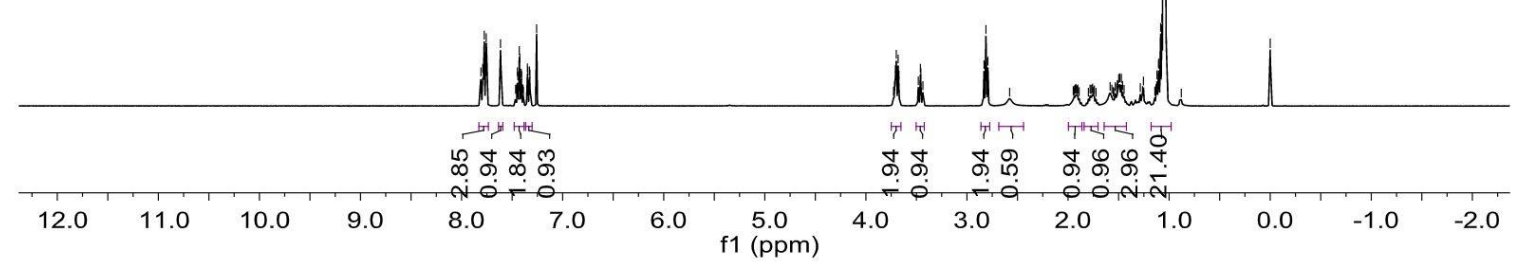

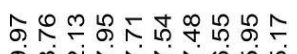

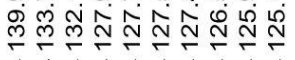

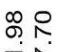

זे

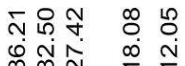

mim 110

${ }^{13} \mathrm{C}$ NMR (100 MHz, $\left.\mathrm{CDCl}_{3}\right)$

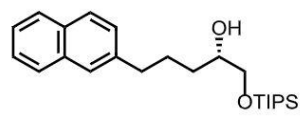

$3 \mathbf{j}$

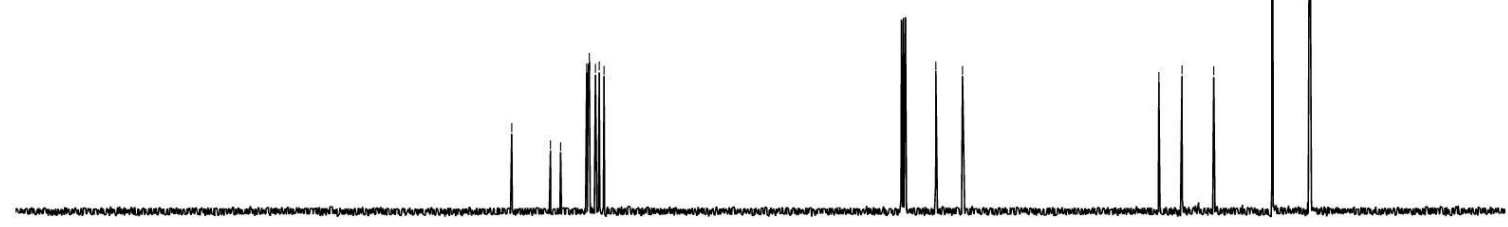

$\begin{array}{llllllllllllllllllllllll}210 & 200 & 190 & 180 & 170 & 160 & 150 & 140 & 130 & 120 & 110 & 100 & 90 & 80 & 70 & 60 & 50 & 40 & 30 & 20 & 10 & 0 & -10\end{array}$ $\mathrm{f1}(\mathrm{ppm})$ 


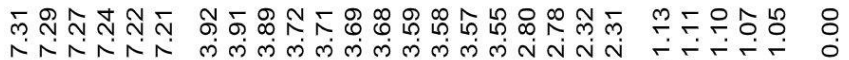

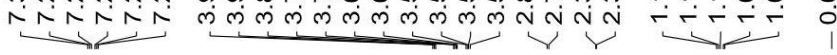

${ }^{1} \mathrm{H}$ NMR (400 MHz, $\left.\mathrm{CDCl}_{3}\right)$

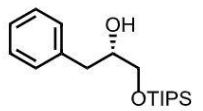

$3 k$

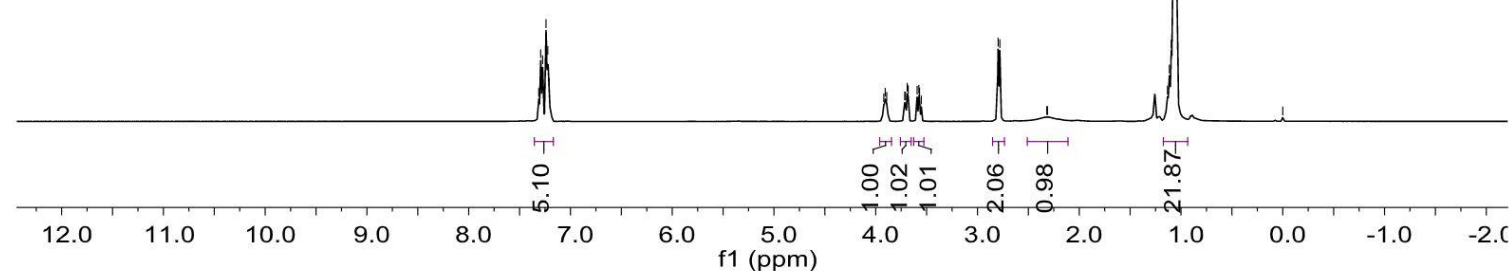

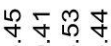

产 $\stackrel{\sim}{N} \stackrel{\sim}{N} \stackrel{0}{N}$

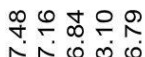

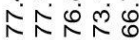

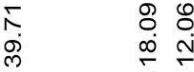

${ }^{13} \mathrm{C} \mathrm{NMR}\left(100 \mathrm{MHz}, \mathrm{CDCl}_{3}\right)$

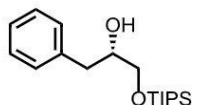

$3 \mathbf{k}$

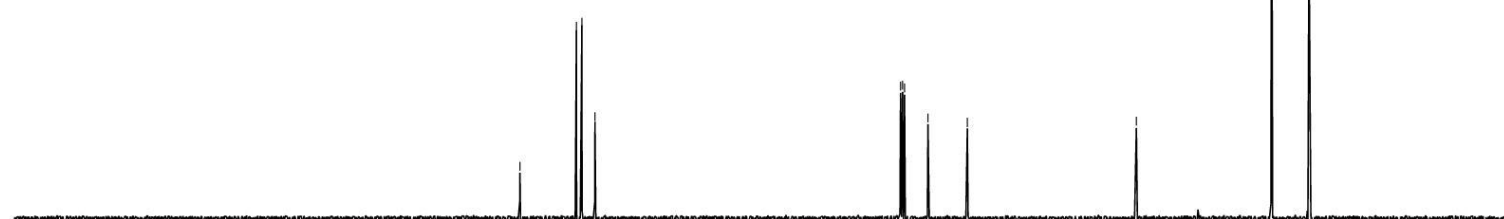

$\begin{array}{lllllllllllllllllllllll}210 & 200 & 190 & 180 & 170 & 160 & 150 & 140 & 130 & 120 & 110 & 100 & 90 & 80 & 70 & 60 & 50 & 40 & 30 & 20 & 10 & 0 & -10\end{array}$ f1 (ppm) 


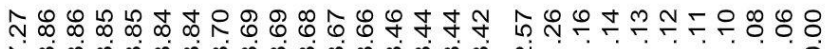

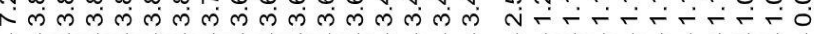

${ }^{1} \mathrm{H}$ NMR (400 MHz, $\left.\mathrm{CDCl}_{3}\right)$<smiles>C[C@@H](O)C[18OH]</smiles>

31

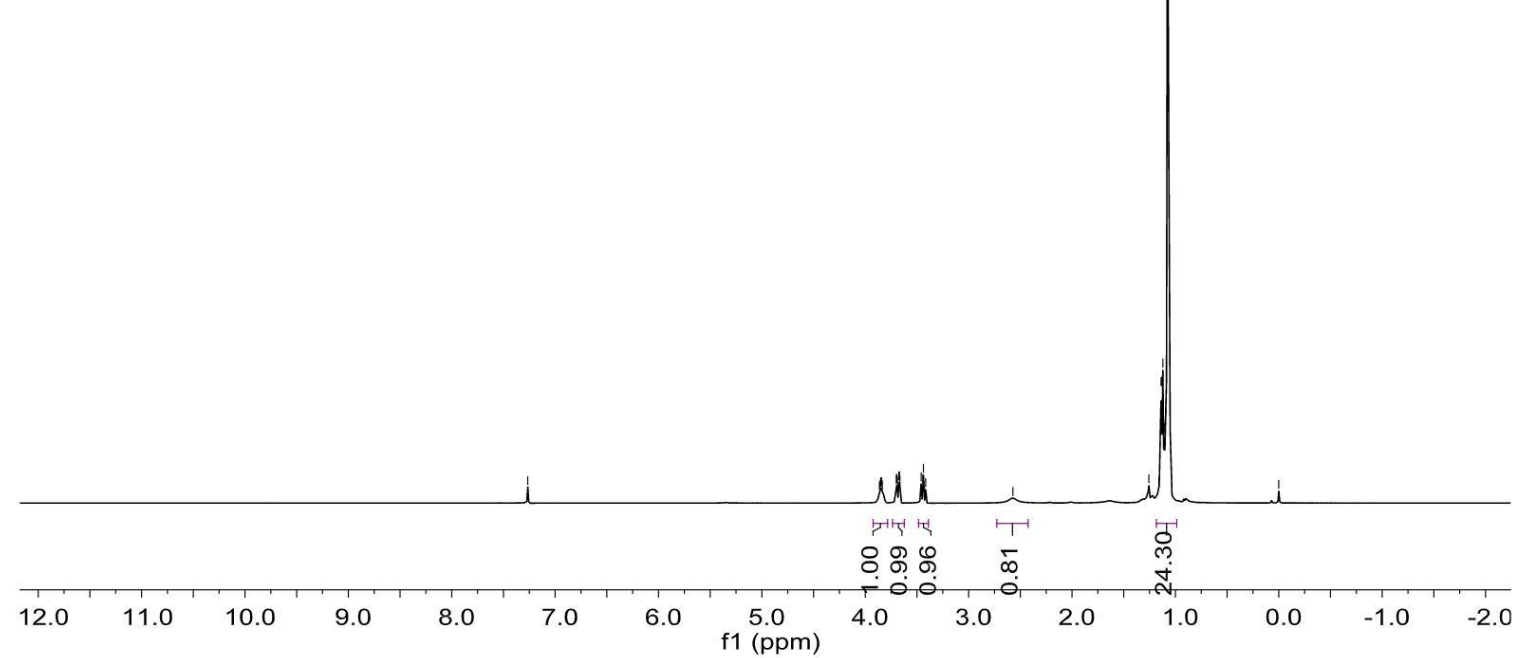

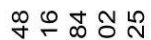

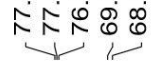

ํำ

$\stackrel{\infty}{\sim} \sim$

${ }^{13} \mathrm{C} \mathrm{NMR}\left(100 \mathrm{MHz}, \mathrm{CDCl}_{3}\right)$<smiles>C[C@@H](O)C[18OH]</smiles>

31

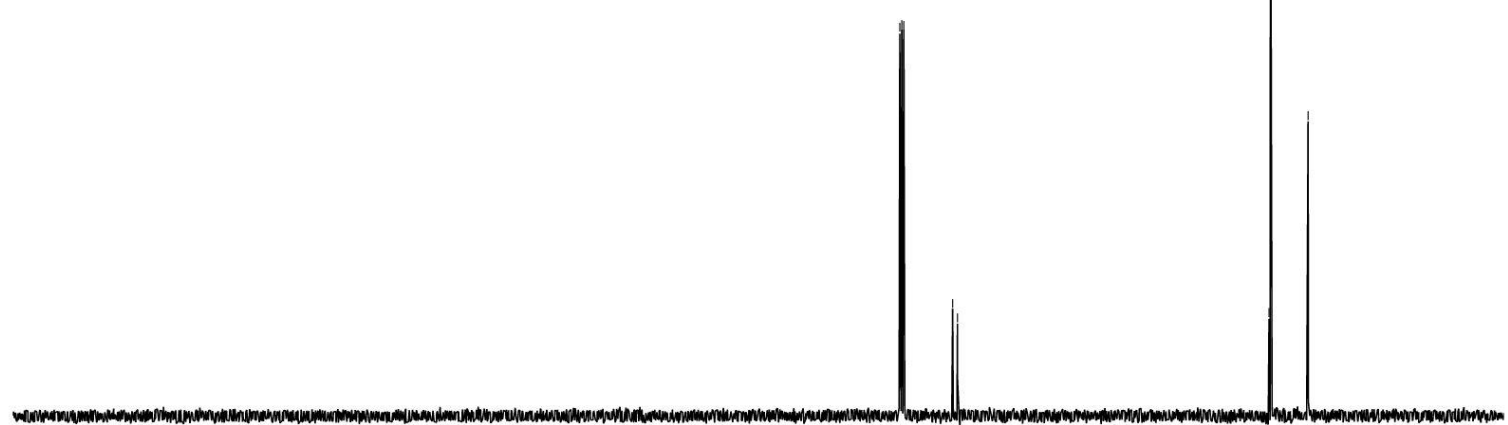

$\begin{array}{lllllllllllllllllllllll}210 & 200 & 190 & 180 & 170 & 160 & 150 & 140 & 130 & 120 & 110 & 100 & 90 & 80 & 70 & 60 & 50 & 40 & 30 & 20 & 10 & 0 & -10\end{array}$ f1 (ppm) 


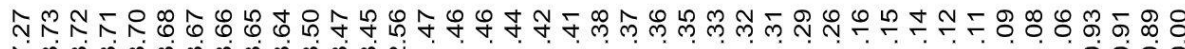

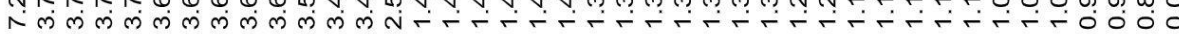

${ }^{1} \mathrm{H}$ NMR $\left(400 \mathrm{MHz}, \mathrm{CDCl}_{3}\right)$

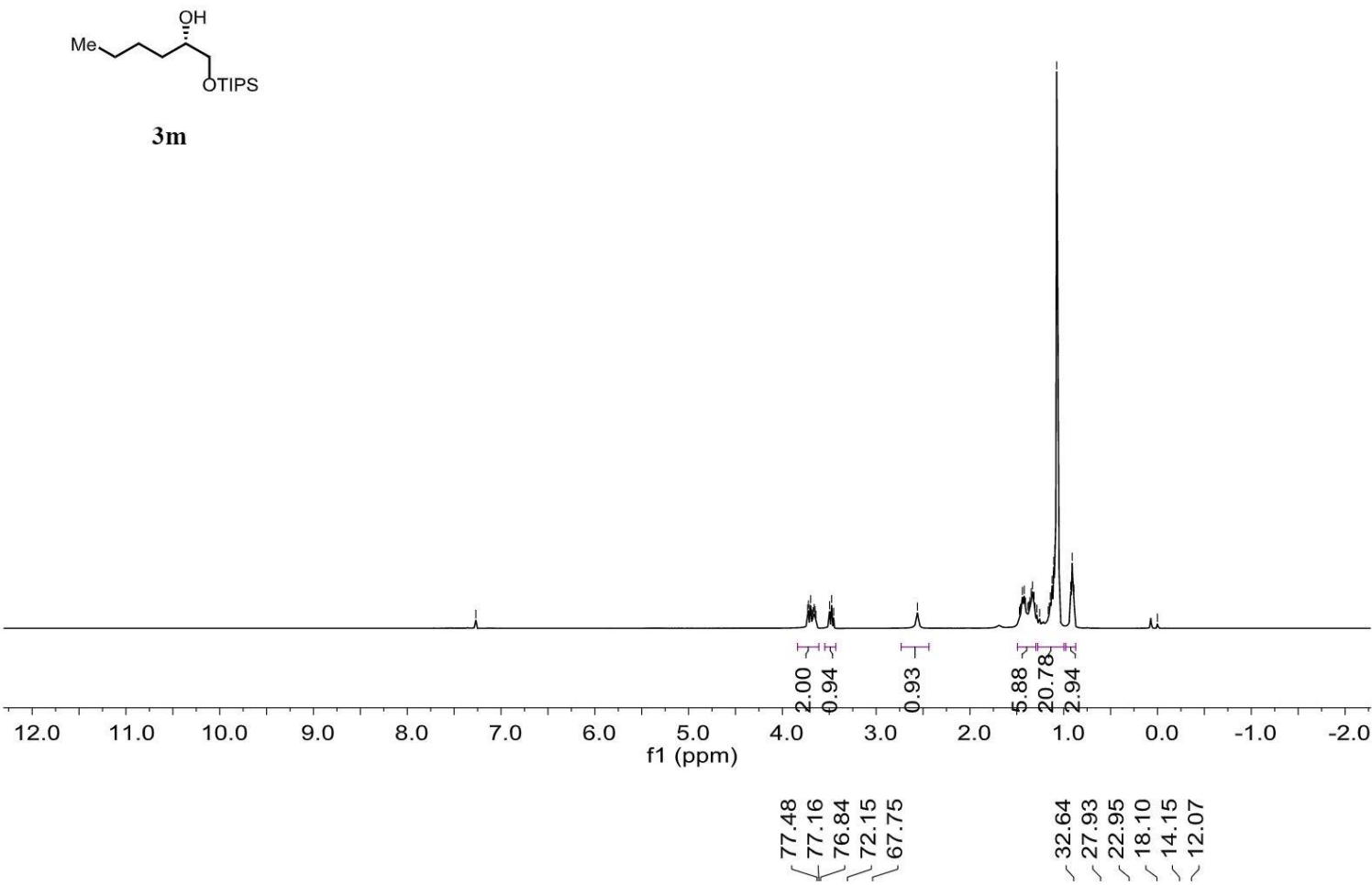

${ }^{13} \mathrm{C} \mathrm{NMR}\left(100 \mathrm{MHz}, \mathrm{CDCl}_{3}\right)$<smiles>CCCC[C@H](O)C[Hg]C</smiles>

$3 \mathbf{m}$

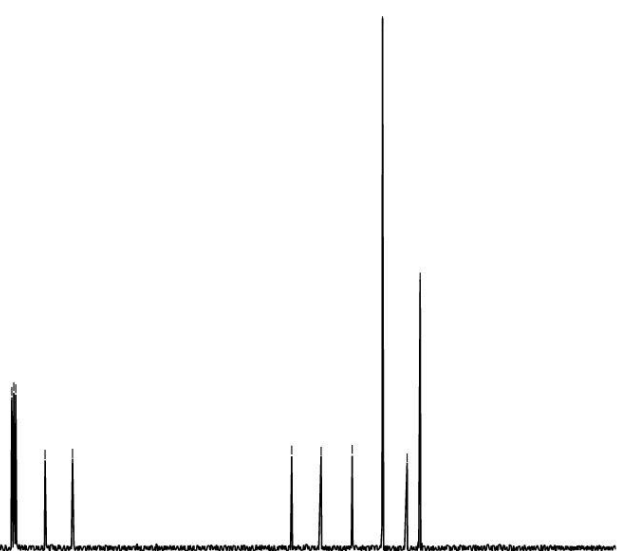

$\begin{array}{lllllllllllllllllllllll}210 & 200 & 190 & 180 & 170 & 160 & 150 & 140 & 130 & 120 & 110 & 100 & 90 & 80 & 70 & 60 & 50 & 40 & 30 & 20 & 10 & 0 & -10\end{array}$ f1 (ppm) 


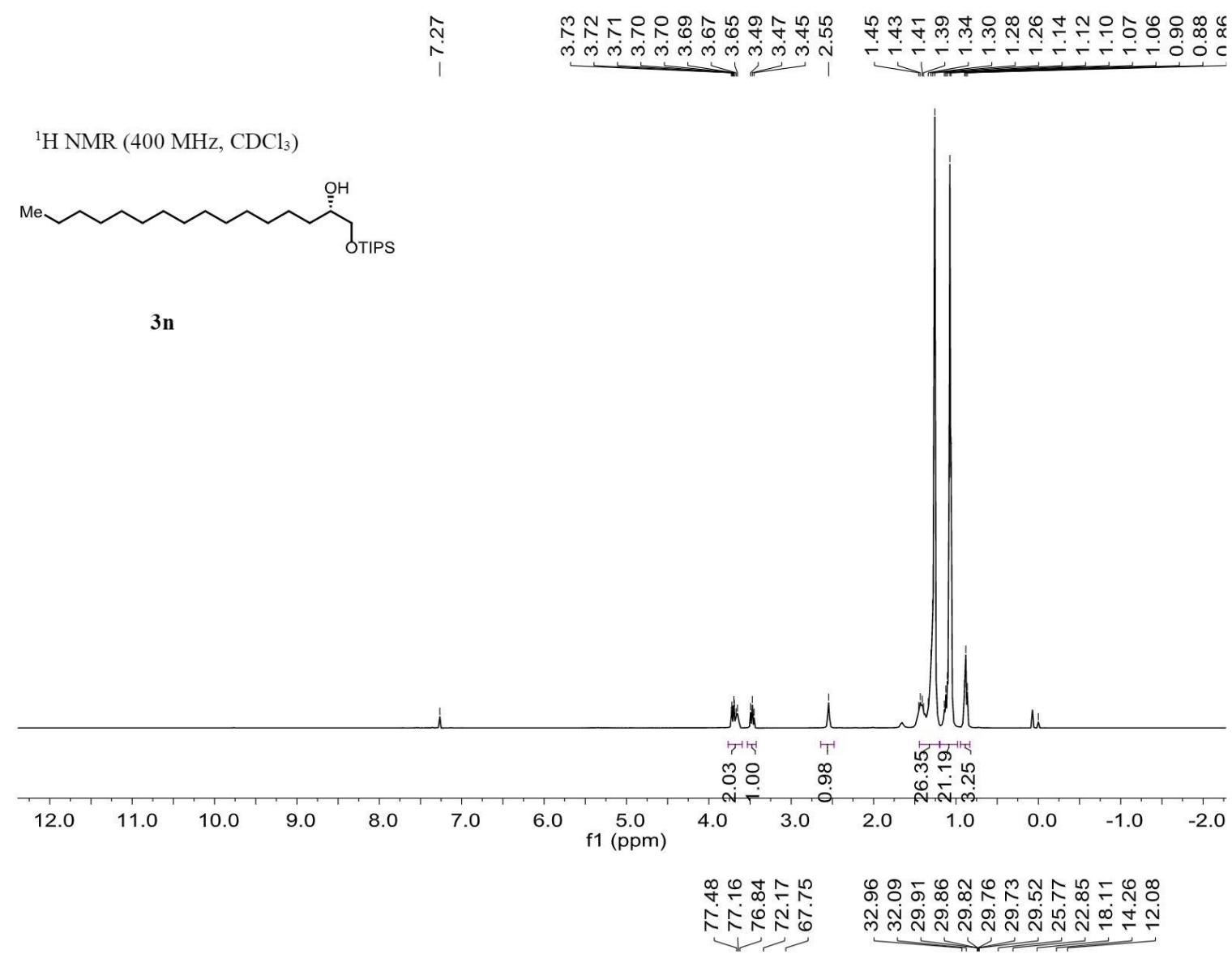

${ }^{13} \mathrm{C} \mathrm{NMR}\left(100 \mathrm{MHz}, \mathrm{CDCl}_{3}\right)$
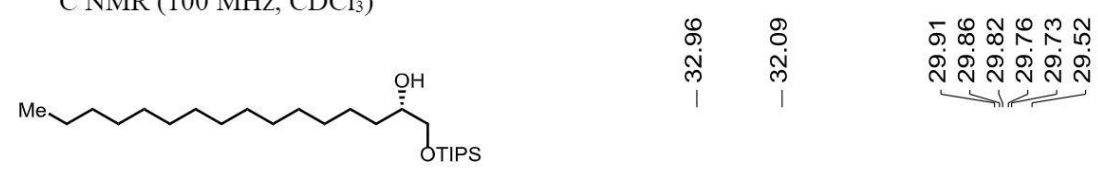

$3 n$
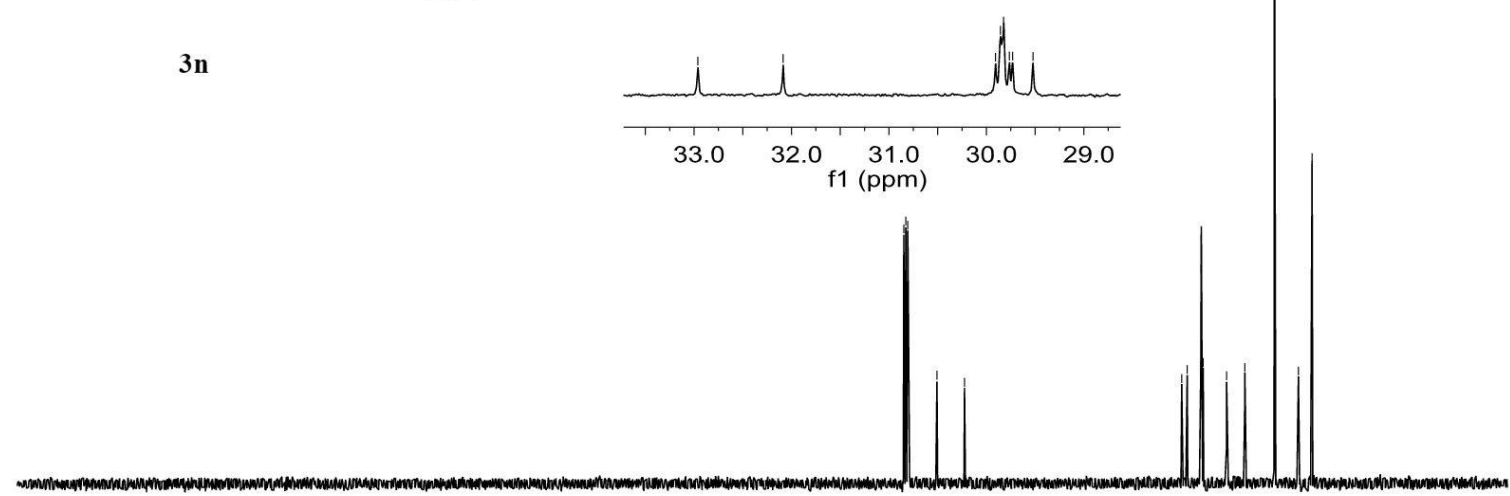

$\begin{array}{lllllllllllllllllllllllll}210 & 200 & 190 & 180 & 170 & 160 & 150 & 140 & 130 & 120 & 110 & 100 & 90 & 80 & 70 & 60 & 50 & 40 & 30 & 20 & 10 & 0 & -10\end{array}$ 


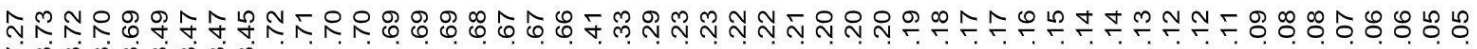

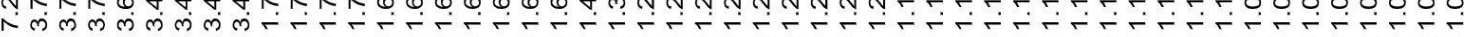

${ }^{1} \mathrm{H}$ NMR $\left(400 \mathrm{MHz}, \mathrm{CDCl}_{3}\right)$<smiles>OCCC(O)CO</smiles>

30
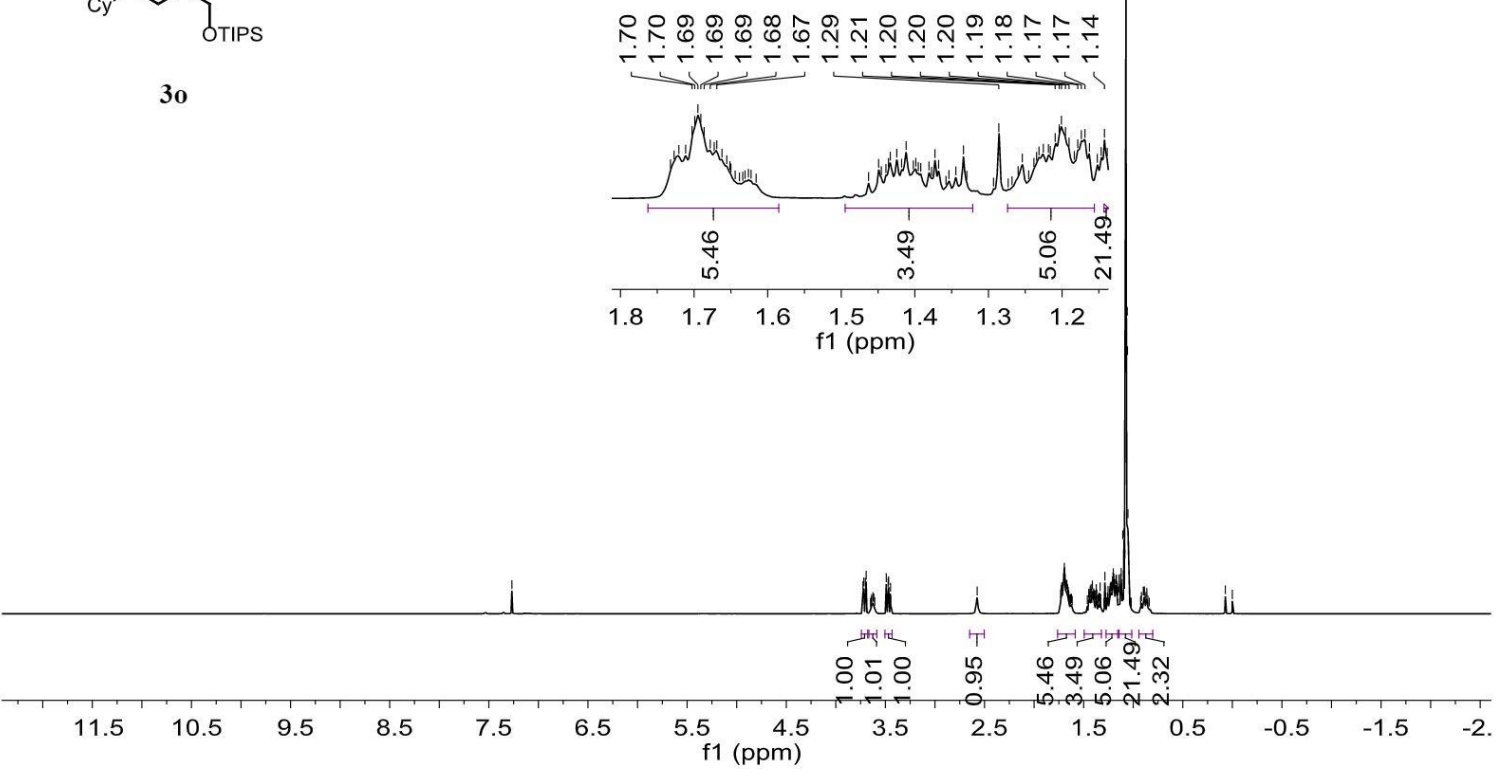

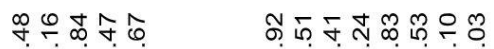

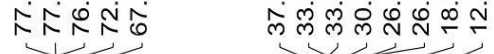

舟

${ }^{13} \mathrm{C}$ NMR (100 MHz, $\left.\mathrm{CDCl}_{3}\right)$<smiles>C[Hg]C[C@H](O)CC[Hg]</smiles>

30

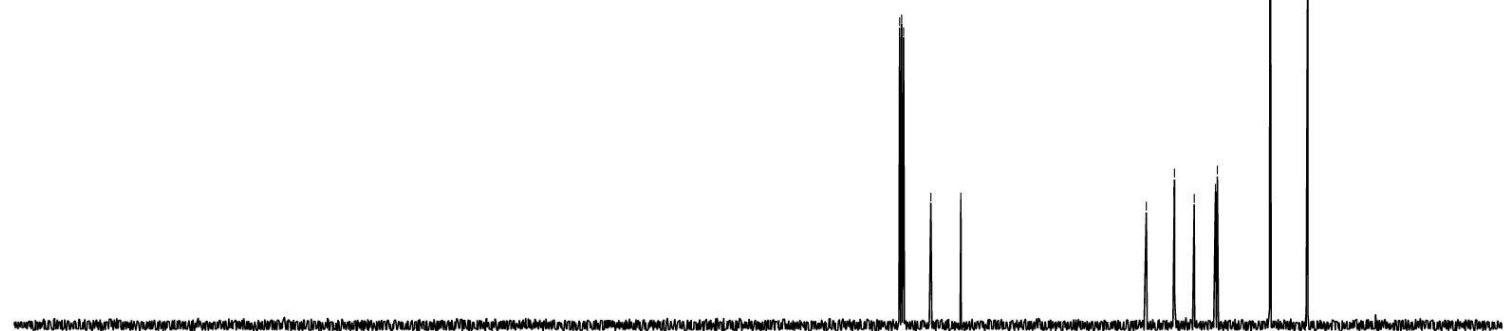

$\begin{array}{lllllllllllllllllllllll}210 & 200 & 190 & 180 & 170 & 160 & 150 & 140 & 130 & 120 & 110 & 100 & 90 & 80 & 70 & 60 & 50 & 40 & 30 & 20 & 10 & 0 & -10\end{array}$ f1 (ppm) 


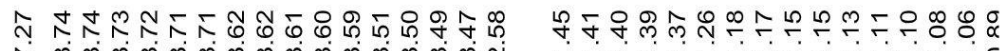

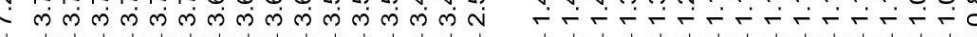

${ }^{1} \mathrm{H} \mathrm{NMR}\left(400 \mathrm{MHz}, \mathrm{CDCl}_{3}\right)$

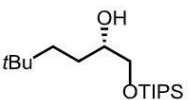

$3 p$

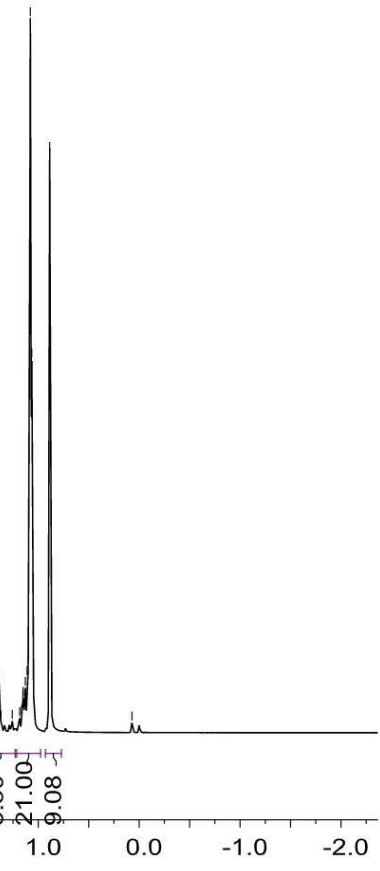

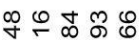

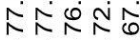

क.

ஓं

I

il

${ }^{13} \mathrm{C} \mathrm{NMR}\left(100 \mathrm{MHz}, \mathrm{CDCl}_{3}\right)$

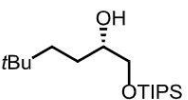

$3 p$

$\begin{array}{lllllllllllllllllllllll}210 & 200 & 190 & 180 & 170 & 160 & 150 & 140 & 130 & 120 & 110 & 100 & 90 & 80 & 70 & 60 & 50 & 40 & 30 & 20 & 10 & 0 & -10\end{array}$ f1 $(\mathrm{ppm})$ 


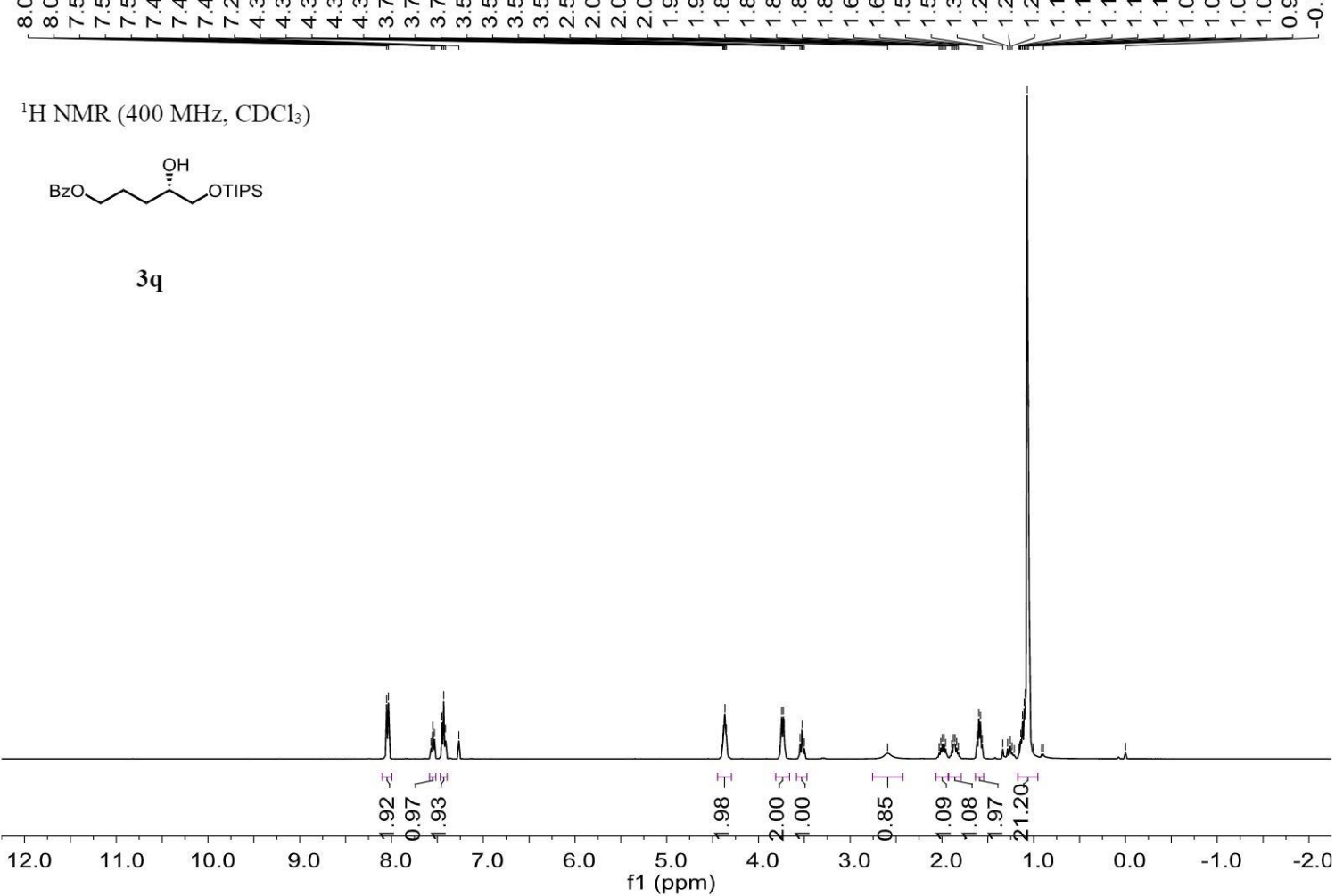

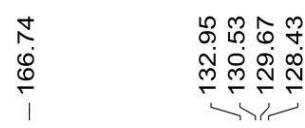

\section{舟 $\stackrel{0}{\sim}$}

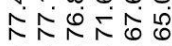

จำำ ㅇำ

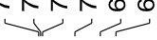

ค่ $\stackrel{\text { N }}{\infty} \stackrel{\infty}{\sim}$

${ }^{13} \mathrm{C}$ NMR (100 MHz, $\left.\mathrm{CDCl}_{3}\right)$<smiles>O[C@@H](C[18OH])CCC[18O]</smiles>

$3 q$

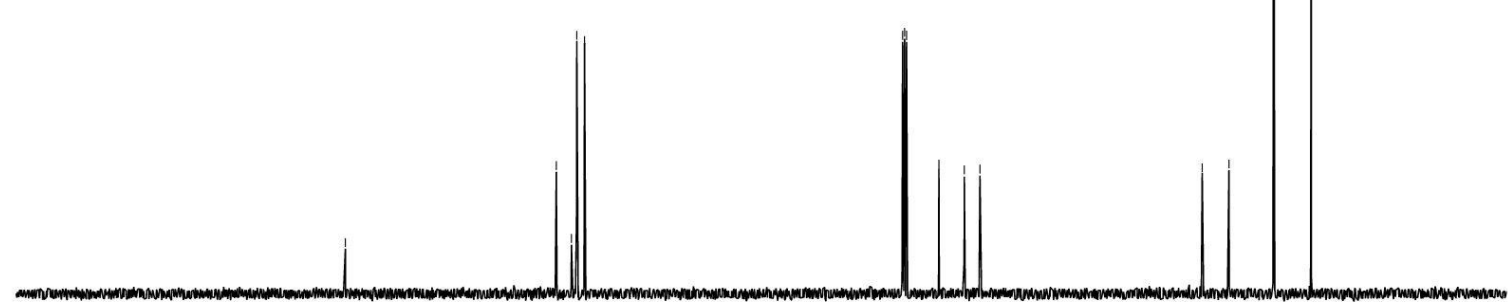

$\begin{array}{lllllllllllllllllllllll}210 & 200 & 190 & 180 & 170 & 160 & 150 & 140 & 130 & 120 & 110 & 100 & 90 & 80 & 70 & 60 & 50 & 40 & 30 & 20 & 10 & 0 & -10\end{array}$ f1 (ppm) 


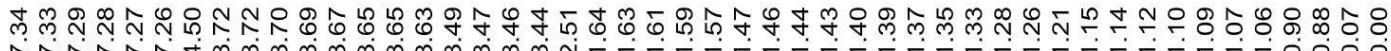

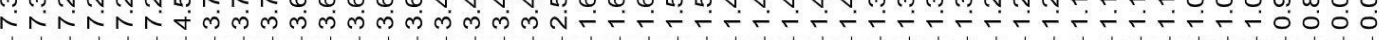

${ }^{1} \mathrm{H}$ NMR $\left(400 \mathrm{MHz}, \mathrm{CDCl}_{3}\right)$

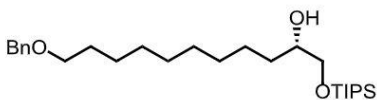

$3 \mathbf{r}$

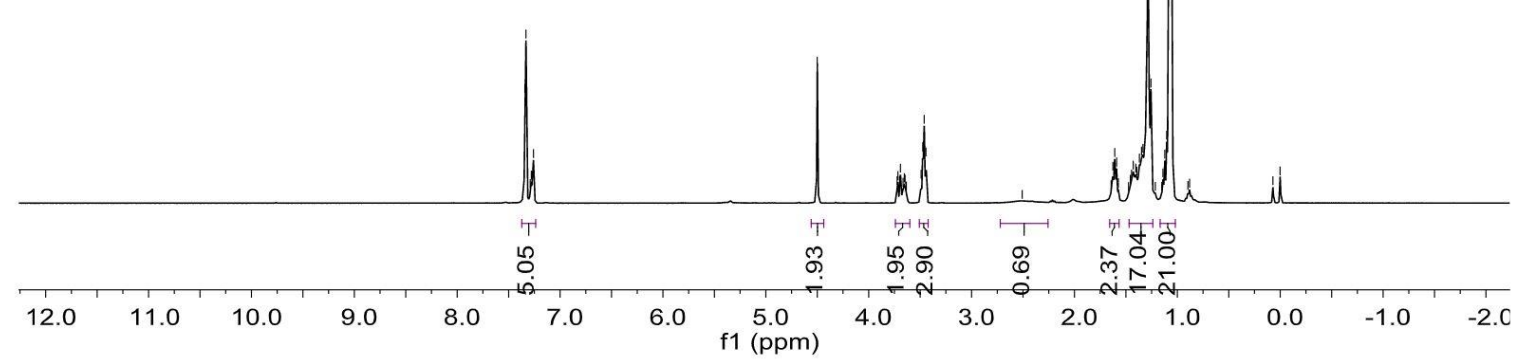

$\infty$ 守只员

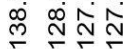

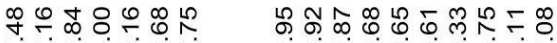

Nरํำ

r

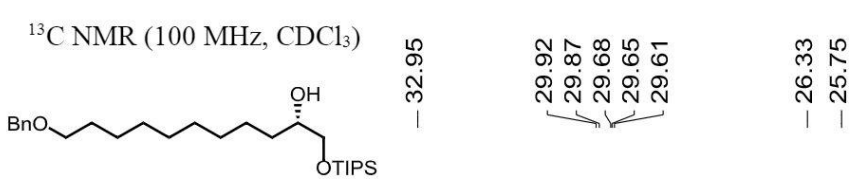

$3 \mathbf{r}$

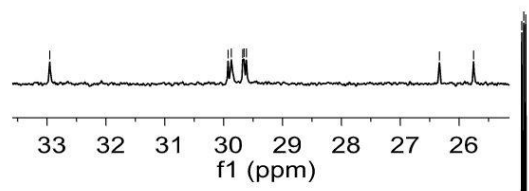

$\begin{array}{lllllllllllllllllllllll}210 & 200 & 190 & 180 & 170 & 160 & 150 & 140 & 130 & 120 & 110 & 100 & 90 & 80 & 70 & 60 & 50 & 40 & 30 & 20 & 10 & 0 & -10\end{array}$ 
స়下

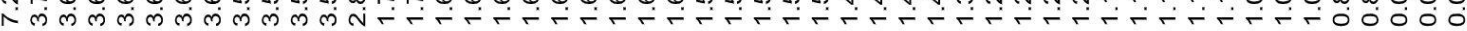

${ }^{1} \mathrm{H}$ NMR $\left(400 \mathrm{MHz}, \mathrm{CDCl}_{3}\right)$

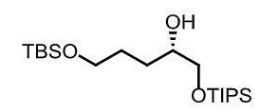

$3 s$

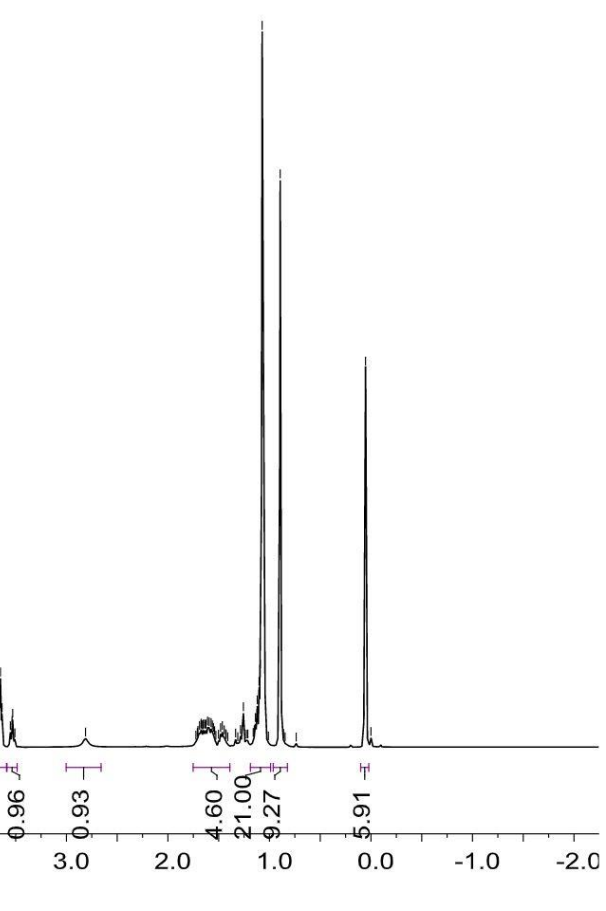

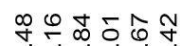

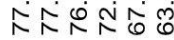

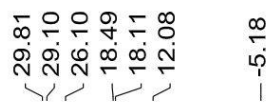

${ }^{13} \mathrm{C}$ NMR $\left(100 \mathrm{MHz}, \mathrm{CDCl}_{3}\right)$

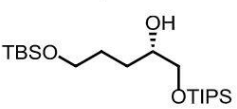

$3 s$

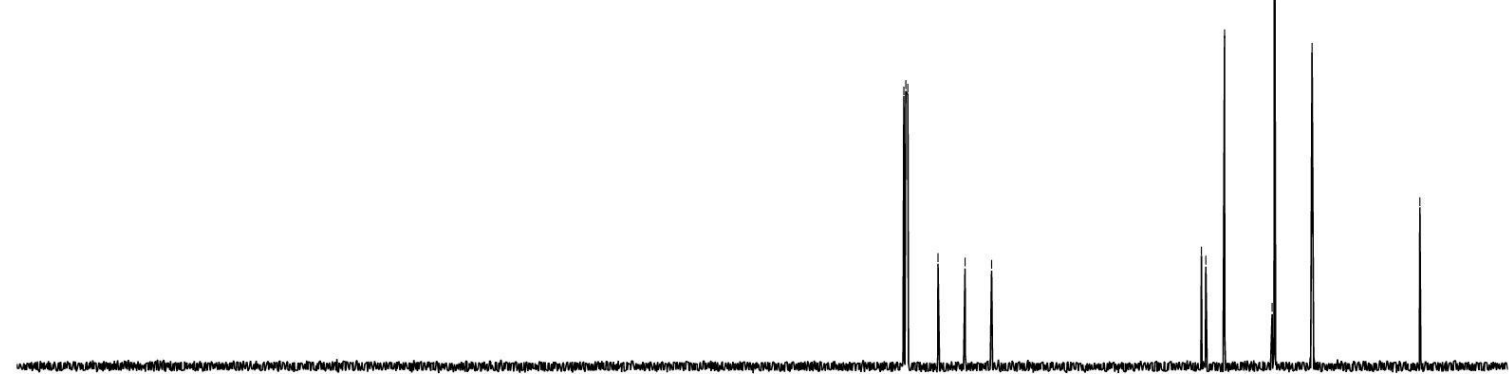

$\begin{array}{lllllllllllllllllllllll}210 & 200 & 190 & 180 & 170 & 160 & 150 & 140 & 130 & 120 & 110 & 100 & 90 & 80 & 70 & 60 & 50 & 40 & 30 & 20 & 10 & 0 & -10\end{array}$ f1 (ppm) 
${ }^{1} \mathrm{H} \mathrm{NMR}\left(400 \mathrm{MHz}, \mathrm{CDCl}_{3}\right)$

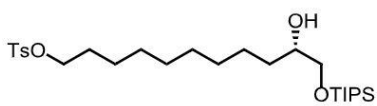

$3 t$

OTIPS

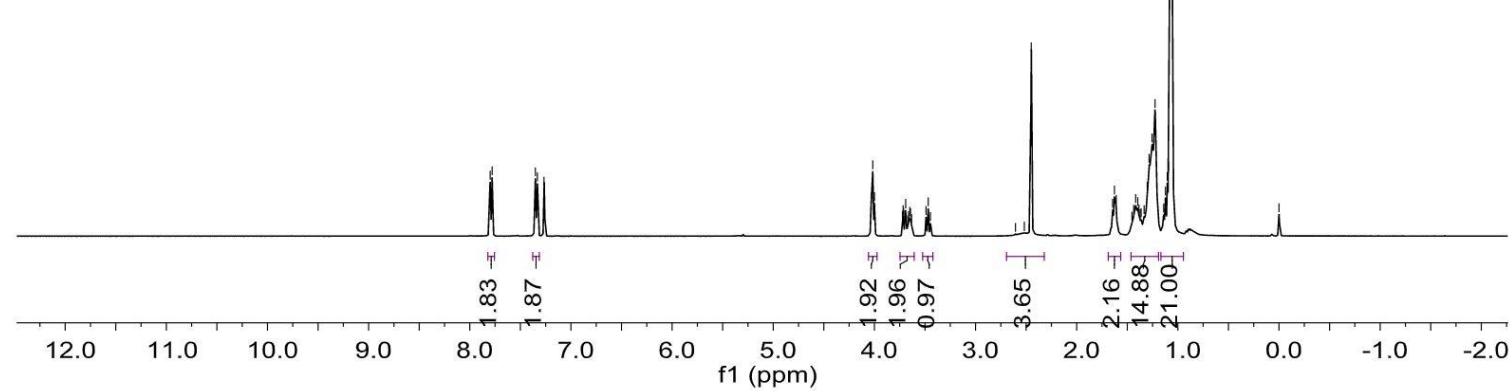

ก.

孛

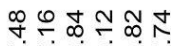

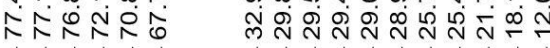

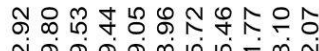

${ }^{13} \mathrm{C} \mathrm{NMR}\left(100 \mathrm{MHz}, \mathrm{CDCl}_{3}\right)$<smiles>O[C@@H](C[OH2+])CCCCCCCCCO[Na]</smiles>

$3 t$
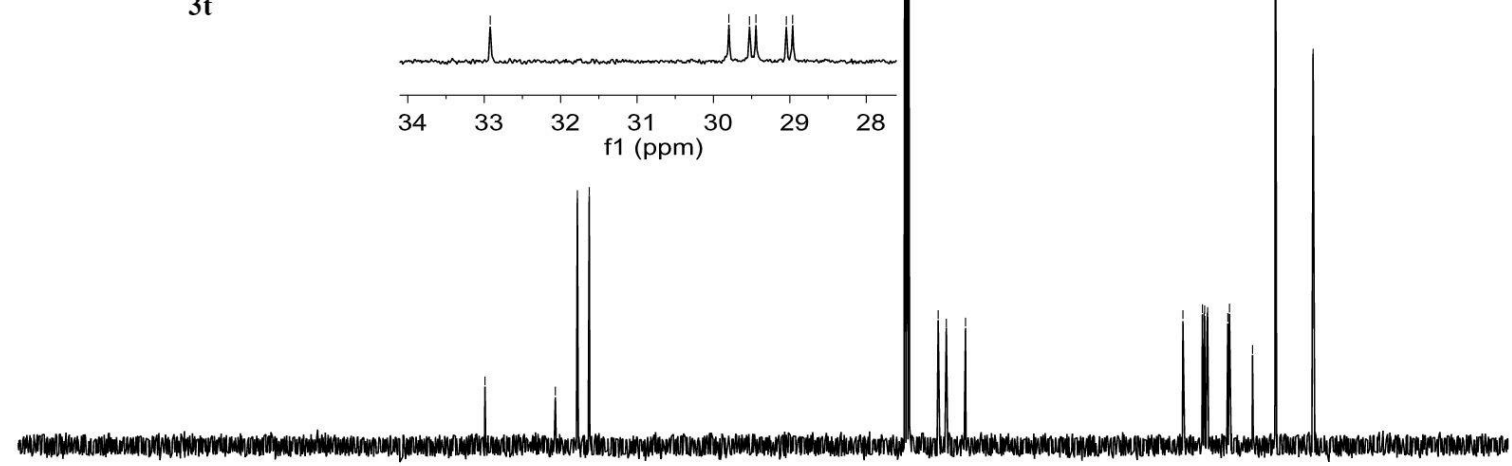

$\begin{array}{lllllllllllllllllllllll}210 & 200 & 190 & 180 & 170 & 160 & 150 & 140 & 130 & 120 & 110 & 100 & 90 & 80 & 70 & 60 & 50 & 40 & 30 & 20 & 10 & 0 & -10\end{array}$ 
${ }^{1} \mathrm{H}$ NMR (400 MHz, $\left.\mathrm{CDCl}_{3}\right)$

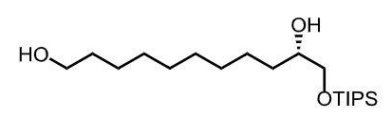

$3 \mathbf{u}$

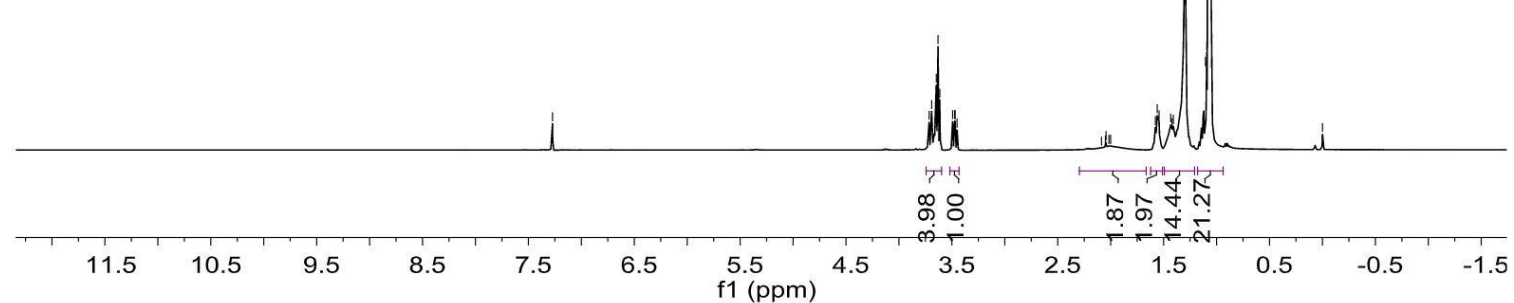

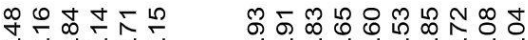

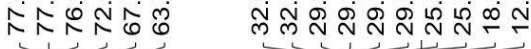

${ }^{13} \mathrm{C}$ NMR (100 MHz, $\left.\mathrm{CDCl}_{3}\right)$

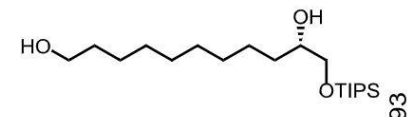

$3 \mathbf{u}$

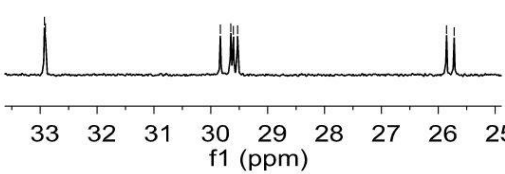


${ }^{1} \mathrm{H}$ NMR $\left(400 \mathrm{MHz}, \mathrm{CDCl}_{3}\right)$

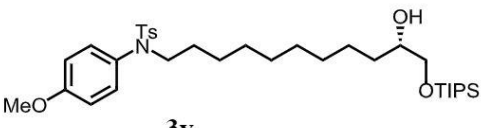

$3 \mathbf{v}$
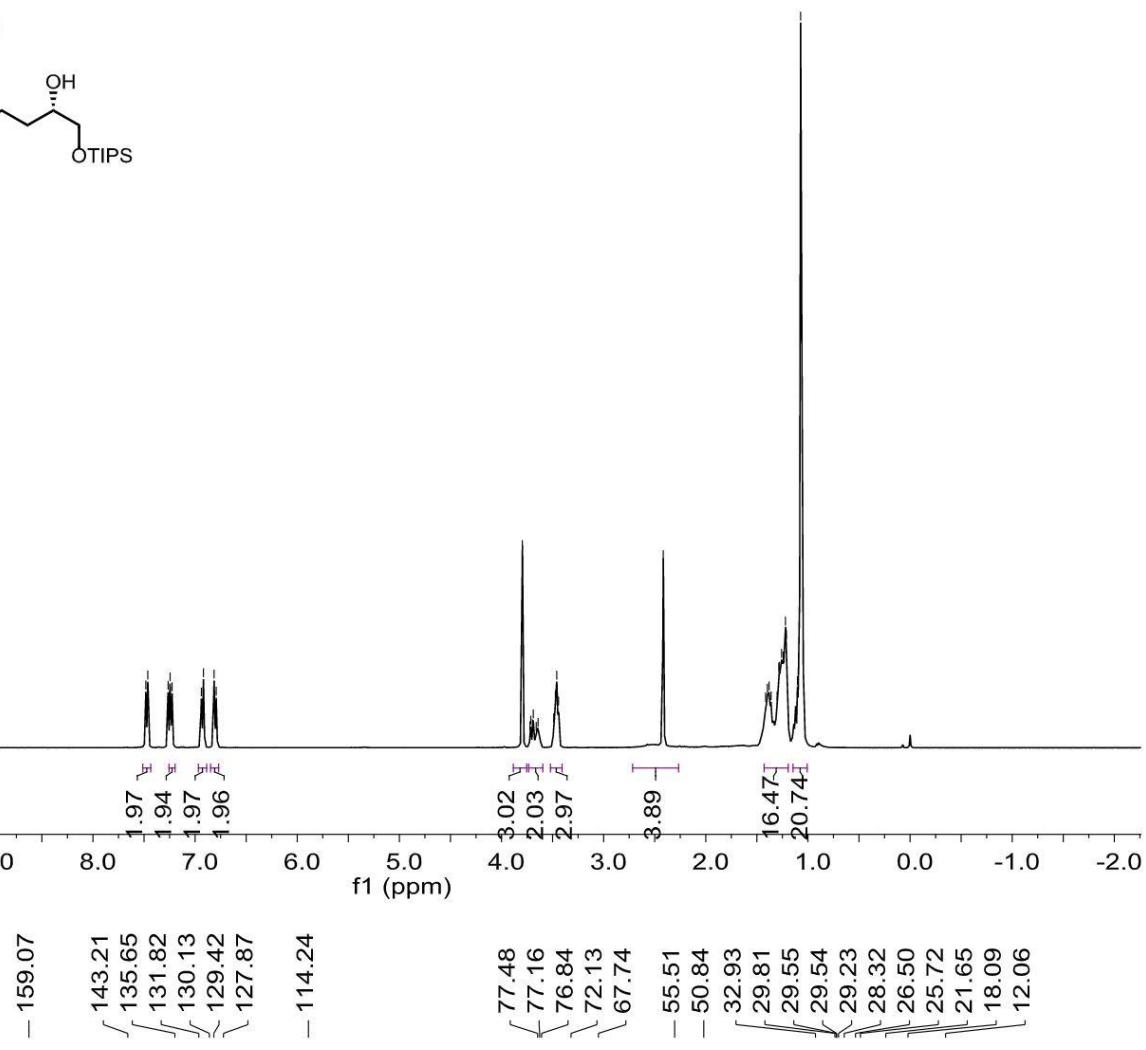

${ }^{13} \mathrm{C} \mathrm{NMR}\left(100 \mathrm{MHz}, \mathrm{CDCl}_{3}\right)$<smiles>COc1ccc([NH2+]CCCCCCCCCC(O)CO[Na])cc1</smiles>

$3 \mathbf{v}$

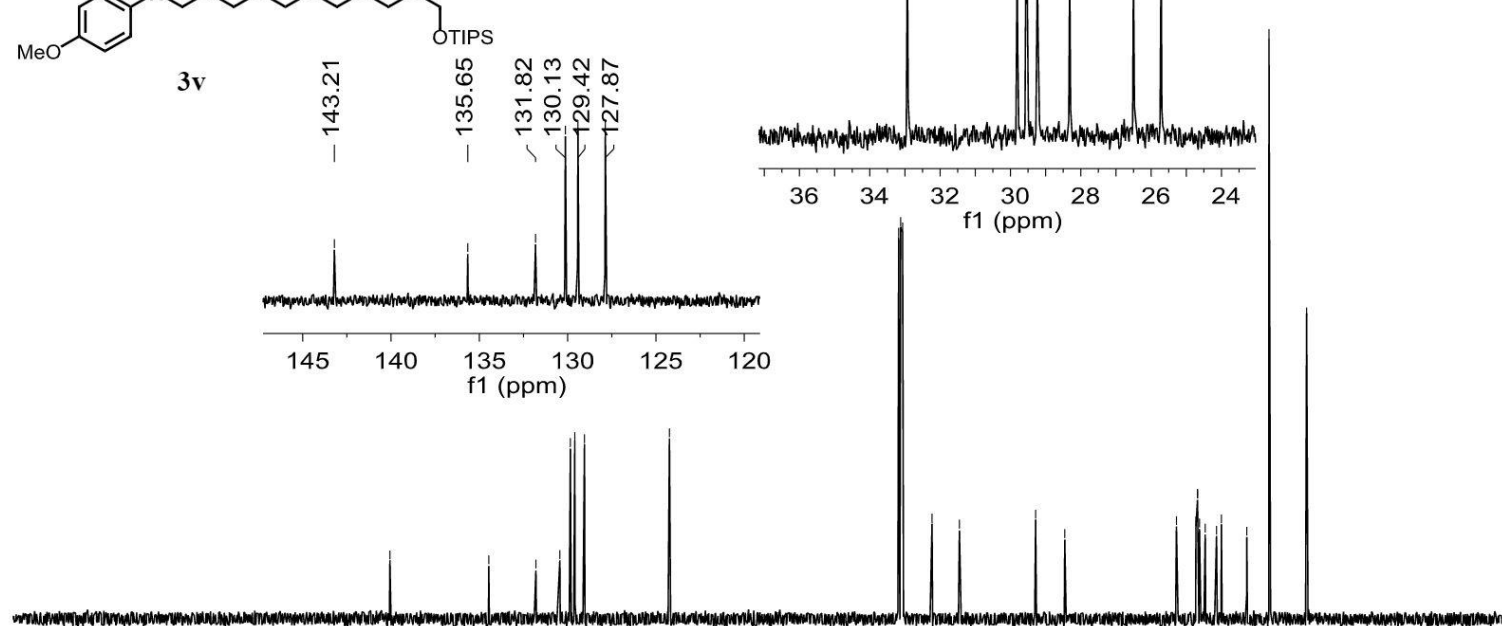

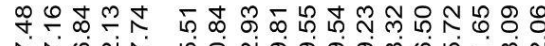
ヘトง

माप्रा मो

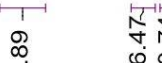

\begin{tabular}{|c|c|c|c|c|c|c|c|c|c|c|c|c|c|}
\hline \multirow[t]{2}{*}{12.0} & \multirow[t]{2}{*}{11.0} & \multirow[t]{2}{*}{10.0} & 9.0 & 8.0 & 7.0 & 6.0 & \multirow[t]{2}{*}{$\begin{array}{c}5.0 \\
\mathrm{f} 1(\mathrm{ppm})\end{array}$} & \multicolumn{2}{|c|}{$\begin{array}{ll}4.0 & 3.0\end{array}$} & 2.0 & 1.0 & 0.0 & -1.0 \\
\hline & & & $\begin{array}{l}\hat{0} \\
0 \\
0\end{array}$ & 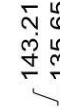 & 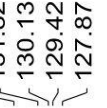 & 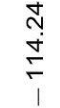 & & \begin{tabular}{l} 
Dos \\
\multirow{2}{*}{$\hat{N}$}
\end{tabular} & 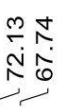 & 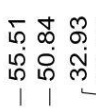 & & 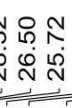 & 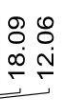 \\
\hline
\end{tabular}

$\begin{array}{lllllllllllllllllllllll}210 & 200 & 190 & 180 & 170 & 160 & 150 & 140 & 130 & 120 & 110 & 100 & 90 & 80 & 70 & 60 & 50 & 40 & 30 & 20 & 10 & 0 & -10\end{array}$ f1 (ppm) 


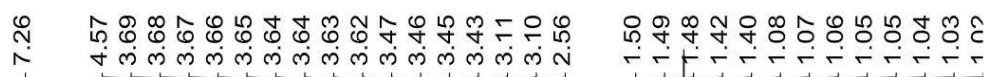

${ }^{1} \mathrm{H}$ NMR $\left(400 \mathrm{MHz}, \mathrm{CDCl}_{3}\right)$

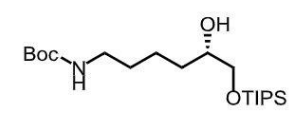

$3 w$
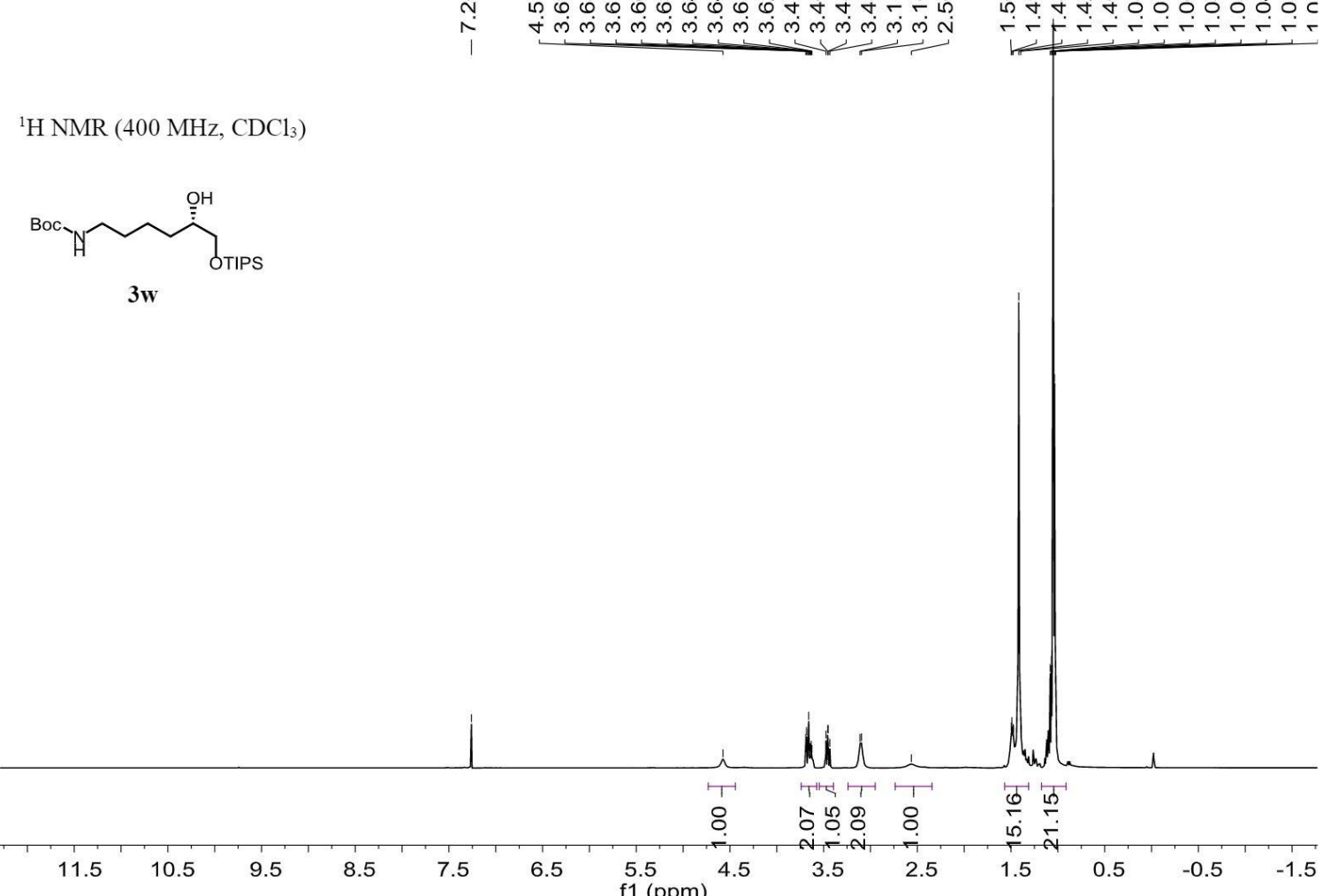

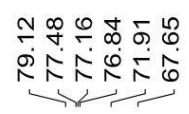

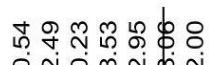

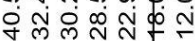
1) $1,1,1$

${ }^{13} \mathrm{C}$ NMR (100 MHz, $\left.\mathrm{CDCl}_{3}\right)$

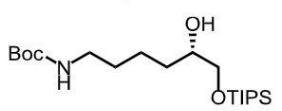

$3 w$

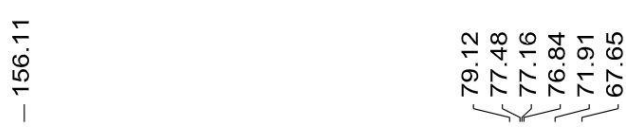
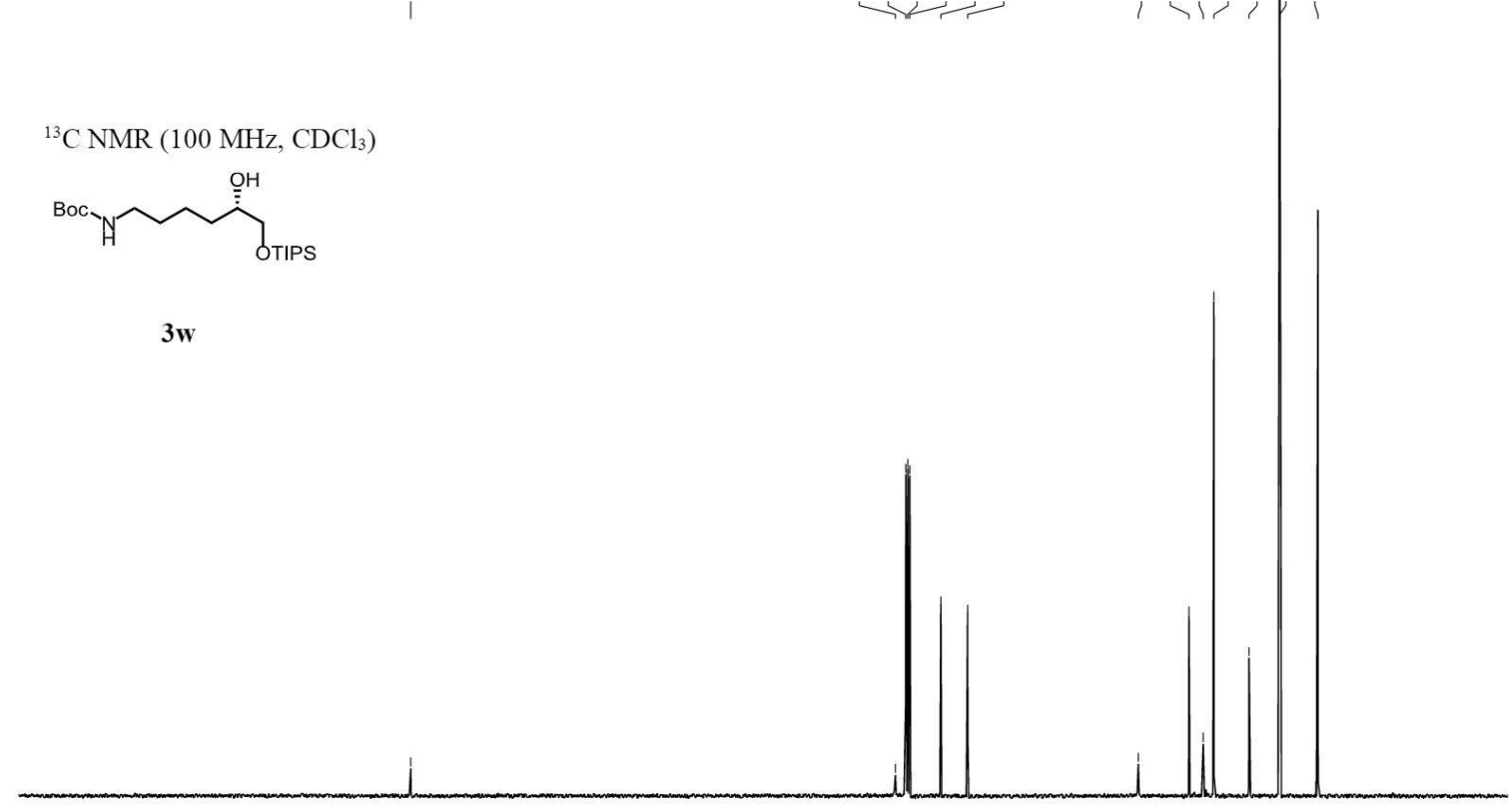

$\begin{array}{lllllllllllllllllllllllllllllllll}210 & 200 & 190 & 180 & 170 & 160 & 150 & 140 & 130 & 120 & 110 & 100 & 90 & 80 & 70 & 60 & 50 & 40 & 30 & 20 & 10 & 0 & -10\end{array}$ 


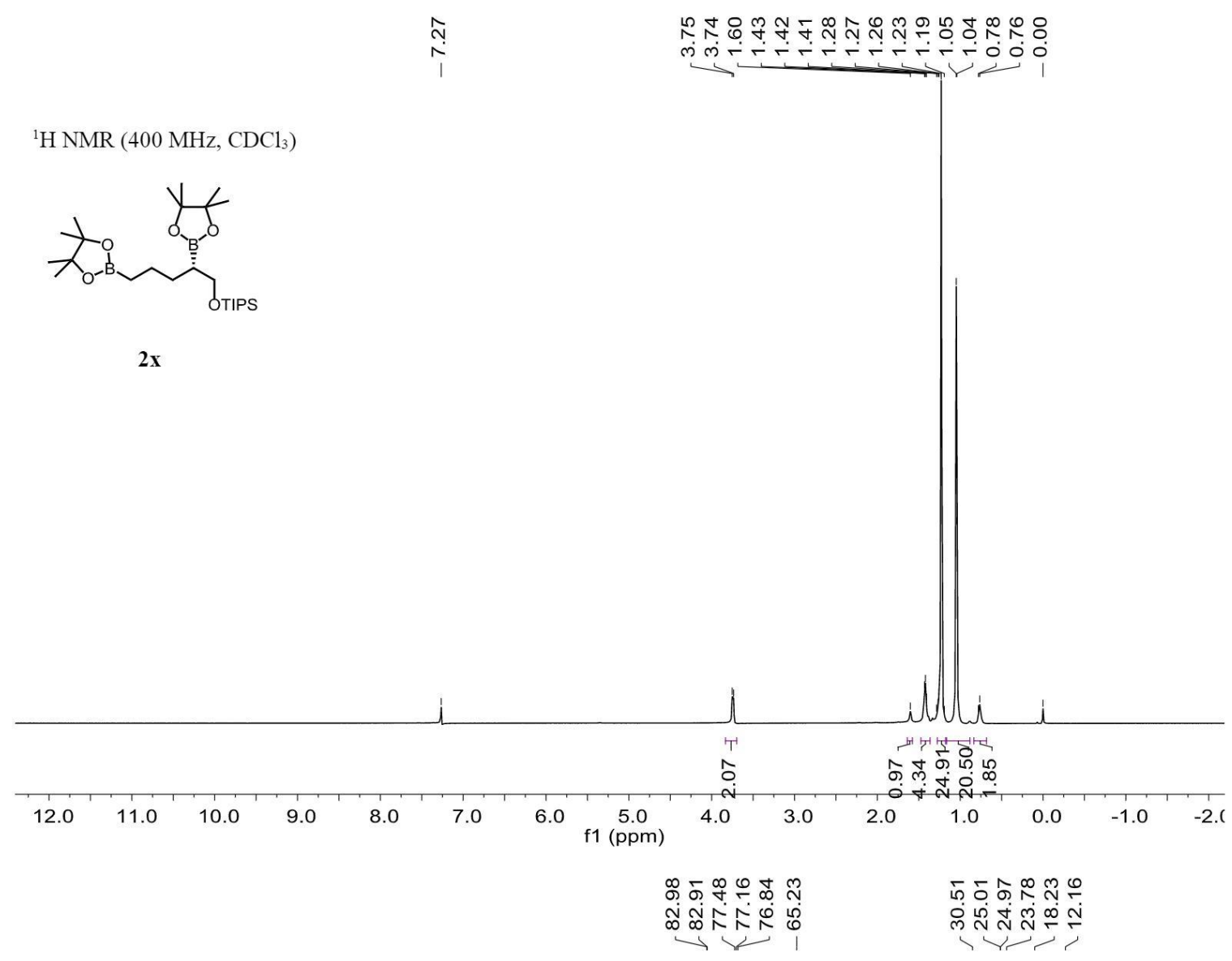

${ }^{13} \mathrm{C} \mathrm{NMR}\left(100 \mathrm{MHz}, \mathrm{CDCl}_{3}\right)$

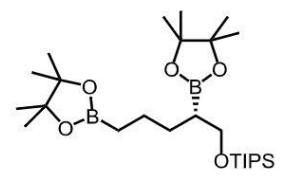

2x

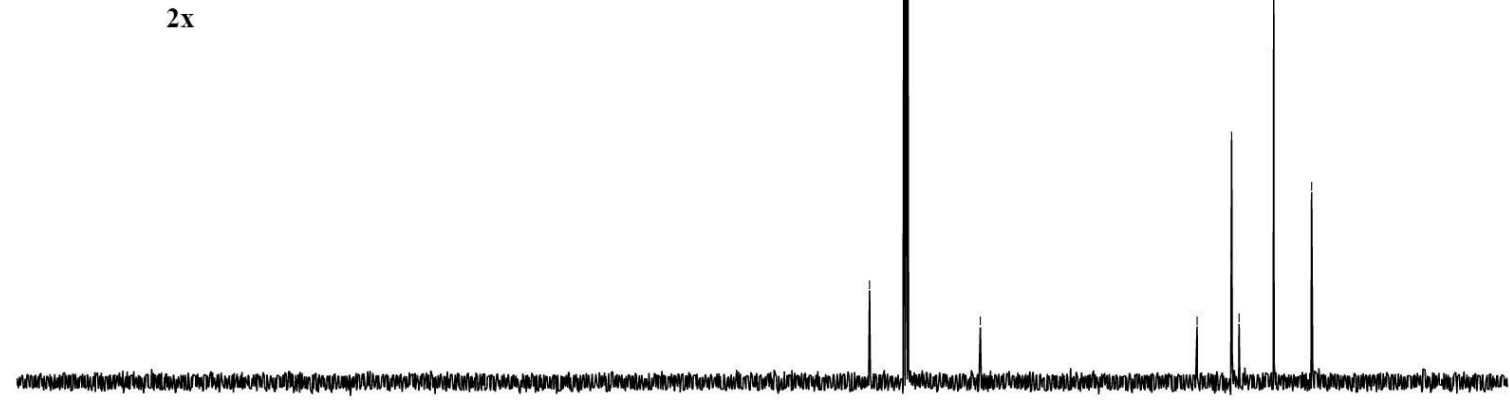

$\begin{array}{lllllllllllllllllllllll}210 & 200 & 190 & 180 & 170 & 160 & 150 & 140 & 130 & 120 & 110 & 100 & 90 & 80 & 70 & 60 & 50 & 40 & 30 & 20 & 10 & 0 & -10\end{array}$ 
${ }^{1} \mathrm{H}$ NMR $\left(400 \mathrm{MHz}, \mathrm{CDCl}_{3}\right)$

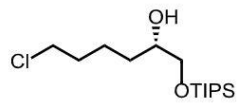

$3 \mathbf{y}$

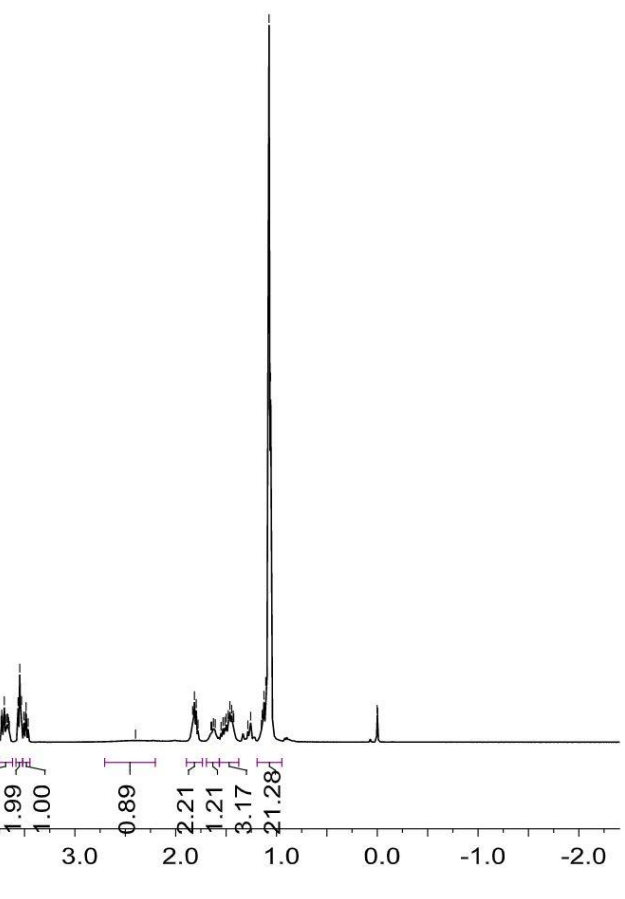

${ }^{13} \mathrm{C} \mathrm{NMR}\left(100 \mathrm{MHz}, \mathrm{CDCl}_{3}\right)$

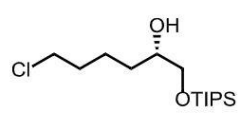

$3 \mathbf{y}$

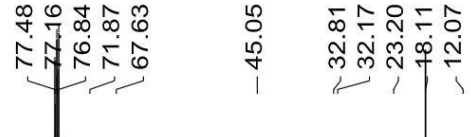

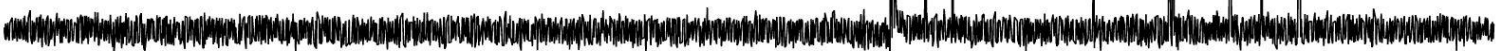

$\begin{array}{lllllllllllllllllllllll}210 & 200 & 190 & 180 & 170 & 160 & 150 & 140 & 130 & 120 & 110 & 100 & 90 & 80 & 70 & 60 & 50 & 40 & 30 & 20 & 10 & 0 & -10\end{array}$ 


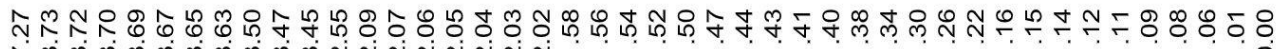

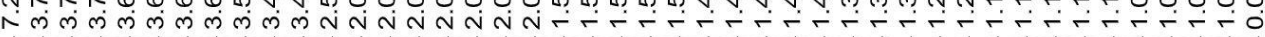

${ }^{1} \mathrm{H}$ NMR $\left(400 \mathrm{MHz}, \mathrm{CDCl}_{3}\right)$

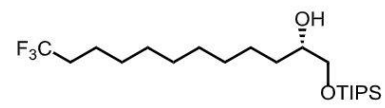

$3 z$

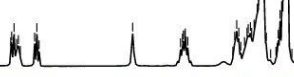

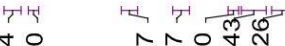
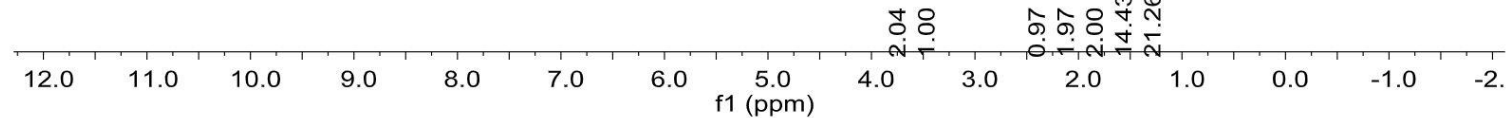

Lீ

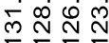

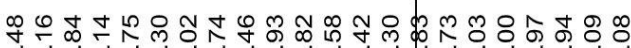

ト๋

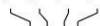

${ }^{13} \mathrm{C} \mathrm{NMR}\left(100 \mathrm{MHz}, \mathrm{CDCl}_{3}\right)$

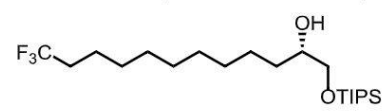

$3 z$

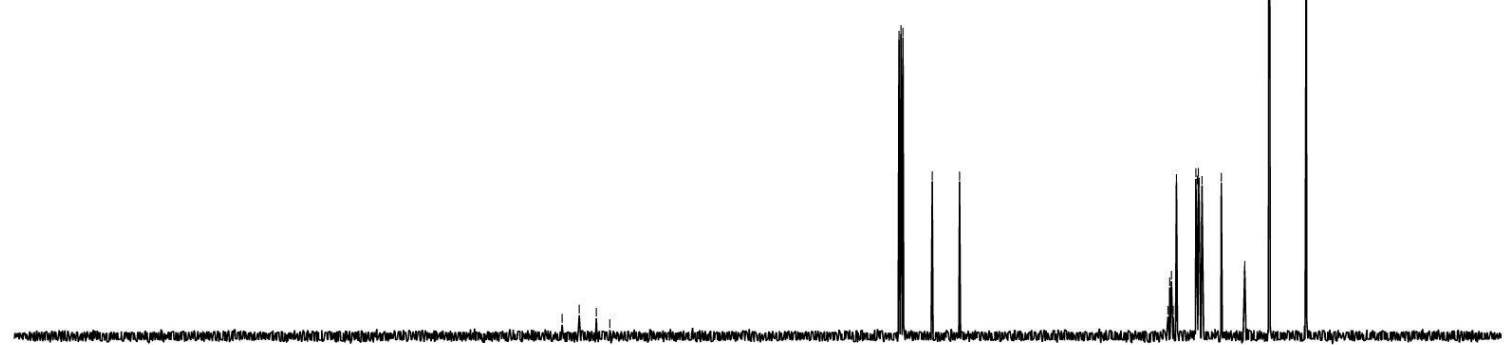

$\begin{array}{lllllllllllllllllllllll}210 & 200 & 190 & 180 & 170 & 160 & 150 & 140 & 130 & 120 & 110 & 100 & 90 & 80 & 70 & 60 & 50 & 40 & 30 & 20 & 10 & 0 & -10\end{array}$ $\mathrm{f} 1(\mathrm{ppm})$ 


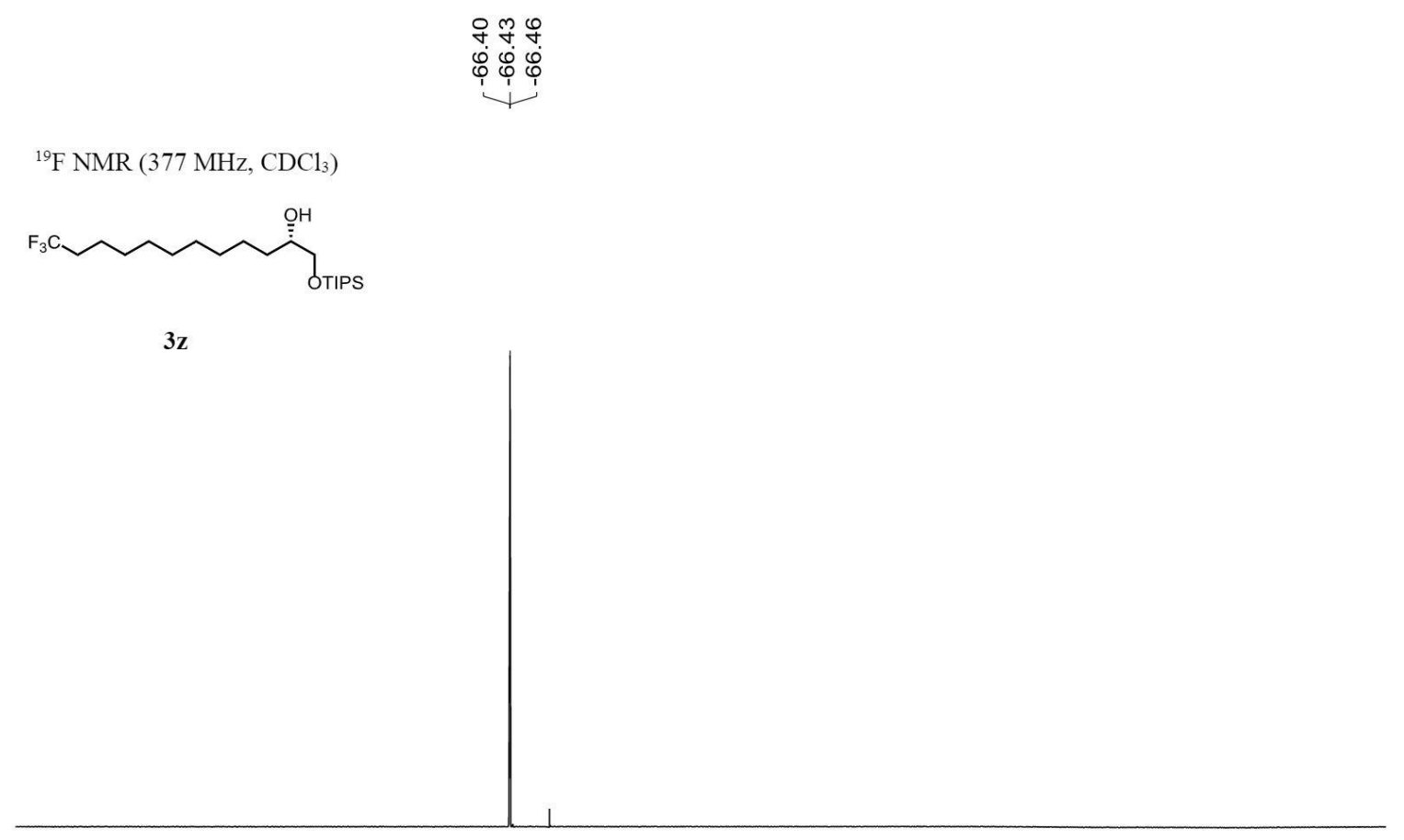

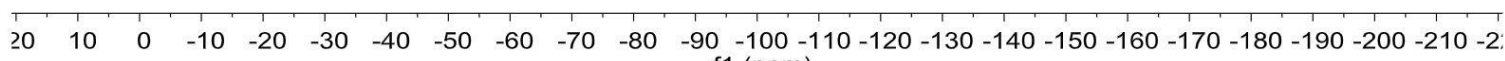
$\mathrm{f} 1(\mathrm{ppm})$

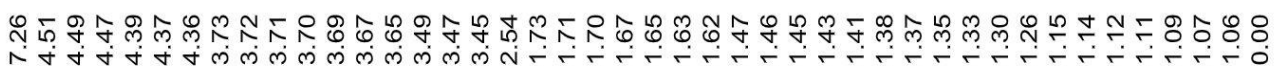

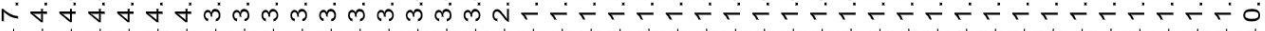

${ }^{1} \mathrm{H} \mathrm{NMR}\left(400 \mathrm{MHz}, \mathrm{CDCl}_{3}\right)$

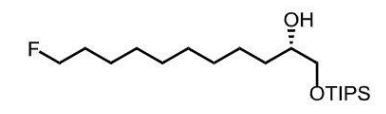

3aa

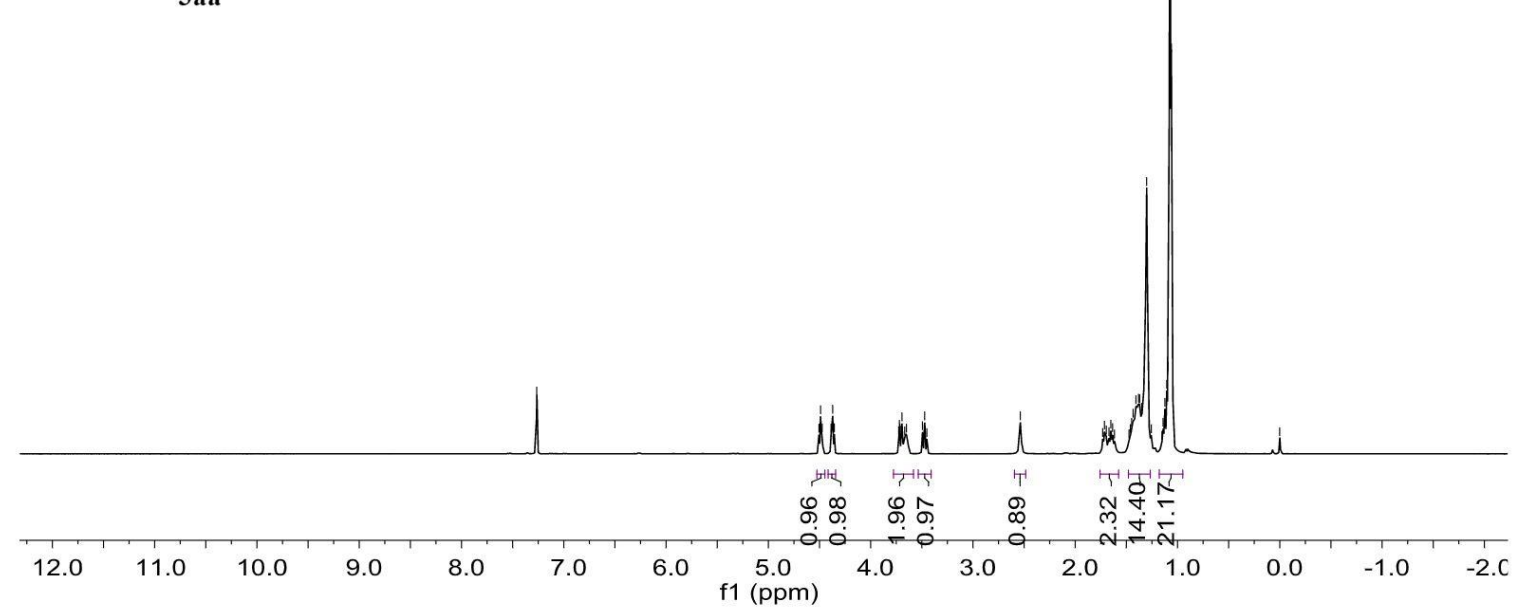


${ }^{13} \mathrm{C}$ NMR (100 MHz, $\left.\mathrm{CDCl}_{3}\right)$

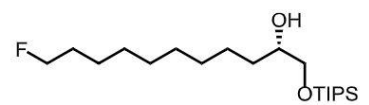

3aa

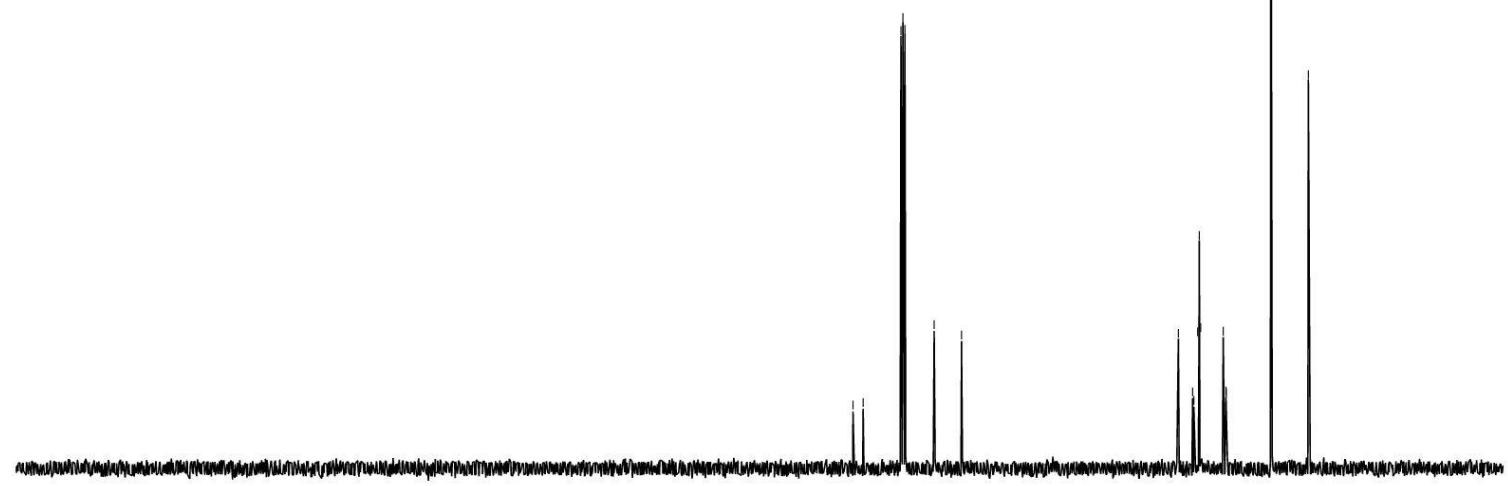

$\begin{array}{lllllllllllllllllllllll}210 & 200 & 190 & 180 & 170 & 160 & 150 & 140 & 130 & 120 & 110 & 100 & 90 & 80 & 70 & 60 & 50 & 40 & 30 & 20 & 10 & 0 & -10\end{array}$ $\mathrm{f} 1(\mathrm{ppm})$

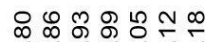

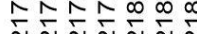

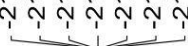

${ }^{10} \mathrm{~F} \mathrm{NMR}\left(377 \mathrm{MHz}, \mathrm{CDCl}_{3}\right)$

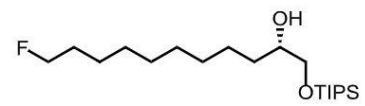

3aa

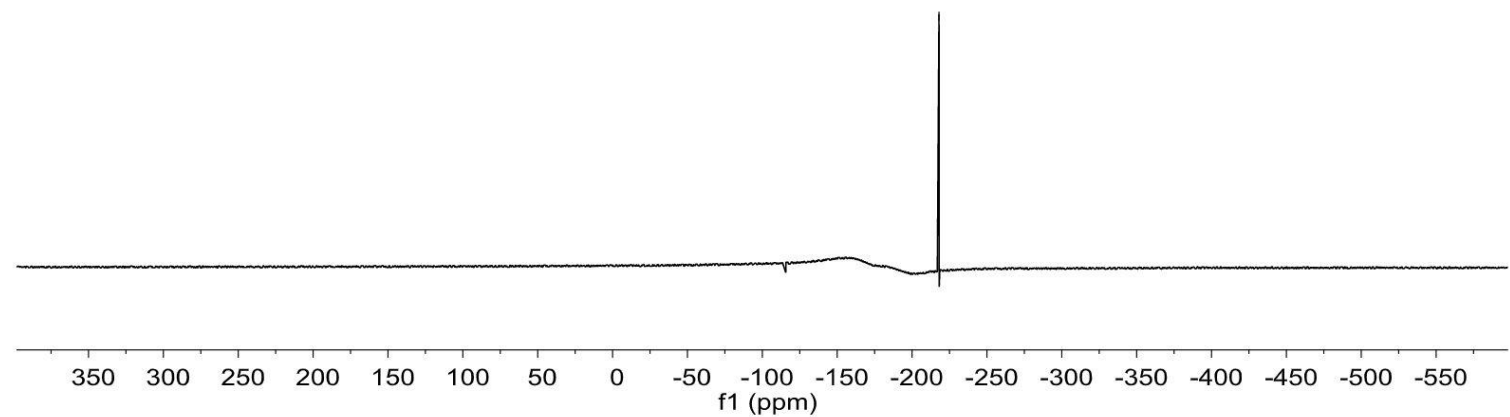




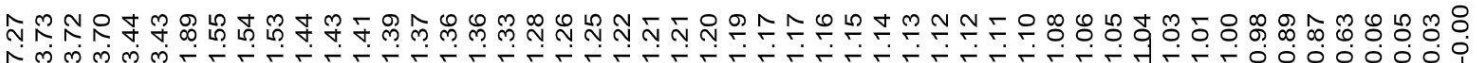

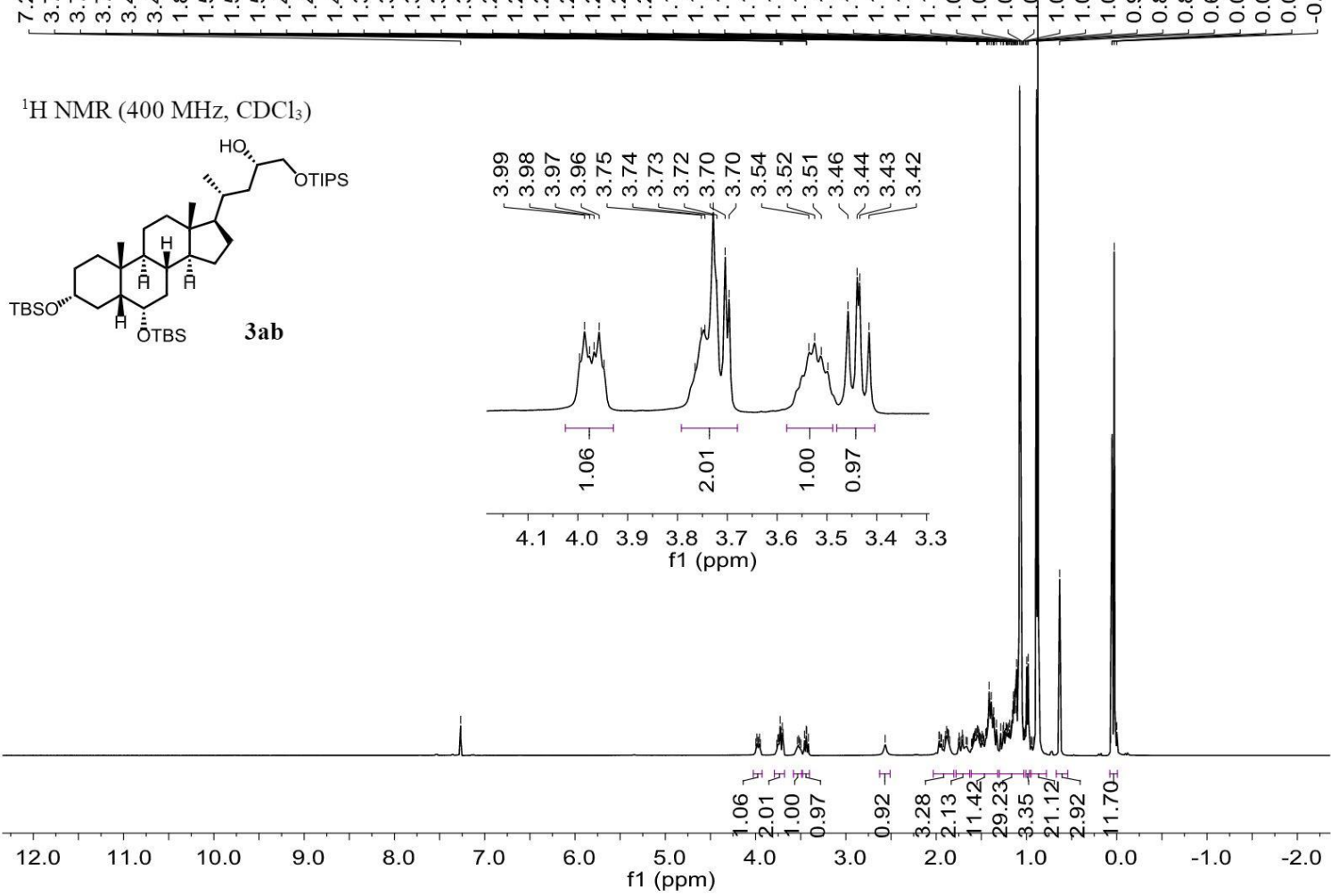

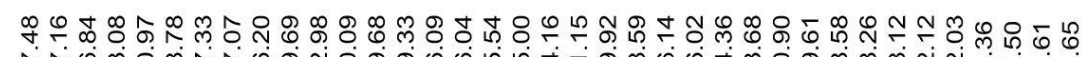

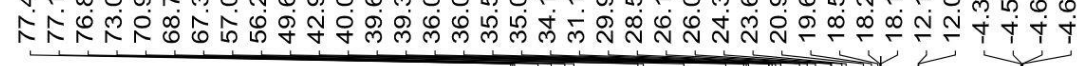

${ }^{13} \mathrm{C}$ NMR $\left(100 \mathrm{MHz}, \mathrm{CDCl}_{3}\right)$

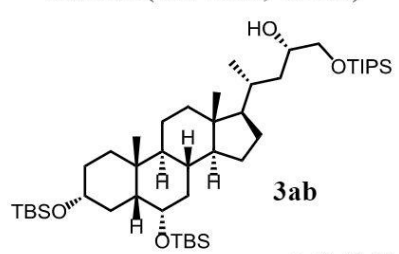

ஜำ

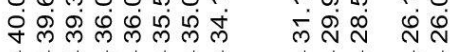

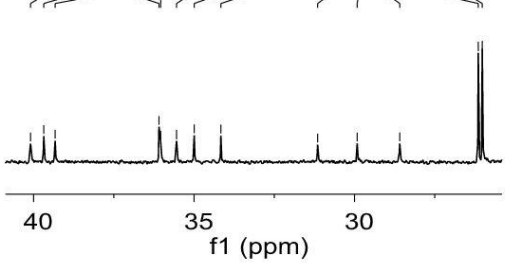

$\begin{array}{llllllllllll}210 & 200 & 190 & 180 & 170 & 160 & 150 & 140 & 130 & 120 & 110 & 100 \\ \mathrm{f} 1(\mathrm{ppm})\end{array}$ 


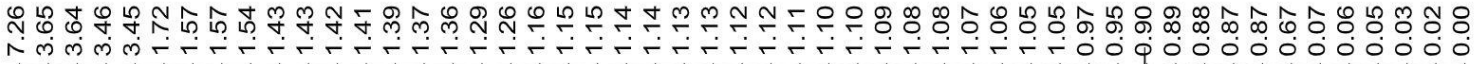

${ }^{1} \mathrm{H}$ NMR $\left(400 \mathrm{MHz}, \mathrm{CDCl}_{3}\right)$

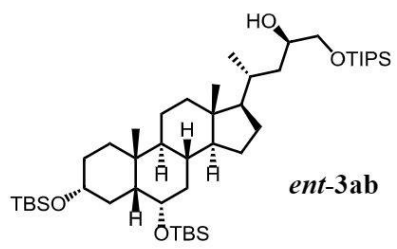

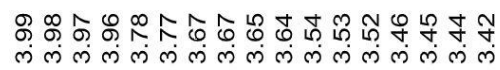

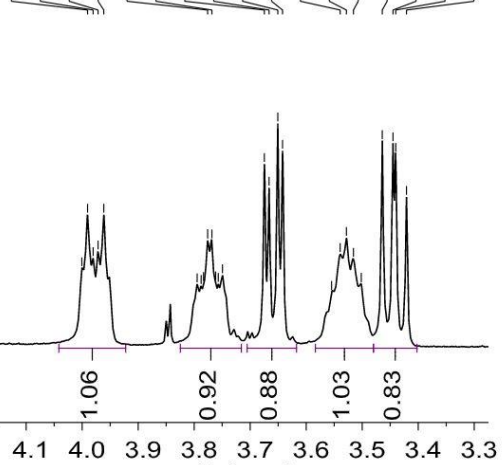

$\begin{array}{lllllllll}4.1 & 4.0 & 3.9 & 3.8 & 3.7 & 3.6 & 3.5 & 3.4 & 3.3\end{array}$ f1 (ppm)

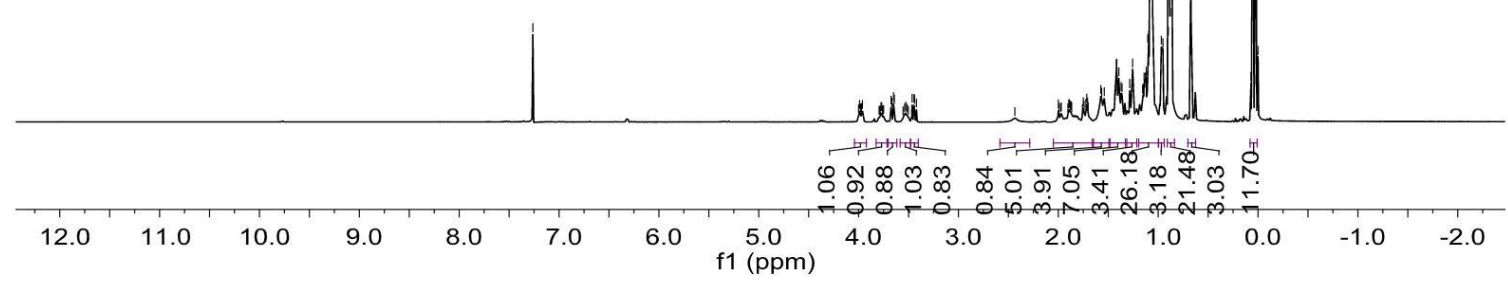

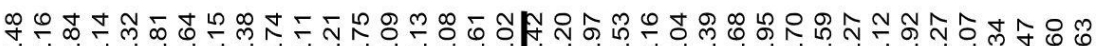

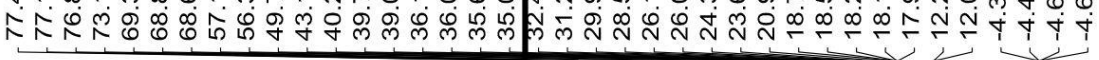

${ }^{13} \mathrm{C}$ NMR (100 MHz, $\left.\mathrm{CDCl}_{3}\right)$

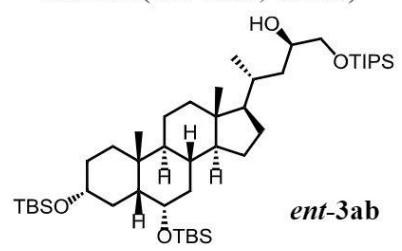

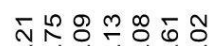

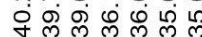

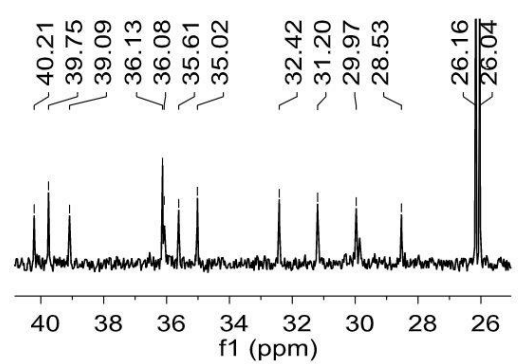

స్ల

$\begin{array}{llllllllllll}210 & 200 & 190 & 180 & 170 & 160 & 150 & 140 & 130 & 120 & 110 \quad 100 \\ \mathrm{f} 1(\mathrm{ppm})\end{array}$ 
${ }^{1} \mathrm{H}$ NMR $\left(400 \mathrm{MHz}, \mathrm{CDCl}_{3}\right)$<smiles>O[C@H](CO[SnH])c1ccccc1</smiles>
$3 a c$

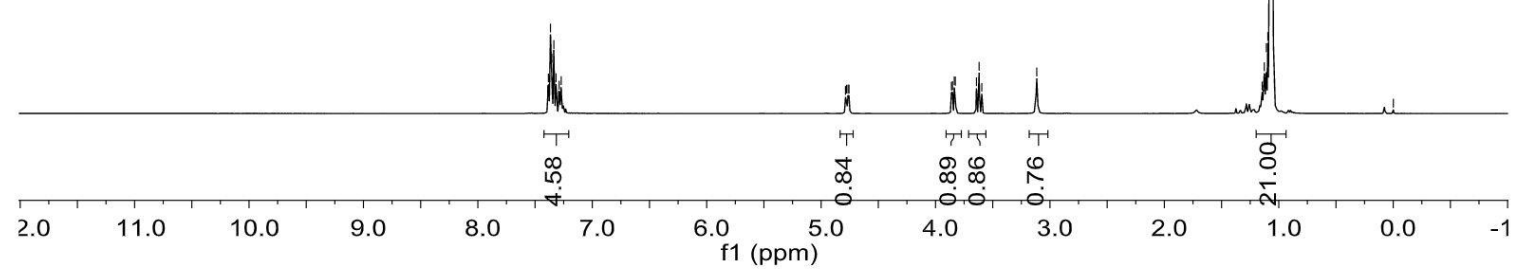

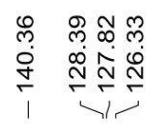

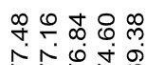

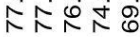

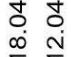
$\stackrel{i}{i} \underset{\sim}{\stackrel{1}{r}}$

${ }^{13} \mathrm{C}$ NMR (100 MHz, $\mathrm{CDCl}_{3}$ )<smiles>O[C@H](CO[I-])c1ccccc1</smiles>

3 ac

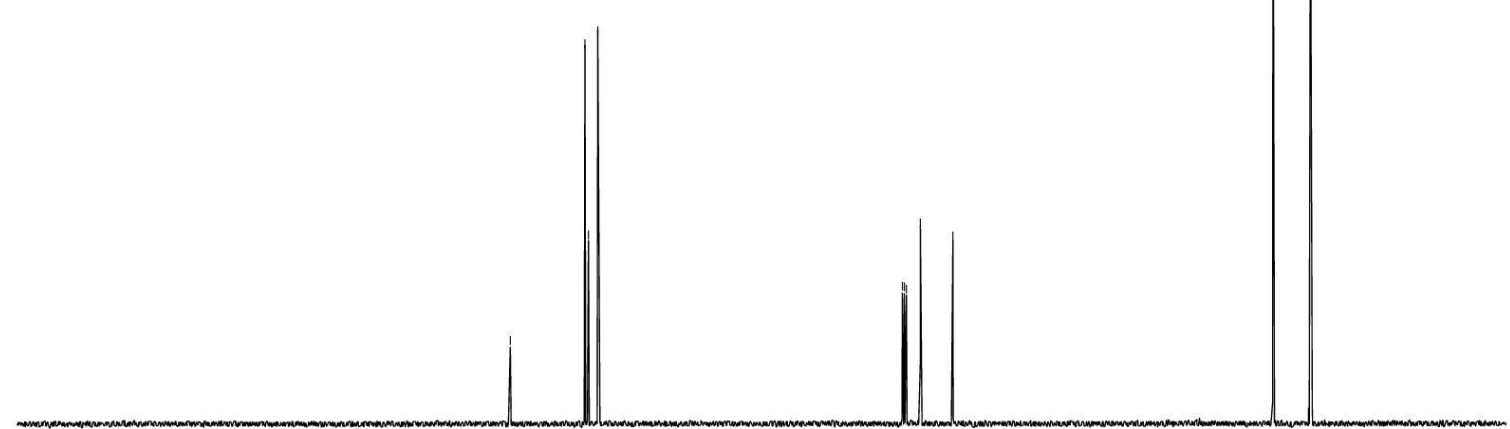

$\begin{array}{lllllllllllllllllllllll}210 & 200 & 190 & 180 & 170 & 160 & 150 & 140 & 130 & 120 & 110 & 100 & 90 & 80 & 70 & 60 & 50 & 40 & 30 & 20 & 10 & 0 & -10\end{array}$ 


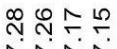

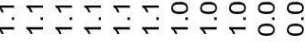

${ }^{1} \mathrm{H}$ NMR $\left(400 \mathrm{MHz}, \mathrm{CDCl}_{3}\right)$<smiles>Cc1ccc([C@@H](O)C[OH+][SH2])cc1</smiles>

$3 a d$

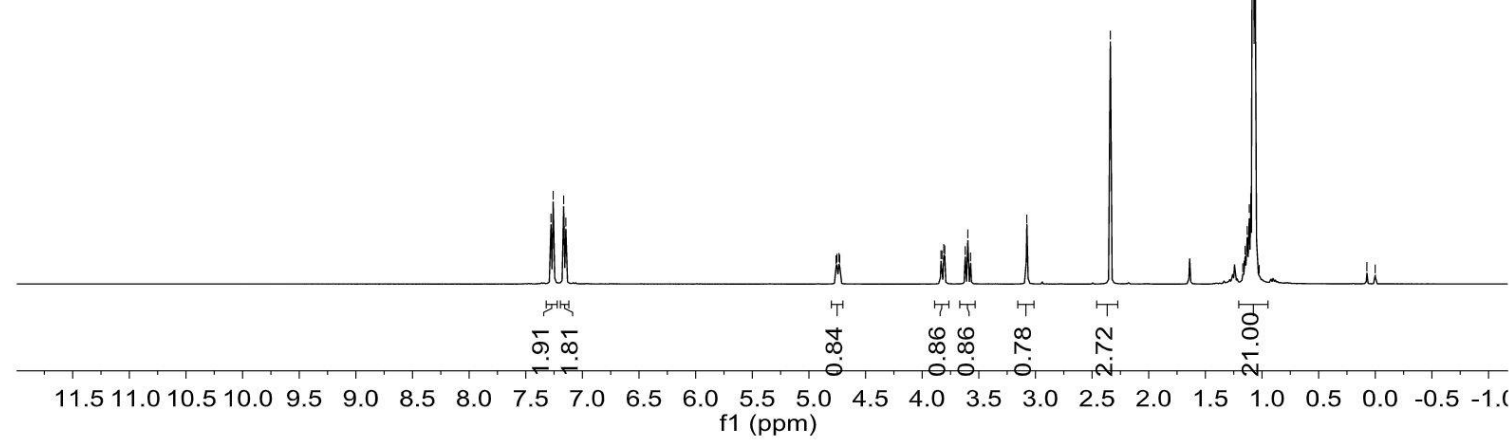

เป็ 유ำ

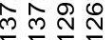

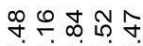

ヘペ市。

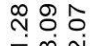

ஸे ฺ

舟

। !

${ }^{13} \mathrm{C} \mathrm{NMR}\left(100 \mathrm{MHz}, \mathrm{CDCl}_{3}\right)$<smiles>Cc1ccc([C@@H](O)C[OH+][S-](F)(F)F)cc1</smiles>

3ad

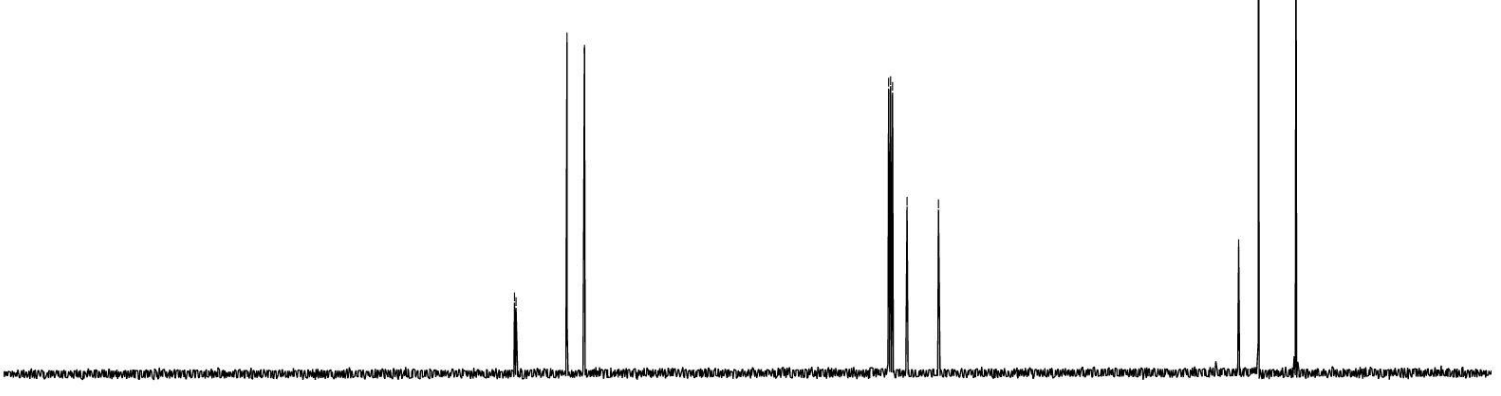

$\begin{array}{lllllllllllllllllllllll}210 & 200 & 190 & 180 & 170 & 160 & 150 & 140 & 130 & 120 & 110 & 100 & 90 & 80 & 70 & 60 & 50 & 40 & 30 & 20 & 10 & 0 & -10\end{array}$ f1 (ppm) 


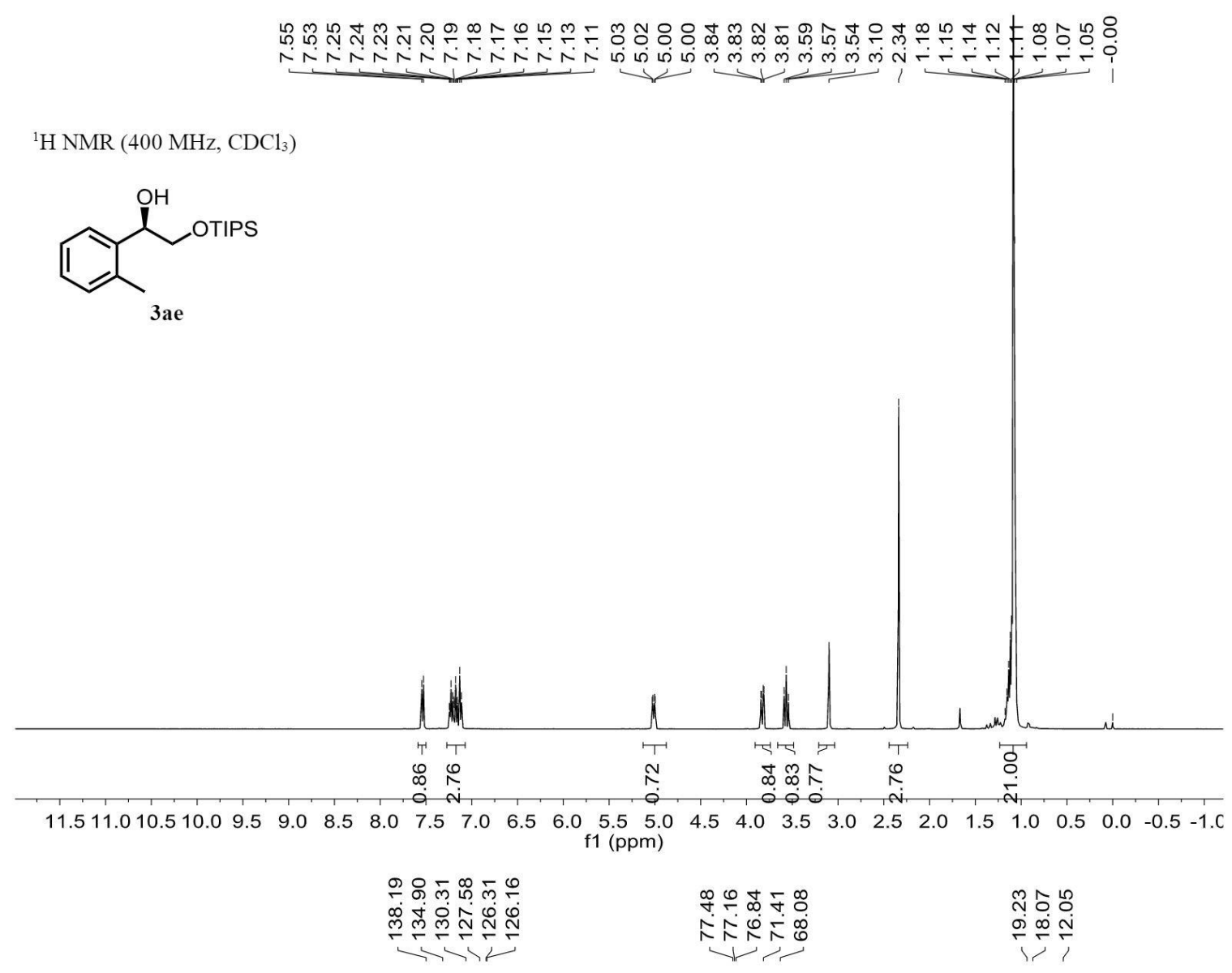

$\left.{ }^{13} \mathrm{C} \mathrm{NMR} \mathrm{(100} \mathrm{MHz,} \mathrm{CDCl}_{3}\right)$

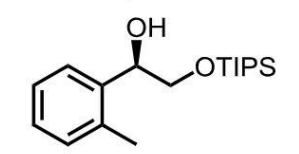

3 ae

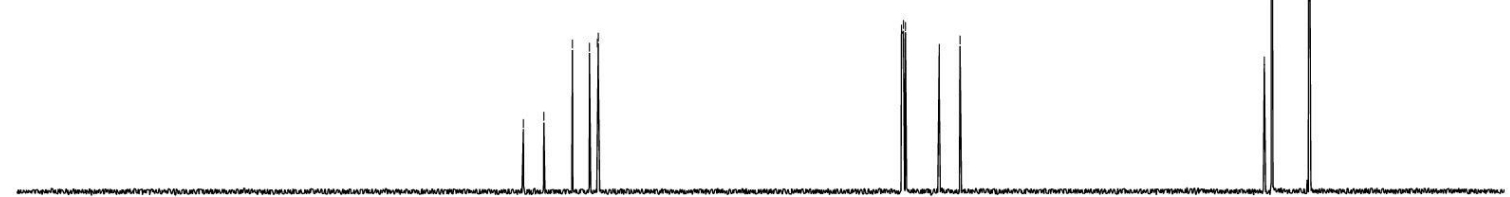

$\begin{array}{llllllllllllllllllllllllll}210 & 200 & 190 & 180 & 170 & 160 & 150 & 140 & 130 & 120 & 110 & 100 & 90 & 80 & 70 & 60 & 50 & 40 & 30 & 20 & 10 & 0 & -10\end{array}$ 


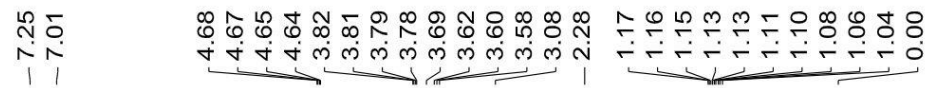

${ }^{1} \mathrm{H}$ NMR (400 MHz, $\mathrm{CDCl}_{3}$ )

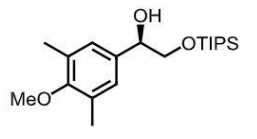

3af

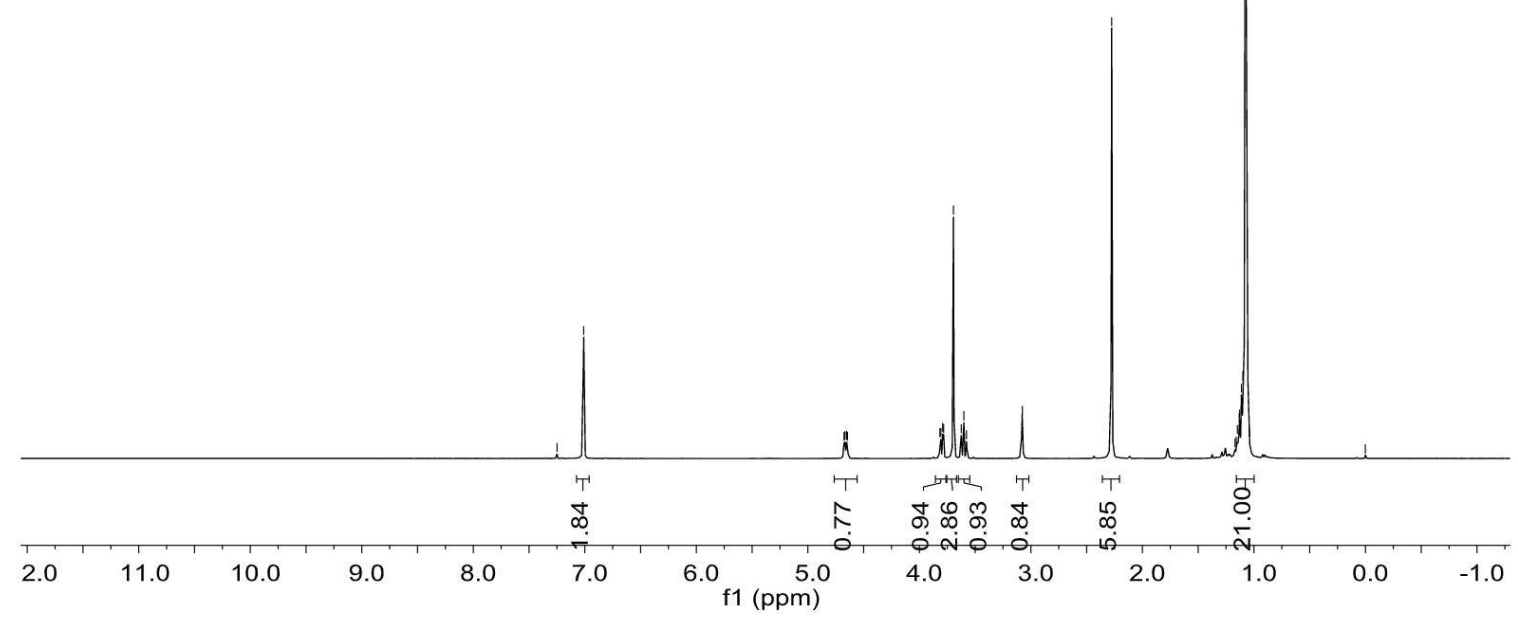

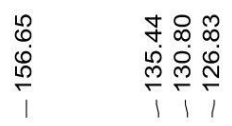

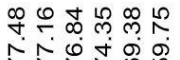

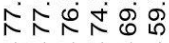

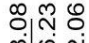

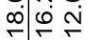

11

${ }^{13} \mathrm{C} \mathrm{NMR}\left(100 \mathrm{MHz}, \mathrm{CDCl}_{3}\right)$

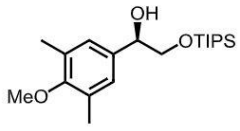

3af

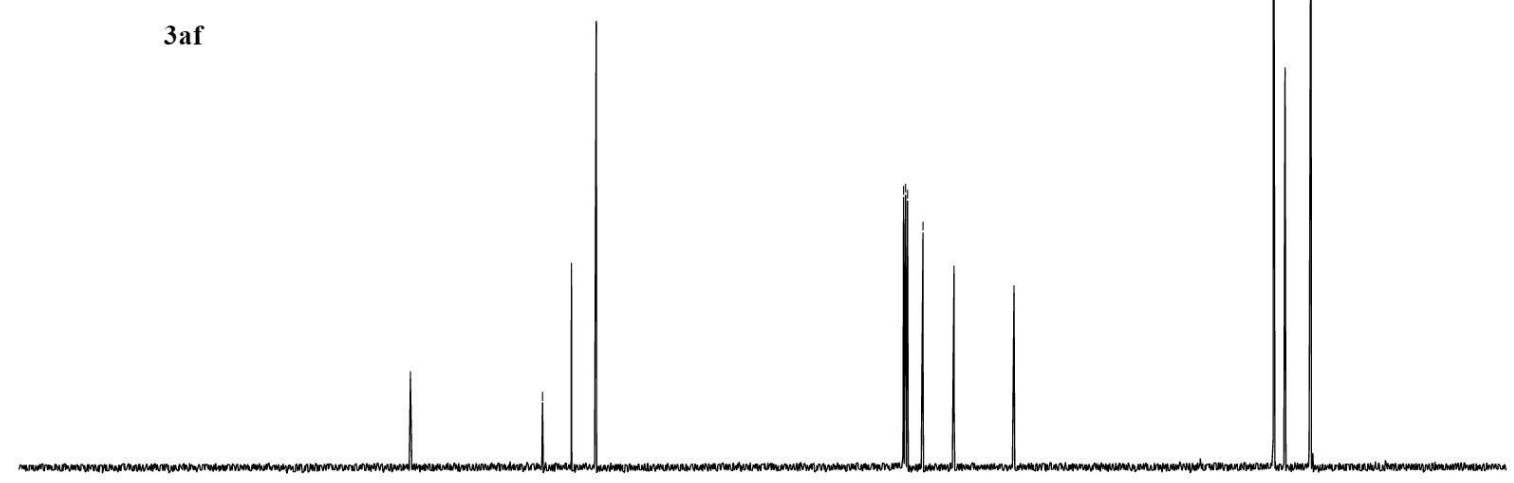

$\begin{array}{lllllllllllllllllllllll}210 & 200 & 190 & 180 & 170 & 160 & 150 & 140 & 130 & 120 & 110 & 100 & 90 & 80 & 70 & 60 & 50 & 40 & 30 & 20 & 10 & 0 & -10\end{array}$ 


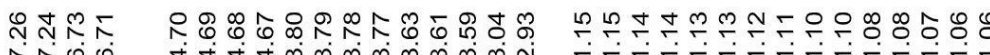

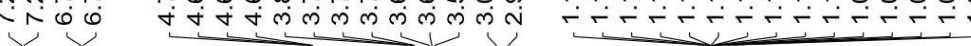

${ }^{1} \mathrm{H}$ NMR $\left(400 \mathrm{MHz}, \mathrm{CDCl}_{3}\right)$

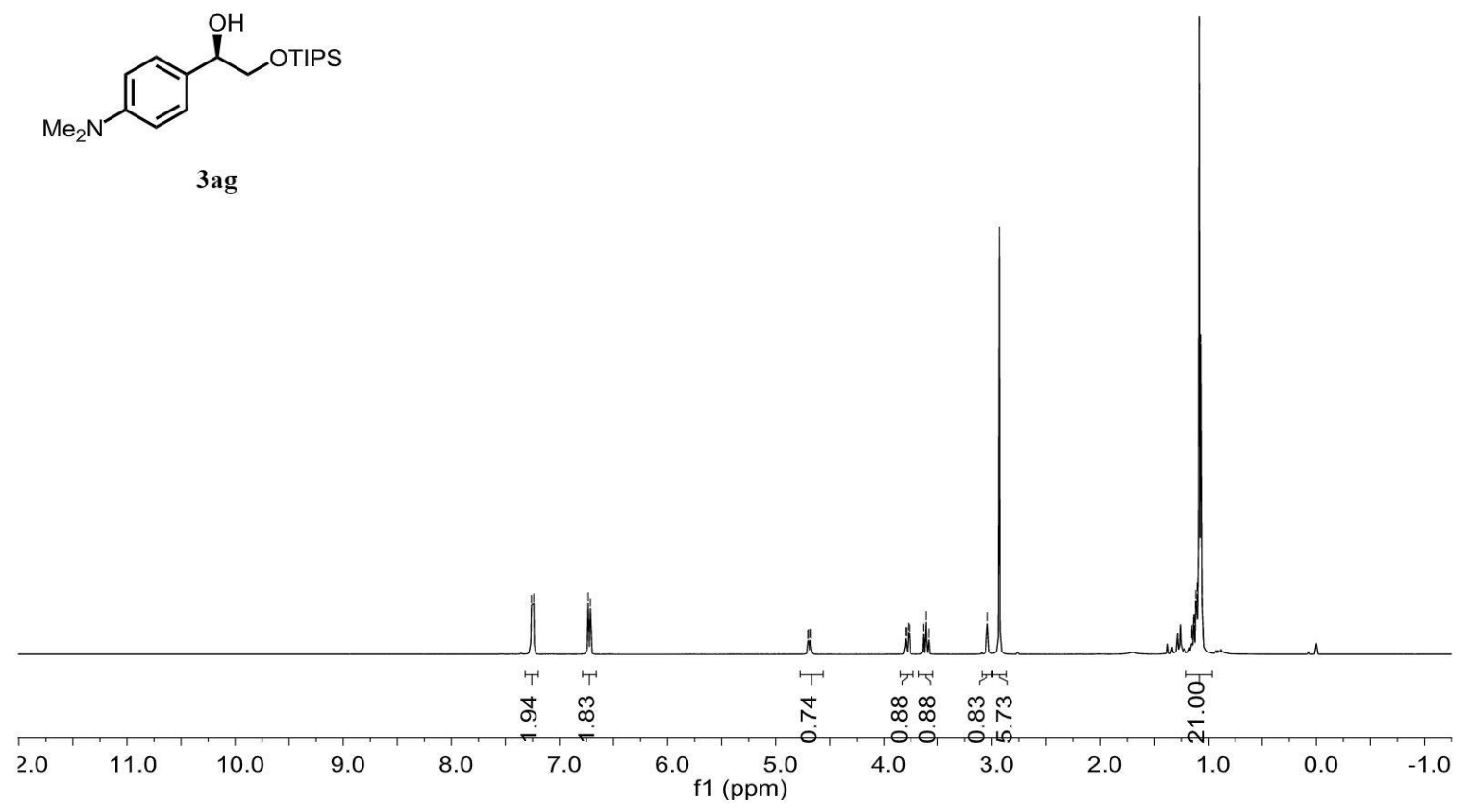

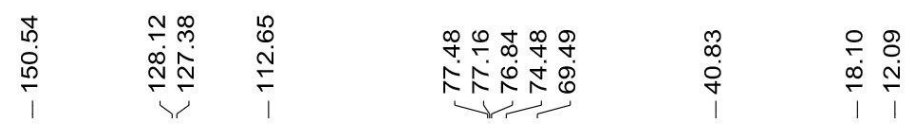

${ }^{13} \mathrm{C}$ NMR $\left(100 \mathrm{MHz}, \mathrm{CDCl}_{3}\right)$<smiles></smiles>

3ag

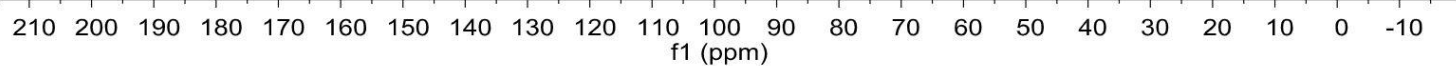




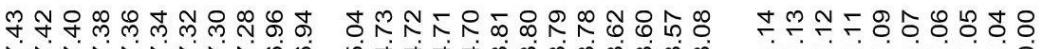

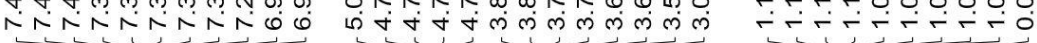

${ }^{1} \mathrm{H}$ NMR $\left(400 \mathrm{MHz}, \mathrm{CDCl}_{3}\right)$

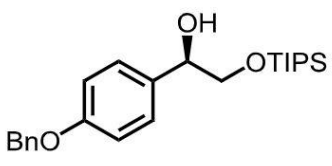

3ai

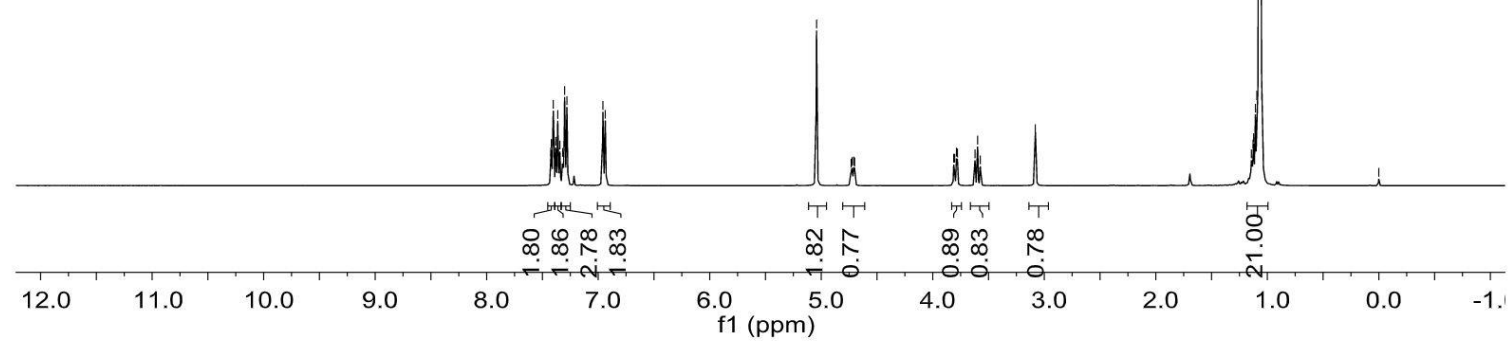

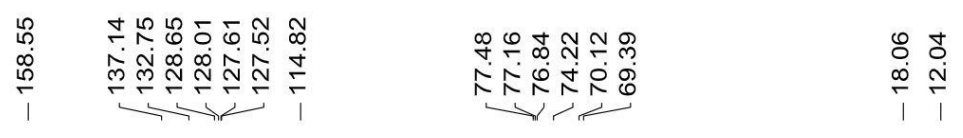

${ }^{13} \mathrm{C} \mathrm{NMR}\left(100 \mathrm{MHz}, \mathrm{CDCl}_{3}\right)$<smiles>O[C@H](CO[PbH])c1ccc(OCc2ccccc2)cc1</smiles>

3ai

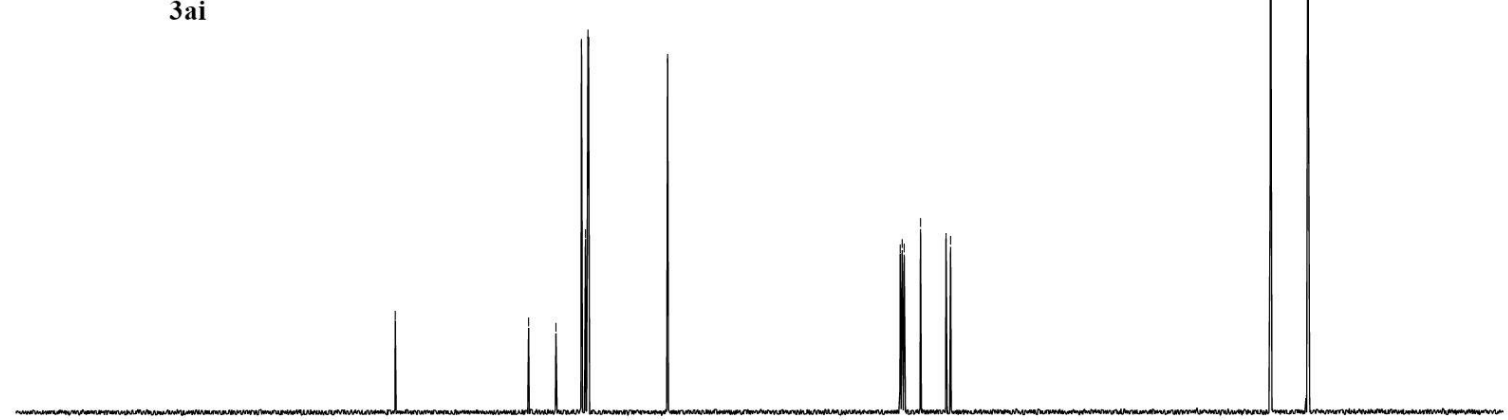

$\begin{array}{lllllllllllllllllllllll}210 & 200 & 190 & 180 & 170 & 160 & 150 & 140 & 130 & 120 & 110 & 100 & 90 & 80 & 70 & 60 & 50 & 40 & 30 & 20 & 10 & 0 & -10\end{array}$ 


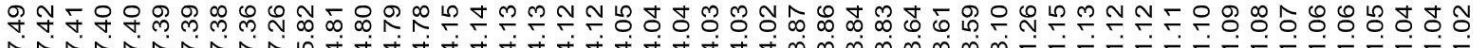

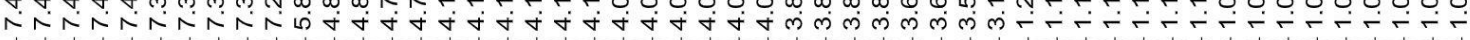

${ }^{1} \mathrm{H}$ NMR $\left(400 \mathrm{MHz}, \mathrm{CDCl}_{3}\right)$<smiles>O[C@H](CO[SnH3])c1cccc(C2OCCO2)c1</smiles>
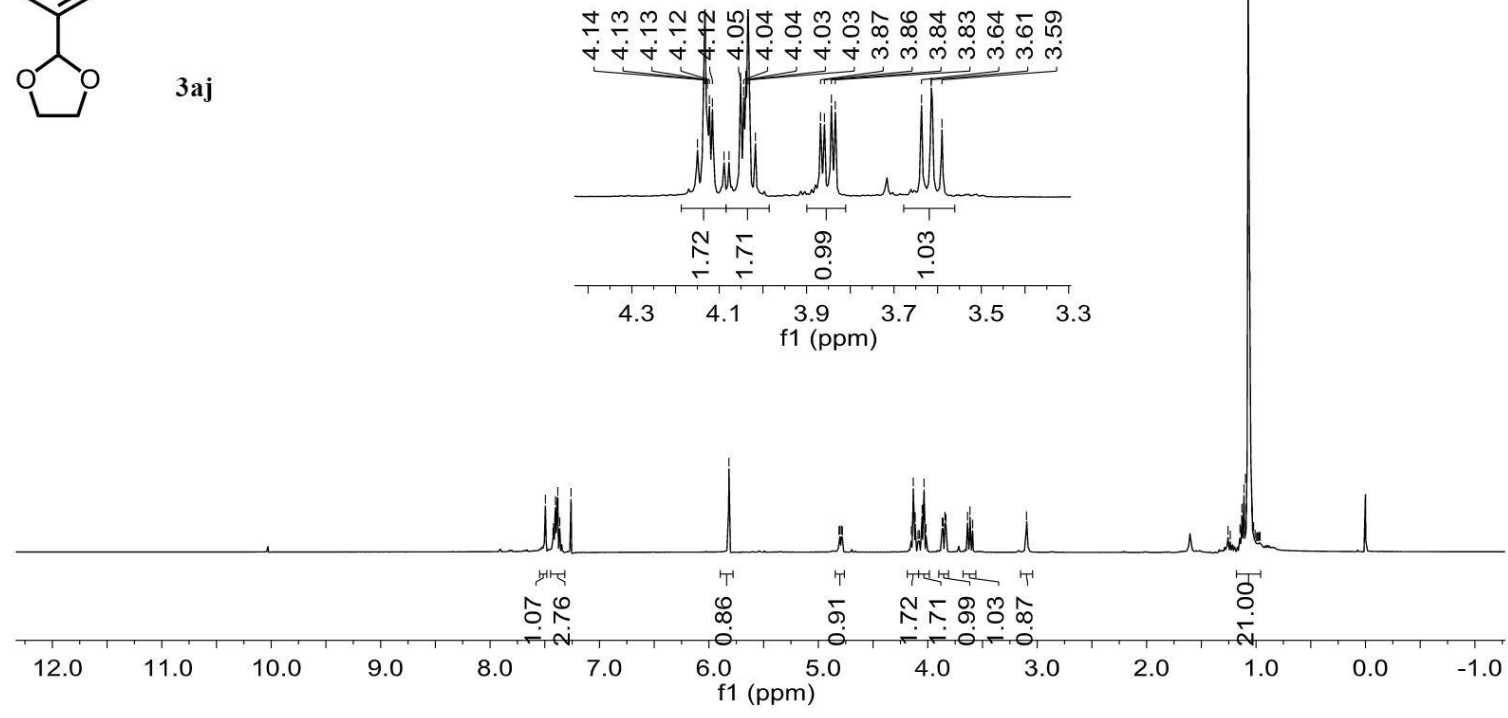

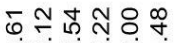

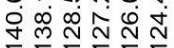
$\begin{array}{ll}1 & 0 \\ 0 & 0\end{array}$

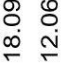

${ }^{13} \mathrm{C} \mathrm{NMR}\left(100 \mathrm{MHz}, \mathrm{CDCl}_{3}\right)$<smiles>O[C@H](CO[InH])c1cccc(C2OCCO2)c1</smiles>

3aj

$\begin{array}{lllllllllll}210 & 200 & 190 & 180 & 170 & 160 & 150 & 140 & 130 & 120 & 110 \\ \mathrm{f} 1(\mathrm{ppm})\end{array}$ 


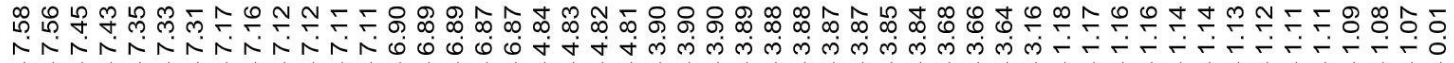

${ }^{1} \mathrm{H}$ NMR $\left(400 \mathrm{MHz}, \mathrm{CDCl}_{3}\right)$<smiles>COc1cccc(-c2ccc(C(O)CO[Na])cc2)c1</smiles>

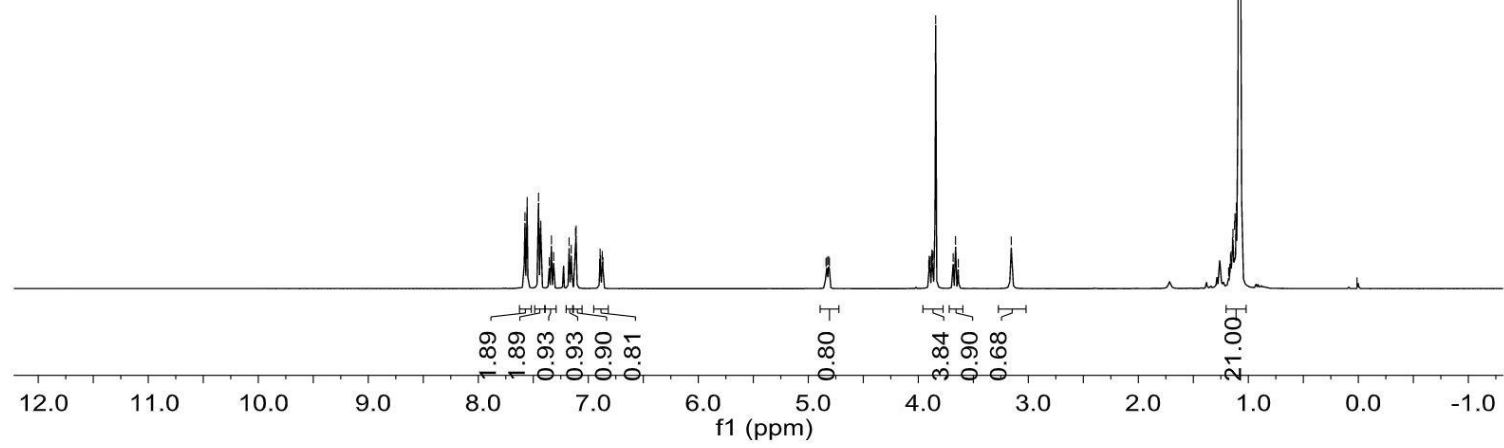

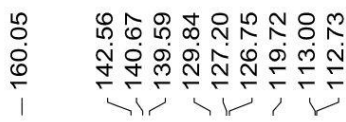

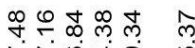

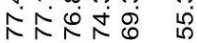

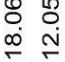

${ }^{13} \mathrm{C}$ NMR (100 MHz, $\left.\mathrm{CDCl}_{3}\right)$<smiles>COc1cccc(-c2ccc([C@@H](O)C[OH+])cc2)c1</smiles>
$\begin{array}{llllllllllll}210 & 200 & 190 & 180 & 170 & 160 & 150 & 140 & 130 & 120 & 110 & 100 \\ \mathrm{f} 1(\mathrm{ppm})\end{array}$ 
${ }^{1} \mathrm{H}$ NMR (400 MHz, $\mathrm{CDCl}_{3}$ )<smiles>O[C@H](CO[I+]S(F)(F)F)c1ccc(F)cc1</smiles>

3al

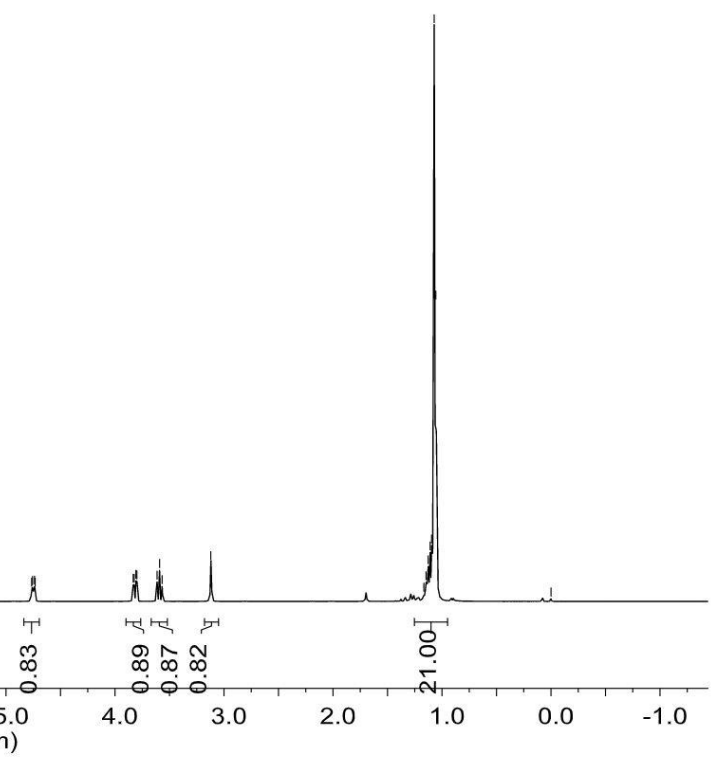

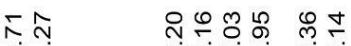

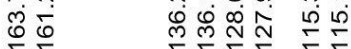

+ִ

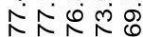

¿

in

$\stackrel{\infty}{\sim} \stackrel{1}{\leftarrow}$

${ }^{13} \mathrm{C} \mathrm{NMR}\left(100 \mathrm{MHz}, \mathrm{CDCl}_{3}\right)$<smiles>O[C@H](CO[SnH3])c1ccc(F)cc1</smiles>

3al

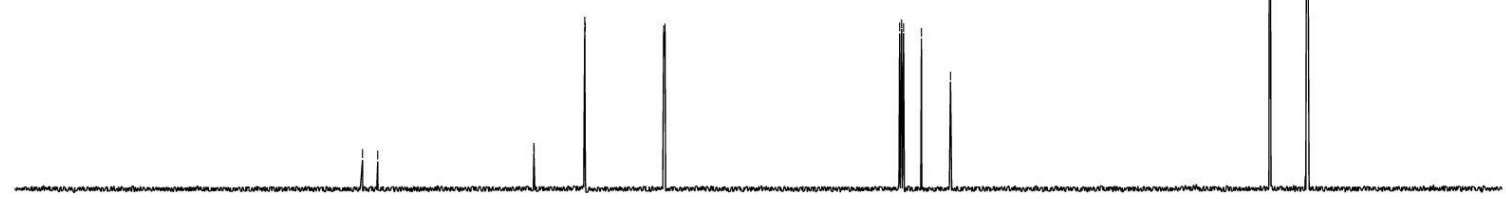

$\begin{array}{lllllllllllllllllllllll}210 & 200 & 190 & 180 & 170 & 160 & 150 & 140 & 130 & 120 & 110 & 100 & 90 & 80 & 70 & 60 & 50 & 40 & 30 & 20 & 10 & 0 & -10\end{array}$ 


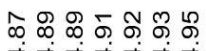

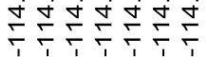

${ }^{19} \mathrm{~F} \mathrm{NMR}\left(377 \mathrm{MHz}, \mathrm{CDCl}_{3}\right)$<smiles>O[C@H](C[OH2+])c1ccc(F)cc1</smiles>

3al

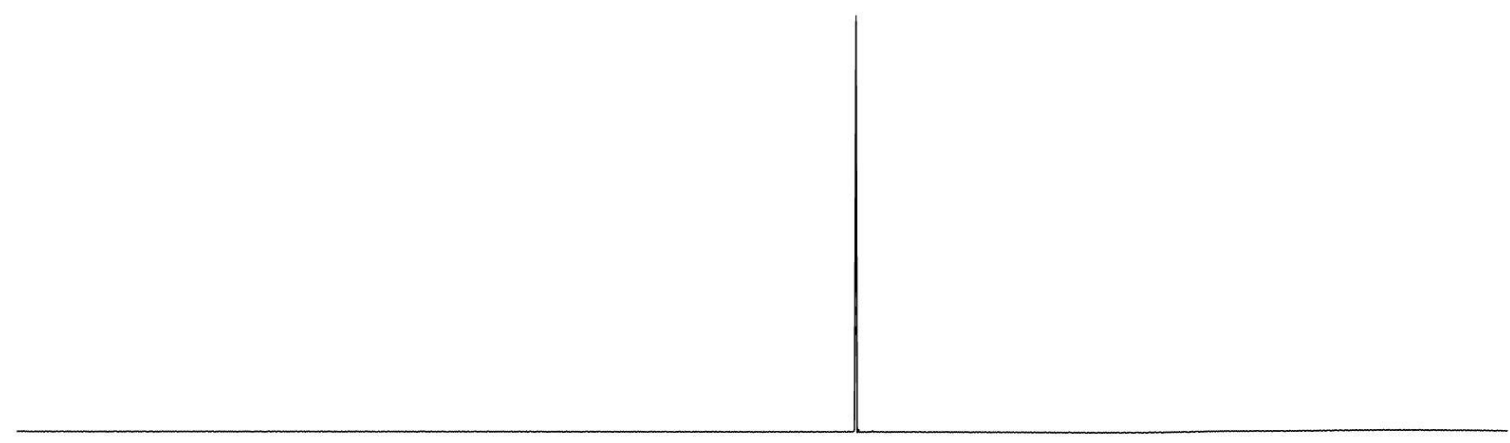

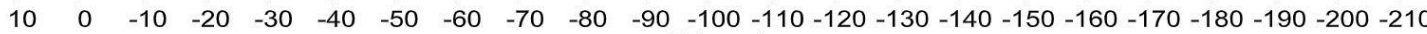
f1 (ppm) 
${ }^{1} \mathrm{H}$ NMR (400 MHz, $\left.\mathrm{CDCl}_{3}\right)$<smiles>O[C@H](CO[SnH3])c1ccc(Cl)cc1</smiles>

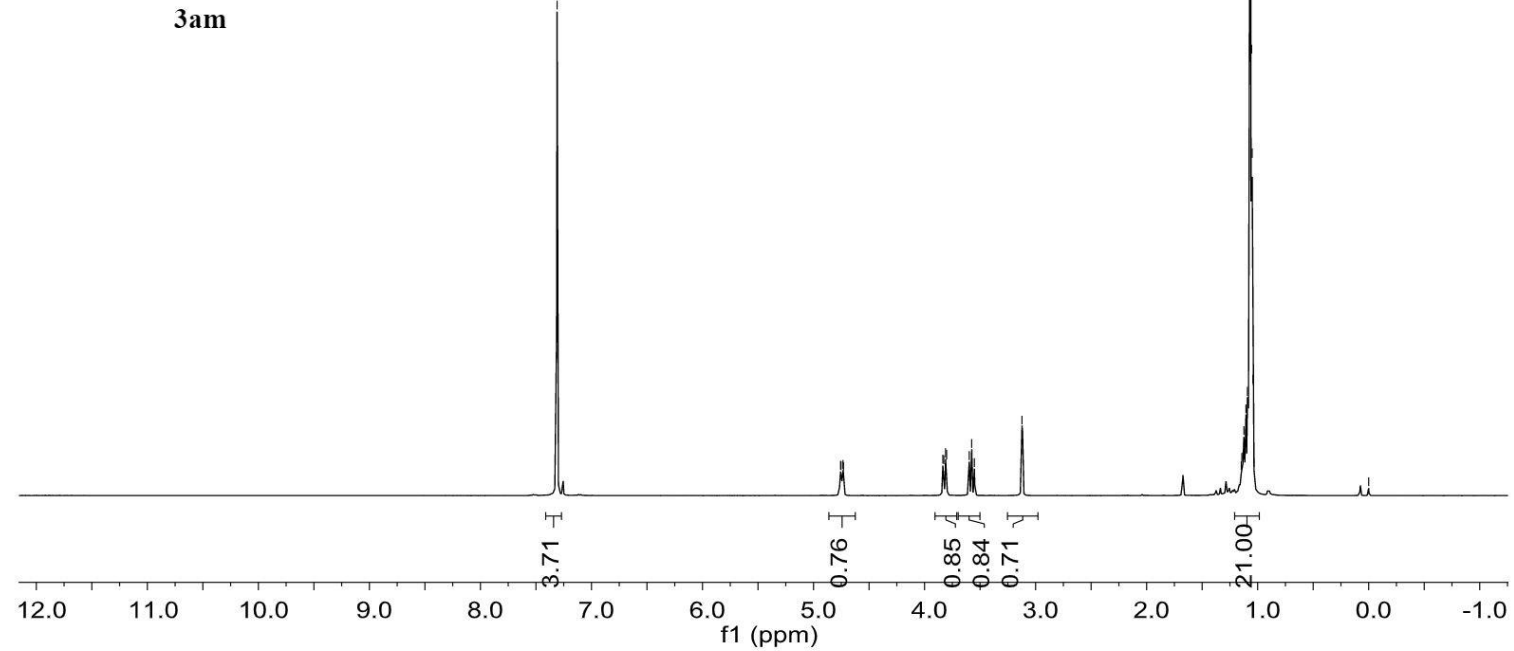

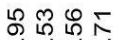

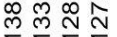

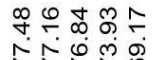

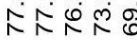

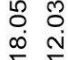

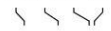

तr

${ }^{13} \mathrm{C} \mathrm{NMR}\left(100 \mathrm{MHz}, \mathrm{CDCl}_{3}\right)$<smiles>O[C@H](CO[SbH2])c1ccc(Cl)cc1</smiles>

$3 a m$

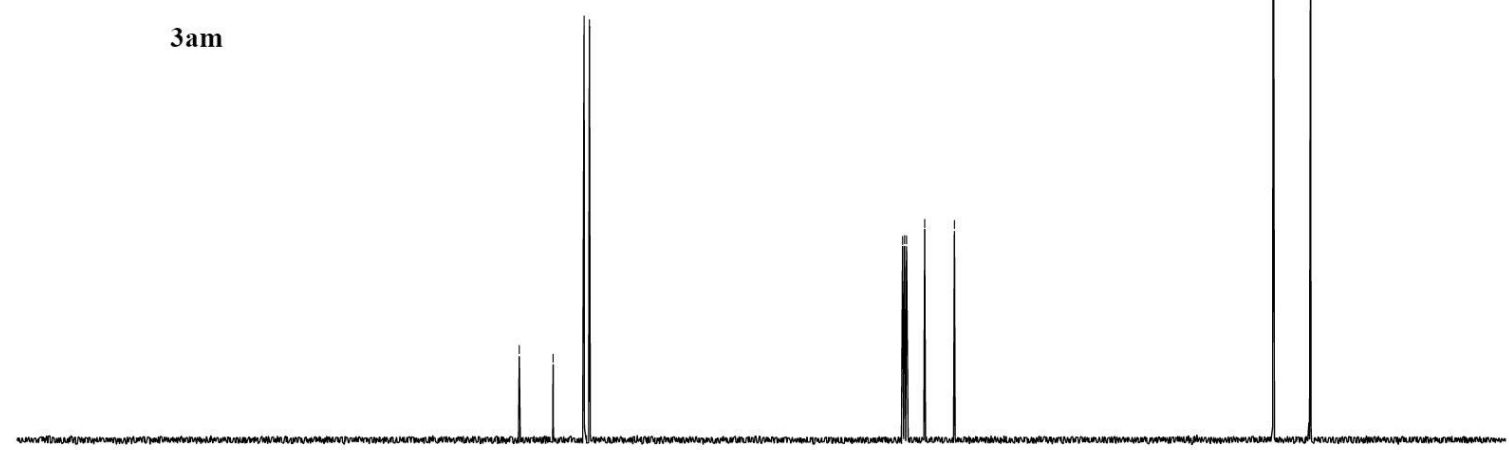

$\begin{array}{lllllllllllll}210 & 200 & 190 & 180 & 170 & 160 & 150 & 140 & 130 & 120 & 110 & 100 & 90\end{array}$

f1 $(\mathrm{ppm})$ 
${ }^{1} \mathrm{H}$ NMR $\left(400 \mathrm{MHz}, \mathrm{CDCl}_{3}\right)$<smiles>O[C@H](CO[PbH])c1ccc(Br)cc1</smiles>

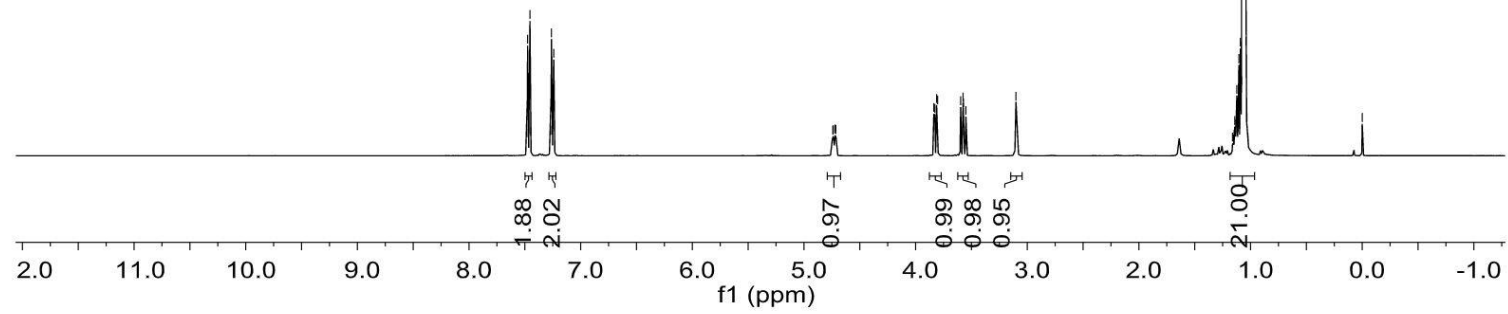

ㄴ.

ले

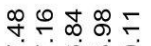

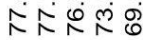

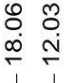

${ }^{13} \mathrm{C}$ NMR (100 MHz, $\left.\mathrm{CDCl}_{3}\right)$<smiles>O[C@H](CO[SbH2])c1ccc(Br)cc1</smiles>

3an

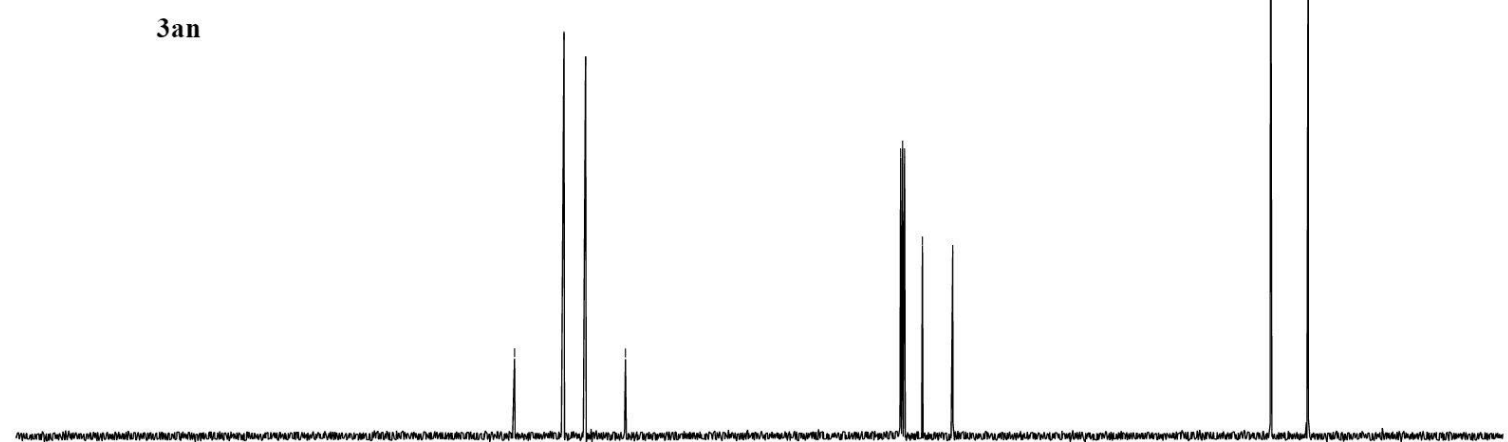

$\begin{array}{lllllllllllllllllllllll}210 & 200 & 190 & 180 & 170 & 160 & 150 & 140 & 130 & 120 & 110 & 100 & 90 & 80 & 70 & 60 & 50 & 40 & 30 & 20 & 10 & 0 & -10\end{array}$ f1 (ppm) 
ํำ

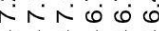

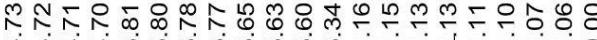

ait if

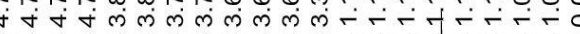

${ }^{1} \mathrm{H}$ NMR $\left(400 \mathrm{MHz}, \mathrm{CDCl}_{3}\right)$<smiles></smiles>

3ao
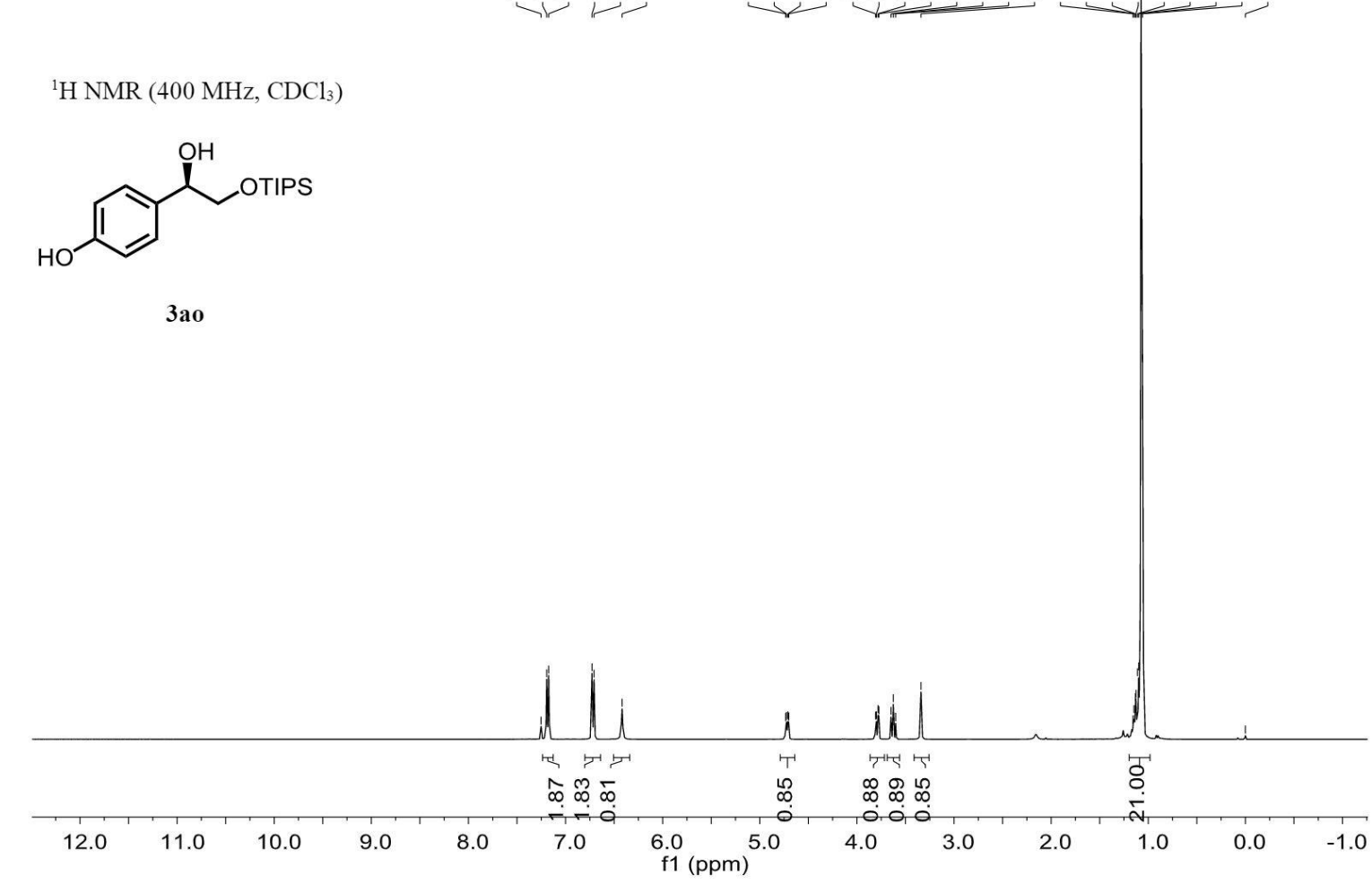

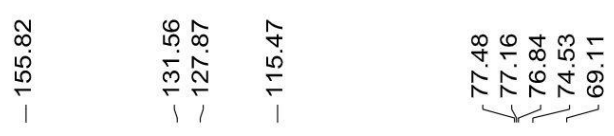

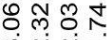

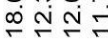

${ }^{13} \mathrm{C}$ NMR (100 MHz, $\left.\mathrm{CDCl}_{3}\right)$<smiles></smiles>

3 ao

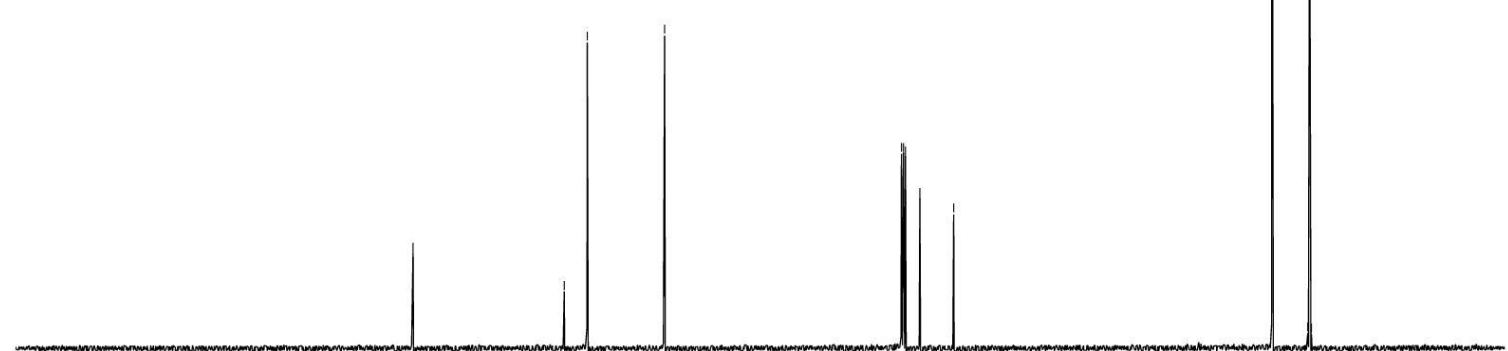

$\begin{array}{lllllllllllllllllllllll}210 & 200 & 190 & 180 & 170 & 160 & 150 & 140 & 130 & 120 & 110 & 100 & 90 & 80 & 70 & 60 & 50 & 40 & 30 & 20 & 10 & 0 & -10\end{array}$ 
${ }^{1} \mathrm{H}$ NMR (400 MHz, $\left.\mathrm{CDCl}_{3}\right)$<smiles>O[C@H](CO[I-]S)c1ccc(C(F)(F)F)cc1</smiles>

3ap
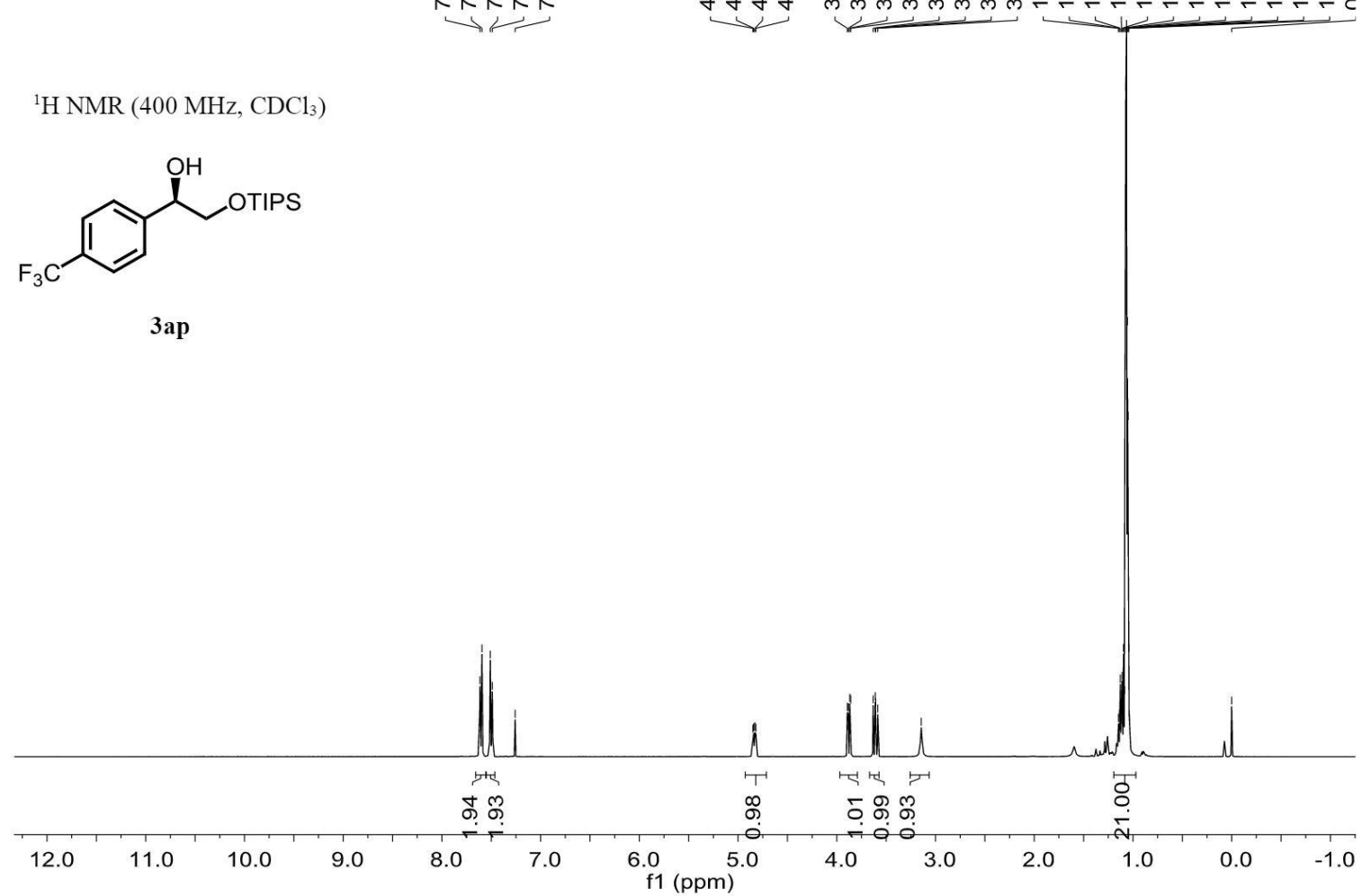

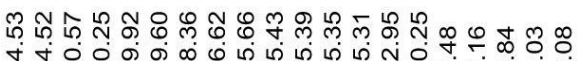

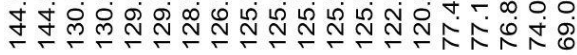

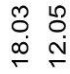

${ }^{13} \mathrm{C} \mathrm{NMR}\left(100 \mathrm{MHz}, \mathrm{CDCl}_{3}\right)$<smiles>O[C@H](C[OH+]S)c1ccc(C(F)(F)F)cc1</smiles>

3ap

(1)

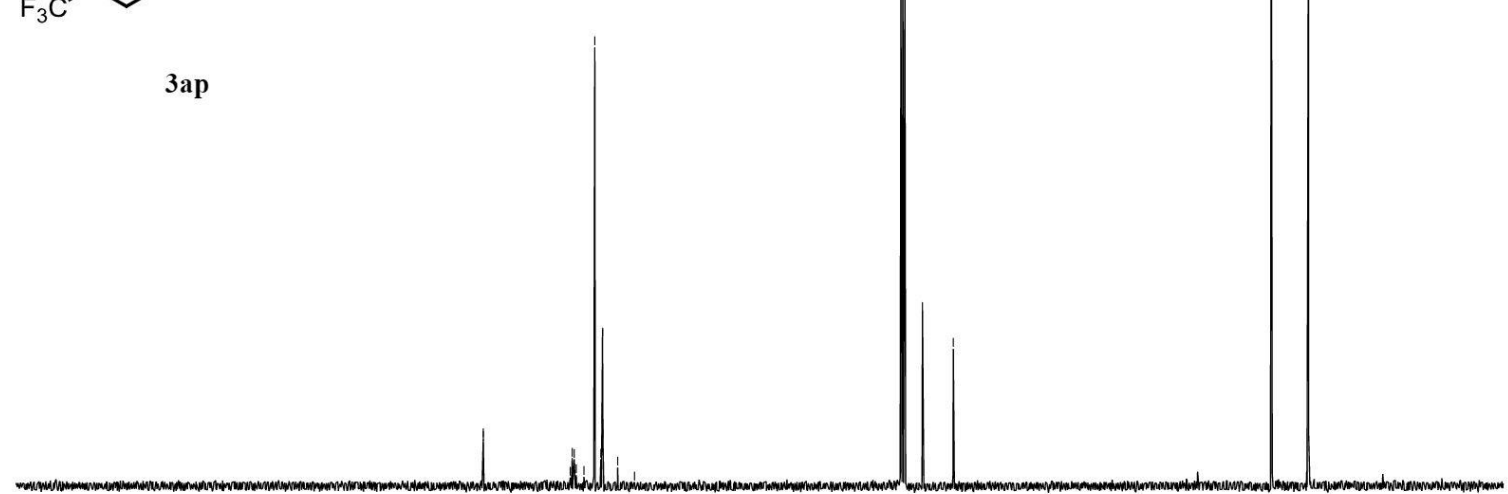

$\begin{array}{lllllllllllllllllllllll}210 & 200 & 190 & 180 & 170 & 160 & 150 & 140 & 130 & 120 & 110 & 100 & 90 & 80 & 70 & 60 & 50 & 40 & 30 & 20 & 10 & 0 & -10\end{array}$ 
${ }^{19} \mathrm{~F}$ NMR $\left(377 \mathrm{MHz}, \mathrm{CDCl}_{3}\right)$<smiles>O[C@H](CO[In])c1ccc(C(F)(F)F)cc1</smiles>

3ap

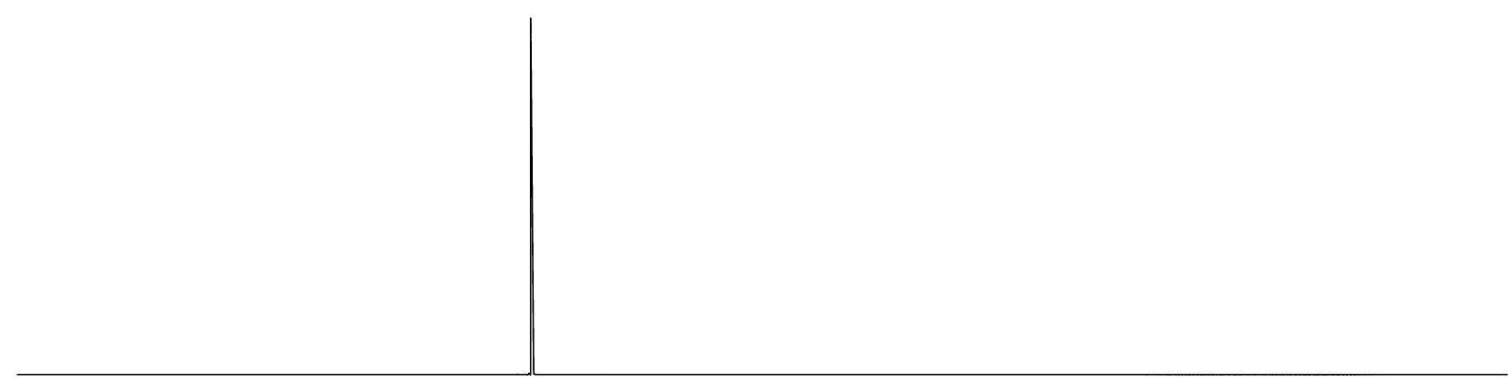

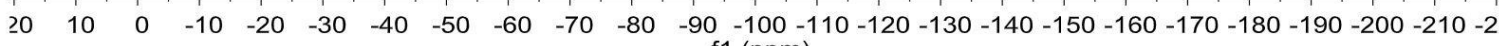
f1 (ppm) 
${ }^{1} \mathrm{H}$ NMR $\left(400 \mathrm{MHz}, \mathrm{CDCl}_{3}\right)$<smiles>N#[N+]c1ccc(C(O)COP)cc1</smiles>

$3 a q$

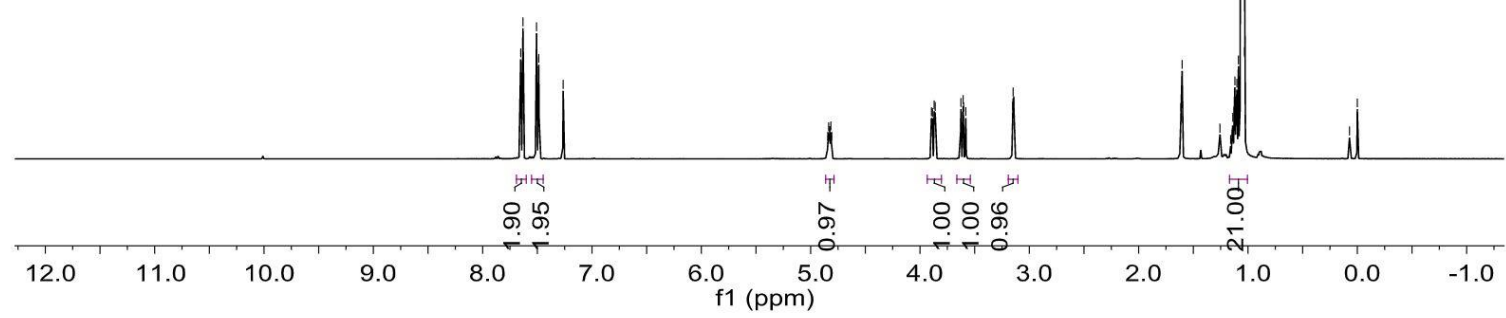

${ }^{13} \mathrm{C} \mathrm{NMR}\left(100 \mathrm{MHz}, \mathrm{CDCl}_{3}\right)$

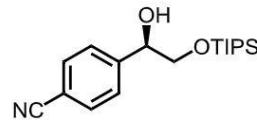

$3 a q$

D.

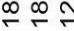

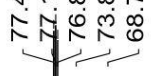

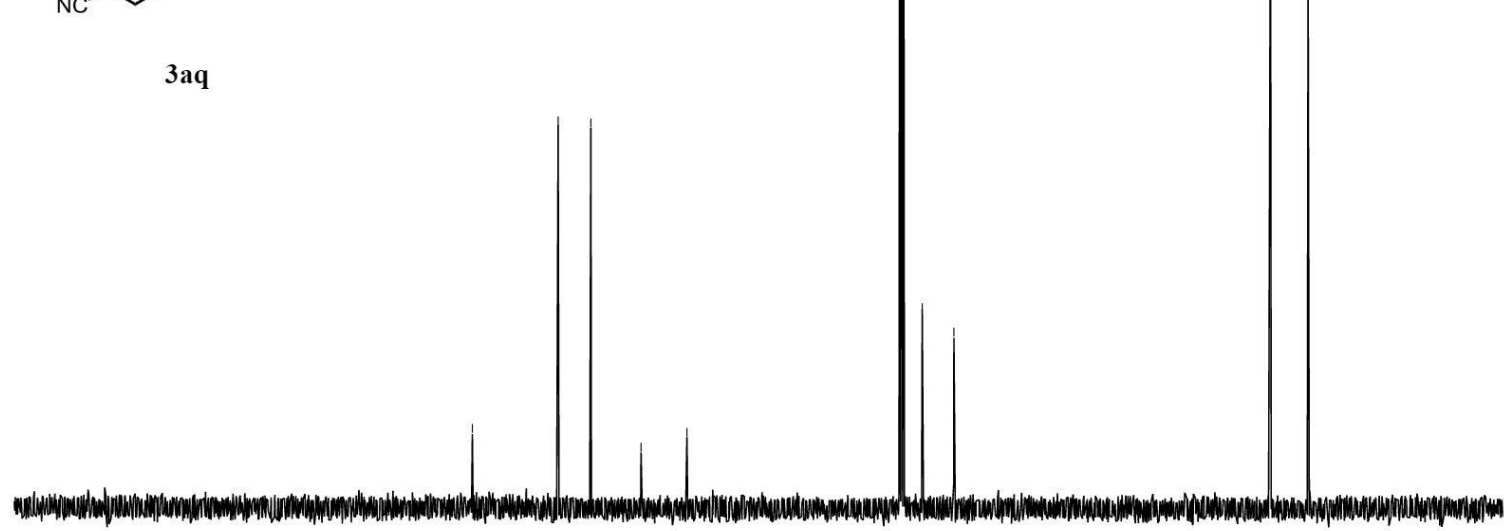

$\begin{array}{lllllllllllll}210 & 200 & 190 & 180 & 170 & 160 & 150 & 140 & 130 & 120 & 110 & 100 & 90\end{array}$ $\mathrm{f1}(\mathrm{ppm})$ 


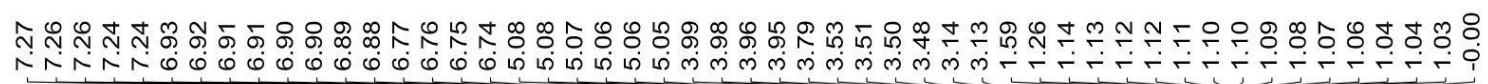

${ }^{1} \mathrm{H}$ NMR $\left(400 \mathrm{MHz}, \mathrm{CDCl}_{3}\right)$

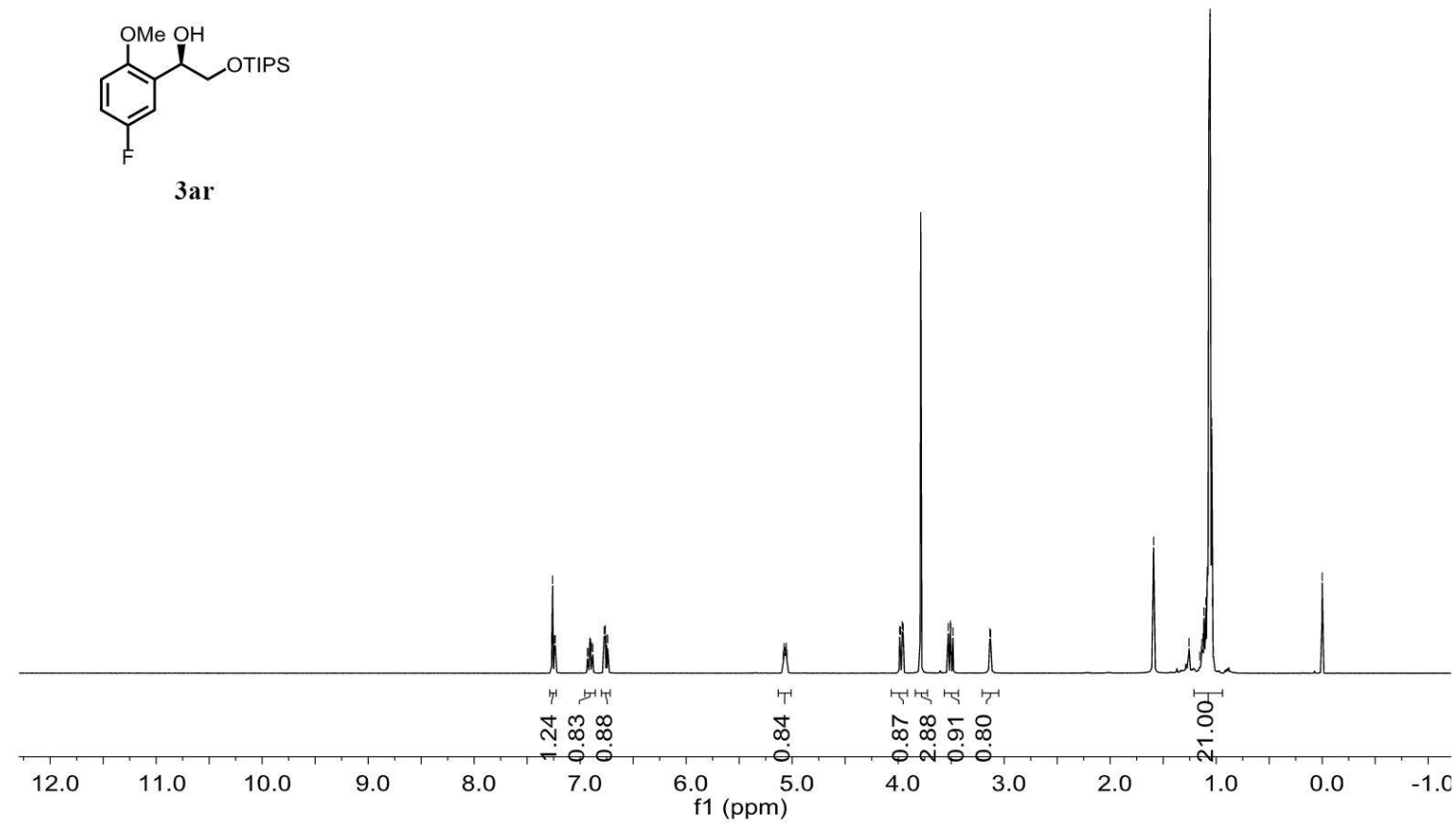

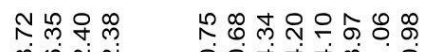

旁织

$\stackrel{\infty}{+\infty} \oplus$

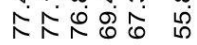

ํํㅇำ

$\stackrel{\infty}{\sim} \stackrel{\infty}{\sim}$

${ }^{13} \mathrm{C} \mathrm{NMR}\left(100 \mathrm{MHz}, \mathrm{CDCl}_{3}\right)$<smiles>COc1ccc(F)cc1[C@@H](O)CO[AsH3]</smiles>

$3 a r$

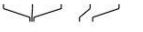

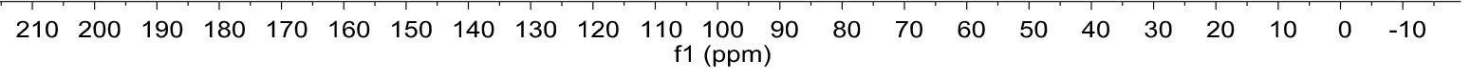


${ }^{19} \mathrm{~F} \mathrm{NMR}\left(377 \mathrm{MHz}, \mathrm{CDCl}_{3}\right)$<smiles>OCCOCc1cc(F)ccc1O</smiles>

3ar

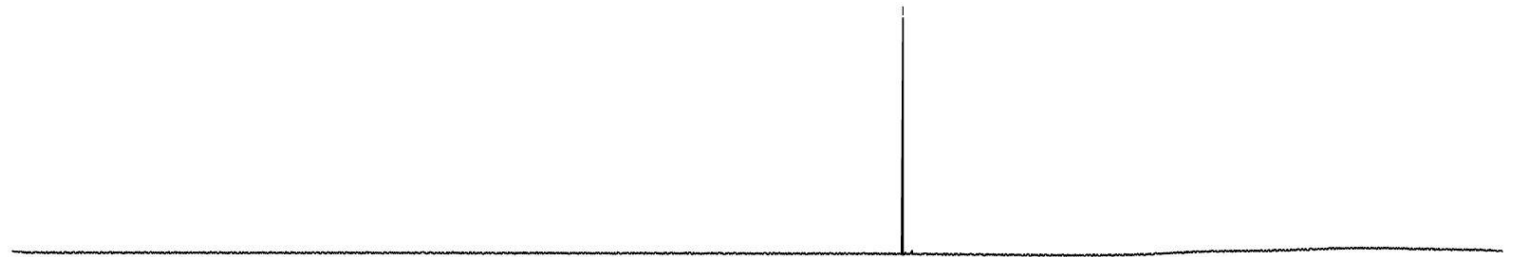

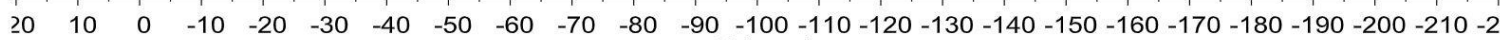
f1 (ppm) 


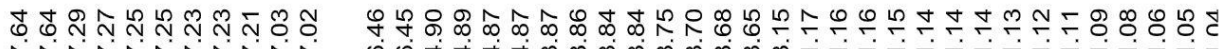

${ }^{1} \mathrm{H}$ NMR $\left(400 \mathrm{MHz}, \mathrm{CDCl}_{3}\right)$
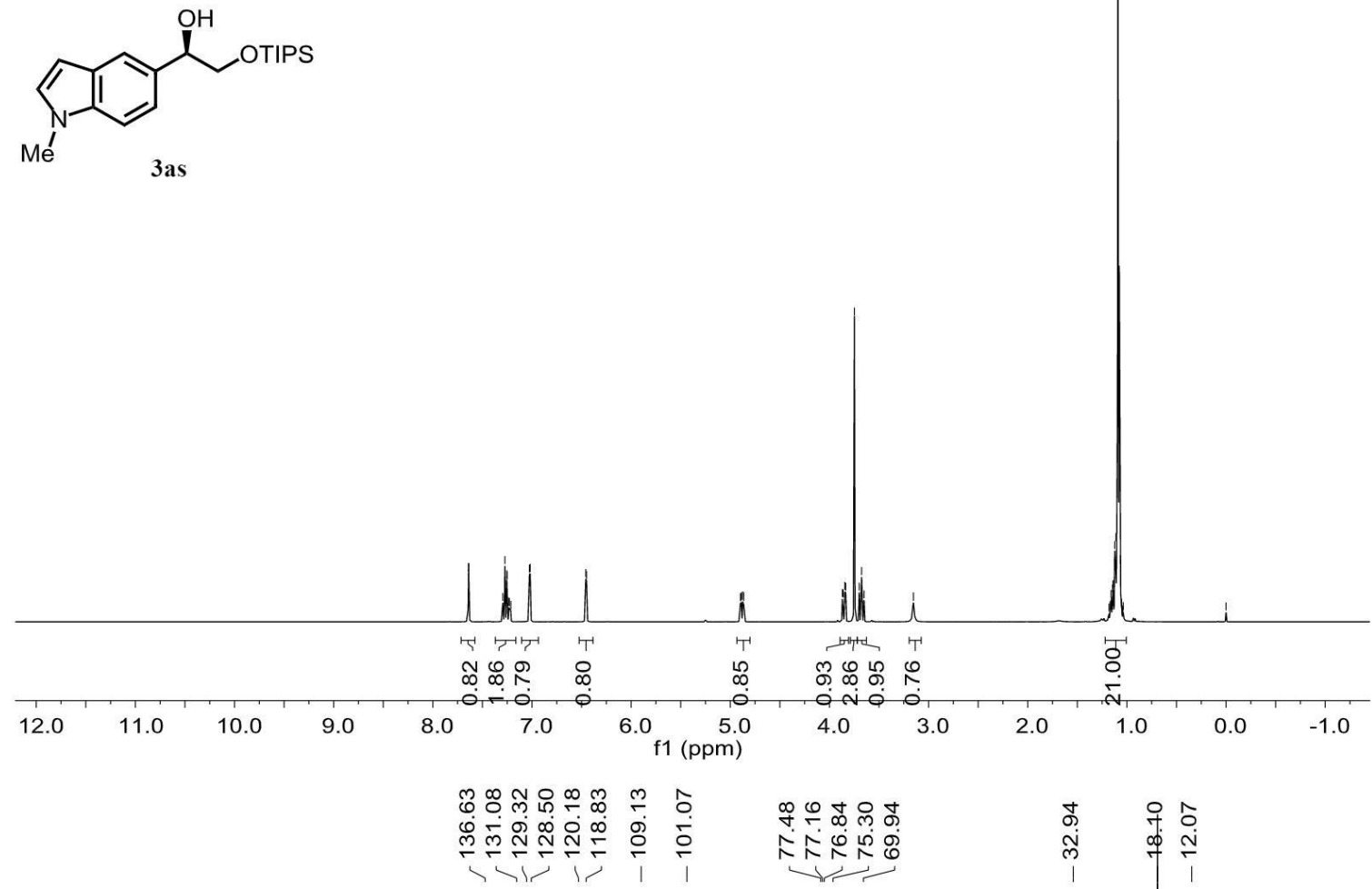

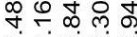

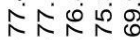

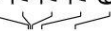

${ }^{13} \mathrm{C}$ NMR $\left(100 \mathrm{MHz}, \mathrm{CDCl}_{3}\right)$

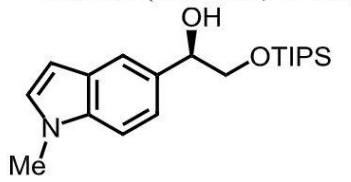

3as

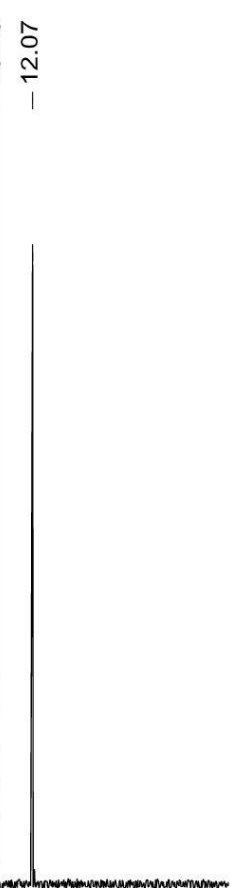

$\begin{array}{lllllllllllllllllllllll}210 & 200 & 190 & 180 & 170 & 160 & 150 & 140 & 130 & 120 & 110 & 100 & 90 & 80 & 70 & 60 & 50 & 40 & 30 & 20 & 10 & 0 & -10\end{array}$ 
${ }^{1} \mathrm{H}$ NMR (400 MHz, $\left.\mathrm{CDCl}_{3}\right)$<smiles>O[C@H](CO[In]S)c1ccoc1</smiles>

3at

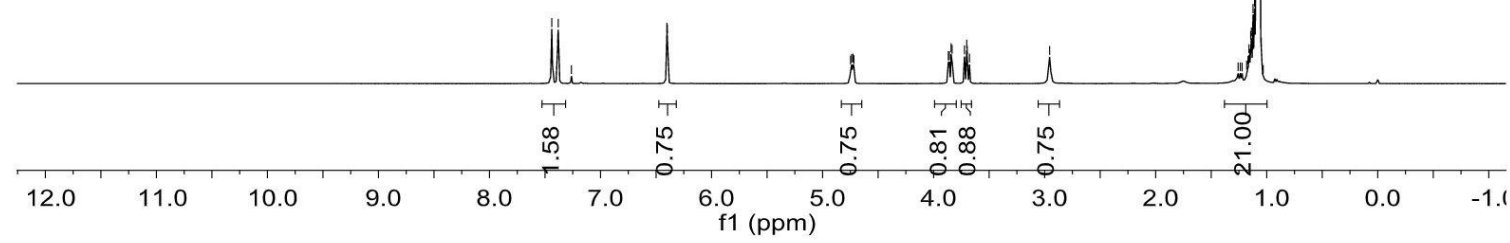

\begin{tabular}{|c|c|c|c|c|}
\hline $\begin{array}{l}\stackrel{0}{\infty} \bar{\infty} \\
\stackrel{m}{\sim} \\
\stackrel{m}{\sim}\end{array}$ & $\begin{array}{l}\stackrel{2}{0} \\
\stackrel{\text { Dे }}{\mathrm{N}}\end{array}$ & $\begin{array}{l}\infty \\
\infty \\
\infty \\
0 \\
0\end{array}$ & 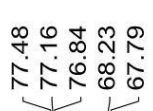 & 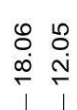 \\
\hline
\end{tabular}

${ }^{13} \mathrm{C} \mathrm{NMR}\left(100 \mathrm{MHz}, \mathrm{CDCl}_{3}\right)$

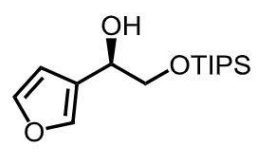

3at

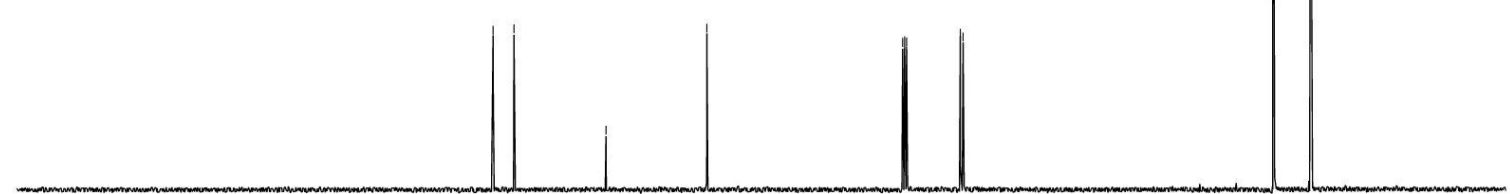

$\begin{array}{llllllllllllllllllllllllllllllll}210 & 200 & 190 & 180 & 170 & 160 & 150 & 140 & 130 & 120 & 110 & 100 & 90 & 80 & 70 & 60 & 50 & 40 & 30 & 20 & 10 & 0 & -10\end{array}$ 
${ }^{1} \mathrm{H}$ NMR (400 MHz, $\mathrm{CDCl}_{3}$ )

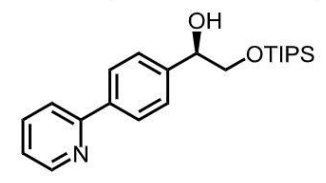

3au

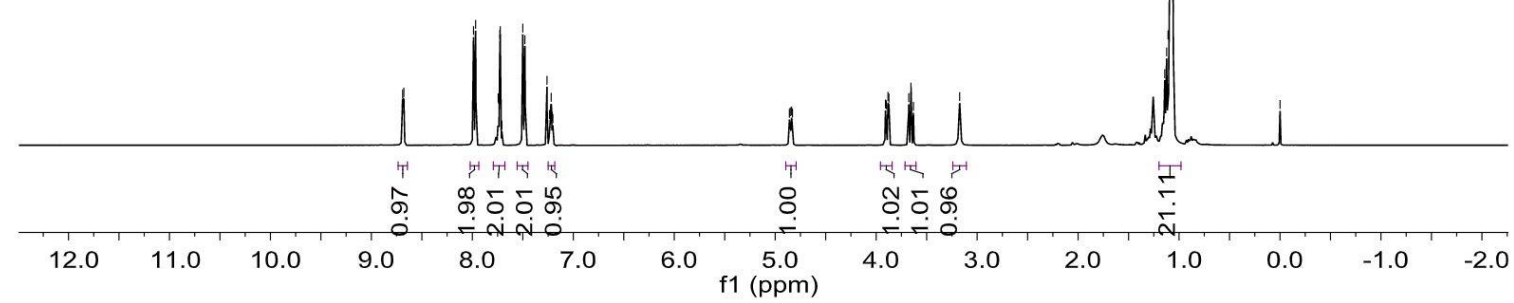

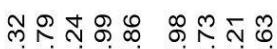

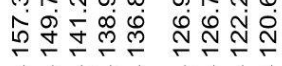

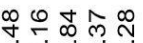

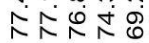

요

슬

$\stackrel{\infty}{\infty}$

${ }^{13} \mathrm{C}$ NMR $\left(100 \mathrm{MHz}, \mathrm{CDCl}_{3}\right)$<smiles>[13CH3]OC[C@@H](O)c1ccc(-c2ccccn2)cc1</smiles>

3au

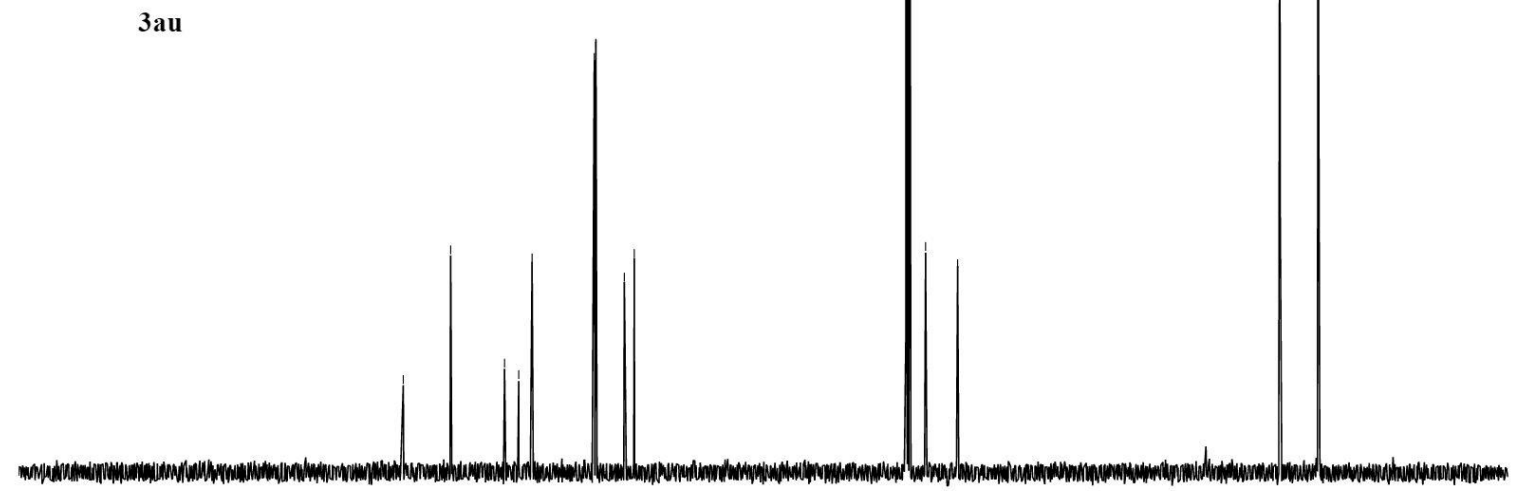

$\begin{array}{lllllllllllllllllllllll}210 & 200 & 190 & 180 & 170 & 160 & 150 & 140 & 130 & 120 & 110 & 100 & 90 & 80 & 70 & 60 & 50 & 40 & 30 & 20 & 10 & 0 & -10\end{array}$ 


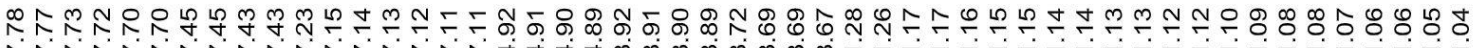

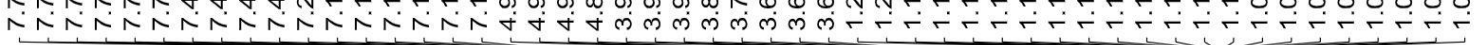
${ }^{1} \mathrm{H}$ NMR $\left(400 \mathrm{MHz}, \mathrm{CDCl}_{3}\right)$

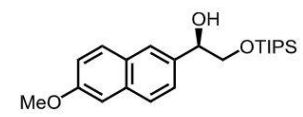

3av

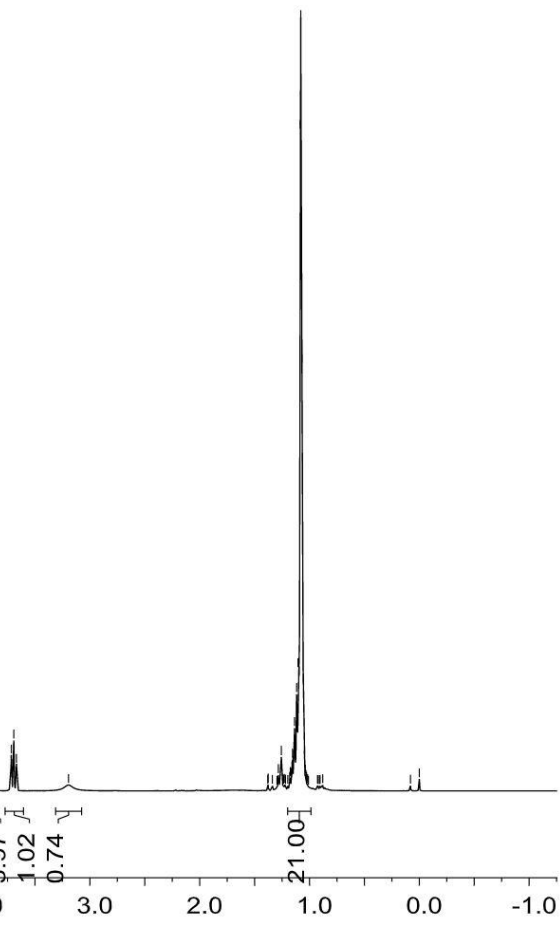

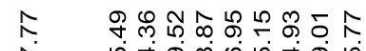

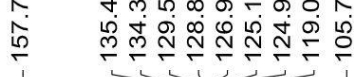

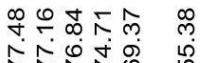

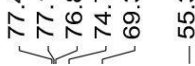

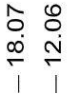

${ }^{13} \mathrm{C}$ NMR (100 MHz, $\mathrm{CDCl}_{3}$ )

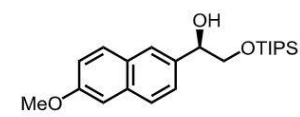

3av
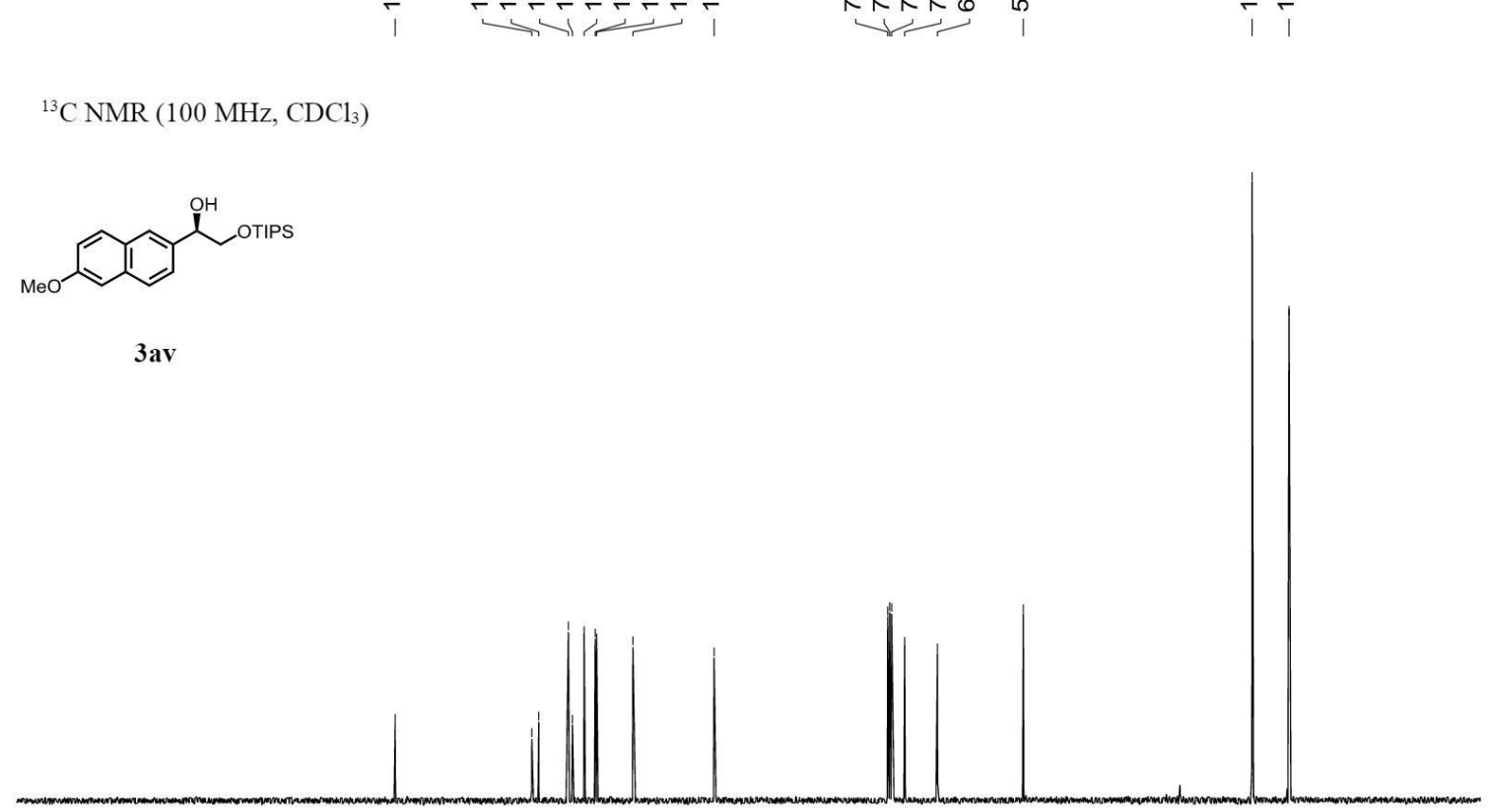

$\begin{array}{lllllllllllllllllllllll}210 & 200 & 190 & 180 & 170 & 160 & 150 & 140 & 130 & 120 & 110 & 100 & 90 & 80 & 70 & 60 & 50 & 40 & 30 & 20 & 10 & 0 & -10\end{array}$ 


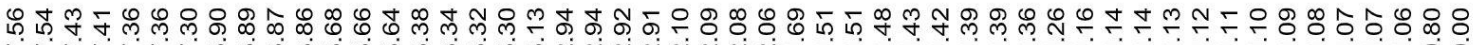

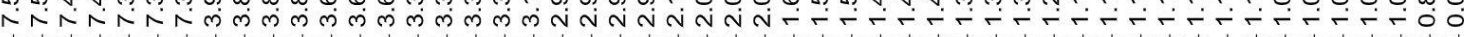

${ }^{1} \mathrm{H}$ NMR $\left(400 \mathrm{MHz}, \mathrm{CDCl}_{3}\right)$

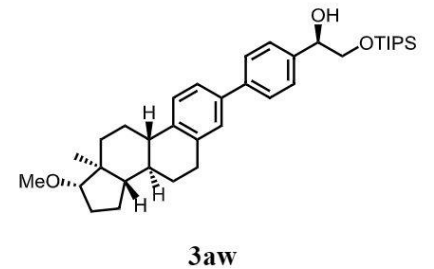

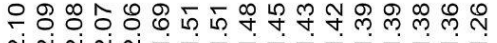

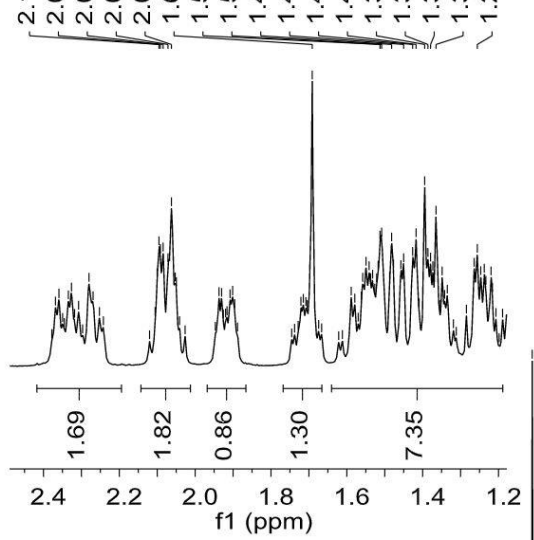

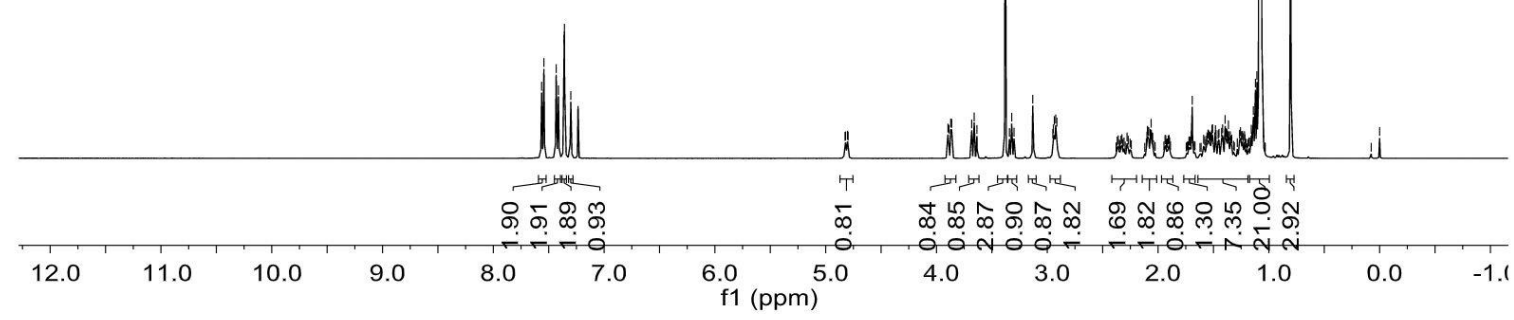

t.5. 守

\&

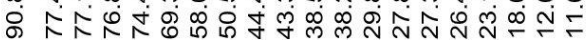
d

$\left.{ }^{13} \mathrm{C} \mathrm{NMR} \mathrm{(100} \mathrm{MHz,} \mathrm{CDCl}_{3}\right)$

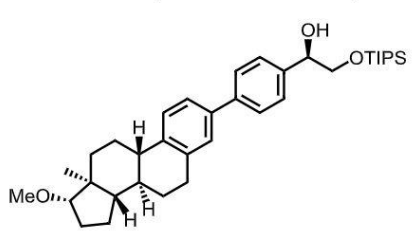

3aw
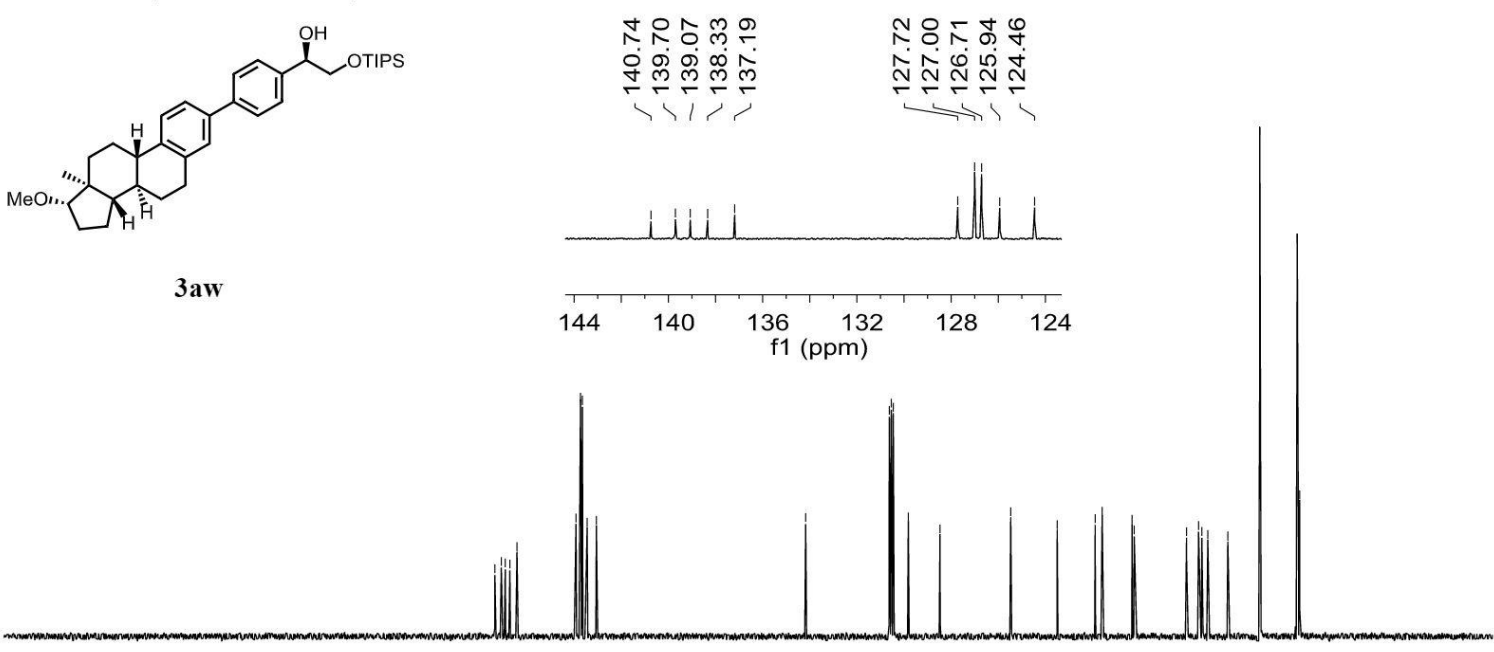
$\begin{array}{llllllllllll}210 & 200 & 190 & 180 & 170 & 160 & 150 & 140 & 130 & 120 & 110 \quad \begin{array}{l}100 \\ \mathrm{f} 1\end{array}(\mathrm{ppm})\end{array}$ 


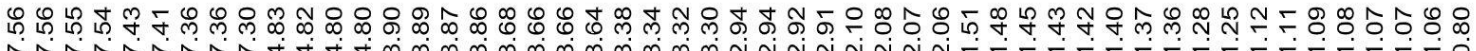

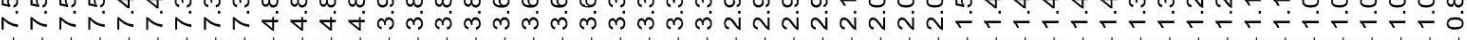

${ }^{1} \mathrm{H}$ NMR $\left(400 \mathrm{MHz}, \mathrm{CDCl}_{3}\right)$

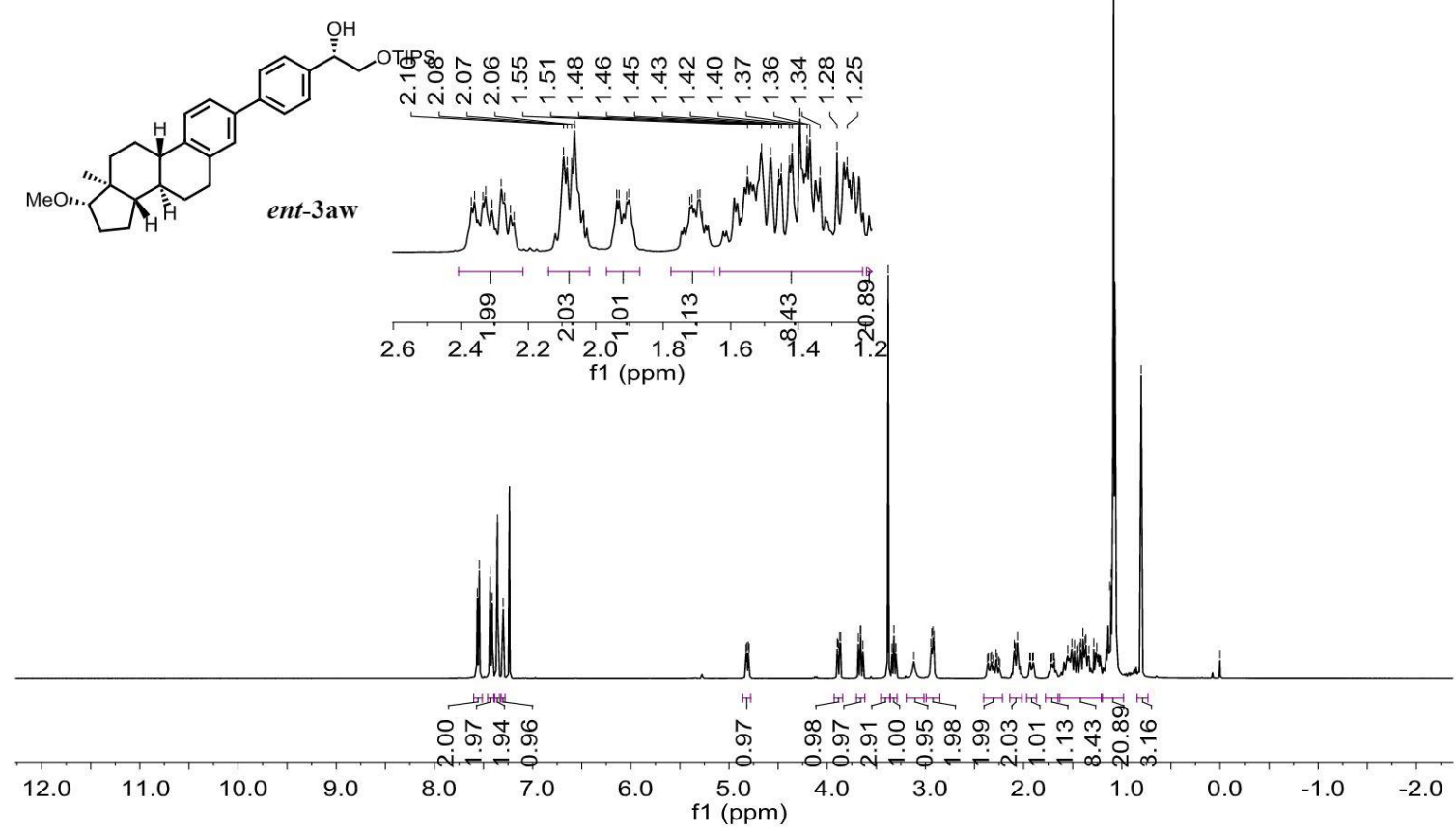

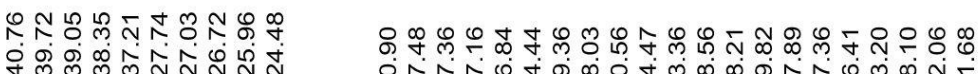

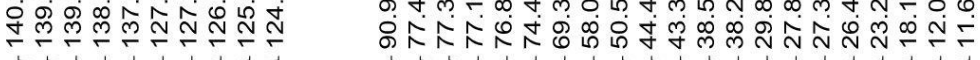

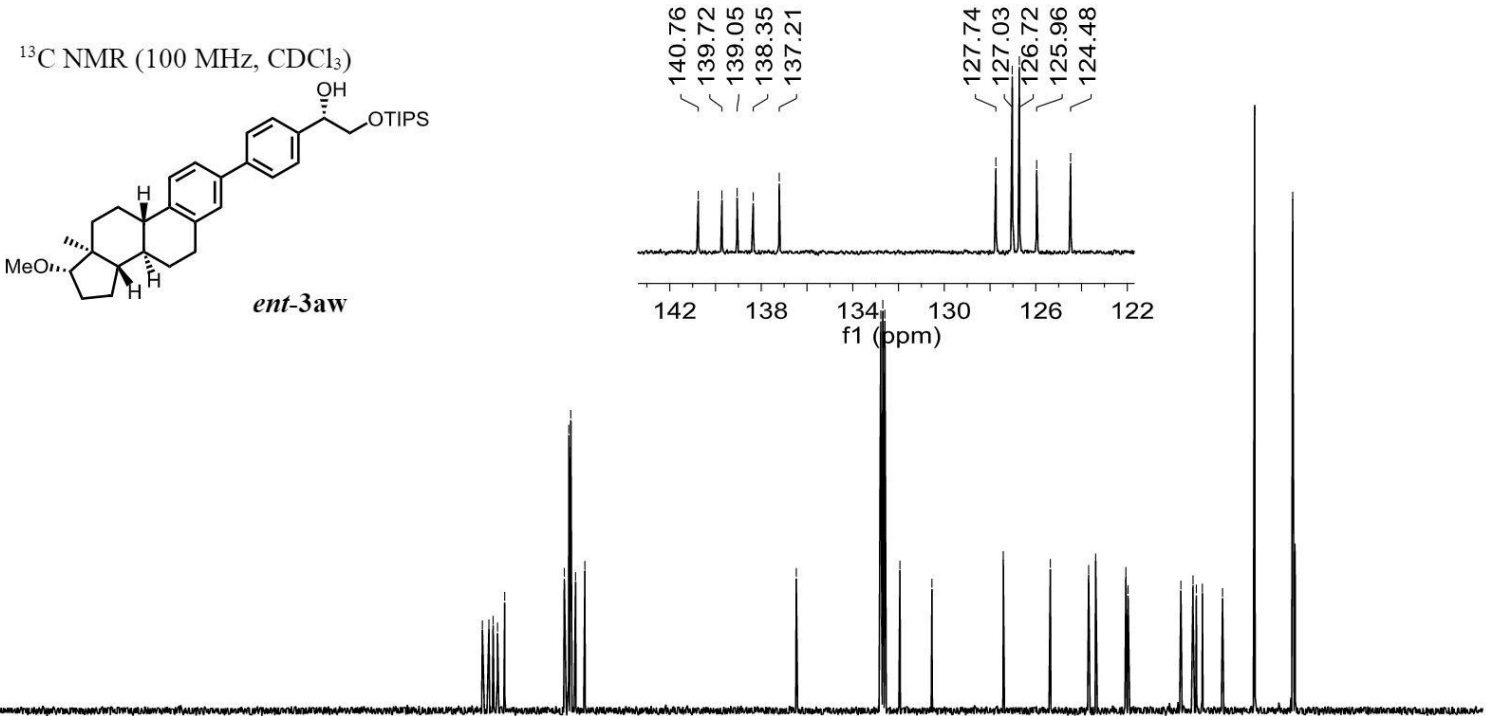

$\begin{array}{lllllllllllllllllllllll}210 & 200 & 190 & 180 & 170 & 160 & 150 & 140 & 130 & 120 & 110 & 100 & 90 & 80 & 70 & 60 & 50 & 40 & 30 & 20 & 10 & 0 & -10\end{array}$ 


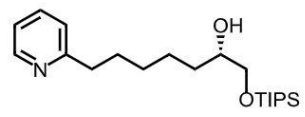

3ax
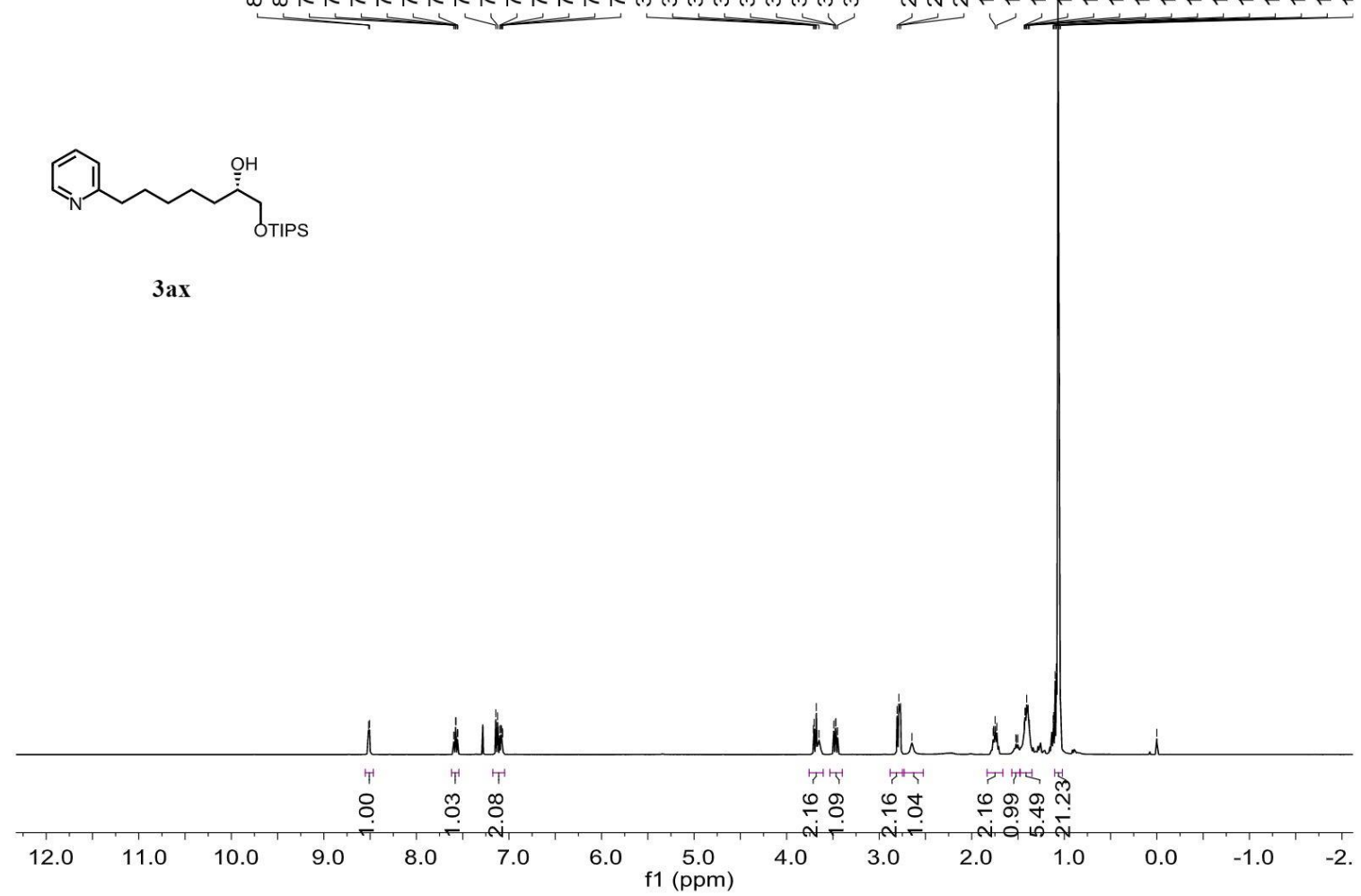

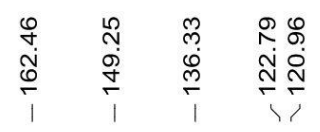

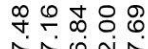

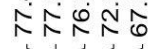

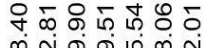

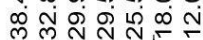

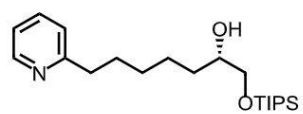

$3 a x$
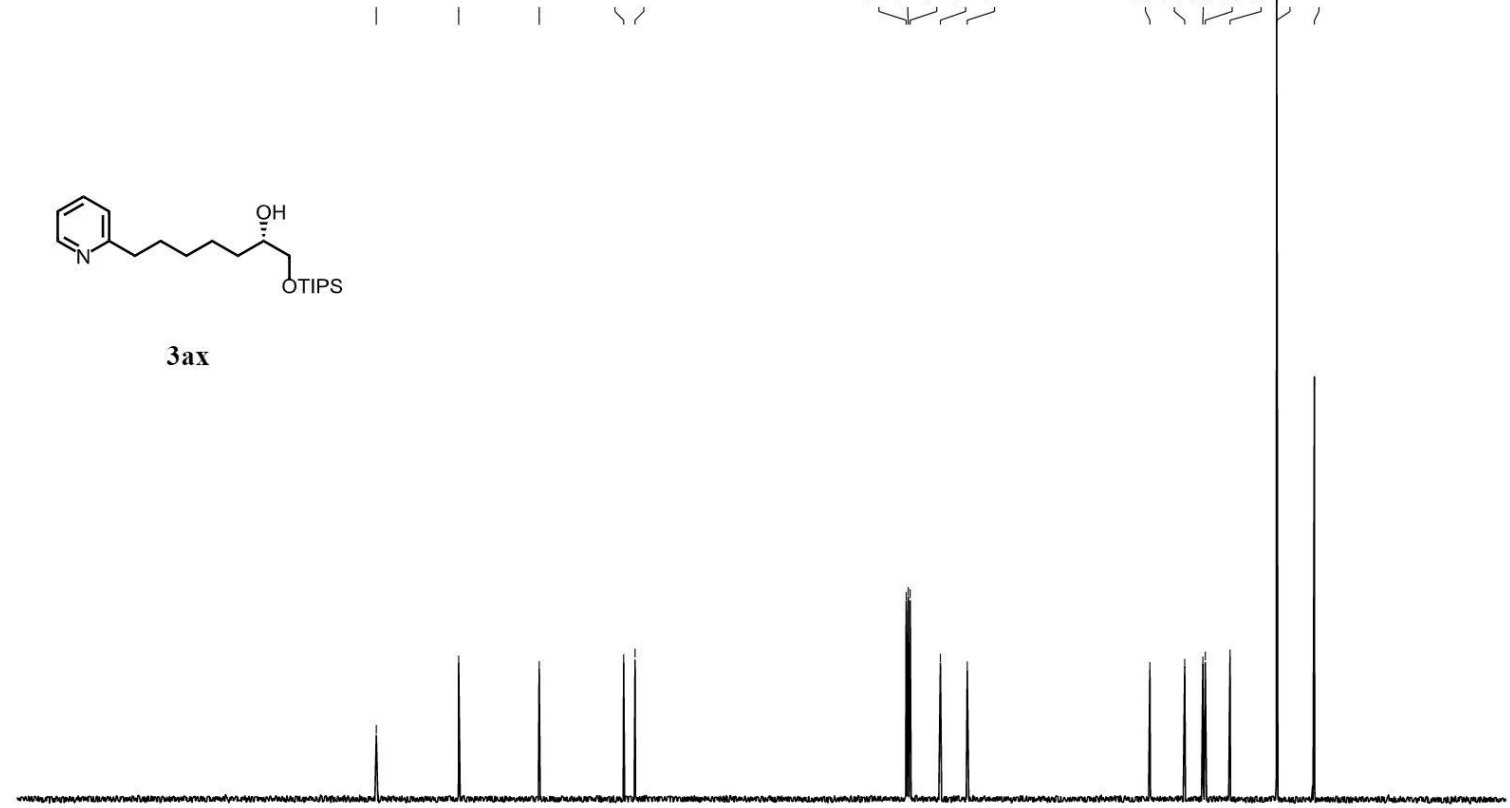

$\begin{array}{lllllllllllllllllllllllllllllll}20 & 210 & 200 & 190 & 180 & 170 & 160 & 150 & 140 & 130 & 120 & 110 & 100 & 90 & 80 & 70 & 60 & 50 & 40 & 30 & 20 & 10 & 0 & -10\end{array}$ f1 $(\mathrm{ppm})$ 


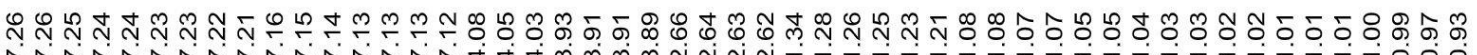

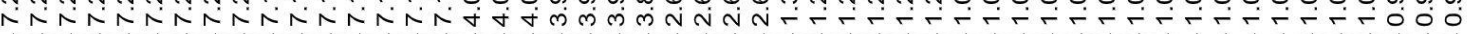

${ }^{1} \mathrm{H}$ NMR $\left(400 \mathrm{MHz}, \mathrm{CDCl}_{3}\right)$

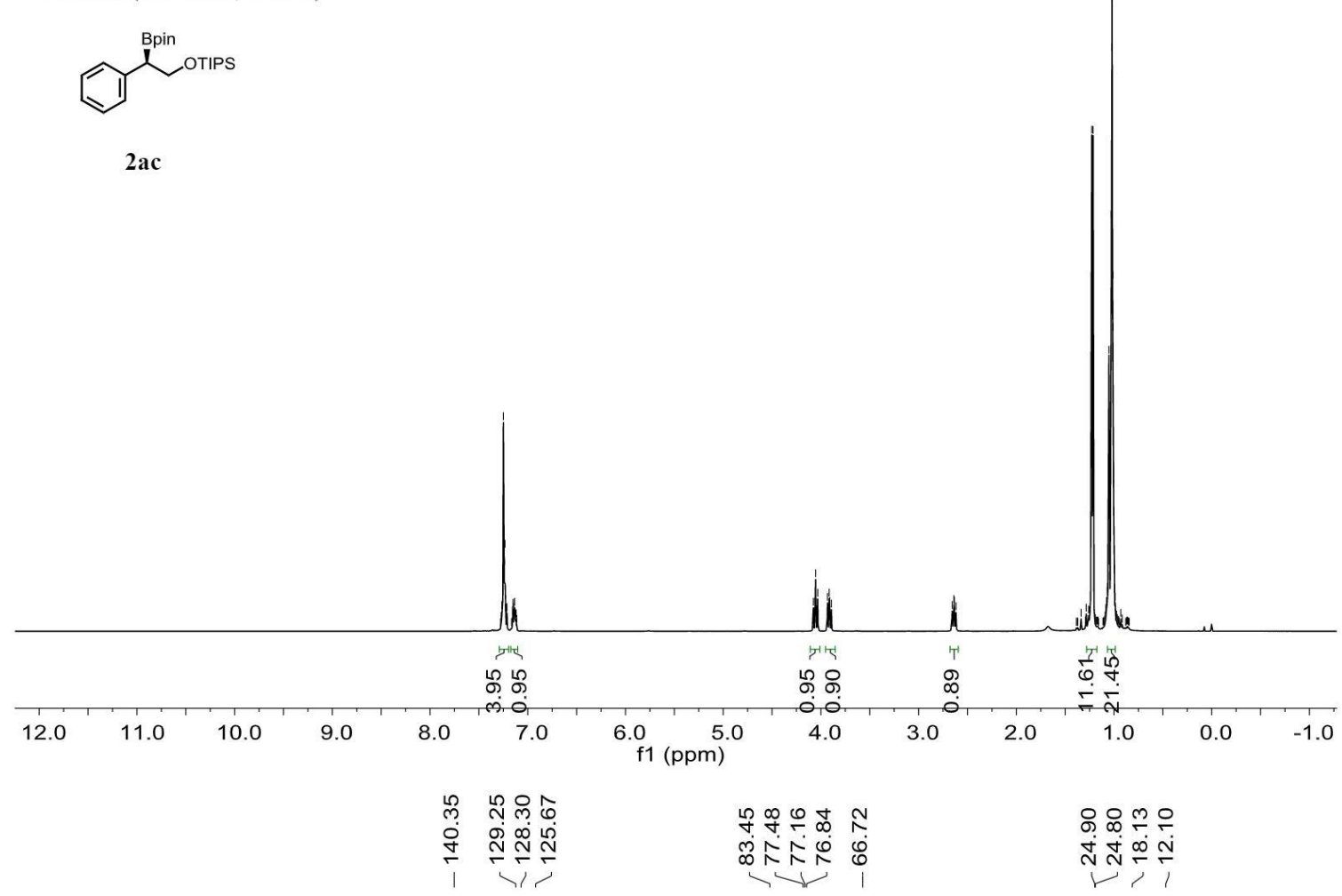

${ }^{13} \mathrm{C}$ NMR $\left(100 \mathrm{MHz}, \mathrm{CDCl}_{3}\right)$

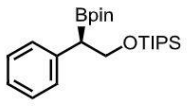

$2 a c$

miNo

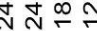

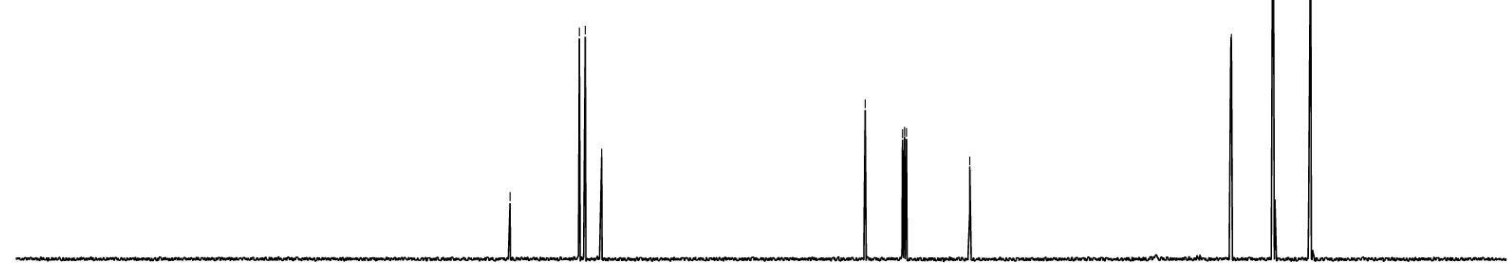

$\begin{array}{lllllllllllllllllllllll}210 & 200 & 190 & 180 & 170 & 160 & 150 & 140 & 130 & 120 & 110 & 100 & 90 & 80 & 70 & 60 & 50 & 40 & 30 & 20 & 10 & 0 & -10\end{array}$ 


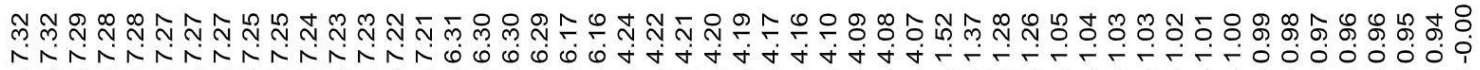

${ }^{1} \mathrm{H}$ NMR (400 MHz, $\mathrm{CDCl}_{3}$ )<smiles>OCC(c1ccccc1)c1ccco1</smiles>

5

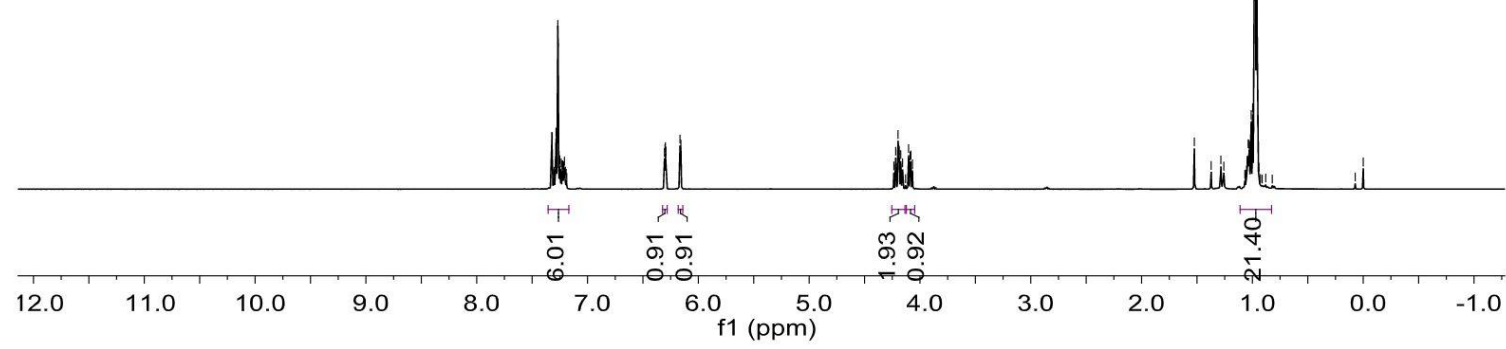

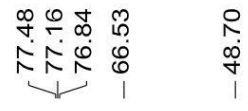

$\stackrel{\substack{0 \\ \infty}}{\substack{\infty \\ \infty}}$

잉으

$\stackrel{\infty}{\stackrel{1}{\leftarrow}}$

${ }^{13} \mathrm{C}$ NMR $\left(100 \mathrm{MHz}, \mathrm{CDCl}_{3}\right)$<smiles>OCC(c1ccccc1)c1ccco1</smiles>

5

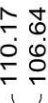

\'

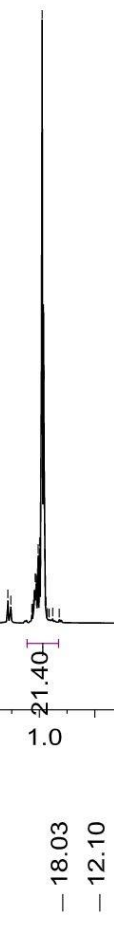

\section{$\frac{i}{\text { i }}$}

$\frac{i}{\stackrel{2}{\circ}}$




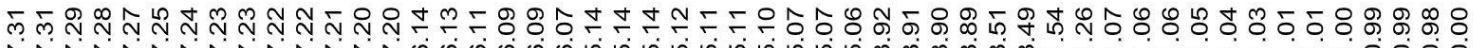
ง

${ }^{1} \mathrm{H}$ NMR $\left(400 \mathrm{MHz}, \mathrm{CDCl}_{3}\right)$<smiles>C=C[C@@H](CO[Na])c1ccccc1</smiles>

6

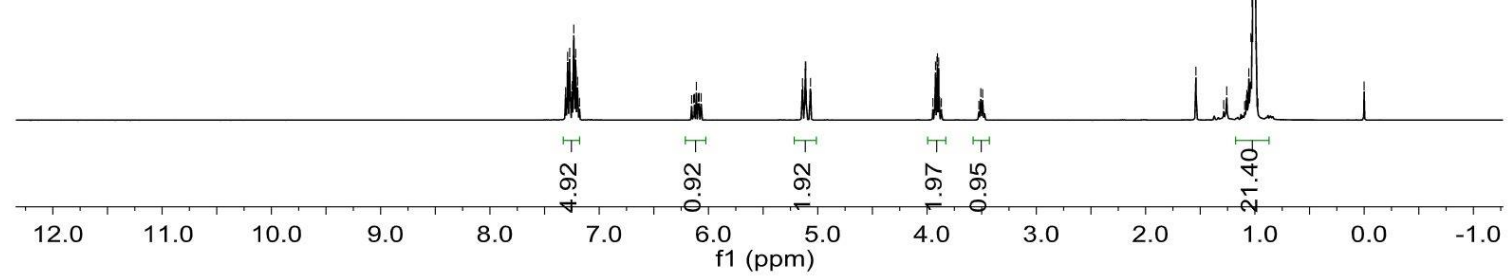

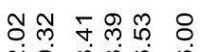

岁

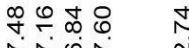

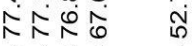

$\stackrel{i}{\stackrel{4}{4}}$

i

舟

$\stackrel{1}{+}$

${ }^{13} \mathrm{C} \mathrm{NMR}\left(100 \mathrm{MHz}, \mathrm{CDCl}_{3}\right)$<smiles>C=C[C@@H](CO[PbH3])c1ccccc1</smiles>

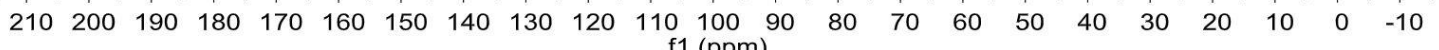




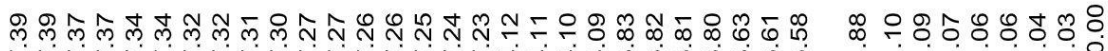

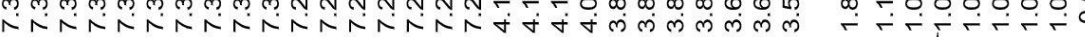

${ }^{1} \mathrm{H} \mathrm{NMR}\left(400 \mathrm{MHz}, \mathrm{CDCl}_{3}\right)$<smiles>NC(CO[Na])c1ccccc1</smiles>

7
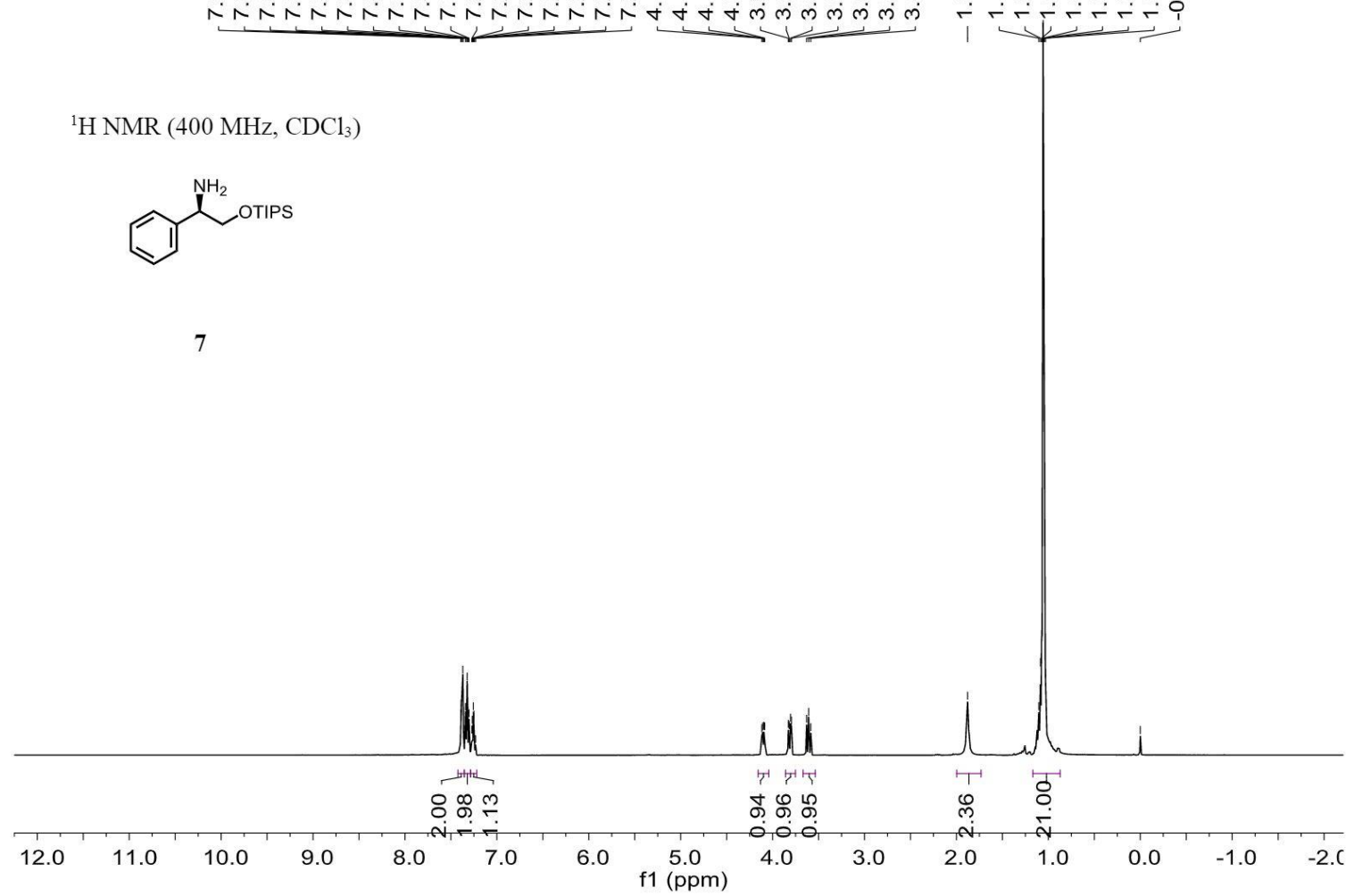

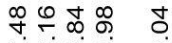

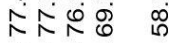

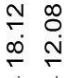

${ }^{1} \mathrm{H} \mathrm{NMR}\left(400 \mathrm{MHz}, \mathrm{CDCl}_{3}\right)$<smiles>NC(CO[AlH2])c1ccccc1</smiles>

7

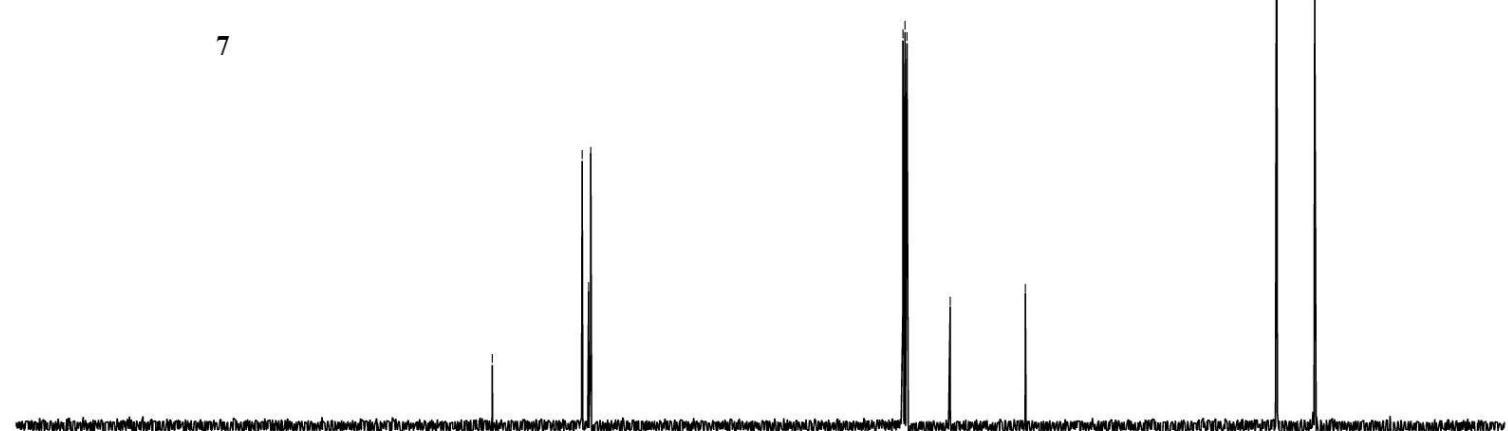

$\begin{array}{lllllllllllllllllllllll}210 & 200 & 190 & 180 & 170 & 160 & 150 & 140 & 130 & 120 & 110 & 100 & 90 & 80 & 70 & 60 & 50 & 40 & 30 & 20 & 10 & 0 & -10\end{array}$ 
${ }^{1} \mathrm{H}$ NMR $\left(400 \mathrm{MHz}, \mathrm{CDCl}_{3}\right)$

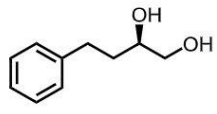

8

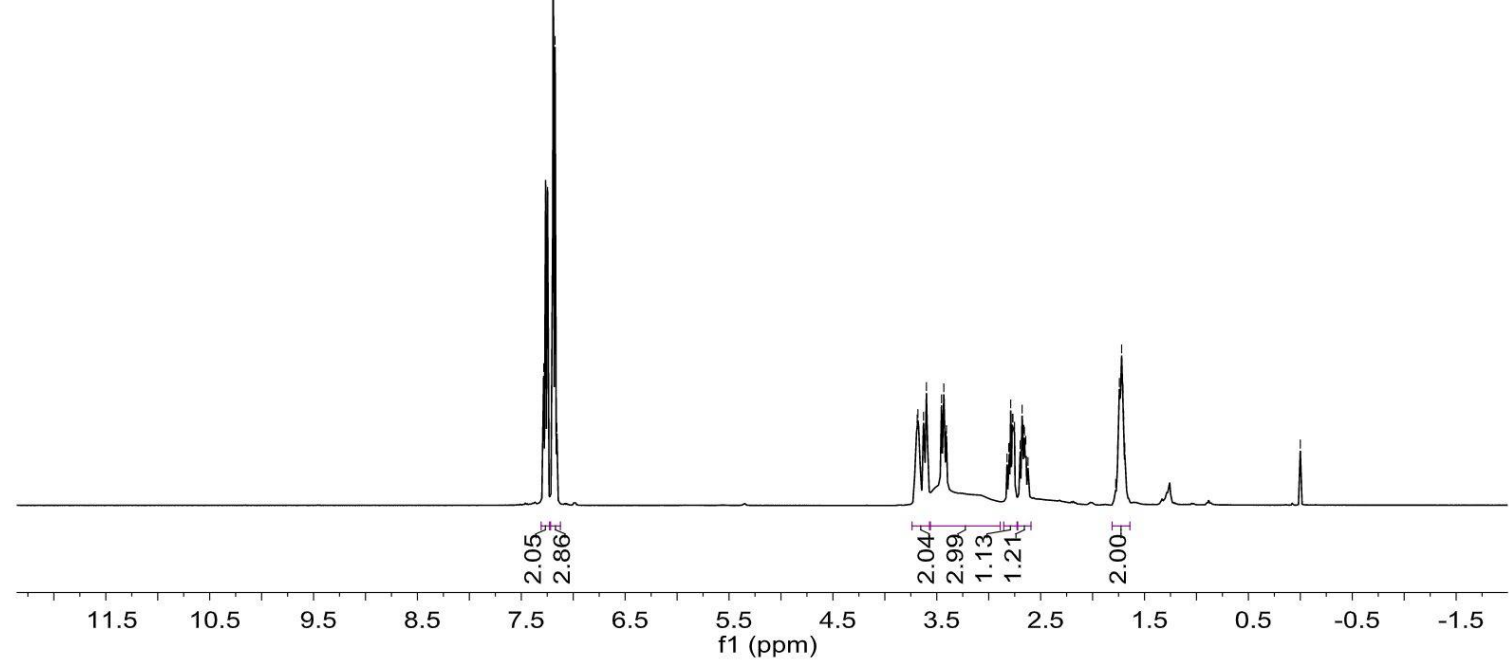

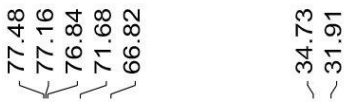

कृ

${ }^{13} \mathrm{C} \mathrm{NMR}\left(100 \mathrm{MHz}, \mathrm{CDCl}_{3}\right)$

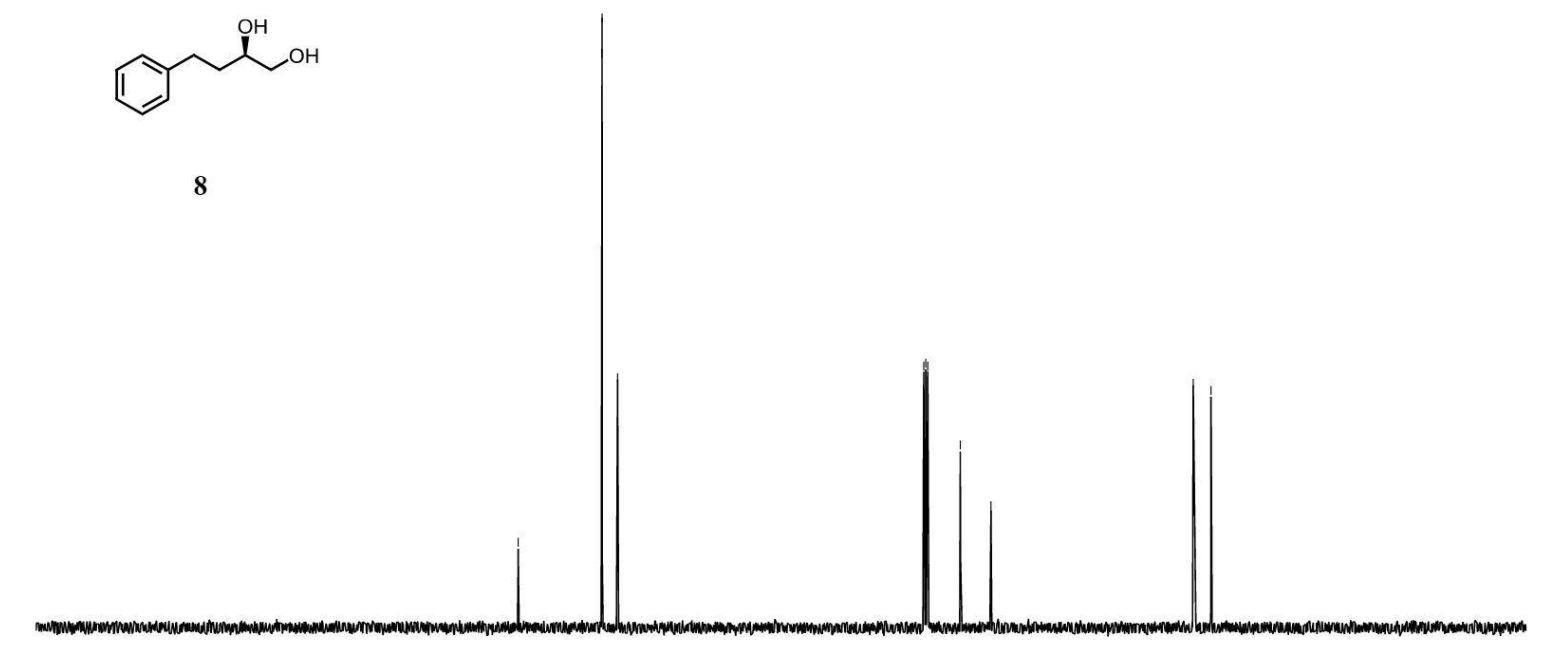

8

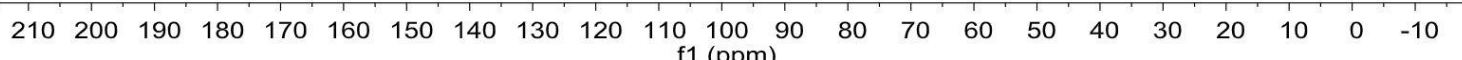


${ }^{1} \mathrm{H}$ NMR $\left(400 \mathrm{MHz}, \mathrm{CDCl}_{3}\right)$

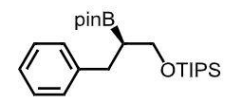

$2 \mathbf{k}$

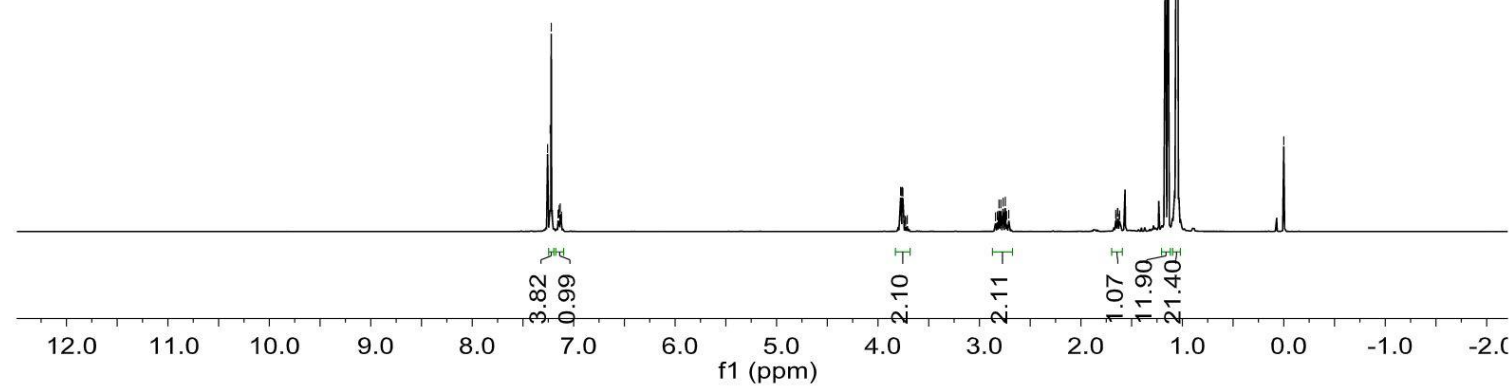

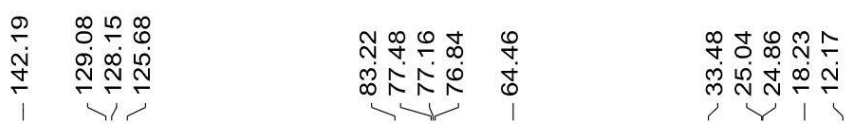

${ }^{13} \mathrm{C} \mathrm{NMR}\left(100 \mathrm{MHz}, \mathrm{CDCl}_{3}\right)$

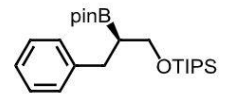

$2 \mathbf{k}$

$\begin{array}{llllllllllllllllllllll}210 & 200 & 190 & 180 & 170 & 160 & 150 & 140 & 130 & 120 & \begin{array}{c}110 \\ \mathrm{f} 1\end{array} \underset{(\mathrm{ppm})}{100} & 90 & 80 & 70 & 60 & 50 & 40 & 30 & 20 & 10 & 0 & -10\end{array}$ 
${ }^{1} \mathrm{H}$ NMR $\left(400 \mathrm{MHz}, \mathrm{CDCl}_{3}\right)$

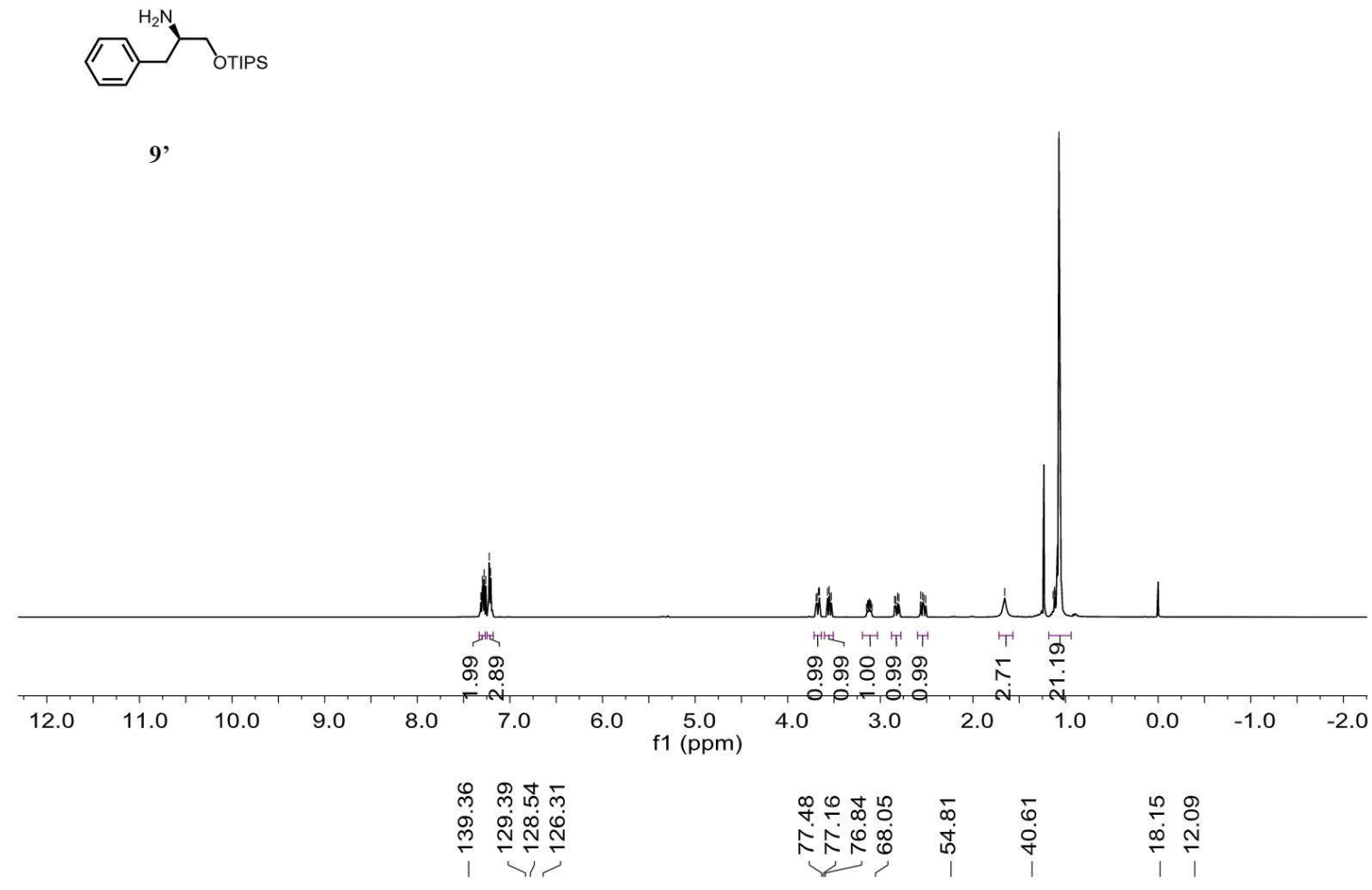

${ }^{13} \mathrm{C}$ NMR (100 MHz, $\left.\mathrm{CDCl}_{3}\right)$

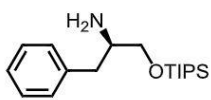

9'

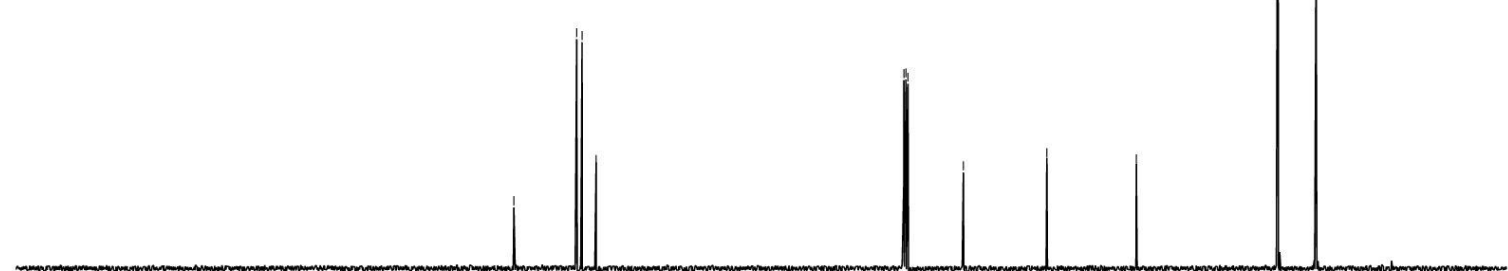

$\begin{array}{lllllllllllllllllllllll}210 & 200 & 190 & 180 & 170 & 160 & 150 & 140 & 130 & 120 & 110 & 100 & 90 & 80 & 70 & 60 & 50 & 40 & 30 & 20 & 10 & 0 & -10\end{array}$ 
Mำ ㅅNN N N N N N N

${ }^{1} \mathrm{H}$ NMR $\left(400 \mathrm{MHz}, \mathrm{CDCl}_{3}\right)$

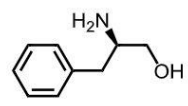

9 ,

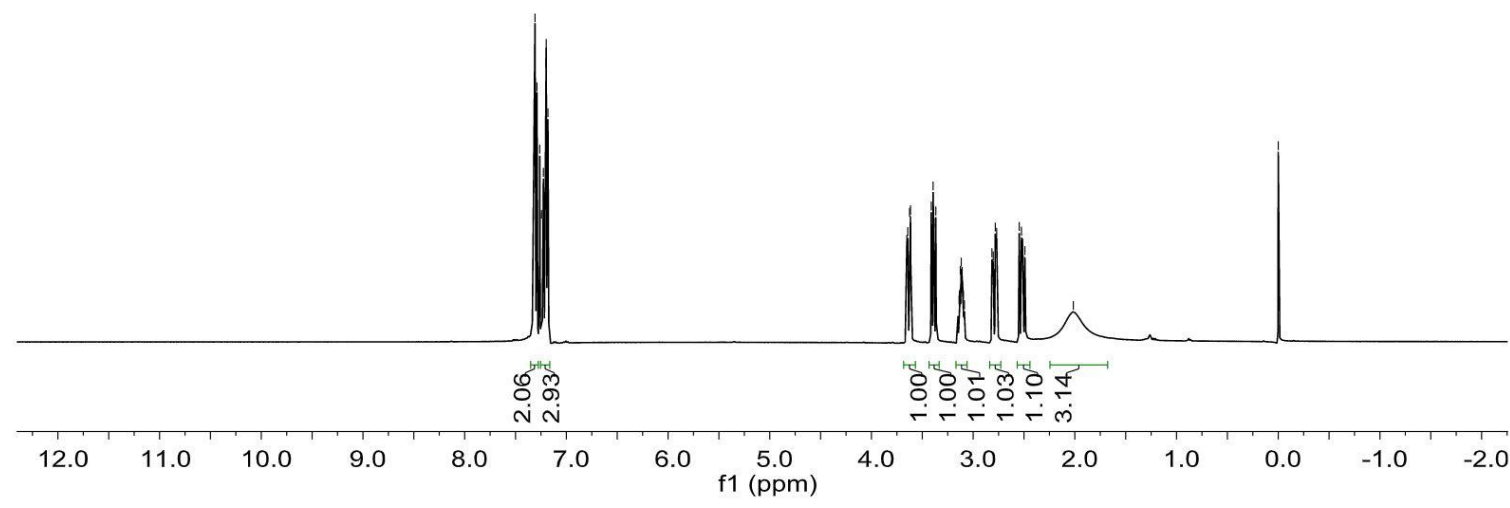

d

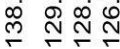

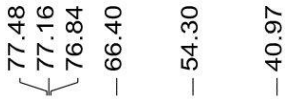

${ }^{13} \mathrm{C} \mathrm{NMR}\left(100 \mathrm{MHz}, \mathrm{CDCl}_{3}\right)$<smiles>N[C@@H](CO)Cc1ccccc1</smiles>

9"9

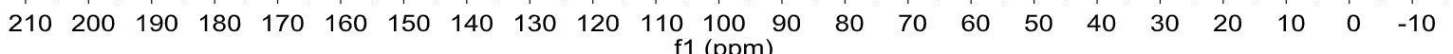


${ }^{1} \mathrm{H}$ NMR $\left(400 \mathrm{MHz}, \mathrm{CDCl}_{3}\right)$

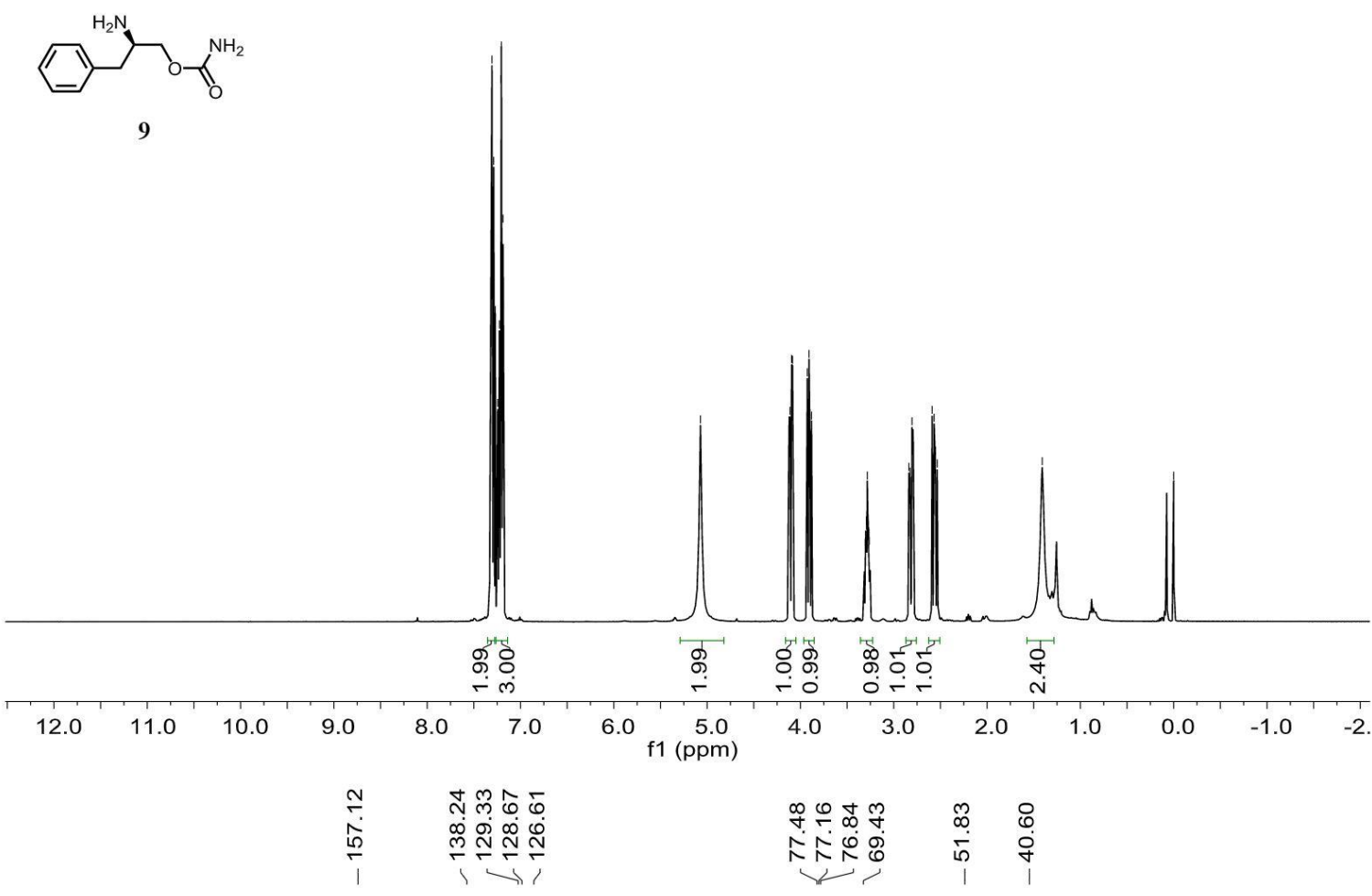

${ }^{13} \mathrm{C}$ NMR (100 MHz, $\mathrm{CDCl}_{3}$ )

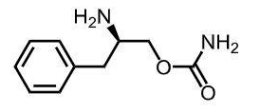

9

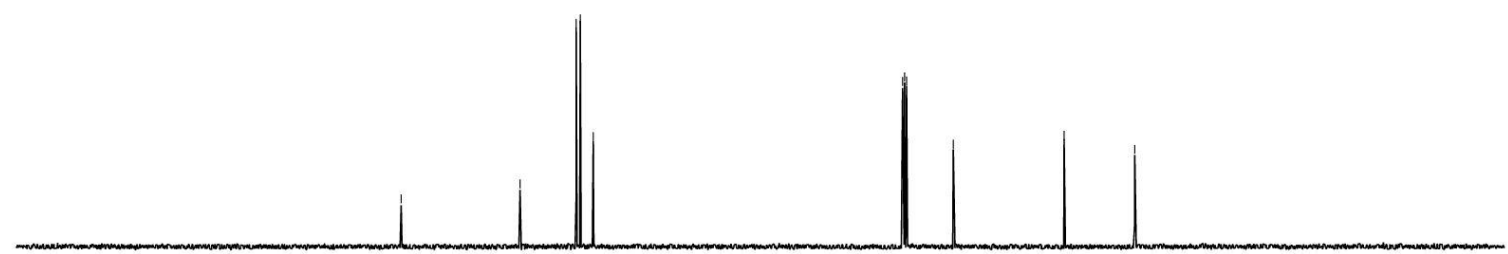

$\begin{array}{llllllllllllllllllllllll}210 & 200 & 190 & 180 & 170 & 160 & 150 & 140 & 130 & 120 & \begin{array}{c}110 \\ \mathrm{f} 1(\mathrm{ppm})\end{array} & \mathbf{1 0 0} & 80 & 70 & 60 & 50 & 40 & 30 & 20 & 10 & 0 & -10\end{array}$ 